\title{
The Impact of Global Climate Change on Tropical Forest Biodiversity in Amazonia
}

\author{
Lera Jane Miles \\ Submitted in accordance with the requirements for the degree of $\mathrm{PhD}$ \\ University of Leeds \\ Centre for Biodiversity and Conservation \\ School of Geography \\ January 2002
}

The candidate confirms that the work submitted is her own and that appropriate credit has been given where reference has been made to the work of others. 


\section{ACKNOWLEDGEMENTS}

The first thanks must go to the National Environment Research Council, who were kind enough to fund the first three years of this project. My supervisors Oliver Phillips and Alan Grainger deserve the strongest possible thanks for all their ideas, feedback and support. Thanks too to Steve Carver, a helpful member of the supervisory team in the first phase of the project. I am grateful for the constructive criticism of RSG members Mike Kirkby and Steve Compton.

On the climate modelling side, Richard Betts and Peter Cox from Hadley Centre were supportive with advice, elucidation and most importantly, climate change scenario outputs. Thanks for taking my elementary questions seriously!

I owe a debt to taxonomists from all over the world, who contributed plant distribution data that was vital for this project - Andre Chanderbali at the Missouri Botanical Garden, Terry Pennington, Ghillean Prance and Vanessa Plana at the Royal Botanic Gardens, Kew and Susanne Renner at University of Missouri St. Louis all took the trouble to send data in electronic format. Paul Wilkin kindly helped me to find my way around the herbarium at Kew. In Brazil, I was helped by the staff of the beautifully run herbaria at MPEG and IAN in Belém, and INPA in Manaus. I am extremely grateful to Samuel Almeida, Regina Martins-Da-Silva and Carlos Alberto 'Cid' Ferreira for helping me access the respective herbaria and their BRAHMS databases. Thanks too to the welcoming researchers at INPA - Patricia Delamonica, who introduced me to lots of folk and organised a place to stay in Manaus; Mike Hopkins and Susan Laurance for giving me access to their respective databases; and Emilio Bruna, for letting me tag along on his visit to the $\mathrm{km}-41$ forest!

Miguel Bastos Araújo, then of the Natural History Museum, collaborated in the planning and carrying out of the comparison of the effectiveness of similarity and autologistic distribution models, as detailed in the Discussion. Paul Williams, also of the Natural History Museum, generously allowed me to use his WORLDMAP software (Williams 1998b) for data transformations and GIS.

Thanks to successive darling office-mates Sarah Benson, Dimitris Ballas, Steffen Fritz, Crewenna Dymond and Naomi van der Velden, for making work-life much more fun. It was good sharing food and many diverse beverages in room 101 !

The last months of writing-up overlapped Christmas and a New Year's break in Devon. So, final thanks to all my relatives and friends for putting up with my disappearance into the shed or top room, I look forward to fully participating in next year's festivities! 


\begin{abstract}
Species' ranges are configured according to their tolerance of environmental conditions, especially climate, and their history of dispersal since speciation. Previous studies of the potential impact of climate change on biodiversity have been biased towards species of high latitudes. This situation results from a lack of detailed knowledge about the distribution of tropical biodiversity, and from the smaller degree of warming expected at low latitudes. However, various General Circulation Models (GCMs) simulate regional drying and increasing seasonality for parts of the tropics, including Amazonia. This may have a greater impact on tropical forest flora than temperature change alone. The Amazon region holds a high proportion of global biodiversity, yet conservation plans rarely consider possible climate change impacts.
\end{abstract}

This thesis presents a methodology for projecting a set of Amazonian plant species' ranges from limited data, and estimating their response to climate change scenarios. Species are classified into plant functional types (PFTs), which share traits such as growth form and reproductive strategy. Species' current distributions are modelled over a coarse scale (a $1^{\circ}$ latitude-longitude grid), using a suitability index based on bioclimate variables. Distributions are additionally limited by species' absolute tolerances to extreme values, and by dispersal barriers. A sizestructured population is simulated for each cell, to enable modelling of lags in response to climate change.

In the standard impact scenario (SIS), future population processes are simulated over 100 years, with changes in the variables governing cell suitability being applied annually according to anomalies from a selected GCM. The run is repeated for each species using anomalies of half that magnitude, as a reduced-impact scenario (RIS).

The range of potential outcomes for each species and PFT is evaluated. Widespread impacts are seen under both scenarios. An alternative impact scenario (AIS) is devised to examine the effects of allowing some "c-species" to thrive under heightened AET. The most vulnerable taxonomic groups, PFTs and geographical regions are identified as targets for monitoring and conservation action. In particular, there is a dramatic loss of species' viability in much of northeastern Amazonia at 2095 under all scenarios. The far western part of Amazonia is identified as important for persistence of the greatest number of species. Areas falling between the major rivers of the region have very limited distribution data, so are highlighted for future biodiversity survey work. 


\section{Contents}

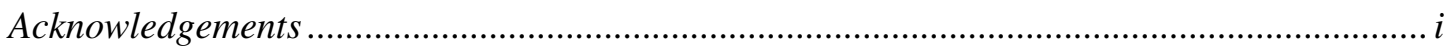

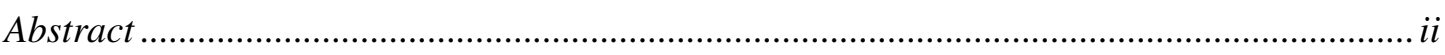

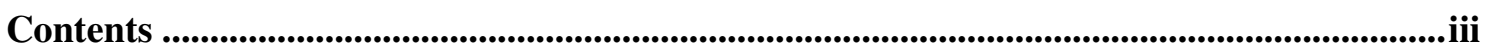

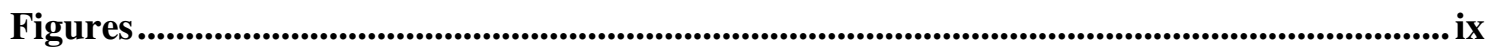

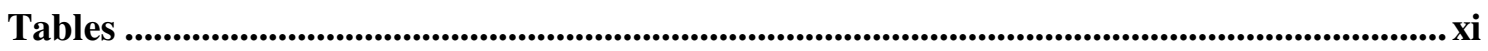

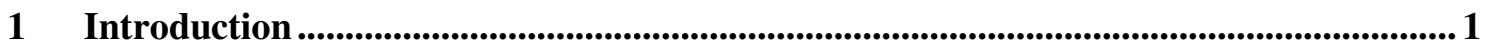

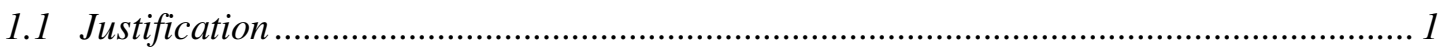

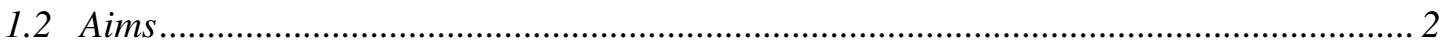

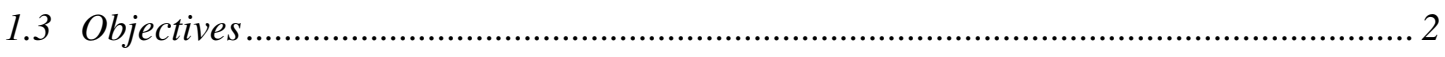

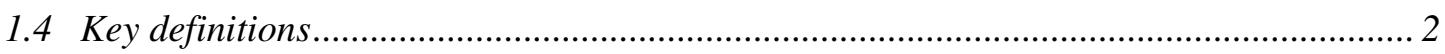

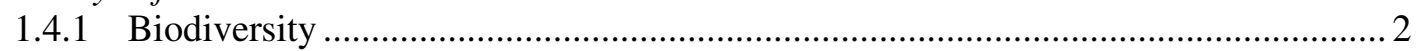

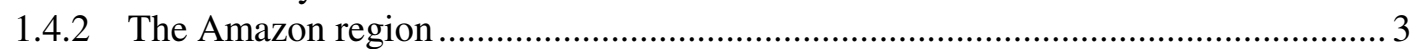

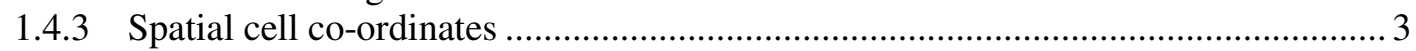

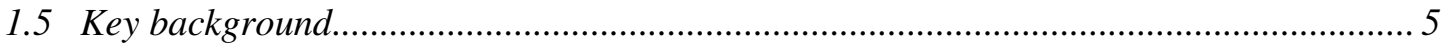

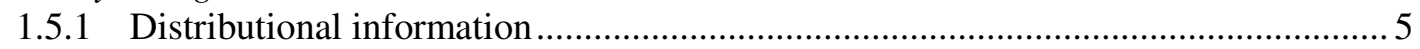

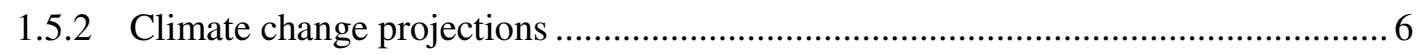

2 Geography and biodiversity of the Amazon region ......................................................

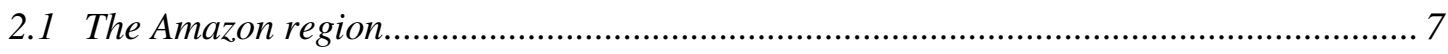

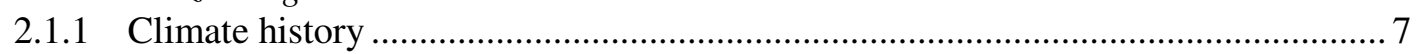

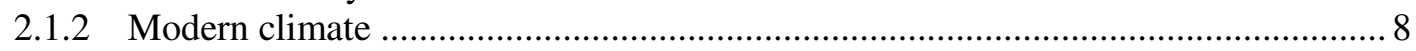

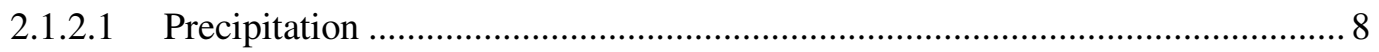

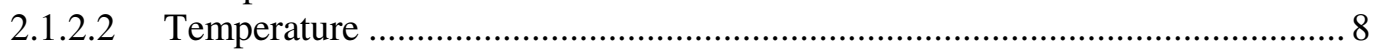

2.1.2.3 El Niño Southern Oscillation (ENSO) …….................................................. 10

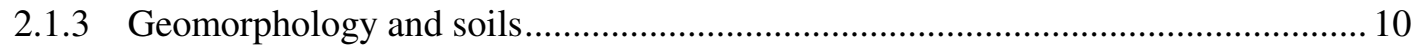

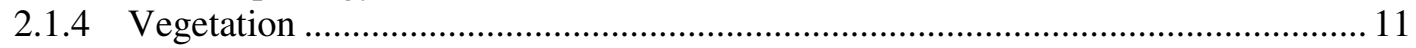

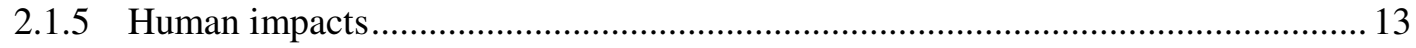

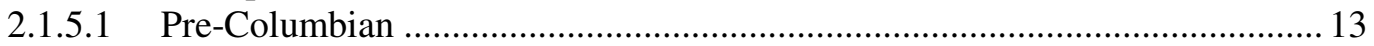

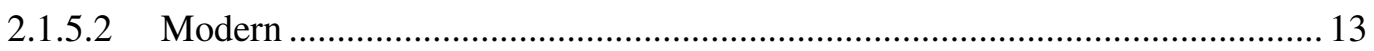

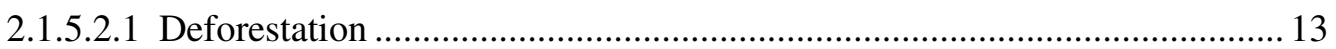

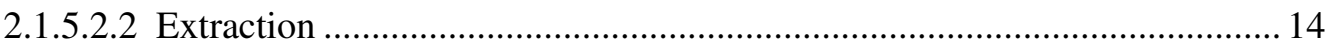

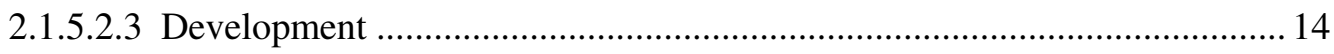

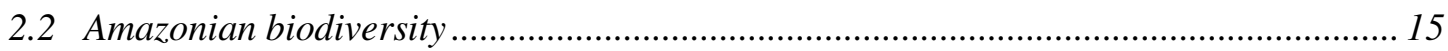

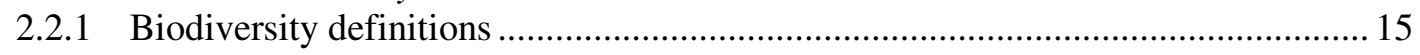

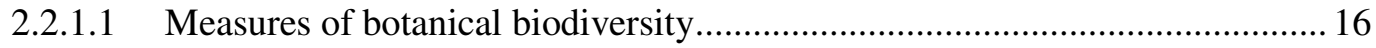

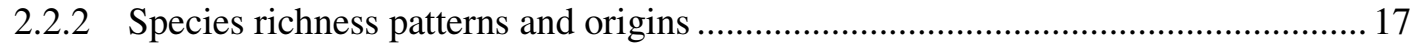

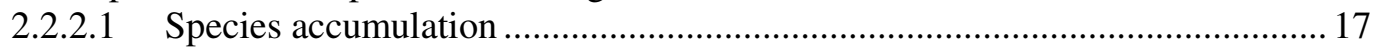

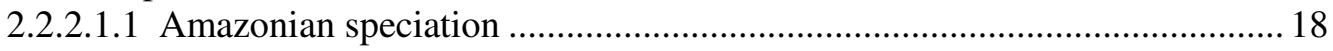

2.2.2.2 Stability and disturbance ……................................................................... 19

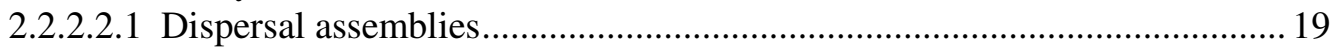

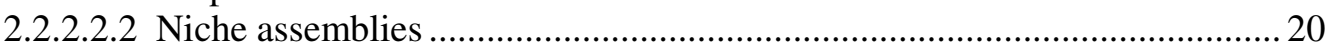

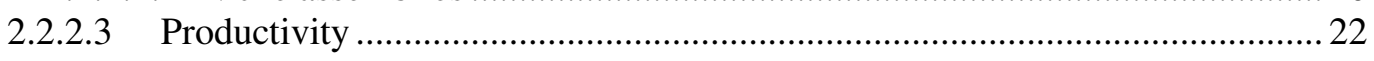

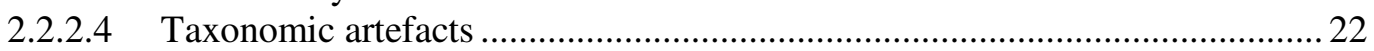




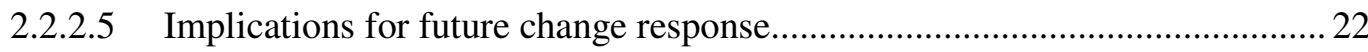

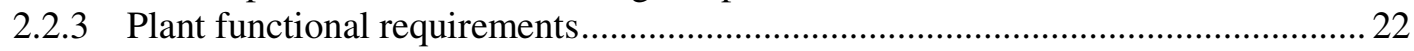

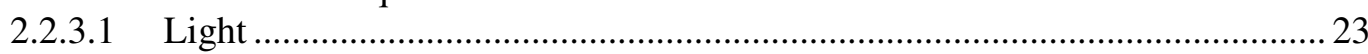

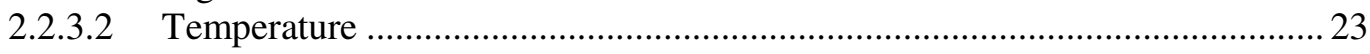

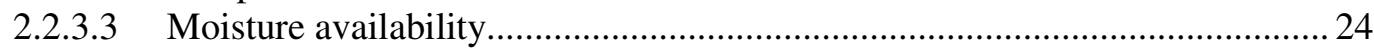

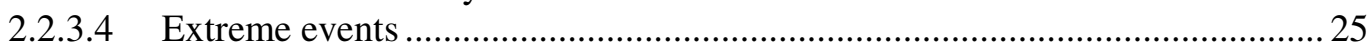

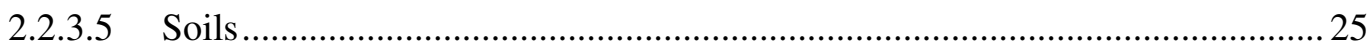

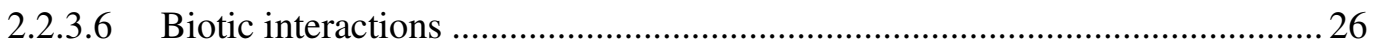

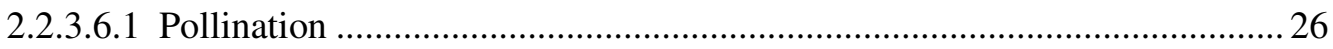

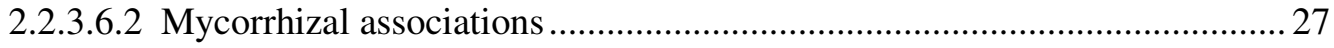

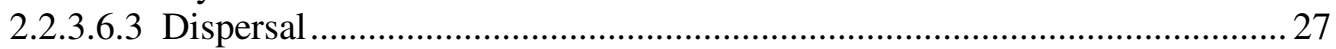

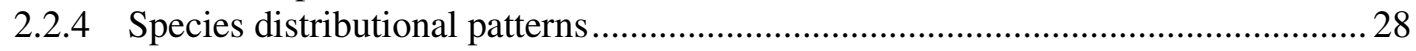

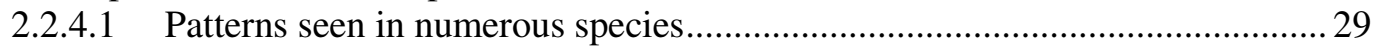

2.2.4.1.1 Common: ubiquitous distribution, high density ..........................................2 29

2.2.4.1.2 Common endemic: restricted distribution, high density............................29

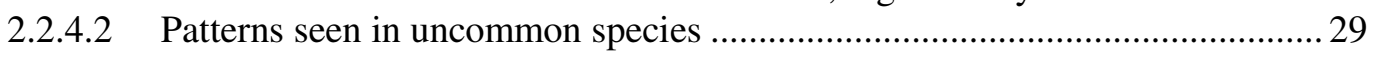

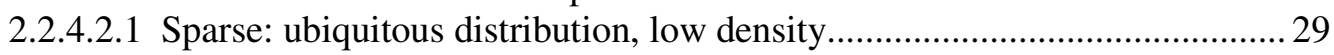

2.2.4.2.2 Rare: restricted distribution, low density................................................. 30

3 Modelling climate change

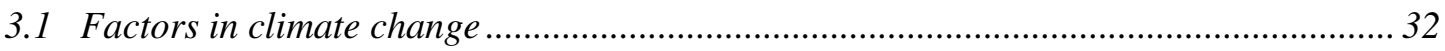

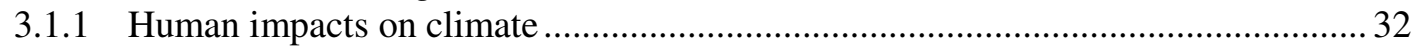

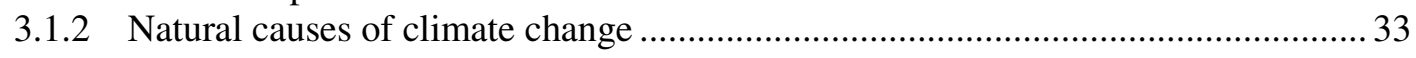

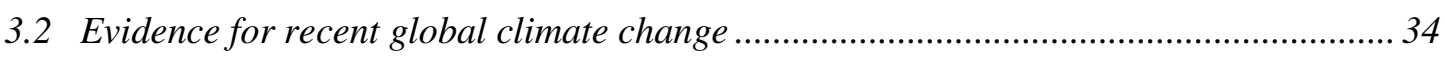

3.2.1.1.1 Climate change impacts on tropical forests .................................................. 35

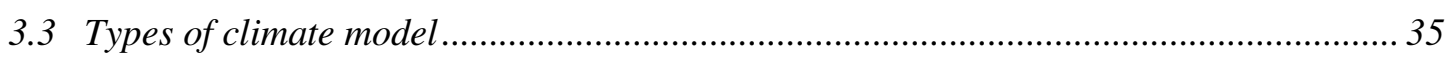

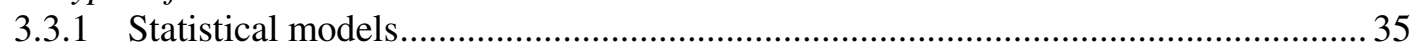

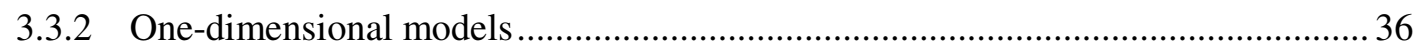

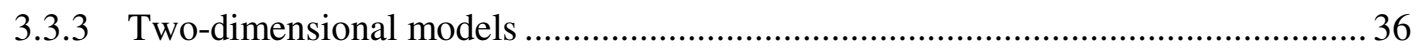

3.3.4 General Circulation Models (GCMs) ………......................................................... 36

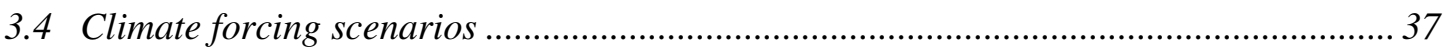

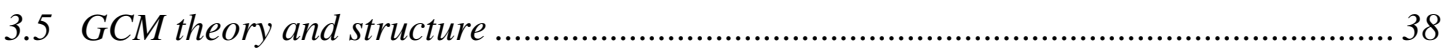

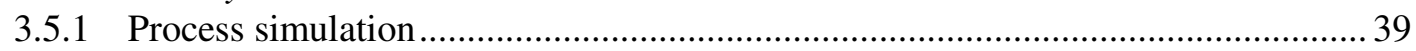

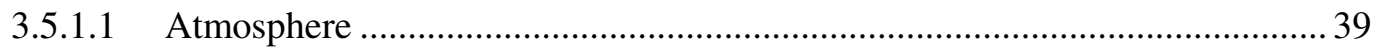

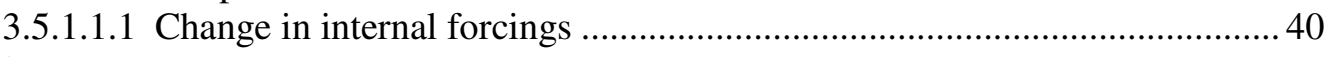

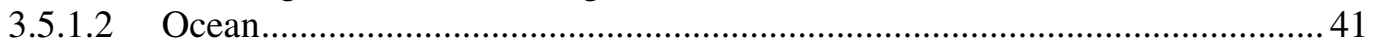

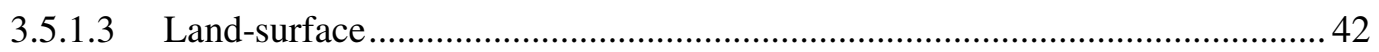

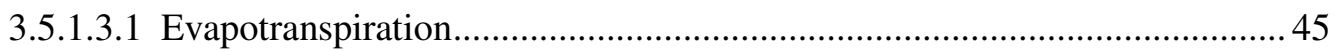

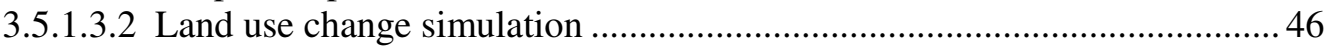

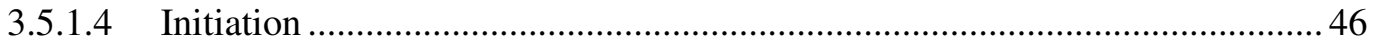

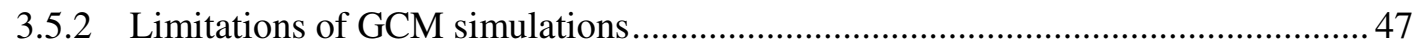

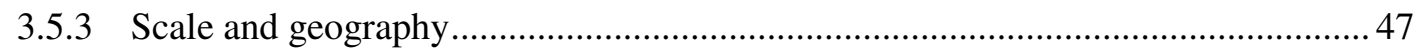

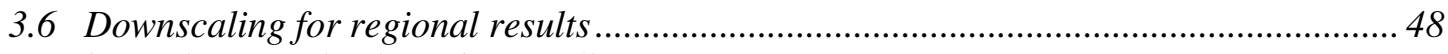

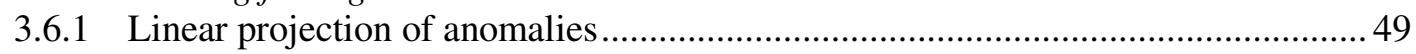

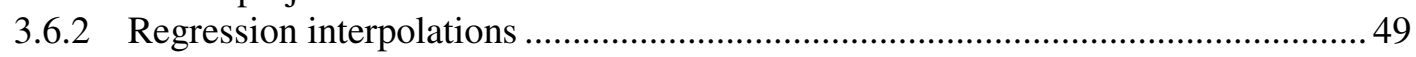

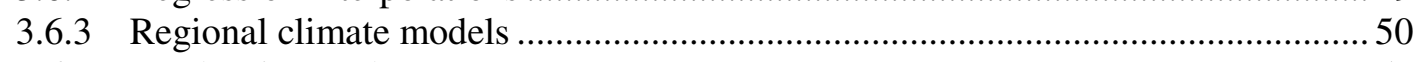

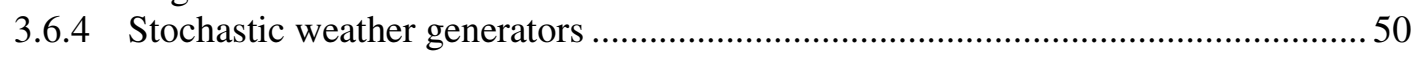

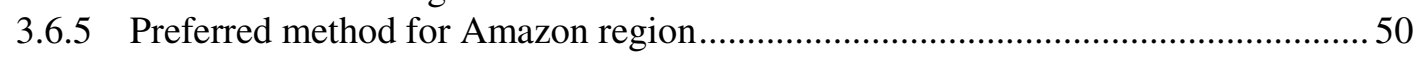

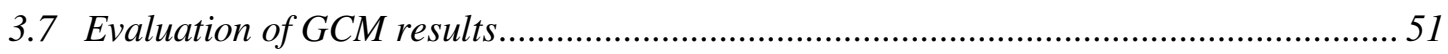

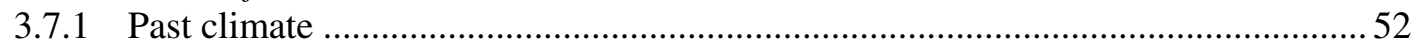

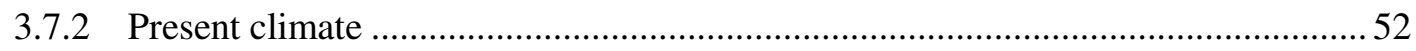

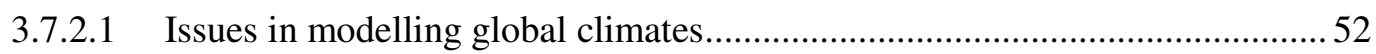

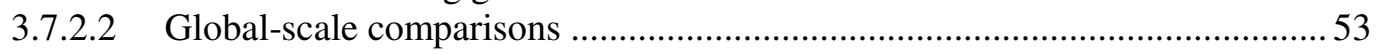




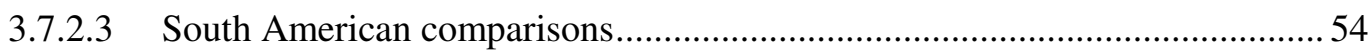

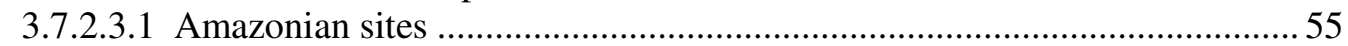

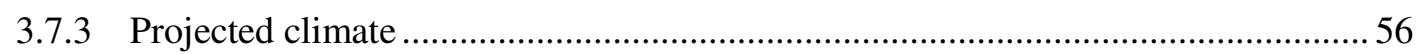

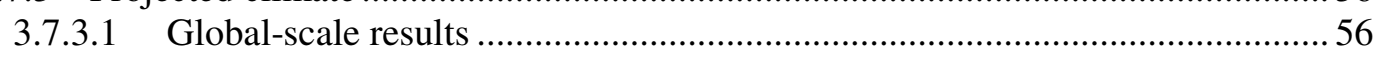

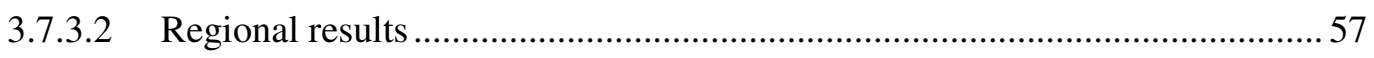

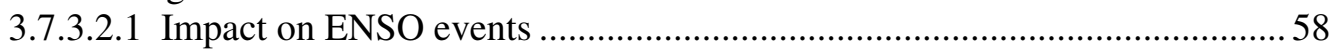

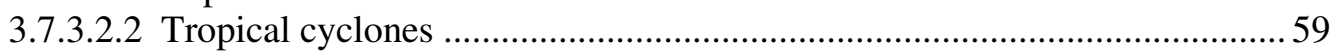

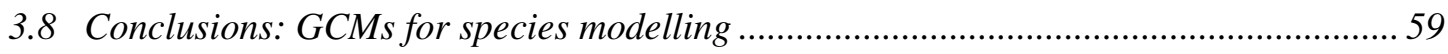

$4 \quad$ Plant response to climate .................................................................................................................. 61

4.1 Variation in climate response between species ............................................................ 61

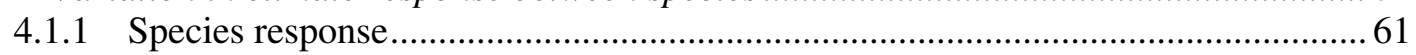

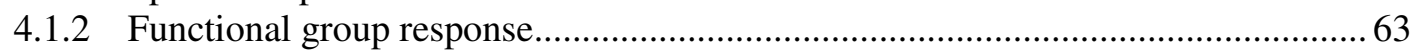

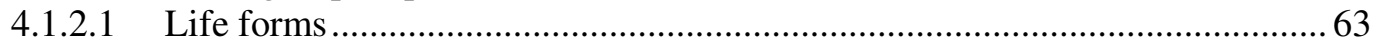

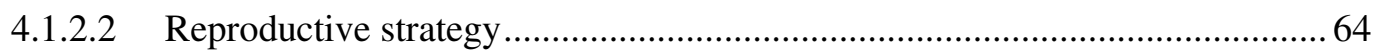

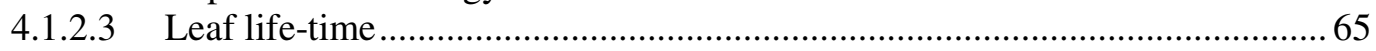

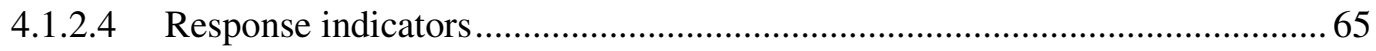

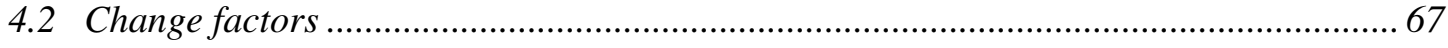

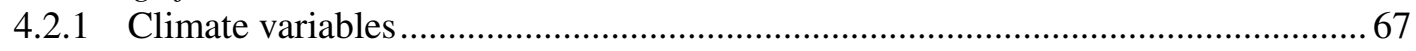

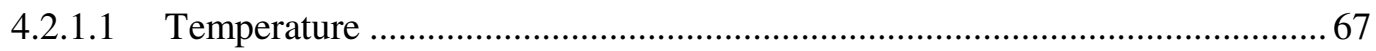

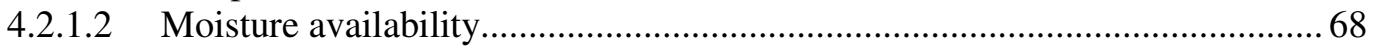

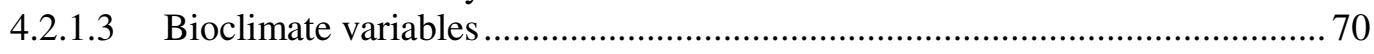

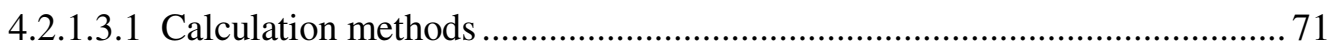

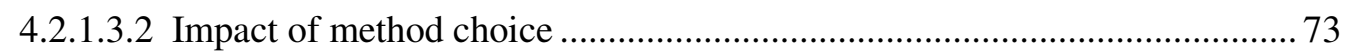

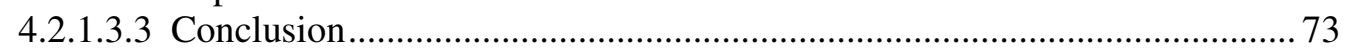

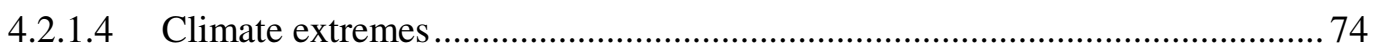

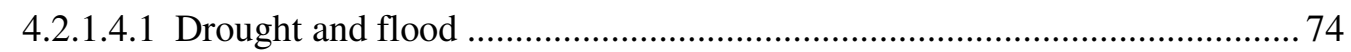

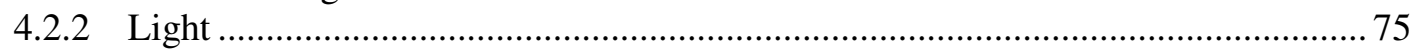

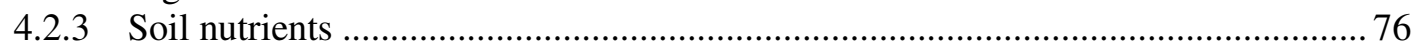

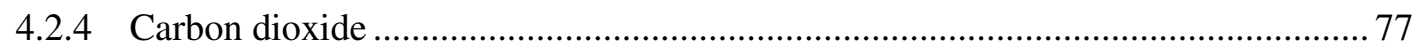

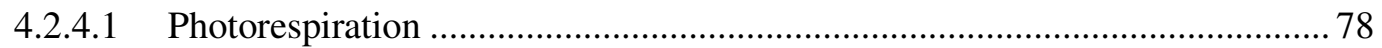

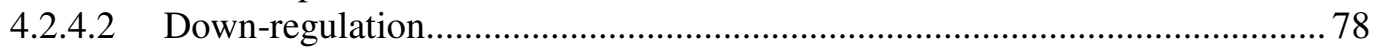

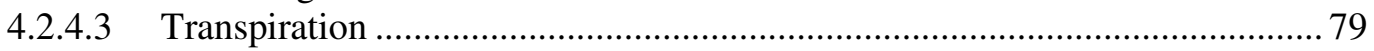

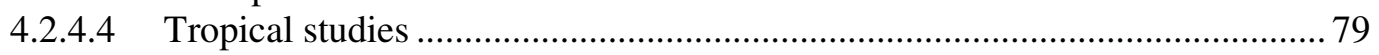

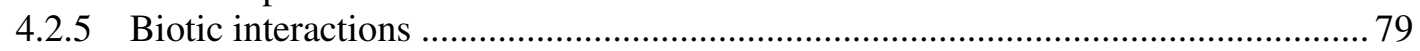

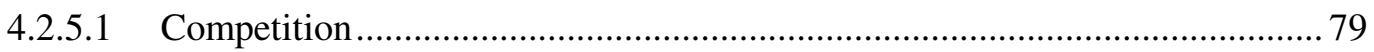

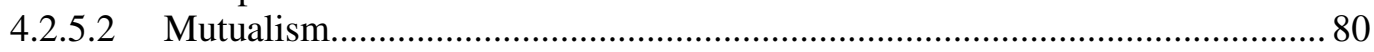

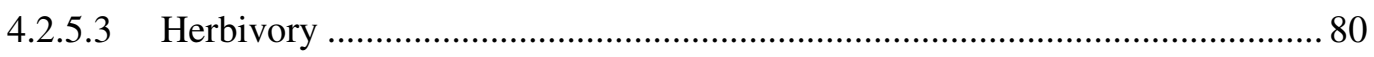

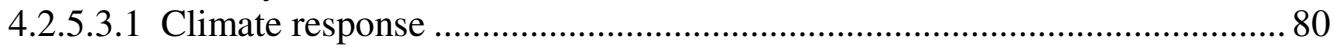

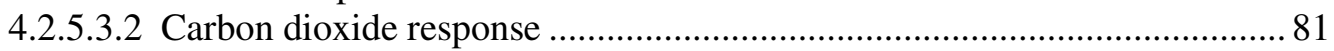

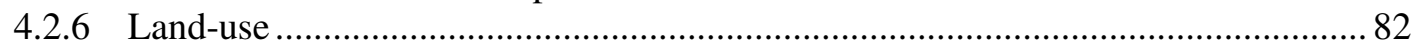

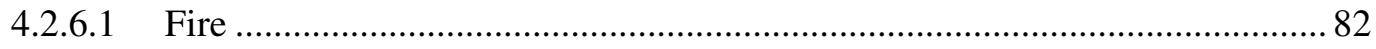

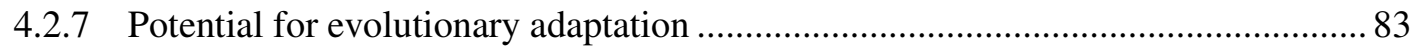

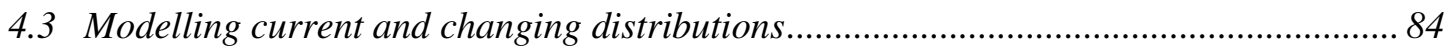

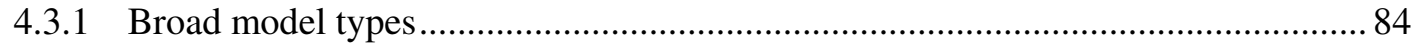

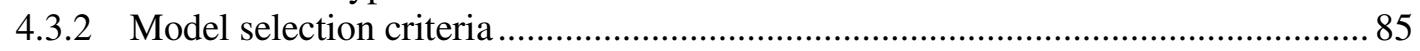

4.3.3 General form of plant-climate relationships ......................................................... 85

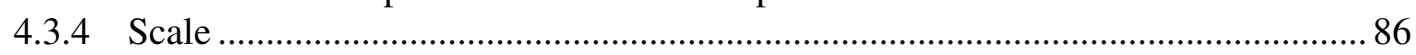

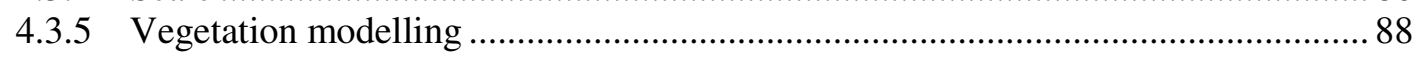

4.3.5.1 Biome models and species modelling ........................................................... 90

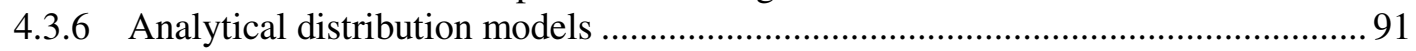

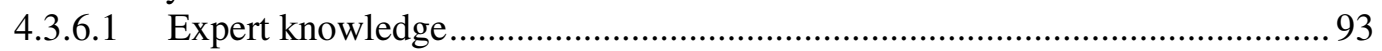

4.3.6.2 Selection of variables for pattern analysis ........................................................ 93

4.3.6.3 Pattern analysis without independent variables................................................ 94

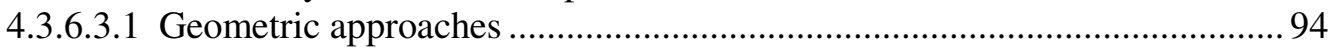




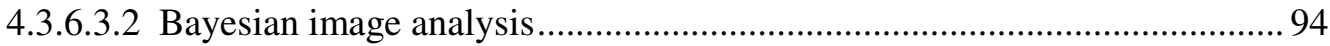

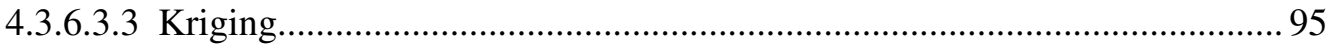

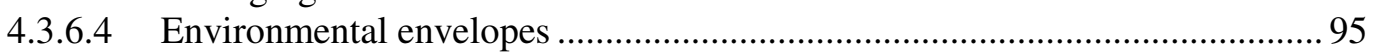

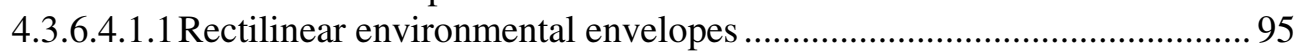

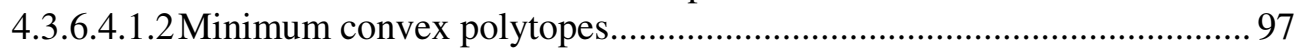

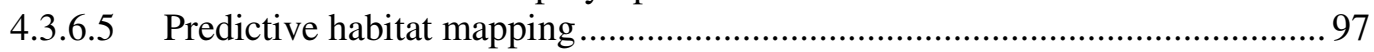

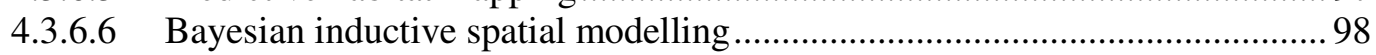

4.3.6.7 GLMs: Generalised linear (regression) models............................................. 99

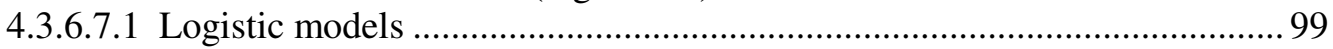

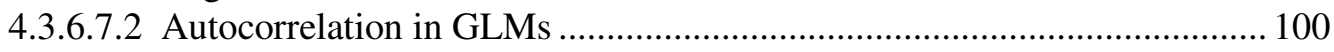

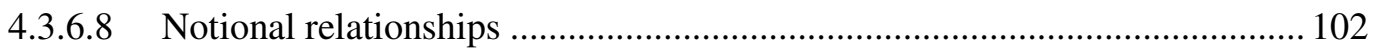

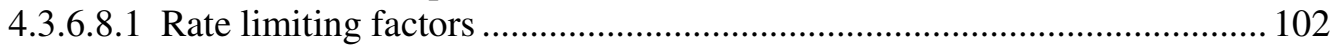

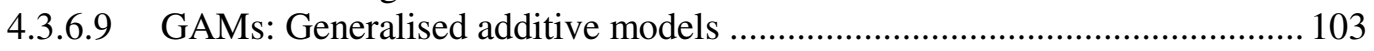

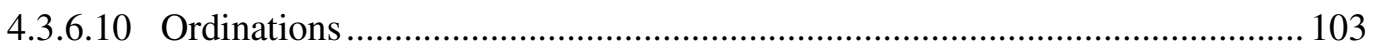

4.3.6.11 Constrained ordination: canonical correspondence analysis ........................ 104

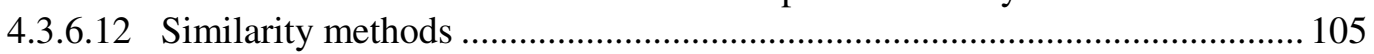

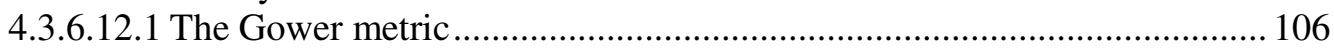

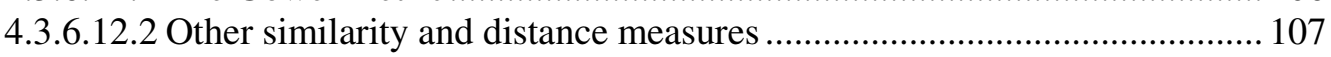

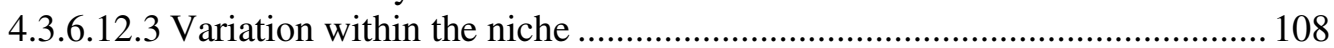

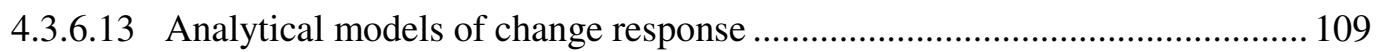

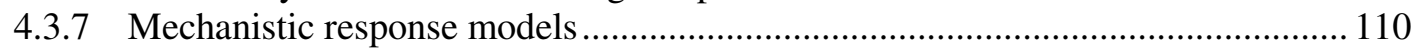

4.3.7.1 Productivity and leaf area index (LAI) ...................................................... 110

4.3.7.2 Forest growth models ............................................................................. 111

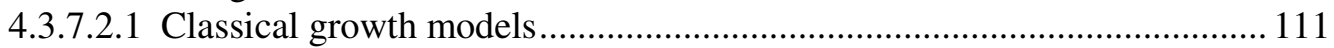

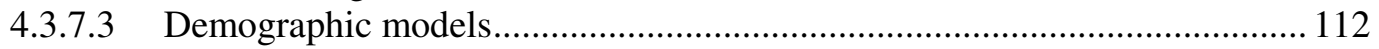

4.3.7.3.1 Structured population models ................................................................... 112

4.3.7.3.1.1 Stage-classified models .................................................................... 113

4.3.7.3.1.2Continuous models............................................................................. 115

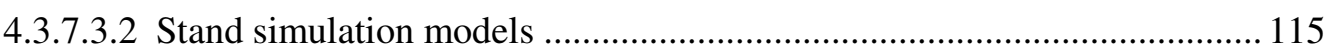

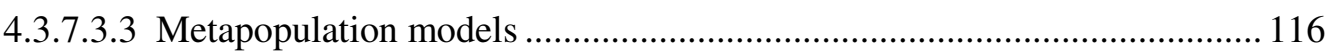

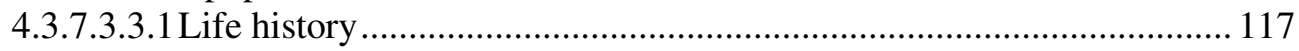

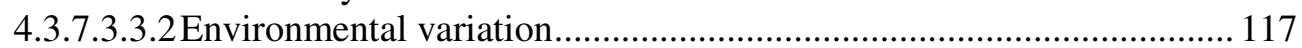

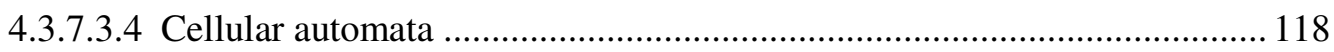

4.4 Conclusions: Modelling distribution and change .................................................... 119

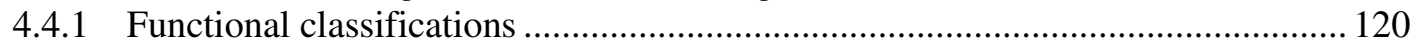

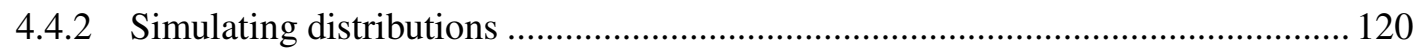

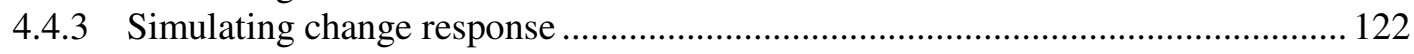

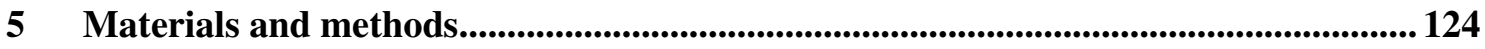

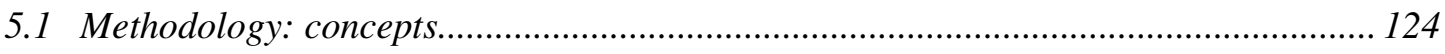

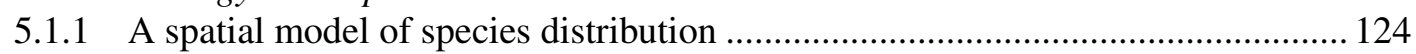

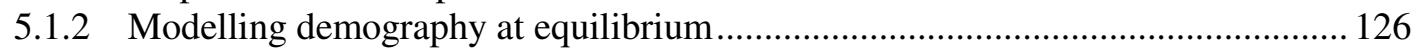

5.1.2.1 The balance between survival, growth and reproduction .............................. 126

5.1.2.2 Variation between patches......................................................................... 128

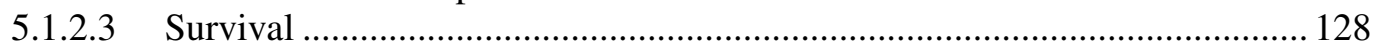

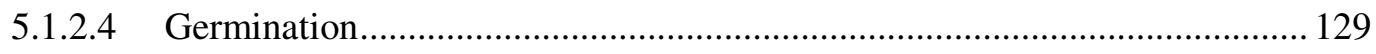

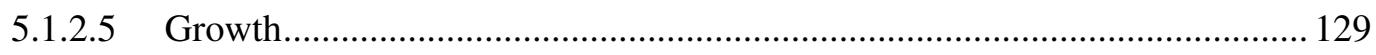

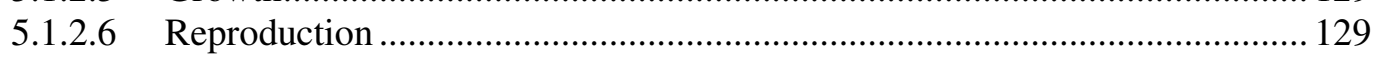

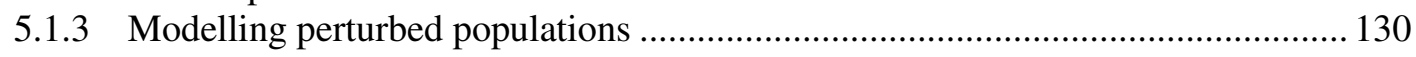

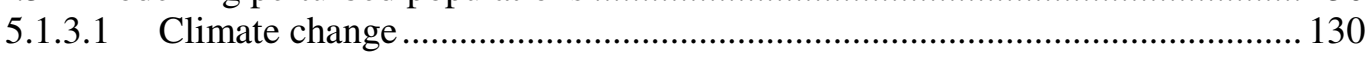

5.1.3.2 Impacts on population processes ............................................................. 131

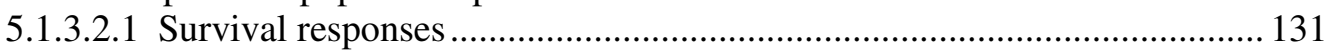

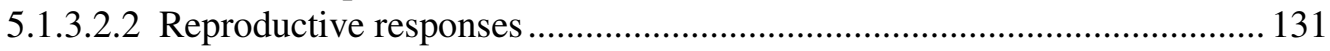

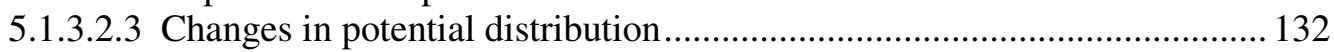

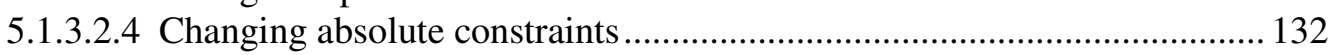

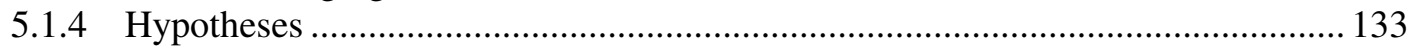




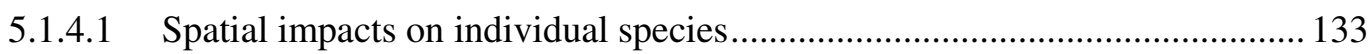

5.1.4.2 Spatial impacts on the set of species ........................................................... 133

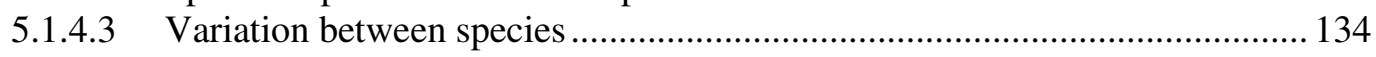

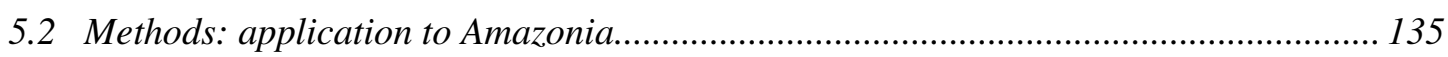

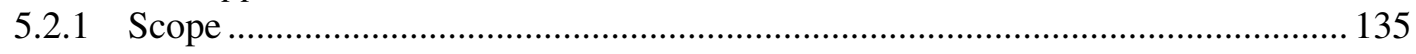

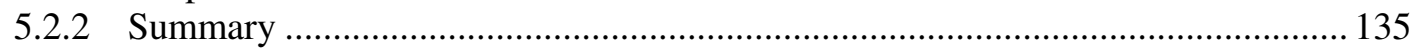

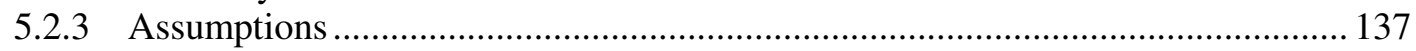

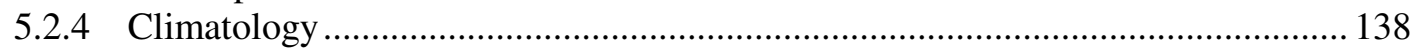

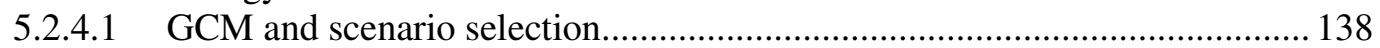

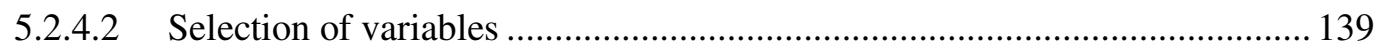

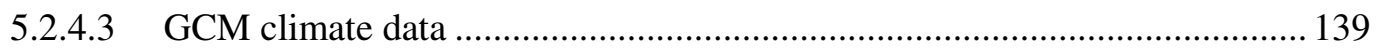

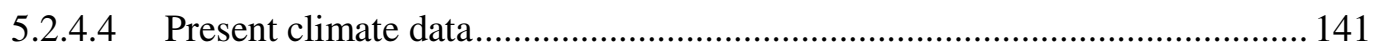

5.2.4.5 Model of present climate ..................................................................... 143

5.2.4.5.1 Derivation of land-surface parameters ...................................................... 145

5.2.4.6 Simulated change ................................................................................ 146

5.2.4.7 Neotropical and Amazonian reference bioclimates................................... 149

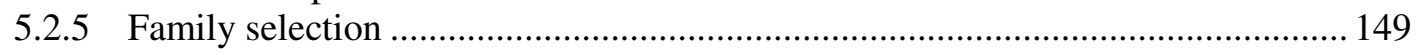

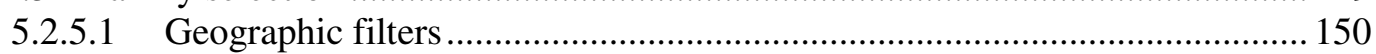

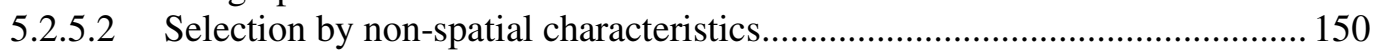

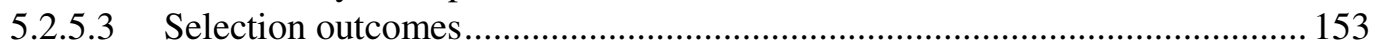

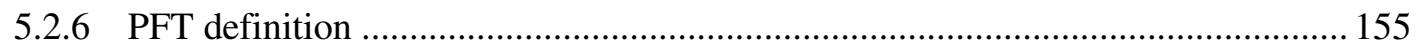

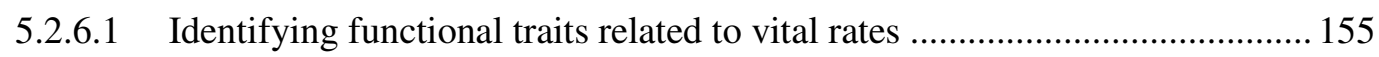

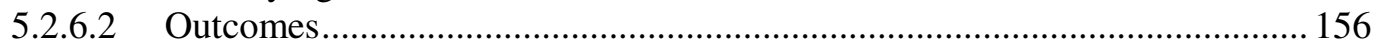

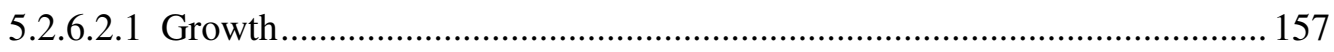

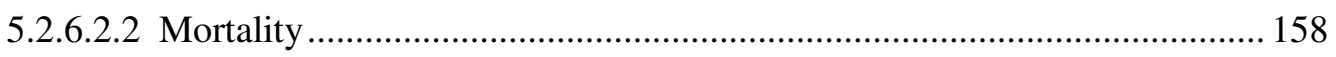

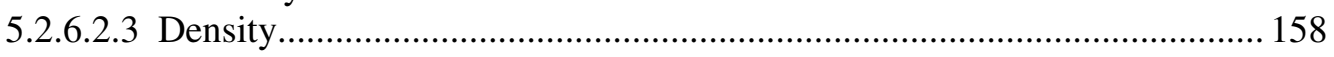

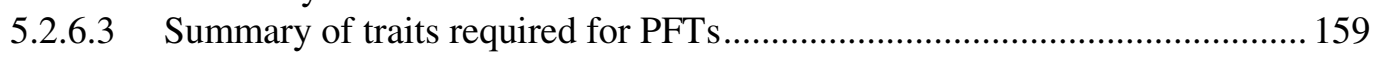

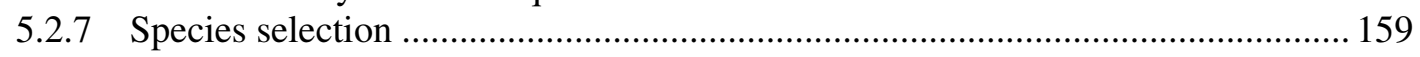

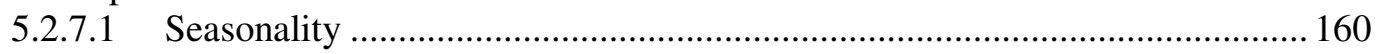

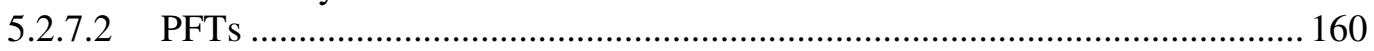

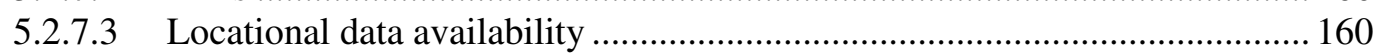

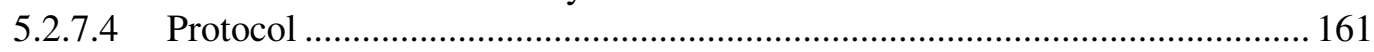

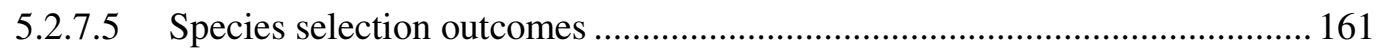

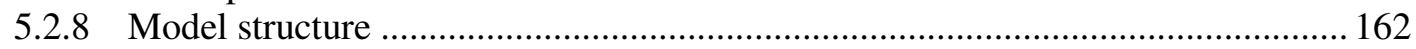

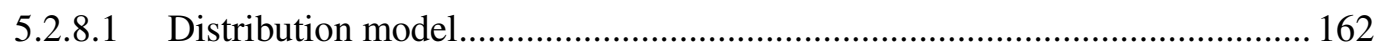

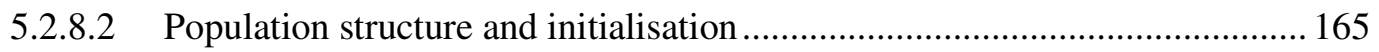

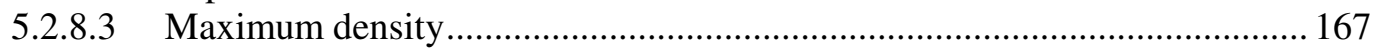

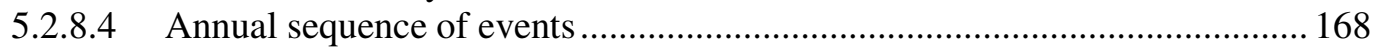

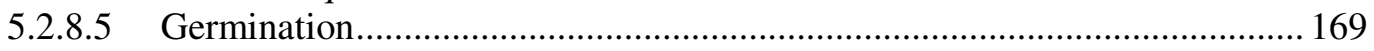

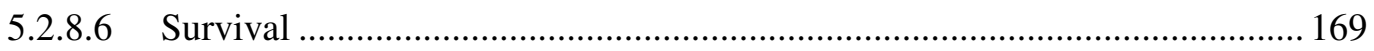

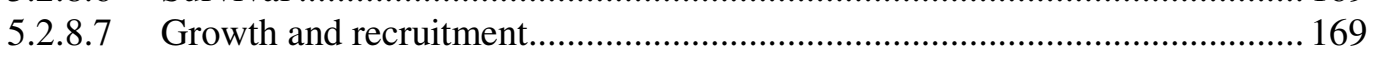

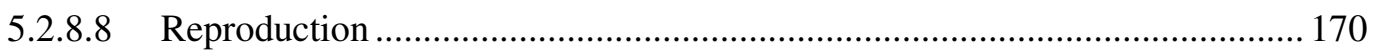

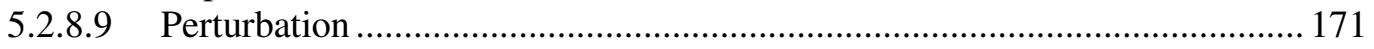

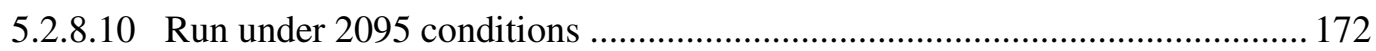

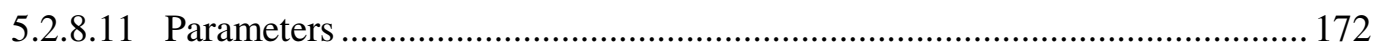

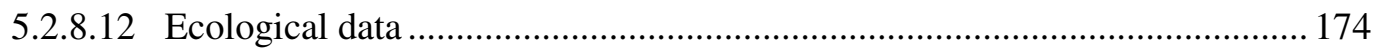

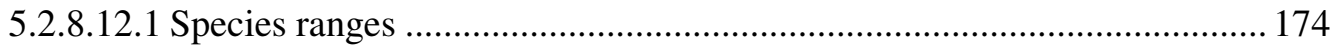

5.2.8.12.2 Diameter-height classes for growth estimates ...................................... 175

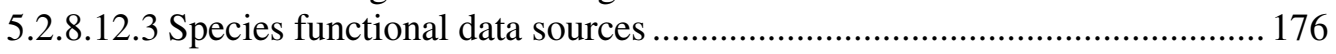

5.2.8.12.3.1Estimating vital rate relationships with climate ................................ 176

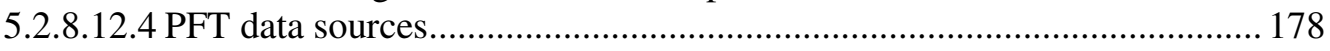

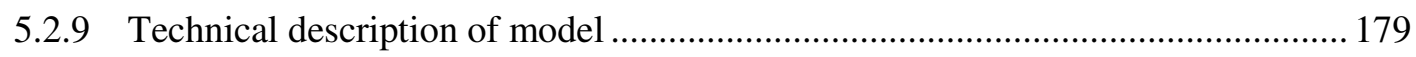

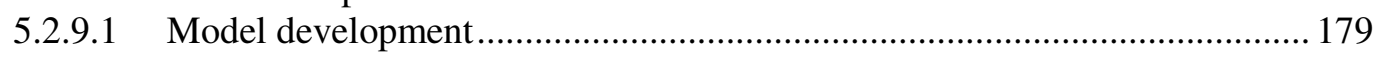

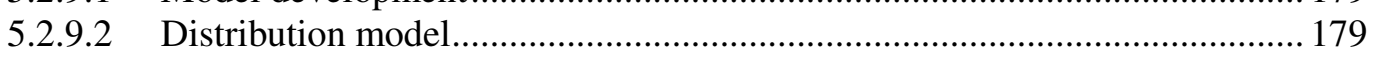

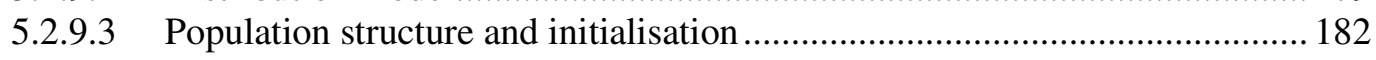

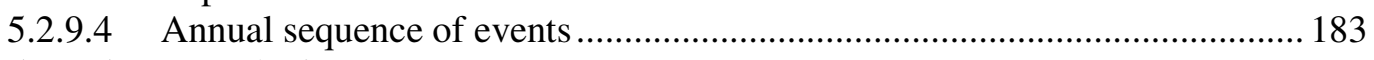

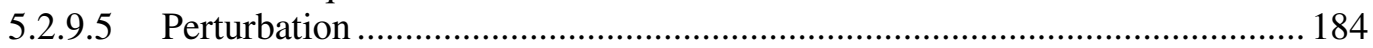




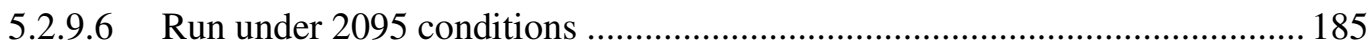

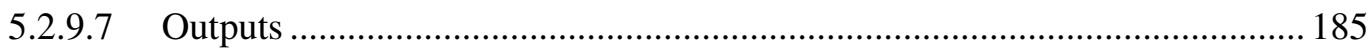

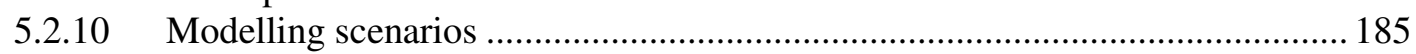

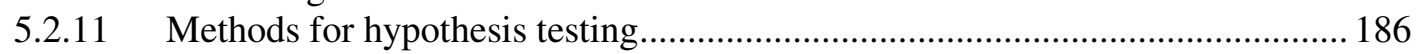

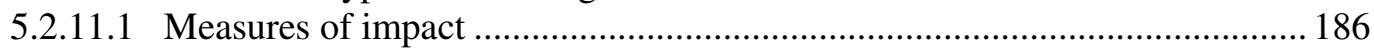

5.2.11.2 Parametric and non-parametric tests employed........................................ 187

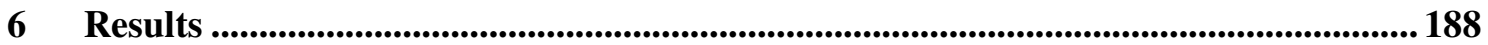

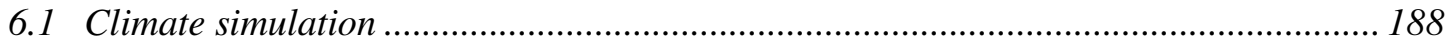

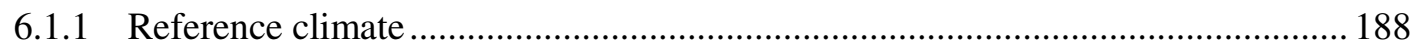

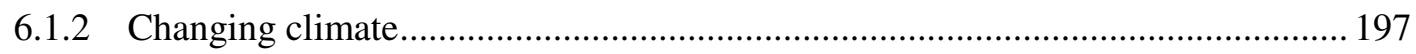

6.1.2.1 Temporal change over the Amazon basin .................................................... 197

6.1.2.2 Variables most affected by climate change .................................................... 197

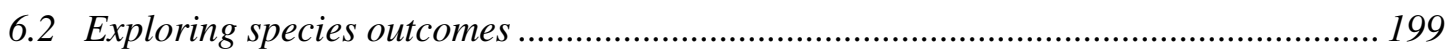

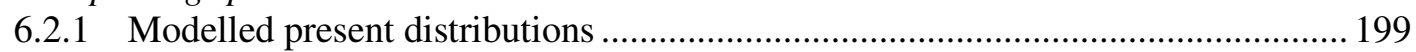

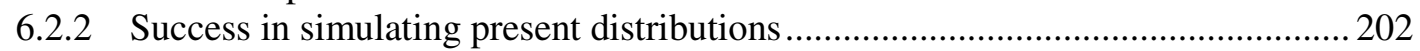

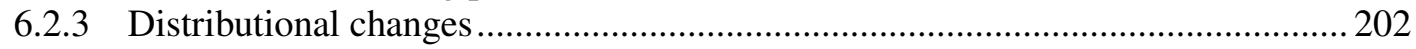

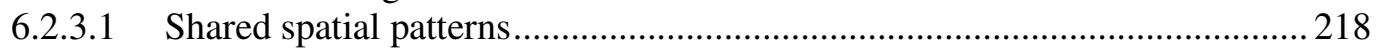

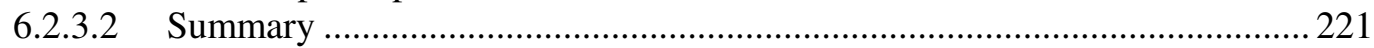

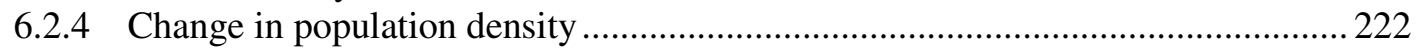

6.3 Effect of family and functional traits on species' outcomes .......................................... 227

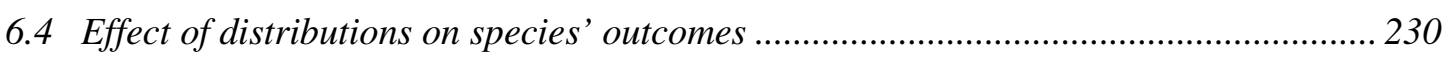

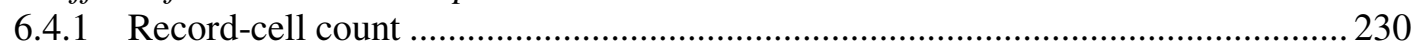

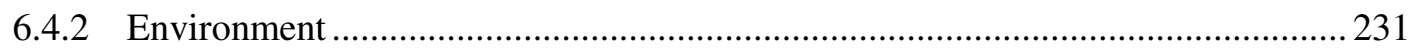

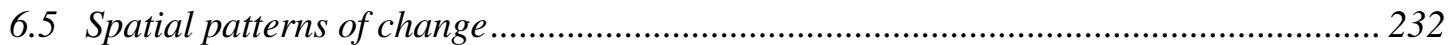

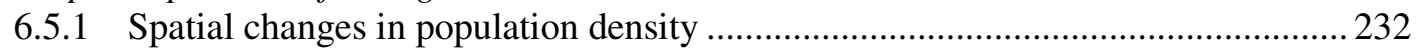

6.5.2 Spatial changes in population viability.............................................................. 234

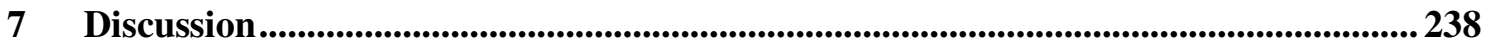

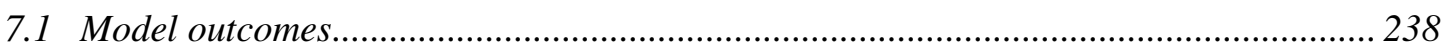

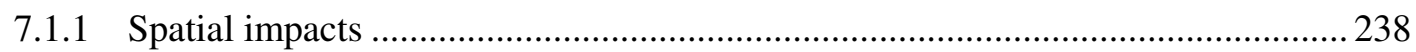

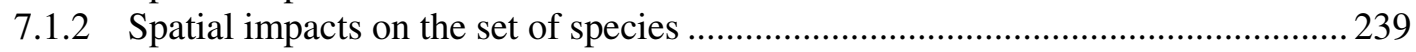

7.1.3 Correlates of variation between species ............................................................ 240

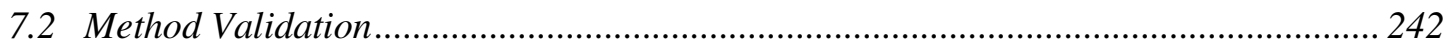

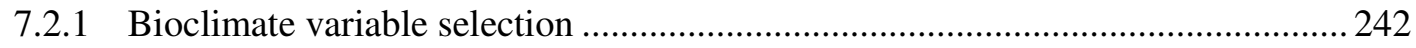

7.2.1.1 Relationships between bioclimate variables............................................ 242

7.2.1.2 Species frequency and bioclimate variables............................................. 243

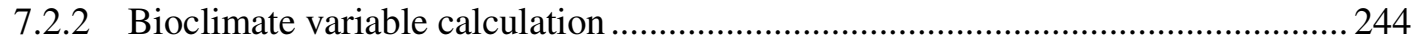

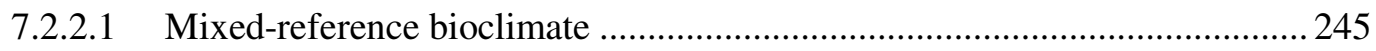

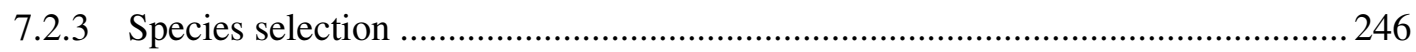

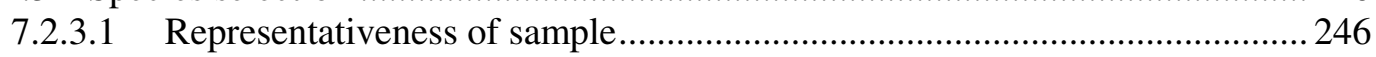

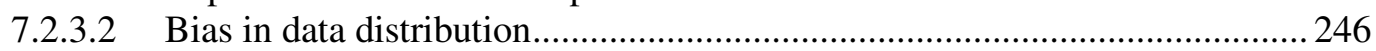

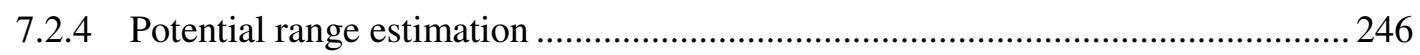

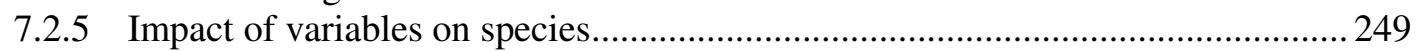

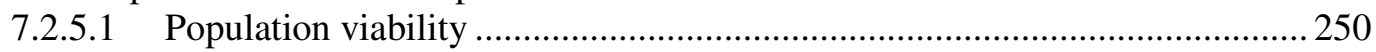

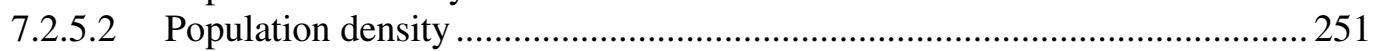

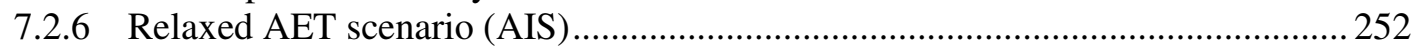

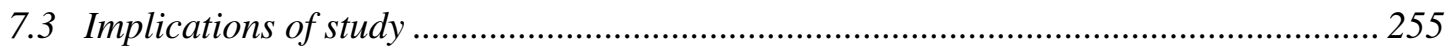

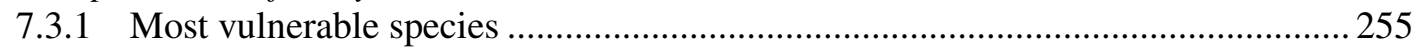

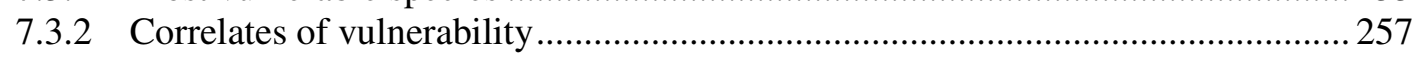

7.3.3 Areal targets for conservation and monitoring .................................................. 258

7.3.4 Comparison with vegetation model outcomes .....................................................2. 259 
7.3.5 Implications for GCM land surface parameterisations ......................................... 260

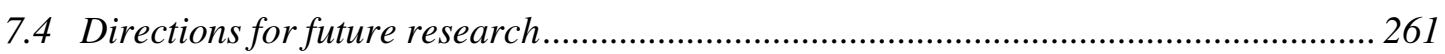

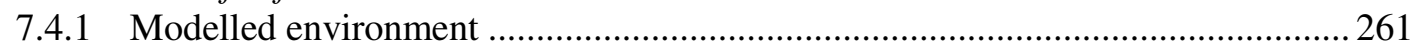

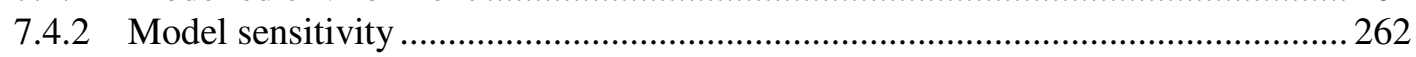

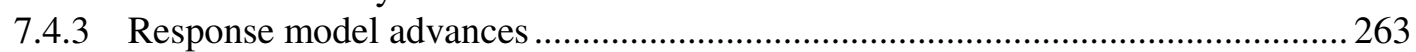

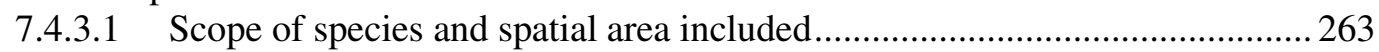

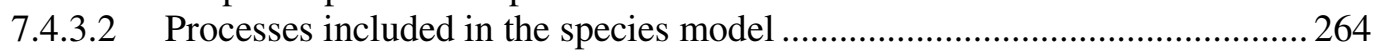

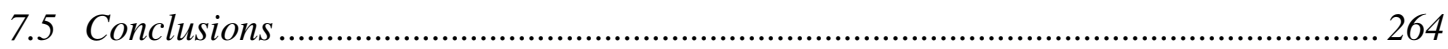

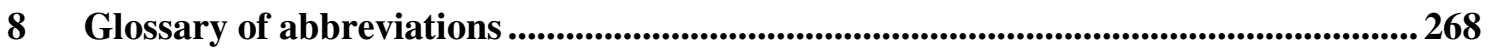

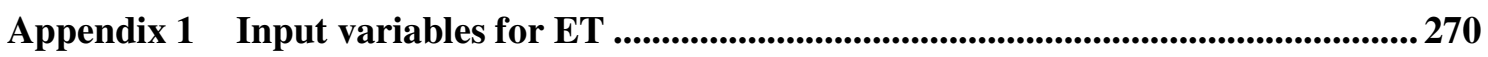

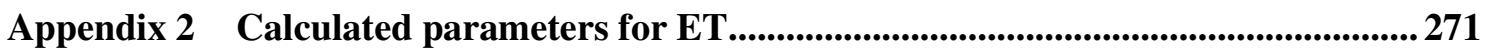

Appendix 3 Family selection ........................................................................................................... 275

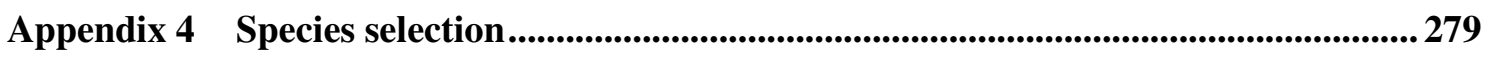

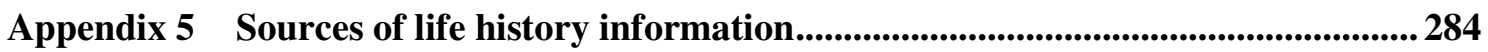

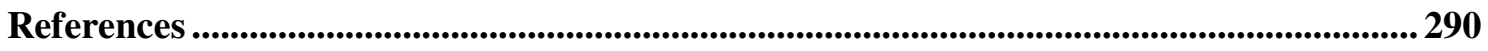

\section{Figures}

Fig. 1:1: Rivers of the extended Neotropics and the Amazon river basin (after EOS-Amazon Project, 1990)

Fig. 1:2: Topology, m above sea level, and 'enclosed-Amazon' 4

Fig. 2:1: Annual precipitation for the extended Neotropics $\left(0-7412 \mathrm{mmy}^{-1}\right.$, light-dark blue), with

EOS-Amazon boundary. Aggregated from New et al. $1999\left(0.5^{\circ}\right)$

Fig. 2:2: Annual temperature means for the extended Neotropics $\left(1-28^{\circ} \mathrm{C}\right.$, blue-red). Aggregated from $0.5^{\circ}$ map of New et al. 1999

Fig. 2:3: Grime's competition-stress-disturbance triangle

Fig. 4:1: Records for three Licania spp., (Chrysobalanaceae) (Prance \& Plana 1997)

Fig. 4:2: Generalised unimodal response to a single variable 86

Fig. 4:3: Regional and local processes affecting species' distribution 122

Fig. 5:1: (a) Homogenous region; (b) Variation in suitability within region; (c) Patches of $\begin{array}{ll}\text { potential distribution divided by unsuitable zone } & 124\end{array}$

Fig. 5:2: Relationship between variables used to derive cell suitability 139

Fig. 5:3: GCM trajectories in (a) temperature and (b) precipitation for Amazon region from HADCM2GSa1, showing mean values for three groups of cells segregated by longitude 140

Fig. 5:4: Data flow for daily Penman-Monteith PET $\quad 142$

$\begin{array}{ll}\text { Fig. 5:5: Data flow for daily AET } & 143\end{array}$

Fig. 5:6: Example of derivation of land-surface parameters 145

Fig. 5:7: Percentage cover for vegetation classes (coded as in Wilson \& Henderson-Sellers 1985) estimated from LAI, with QEN cover type 146

Fig. 5:8: Decadal HADCM2 (HD), and annual downscaled (AN), January \& June precipitation for cell SW002060 
Fig. 5:9: Decadal HADCM2 (HD), and annual downscaled (AN), January \& June temperature for cell SW002060

Fig. 5:10: HADCM land grid for Amazon region, with EOS-Amazon boundary 147

Fig. 5:11: Cladogram (APG 1998). Bold indicates inclusion in the final selection 151

Fig. 5:12: Family selection protocol $\quad 152$

$\begin{array}{lr}\text { Fig. 5:13: Range of mortality rates in PSPs sampled } & 158\end{array}$

Fig. 5:14: Distribution of records for (i) the 'pool' and (ii) selected species sets 162

Fig. 5:15: Example of application of Gower similarity metric to a single variable 163

Fig. 5:16: Potential and realised distribution for Couepia dolichopoda, SIS 165

Fig. 5:17: Example of Gaussian curve and underlying relationship 167

Fig. 5:18: Steps to simulating realised distribution, for Caryocar brasiliensis under SIS: (a) recorded and accepted records; (b) maximum similarity to accepted records under mixedreference bioclimate; (c) $5^{\circ}$ spatial buffer from accepted records; (d) areas inside all rectilinear envelopes; (e) Simulated initial distribution for extended Neotropics and extended Amazon. Records: Prance \& Plana (1997), INPA (2000b) \& EMBRAPA (2000b)

Fig. 5:19: Mean and minimum of the maximum similarity values for each jack-knifed record, plotted against record count, for all 69 species

Fig. 5:20: Model processes through simulated time

Fig. 6:1: Histograms of values over the extended Neotropics for (a) AET (annual sum of daily evapotranspiration), (b) $M D$ (annual sum of daily PET - AET), (c) SMA (maximum consecutive days where AET $<$ PET), and (d) $T_{\text {min }}$ (lowest mean monthly temperature) 189

Fig. 6:2: AET, extended Neotropics (linear colour scale) 190

Fig. 6:3: $M D$, extended Neotropics (linear colour scale) 190

Fig. 6:4: SMA, extended Neotropics (linear colour scale) 191

Fig. 6:5: $T_{\min }$, extended Neotropics (linear colour scale) 191

Fig. 6:6: AET, PRES and changing values under SIS 193

Fig. 6:7: $M D$, PRES and changing values under SIS 194

Fig. 6:8: SMA, PRES and changing values under SIS 195

Fig. 6:9: $T_{\min }$, PRES and changing values under SIS 196

Fig. 6:10: CVs and mean relative change in DOWNS bioclimate variables, SIS 198

Fig. 6:11: Species frequency (realised distributions) at 1990 under (i) SIS and (ii) RIS 199

Fig. 6:12: Jack-knifed similarity under SIS for the two species with most record-cells, (a) Licania heteromorpha (220 cells); (b) Hirtella triandra (214 cells)

Fig. 6:13: Jack-knifed similarity under SIS for (a) Roupala montana (213 cells); (b) a selection of species with a small number of record-cells: Inga plumifera, Chamaedorea fragrans, Tecoma tenuiflora and Geonoma oldemanii

Fig. 6:14: Coded SIS \& RIS outcomes for 6 Arecaceae species 206

Fig. 6:15: Coded SIS \& RIS outcomes for a further 6 Arecaceae species 207

Fig. 6:16: Coded SIS \& RIS outcomes for 2 Balanophoraceae (top row) and 4 Bignoniaceae $\begin{array}{ll}\text { (centre \& bottom rows) species } & 208\end{array}$

Fig. 6:17: Coded SIS \& RIS outcomes for 6 Bignoniaceae species 209

Fig. 6:18: Coded SIS \& RIS outcomes for 4 Caryocaraceae (top \& centre rows) and 2 $\begin{array}{ll}\text { Chrysobalanaceae (bottom row) species } & 210\end{array}$

Fig. 6:19: Coded SIS \& RIS outcomes for 6 Chrysobalanaceae species 211

Fig. 6:20: Coded SIS \& RIS outcomes for a further 6 Chrysobalanaceae species 212

Fig. 6:21: Coded SIS \& RIS outcomes for 6 Lauraceae species 213

Fig. 6:22: Coded SIS \& RIS outcomes for 6 Fabaceae species 214

Fig. 6:23: Coded SIS \& RIS outcomes for 2 Fabaceae (top row) and 3 Proteaceae (centre \& $\begin{array}{ll}\text { bottom rows) species } & 215\end{array}$

Fig. 6:24: Coded SIS \& RIS outcomes for 5 Rubiaceae species 216

Fig. 6:25: Coded SIS \& RIS outcomes for 5 Sapotaceae species 217

Fig. 6:26: (a) Limiting factor level \& suitability index; (b) Frequency in stage classes ( $\mathrm{S} 0=$ seed, $\mathrm{S} 1=$ seedling, $\mathrm{S} 2=$ bush) ('c') both for Chamaedorea fragrans in cells SW010075 ('a'), SW011074 ('b') \& SW012073 ('c')

Fig. 6:27: $K_{\mathrm{kp}} / K_{\mathrm{kmax}}$, SIS- Balanophoraceae, Proteaceae \& Chrysobalanaceae 224

Fig. 6:28: $K_{\mathrm{kp}} / K_{\mathrm{kmax}}$, SIS - Sapotaceae, Lauraceae \& Fabaceae 224 
Fig. 6:29: $K_{\mathrm{kp}} / K_{\mathrm{kmax}}$, SIS - Caryocaraceae, Arecaceae, Rubiaceae \& Bignoniaceae 225

Fig. 6:30: Count of cells with surviving populations at three points in time 226

Fig. 6:31: Count of cells with $S_{\mathrm{p}}>S_{\min }$ at 2 points in time 226

Fig. 6:32: Species-mean change in density relative to 1990 levels 227

Fig. 6:33: Species-mean $\Delta K_{\text {kp }}(1990 \rightarrow 2095$ or 2195), by height class, SIS 229

Fig. 6:34: Distribution of species-mean population non-viability by family, SIS, 2095; mean with confidence limits (left series) and median with boxplots (right series)

Fig. 6:35: Percentage of non-viable populations (2095) by population count (1990), per species. SIS \& RIS

Fig. 6:36: (I) Wilcoxon tests comparing $K_{\mathrm{kp}} / K_{\mathrm{kmax}}$ for 1990 versus 2095 and 2195, (II) mean change by 2095 and 2195 for increasing and decreasing populations. SIS \& RIS 233

Fig. 6:37: (a) Proportion of viable populations at 2095, and 'best' cells for (b) 1990 climate and (c) 2195. SIS \& RIS

Fig. 7:1: DOWNS $M D$ versus (a) $A E T$, (b) $T_{\min }$ and (c) $S M A$ for 1980 s and 2080 s decadal means, and (d) HADCM2 versus DOWNS AET. SIS

Fig. 7:2: Observed record count versus mixed-reference (a) $A E T$, (b) $S M A$, (c) $M D$, (d) $T_{\min }$. Extended Neotropics, SIS

Fig. 7:3: Total number of records found in cells with few or many records

Fig. 7:4: Success of simulation of actual presences and absences by SM, LM and AM, based on bootstrapping of $20 \%$ samples, for four European tree species

Fig. 7:5: Causes of absolute exclusion by rectilinear envelope, all non-viable populations and most-common cause per species, 2095 SIS \& RIS

Fig. 7:6: Distribution of MLF across viable populations at 1990 and 2095, SIS \& RIS (population frequency)

Fig. 7:7: Most common MLF per species for viable populations at 1990 and 2095, SIS \& RIS (species frequency)

Fig. 7:8: (a) Outcomes for the 15 'c-species', SIS \& AIS; (b) Outcomes for all species, formed by replacing SIS with AIS outcomes set for those in (a); (c) 'Best cells under AIS. 254

Fig. 7:9: (a) Proportion of modelled species to fall into ' victim' category under SIS (and AIS); (b) minimum distance between existing and new potential distribution for 'victims'

\section{Tables}

Table 3:1: IPCC 'equivalent $\mathrm{CO}_{2}$ ' emission scenarios (Leggett et al. 1992) 38

Table 3:2: Structural characteristics of a sample of GCMs 48

Table 3:3: Mean anomalies between GCM simulations and three Amazonian sites (Culf et al. 1998)

Table 3:4: GCM results for IS92a - temperature and precipitation anomalies 56

Table 3:5: Selected monthly output variables for GCMs

Table 4:1: Summary of biome models' response to GCM forcing

Table 4:2: Interpolation models' data requirements

Table 5:1: Family selection, summary with species and records available, and data sources 154

Table 5:2: Climate for cells holding PSPs

Table 5:3: Relationship between mean species growth rates for each site and traits

Table 5:4: Relationships between mean species mortality rate for each site and traits $\quad 157$

Table 5:5: Relationships between species' density per hectare for each site and traits $\quad 159$

Table 5:6: Traits for PFT construction

Table 5:7: Derivation of model parameters from surrogate traits 174

Table 5:8: Model parameters with default values in the absence of species data $\quad 174$

Table 5:9: Diameter values for height classes

Table 5:10: Maximum recorded vital rates for Hirtella triandra 177

Table 5:11: Statistical tests (Siegel \& Castellan 1998, SPSS 1998) 187

Table 6:1: Species outcomes at 2095, SIS and RIS 
Table 6:2: One-way ANOVA results - traits versus 2095 and 2195 mean outcomes

Table 6:3: Spearman's correlation coefficients $(\rho)$ between environment variables and speciesmean relative change in final-stage density $\left(\Delta K_{\mathrm{kp}}\right)$

Table 7:1: Spearman's correlation coefficients ( $\rho$ ) for AET, SMA, $M D$ and $T_{\min }, 1980 \mathrm{~s}$ and 2080s cell means (two-tailed, $\mathrm{p}<0.01$ in each case). SIS $\quad 242$

Table 7:2: Evapotranspiration and precipitation estimates for cell SW003060 244

Table 7:3: European tree species used in the tests $\quad 247$

Table 7:4: 'c-species' (AET assumption relaxed in AIS) 253

Table 7:5: Most threatened species: SIS \& AIS (not bold); SIS, AIS \& RIS (bold); endemic to Amazonia (underlined) 


\section{Introduction}

\subsection{JUSTIFICATION}

Tropical forest conservation and global warming are two urgent environmental concerns (Moore 1996), but until recently, little attention has been paid to the potential contribution of climate change to biodiversity loss. The rate of environmental change in the Neotropics means that it is desirable to use existing limited knowledge to explore likely response to change. A partially informed answer based on ecological theory is more useful for conservation planning than a guess (Lawton 1999).

The global sum of biodiversity is valued for scientific, aesthetic, ethical, functional and economic reasons (Groombridge 1992, Walker 1992, Fearnside 1995a, Williams 1998a). This project focuses on Amazonian rain forests, which are especially valued because of their high species richness and the perception that they represent a large area of relatively undisturbed wilderness. In addition, the forest has moisture cycling and carbon storage (estimated to be equivalent to $20 \%$ of current atmospheric carbon, Barry \& Chorley 1998) roles that stabilise the local and global climate.

Widespread and narrow endemic species live alongside one another in Amazonian forests, providing an interesting system to explore a range of climate change impacts in the tropics. There has been some speculation about the effects on Amazonian biodiversity (Hartshorn 1992, Bawa \& Markham 1995, Fearnside 1995c, Borchert 1998), but no evaluation of the impacts through species response modelling. The earliest conclusions are that global warming will push species towards cooler regions of higher altitude and further from the equator (Peters and Darling 1985, Halpin 1997). The relatively recent improvements in general circulation model (GCM) resolution and in access to species' locational data provide an opportunity to investigate this issue.

The potential scope for analysis of species' distribution patterns is large, with an estimated 30,000 plant species in the Amazon region (Gentry 1982), contributing about $10 \%$ to the approximate global total of 250,000 angiosperm species (Groombridge 1992, p. 65). Therefore, a small subset is selected to be representative of wider Amazonian biodiversity. A single GCM scenario has been selected (Johns et al. 1997) to simulate outcomes for individual species, and adapted to provide an alternative reduced-impact model. 


\subsection{AIMS}

This project's aims are as follows:

- To develop a model to simulate the impact of possible future climate change on Amazonian plant species.

- To apply the model to evaluate potential impact on a set of species selected to represent functional and higher-taxon biodiversity.

- To provide a preliminary risk assessment for the individual species and functional groups.

- To identify areas likely to undergo change in species composition or retain their original species complements.

- To examine the long-term impact of these levels of climate change by hypothesising a new equilibrium climate under 2095 conditions.

\subsection{OBJECTIVES}

The aims give rise to a set of more specific objectives.

- To develop a methodology that allows interpolation of Amazonian species' ranges from limited locational data at a relatively low resolution.

- Following this approach, to map current neotropical distributions under equilibrium conditions, for a species set selected to broadly represent Amazonian biodiversity.

- To use the same approach to map the species' potential distributions for the year 2095 in response to climate change, using a stronger and a milder change scenario.

- To identify and simulate potential lags in extinction of populations following change.

- To map the distribution of viable and non-viable populations, and alternative suitable sites for each species (potential range) up to 2095 and beyond.

- To assess probable threat levels for different species and areas and contribute to the formulation of management objectives, especially monitoring, research and conservation priorities including protected area designation.

- To construct generic predictive profiles of plant groups most at risk from climate change.

\subsection{KEY DEFINITIONS}

\subsubsection{Biodiversity}

Biodiversity is defined here as 'the sum of biological variation across genes, species and ecosystems'. Biodiversity results from the adaptive evolution of organisms in response to environmental and biotic selection pressures. It therefore has genetic and phenotypic aspects. This project selects a species set to provide a good coverage of genetic variation, through a 
sample of taxa from a cladogram (APG 1998), and of phenotypic (functional) variation, through including a diversity of plant functional traits (PFTs, section 4.1.2).

\subsubsection{The Amazon region}

Amazonia, or the Amazon region, is defined here as the hydrological basin mapped as a vector envelope in the EOS-Amazon Project (1998, Fig. 1:1). The area of this vector envelope is approximately $6,000,000 \mathrm{~km}^{2}$, which is close to previous areal measurements (e.g. 6,500,000 $\mathrm{km}^{2}$, da Silva \& Patton 1998). It is a largely lowland region half-surrounded by mountains: the Andean mountain ranges to the west and Guiana Shield to the north.

The Amazon region intersects Bolivia, Brazil, Colombia, Ecuador and Peru. The area of $1^{\circ}$ grid cells entirely enclosed is $5,189,538 \mathrm{~km}^{2}$. These 426 cells are defined as the 'enclosed-Amazon' (Fig. 1:2) A further 134 cells sit on the vector boundary. All 560 cells are defined as the 'inclusive-Amazon'. A $1000 \mathrm{~m}$ contour is the standard boundary between lowland and lower montane rain forest (Flenley 1998). The boundary obtained at this level from a global topology map (Row \& Hastings 1989) does not match either Amazon region definition well (Fig. 1:2).

This project considers Amazonian species, but as the extent of climate variation tolerated by each species is of interest (Bartlein et al. 1997), locational records for the 'extended' neotropical region (latitudinal range $30^{\circ} \mathrm{S}-30^{\circ} \mathrm{N}$, Fig. 1:1) are included. This further encompasses part or all of Argentina, the Caribbean islands (including Cuba), Chile, Costa Rica, French Guiana, Guatemala, Guyana, Honduras, Mexico, Nicaragua, Panama, Paraguay, El Salvador, Suriname, the USA and Venezuela.

\subsubsection{Spatial cell co-ordinates}

Where a $1^{\circ}$ latitude-longitude grid cell is referred to in this thesis, it is named using the coordinates of the corner closest to the map origin. For example, the cell whose centre is $1.5^{\circ} \mathrm{S}$, $70.5^{\circ} \mathrm{W}$ is referred to as 'SW001070', and the cell centred on $2.5^{\circ} \mathrm{N} 63.5^{\circ} \mathrm{W}$ as 'NW002063'. 


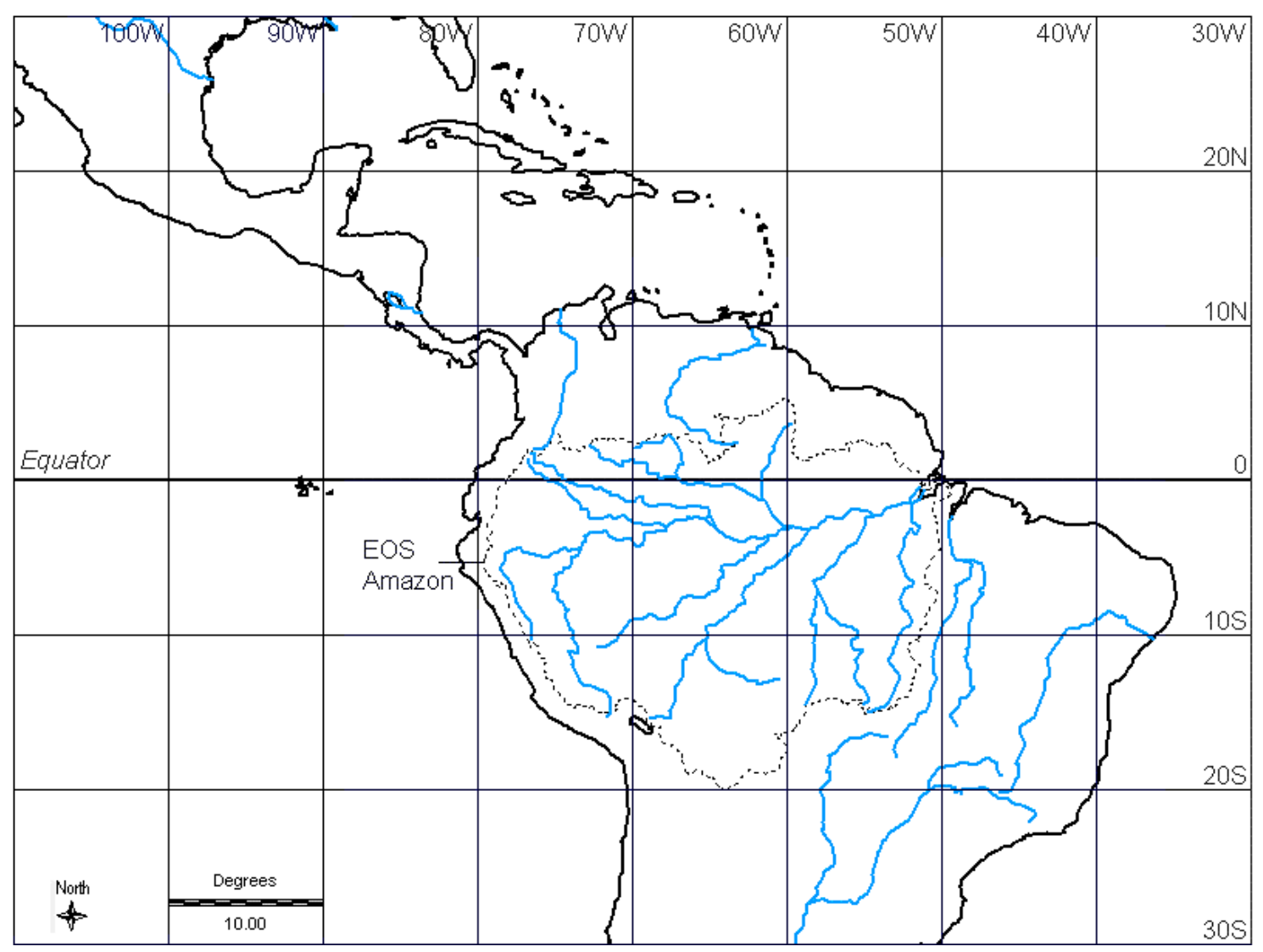

Fig. 1:1: Rivers of the extended Neotropics and the Amazon river basin (after EOS-

Amazon Project, 1990)

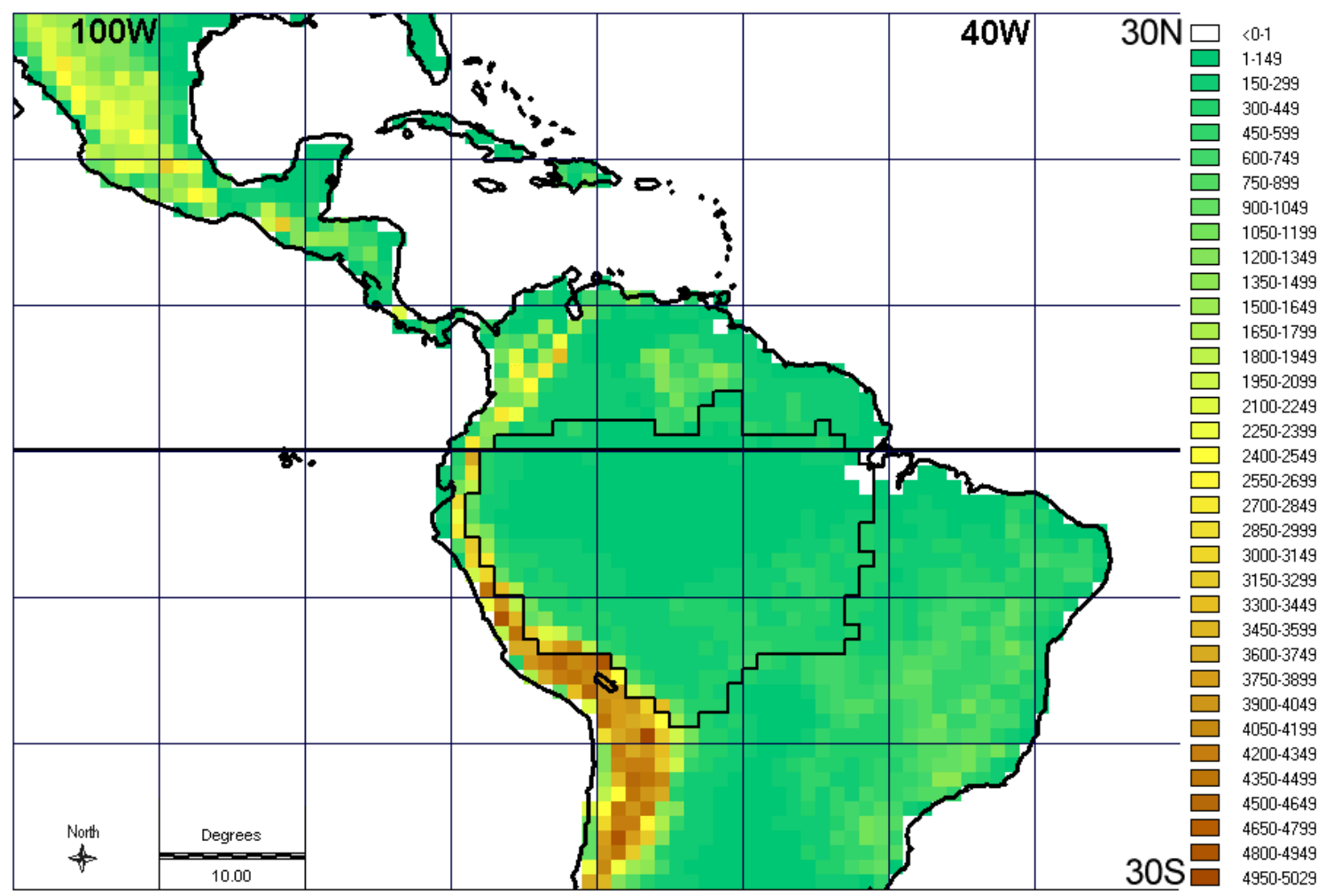

Fig. 1:2: Topology, m above sea level, and 'enclosed-Amazon' 


\subsection{KEY BACKGROUND}

\subsubsection{Distributional information}

Information on the spatial distribution of biodiversity is vital for various ecological, biogeographic, phylogeographic and conservation-oriented fields including distributional studies, community ecology, evolutionary biogeography and conservation priority analyses. However, resources for information gathering and conservation are restricted: it is difficult to obtain funding for an open-ended enterprise.

It follows that our knowledge of species-rich, tropical biodiversity is limited and patchily distributed in space. Heterogeneous sampling of both species and sites results from recorder bias. This encompasses variation in observation and identification skills, interest in certain taxa, difficulties in recording individual taxa caused by inconspicuousness and the relative accessibility of different survey locations to recorders. Recorder bias also applies at an international scale, with research effort concentrated in temperate areas. As overall species richness is lower here than in the tropics (Groombridge 1992), a greater proportion of temperate taxa are well recorded.

Survey effort within the Neotropics tends to be concentrated around field stations, urban areas and roads, which can give rise to misleading conclusions about biodiversity distribution (Gentry 1992, Prance 1987, Nelson et al. 1990). Many field surveys are conducted in the dry season, resulting in more collections from species that are flowering or fruiting then (ter Steege et al. 2000). Taxonomic artefacts resulting from species being classified differently by different local specialists, inaccurately separated or merged, are a further source of error, of particular concern for species that have not been recently monographed (Gentry 1992).

The availability of abundance measures and absence records also affects a dataset's utility from a niche modelling or conservation planning perspective. However, most locational records, and therefore species' atlases, are presence-only (van Jaarsveld et al. 1998).

The global warming scenarios generated by GCMs (Hulme \& Viner 1995, Houghton et al. 1996, 2001) increase the need to understand the factors determining species' distributions, so that their vulnerability and the viability of 'future-proofing' protected-area systems can be assessed. Current conservation plans do not accommodate the likely ecological change resulting from regional warming and drying (Bush 1996). Researchers must identify approaches that make the best use of existing tropical data. 
To assess vulnerability within the short time-scale envisaged for the onset of climate change, modelled distributions and response estimates must be based on existing presence data (van Jaarsveld et al. 1998). Ongoing tropical deforestation increases the urgency of the question: which areas and species are most in need of protection?

\subsubsection{Climate change projections}

The chaotic nature of climate systems means that it is not possible to confidently model climate variables far into the future, so climate models offer projections rather than predictions. These are possible futures based on assumptions about relationships between different atmospheric, ocean and surface processes and concentrations of various atmospheric gases.

The current generation of coupled GCMs yield similar global results when modelling climate change as a consequence of increasing concentrations of greenhouse gases (GHGs, section 3.1). Regional impacts are more varied, because of the models' low spatial resolution. For Amazonia, there is a general trend towards warming and drying, but the degree of impact varies between GCMs (Mata \& Campos 2001). 


\section{Geography and biodiversity of the Amazon region}

\subsection{The AmAZON REgION}

The climate of the Amazon basin is warm and humid, with an east-west gradient in seasonality of precipitation. Every few years the El Niño Southern Oscillation produces localised extremes in temperature and precipitation. These conditions are thought to have prevailed for approximately 9,000 years. Previous global climate changes affected the regional climate: according to palynological and ground-water analyses, the previous era was colder and drier, reaching a peak at the Last Glacial Maximum, 20,000 years ago. Global climate models consistently suggest that in future the climate will become warmer and drier.

\subsubsection{Climate history}

The rate of climate change in earlier periods and of plant migration in response can help us to understand the potential impacts of a modern warming episode. Considering the Quaternary period (1.6 my BP to present), most tropical evidence for climate and ecological changes is palynological. There is more information for the current Holocene epoch (10,000 BP to present) than the earlier Pleistocene epoch (1.6 my BP to 10,000 BP).

Tropical cooling has been associated with repeated glaciation episodes within the northern hemisphere during the Pleistocene. The last glacial maximum (LGM) occurred at 20,000 BP. This glaciation ended around 14,700 BP, with 'modern conditions' being established by 10,000 BP (Barry \& Chorley 1998). Only about 100 pollen diagrams are available for the period for the tropics as a whole (Flenley 1998), so mapping the movement of individual genera and vegetation types is difficult.

There is some evidence for drying as well as cooling at the LGM, with extension of savanna into some forest areas and a downwards movement of the altitudinal forest limit. Other areas retained forest but experienced invasion of plants from drier, cooler habitats. A decrease of 6 to $10^{\circ} \mathrm{C}$ from the modern mean annual temperature has been proposed to explain these vegetational changes (Colinvaux 1998, Flenley 1998). Supporting evidence using noble gases dissolved in

$\mathrm{C}^{14}$-dated ground water gives a record of $-5.4(+/-0.6)^{\circ} \mathrm{C}$ in lowland Brazil (Stute et al. 1995).

In the early Holocene, 8-9000 BP, as the interglacial period became established, a higher temperature than present arose. Archaeological evidence suggests that droughts linked to extreme El Niño events occurred at least in northern lowland South America in the years 400, 700, 1200 and 1500 BP (Meggers 1994). However, apart from short-lived extreme events, the 
rate of change has rarely or never been as fast as that expected from current global warming scenarios (Flenley 1998).

\subsubsection{Modern climate}

\subsubsection{Precipitation}

The mean annual rainfall measured at different Amazonian forest sites ranges from 1,500 to $6,000 \mathrm{~mm}$ (Henderson et al. 1995). Mean interpolated precipitation for the inclusive-Amazon ranges from 333 to $3890 \mathrm{mmy}^{-1}$, with a mean of $2118 \mathrm{mmy}^{-1}$ (from data of New et al. 1999, Fig. 2:1). The lowest values are seen on the mountainous western edge of the region.

Much precipitation is derived from 'mesoscale lines of instability' forming near the east coast and moving westwards. This process occurs faster in January than July. Some of the resulting precipitation is deposited as far as the Andes (Barry \& Chorley 1998). A large orographic convergence at the Andes combines with the high regional evapotranspiration to increase rainfall locally.

Western Amazonia has an aseasonal moist climate. Most of Amazonia experiences one seasonal cycle: the rainy season between October-April over central tropical and southern Amazonia, caused by the contraction of the South Atlantic subtropical high-pressure cell (Barry \& Chorley 1998). This cell expands in July and causes a dry season. There is also a North Atlantic cell, which is less mobile. A monsoon (wide-ranging seasonal reversal of the wind system) occurs over two bands of South America in the northeast \& southeast. At Manaus, surface winds are predominantly southeast from May - August and northeast from September - April.

\subsubsection{Temperature}

Interpolated mean annual temperature for the inclusive-Amazon ranges from 5 to $27^{\circ} \mathrm{C}$ (Fig. 2:2, New et al. 1999). The lower temperature values are seen on the western edge of the EOS region, where cell means are influenced by very cold, high mountain peaks. Amazonian diurnal temperatures generally range from about $24-28^{\circ} \mathrm{C}$, though in the winter, surges of cold air (friagens) can cause cooling to $10^{\circ} \mathrm{C}$ even in the lowlands (Barry \& Chorley 1998). 


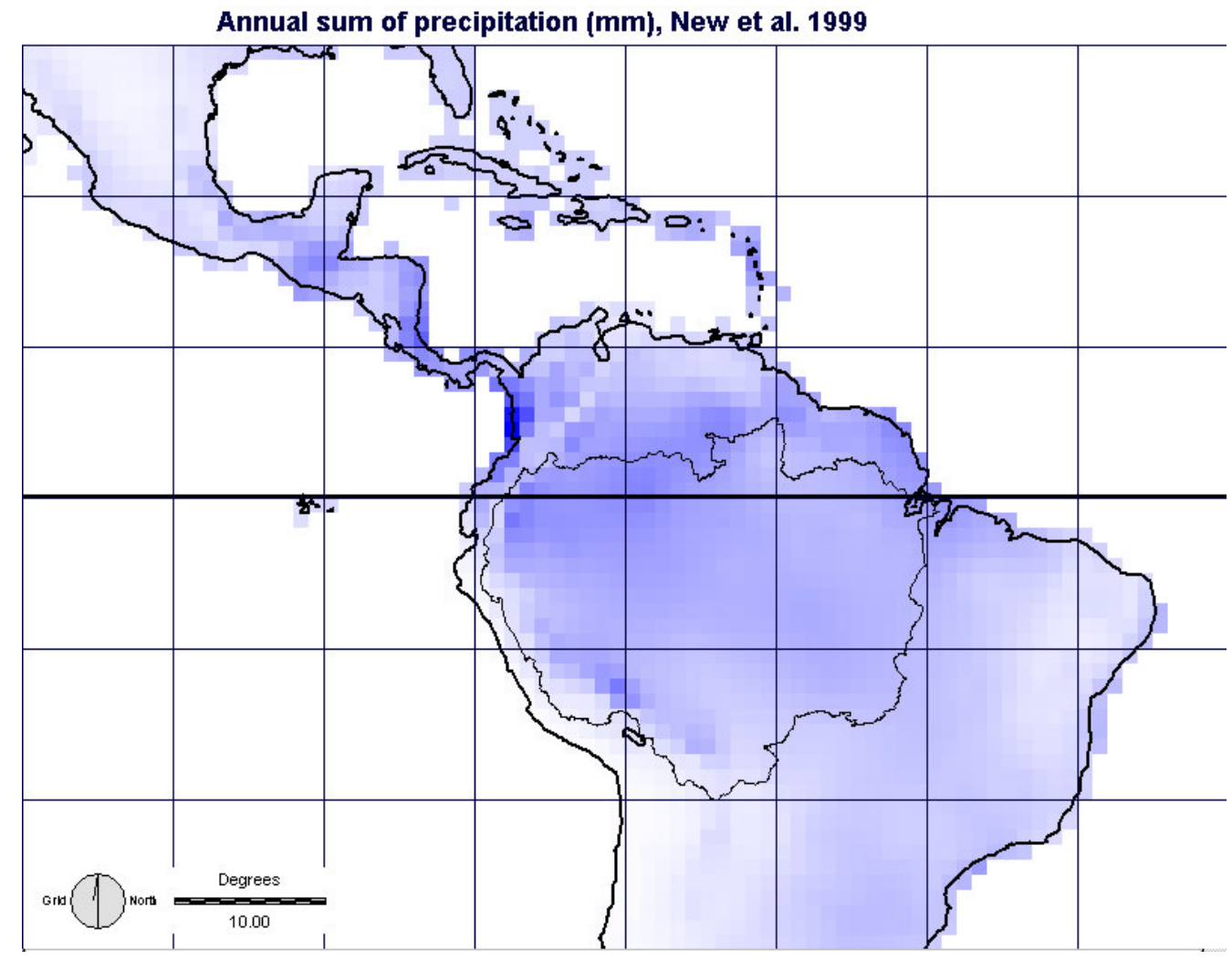

Fig. 2:1: Annual precipitation for the extended Neotropics (0-7412 $\mathrm{mmy}^{-1}$, light-dark blue), with EOS-Amazon boundary. Aggregated from New et al. $1999\left(0.5^{\circ}\right)$

Mean annual temperature (degrees C), based on New et al. 1999

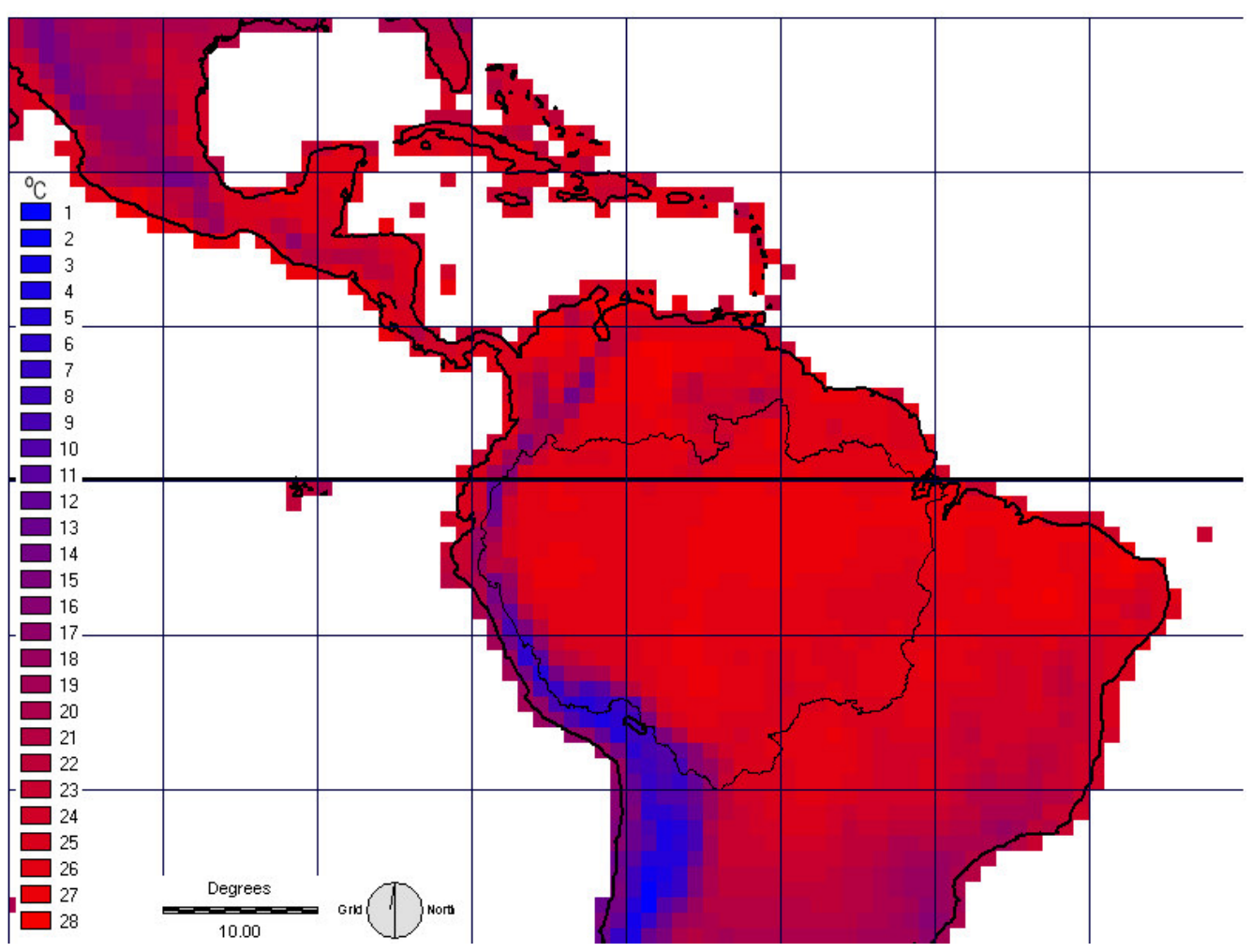

Fig. 2:2: Annual temperature means for the extended Neotropics $\left(1-28^{\circ} \mathrm{C}\right.$, blue-red). Aggregated from $0.5^{\circ}$ map of New et al. 1999 


\subsubsection{El Niño Southern Oscillation (ENSO)}

'El Niño' is an annual southward oceanic flow of warm water along the west coast of South America to about $6^{\circ} \mathrm{S}$, which replaces the northward Peru Current each December (Barry \& Chorley 1998). Every 2 to 10 years, this flow strengthens in March and April, preventing coastal upwelling of nutrient-rich water and limiting the growth of marine life, in an 'El Niño warm episode'. The mutually exclusive 'La Niña cold episode' is a high phase in the Peru Current bringing nutrient-rich water, occurring on average $75 \%$ of the time. At times, neither phase is dominant. The entire cycle is the 'El Niño Southern Oscillation' (ENSO).

These events have a complex influence on regional climates through teleconnections - longdistance linkages between atmospheric and oceanic variables. El Niño events tend to increase drought over northeast Brazil via an effect on the Atlantic atmosphere-ocean system, reducing sea-surface temperatures and evaporation. Northeastern Argentina, Uruguay and the three southernmost states of Brazil experience wet anomalies during the austral spring, with opposite effects during a La Niña year (Grimm et al. 1998). Additional precipitation in southern Brazil is associated with the autumn to winter of the year following an El Niño event. The ENSO cycle is thus linked to some of most frequent, spatially predictable climate extremes experienced by neotropical organisms.

Detailed long-term records of ENSO cyclicity do not exist, but El Niño episodes have been more frequent, long-lasting and intense from the mid-1970s to the turn of the century compared to the previous 100 years (Houghton et al. 2001).

\subsubsection{Geomorphology and soils}

Spatial variation in substrates arises both from the geological past (parent material, age and weathering) and contemporary processes (fluvial deposition, build-up of organic material). Soil type is therefore locally heterogeneous in distribution, but some broad generalisations can be made (Tuomisto \& Poulsen 1996, Brown \& Prance 1987, Kricher 1997). Much of the eastern and central Amazon region has predominantly sandy, well-weathered oligotrophic (nutrientpoor) soils. These areas are drained by 'black-water' rivers such as the Rio Negro, which gain their dark colour from decomposing leaf defence compounds such as tannins. Plants undergoing greater competition for soil nutrients tend to put more resources into defending their leaves from herbivores.

To the west of the Amazon basin, the slightly elevated Planalto region is composed of sediments deposited during the late Tertiary period (up to about 1.6 my BP). These are clay- 
rich, but still rather oligotrophic. Where more recent fluvial deposition of volcanic soils from the Andes has occurred, nutrient-rich conditions prevail. These soils are drained by muddier 'white-water' rivers, such as the Amazon. The nutrient-rich sediments that colour these rivers are deposited as they widen and slow down, as far east as Belém. Further depositions of silt occur along long stretches of the Amazon and smaller rivers in annual flooding episodes. These floodplains cover up to 1.3 million $\mathrm{km}^{2}$ (Richey et al. 1997) - over a fifth of the Amazon region. Thus, local patterns of soil nutrient availability are highly dependent upon current and historical river courses.

This contrast between the generally more dynamic soil deposition in the west and stable eastern Amazonian landscape reinforces the resource availability gradient already created by the difference in moisture seasonality. Although both sand and clay-based soils can be oligotrophic, sandy soils are more freely draining, so that seasonality of moisture availability is accentuated. Forests tend to be taller and more productive towards the west of the basin, having greater access both to moisture and soil nutrients. However, the ongoing fluvial deposition of younger sediments brings corridors of nutrient rich soils into the eastern Amazon.

\subsubsection{Vegetation}

Variation in vegetation type arises both from localised diversity in geology and topology, and from local to regional scale climate patterns. A standard categorisation of the natural vegetation of lowland tropical South America (Brown \& Prance 1987) can be summarised as follows:

The Amazonian rain forest on terra firme is closed canopy forest occurring above the level of the riverine floodplains, in areas where there is a very limited dry season. It is bordered by cerrado (central Brazilian savanna and savanna forest) on upland (mainly $>500 \mathrm{~m}$, with highly seasonal rainfall) areas to the south. Dry caatinga (tropical thorn scrub) areas appear to the east. The forest is broken by the Andean Mountains to the west. Terra firme forest occupies about half of the area of the Amazon basin. It can be divided into hill and lowland types; both dominated by evergreen species with characteristically high species richness per unit area (Gentry 1988). In lowland forests, which grow at altitudes up to about $250 \mathrm{~m}$, canopy height varies from 25 to $50 \mathrm{~m}$, with $30 \mathrm{~m}$ being a typical height. Hill forest height is rarely greater than $25 \mathrm{~m}$, with a more open canopy and denser understorey.

Amazonian savannas occur within and to the north of the lowland rain forest, in regions with annual rainfall under $2000 \mathrm{~mm}$. White sand formations including campina and campina forest also appear in patches within the Amazon basin, on the nutrient-depleted substrate of podzols of leached white quartz sand, or regosols. Transition forests represent gradations from rain forest 
to savanna, whilst gallery forests of savanna areas occur alongside rivers. It has been speculated that gallery forests could have acted as refugia for lowland rain forest species in the time of the last glaciation (section 2.2.1).

Swamp (inundated) forests are supported on permanently or seasonally flooded riverine habitats. Black-water rivers such as the Rio Negro, which are low in mineral sediment but carry dark organic matter, support permanent or seasonal igapo forests, as do the clearwater rivers such as the Tapajós, Xingo and Tocantins. White-water rivers, including much of the Amazon and some of its major tributaries, are rich in Andean sediments. Here seasonal várzea or permanent swamp forest occurs. These forests generally have lower species diversity than the terra firme forest, but diversity is higher where there are irregular flash floods of short duration rather than long-term immersion, because more terra firme species can colonise.

Montane rain forest occurs on higher slopes of over $700 \mathrm{~m}$. It supports a high level of narrowrange endemics (Churchill et al. 1995, Gentry 1992). At about $1000 \mathrm{~m}$ the lower montane rain forest grades into cloud forest, a humid environment supporting many epiphytic plants.

Finally, secondary forest, sometimes described as 'jungle', is a pioneer community occupying areas where forest has been cleared. Its species are typically fast-growing, light-demanding and insensitive to moisture variation (Condit et al. 1996a). Species richness in secondary forest of 50-80 years may be similar to that in 'undisturbed' mature forest, but composition differs, and can become increasingly dissimilar as long-lived remnant trees die out (Finegan 1996). Tree-fall gaps in mature forest exhibit a different regeneration pattern to these clearings, because seed banks and vegetative regrowth have not been affected by fire, and because there is a greater diversity of potential parents in the vicinity.

Another commonly used category, 'tropical moist forest' is defined by the environmental niche it occupies (Myers 1980). It groups together terra firme, montane and inundated forests with some white sand formations, transition and secondary forests. Amazonian tropical moist forests contain evergreen or partly evergreen trees and occur at altitudes up to $1,800 \mathrm{~m}$ where there is not less than $100 \mathrm{~mm}$ of precipitation in any month for two out of three years. Mean annual temperatures are at least $24^{\circ} \mathrm{C}$ and the environment is essentially frost-free. When this classification was mapped for neotropical South America, $123 \times 1^{\circ}$ cells were identified, mainly in northwest Amazonia (based on cell mean values from Legates \& Wilmott 1990a, 1990b; Row \& Hastings 1989). The precipitation requirement was particularly restrictive. Eight hundred cells in the same region contained at least $1000 \mathrm{~km}^{2}$ of evergreen broadleaf forest (based on USGS et al. 1998). The definition may be more appropriate at a finer spatial scale. 
This project takes forest species from the Amazon basin as its scope, rather than selecting a particular Amazonian community. Some specialise in moist rain forest environments, and some are also found in drier habitats such as cerrado.

\subsubsection{Human impacts}

\subsubsection{Pre-Columbian}

The first arrival of human populations in Latin America is thought to have coincided with the end of the last Ice Age, around 13,000 BP. These were the descendants of 'Clovis' peoples colonising North America, although there are claims for earlier colonisers (Meltzer 1995). The subsequent rapid extinction of megafauna from the Americas, including mammoths, large cats, horses, camels, mountain goats and giant ground-sloths was probably anthropogenic, but this cannot be demonstrated from archaeological evidence (Diamond 1998). It is hypothesised that the widespread distribution of certain fruit trees is the result of seeding and clearing around preferred trees.

The arrival of Europeans in 1492 led to a rapid initial decrease in human population densities (an estimated $95 \%$ of Native Americans were killed by exotic diseases, Diamond 1998). PreColumbian population densities over most of Amazonia are difficult to estimate with any degree of certainty, but it is thought that up to 5 million people were present, with populations concentrated along major rivers (Denevan 1992, Clement 1997). The area of forest altered by swidden farming and agroforestry will have declined accordingly.

\subsubsection{Modern}

Threats to tropical forest from modern humans are more immediate than longer-term threats from climate change and may have a greater overall impact: where deforestation has occurred, the occurrence of suitable climates is irrelevant (Bawa \& Markham 1995). However, a less optimal climate can decrease the rate of recovery from damage. The combined impacts of climate and land use change may particularly affect the seasonal forest of southeastern Amazonia, which is at the physiological limits of tropical rain forest, and is the part most threatened by population pressure and associated fires (Laurance 1998).

\subsection{Deforestation}

Deforestation and forest degradation is largely the result of agricultural conversion by subsistence and large-scale farmers. Roads built as highways, or for access to logging areas, hydroelectric plants or mineral extraction, provide access to land. Some of this land take is illegal, some is officially sanctioned. $12.9 \%$ of the Brazilian Amazon region has been now 
deforested, with a further 6,000,000 ha of roadside land officially designated available for agricultural use from 1997 (Laurance 1998).

Deforestation leads to the extinction of small-range endemics. Adjacent regions are susceptible to drying and other edge effects such as wind turbulence leading to tree fall. Fragmentation of forests can lead to further biodiversity loss (section 4.2.6).

\subsection{Extraction}

12 million ha of forest in Brazil, Guyana, Suriname and Bolivia are owned or controlled by multinational companies. In Brazil, an estimated $80 \%$ of logging is thought to be conducted illegally (Laurance 1998), although some of this occurs on multinational land. Most of this logging is selective extraction rather than clear-felling.

Extraction has direct effects on the target populations unless it is sustainably managed. Commercially favoured timber species are taken, with the larger mammals also being harvested by the logging crews and support workers. Species with low reproductive rates are most threatened by uncontrolled harvesting. Changes in the numbers of herbivores, predators and mutualists (e.g. seed dispersers) can have longer-term effects on plant community composition.

Selective extraction causes structural change, ranging from construction of logging roads and trails and incidental destruction of vegetation surrounding target trees. When a canopy tree is felled, two gaps arise: one where the crown was located, and one at the site of impact of the falling crown. In one Brazilian location, $1.7 \%$ of trees of $>10 \mathrm{~cm}$ diameter were harvested, but $26 \%$ of the remainder were killed or damaged, including $11 \%$ that were bulldozed (Uhl \& Kaufman 1990). This damage affects $10-40 \%$ of forest biomass over an estimated 10,000 to $15,000 \mathrm{~km}^{2} \mathrm{y}^{-1}$ (Nepstad et al. 1999).

The creation of multiple canopy gaps leads to denser forest floor vegetation, which together with debris from the logging operation and greater sunlight penetration increases fire risk. In logged forest, fuels were dry enough to burn after 5-6 rainless days (Uhl \& Kaufman 1990).

\subsection{Development}

The mineral and hydrological resources of the Amazon basin have a high potential economic value. There is considerable investment in economic development in the region, largely in areas spreading out from the farms. In Brazil alone in 1995 there were plans for 79 new 100-13000 MW hydroelectric dams, each with its own access roads (Fearnside 1995b). Some existing dams have flooded river valleys hundreds of kilometres long (Fundação Instituto Brasilieiro de 
Geografia e Estatística 1995). Current plans exist for oil exploitation with export pipelines stretching across several degree squares (Laurance, pers. comm. 2000). Major road-building plans also exist. The area of land taken by each of these activities is likely to be far outweighed by the area subject to secondary deforestation.

\subsection{AMAZONIAN BIODIVERSITY}

Environmental variation and change has been instrumental in the development of the diversity of species found in the Amazon basin, by stimulating different physiological, morphological and phenological adaptations. As the aim is to assess the possible effects of climate change on overall biodiversity, aspects of that biodiversity should first be explored. Possible mechanisms for the high species richness, and the types of adaptations employed by species in the Amazon basin are introduced here.

\subsubsection{Biodiversity definitions}

The biodiversity definition given in section 1.4 .1 is only one interpretation of the concept. The particular field of interest affects the definition chosen. A phylogenetic definition is:

“...The sum of genetic variation held in the individuals present at a site" (Williams 1998a).

A functional ecology definition concentrates instead on the role of the phenotype:

"...The processes that contribute to ecosystem function, and not the individual species present." (Silver et al. 1996).

Whereas this dictionary definition avoids functional aspects, but includes the abiotic environment:

"The variety of organisms considered at all levels, from genetic variants of a single species through arrays of species to arrays of genera, families and still higher taxonomic levels; includes the variety of ecosystems, which comprise both the communities of organisms within particular habitats and the physical conditions under which they live; the totality of biological diversity" (Lincoln et al. 1998).

Despite the different interpretations, taxonomic and functional concepts of biodiversity are closely related in practice, as morphology is largely the basis for classifying individuals into both species and functional groups. 


\subsubsection{Measures of botanical biodiversity}

The species is the most common unit of measurement in biodiversity indices. All species definitions are slightly fuzzy, but it is usually agreed that if two individuals are able to produce fertile offspring, they belong to the same species ('the biological species concept'). Obligate asexual plant species are infrequent, but individuals are assigned to these species, as others, using morphology or genetic analysis.

Biodiversity indices have been developed to measure community diversity, or to weight species according to their local abundance. Each can be applied at $\alpha$ (inside a site or community), $\beta$ (between sites) and $\gamma$ (regional) levels (Whittaker 1965).

The simplest measure is plant species richness. The measure is always applied using a subset of the taxa found at a site, because of the difficulty of assembling specialist knowledge to identify all groups present. It is often assumed this reflects overall richness. As trees are often surveyed because of their contribution to forest structure and biomass, and the available taxonomic expertise, inter-site comparisons of tree data are most common.

Indices for one taxonomic or functional group are often not reliable for predicting the spatial behaviour of other groups. It cannot be assumed that one group acts as a surrogate for others at the same taxonomic level (Brown 1995 p.144; Faith \& Walker 1996). It has been suggested that at a species level, bushy plants and ferns would make better richness indicators than trees, because overall richness is lower (at least in western Amazonia) and it is simpler to obtain specimens (Ruokolainen et al. 1997). However, it appears that diversity of non-tree taxa is almost inversely correlated with diversity of tree taxa (Gentry 1992).

Where a phylogenetic biodiversity measure is the main requirement, the use of higher taxa could result in surveys that are more inclusive with less recording effort. However, higher taxon richness does not correlate well with species richness (Williams \& Gaston 1994) or functional diversity (Gaston 1994) across sites.

Several indices measure evenness in the allocation of individuals or biomass across species. The most common is the Shannon-Wiener index, which incorporates the abundance or percentage cover of all species in the set (Goldsmith et al. 1996). The Chao index (Chao 1984) is intended to be less arbitrary in its weighting. These indices are inappropriate for use with presence data such as that available for Amazonian species.

Another measure encompasses the concept of taxonomic distinctiveness (Vane-Wright et al. 1991). An area containing a large number of closely related species is defined as less diverse 
than an area containing a smaller number of distantly related species. It would be possible to weight impacts on species from speciose families less highly because of their reduced contribution to genetic diversity, or to assign a greater biodiversity value to regions holding species from deeply divergent lineages. The incomplete and changing tropical phylogeny means that this approach would be rather imprecise for Amazonian species. Species in this study are therefore treated equally regardless of phylogeny.

\subsubsection{Species richness patterns and origins}

A latitudinal gradient in richness, with higher counts at lower latitudes, has been observed in many taxa, though some show an asymmetrical pattern about the equator (Woodward 1987, Brown 1995, Blackburn \& Gaston 1996, Lawton 1999). Why are there so many more species in the tropical than the temperate zone, and why so many in Amazonia? Various hypotheses exist, based upon different theories of biodiversity maintenance. These concepts provide a background for potential climate change impacts on rates of species loss and accumulation.

Within Amazonian forest, $\alpha$ and $\beta$ diversity have been thought to follow different patterns. At $\alpha$ level, a few species usually predominate in individual counts (an 'oligarchic forest', Campbell 1993, Hubbell \& Foster 1986). At $\beta$ level, the identities of some of these oligarchic species differ between patches (e.g. Hubbell \& Foster 1986). Biomass is thus more evenly allocated over more plant species, although most are rare in all occupied habitats (section 2.2.4.2). For example, $63 \%$ of tree species in a 1 ha plot of aseasonal lowland rain forest at Yanamono, Peru were represented by one individual (Gentry 1988). Recent analysis of Peruvian and Ecuadorian forest inventories suggests that some species predominate over thousands of $\mathrm{km}^{2}$ (Pitman et al. 2001), producing a few common and many rare species at both $\alpha$ and $\beta$ scales. The majority of species are rare, but the majority of individual trees belong to a much smaller set of perhaps a few hundred common species.

\subsubsection{Species accumulation}

Species richness increases when new species appear through immigration or speciation, and the rate of addition is higher than the local extinction rate. One explanation for the relatively high species richness in tropical forests is that many opportunities for speciation have occurred, with few losses. For example, 70\% of genera from Oligocene times (38-26 my BP) are still found on the island of Puerto Rico (Bawa \& Markham 1995). Various mechanisms for a comparatively high speciation:extinction ratio have been proposed.

Speciation events can be classified as sympatric (a new species emerges in situ), allopatric (a new species emerges from an isolated population) and parapatric (divergence occurs in spatial 
segregation, but the resulting populations are contiguous) (Lincoln et al. 1998). Speciation occurs because of changing environmental opportunities (an open niche), or selection pressure on existing species (a closing or shifting niche). Adaptation to one factor may open unrelated new opportunities. The speciation rate and direction of adaptive response is influenced by the original species' genome. Selection pressure leads to local extinction if the rate of change exceeds the rate of adaptive response.

\subsection{Amazonian speciation}

The proposed tropical speciation mechanisms include (Haffer 1997):

- Parapatric speciation along current environmental gradients, with low levels of disturbance minimising extinction rates, including specialisation for marginal habitats (Gentry 1992);

- Allopatric speciation on either side of natural barriers, especially shifting river meanders;

- The 'refuge hypothesis', in which allopatric speciation occurs in isolated centres of endemism during dry periods of the Pleistocene epoch;

- The 'disturbance-vicariance hypothesis', in which shifting vegetation zones caused by periodic Pleistocene cooling promote adaptive parapatric speciation;

- Allopatric speciation resulting from tectonic changes resulting from the last Andean uplift in the Miocene epoch, 26-5 my BP (da Silva \& Patton 1998);

Contentious subjects include the locations of proposed centres of speciation, the effect and historical location of dispersal barriers such as rivers and mountains, and the effects of environmental change over the Cenozoic era (65 my BP - present). The mechanisms that invoke speciation in the Pleistocene or earlier suggest that species survived at least one major climate change (warming to reach the current climate). Survival responses may have included dispersal or adaptation. Available palaeoecological and present data are far from sufficient to give an overall biogeographic history (Tuomisto \& Ruokolainen 1997).

The evidence for dry climate periods and associated changes in vegetation type during the Late Pleistocene is increasing, which supports the allopatric speciation hypotheses (Haffer 1997). Pollen samples taken from Lake Pata in northwestern Brazil contradict this evidence (Colinvaux et al. 1996). There is no evidence of the colonisation by grasses expected under the refuge hypothesis' assumption that the forest was replaced by savanna. The reduction of the lake level at this time agrees with the hypothesis that drying took place, but the pollen spectrum suggests a lowland rain forest invaded by additional species from higher altitudes - the forest persisted despite the cold temperatures and drying. 
Soil carbon typing over 14 Brazilian sites supports the presence of $\mathrm{C}^{3}$ (forest) rather than $\mathrm{C}^{4}$ (savanna) vegetation in mid-Holocene times (Martinelli et al. 1996) - an alternative possibility being that $\mathrm{C}^{3}$ grasses were dominant.

Centres of endemism proposed as refugia do not match well between taxa (Tuomisto \& Ruokolainen 1997), and the limited data hinders conclusive analysis. In addition, Amazonian palynological diversity during the Miocene was double that during the Holocene (Flenley 1998). This suggests that the Plio-Pleistocene epoch (5-0.01 my BP) showed a regional decrease in biodiversity. If high present diversity derived from refugia, one would expect the reverse to occur, following repeated dry phases and speciation within refugia.

\subsubsection{Stability and disturbance}

Even if many opportunities for speciation exist, how are such high levels of biodiversity retained? It may be that ongoing change in community composition maintains biodiversity by preventing competitive elimination by more vigorous species. Another hypothesis suggests that a stable environment promotes the specialisation of many species fulfilling subtly different niche requirements. The communities that would be formed according to these opposing paradigms have been named the 'dispersal assembly' and the 'niche assembly' (Hubbell 1997).

\subsection{Dispersal assemblies}

The intermediate disturbance hypothesis states that other things being equal, diversity should be highest at an intermediate level of environmental disturbance (Connell 1978). There is no need for an increased speciation rate. In undisturbed environments, the better competitors exclude other species, and at high levels of disturbance, most species are not able to complete their lifecycles. This hypothesis explains high Amazonian species richness as the result of gap formation processes in an otherwise stable environment. The rate of species accumulation is higher than the rate of species loss.

The 'gradual change' hypothesis suggests that environmental fluctuations or gradual changes lead to variation in competitiveness between species, favouring different species at different times (Connell 1978).

The 'equal chance' or 'drift' hypothesis suggests that a regional pool of equally competitive species with similar life strategies is maintained by random colonisation of local gaps (Connell 1978, Condit 1996, Condit et al. 1996c). It shares the island biogeography theory assumption of equal extinction probability between species (MacArthur \& Wilson 1967). Disturbances, speciation events, limited dispersal and the chaotic dynamics of a complex system with high 
species diversity can contribute to heterogeneity in local community composition independent of any environmental variation (Condit 1996). Support comes from work on secondary forest regeneration, where the initial availability of diaspores affects long-term forest composition (Finegan 1996), and from the low proportion of suitable gaps colonised by each species at Barro Colorado Island (BCI, in the Panama Canal) (2-6\% per species, Hubbell et al. 1999). Recruitment limitation of this type can mean that the species that occupies a gap is not the 'best' competitor in terms of growth.

\subsection{Niche assemblies}

Niche differentiation (or diversification) theory holds that each species occupies a unique environmental niche, however functionally similar it is to other species. Species in a stable productive environment diverge to exploit many different niches. Each species is superior at exploiting a particular range of conditions.

The niche is often defined as a discrete 'environmental envelope' for modelling purposes, but can more realistically be thought of as a fuzzy-edged zone in environment space, with internal gradients of occupancy probability. The 'core niche' is the zone with the highest probability of presence, and is likely to sustain the highest densities of individuals (Brown et al. 1995). 'Marginal' niche-space may be occupied by populations that are not self-perpetuating, but are replenished from the core area. In metapopulation theory, these core and marginal zones are termed sources and sinks.

Species may share preferences for environmental conditions, existing alongside one another and competing for the same resource. The fundamental niche is the set of all environments to which a species is physically adapted. The realised niche is the set of environments that it is able to occupy, given the effects of other species (MacArthur 1972). A guild is a set of species that share a modal niche and have similar ecosystem functions.

Two lines of thought suggest that biotic competition is important in defining niches in tropical forest. The 'Dobhansky-MacArthur hypothesis' is that range size is limited more by biotic than environmental factors as diversity increases, allowing more species per unit area at a small scale (Brown 1995). One mechanism for this is provided by the 'compensatory mortality hypothesis', which suggests that disease and herbivory diminish the dominance of good competitors within a guild, augmenting species diversity by permitting niche overlap (Wills et al. 1997). As a special case of the gradual change hypothesis, this places the dispersal assembly in a niche assembly context, but does not help to explain the latitudinal gradient. 
Species within a guild generally have different niche breadths. Some occupy environments outside the optimal niche and others ('intolerant' species) are restricted to it (MacArthur 1972, Kunin \& Gaston 1997). There is a trade-off between competitive ability under optimal conditions and niche breadth: specialised species must be able to outcompete generalists, in order to survive. Strategies for success can include interference competition (e.g. production of allelopathic chemicals that obstruct other plant growth), or an ability to colonise preferred patches faster.

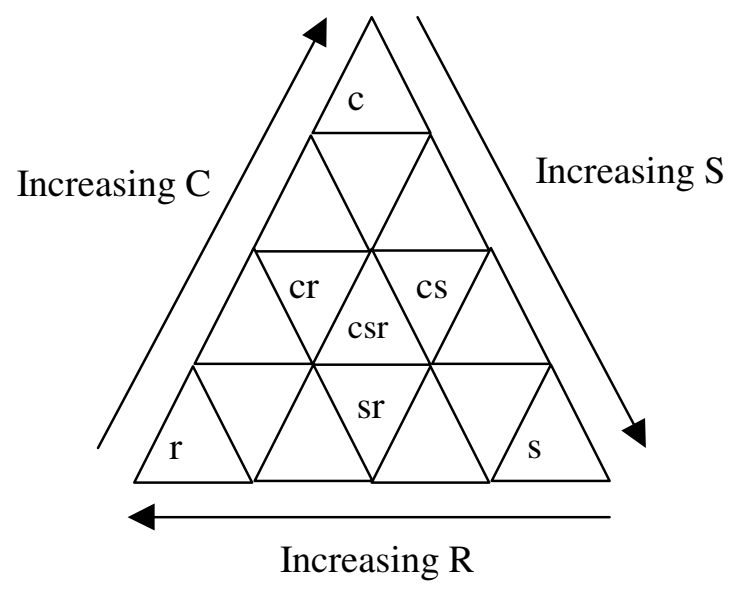

Fig. 2:3: Grime's competition-stress-disturbance triangle

During adaptive speciation, plants adopt new strategies in response to environmental variation. The range of possible strategies is summarised in the CSR triangle (Fig. 2:3, Grime 1977), which places species along three gradients based on resource availability and degree of disturbance. Rain forest habitat would be placed on the $\mathrm{C}$ sector of the global triangle. Within this zone, competitive (c) plants such as canopy trees succeed in productive, low-disturbance environments, stress-tolerant (s) plants such as non-vascular epiphytes in low-resource, lowdisturbance environments, and ruderal (r) plants such as riverine species in productive, disturbed environments. An earlier scheme based on the intrinsic rate of increase of a population differentiates only climax ('k-selected' $=\mathrm{c}$ and s) and pioneer ('r-selected' $=\mathrm{r}$ ) strategists (MacArthur \& Wilson 1967). However, many tropical rain forest species behave as pioneer or climax types depending on local conditions (Gillison \& Carpenter 1997).

The breadth of a species' environmental niche determines how much of the CSR triangle area it occupies. A high degree of overlap between two co-occurring species from the same canopy level (e.g. two bushy species) suggests competitive (or mutualist) interactions.

Stress is a relative term, as 'stress-tolerant' (s) plants may be poorly adapted for 'low-stress' conditions (Stott 1998). However, few plants can withstand both low resources and frequent disturbance; hence the diagram has only three apices. 


\subsubsection{Productivity}

Low ecosystem productivity may place some limits on species richness in temperate regions, whilst fluctuating environments limit species' ranges. Very low productivity certainly reduces structural diversity, and there will be fewer species where there are fewer vegetation layers. However, the relationship between productivity and biodiversity seems to be hump-shaped, with the most productive areas not always hosting the most species (Blackburn \& Gaston 1996). There is also a latitudinal gradient in the division of biomass between species: individuals are allocated more evenly between species at higher latitude.

\subsubsection{Taxonomic artefacts}

It is sometimes suggested that systematic differences in the way in which tropical and temperate workers classify species lead to a greater number of species being allocated to individual tropical genera (Walters 1986). Conversely, specimens are often classified into 'morphospecies' pending further work, pooling sibling species and decreasing biodiversity estimates (Campbell 1993). The decision to split or lump particular groups into species based on morphology affects richness estimates to an unknown degree, but cannot provide a sole explanation for high Amazonian biodiversity.

\subsubsection{Implications for future change response}

It is difficult to support one set of ecological hypotheses over another. As in other widelyobserved patterns, it is likely that more than one of the causes postulated is operating or has operated to maintain to regional biodiversity (Gaston \& Blackburn 1999). The hypotheses suggest possible responses of biodiversity to change. For example, if refugia form in response to climate change, a new wave of speciation may be encouraged in the long-term. If stability is more important in species accumulation, then climate change will be wholly negative for richness. The greatest uncertainty surrounds the likely response to environmental change of narrow-range species: widespread species can be seen to be limited primarily by environmental factors (Pitman et al. 2001).

\subsubsection{Plant functional requirements}

When considering variations in response to climate change, it is desirable to include species with a range of environmental tolerances and life history modes. If information on the timing of speciation and subsequent distributional history were available, estimated historical climate data would identify not only the range, but also the rates of change of conditions tolerated. In the absence of this information, current distribution patterns and functional traits can be used to ensure that this functional diversity is represented. 
Grime's triangle offers a simple basis for classifying plants whose ecology is well-understood (section 2.2.2.2.2). However, autecological knowledge of the vast majority of Amazonian forest species is very limited. As similar forms are adopted for similar purposes across unrelated taxa, many assumptions about species preferences are based on their morphology. So, how are the environmental niches of different Amazonian groups differentiated, and how does this provide a basis for modelling their distribution and response to change?

Plant survival depends upon the physical resources of light, water, nutrients, carbon dioxide $\left(\mathrm{CO}_{2}\right)$ and a physiologically optimum temperature. Other organisms may assist or hinder the process of biomass accumulation and storage as discussed, but this is a secondary filter on distribution (Lawton 1999). This section briefly considers the environmental requirements common to all plants, and the range of adaptations employed.

\subsubsection{Light}

In tropical rain forest, light availability depends more upon the position of an individual plant within the forest structure than upon seasonal variations. Incident radiation diminishes with the density and structure of the canopy levels above the plant, and the spectrum changes due to preferential absorption by higher levels. Taller plants therefore experience greater light availability (Eqn. 1):

$$
I=I_{o} e^{-K L}
$$

where $I=$ irradiance reaching level, $I_{\mathrm{o}}=$ irradiance outside canopy, $k=$ coefficient of canopy architecture (from angle of leaves), $L=$ leaf area index (LAI).

When working at a coarse scale, one can assume that canopy gaps are homogeneously distributed between cells. Colonisation opportunities for species with a particular light requirement are limiting at a finer scale. Hence, this factor is more useful in simulating very local variation than in comparing environments across the Amazon basin.

\subsubsection{Temperature}

Temperature is a fundamental control on plant distributions, and is included in many predictive models (e.g. Huntley et al. 1995, Jeffree \& Jeffree 1996). It is a direct factor in hydrological cycles and is a surrogate variable expressing the availability of solar energy. Physiological processes such as photosynthesis can only occur within certain temperature ranges. High temperatures may limit pollen tube survival or formation relative to flower persistence time. 
Temperature optima and constraints vary between species (e.g. Hill et al. 1988). The optimum temperature for photosynthesis may even vary between leaves on a plant - there is a high level of adaptation to the microenvironment. High temperature tolerance is related to the ability to retain moisture under warm conditions, and to physiological temperature dependence. In addition to metabolic tolerances, some plants are adapted to high temperatures by reflecting incident radiation with a high albedo, absorbing it with a hairy or spiny surface, or avoiding it with a low surface-area:volume ratio.

Tolerance ranges increase with latitude in many groups, following the latitudinal increase in seasonality, but this effect has not been investigated for neotropical species (Gaston et al. 1998).

\subsubsection{Moisture availability}

Plants require water in photosynthetic reactions, as a transport medium, for cell turgidity and evaporative cooling. Different selection pressures are exerted by gradients in annual water availability and gradients in seasonal precipitation. That is, different adaptive responses are required for constantly wet or dry environments, and environments that undergo seasonal change in water availability. Aseasonal and seasonal forest types have distinctive floras, with some species occurring throughout the seasonality gradient and others being highly restricted (Pitman et al. 2001). High neotropical species richness is independently related to low seasonality and high annual rainfall - it seems that water is a stronger limiting factor than temperature (Clinebell et al. 1995).

There is a direct trade-off between growth rate (competitive ability), favoured in moist environments, and water efficiency, favoured in more arid environments. Photosynthetic mechanisms affect water requirements, with $\mathrm{C}^{3}$ plants being least efficient, $\mathrm{C}^{4}$ plants moderately efficient and CAM (Crassulacean Acid Metabolism) plants highly efficient. In seasonal environments, reproduction can be induced by the onset or end of the dry season. Deciduousness is also strongly linked with seasonality in moisture availability (Condit 1998). However, in the seasonal moist forest on BCI, only $37 \%$ of canopy species were deciduous.

Very moist environments also require adaptations - for example, many species have leaves with 'drip tips' whose function is to shed excess water. Presumably, their primary aims are to lose the additional weight which endangers leaf survival, to reduce scorching and to avoid supporting pathogens and parasites.

Water availability can be incorporated into distribution models as precipitation, humidity, soil moisture content, evapotranspiration or water balance. 


\subsubsection{Extreme events}

Amazonian forest species encounter extreme events in the form of fire, drought, flooding and tropical storms. El Niño events have a tendency to increase drought over northeast Brazil, accounting for $10 \%$ of the variation in precipitation in the region (Barry \& Chorley 1998). This also increases the likelihood of fire. In southern Brazil, Paraguay and northern Argentina, a strong El Niño event causes heavy rainfall. The effect on species' survival and reproductive success is strong and localised.

Fire frequency is controlled by fuel load in the understorey, moisture levels dependent on climate conditions and vegetation structure, and human activity. Fires spread from Amazonian agricultural plots into adjacent forest, with vegetation in partially logged areas being much more vulnerable to fire (Uhl \& Buschbacher 1985, Whitmore 1990, Fearnside 1995c). A low-level fire in moist forest opens the canopy, thus decreasing humidity. The fuel load in the understorey and forest floor increases because of leaf and branch fall with rapid seedling growth in response to the nutrient flush and canopy gaps (Cochrane \& Schulze 1998). The likelihood and potential impacts of future fires are thus increased: repeated fires are stronger and damage larger trees. Vegetation may shift from forest to savanna or scrub - it is hypothesised that frequent fire maintains areas in West Africa as savanna that would otherwise suitable for rain forest (Gignoux et al. 1997).

Extreme events are not easy to model because of the unpredictable nature of their temporal and spatial occurrence: for example, the ENSO cycle recurs every 2 - 10 years. Without a detailed history of past occurrence, it is difficult to simulate effects on species' distribution. In a deterministic modelling framework, the event could be applied cyclically at its return frequency, or the model iterated in supra-annual blocks and the impact of extreme events over the set of years applied according to the probability of occurrence. The events are best modelled using a stochastic model that must be run many times to obtain a standardised result, and generate a probability estimate of species loss per cell.

\subsubsection{Soils}

Non-climate variables such as soil type and nutrient status are often omitted from species' distribution hypotheses. Plant colonisation and survival is affected by the $\mathrm{pH}$, mineral content, soil water holding capacity (WHC) and texture of the soil. On a local level, edaphic variation can explain the distribution of different communities (e.g. Ruokolainen et al. 1997). Fern communities have been correlated with soil fertility classes on a regional level in the northern Neotropics (Tuomisto \& Poulsen 1996). 
However, when species richness was examined over a large area of the Neotropics, the impact of soil variables was explained by correlation with water availability (Clinebell et al. 1995). Nutrient content was of secondary importance. Drier conditions were associated with more fertile soils, presumably because of limited leaching. As tropical rain forest cycles nutrients from leaf litter, it is less dependent upon nutrient availability than are more moisture-limited vegetation types such as cerrado.

Soil drainage levels together with precipitation and temperature do control water availability. As much of the Amazonian forest subsists on a sandy, infertile soil, a small decrease in water availability might threaten its persistence (Brown \& Prance 1987). The fine scale of variation of soil type rules out the inclusion of soil as a categorical variable in the current project, but its mean influence on moisture availability can be taken into account at a coarser scale.

\subsubsection{Biotic interactions}

Individuals are broadly dependent upon a web of interactions between other organisms that is dynamic, variable between sites, and contingent upon the community composition and the species' characteristics (Lawton 1999). Some interactions do have predictable effects. Seeds and seedlings are particularly likely to experience fatal disease, herbivory and shading. These are density dependent according to optimal foraging theory and field evidence (Augspurger 1983, Křiven 1996), so that seedlings at high density, or near to adult conspecifics suffer increased levels of background mortality.

Interspecific interactions can be more complex. Specific pollinators or dispersers can act to limit distribution, but most are generalists. 'Myrmecophytes' have a mutualist interaction with ants. The plants provide shelter ('domatia' for nest-building) or food (extrafloral nectaries or seed appendices), whilst the ants remove herbivorous insects, epiphytes or plant competitors (e.g. Morawetz et al. 1992).

\subsection{Pollination}

One might assume that the low population densities of many tropical trees would be linked to high levels of selfing, but self-fertilisation is uncommon (Nason et al. 1997). A reduced reproductive rate at low density has been observed in dioecious species at BCI (Hubbell \& Foster 1986). Pollination agents and the availability of non-self pollen are therefore important requirements for reproduction.

High levels of insect pollination, weak winds inside the forest structure, and heavy rains during peak flowering times that precipitate wind-disseminated pollen have been thought to result in 
generally short pollination distances. In a BCI study, 65\% of pollen-species had an estimated pollination distance $>20 \mathrm{~m}$, but only $20 \%>40 \mathrm{~m}$ (Bush \& Rivera 1998). The maximum recorded insect transfer distance is $4 \mathrm{~km}$, for fig wasps on a rare host. At the low extreme, the vine Capparis frondosa has a maximum measured pollination distance $<5 \mathrm{~m}$.

Species pollinated by generalist insects suffer the disadvantage that their pollen may be deposited with another species. This is increasingly likely at low density. However, they may be better able to survive forest fragmentation and the corresponding reduction in insect species richness (Cane 2001). One set of trees in fragmented Amazonian forests found a new pollinator in non-native Africanised honey bees (Dick 2001).

A study of 232 Venezuelan species from several habitats showed that pollination mode is also linked to the proportion of seeds formed and aborted per fruit. More viable seeds per fruit were formed by wind, beetle and moth pollinated species than fly, bird, bat or hymenopteranpollinated species (Ramírez 1995). Flies seem to be particularly poor pollinators, but the flypollinated species provided a relatively high number of ovules, presumably in compensation.

\subsection{Mycorrhizal associations}

A common mutualism occurs between plant roots and mycorrhizal fungi. Mycorrhizae fall into two types: ectomycorrhizae (EM) and arbuscular (endo-) mycorrhizae (vesicular arbuscular mycorrhizae or VAM) (Guehl et al. 1998). VAM are non-specific, and tend to occur in high richness habitats. EM are more specific and more frequently found in low richness habitats. Most tropical forest mycorrhizae are VAM, therefore plants dispersing between forest patches are likely to find suitable mycorrhizae. The fungi migrate separately from the plant, re-infecting seedlings. VAM produce their spores underground, so that dispersal rates are slow, often mediated by animals (Wilkinson 1998), whilst some EM have above-ground fruiting bodies. Specificity, reproduction and dispersal of mycorrhizal associations, as well as the environmental tolerances of mycorrhizae, may limit plant dispersal and eventual range to an unknown degree.

\subsection{Dispersal}

Seed morphology is linked to the current or ancestral dispersal mode. Seeds can be dispersed by wind, animals, projection from the plant, flowing water or gravity. Most tropical forest species are vertebrate-dispersed. Most fruit trees are dispersed by frugivorous guilds rather than hostplant specialists. Many seeds are secondarily dispersed (or eaten) by ants or ground mammals, reducing concentrations around frugivore perches or latrines (Byrne \& Levey 1993, Fragoso 1997). It is in a plant's interest to restrict seed dispersal to sites within its ecological niche, whilst distributing seeds widely enough to avoid density-induced competition, and densely 
enough to allow pollination between individuals if necessary. Plants specialising in stable habitats are likely to have small dispersal distances. Opportunists and more generalist plants are likely to have longer distances. This affects the rate of distributional response to change.

Low sub-canopy wind speeds limit dispersal distance, and under shaded conditions a seed mass larger than can be wind-dispersed has a competitive advantage (Lord et al. 1997, Howe \& Smallwood 1998). Most wind-dispersed species are canopy to emergent trees or vines. In seasonal forests, wind dispersed fruits tend to be produced in the dry season, when dry, light seeds can most easily be produced, and animal dispersed fruits in the wet season, when water is available to build seed mass (Howe \& Smallwood 1998).

Demographic models suggest that long distance dispersal events govern the rate of spatial spread, even when these are uncommon (Neubert \& Caswell 2000). There is much debate around the appropriate functions to estimate rates of spread (Clark et al. 2001), so historical data would be useful to assess past performance. Neotropical plant migration rates have not been recorded, but the European palynological record following the end of the last ice age from 12,000 to $6,000 \mathrm{BP}$ indicates that the mean rate for forest tree species was $300 \mathrm{my}^{-1}$ (Huntley \& Birks 1983). Climate changed little after 9000 BP, but it took an additional 3000 years before vegetation reached equilibrium.

\subsubsection{Species distributional patterns}

A species' occupied range depends upon its dispersal history within its potential range, which is defined by its tolerance of existing environmental conditions, especially climate and soils. As seen above, both the potential and realised niche can be affected by multiple factors. Monographs describe realised range at the macro-scale, and site surveys describe abundance at the microscale. It is not always clear whether a restricted range is real or a recording artefact, but neotropical forest surveys illustrate a predominance of uncommon species. This section considers the types and causes of spatial distribution seen in Amazonian species, focusing on uncommon species.

The definition of rarity is dependent upon geographical scale, an abundance threshold and the relative rarity of other species. Nonetheless, a high proportion of Amazonian forest species appear to be rare, with a restricted abundance and range size, or sparse, with a wider range but a restricted number of occurrences in each site. Range size is often positively correlated with maximum local population abundances, so that repeated rarity is more usually an indicator of restricted range (Gaston \& Blackburn 1999, Lawton 1999). 


\subsubsection{Patterns seen in numerous species}

\subsection{Common: ubiquitous distribution, high density}

There are three main causes for ubiquity at high density:

1) A broad or very common environmental niche, leading to widespread distribution.

2) Successful adaptation to widespread stressful conditions that few species can tolerate - e.g. savanna grasses.

3) A successful recent invasion, to which the original species community has not yet adapted. These species are most likely to be resilient to climate change.

\subsection{Common endemic: restricted distribution, high density}

These endemics are 'intolerant' of environmental conditions outside a narrow range, but highly successful within this range. This pattern can develop when:

1) The niche is narrow and regionally rare.

2) The niche is narrow; the species appears in an isolated patch and has a low dispersal ability. The high density indicates that the species is probably not under current threat, but these species are vulnerable to climate change.

\subsubsection{Patterns seen in uncommon species}

\subsection{Sparse: ubiquitous distribution, low density}

To maintain this type of metapopulation, a highly dispersed species must be capable of long distance propagule dispersal (and pollination where required). Potential causes of this 'hyperdispersion' are:

1) The species has rarely fulfilled requirements. For example, extreme heliophiles require large gaps for regeneration, which only occur in closed forest following extreme events like fire or hurricane. Heliophiles may have population sources outside the forest. Other hyperdispersed species may specialise in colonising uncommon substrates or flooded areas.

2) The species is subject to predation (herbivory) or disease pressure when clumped. Selective removal by humans is a potential factor.

3) Hyperdispersion at a microscale can result from inverse density dependence because of interference competition.

The dispersal capabilities of these species make them resilient to change in the location of their niches, but the specialisation renders them vulnerable to change in patch frequency. 


\subsection{Rare: restricted distribution, low density}

The classical log-normal distribution of frequency by species number in an undisturbed stable community (Preston 1948) suggests that relative abundance for any one species is determined accidentally, resulting in a standard distribution of abundance. A set proportion of species will thus always be approaching extinction (Kunin \& Gaston 1997). There are many more species in the rarest portion of the neotropical curve than would be expected from Preston's distribution (Hubbell \& Foster 1986), indicating that rarity is not randomly determined.

Potential causes of rarity, and their implications for population status, include (Hubbell \& Foster 1986, Kunin \& Gaston 1997):

1) Resource dependency:

a) Specialisation to a restricted environmental niche, leading to a restricted distribution that mirrors the habitat. Restricted species are expected to be highly competitive in their favoured niche (section 2.2.4.1.2). The population is probably stable.

b) Adaptation to a decreasing available niche, for environments that were common under historical climates. Population is probably becoming increasingly rare.

c) Infrequent occurrence of regeneration requirements. The population is senescent, but may recover if requirements recur. Population is probably becoming increasingly rare.

d) Population has not recovered from reduction in numbers following an extreme event. Population may be becoming increasingly rare or recovering.

e) Large size relative to resource availability limits number of individuals. Population is probably stable.

2) Competitive interactions:

a) The species is favoured by a predator or pathogen and has low resistance levels at high densities. If this mortality source is novel, the population may be in terminal decline.

b) Outcompetition by other species in the most freely available niches. Population is probably stable.

3) Evolution and demography:

a) Low numbers because species is a recent or occasional immigrant. Population may be becoming more common. If there are low regeneration rates, the population may be a sink in metapopulation terms (Bowers \& Harris 1994).

b) The species may be poorly adapted to its environment and less able to respond to natural selection because of high levels of self-fertilisation or asexual reproduction. Population is probably becoming increasingly rare.

c) Low numbers may lead to narrow genetic pool through inbreeding and genetic drift, reducing survival probabilities and reproductive rate. Population becomes increasingly rare. Substantial drift is expected in populations smaller than 20 individuals, moderate drift with 200 individuals, and insignificant levels with over 1000 (Nason et al. 1997). 
d) Recent speciation. Population may be becoming more common. Species able to reproduce asexually (e.g. by vegetative propagation) are more likely to survive the demographic stochasticity found in small populations.

4) Spurious rarity:

a) The species is rarely observed (e.g. cryptic) or rarely appears in the size class that is being collected, without having a low abundance. Population is not rare.

Some causes of rarity imply a declining population, and some a stable one that is adapted to persist at low numbers. Rarity always renders a species more vulnerable to extreme or stochastic events. Extinction results from a reduced reproductive capacity and increased susceptibility to genetic and pathogenic disease. In small populations, increased localised extinction probabilities result from the loss of adaptive capacity following genetic bottlenecks (the founder effect) and drift (stochastic loss of diversity), together with Muller's ratchet (inbreeding effects). These positive feedback mechanisms are collectively termed Allee effects. Vegetatively reproducing species are not affected by Muller's ratchet, so may survive as rare populations for longer (Kunin \& Gaston 1997).

These factors should be considered when modelling species' distribution or demography. At the least, the recognition of processes excluded from a model helps understanding of when it may be applied! 


\section{Modelling climate change}

The assumption that changes in global climate will impact species' success and distribution is based on projections from General Circulation Models (GCMs). This section reviews the evidence for climate change, variations seen in recent and current GCMs, and the limitations of climate modelling. GCM structure, processes modelled, spatial resolution, outputs and assumptions are considered. Methods for selecting GCMs for species modelling, evaluating GCM results, obtaining fine-scale data from coarse GCM outputs are also evaluated. Key considerations are GCM success at representing current Amazonian climate, its variability and the extremes seen in the El Niño cycle.

\subsection{FACTORS IN CLIMATE CHANGE}

The climate system is dynamic, with a transient balance in energy flows over longer periods. This balance shifts in response to external or internal forcing. Potential changes include altered levels of incoming radiation (external forcing and altered levels of tropospheric gases blocking flow of longwave (infrared) radiation out of the system(internal forcing).

\subsubsection{Human impacts on climate}

Anthropogenic changes in atmospheric composition have an impact on heat transfers. The principal greenhouse gases (GHGs) involved in internal forcing of the climate system are water vapour and carbon dioxide $\left(\mathrm{CO}_{2}\right)$. Smaller volumes of methane $\left(\mathrm{CH}_{4}\right)$, nitrous oxide $\left(\mathrm{N}_{2} \mathrm{O}\right)$, chlorofluorocarbons (CFCs, released since the 1930s) and tropospheric ozone $\left(\mathrm{O}_{3}\right)$ also play a part (Houghton et al. 1996). Carbonaceous and sulphate particulate aerosols can also affect atmospheric processes.

Anthropogenic $\mathrm{CO}_{2}$ emissions primarily result from combustion of fossil fuels and the reduction of photosynthetic biomass, i.e. by conversion of forests to pasture. The rate of increase in atmospheric $\mathrm{CO}_{2}$ concentration also varies due to the effect of climate on natural uptake. Annual increase in the $1990 \mathrm{~s}$ has been between $0.4 \%$ to $0.8 \%$ per annum (Houghton et al. 2001). As $\mathrm{CO}_{2}$ appears to be the most influential GHG, the impact of other gases is often described in units of $\mathrm{CO}_{2}$-equivalence.

In addition to their GHG status, CFCs have a secondary role in global warming via the decomposition of stratospheric $\mathrm{O}_{3}$ molecules by chlorine. Nitrogen oxides (NOx) emitted by jet aircraft or derived from $\mathrm{N}_{2} \mathrm{O}$ are required for this reaction. The use of fossil fuels and fertilisers increases $\mathrm{N}_{2} \mathrm{O}$ concentrations. 
Stratospheric $\mathrm{O}_{3}$ at high level (above $30 \mathrm{~km}$ ) absorbs more incoming shortwave than outgoing longwave radiation, so a reduction in stratospheric $\mathrm{O}_{3}$ may increase surface temperature. The Montreal Protocol of 1988 halted CFC production in order to reduce $\mathrm{O}_{3}$ depletion. Hydrogenated halocarbons (HFCs and HCFCs) are now substituted for CFCs. These have shorter lifetimes in the atmosphere, but retain strong greenhouse effects. CFC emissions are now very limited, but as their lifetime is 65-130 years, the atmospheric impacts are ongoing.

At tropospheric levels, $\mathrm{O}_{3}$ absorbs proportionately more outgoing longwave radiation and so increases heat retention (Barry \& Chorley 1998). This $\mathrm{O}_{3}$ results from the reaction of NOx and $\mathrm{CH}_{4}$ (Trenberth 1992). Two-thirds of atmospheric $\mathrm{CH}_{4}$ is estimated to derive from human activities such as enteric fermentation in livestock, anaerobic decomposition in rice paddies and biomass burning (Barry \& Chorley 1998).

The temperature rise resulting from global warming leads to increased evaporation of surface water. If the water vapour generated enters the troposphere, a positive feedback results. Negative feedbacks are also possible if the new vapour forms stratiform clouds at low level: the reflective effect of the increased albedo over a large area may be greater than the greenhouse effect of the water vapour (McGuffie \& Henderson-Sellers 1996).

Aerosols are small airborne particles derived from volcanic dust, industry, transport and agriculture. Aerosols are less clumped than clouds, and their effects depend on their composition and their location relative to clouds or the land surface. They alter local albedo, absorb outgoing radiation and scatter radiation in various directions (Kacholia \& Reck 1997). In addition, they form nuclei for condensation of moisture vapour, creating clouds composed of smaller drops in greater densities, which also deflect radiation. Cloud longevity may also be extended (Hulme et al. 1999). Their overall effect is thought to be cooling, but this is uncertain (Barry \& Chorley 1998). Fossil fuel black carbon aerosols are the only type thought to cause a positive direct radiative forcing (Houghton et al. 2001). Sulphate aerosols, which have a negative effect, are included in some GCM scenarios, but the uncertainty surrounding their interaction with clouds them difficult to model.

\subsubsection{Natural causes of climate change}

The natural variation in climate related to solar activity is not fully understood, which makes it impossible to definitively partition the human influence on climate. One theory is that although observed changes are correlated with increased greenhouse gas concentrations, they are caused by variation in solar forcing following sunspot cycles. An 11-year cycle of changes in solar 
brightness is well documented, and terrestrial temperature changes on the decade-to-century scale have shown some correlation with changes in solar cycle length, sunspot structure and related variables. The record also shows terrestrial changes that occur before changes in the solar envelope, indicating that long-term solar forcing is not the only determinant of climate change (Hoyt \& Schatten 1997).

This theory was tested using a historical climate model, which found that solar forcing had to be unrealistically high to act as a sole causative agent for the observed climate changes (Kelly \& Wrigley 1990). The authors suggest that a small fraction of warming may be related to solar activity, but that this is not great enough to account for observed trends.

Stratospheric aerosols produced by volcanic eruptions have a cooling effect. The combination of radiative forcing due to both sources of natural variation is thought to be negative over at least the 1980s-1990s (Houghton et al. 2001).

\subsection{EVIDENCE FOR RECENT GLOBAL CLIMATE CHANGE}

Global temperatures have been rising since the end of the Little Ice Age, around 1700. The 20th century warming of $0.5^{\circ} \mathrm{C}$ seems to exceed this trend by about $0.2^{\circ} \mathrm{C}$ (Barry \& Chorley 1998). There is a correlation with anthropogenic climate forcing mechanisms. It remains possible that natural variability is responsible for part or all of the increase, but the internal variability seen in climate models and reconstructions of climate data over the past millennium indicate that this is unlikely (Houghton et al. 2001).

Departing from these mean changes, the diurnal temperature range decreased by $0.8^{\circ} \mathrm{C}$ over at least half of the northern land areas during 1951-1990. One explanation is that increased cloudiness resulting from increased GHGs and tropospheric aerosols has reduced nocturnal heat loss (Barry \& Chorley 1998). The strongest temperature increase has been seen in winter, and has been accompanied by changes in latitudinal sea-level pressure gradients. Global circulation models (GCMs) with a detailed representation of the stratosphere have been able to simulate both trends. This supports the view that GHGs are exerting an effect via atmospheric circulation changes in addition to the standard warming mechanisms (Shindell et al. 1999).

According to the Intergovernmental Panel on Climate Change (IPCC) science working group (Houghton et al. 1996),

"the balance of evidence suggests that there is a discernible human influence on global climate." 
A trend of temperature increase by $0.56^{\circ} \mathrm{C}$ per century over the Amazon basin has been estimated based on data from 17 weather stations operating from 1913 to 1995 (Victoria et al. 1998). The analysis indicates that the trend became significant in the 1960s-70s, and so may be related to urbanisation and deforestation in the basin rather than global change. However, it corresponds to a similar trend, starting three decades earlier, for the whole of the Southern Hemisphere.

\subsection{Climate change impacts on tropical forests}

There is some evidence that bird, lizard and amphibian populations on higher ground at the Monte Verde Cloud Forest Reserve of Costa Rica have been affected by climate change (Pounds et al. 1999). Population changes are correlated with decreases in dry-season mist frequency, over and above the variation resulting from ENSO events. These are the type of decreases that might be expected from GCM scenarios.

However, the likely immediate cause is a change in cloud patterns following deforestation in lowland forests (Lawton et al. 2001). Various bird species from the lower mountain slopes have colonised the new dry areas upslope, and many herptile species have declined in abundance or vanished. Three frog mortality events coincided with the three annual peaks in dry day frequency. There is circumstantial evidence that these declines are linked to increased activity of parasitic flies following frog concentrations around water sources. These changes serve to highlight the complex relationships between local climate, land use and species survival.

\subsection{TYPES OF CLIMATE MODEL}

The history of climate modelling has involved an increase in the complexity of climate models, but the earliest models were not the simplest (McGuffie \& Henderson-Sellers 1996). Simpler statistical, one-dimensional or two-dimensional models may be used to examine aspects of a system in isolation and with greater computational efficiency.

\subsubsection{Statistical models}

The extrapolation of a time series of a climate variable via regression, with or without controlling variables, gives an estimation of future trends. If controlling variables are not included, it is assumed that the future rate of change of the causative factors (e.g. $\mathrm{CO}_{2}$ concentration) will remain identical to that of the period from which the response is extrapolated. This approach has been criticised for the lack of fit of the curves applied to the climate data, and the assumption that all causative variables act independently (Barry \& Chorley 1998). 


\subsubsection{One-dimensional models}

One-dimensional models provide hypotheses about how perturbations might affect processes. The simplest empirical model is the one-dimensional 'energy balance model' (EBM, Viner \& Hulme 1997). Variation in surface temperature is modelled over latitude. Units of energy move between cells at different latitudes by diffusion, and energy from insolation leaves and enters the system. Albedo is parameterised according to a simple formulation of cloud likelihood clouds in warm areas are not modelled. EBMs may be used to investigate the affects of different external forcings.

Radiative-convective models (RCMs) also simulate surface temperature, but consider vertical transfer of energy through the atmosphere rather than surface flow. Convection (mixing between layers) is applied if a threshold lapse rate is passed. These models can be used to study the effects of different atmospheric composition on energy flow.

\subsubsection{Two-dimensional models}

A two-dimensional model may simulate energy transfer over latitude-longitude, or more commonly, latitude-altitude. There are intrinsic climate gradients for latitude and altitude. Although the rotation of the earth causes any point's climate to have an additional influence on the climate due east of it, longitudinal variation is more related to surface geography and atmospheric circulation.

These 'statistical dynamical' (SD) models have been used to specify temperature diffusion coefficients for different latitudes, and to investigate baroclinic (pressure gradient) waves. Twodimensional models have also been used to simulate the effects of hundreds of atmospheric chemicals and reactions.

\subsubsection{General Circulation Models (GCMs)}

GCMs simulate climate and ocean processes over three-dimensions. Parcels of energy, water and mass move through a grid, simulating patterns of flow and their interaction with surface processes. The models are calibrated using historical data, and forcing mechanisms may be applied to stimulate change. Asynchronous GCMs treat atmosphere and ocean as separate submodels (AGCMs and OGCMs). Coupled GCMs (CGCMs) specify continuous interactions between the submodels. AGCMs and CGCMs may include spatially heterogeneous, interactive land-surface processes. 
Criticisms of the GCM approach include the uncertainty that the processes used to simulate the present climate system will accurately portray a response to changing conditions, and the difficulty inherent in testing models of future conditions. There is concern that models are 'tuned' to match current conditions, rather than based purely on known physical processes.

The coarse horizontal scale of the models means that critical interactions on a finer scale may be neglected. Unexpected feedbacks from processes intentionally not covered, because of the inevitable trade-off between complexity and efficiency, are also possible. GCMs are reviewed further below.

\subsection{Climate FORCING SCENARIOS}

The IPCC emissions scenarios offer a common set of modelling objectives for climate change researchers (Leggett et al. 1992). These scenarios simulate a range of GHG emissions from 1990 to 2100, denominated as IS92a-f (Table 3:1). Thus, most GCM change projections are run over this period. $\mathrm{CO}_{2}$ levels for 1850 are shown for a pre-industrial comparison.

The scenarios are based on various assumptions about future patterns of population and economic growth, and their effect on GHG emissions. Emissions are referred to in terms of 'equivalent $\mathrm{CO}_{2}$ ', using $\mathrm{CO}_{2}$ concentrations as a surrogate for the effects of all GHGs. The IPCC specify concentration-radiative forcing relationships for each GHG, which changed between 1992 and 1996, creating slightly smaller forcing scenarios for later models (Hulme et al. 1999).

IS92a became the standard scenario for low levels of international action on climate change. It uses a World Bank projection on population growth. Under this scenario, $73 \%$ of remaining tropical forests (1.4 billion ha) are cleared by 2100 , and only 118 million ha of forest planted.

IS92d represents the standard scenario for stronger international action. It reduces $\mathrm{CO}_{2}$ emissions from fossil fuels and deforestation, and also assumes a phase-out of HCFC-22 and a greater usage of HFC-134a, in full compliance with the Montreal Protocol and the London and Copenhagen Amendments. The IS92a halocarbon assumptions were later also adjusted to show full compliance (Houghton et al. 1996). The other scenarios are used less frequently. 
Table 3:1: IPCC 'equivalent $\mathrm{CO}_{2}$ ' emission scenarios (Leggett et al. 1992)

\begin{tabular}{|c|c|c|c|c|c|}
\hline $\begin{array}{l}\text { Scenario } \\
(1990 \rightarrow \\
2100)\end{array}$ & $\begin{array}{l}\text { World } \\
\text { population } \\
\text { (billions) }\end{array}$ & $\begin{array}{l}\text { Economic } \\
\text { growth } \\
\text { (annual \% } \\
\text { GNP) }\end{array}$ & $\begin{array}{l}\text { co2 } \\
\text { concentration } \\
(\mathrm{ppmv})\end{array}$ & $\begin{array}{l}\mathrm{CO}_{2} \\
\text { emissions } \\
(\mathrm{GtC} / \mathrm{y})- \\
\text { Kattenberg } \\
\text { et al. } 1996\end{array}$ & $\begin{array}{l}\text { Other scenario } \\
\text { conditions }\end{array}$ \\
\hline 1850 & - & - & $\begin{array}{l}270 \text { (McGuffie } \\
\& \text { Henderson- } \\
\text { Sellers 1996) }\end{array}$ & - & - \\
\hline 1990 & 5.25 & - & 355 & 7.40 & - \\
\hline IS92a & 11.3 & 2.3 & $\begin{array}{l}733 \text { by } 2100 \\
(1 \% \text { of } 1990 \\
\text { values per } \\
\text { annum) }\end{array}$ & $\begin{array}{l}20.8 \text { by } \\
2100\end{array}$ & $\begin{array}{l}\text { 'Business as usual' } \\
\text { pathway. Controls on } \\
\mathrm{NOx}, \mathrm{SO}_{2} \text { (causes } \\
\text { acid precipitation) \& } \\
\text { non-methane volatile } \\
\text { organic compounds } \\
\text { (NMVOCs). Mix of } \\
\text { conventional and } \\
\text { renewable energy } \\
\text { sources. }\end{array}$ \\
\hline IS92b & 11.3 & 2.3 & 710 by 2100 & $\begin{array}{l}19.11 \text { by } \\
2100\end{array}$ & $\begin{array}{l}\text { As 'a' plus } \mathrm{C} \\
\text { limitations on } \mathrm{CO}_{2} \\
\text { emissions. }\end{array}$ \\
\hline IS92c & 6.4 & 1.2 & 485 by 2100 & $\begin{array}{l}4.61 \text { by } \\
2100\end{array}$ & $\begin{array}{l}\text { As 'a' plus limited } \\
\text { oil \& gas supply, } \\
\text { reduced nuclear } \\
\text { costs. Population } \\
\text { declines after an } \\
\text { initial growth. }\end{array}$ \\
\hline IS92d & 6.4 & 2.0 & $\begin{array}{l}568 \text { by } 2100 \\
(0.5 \% \text { per } \\
\text { annum })\end{array}$ & $\begin{array}{l}10.33 \text { by } \\
2100\end{array}$ & $\begin{array}{l}\text { Halt deforestation, } \\
\text { capture coal-mining } \\
\text { emissions. }\end{array}$ \\
\hline IS92e & 11.3 & 3.0 & 986 by 2100 & $\begin{array}{l}35.84 \text { by } \\
2100\end{array}$ & $\begin{array}{l}30 \% \text { charge on } \\
\text { fossil-based energy, } \\
\text { nuclear phased out } \\
\text { by } 2075 .\end{array}$ \\
\hline IS92f & 17.6 & 2.3 & 848 by 2100 & $\begin{array}{l}26.59 \text { by } \\
2100\end{array}$ & As 'a'. \\
\hline
\end{tabular}

\subsection{GCM THEORY AND STRUCTURE}

Modern GCMs have a shared theoretical basis, using a set of equations to represent the laws governing climatic processes. However, variations in the mechanisms employed to simulate these processes, together with differences in initial parameterisation and physical structure, result in different outcomes from GCMs using the same emissions scenario. Much of the GCM information in this section was drawn from McGuffie \& Henderson-Sellers (1996). 


\subsubsection{Process simulation}

\subsubsection{Atmosphere}

The atmosphere is warmed mainly from the earth's surface, as energy from sunlight is reflected back upwards in the form of heat. Atmospheric GCMs (AGCMs) can model atmospheric energy transports via a 'finite grid' or a 'spectral' representation. That is, the transports are conducted either in gridded physical space, or in spectral space.

In a finite grid AGCM, the atmosphere is modelled as a set of boxes, which store the values of multiple variables (e.g. HADCM2, Johns et al. 1997). Transports are allowed on a horizontal and vertical plane. The latitudinal variation in each of these continuous variables can be considered as a periodic function. In a spectral AGCM, atmospheric variables within each horizontal layer are manipulated as waveforms (e.g. CSIRO Mark 2b, Hirst et al. 1996). Fourier transforms of variables in each timestep convert the continuous function (in the spatial domain) to multiple sine and cosine waveforms that hold the same information (in the frequency domain), and back again. Vertical radiative transfer and surface processes are still modelled in the gridded domain. The spatial resolution is set by the number of waves used to represent each variable in each latitude zone and vertical level. This is the 'wave-number of truncation', which is prefaced in the literature by an $\mathrm{R}$ for rhomboidal or $\mathrm{T}$ for triangular truncation, e.g. Table 3:2, p.48.

Spectral AGCMs have the advantage of elegance and reduced effort in the computation of gradients, but the disadvantage that the wave form variables stray outside their physical limits at steep gradients, for example simulating negative humidity. Alternative schemes for moisture vapour transport are employed in some spectral AGCMs to avoid this particular issue.

Atmospheric behaviour is modelled through a set of six basic equations, controlling temperature, moisture, surface pressure, two horizontal wind components (from which the vertical component is also derived) and geopotential height ${ }^{1}$. These are the hydrostatic equation, two horizontal motion equations, the thermodynamic equation, water vapour and mass continuity equations. These equations enforce the laws of conservation of momentum, mass, energy and enstrophy (the root mean square of vorticity ${ }^{2}$ ), and the ideal gas law. If the enstrophy conservation law is broken, the energy of motion is transferred to smaller and smaller spacescales, rendering the model unstable.

\footnotetext{
${ }^{1}$ Geopotential: the potential energy of a unit mass relative to mean sea level, equal to the work required to lift it to its current height, and varying with temperature and pressure. Geopotential height is the height above sea level measured in terms of atmospheric pressure.

${ }^{2}$ Vorticity: twice the angular velocity of a body (e.g. an air column) about the vertical.
} 
Radiative transfer in AGCMs deals with shortwave and longwave energy components. Incoming solar radiation is absorbed or reflected by atmospheric gases, clouds and the earth's surface. Cloud dimensions can be primarily vertical (convective) or horizontal (stratiform, most models assume these occupy the entire depth of an atmospheric layer). Cloud formation is not well understood, and occurs at scales well below that of the GCM. Cloud distributions, optical properties and the radiative effects of cloud height vary between models. . This variation is not reflected in the top-of-atmosphere and surface level radiation fluxes, because the differences in distribution and optical properties compensate for one another to achieve realistic model results in each case. This is therefore a strong source of simulation uncertainty.

Longwave radiation transfer is generally treated in several wavelength bands. Different gases are parameterised as having different abilities to transmit and emit energy in each band, which varies with temperature. This part of the model responds directly to increasing GHG concentrations, so that planetary warming ensues.

Convection is another sub-grid-cell process whose representation suffers from the GCM's scale. Mean atmospheric conditions over several degrees might not cause convection in a given instance, but convection might occur over a smaller area. Therefore, the effect of relatively small cumulus clouds may be omitted. Modern GCMs tend to use mass flux schemes to separate clouds and non-cloud parcels of atmosphere into two units, and treat fluxes of energy, moisture and mass within these units separately (e.g. Hulme et al. 1999).

Precipitation is modelled on a grid-cell basis, occurring when air in a layer becomes supersaturated with moisture.

The atmospheric boundary layer is the lowest layer of the AGCM, where it interacts with the OGCM and land-surface model (section 3.5.1.3). The representation of interactions with surface topography and vegetation is limited by GCM spatial scale.

\subsection{Change in internal forcings}

GHG concentration is altered in GCMs to simulate its impact on historical or future climate trends. This may be simulated as an instantaneous (an 'equilibrium' run) or incremental change (a 'transient' run). Transient simulations can be more useful for impact models, because they model ongoing change in climate rather than system response to a sudden change.

The primary role of GHGs in GCMs is their limitation of longwave energy transfer from the planet (section 3.1.1). Models usually specify gas concentrations in terms of $\mathrm{CO}_{2}$-equivalence, 
using the combined radiative forcing of all the greenhouse gases. Negative forcing from sulphate aerosols may be included via an increase in clear-sky surface albedo proportional to the local sulphate loading (Mitchell et al. 1995).

Variation in model outcomes resulting from a given degree of climate forcing also depend upon physical parameterisations that vary between models, particularly in interactions between radiation and clouds. Oceanic and land absorption and release of $\mathrm{CO}_{2}$ and moisture vapour control atmospheric concentrations of these GHGs, and are incorporated to varying degrees in different GCMs.

\subsubsection{Ocean}

Unlike the atmosphere, the ocean receives most of its energy from above. It conducts energy more slowly due to its greater thermal inertia. It also covers a more limited area of the planet and has currents affected by wind forcing and salinity levels. The ocean's heat storage and transfer capacities are important components of global climate. Ocean GCMs (OGCMs) can be categorised as slab and dynamic.

A slab (mixed-layer) model has a single ocean layer, well-mixed by interaction with the atmospheric boundary layer, often with homogeneous depth. Moisture and energy transfers can be coupled with the AGCM. It has fixed energy transports (ocean currents), but has the ability to absorb and transport additional energy. A detailed mixed layer model has vertical fluxes. OGCMs using a slab model have a limited response to simulated warming.

A dynamic ocean model has spatially varying total depth, modelling ocean-floor topography. Vertical heat transports into the deep ocean can be simulated (Labraga \& López 1997). Deep ocean processes are still not well understood, and thus have limited integration into GCMs. The depth of individual layers is often smaller near to the surface to resolve the mixed layer (Hulme et al. 1999). Although modern GCMs use dynamic models, slab models are often revived for specific experiments because of their greater computational efficiency (e.g. Betts et al. 1997).

Moisture, energy and momentum are coupled to the AGCM, with transfers being made daily (Gordon et al. 2000) or less frequently. The OGCM currents respond to energy inputs and other drivers, such as salinity-determined water density. Changes in the rate of upwelling or circulation can alter regional climates in unexpected ways. Final equilibration following increased atmospheric $\mathrm{CO}_{2}$ may take thousands of years, as vertical heat transports to the deep ocean are slow (Kacholia \& Reck 1997). 
The difference in time scales between movement of energy through air and water is an issue in integrated oceanic-atmospheric modelling. It causes a sizeable discrepancy in equilibration and response times. Some CGCMs (e.g. GENESIS) have been run asynchronously, following an initial coupled period to derive an average climate for the ocean run. The particularly slow response of the deep ocean can be countered by using 'distorted physics ${ }^{3}$ in the spin-up period an undistorted run is then required to regain normal ocean dynamics. The aim may be to produce an equilibrium model of present, prehistoric or projected climate.

A second issue is climate drift: discrepancies in the heat, freshwater and momentum fluxes calculated by the ocean and atmospheric components (Labraga \& López 1997). For example, coupling has to deal with sea surface temperature (SST) and lower atmosphere temperature, which are involved in different processes and generated differently. As these discrepancies increase over time, flux adjustments are often applied to standardise the values and prevent impacts on ocean circulation. This avoids movement away from the reference SST and salinity (Hulme et al. 1999). Some recent CGCMs have eliminated this problem through heightened OGCM resolution (e.g. HADCM3, Gordon et al. 2000).

\subsubsection{Land-surface}

Climate change influences vegetation type, which leads to changes in albedo, surface roughness and evapotranspiration (ET) with climatic feedbacks. Additional $\mathrm{CO}_{2}$ production may result from vegetation death and decay and increased decomposition rates in wetlands. $\mathrm{CO}_{2}$ influences on vegetation may alter growth rates. The land-surface parameterisation (LSP) used in the GCM determines whether these interactions are modelled.

The range of LSPs is considered here in some detail for the following reasons:

1) the bioclimate variable scheme used to drive changes in an offline species model should be compatible with the LSP of the GCM. It is especially important that the species model should be compatible with the vegetation model, because this is where the species model fits into the GCM layer structure.

2) surface processes influence continental climate both at the local and regional scale (Houghton et al. 2001)

3) climate-driven changes in the LSP give an indication of the type of change that might be seen in individual species' models.

${ }^{3}$ This 'distorted physics' involves a reduction in the specific heat capacity by a factor of up to 10 , to speed heat transfer, and a similar distortion to the transport of salinity. 
AGCMs without a vegetation model still have an LSP, prescribing albedo, roughness length and surface resistance (Sellers et al. 1996b). Moisture transfer is typically governed via a bucket model' of evaporation and runoff based on one or more soil layers (Sato et al. 1989).

LSPs range from treatment of all land surfaces as static entities, through equilibrium vegetation that reacts instantaneously to change, to dynamic vegetation models with delayed change responses and/or physiological response to $\mathrm{CO}_{2}$ concentration. Vegetation model cells do not usually interact with one another.

The biophysical traits of the vegetation specify various parameters governing energy fluxes between the surface and the GCM atmospheric boundary layer. Roughness length, leaf area index (LAI), fractional vegetation cover and stomatal resistance to water loss seem to be the most influential traits (Henderson-Sellers \& McGuffie 1995).

Offline biosphere models were first developed to investigate relationships between changing climate and vegetation. These models, such as the Simple Biosphere Model (SiB, Sellers et al. 1986, 1989) and the Biosphere-Atmosphere Transfer Scheme (BATS, Dickinson et al. 1986) are forced by the GCM climate without providing feedback to it. SiB models 12 vegetation types at two canopy levels (grass and forest), plus permanent ice, at a scale of $1.8^{\circ}$ x $2.8^{\circ}$. BATS has 18 types and a scale of $4.5^{\circ} \times 7.5^{\circ}$. Both models were later integrated into GCMs.

Vegetation model integration began with asynchronously meshed climate-vegetation models (Foley et al. 1998). In each iteration, the land surface parameters used by the GCM were specified by the vegetation model, or derived from it, and the GCM climate was supplied to the vegetation model. Potential lags in migration of vegetation types and the direct effects of $\mathrm{CO}_{2}$ on plants were largely ignored. An example is the integration of BATS with the NCAR CCM1Oz GCM, via a 'meshing' period of one year (Henderson-Sellers 1993, Henderson-Sellers \& McGuffie 1995). The impact of instantaneous vegetation change on modelled climate, and GCM stability, were explored using several scenarios. The potential area and location of South American tropical forests remained reasonably constant in the doubled $\mathrm{CO}_{2}$ scenarios, but the changing vegetation did intensify tropical atmospheric circulation and increase continental and oceanic evaporation, with concomitant increase in precipitation.

When SiB was integrated into the NMC spectral GCM, the simulation of the surface energy budget over Amazonia was improved (Sato et al. 1989). The GCM had previously used a bucket model for soil moisture, which limited evaporation by aerodynamic surface resistance. The additional stomatal resistance to transpiration modelled in SiB decreased the ratio of latent heat flux (lost with transpired water) to sensible heat flux (lost as longwave radiation) so that it was 
close to observed values. As a consequence, precipitation decreased to better match the climatology, though its spatial pattern was still a poor fit. Albedos were also higher than in the NMC GCM, further decreasing energy available for evapotranspiration.

Later work using the Integrated Biosphere Simulator biome model (IBIS, $2^{\circ} \times 2^{\circ}$ resolution) with the GENESIS GCM $\left(4.5^{\circ} \times 7.5^{\circ}\right)$ had a meshing period of only 30 minutes, so can be described as synchronously coupled (Foley et al. 1998). The GCM's land-surface model was integrated into the biosphere model, so that factors such as ET were calculated only once. Vegetation dynamics were simulated with separate $\mathrm{C}^{3}$ and $\mathrm{C}^{4}$ photosynthetic systems, deciduousness, and competition between layers for light and soil moisture. Nine global plant functional types (PFTs) with simple climate constraints are used, giving the model a process approach overlaid on an analytical model. When run over 30 years from present conditions, existing forest cover was not simulated over some southern parts of South America. Annual rainfall was much underestimated over this region and the Amazon basin.

In the SiB2 single layer vegetation model, monthly LAI, fraction of photosynthetically active radiation absorbed by the canopy (FPAR) and canopy greenness fraction for the present day were derived from NDVI (normalised difference vegetation index) satellite data (Sellers et al. 1996b). This was the first model to use satellite data, and it was found to be problematic in tropical regions because of increased cloud cover limiting NDVI measurements. The highest monthly measured values were applied to evergreen broadleaf forests, but this still left a question over drought-deciduous broadleaf areas. The other surface parameters were derived from literature sources as is usual.

Finally, biotic feedbacks arising from the direct impacts of $\mathrm{CO}_{2}$ on vegetation were incorporated into models. In $\mathrm{SiB} 2$, stomatal conductance fell when additional $\mathrm{CO}_{2}$ availability reduced the required diffusion time for $\mathrm{CO}_{2}$ uptake (Sellers et al. 1996a, d). A SiB2 run including downregulation of $\mathrm{CO}_{2}$ assimilation was also performed. Canopy conductance values (a function of LAI and stomatal conductance) were reduced relative to the control case, leading to an even greater temperature increase.

The Sheffield University Dynamic Global Vegetation Model (SDGVM) was coupled with a simplified HADCM2 to measure the effects of physiological and structural response to a doubled $\mathrm{CO}_{2}$ climate, including a $\mathrm{CO}_{2}$ fertilisation effect on LAI (Betts et al. 1997). SDGVM is an equilibrium model, so does not measure lags in structural change. Increasing LAI was seen in some regions, leading to decreasing albedo and increasing surface roughness, root depth and canopy conductance. LAI change was negatively correlated with temperature change, except in snowy regions where afforestation led to a more dramatic decrease in albedo. The increased 
LAI in areas of increased rainfall offset the reduced stomatal conductance per unit leaf. LAI change was negligible for tropical forests, so transpiration was still reduced under doubled $\mathrm{CO}_{2}$.

The HADCM2 vegetation parameterisation is fixed, but the MOSES biosphere model incorporated into HADCM3 calculates photosynthesis, carbon storage and canopy conductance (Cox et al. 1999). It uses one canopy level and vegetation types based on the Wilson \& Hendersen-Sellers classification (1985). Similar stomatal conductance effects are seen.

In all cases, the largest impacts of changing stomatal conductance were in the tropics, where canopy conductance is high. GCMs not including this response to $\mathrm{CO}_{2}$ concentration may be under-estimating potential changes in tropical temperature. Further discussion on the changes in tropical vegetation simulated by GCM-forced vegetation models can be found in section 4.3.5.

\subsection{Evapotranspiration}

Most GCMs do not calculate potential evapotranspiration (PET), though some vegetation models (e.g. SiB) do. Calculation of actual evapotranspiration (AET) in the Hadley Centre models is broadly compatible with the Penman-Monteith approach for PET (section 4.2.1.3; Eqn. 11 from Cox et al. 1999, Cox, pers. comm. 2000). AET is calculated as a sum of evaporation from canopy and soil, and transpiration from vegetation. In the GCM, evaporating water meets with aerodynamic but not surface resistance; transpirating water meets with both.

In HADCM2, surface resistance is fixed according to vegetation type. MOSES in HADCM3 incorporates photosynthesis and transpiration, meaning that surface resistance is continuously updated (Cox et al. 1999). Daily values are output, but at the time of writing, only the fixed HADCM2 values were freely available.

HADCM3 vegetation responds to changing $\mathrm{CO}_{2}$ concentrations, with reduced stomatal opening and thus reduced ET under increasing $\mathrm{CO}_{2}$ (Cox et al. 1999). This adds to surface temperature, but this does not impact upon plant moisture stores as it would with unaffected stomata. If stomatal $\mathrm{CO}_{2}$ response is represented in a $\mathrm{GCM}$, species response models should incorporate a corresponding increase in plant tolerance to apparent moisture deficit. This could rely on empirical tests of water retention under different $\mathrm{CO}_{2}$ levels, or could incorporate a model of photosynthesis directly. To distinguish between species, a photosynthesis model would require a good assessment of individual structure (LAI, size) and phenology (growth, deciduousness). 


\subsection{Land use change simulation}

Several simulations of the impact on climate of instantaneous tropical deforestation have produced broadly the same changes in the Amazon region (Salati \& Nobre 1991, McGuffie et al. 1995 (BATS), Sud et al. 1996 (SiB), O’Brien 1996). ET and precipitation decreased with deforestation, and surface temperature increased. These are similar regional trends to those simulated following GHG-forced change. All studies have made the simple but unrealistic assumption of sudden widespread deforestation over one or all continents rather than incremental increases in deforested land, and have replaced modelled forest by grassland rather than allowing any secondary growth.

Until now, GCM simulations have either considered climate forcing from change in atmospheric composition, or from land use change. It is not appropriate to simply sum the climate impacts of deforestation and climate change, so until dual-impact models are available, the climate impacts of land use change cannot be considered in species modelling. Habitat loss can only be considered in terms of availability for species colonisation or population persistence (section 4.2.6).

\subsubsection{Initiation}

In a 'warm-start' transient experiment, the GCM is started with radiative forcings and an appropriate monthly climate from a historical era (e.g. 1860, the middle industrial era, Johns et al. 1997, Gordon et al. 2000). There were fewer anthropogenic influences on atmospheric composition, so these forcings are lower than present levels (Table 3:1). Historical forcings are then applied. A period of slow warming due to the thermal inertia of the oceans occurs, as in the real world. In a 'cold-start' experiment, forcing is started at 1990, and this slow initial warming will affect the outcome (McGuffie \& Henderson-Sellers 1996). For this reason, a warm-start is now the norm. Presumably, this is a factor in the increasing global mean temperature change seen in climate projections (Kacholia \& Reck 1997).

An ensemble of GCM simulations of the same change process will be initiated separately from different versions of an unforced control simulation. For HADCM2, the control models were taken from the same equilibrium run, 100 years apart from one another (Hulme et al. 1999). The different initial conditions arise from the chaotic (probabilistic) processes within the GCM, and will lead to at least slightly different evolutions of climate change. The climate biases and sensitivities found in four HADCM2 ensemble members were similar to the ensemble means values, and usually did not differ from one another at the 5\% confidence level (Giorgi \& Francisco 2000). Therefore, it is hoped that a small number of ensemble members should be sufficient to evaluate the potential range of change for a scenario-GCM combination. 


\subsubsection{Limitations of GCM simulations}

Fine scale physical processes can only be represented as mean outcomes in a large GCM cell. For example, current models are too coarse to simulate the localised convection that initiates most tropical rainfall (Scholes \& van Breemen 1997), and cloud formation, which is important in radiative and moisture transfer, is poorly simulated.

At ground level, hydrological flow between cells in the same layer is rarely included (Houghton et al. 2001). This could exacerbate localised droughts in the models. In most GCMs (e.g. HADCM2), runoff is released immediately into the ocean from cells containing river mouths (e.g. the Amazon). Some recent GCMs include a specified delay period before moisture is released, which improves representation of flows into the ocean, but still ignores surface flows. The calving of icebergs is not represented at all, resulting in a small decrease in the volume of water and increase in salinity in the ocean as snow accumulates at the poles (Gordon et al. 2000).

Land use change over recent years is not included in GCMs, which are typically initiated with climax vegetation schemes. Human impacts on this natural cover, producing current conditions over a 17 year period, were simulated using BATS with the NCAR CCM3 GCM. (Zhao et al. 2000). Statistically significant regional changes in temperature and precipitation were seen. This corresponds to the type of change observed in complete deforestation experiments, but the global mean climate was not affected. Simulation of $20^{\text {th }}$ century climate might be improved by incorporating land cover change feedbacks.

Lags in equilibration of vegetation to new climates are generally not well simulated in GCMs. Even those using plant functional type schemes tend to assume an immediate ability of plants to colonise new areas.

\subsubsection{Scale and geography}

As well as the difference between spectral and finite grid AGCMs, and slab and dynamic OGCMs, structural variation on the horizontal plane includes the size and shape of grid-cells and placement of ocean-land boundaries. On the vertical plane, the number of and level of integration between oceanic and atmospheric layers vary. The vertical complexity and large spatial area of GCMs together with limited information about underlying processes initially led to an extremely coarse horizontal resolution, $3.5^{\circ} \times 2.75^{\circ}$ latitude-longitude at best. At greater resolutions, finer scale processes can be represented, but computational restrictions mean that it is easier to adapt and test scenario variations on a faster-running coarse-scale model. 
Table 3:2: Structural characteristics of a sample of GCMs

\begin{tabular}{|c|c|c|c|c|}
\hline$\overline{\text { GCM }}$ & ECHAM4 & HADCM2 & HADCM3 & CSIRO Mark 2b \\
\hline Creation date & 1996 & 1995 & 1998 & 1996 \\
\hline Institution & DKRZ, Germany & \multicolumn{2}{|c|}{ CRU, UK } & CSIRO, Australia \\
\hline $\begin{array}{l}\text { Grid resolution } \\
\text { (latitude-longitude) }\end{array}$ & T42: about $2.8^{\circ}$ & $\begin{array}{c}2.5^{\circ} \times 3.75^{\circ} \\
\text { (Fig. 5:8, p. 147) }\end{array}$ & $\begin{array}{l}2.5^{\circ} \times 3.75^{\circ} \\
\text { atmosphere; } \\
1.25^{\circ} \text { ocean }\end{array}$ & $\mathrm{R} 21: 5.6^{\circ} \times 3.2^{\circ}$ \\
\hline $\begin{array}{l}\text { Vertical Levels } \\
\text { (atmosphere) }\end{array}$ & 19 & \multicolumn{2}{|c|}{19} & 9 \\
\hline $\begin{array}{l}\text { Vertical Levels } \\
\text { (ocean) }\end{array}$ & & \multicolumn{2}{|c|}{20} & 21 \\
\hline Timestep & 24 minutes & $\begin{array}{c}\text { Atmosphere: } \\
30 \text { minutes, } \\
\text { Ocean: } 60 \\
\text { minutes }\end{array}$ & 30 minutes & $?$ \\
\hline
\end{tabular}

Variable resolution models are being developed, with finer latitude-longitude grids at the tropics to compensate for the greater width of a tropical degree cell. The coarse horizontal scale of most existing climate models means that it is necessary to consider methods of generating finer-scale outputs for local applications.

\subsection{DOWNSCALING FOR REGIONAL RESULTS}

GCM output is often 'downscaled' for comparison with finer-resolution climate maps. It is also sometimes employed to reduce GCM computational requirements, 'spinning-up' a model at a coarser resolution, and then adjusting to a finer resolution at a later stage (e.g. OPYC OGCM, Oberhuber 1993). No studies involving downscaling of GCM outputs for the Amazon region have been identified, but some form of downscaling is necessary in any application of GCM results to smaller grid cells. The challenge of obtaining local results from a coarse-scale GCM is known as the 'climate inversion' problem.

Aspects to this problem are:

- GCMs are designed to evaluate regional, not fine-scale changes - so there is less effort to ensure grid-cell level concurrence between the control model and reality.

- Columns on the grid participate in different processes, and have different vertical resolutions, depending upon whether the GCM treats them as oceanic or land-based. Therefore, a naive interpolation of a coastal grid cell onto a finer scale map may apply oceanic results to the land-based cells (Goodess \& Palutikof 1992). Seasonal cycles of temperature, for example, are less pronounced over the ocean.

- Downscaling methods must assume that there is no difference in the relationship between the recorded station point or grid cell value and the GCM grid cell average in the future and present scenarios. That is, that the relationship between climate patterns at different scales remains the same. 
- GCM outputs are intended for use as decadal averages, whilst the time frame of interest for many end-users is annual (Russo \& Zack 1997).

\subsubsection{Linear projection of anomalies}

Cells where future scenario climates differ from those in the GCM control climate, or 'anomalies', are projected directly onto the current (reference) climate in a map overlay operation. For each fine-resolution cell, the mean anomalies from nearby GCM cells are applied to the present data (Bartlein et al. 1997, Hulme et al. 1999). Distance weighting or other smoothing techniques may be applied (Huntley et al. 1995). If there is no anomaly, the unweighted reference values are retained. This technique provides a more plausible interpretation of fine-scale climate change than is obtained through an imposition of the GCM cells onto a finer grid, but fails to resolve the above points.

This technique may cause problems when, because of the variation in the fine-resolution grid, a negative anomaly exceeds the reference climate value for a variable that must remain positive. To avoid this issue, a percentage change rather than an absolute anomaly is frequently applied to such variables (pers. comm. Betts 2000).

Temporal interpolation is also an issue. If decadal means were not available from the GCM, an acceleration of change could be simulated using a square interpolation over the entire GCM period (Belotelov et al. 1996). Linear interpolation is more suitable for simulation from decadal data. Stochastic variation could be applied to simulate a more realistic change pattern.

\subsubsection{Regression interpolations}

There are two types of GLM-based interpolation. The first is based on the reference climate. The variable of interest is explained via regression against regionally averaged variations in itself and other climate variables (Aspinall \& Matthews 1994, Wilby \& Wigley 1997). For example, temperature may be explained using temperature, precipitation and surface pressure. Climate anomalies are then assigned proportionately to the variation at each point.

The second type relates the reference climate to GCM outputs for the present day (e.g. by first identifying a set of meta-variables explaining interannual GCM variation, Fischlin \& Gyalistras 1997). These empirical relationships, matching GCM anomalies to local conditions, are then used to downscale the change. 


\subsubsection{Regional climate models}

A regional climate model (RegCM), or mesoscale model, can be 'nested' within a GCM, or driven by boundary conditions stored from a GCM run. This allows high-resolution simulation for a specified area within on the GCM. There is no feedback to the global model (Cubasch et al. 1995, Russo \& Zack 1997). Typically the RegCM occupies in lower atmosphere of the GCM. The RegCM describes fine-scale transfers of heat, momentum and moisture, and translates GCM variables into the variables of interest.

This resource-intensive approach yields a plausible simulation of local atmospheric processes. The coupling allows details that cannot be captured by a coarse-scale GCM to undergo climate forcing, and provide feedbacks to the GCM. This offers a better approximation of local change related to existing variation. There is still a reliance on the cell-by-cell accuracy of the GCM outputs, whereas GCM validations generally consider regional rather than cellular accuracy. The RegCM and GCM may converge in future, as GCM spatial resolution and computational capacity continue to improve.

\subsubsection{Stochastic weather generators}

Where a GCM is inaccurate at simulating a local variable, it may be preferable to use other more accurate variables to force the regional simulation, using a stochastic model (Bárdossy 1997). Rather than using GCM variables to directly feed a finer-scale model, air circulation patterns from a group of GCM cells can be associated with observed local data. The daily patterns seen are classified, and seasonal linkages between pattern type and observed climate (e.g. precipitation) are developed. In a typical stochastic model, diurnal variations are randomised, outputting longer-term variability. However, these models do not appear to consider the availability of water for precipitation.

\subsubsection{Preferred method for Amazon region}

As there is a high level of uncertainty about the sub-regional accuracy of GCM outputs, one downscaling route might be to apply regionally averaged results to each local cell. This method would lose much of the variation in the GCM results, and reduce the modellers' ability to compare sub-regional patterns of results between scenarios.

Nested RegCMs are a more satisfactory option, as they provide local outputs that come from a partial integration with the GCM. However, these are not widely available to impact modellers. Regression models are the next option, considering spatial relationships between local climate and GCM variability. Stochastic models provide a more complex but similar function. 
In the Amazon region, the available climate data are derived from interpolations between climate stations. These stations are sparsely and unevenly distributed (New et al. 1999). Regression modelling is impracticable where climate station data are poor - it would have to be based on pre-interpolated data, adding another layer of smoothing.

The remaining choice is an area-weighted linear projection of anomalies. The method is simple in application and is guaranteed to retain local climate patterns. This method is chosen for use in the current project.

\subsection{EVALUATION OF GCM RESULTS}

Ideally, evaluation of a complex model should consider its theoretical basis, statistical properties, error characteristics and include sensitivity analyses (Vanclay \& Stovsgaard 1997). Various model intercomparison projects have looked at one part of the model system whilst fixing the other units at prescribed levels (e.g. AMIP, Gates 1992; PILPS, Chen et al. 1996). No one GCM is the overall leader - and the mean values from all models are usually closer to observed data than any single model (McGuffie \& Henderson-Sellers 1996).

Once any advantages pertaining to the main differences in approach (such AGCM type) are considered, the simplest way for a user to evaluate model effectiveness is to examine its performance under different conditions. Typically, such error evaluations involve comparisons with alternative data not used in parameterisation. Some care does need to be taken - for example, the surface level for air temperature may be defined as the soil skin surface, first soil layer, lowest atmospheric layer, or 'screen height' (1.5-2 m) (Kittel et al. 1998, Williams et al. 1998, Betts, pers. comm. 2000). Unadjusted comparison is therefore not always appropriate.

Aggregated climates for the GCM cell, or representative sites, can be used in a present-day comparison. However, success in replicating the present system does not guarantee that the mechanisms modelled are those operating in reality, but simply that this is one set of possible rules that generates the same pattern of results. The difference in representation of cloud systems is a good example of this (section 3.5.1.1). If the observed processes on which the model is based are useful in 'retro-predicting' past climates, the model is more plausible. To test against past climate, GCMs can be run using estimated historical forcings. Some examinations of GCM performance focusing on different areas are reviewed below.

GCM results may be compared with one another to see what degree of consistency there is between simulations of the same scenarios. A similar pair of outcomes can result from a shared 
theoretical or structural basis (e.g. via a common ancestor), but do not necessarily imply conformity with reality. These comparisons may be valuable in identifying the features that lead to a good correspondence with field data.

\subsubsection{Past climate}

Comparisons with climate values and forcings between 1860 and the present are routine. It is difficult to judge exactly how closely a hindcast' should fit the historical climate, as natural variability results in stochastic differences (Johns et al. 1997). Simulation of earlier climates more dissimilar from ours may better test the model's capability to represent the impacts of atmospheric gases on climate.

Some palaeoclimate estimates indicate that heightened levels of $\mathrm{CO}_{2}$ and $\mathrm{CH}_{4}$ have accompanied historical transitions from glacial to interglacial climates (Petit et al. 1999). Palynological data may be used to estimate past vegetation from these earlier periods, and climates are inferred from this. Geological data such as evaporite, peat, coal or bauxite distribution may also be useful proxies (Pollard \& Schulz 1994, Price et al. 1997). The GCM can be parameterised for contemporaneous climates using GHG concentrations from ice cores, but otherwise run with a standard configuration. Where surface models forced by a GCM palaeoclimate do produce flora or geology matching the observed distributions, the model data can be used to estimate the climate for areas without such deposits (Price et al. 1997).

Recent palaeoclimate comparisons using the GENESIS GCM have used both floral and geological indicators for the Permian, together with a range of heightened $\mathrm{CO}_{2}$ levels (Rees et al. 2002). The models matched reasonably well except in low southern latitudes, and it was concluded that possible culprits were limited ocean heat transport and the unidirectional nature of the vegetation model.

\subsubsection{Present climate}

\subsubsection{Issues in modelling global climates}

To obtain the high data density needed for accurate interpolations, relatively recent records must be included because of the lack of older data (Leemans \& Cramer 1991). This makes it difficult to produce a pre- $\mathrm{CO}_{2}$ dataset for comparison with climate model outputs, because of modern increases in atmospheric $\mathrm{CO}_{2}$ concentration. When a long period is selected to maximise the use of records for sparse areas, climate is assumed to be stable over an interval in which climate models would predict a change. Compilers must also decide whether to take mean values from all climate stations for a cell, recognising that operative stations may differ through time, or 
select the stations with the longest run of data or 'most typical' climate. Aggregating data reduces the effect of single-site outliers, by increasing the number of observations contributing to each comparison. Recording techniques for a single-site are more likely to be standard, and the series may track the trend of variation better than aggregations from sparsely distributed climate stations.

Most dataset compilers have taken the pragmatic approach of restricting the mass of records to a set period of several decades this century, but accepting additional records from outside the period for data-poor regions. Legates and Wilmott (1990) opted to maximise spatial coverage rather than restricting the temporal period.

The heterogeneous nature of the data means that inconsistencies and errors are introduced when standardising measurements from different countries and periods to one global database. Procedures and instrumentation at climate stations are not identical, and can influence the raw data collected (Hoyt \& Schatten 1997). Data manipulation can introduce further inconsistencies, for example via different formulae used for monthly mean temperature , or corrections for factors affecting rain gauges such as wind speed or snow drift. A loss in precision may also occur when older measurements in ${ }^{\circ} \mathrm{F}$ are converted to ${ }^{\circ} \mathrm{C}$ (Leemans \& Cramer 1991).

Although the ocean covers most of the planet's surface, oceanic climate records are less consistently available than land-surface records. Methods have been developed to correct precipitation records sourced from shipboard gauges, because ships (a) avoid storms and (b) alter the microclimate through their own structure, movement and capture of spray (Legates \& Wilmott 1990). These formulae include coastal station records for validation, and make adjustments for latitude, air temperature and month. A variety of different treatments have therefore gone into the construction of any global dataset.

Some climate interpolations include altitude as an independent variable, because there is a wellestablished pattern of increasing precipitation and decreasing temperature with altitude (e.g. New et al. 1999). It is also possible to perform an adiabatic correction for a climate variable as a separate measure (e.g. Leemans \& Cramer 1991).

\subsubsection{Global-scale comparisons}

Comparison of GCM control runs for the present day with gridded global climate data gives an indication of success of the current simulation. Comparisons are usually carried out using monthly data, which is more easily obtained than daily data. It is possible to use data originating from individual weather stations, or to produce a climate mean from all weather stations found within that GCM cell. The advantages pertaining to these alternatives are as for current climate 
simulation. Certain criteria have been suggested to increase correspondence with the GCM cell mean when single sites are used in validations: sites should be large, well-watered, fairly uniform (especially in precipitation) and hold long data series (Shuttleworth 1991).

The daily global results from six AGCM simulations (a BMRC model, 3 CCM versions and a GENESIS run) showed a tendency to overestimate the strength of the hydrological cycle, in particular, moisture exchange between oceans and land (CIC 1996). Both evaporation and precipitation were consistently overestimated. The worst example was GENESIS, which overestimated global values by up to about $70 \%$. In the tropics, runoff was underestimated by all but one GCM (CCM10Z). The patterns of the global hydrological cycle were much better simulated than the actual volumes.

In an assessment of the spatial representation of current climate, seven regions (not including Amazonia) were considered (Kittel et al. 1998). The CSIRO and HADCM2 models were best at simulating precipitation and temperature over these regions. There was greater agreement between GCMs in sensitivity to $\mathrm{CO}_{2}$ than between GCM controls and observed data - i.e. the global result did not depend upon details of the regional representation. Regional precipitation is one of the most variable and difficult to simulate GCM outputs (Hulme et al. 1998).

\subsubsection{South American comparisons}

Models may place more emphasis on optimising simulation of particular regions. As GCMs are designed and maintained in the richer countries, less effort may go into ensuring the accuracy of tropical representation. However, the Amazon basin is of interest to climate modellers because of potential deforestation feedbacks on climate, so has received more attention than some other less-developed regions.

Several CGCMs with dynamic OGCMs were compared with observed climate patterns (Legates \& Wilmott 1990a, b) and features for South America (Labraga \& López 1997). Amongst the features considered were the location and intensity of the continental summer maximum temperature, and the seasonality and distribution of precipitation. Observed and modelled fields were compared using root mean square error and correlation coefficients. The GCMs compared were CSIRO Mark 2 (Gordon \& O'Farrell 1997), GFLD R-15 (Manabe et al. 1991), ECHAM-1 and -2 (Cubasch et al. 1992, Lunkeit et al. 1996) and UKTR, the first Hadley Centre transient CGCM (Murphy 1995). It is not stated what type of downscaling was used.

Correlations in temperature and pressure simulation were generally high, though GFLD R-15 had higher error values for pressure. Precipitation simulation was much more variable. UKTR 
had the best overall correlations for the two 3-month means sampled ( 0.79 for DJF and 0.72 for JJA), although ECHAM2 had a better correlation at JJA (0.7). Of the corresponding set of GCMs with slab OGCMs that were also tested, the Hadley Centre model also performed best for precipitation. HADCM2 shows further improvements in simulation of tropical precipitation, though it produced excessive rainfall over the high ground on the west of South America (Johns et al. 1997).

\subsection{Amazonian sites}

The radiation and precipitation outputs from three GCM simulations were evaluated using three years' data from a set of three Amazonian sites from the ABRACOS climate monitoring project (Culf et al. 1998). The GCMs were versions designed to simulate current neotropical conditions, from the Hadley Centre (UKMO), the European Centre for Medium-Range Weather Forecasts (ECMWF) and the Laboratoire du Météorologie Dynamique (LMD). The mean percentage differences between simulated and recorded data are summarised in Table 3:3. A positive value indicates over-estimation by the GCM.

If the sites are representative of the mean climate of the appropriate GCM cells, the potential error is large. The mean anomalies mask greater disparities for individual sites. The precipitation anomaly is most striking. In fact, the seasonal trends of precipitation were well represented by the UKMO and LMD models, although they consistently over- or underestimated the volume. The ECMWF model radically overestimated precipitation at one site, Marabá, which largely accounts for its high error.

Table 3:3: Mean anomalies between GCM simulations and three Amazonian sites (Culf $\boldsymbol{e t}$

al. 1998)

\begin{tabular}{|l|l|l|l|l|l|}
\hline GCM & $\begin{array}{l}\text { Net } \\
\text { radiation } \\
\mathbf{( \%} \\
\text { difference) }\end{array}$ & $\begin{array}{l}\text { Incoming } \\
\text { solar } \\
\text { radiation } \mathbf{( \% )} \\
\text { difference) }\end{array}$ & $\begin{array}{l}\text { Reflected } \\
\text { solar } \\
\text { radiation } \\
\left.\mathbf{( M J m}^{-2} \mathbf{d}^{-\mathbf{1}}\right)\end{array}$ & $\begin{array}{l}\text { Net longwave } \\
\text { radiation (net } \\
\text { all-wave - net } \\
\text { solar) } \mathbf{( \%}\end{array}$ & $\begin{array}{l}\text { Precipitation } \\
\text { (\%) difference) }\end{array}$ \\
\hline UKMO & 18.2 & 13.7 & 0.33 & -17.2 & 12.4 \\
\hline ECMWF & -14.1 & -15.4 & -0.39 & -18.4 & 72.8 \\
\hline LMD & 25.4 & 31.2 & 0.8 & 48.4 & -29 \\
\hline
\end{tabular}

These results demonstrate the need for downscaling of GCM anomalies to accommodate local variability (section 3.6). Further evaluation of the regional distribution of error would be useful.

In both the South American (Labraga \& López 1997) and the Amazon site comparisons, the Hadley Centre family of models has performed well in simulating precipitation, a difficult climate field. HADCM2 was also competent at simulating precipitation at a global scale (Kittel et al. 1998). Not all GCMs were included in each comparison, but this is a good indication that 
HADCM2 is a reasonable choice for simulating the Amazon region. The remainder of the review will focus on HADCM2 as a target model, and highlight differences between it and other GCMs.

\subsubsection{Projected climate}

\subsubsection{Global-scale results}

Temperature change projections from GCMs have been based on increasingly complex models, which have had a reasonably stable climate sensitivity. In all the experiments, the land warms more than the ocean. When the published results from 108 GCMs dated between 1980 and 1995 and run under a doubled $\mathrm{CO}_{2}$ scenario were compiled, the mean anomaly was found to be $2.62^{\circ} \mathrm{C}$ (range $0.16^{\circ} \mathrm{C}$ to $8.7^{\circ} \mathrm{C}$ ) (Kacholia \& Reck 1997). GCM mean temperature change is greater than that for the one-dimensional models.

Similar results can be produced for different reasons - for example, improving representation of tropical cloud feedback versus increasing sea ice increased temperature by a comparable amount in different GCMs (Kacholia \& Reck 1997). The authors feel that it is reassuring that most models produce mean doubled $\mathrm{CO}_{2}$ (IS92a) projections in the $2^{\circ} \mathrm{C}-3^{\circ} \mathrm{C}$ range, given that it is difficult to decide which has the most useful representation of reality. The very latest range of projections based on a new set of IPCC scenarios include temperature increases ranging from $1.4^{\circ} \mathrm{C}$ to $5.8^{\circ} \mathrm{C}$ by 2100 , greater than those seen under IS 92 .

Precipitation anomalies are also important for species response. Two sets of global temperature and precipitation results for each of three GCMs under the IS92a scenario, which simulates approximately doubled $\mathrm{CO}_{2}$ by 2099, are rather divergent (Table 3:4). $\mathrm{CO}_{2}$ concentrations rise to 697 ppmv by the 2080s. The GGa and GSa runs cited do or do not include negative forcing from sulphate aerosols respectively. Global temperature anomalies vary from $2.5^{\circ} \mathrm{C}$ to $3^{\circ} \mathrm{C}$, and precipitation from 2-6\%. The simulated temperature increase is higher with latitude, and minimum temperature increases more than the maximum.

Table 3:4: GCM results for IS92a - temperature and precipitation anomalies

\begin{tabular}{|l|ll|l|llll|}
\hline \multirow{2}{*}{ GCM } & \multicolumn{2}{|l|}{ ECHAM4 } & HADCM2 & \multicolumn{3}{l|}{ CSIRO Mark 2b } \\
\cline { 2 - 7 } & GGa & GSa & GGa1-4 & GSa1-4 & GGa & GSa \\
\hline Global change with respect to 1961-1990, IS92a, thirty-year means: & & \\
\hline Temperature anomaly, 2020s $\left({ }^{\circ} \mathrm{C}\right)$ & 1.22 & 1.02 & 1.19 & 0.92 & 1.21 & 1.06 \\
\hline Temperature anomaly, 2050s $\left({ }^{\circ} \mathrm{C}\right)$ & 2.13 & 1.35 & 2.05 & 1.56 & 2.05 & 1.84 \\
\hline Temperature anomaly, 2080s $\left({ }^{\circ} \mathrm{C}\right)$ & 3.02 & - & 3.01 & 2.51 & 3.07 & 2.72 \\
\hline Precipitation anomaly, 2020s $(\%)$ & 0.7 & 0.4 & 2 & 1.4 & 2.5 & 1.8 \\
\hline Precipitation anomaly, 2050s $(\%)$ & 1.4 & 0 & 3.3 & 2.2 & 3.9 & 3.2 \\
\hline Precipitation anomaly, 2080s $(\%)$ & 2.1 & - & 4.87 & 3.73 & 6.1 & 5.1 \\
\hline
\end{tabular}


The results of the latest Hadley Centre experiment, $\operatorname{HADCM} 3$, are not suitable for the current project because they simulate stomatal response to $\mathrm{CO}_{2}$ concentration, using the MOSES land surface scheme (Cox et al. 1999). A new radiation scheme represents 'minor trace gases' as well as $\mathrm{CO}_{2}, \mathrm{H}_{2} \mathrm{O}$ and $\mathrm{O}_{3}$. Convection, cloud, boundary layer mixing, gravity wave drag and various ocean processes have also been altered, and new components added to represent orographic drag (the effect of mountains on precipitation) and a background aerosol climatology (Gordon et al. 2000). The equilibrium climate sensitivity to doubled $\mathrm{CO}_{2}$ under IS92a is about $3.3^{\circ} \mathrm{C}$ (Hulme et al. 1999). This global change is within the same range as earlier models, but there are differences in regional patterns.

For greater detail on global patterns of climate change, I refer the reader to Houghton et al. 2001, which includes the results of models based on the new SRES emissions scenarios from the IPCC. These were not available at the time of this study. The projected increases in global mean temperature are generally higher because the new scenarios expect smaller $\mathrm{SO}_{2}$ emissions.

\subsubsection{Regional results}

The mean South American outcomes of five slab and five coupled dynamic GCMs were compared to evaluate climate change patterns (Labraga \& López 1997). Mean outcomes were used because the authors wanted to find patterns that were consistent between the GCMs. They mapped areas with an equal sign change amongst the GCMs, to indicate 'robust trends'. The different ocean representations did affect the outcomes, especially in oceanic regions and to the south of the continent. Amazon-region patterns will be discussed here. Mean outcomes would not be a sensible option for modelling ongoing change, as the additional smoothing in the climate patterns from the different runs can make them less internally consistent.

In mid to high South American latitudes, a greater rate of increase in temperature, evaporation and precipitation was observed in slab than dynamic GCMs, largely due to heat transports into the deep ocean. Shared patterns included an increase in precipitation in the northwest of the continent following increased oceanic evaporation. A negative pressure change extends from the tropical North Atlantic to the equatorial zone. In the summer (DJF), precipitation decreases between the Orinoco and lower Amazon river basins. This is expected to increase warming and moisture deficit, and is the region where the relative maximum warming is simulated. In the winter (JJA), a similar change in precipitation is simulated, but it is shifted slightly to the north.

The tropical effects of climate change according to UKTR included decreased rainfall, mean temperature increase, a longer dry season and increased interannual variability in rainfall over the Amazon basin, by approximately the 2060s (Hulme \& Viner 1995). A marked decrease in soil moisture content $(30 \mathrm{~mm})$ was observed. Central America was warmed by less than $2^{\circ} \mathrm{C}$, 
which is less than comparable African latitudes. A high degree of confidence was not attached to these results, partly because the GCM did not simulate cloud cover dynamics very well.

The natural variability simulated in the GCM is much higher for precipitation than for temperature. Over much of the tropics, the range of precipitation change between ensemble members exceeds $30 \%$ of the ensemble mean, though the greatest differences were in arid regions (Hulme et al. 1999). Northwestern Amazonia is particularly affected by increased mean temperature and decreased precipitation.

Although in general, HADCM2 warms more in the tropics than HADCM3, because of the differences in land surface process and cloud representation, this is not true for the Amazon region (CRU 1999, Hulme et al. 1999). Cloud cover increases in the southern extra-tropics in both models. HADCM3 has a greater area of precipitation decrease over the northern part of South America. This drying for the Amazon region is much greater than in HADCM2, and not seen in other GCMs.

\subsection{Impact on ENSO events}

El Niño events are important to neotropical climate and vegetation because of their impact on precipitation (section 2.1.2.3). Although the ENSO cycle is difficult to simulate in GCMs with a coarse equatorial resolution, HADCM2 has 'reasonable' ENSO-like behaviour, which changes little under doubled $\mathrm{CO}_{2}$ (Collins 2000). Two opposed speculations have been made about climate change impacts on the ENSO cycle, both focusing on relative changes in temperature between the western and eastern Pacific. One suggestion is that a strong equatorial upwelling in the east will reduce warming, so that the west will be disproportionately affected by climate change (Cane et al. 1997). Stronger winds along the equator would follow, with further upwelling and cooling in the east, and more frequent La Niña-type conditions. This would slow global warming.

It is also argued that the equatorial west Pacific is currently so warm that a small further warming will create clouds that buffer incoming radiation preventing additional warming. Under this scenario, the east Pacific would warm more than the west (Meehl \& Washington 1996). This would reduce winds along the equator and result in El Niño-type conditions being more prevalent.

A 1999 version of ECHAM features a meridional resolution of $0.5^{\circ}$ in the tropics, and claims to closely simulate an ENSO cycle, including both possibilities for oceanic feedback (Timmermann et al. 1999). When greenhouse gas forcing is applied in accordance with scenario 
IS92a, there is stronger eastern Pacific warming. The interannual variability in sea surface temperature (SST) also increases towards the end of the simulation, with a bias towards cold extremes (relative to the mean).

With the mean tropical Pacific climate becoming closer to El Niño conditions (Houghton et al. 2001), and an eastward shift of precipitation, the frequency of El Niño events may increase, though the degree of change is not quantifiable. Most recent projections show a small or no increase in frequency over the next century (Houghton et al. 2001).

\subsection{Tropical cyclones}

It is suggested that cyclones could act as a vector for seed transmission over long distances (Melillo et al. 1996), but their impact on non-opportunist species is entirely negative. Several models of tropical cyclone generation (TCG) under current and climate change conditions have been formulated. TCG increases with the heat content of the ocean. The later models (Bengtsson et al. 1996, Royer et al. 1998) do not show a significant increase in the generation of tropical cyclones in the Southern Hemisphere. In the Royer model, the climate anomaly conditions simulated at doubled $\mathrm{CO}_{2}$ in two GCMs (ECHAM1/LSG and HADCM2) were applied to the ARPEGE-Climate GCM, which generated the parameters required for TCG simulation. The simulated increase in sea surface temperature is countered by an increase in the TCG threshold temperature. Cyclone frequency was found to decrease slightly over the southern Hemisphere, especially in the vicinity of Latin America.

These studies suggest that under a scenario of an annual $1 \%$ (of 1990 values) rise in $\mathrm{CO}_{2}$, Neotropical cyclone frequency is likely to remain unchanged until at least 2050.

\subsection{CONCLUSIONS: GCMS FOR SPECIES MODELLING}

It is desirable that the processes simulated include those most relevant to tropical climates, and to the accurate simulation of the variables affecting plant response. A good agreement with present-day neotropical climate data increases confidence in that GCM's ability to simulate future trends, with the proviso that there is much potential for error and scope for improvement in the present-day data itself.

As the Hadley Centre model has performed well at the regional and global scale relative to the other GCMs considered, it was of particular interest in the latter part of this review. HADCM3 had a particularly strong change response in the Amazon region, whilst HADCM2 fell more within the range of response seen in other GCMs (Hulme et al. 1999). It is also preferable to 
HADCM3 because the HADCM2 land surface scheme does not parameterise $\mathrm{CO}_{2}$ impacts on stomatal closure.

The other criteria concern output data. It is preferable that the data have a high horizontal resolution. It is also desirable that the output variables include the climate variables for species modelling or those required to calculate these offline. In this project, the variables are based on daily PET and AET. For consistency, both AET and PET should be derived in the same way: so even if AET is available as a GCM output, it should be calculated offline if PET is not.

Table 3:5 compares the output variables for the models discussed above: an $\mathrm{X}$ or height measure indicates availability at unknown or specified height respectively. This table can be crossreferenced with Table A1:1 to identify which GCMs offer input variables required for the calculation of the bioclimate variables preferred for distribution modelling. HADCM2 is satisfactory in all instances.

GCMs simulate, rather than predict, possible future climates. However, the similarity in the direction of change forecast for the Amazon region gives a degree of confidence. By modelling response to a range of scenarios, best and worst-case outcomes can be simulated.

Table 3:5: Selected monthly output variables for GCMs

\begin{tabular}{|c|c|c|c|c|c|c|c|}
\hline $\begin{array}{l}\qquad \text { GCM } \rightarrow \\
\text { Variable } \\
\end{array}$ & $\begin{array}{l}\text { ECHAM4 } \\
\text { GGa }\end{array}$ & $\begin{array}{l}\text { ECHAM4 } \\
\text { GSa }\end{array}$ & $\begin{array}{l}\text { HADCM2 } \\
\text { GGa1-4 }\end{array}$ & $\begin{array}{l}\text { HADCM2 } \\
\text { GSa1-4 }\end{array}$ & HADCM3 & \begin{tabular}{|l} 
CSIRO \\
Mark 2b \\
GGa \\
\end{tabular} & \begin{tabular}{|l|} 
CSIRO \\
Mark 2b \\
GSa \\
\end{tabular} \\
\hline $\begin{array}{l}\text { Radiation } \\
\text { measures }\end{array}$ & \multicolumn{2}{|c|}{$X$} & \multicolumn{2}{|c|}{$\begin{array}{c}\text { X (including net } \\
\text { shortwave \& } \\
\text { longwave) } \\
\end{array}$} & & \multicolumn{2}{|c|}{$\mathrm{X}$} \\
\hline $\begin{array}{l}\text { Specific } \\
\text { humidity }\end{array}$ & & & \multicolumn{3}{|c|}{$X$} & & \\
\hline $\begin{array}{l}\text { Relative } \\
\text { humidity }\end{array}$ & & & \multicolumn{3}{|c|}{$1.5 \mathrm{~m}$} & \multicolumn{2}{|c|}{$2 \mathrm{~m}$} \\
\hline $\begin{array}{l}\text { Mean } \\
\text { temperature }\end{array}$ & \multicolumn{2}{|c|}{$\mathrm{X}$} & \multicolumn{2}{|c|}{ Surface, $1.5 \mathrm{~m}$} & $1.5 \mathrm{~m}$ & \multicolumn{2}{|c|}{$2 \mathrm{~m}$} \\
\hline $\begin{array}{l}\text { Max/min } \\
\text { temperature }\end{array}$ & \multicolumn{2}{|c|}{$\mathrm{X}$} & \multicolumn{2}{|c|}{$1.5 \mathrm{~m}$} & & \multicolumn{2}{|c|}{$2 \mathrm{~m}$} \\
\hline $\begin{array}{l}\text { Total } \\
\text { precipitation }\end{array}$ & \multicolumn{2}{|c|}{$\mathrm{X}$} & \multicolumn{3}{|c|}{$X$} & \multicolumn{2}{|c|}{$\mathrm{X}$} \\
\hline Surface pressure & $?$ & $?$ & $X$ & $\mathrm{X}$ & $?$ & & $?$ \\
\hline PET & & & & & & & $X$ \\
\hline $\begin{array}{l}\text { AET / Surface } \\
\text { moisture flux }\end{array}$ & \multicolumn{2}{|c|}{$\mathrm{X}$} & \multicolumn{3}{|c|}{ X (soil \& canopy) } & \multicolumn{2}{|c|}{$\mathrm{X}$} \\
\hline Cloud cover & \multicolumn{2}{|c|}{$\bar{X}$} & & & $\mathrm{X}$ & & $X$ \\
\hline Runoff & & & \multicolumn{3}{|c|}{ Surface, subsurface } & \multicolumn{2}{|c|}{ Surface } \\
\hline $\begin{array}{l}\text { Soil moisture } \\
\text { content }\end{array}$ & & & \multicolumn{3}{|c|}{$X$} & \multicolumn{2}{|c|}{2 levels } \\
\hline Wind-speed & & & \multicolumn{3}{|c|}{$\begin{array}{c}10 \mathrm{~m} \text { (W-E and N-S } \\
\text { components) }\end{array}$} & \multicolumn{2}{|c|}{ Surface } \\
\hline
\end{tabular}




\section{Plant response to climate}

This chapter considers plant populations' potential responses to climate change, and methods of simulating current and changing species distributions, with a view to identifying the most useful methods for Amazonian species simulation.

There is a wide literature on the spatial interpolation of species distributions. Many techniques are inapplicable because available data for most Amazonian species does not meet their requirements. In addition, a species' ability to occupy the potential range given what is actually known about its spatial distribution is not usually considered; a simple solution is proposed.

Various options for predictive variables are available. Bioclimate variables, which most directly represent resource availability, are considered most appropriate. Evapotranspiration (ET), moisture deficit and seasonality of moisture availability together represent the variability in energy and moisture supply over the extended Neotropics. These variables can be calculated from daily measures of actual and potential ET, based on the vegetation present.

Modelling a changing distribution can also involve considering the effects of distributional barriers and lag in population response to change. The impact of a climate change depends upon whether it shifts the current climate towards or away from species optima. Typically, estimates of population response to change are based on field or laboratory studies of behaviour under different conditions. Alternative estimation methods based on more limited data are considered.

\subsection{VARIATION IN CLIMATE RESPONSE BETWEEN SPECIES}

\subsubsection{Species response}

Niche segregation and differential response to change can be seen at the species level. Distributional impacts on biodiversity do not automatically have to be approached at this level. Variation also exists within species, so why not work with subspecies, ecotypes or varieties? The taxonomic value equivalence of species is questionable, as the relatedness of species within and between families is variable (Vane-Wright et al. 1991, Mishler 1999). The biological species concept does imply a certain level of genetic homogeneity, together with the ability for successful genes to flow within a population. Given sufficient time, the genes that enable individuals to cope with environmental extremes are theoretically available to their entire population, which supports the concept of a shared environmental niche-space. Additionally, the species resolution is usually the highest available, although it is possible to identify ecotypes within a well-recorded species (Jones et al. 1997). 
The genus or family level would provide more spatial locations per taxon. Spatial biases in the data are similarly distributed at each level, because the higher-taxon distribution information is simply the sum of the species information, but the spatial coverage for each unit should increase with taxonomic level.

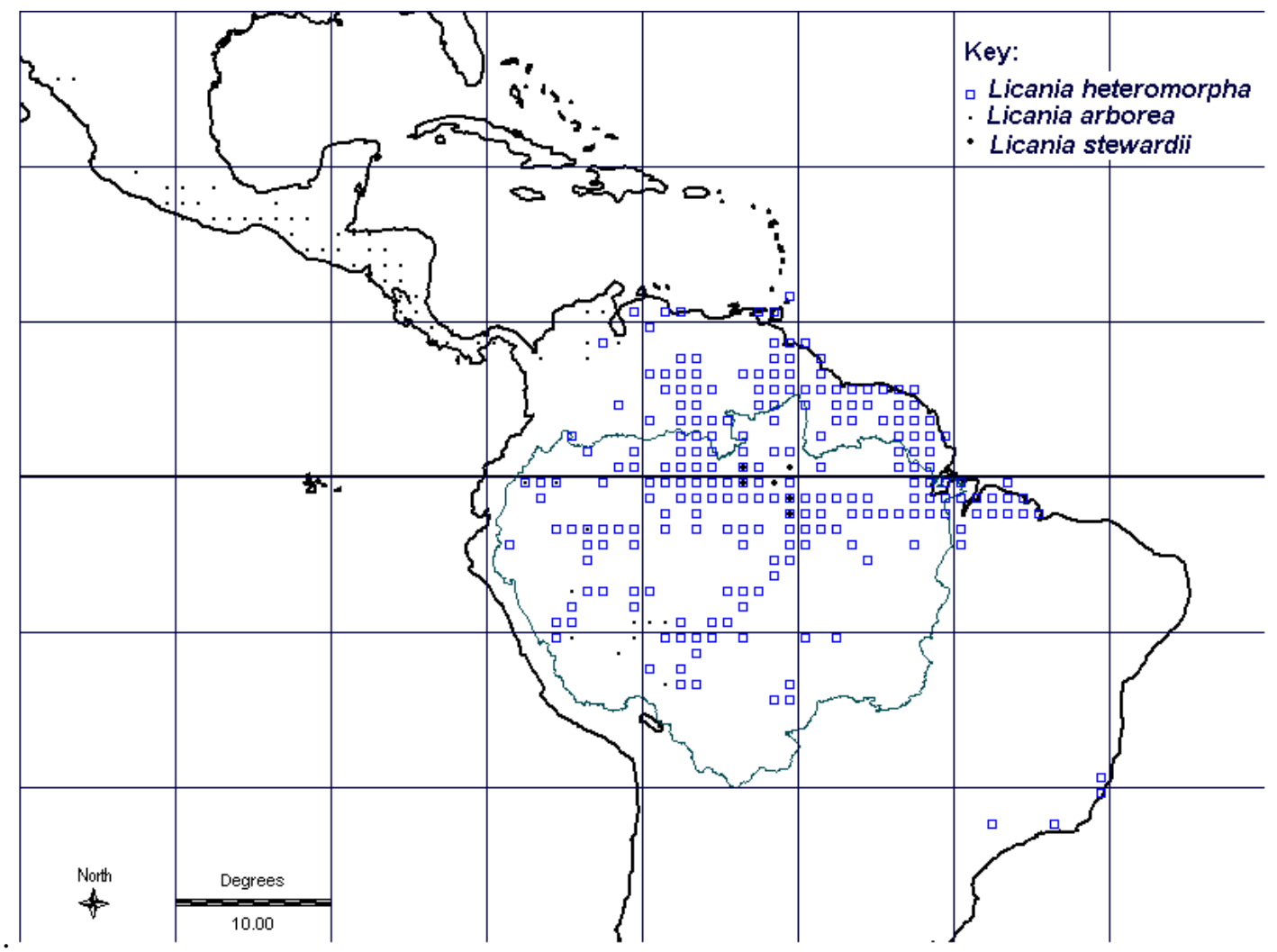

Fig. 4:1: Records for three Licania spp., (Chrysobalanaceae) (Prance \& Plana 1997)

Functional diversity within families and genera is such that an assessment of climate change response at this taxonomic level would give a falsely optimistic outlook for biodiversity conservation. The spatial distributions of species within a genus can be very variable (Fig. 4:1), increasing the likelihood of mixed response to a given environmental change. A species-level analysis still allows assessment of variation in higher taxon response.

Another alternative is to model impacts on vegetation types or biomes (e.g. white sand formations). A species focus is likely to simulate new assemblages following differential distributional change from present associations (Prentice et al. 1991, Fischlin \& Gyalistras 1997, Colinvaux et al. 1997), as species display non-random individual responses to spatial and temporal gradients. Where a community type survives, component species may be lost.

The potential for biodiversity loss can only be quantified by examining species-level change. Hence, this project takes the approach of studying Amazonian species' distributional response to climate change. 


\subsubsection{Functional group response}

Species may also be grouped by function, using guilds or plant functional types (PFTs ${ }^{4}$ ). PFTs interpret ecosystem function through morphology, phenology and life history characteristics (traits). It is arguable that the search for patterns amongst groups of species with similar characteristics would reduce the influence of error in species data (Gaston \& Blackburn 1999).

Functional classifications can be a priori systems, or be derived from a set of species and traits using a classification algorithm (Rutherford et al. 1995, Condit et al. 1996a, Díaz \& Cabido 1997). In an a priori system the classification is rigid, whilst with a data-driven approach the number of PFTs and the traits shift according to the species used. The advantage of a datadriven approach is that real patterns of shared traits are identified.

\subsubsection{Life forms}

Species can be grouped into life forms based on the size and physiognomy of mature individuals. The earliest classification for all higher plants used the positioning and protection of apical meristems, labelling plants as phanerophytes, chamaephytes, geophytes, helophytes, hydrophytes, hemicryptophytes, cryptophytes or therophytes (Raunkiaer 1934).

Height classes are often more useful to classify freestanding woody plants (typically phanerophytes, whose buds are borne on shoots projecting into the air, and chamaephytes, whose buds are close to the ground). Herbaceous plants, climbers and epiphytes are treated separately, with the exception of strangler figs, which originate as lianas and pass into the freestanding overstorey category on maturity. Classes depend upon the height and structure of the forest. For example, Hubbell \& Foster (1986) use the following classification:

- Shrub/bush: $<4$ m high

- Understorey tree (treelet): 4-10 m high

- Midstorey tree: 10-20 m high

- Overstorey tree (canopy and emergent): $>20 \mathrm{~m}$ high.

Height classes are imposed on a continuous gradient, whilst Raunkiaer's classes are intrinsically discrete. Many tree surveys exclude individuals below $10 \mathrm{~cm}$ diameter at breast height (dbh,

\footnotetext{
${ }^{4}$ The terms 'plant functional type' and 'functional vegetation type' (sensu Henderson-Sellers \& McGuffie 1995) are sometimes used interchangeably. In the current study, PFTs (e.g. 'deciduous understorey tree') are regarded as components of vegetation types (e.g. 'evergreen broadleaf forest') rather than equivalents.
} 
typically 1.3 m, e.g. Condit et al. 1996a, Phillips et al. 1998, Finegan et al. 1999). Dbh is a standard measure, but because it compares upper stem diameter of smaller individuals with lower diameter of taller individuals, it should be used with care (King 1996).

Height classes can be related to individual mass and shade tolerance. Organisms of a smaller mass typically have a higher reproductive rate and turnover time than larger organisms (see $\mathrm{r} / \mathrm{k}$ selection, section 2.2.2.2.2). The production of propagules at different heights implies different dispersal mechanisms (taller plants can exploit long-distance wind dispersal; different animals inhabit different height strata). The taller the mature plant, the greater its demand for light and other resources. Correlated features may include a faster potential growth rate and rapid response to light in seeds and young plants. Experimental work supports this: across six canopy species in Costa Rica, growth response to climate variation was related to individual size rather than species (Clark \& Clark 1994).

The life form system does not definitively separate opportunist and persistent species, seasonal and aseasonal habitats, maturation time or dispersal syndrome (even many emergents are animal- rather than wind-dispersed, Clark \& Clark 1992). It can be used to estimate impacts on ecosystem structure (Woodward \& Lee 1995), but gives a limited view of impacts on other biodiversity values. Life form does usually form part of other PFT classifications.

\subsubsection{Reproductive strategy}

Regeneration modes are widely used to classify tropical species, with light-demanding (pioneer) and shade-tolerant (persistent) species being treated as opposite ends of a continuum. Various field studies have rejected this dichotomy, identifying many intermediate species (Clark \& Clark 1992, Guehl et al. 1998, Kursar 1998).

At BCI, saplings of the more abundant species appeared indifferent to both light availability and slope (Hubbell \& Foster 1986). Rare species were more specialised, but mainly indifferent to slope. These short-term studies are likely to be inadequate in distinguishing tree strategies, because of their long life spans and annual variation in microenvironment (Clark \& Clark 1992). Characteristics from more life-history stages should be included in a trait classification.

A subsequent study (Condit et al. 1996a) therefore allocated additional values for continuous traits (mortality, diameter growth rate, gap colonisation, leaf lifetime, moisture requirement) and Boolean traits (life form, deciduousness) to trees from Barro Colorado Island (BCI, in the Panama Canal). The trees were grouped into 11 PFTs based on these traits. Pioneer and shadetolerant species fell into separate groups according to all the demographic traits, but there was much more variation within the pioneer species. This may support the suggestion that long-lived 
and short-lived pioneer trees have adopted different strategies, with fast growth effectively conferring a short lifespan (Finegan 1996).

The recorded response of species in these PFTs to the recent drying in Panama (section 4.2.1.2) was evaluated. It was predicted that deciduous trees would increase and understorey trees decline in abundance, but neither prediction was borne out. Mesophytic understorey trees, representing a small proportion of species richness, did experience a marked decline in numbers (47\%), a change negatively associated with moisture index. There was a negative association between demographic score $(\lambda)$ and mortality, showing that more pioneer species had declining populations under drought conditions than shade-tolerant species. The fastest-growing populations in understorey and canopy were both pioneers, however. This apparent paradox may result from a change in competitive dominance amongst pioneer species. The most extreme pioneers in the classification were the most successful following climate change.

\subsubsection{Leaf life-time}

Longer-lived leaves hold a greater nutrient store and a higher level of herbivore-repelling secondary metabolites. Shorter lifetimes carry a metabolic cost, but allow more flexibility in response to gap formation and light flecks, as older leaves have a limited capacity to acclimate to new conditions. One study focusing on shade-tolerant species of seasonal forest aimed to identify traits important for in situ climate change response (Kursar 1998). Leaf lifetime (measured in years) was chosen as the sole classifier, because its variation is indicative of differences in physiology. Species with long-lived leaves also had drought adaptations such as long roots and xylem cavitation resistance, and were resistant to the 1991-92 El Niño event.

Leaf lifetime also correlates strongly with demography (mortality, growth rate and gap colonisation) - for all pioneer species in BCI, leaf lifetimes were under one year, and for most shade-tolerant species, they exceeded one year (Condit et al. 1996a). Leaf lifetime was negatively associated with growth.

Were suitable information available for a large number of species, this gradient would be useful in identifying risk and parameterising desiccation response.

\subsubsection{Response indicators}

Combinations of plant traits selected for their responsiveness to climate change can be used to selecting indicator species to monitor the onset of climate change effects. In Argentina, 100 widespread, abundant species were classified into eight PFTs with such an aim (Díaz \& Cabido 1997). Traits such as photosynthetic pathway, seed size, palatability and drought resistance were 
employed. The most common patterns were high investment in photosynthesis and growth (a 'pioneer' strategy), versus longer lifespan, better storage and defence (a 'climax' strategy). Types produced by the classification include 'trees' and 'short graminoids'.

The focus on common species gives a very limited picture of overall response, as the largest fraction of tropical biodiversity is represented by rare species (section 2.2.4). Species survival is likely to be over-estimated, because the more widely spread species will have the greatest range of environmental tolerances. The survey identified a within-group variation in dispersal ability. As each type includes examples of good dispersers, it was concluded that:

"no PFT would be eliminated by climatic change",

No assessment of potential versus required dispersal rates was made to substantiate this conclusion.

In another classification based on factors governing response, four gradients were chosen to identify tree PFTs for a climate change gap model for the boreal and temperate forests of the Northern Hemisphere (Bugmann 1996). These were tolerance to cold, drought and shade, and deciduousness. The species were subjectively placed into six types based on the gradients. There was a good correlation between simulated PFT distributions using mean parameters from the constituent species, and the species themselves. Under IS92a conditions, there were more discrepancies between PFTs and species. This supports the assertion that species will have a unique response to change, differing from that of their guild or vegetation type.

Gillison \& Carpenter (1997) propose a detailed 'functional grammar' based on individuals' attributes: the chlorotype, leaf type, size \& inclination, life form and root type are used to classify each plant into a functional type or 'modus'. Species may appear in multiple modi. They suggest that change response can be predicted both between and within species.

This detailed data is not available for Amazonian species. However, a PFT classification using more frequently recorded traits could be useful both in selecting a species set representative of overall response and in offering parameters for species with insufficient life-history data. It is clear that species-level modelling can demonstrate impacts on range governed by individual species tolerances that would be masked by the wider spatial coverage of PFTs. 


\subsection{Change FaCtors}

This section considers the environmental factors affecting species' survival under changing conditions. The variables most influencing present-day distributions, and those likely to induce changes with climate change, are discussed with a view to response modelling. The chosen variables should represent physiological constraints to plant growth, reproduction and survival. For example, it is better to use the sum or extreme values of a given variable, rather than the mean, as these are the values that plants experience.

Climate change is expected to subject forest plants to decreased moisture availability, increased temperature, increased climate variability, a changed atmospheric composition and if tree mortality rises, a more disturbed, open canopy. Physiological processes affected could include photosynthesis and respiration. Phenological processes affected could include bud-burst, defoliation (dehiscence), the onset of flowering, seed setting and ripening (de Groot et al. 1995). Even species' growth forms can be altered (Bazzaz 1998). In modelling terms, the combination of these impacts would affect the rates of growth, survival and reproduction.

The impact of most types of change is proportional to their intensity, but there are some distinct boundaries at which physiological or phenological processes are halted, such as the temperature at which an enzyme is denatured.

\subsubsection{Climate variables}

Energy and moisture availability are the key climate requirements for photosynthesis. Both limit growth, and temperature can limit MA through high evaporative demand. Temperature tolerance through transpiration is costly in terms of water loss. Drought adaptations include tolerance mechanisms to limit, and avoidance mechanisms to escape, the joint impacts of high temperature and low MA.

\subsubsection{Temperature}

GCMs simulate a regional increase in temperature with increased $\mathrm{CO}_{2}$. As the previous major climate epoch was largely glacial, any inherent adaptations are likely to be biased towards cooler temperatures (Mitchell \& Williams 1996). Species old enough to have persisted through previous warm periods may encounter faster change than before.

Generally, present-day temperature gradients in the tropics are spatially and temporally weaker than moisture gradients (Barry \& Chorley 1998). Plants may therefore be closely adapted to particular thermal regimes, which would render them vulnerable to increases in the level and variability of temperature (Janzen 1967). 
Temperature rises of a few ${ }^{\circ} \mathrm{C}$ may not directly affect tropical plant photosynthetic rates (Scholes \& van Breemen 1997). However, the interaction between temperature and water availability may have effects that are more significant. Photosynthetic response pattern depends upon the photosynthetic pathway. The $\mathrm{C}^{3}$ pathway is most common in tropical forest plants (Bazzaz 1998), while many epiphytes have drought-adapted CAM or $\mathrm{C}^{4}$ pathways (Hogan et al. 1991). CAM plants avoid water loss by nocturnal stomatal opening, and by 'CAM-idling' can keep their stomata closed during dry, hot periods. Most savanna grasses are $\mathrm{C}^{4}$ plants. The $\mathrm{C}^{4}$ pathway is thought to have evolved independently several times under warm conditions and high oxygen concentrations, when it is advantageous to have a shorter period of stomatal opening, and photorespiration reduces photosynthetic efficiency (Darnell et al. 1990, Spicer 1993). These groups may be more tolerant to temperature increase and associated drying (Grime \& Callaghan 1987).

Temperatures above plant tolerances may damage photosynthetic and other enzymatic systems, especially if water availability is restricted, as transpiration reduces temperature damage by cooling leaf surfaces. However, high temperature per se appears to be a less serious limiting factor than moisture availability. The present study has found that Amazonian species are recorded in those $1^{\circ}$ cells with the highest temperatures when precipitation $>1000 \mathrm{~mm}$, but that the combination of high temperatures and low precipitation limits presence.

Cold can also limit distributions (Woodward 1987). Some tropical trees require temperatures constantly greater than $10^{\circ} \mathrm{C}$, so that warming alone would enable them to spread poleward (Melillo et al. 1996). This study will therefore include coldest annual mean temperature as an absolute constraint on species' distribution and climate change response. All other impacts of temperature change on species physiology and distribution are assumed to be mediated through interactions with moisture supply.

\subsubsection{Moisture availability}

Most GCMs simulate a decline in the quantity and frequency of neotropical precipitation with climate change (section 3.7.3.2). Increased temperature may induce a further decline in atmospheric humidity, if precipitation is not sufficient to meet evaporative demand.

Where an increase in precipitation occurs, species survival will depend upon their competitiveness under these higher-resource conditions, and resistance to pathogens associated with increased humidity. At BCI, an unusually wet 'dry' season apparently prevented flowering and fruiting in many plants (Condit 1998). 
Moisture availability (MA) experienced by plants is a combined result of precipitation, soil water holding capacity (WHC) and stores, and evapotranspirative demand. While preadaptations to temperature increase may be unlikely, pre-adaptations to increased MD might be expected because of the evidence of drying at the time of the last glaciation (section 2.1.1). The existing moderate level of interannual variation in rainfall in the Amazon region also means that many species may be able to withstand an increased frequency of extreme mean values. However, Fearnside (1995c) argues that many Amazonian tree species are at the limits of their tolerance to drought stress, as demonstrated by the high turnover of trees at the edge of the forest patches created by the Biological Dynamics of Forest Fragments (PBDFF) near Manaus. Many trees die standing, which points to water stress as a likely cause. Species from more seasonal environments are colonising this fragmented forest.

A decrease in annual rainfall of 14\% was observed between 1966 and 1992 in the centre of the isthmus surrounding BCI. There are signs of species loss at BCI with declining MA (Condit 1998). The overall drying trend has reduced numbers of 33 of 37 originally abundant mesophytic species. The number of individuals of drought-tolerant trees has increased, and species richness is declining. This pattern could be part of a fluctuation in metapopulation distribution associated with rainfall fluctuations, with numbers remaining more stable over a wider area, but even so, the study highlights species' vulnerability to desiccation.

The physiological process most susceptible to chronic water stress seems to be cell growth (Hsiao et al. 1976). All maturation and reproduction processes require new growth and so are inhibited by water deficits. Relationships between rates of cell and cell wall expansion and MA have been formulated. These require measures of cell wall yield threshold and cell turgor potential (Woodward 1987).

Species richness is inversely correlated with dry season length at a neotropical scale (Clinebell et al. 1995). That is, more species are intolerant of, or competitively disadvantaged under, long dry seasons than short or non-existent ones. Data from Panama, where rainfall is seasonal, also suggest that the moisture-demanding (mesophytic) trees are restricted to wet areas, but that fewer drought-tolerant species are restricted to drier zones (Condit 1998).

It has been suggested that an increase in dry season length of just three weeks would affect forest composition, removing 10-20\% of species in wet forests (Condit cited in Bawa \& Markham 1995). A longer dry season would have more immediate effects on seasonal forest than changes in annual volume of precipitation: it is known, for example, that the onset of a dry period stimulates flowering or leaf-fall in many seasonal forest species. A wet period often stimulates germination, and therefore a longer interval between the start of the dry and the wet 
season may reduce seed survival rates. A longer interval between dry-season triggered leaf fall and wet-season triggered leaf flush would reduce plants' annual photosynthetic yield.

An increase in MA variability over shorter periods than a 'dry season', will also affect plant fitness. Where mean precipitation remains constant but variability increases, moisture deficit tolerances will be more frequently exceeded. More dry intervals within the wet season may increase seedling mortality. In mature plants, premature leaf abscission has been related to plant and soil water potential thresholds for various species (Woodward 1987).

Amazonian species whose distributions include savanna areas are least likely to be adversely affected in situ by increased seasonality and MA variability. It may also be advantageous to occupy areas of high soil-water holding capacity, which are buffered against increases in variability. For example, in the 1992 drought in eastern Amazonia, annual rainfall was $1100 \mathrm{~mm}$ rather than the usual $1700 \mathrm{~mm}$. Soil reserves were depleted and took 2 years to be restored. There was no unusual tree mortality (Nepstad et al. 1994).

To represent these different aspects, MA variables for a species response model should represent changes in total MA, but also changes in seasonality at a temporal scale of days or weeks and should incorporate changes in soil moisture following drought ('lag effects').

\subsubsection{Bioclimate variables}

Many studies implicitly assume that temperature and precipitation act independently on plants. Bioclimate variables, often based on actual and potential evapotranspiration (AET and PET), combine their effects to express the 'effective environment' experienced by plants (Mackey 1996). These variables simulate the net availability of water and energy to the study system, so are arguably more useful than precipitation and temperature values, which only simulate inputs (Lenihan \& Neilson 1995, Stephenson 1998; e.g. Aspinall \& Matthews 1994, Prentice et al. 1992, Neilson \& Marks 1994, Sykes et al. 1996). A single composite variable cannot express all variation - the annual availability of water and energy has a different impact depending on whether it is evenly spread or clustered throughout the year.

PET represents energy input, or the atmospheric demand for water over a saturated surface. AET represents the water returned to the atmosphere, constrained by moisture availability and maximum rates of ET. AET is a good surrogate variable for primary production (Stephenson 1998). The difference between PET and AET represents the degree of water stress, or moisture deficit (MD). The combination of AET (measuring biologically available energy and water) and MD (measuring drought stress) is well supported for use in vegetation and species modelling (Shao \& Halpin 1995, Prentice et al. 1992, Stephenson 1998). The pattern of moisture 
availability through the year can concentrate this MD into a short period, or spread it over a longer dry season. In Amazonia there is considerable spatial variation in seasonal moisture availability (SMA). An SMA measure would thus be a useful third variable in determining species' distributions.

A count of 'growing degree days' (GDD, also known as effective temperature sum (ETS), thermal time or accumulated temperature), is often used to estimate climate suitability for a specific crop with known growth constraints (Carter et al. 1991, Kenny et al. 1995). 'Effective growing degree days' (annual GDD - GDD to bud-burst, Sykes et al. 1996) also incorporates species' phenology. MA may be measured separately. One adaptation used to assess climate change impacts on South African opportunist species counted 'optimum growth days', on which AET exceeded half of PET and monthly mean temperature exceeded $24^{\circ} \mathrm{C}$ (Rutherford et al. 1996). The assumptions about optimal growth conditions made by these measures are too standardised for use with little known but variable wild species such as Amazonian plants.

Most measures of dry season intensity or seasonality have been based on the number of dry months, or measures of the annual difference between PET and AET (PET - AET, Walsh 1996; AET - PET Prentice et al. 1992; AET/PET, Lenihan \& Neilson 1995, AET - rainfall, Condit 1998). For example, an 'annual soil moisture deficit index' which sums the monthly differences between PET and AET was a more sensitive measure of Canadian vegetation response to drying in a climate-vegetation model than was annual AET/PET (Lenihan \& Neilson 1995). These two measures do not distinguish between seasonal dryness and dryness that is distributed evenly throughout the year.

I suggest that PET - AET is a logical measure of annual MD, and that the length of time for which PET exceeds AET is a consistent SMA measure to use alongside bioclimate variables. A measure of maximum annual SMA in days should provide a finer differentiation between locales than a monthly measure.

\subsection{Calculation methods}

The different concepts and measures of ET are barely explored in the distribution modelling literature. PET can be based on the moisture absorptive and release properties of the actual surface cover $\left(\mathrm{PET}_{\mathrm{a}}\right)$, or on a 'reference' value $\left(\mathrm{PET}_{\mathrm{r}}\right)$ based on a hypothetical

"short green crop, completely shading the ground of uniform height and never short of water" (Ahn \& Tateishi 1994a),

which is usually grass or alfalfa (Federer et al. 1996). Standing water may also be used as a reference surface (Holdridge 1962). Similarly, AET can be modelled using actual (AET ${ }_{a}$ ) or reference vegetation $\left(\mathrm{AET}_{\mathrm{r}}\right)$. $\mathrm{ET}_{\mathrm{r}}$ may be obtained from an $\mathrm{ET}_{\mathrm{a}}$ formula by using surface 
parameters for the appropriate vegetation type. Conversely, coefficients can be used to adjust the reference ET for a given cover type.

ET estimates thus depend on whether $\mathrm{ET}_{\mathrm{r}}$ or $\mathrm{ET}_{\mathrm{a}}$ is used, the chosen calculation method, and the accuracy of the climate and surface input variables. In a North American study of 9 methods and 5 locations, different methods calculated for the same cover type and location found annual differences of several hundred mm (Federer et al. 1996; see also McKenney \& Rosenberg 1993). For both $\mathrm{PET}_{\mathrm{r}}$ and $\mathrm{PET}_{\mathrm{a}}$, this variation was greatest under conditions of high PET. It was also found that monthly calculations of $\mathrm{PET}_{\mathrm{a}}$ were not a good surrogate for the sum of daily calculations.

$\mathrm{PET}_{\mathrm{r}}$ gives a standardised climate variable of physiological importance to plants, but independent of vegetation type (Stephenson 1998): a neutral measure of evaporative demand. This is especially useful in conditions of climate change where future vegetation types are unknown. However, $\mathrm{AET}_{\mathrm{a}}$ for a well-watered forest conditions is likely to exceed $\mathrm{PET}_{\mathrm{r}}$ for that site, because the greater leaf surface area allows a greater throughput of water in transpiration than would be seen in the reference crop. I submit that $\mathrm{PET}_{\mathrm{r}}$ and $\mathrm{AET}_{\mathrm{r}}$ are most useful where vegetation type is unknown, or is to be simulated, but that $\mathrm{PET}_{\mathrm{a}}$ and $\mathrm{AET}_{\mathrm{a}}$ are more useful when the aim is to model the environmental tolerance, and thus spatial range, of a species as closely as possible.

Two common derivations for PET are the Priestley-Taylor $\left(\mathrm{P}-\mathrm{T}=\mathrm{PET}_{\mathrm{r}}\right)$ and Penman-Monteith $\left(\mathrm{P}-\mathrm{M}=\mathrm{PET}_{\mathrm{a}}\right)$ formulae. $\mathrm{P}-\mathrm{T}$ is often described as a calculation for a short green crop (McKenney \& Rosenberg 1993, Ahn \& Tateishi 1994a), but was originally based on the equilibrium evaporation over standing water, modified by a parameter $\alpha$ to account for advection or entrainment of dry air (Priestley \& Taylor 1972 described in Kim \& Entekhabi 1997). PET is usually derived from temperature and net radiation only (Ahn \& Tateishi 1994a). $\mathrm{P}-\mathrm{M}$ has a stronger mechanistic basis, but parameterisation for individual locations may be difficult because of the requirement for vegetation parameters including height, stomatal resistance and leaf area index where P-T uses only $\alpha$.

P-M is thought to be a more accurate overall measure than P-T (McKenney \& Rosenberg 1993, McAneney \& Itier 1996), but P-T has a similar accuracy to P-M for reference vegetation under humid conditions (McAneney \& Itier 1996). However, it performs poorly under arid conditions, possibly because it does not respond to decreasing vapour pressure (Federer et al. 1996). This renders it less useful when the study region includes seasonally or constantly arid zones, or under conditions of climate change towards aridity. 
AET estimation techniques range from simply assuming that all available water is transpired until evaporative demand is met (Dietrich et al. 1982) through to separate calculations of evaporation from surface and intercepted water and canopy transpiration at different vertical levels (Costa \& Foley 1997). It is sometimes estimated using regressions between air temperature and measured evaporative loss for a particular vegetation type (Neilson \& Marks 1994). GCM methods of calculating $\mathrm{AET}_{\mathrm{a}}$ are closely related to the P-M formula (e.g. Cox et al. 1999), but PET is not calculated inside most GCMs.

An ET simulation for the Amazon basin has been carried out using a land-surface model featuring six soil layers and water flow between cells (Costa \& Foley 1997). The model simulates stomatal conductance based on light and carbon dioxide availability for photosynthesis, as does the biome model in HADCM3 (section 3.5.1.3). The models demonstrate the sense of partitioning evaporation and transpiration, as transpiration rates are limited by surface resistance as well as aerodynamic resistance. As outputs from this type of model are not readily available for all regions, resolutions and climate forcings, there is a need for simpler calculations, especially when the research priority is not hydrological.

\subsection{Impact of method choice}

Where an $\mathrm{ET}_{\mathrm{a}}$ method is chosen, different treatments may give different weights to the same cover types. Where possible, comparisons with field measurements of ET should be carried out. Where a calculation produces generally higher PET and AET values for a vegetation type, the estimate of water deficit over any given period will be higher within Amazonia. The area modelled as having seasonally arid climates will therefore be larger.

If calculated ET values are generally lower, there will be more areas with high moisture availability under present conditions. In a distribution-modelling context, fewer species will appear to tolerate conditions of seasonal or perpetual aridity. The same simulated degree of drying may appear to have a greater effect on potential species richness in humid areas.

\subsection{Conclusion}

After examining the alternatives from an ecological standpoint, it is concluded that a formula that accounts for the impact of different vegetation types on ET is most appropriate. I recommend the use of $\mathrm{P}-\mathrm{M} \mathrm{PET}_{\mathrm{a}}$ and $\mathrm{AET}_{\mathrm{a}}$ for change response simulation. This maximises simulation of actual conditions and compatibility with GCMs processes. $\mathrm{AET}_{\mathrm{a}}$ calculation should follow GCM processes as far as possible. GCM processes involving stomatal responses to $\mathrm{CO}_{2}$ should not be included unless similar feedbacks are replicated in the plant suitability model. Reduced stomatal opening increases apparent MD, but also reduces plant water stress. 


\subsubsection{Climate extremes}

Extreme events include occasions upon which climate conditions fall outside the usual range of variation. The frequency of extreme events is expected to increase with climate change (Houghton et al. 1996). The impact will vary between species, depending upon their innate resistance to the type of stress. Disturbance facilitates the dispersal of opportunistic plants, especially those with a short generation time (Grime 1977). Other short-lived species may be adversely affected by an increased occurrence of extreme events that repeatedly disrupt reproduction or regeneration.

Cyclones do not appear to be a concern for future Amazonian populations (section 3.7.3.2.2).

\subsection{Drought and flood}

Droughts and flood frequency can be estimated from GCM monthly rainfall simulations (Cubasch et al. 1995), or the incidence of heavy rainfall. In a drought, there is an absence of significant precipitation, lasting long enough to cause moisture deficits in the soil (Barry \& Chorley 1998):

$$
\text { Soil water + precipitation < evapotranspiration + runoff }
$$

That is, any instance when evaporative demand is not met by moisture availability can be defined as a drought. With drying and warming, drought frequency is likely to increase.

Severe drought damages xylem cells in hardwood trees by cavitation following withdrawal of water, reducing water absorption and storage abilities (Woodward 1987). This can be a major cause of death in mesophytes of usually aseasonal forest undergoing a drought. In seasonal environments, many trees use mechanisms such as narrower xylem vessels and smaller pores in pit membranes to avoid these embolisms (Walsh \& Newbery 1999). These adaptations are not universally adopted because they reduce maximum conductance rates, and thus growth rates.

Epiphytes may be particularly susceptible to drought, because of their dependence on direct water input from atmosphere (Benzing 1998). Vascular and non-vascular epiphyte richness are both strongly correlated with water availability. It is unclear whether CAM in epiphytes confers an in situ advantage under increased water stress, or indicates a borderline existence. Although CAM occurs in drought-resistant desert plants, stomatal conductance decreases with warmer nocturnal temperatures, reducing $\mathrm{CO}_{2}$ intake and thus limiting photosynthesis. Individuals show some ability to adjust to changing temperature, but the extent is unknown. 
Where ET, drainage and runoff are low enough to allow water to collect aboveground, floods occur, mainly in low-lying and riverine areas. Várzea forests undergo this level of flooding annually. A standard definition of an extreme precipitation event, based on field evidence of flooding and erosion, is $>40 \mathrm{mmd}^{-1}$ (CIC 1996). Soil moisture holding capacity will affect flood probability and persistence, so that populations on well-drained sandy soils will be more resilient to flooding.

Localised flooding in terra firme forest, resulting from heavy rains or disruption of hydrological systems, may cause osmotic loss of cell solutes, scorching damage to photosynthetic tissues or encourage microbial breakdown of plant material. Excessive moisture on surface tissues slows evapotranspirative heat loss and photosynthetic gas exchange, and adds weight that may lead to structural damage. In Colombia, 'flooding' caused by river channel shifting is the main cause of observed mortality in mature trees of the riparian understorey palm Phytelephas seemannii (Bernal 1998). Floods may increase propagule dispersal distances, especially for ground-layer plants.

Much of the variation in drought frequency would be captured in the combination of SMA and MD (section 4.2.1.3). The fine spatial scale at which floods generally occur is not amenable to inclusion in geographical scale models. Extreme precipitation events would only act to recharge soil moisture stores, having both an immediate and ongoing effect on AET and MD.

\subsubsection{Light}

Impacts of light incidence, aside from the energy availability component of ET, are considered below. Light availability per se will not be included in the present study of change, because vegetation structure and plant physiology are not modelled, and because there is a large uncertainty in both the spatial and temporal aspects of change in light availability.

Initially, climate change may increase tree mortality as species' ranges shift, increasing light availability in affected forests. If ET were reduced following the loss of biomass, cloud density would decrease and permit further insolation. Increased light availability affects plants directly through increasing potential photosynthetic rates and increased exposure to UVB, and indirectly through additional rises in temperature. Increased UVB has been shown to have deleterious effects on some tropical species' growth even under elevated $\mathrm{CO}_{2}$ conditions (cassava, rice, Hogan et al. 1991). Decreased stratospheric $\mathrm{O}_{3}$ levels permit increased UVB penetration, but at present, most anthropogenic $\mathrm{O}_{3}$ depletion occurs at higher latitudes. 
Shade-tolerant species are less able to exploit increased light (Woodward 1987) and are likely to be outcompeted, all else being equal. Pioneer species able to exploit canopy gaps should benefit most from increased light availability.

In still, humid conditions with low transpiration rates, sun flecks can raise leaf temperatures to several additional degrees above air temperature (Evans et al. 1974). Transpiration rates increase, potentially putting the plant under moisture stress, but reducing its temperature. This makes shade-adapted species vulnerable in an opened canopy. Above the optimum physiological temperature, respiration rates increase and photosynthetic rates drop. At worst, the photosynthetic system of the plant may be damaged.

\subsubsection{Soil nutrients}

Soil type tends to be much more heterogeneous over the Amazon basin than climate variables, which are more spatially autocorrelated. Soil type itself is a categorical variable, difficult to average over a cell. Therefore, it is of more use in modelling local, fine-scale distributions than geographic ones (Peterson \& Vieglais 2001). Present-day soil-related variables can be used to characterise grid cell mean moisture-holding capacity and soil albedo. GCMs do not model changes in soil composition, due to the uncertainty about overall effects of climate change on nutrient concentrations and $\mathrm{pH}$. It is reasonable to assume that soil-related variables will remain fixed throughout a period of climate change.

Various possible climate change effects on fertility have been discussed. Higher temperatures could decrease soil nitrogen (N) content by stimulating denitrifying soil bacteria (Parry 1990, Melillo et al. 1996), and initial faster plant growth under enhanced $\mathrm{CO}_{2}$ can deplete soil nutrient resources. Experimental work has shown that increasing atmospheric $\mathrm{CO}_{2}$ tends to increase $\mathrm{C}: \mathrm{N}$ ratios in plant tissues and leaf litter (Bazzaz 1998). The additional lignin content of the leaves can slow decomposition, increasing the carbon storage of the soil, but decreasing the available nutrient pool.

If the original vegetation is killed by climate change, the dead organic matter content of surface soils will initially increase, and when rainfall is low, nutrient leaching rates will decrease. However, any lag between mortality and colonisation by better adapted species could expose soil to erosion and leaching when heavier rains occur, losing nutrients from the system and increasing soil acidity.

Some Neotropical species have exceptionally high nitrogen requirements (e.g. Laurelia philippiana, Lusk et al. 1997). Under fertile conditions, these plants have characteristic high 
shoot:root ratios and high surface-area:volume ratios, as they are limited by light availability rather than nutrients. The resultant high transpiration levels would be disadvantageous under increased drought, rendering individuals of these species particularly susceptible to nutrient shortage, or to moisture shortage where nutrients are available.

If there were more competition for soil nutrients, various traits would be adaptive. Tree species that are able to mature at a smaller size class would have a selective advantage over those that only flower when full size. Infertile-site plants with long-lived leaves conserve nutrients (Chabot \& Hicks 1982), but lose the water conservation advantages of seasonal deciduousness. Leguminous species, being able to fix nitrogen, might also become more common.

Conversely, removal of limitations on root and nitrogenous nodule growth would increase overall soil nitrogen levels. Higher $\mathrm{CO}_{2}$ levels have been found to enhance nodulation and levels of mycorrhizal association in several north temperate woody species (Hogan et al. 1991, Bazzaz 1998). It is not known whether the same pattern would be seen in tropical forests.

\subsubsection{Carbon dioxide}

Species and climate models rarely consider photosynthetic and growth responses to increased atmospheric $\mathrm{CO}_{2}$ availability, which are variable and non-linear (Parry 1990, Hattenschwiler \& Körner 1996, Bazzaz 1998). The response may be disproportionate to the low rate of anthropogenic increase in $\mathrm{CO}_{2}$, where availability limits photosynthesis. This is more frequent in $\mathrm{C}^{3}$ than $\mathrm{C}^{4}$ plants (Grime \& Callaghan 1987). $\mathrm{CO}_{2}$ concentrations have approximately doubled since the previous ice ages, so many plants may be close to $\mathrm{CO}_{2}$ saturation (Bawa \& Markham 1995).

The main terrestrial gradients in $\mathrm{CO}_{2}$ concentration are temporal rather than spatial, which reduces the available field evidence for species response to variation. Localised emissions of $\mathrm{CO}_{2}$, as around volcanoes, provide exceptions (e.g. high tree mortality following increased $\mathrm{CO}_{2}$ concentrations in soil, Farrar et al. 1995), but only provide limited data, and the effect of $\mathrm{CO}_{2}$ may be difficult to distinguish from that of other gases emitted.

It is also difficult to carry out manipulative field experiments on $\mathrm{CO}_{2}$ effects without indirectly altering other microclimatic factors such as temperature and humidity. However, new climatecontrolled chambers are being developed (Norby et al. 1997). In addition, free-air carbon enrichment (FACE) experiments manipulate $\mathrm{CO}_{2}$ concentrations around unenclosed stands of up to $10 \mathrm{~m}$ height. The technique uses continuous monitoring and computer-controlled vertical $\mathrm{CO}_{2}$ outlets spaced through the plot (Hendrey et al. 1999). Most laboratory and field 
experiments have hitherto used small plants or seedlings, because of limited resources. Outcomes for long-lived plants and communities are therefore still uncertain.

A wide variation in response has been observed for several tropical species in growth chambers, based on leaf assimilation and leaf area. Predicted mass change varied from -59 to $+447 \%$ (Hogan et al. 1991). This growth response is generally lower where nutrients are limited - there is little understanding of the reasons for the exceptions. Most species with lower intrinsic growth rates have a lesser response to $\mathrm{CO}_{2}$ increase than fast-growing plants, but this is not universal (Poorter 1998).

\subsubsection{Photorespiration}

Photorespiration is a cellular process in competition with photosynthesis, catalysed by a photosynthetic enzyme. It dominates when there is a low $\mathrm{CO}_{2}$ to $\mathrm{O}_{2}$ ratio. In photorespiration, $\mathrm{O}_{2}$ is added to ribulose 1,5-bisphosphate with the eventual release of $\mathrm{CO}_{2}$. This causes $\mathrm{C}^{3}$ species, which include most forest trees, to lose up to $50 \%$ of the carbon fixed in photosynthesis (Darnell et al. 1990). $\mathrm{C}^{4}$ species lose much less because of the high $\mathrm{CO}_{2}$ levels in their bundle sheath cells, where the second stage of $\mathrm{C}^{4}$ photosynthesis occurs, suppress the process.

A similar suppression is expected for $\mathrm{C}^{3}$ species at increased $\mathrm{CO}_{2}$ concentration, improving their photosynthetic efficiency. This may balance the advantage of $\mathrm{C}^{4}$ plants under increased temperatures (section 2.2.3.2).

\subsubsection{Down-regulation}

Temporal response to $\mathrm{CO}_{2}$ is also non-linear: an initial growth spurt is typically replaced by a more moderate response, as the plant 'acclimatises' to the increased levels. This 'downregulation' is strongest in plants with few carbon sinks (such as growing tips or symbionts) (Bazzaz 1998). It is associated with a build-up of sugars and starches, which is linked to reduced inorganic phosphate regeneration, which limits photosynthesis. Phosphorous uptake replaces carbon uptake as the rate-limiting step. Mycorrhizal growth in response to increased carbohydrate availability may limit down-regulation, supplying both a carbon sink and a phosphorous source (Staddon \& Fitter 1998). Again, experimental data are very limited. 


\subsubsection{Transpiration}

In addition to its effect on rates of photosynthesis and photorespiration, increased $\mathrm{CO}_{2}$ is associated with a decrease in stomatal conductance, favouring water conservation. This would mitigate the effects of drought on $C^{3}$ plants (Hogan et al. 1991). Growth chamber studies have shown that doubling ambient $\mathrm{CO}_{2}$ causes a decrease in stomatal aperture of $40 \%$, which reduces transpiration by 23-46\% (Cure \& Acock 1986, Eamus 1991). The reduced transpiration is expected to increase surface air warming (by $0.5^{\circ} \mathrm{C}$ with a halving of stomatal conductance at current leaf area). These climate feedbacks are included in some GCM simulations (e.g. Gordon et al. 2000).

\subsubsection{Tropical studies}

Long-term plot studies suggest that Amazonian forest biomass at undisturbed sites is increasing, possibly in response to $\mathrm{CO}_{2}$ concentrations (Phillips et al. 1998). Manipulative experiments concentrate on pioneer species (Bazzaz 1998). These are fast-growing and tolerate disturbance, so may benefit more than others from increased $\mathrm{CO}_{2}$. Photosynthesis, growth and water use efficiency (WUE) all increased in the experiments.

Due to WUE gains, $\mathrm{CO}_{2}$ concentrations are positively correlated with physiological tolerance to low water availability and temperature extremes. Increased $\mathrm{CO}_{2}$ may partially offset the effects of a changing climate, but the effect on different species is unclear. For this reason, the direct effects of changing $\mathrm{CO}_{2}$ concentration will not be included in the response model.

\subsubsection{Biotic interactions}

Individual response models treat a species as if it existed in isolation. Most experimental work is also autecological. The unpredictable response of model ecosystems when subjected to climate change factors sounds a cautionary note for change response studies, as detailed below.

\subsubsection{Competition}

Where a species' spatial distribution appears to be limited by a functionally similar species, a conservative assumption is that this would persist during periods of directional climate change. A simulation of such interactions would only really be effective if all species in a particular guild were being modelled.

This sort of assumption must be viewed with caution. In temperature and $\mathrm{CO}_{2}$ enhancement trials, interactions between species from the same guild have influenced competitive outcomes under climate change in unexpected ways. A study using several Drosophila fly species over a 
simulated temperature cline found that instantaneous warming led to a change in dominance (Davis et al. 1998a, b). Species' survival time changed depending which other species were present, and interactions shifted species' apparent optimum temperature. Competing species coexisted for longer when a parasitoid wasp was present.

A $\mathrm{CO}_{2}$ experiment again demonstrates the difficulty of predicting community responses to change. When five early successional tropical tree species were grown singly, elevated $\mathrm{CO}_{2}$ levels had similar effects on the growth of each. When the young trees were grown competitively, the $\mathrm{CO}_{2}$ effect on plant architecture and biomass varied considerably between species (Bazzaz 1998). Control trials showed that different plants became dominant under high$\mathrm{CO}_{2}$ conditions than under present-day $\mathrm{CO}_{2}$.

\subsubsection{Mutualism}

Small changes in phenological timing can de-couple plants from their pollinators or dispersers, endangering plants whose tolerances would otherwise permit survival under a changed climate. These effects may take some time to be obvious in tree populations, where the lifetime of the tree is much longer than that of its mutualists. Fortunately, generalist associations are more resilient to change, and occur more frequently in Amazonian plant species.

\subsubsection{Herbivory}

\subsection{Climate response}

Herbivorous animal distributions replicate the distributions of their food plants within the animals' own climate tolerances. If climate change has a stronger impact on populations of one than the other, the ratio of food plants to herbivores will change. For example, some major insect herbivores in US forests currently appear to be climate-limited. Forest composition is expected to alter in response to changing herbivore pressure, but the direction of change is very uncertain. When climate change was applied to a discrimination function relating climate to defoliation, strongly opposed results on the area of affected forest were simulated with similar GCM outputs (100 years at doubled $\mathrm{CO}_{2}$, GISS and GFDL GCMs, Williams \& Liebhold 1995).

Changes in the plant community could increase the proportion of one species in the generalist herbivore diet, reducing that plant's competitive ability and density. Total seed predation seems to be higher in disturbed environments (Finegan 1996). Moisture and nutrient stressed plants can be less resistant to herbivorous insects, which increases mortality (Cammell \& Knight 1992). Species in either trophic level may be lost before a new equilibrium is established. The 
generation time of insect herbivores is generally smaller than that of their hosts, so rates of genetic adaptation may be faster.

There is no clear pattern between plant species' rarity and vulnerability to herbivores under current or changing conditions. On a per-plant basis, sparse populations seem to suffer most from herbivory, though dense populations support most herbivores (Kunin \& Gaston 1997). Fast growing plants are favoured by generalist herbivores, as they have a higher nutritional content in the leaves and fewer toxic or inedible secondary metabolites (Díaz et al. 1998). Specialist herbivores are most vulnerable to environmental change, being dependent upon the survival of their host plant.

\subsection{Carbon dioxide response}

Changes in plant nutritional quality or in feeding requirements would alter the consumption rate of individual herbivores and their consequent impact on plants. If the increased availability of $\mathrm{CO}_{2}$ increases $\mathrm{C}: \mathrm{N}$ ratios in plants, the animals' biomass consumption may have to increase to sustain nitrogen intake, and herbivore preferences may shift towards less affected plants. Single species experiments have demonstrated change in plant-herbivore relationships with increased $\mathrm{CO}_{2}$, but experiments with mixed communities of temperate plants have shown no significant effects on insect herbivore preferences, reproductive potential or feeding (Díaz et al. 1998). The long-term effects on herbivory are unknown.

Many lepidopteran herbivores use $\mathrm{CO}_{2}$ concentrations as cues for leaf selection in egg-laying. Under still conditions in the evening, $\mathrm{CO}_{2}$ concentrations decrease above CAM plants, with the strongest gradients around the most photosynthetically active leaves. Experimental doubled $\mathrm{CO}_{2}$ concentration has been shown to decrease the sensitivity of $\mathrm{CO}_{2}$ detection and reduce oviposition rates in Cactoblastis cactorum (Stange 1997). Increased temperature also affects the sensitivity of the moths' $\mathrm{CO}_{2}$ receptors. It is not clear how quickly organisms using $\mathrm{CO}_{2}$ cues will adapt to increased atmospheric concentrations.

Information on the current occurrence, proximate causes and impact of herbivory would be required for any realistic attempt at incorporating it into a plant climate model. As this information is not available for tropical forest systems, and because the potential impacts are multidirectional, this topic is not pursued further.

For some species, the presence of certain other prey, mutualist or competitor species could be the most important determining factor in distribution. A strong correlation with another species' distribution might also indicate that both are reacting to a third, unmeasured variable. No studies using other species as predictive variables have been identified in this literature survey, although 
broad vegetation categories have been used by some authors (Austin et al. 1996). Where species' distribution knowledge is incomplete, environmental factors are more reliable candidate variables.

\subsubsection{Land-use}

Deforestation for agricultural and industrial purposes jeopardises the survival of many species through restriction of available habitat and depletion of local populations, regardless of the effects of greenhouse gases. Habitat fragmentation decreases the total area available to forest species and generates barriers to gene spread between populations, and to species' migration in response to environmental change (Kellman et al. 1998). Dioecious or self-incompatible plant species and those relying on vertebrate dispersal probably experience the greatest reproductive barriers following fragmentation (Gillespie 1999).

Land-use maps may be used to constrain a potential distribution that has already been defined by environmental datasets. This would not factor in the long-term viability of isolated populations, but would identify them. A map of available future territory can likewise be obtained by overlaying a species' potential range map under simulated climate change with projected future land-use (e.g. Mitchell 1993). This is beyond the scope of the current project, but is a logical extension when considering areas and species under threat.

\subsubsection{Fire}

Species whose distribution overlaps savanna land, typically pioneers found on the forest margin, are most likely to be resistant to fire. 50-60 years is recorded as a standard savanna fire recurrence interval (Kellman \& Meave 1997); but a three-year interval has been recorded for Brazilian cerrado under human impact (Hoffman 1998). Whilst mature plants in cerrado vegetation have some fire resistance, seedlings are more vulnerable (Hoffman 1998), presumably because of their thinner bark, greater surface-area:volume ratio and lower height. Frequent fire at low level can increase the importance of vegetative over sexual reproduction for woody plants, as fire destroys seeds, buds and developing fruit faster than stems, and as root suckers develop fire resistance faster than seedlings. Some cerrado species possess fire-resistant seeds; others adapted to severe infrequent fires have serotinous seeds that are only dispersed or germinate following fire.

Soil temperature increases much less than that above ground, so the plant roots are more likely to survive than their aerial biomass (Gignoux et al. 1997). Following fire, the formation of root buds can be stimulated by reduced auxin resulting from loss of the aboveground parts or from increased ground temperature. Increased use of vegetative reproduction will usually reduce the 
number and mean dispersal distance of offspring (Grime et al. 1988), but increase recruit survival rates.

Forest fires are typically taller, slower and more likely to kill mature plants than savanna fires. Fire occurs very infrequently in undisturbed aseasonal moist forest (Uhl \& Kaufman 1990). However, one study based on soil moisture, leaf dehiscence, fine fuel moisture and experimental fire setting concluded that fire potential is present when soil moisture is depleted to $5 \mathrm{~m}$ depth (Nepstad et al. 1999). 270,000 $\mathrm{km}^{2}$ of Amazonian forest met this criterion during the 1997-8 drought. One study of 126 eastern Amazonian tree species showed that $46 \%$ were unable to re-sprout after fire (Kaufman 1991).

In humid forest species, tests showed a reciprocal relationship between bark thickness and cambium temperature response to fire (Uhl \& Kaufman 1990). Adult survival rates increase with the presence of other insulating features such as complex buttressing and centralised phloem. Bark thickness, stem diameter and proportion of biomass above fire level increase with size. Species whose distribution includes savanna are more likely to possess these features.

Fire occurrence is extremely relevant to plant survival, and clearly linked to MD and land use. Fire should thus be part of a comprehensive change response model. Fire is not modelled in GCM surface models, but some submodels exist (Goldammer \& Price 1998). It is theoretically possible to estimate changes in fire frequency under any scenario by extrapolating from current occurrence under different climate regimes and human populations. Present-day maps of fire frequency in the region exist (Nepstad et al. 1999). However, fire frequency is not included in the present study, because fire usually occurs at a finer scale than the level of interest.

\subsubsection{Potential for evolutionary adaptation}

Evolutionary changes including speciation are precipitated by changes in selection pressure. It is often asserted that species will be unable to adapt to simulated levels of climate change, because of slow mutation rates and relatively long generation times . In addition, before a species' genotype can respond, other species already adapted to the new environment may competitively exclude it. Loss of genetic diversity following reductions in plant density may also endanger populations under stress (section 2.2.4.2.2).

Little information on the actual potential for adaptation is available, but several directional selection experiments with isolated populations have obtained response plateaux within 10-20 generations (Holt 1990). This may be because the limited additive genetic variance available for the selected trait has been exhausted, because there are disadvantageous traits genetically linked 
to the selected traits, or because genetic drift reduces the available variation in small experimental populations.

The species expected to have the greatest adaptive response to change are those with high abundance, wide distribution, short generation time and strong dispersal abilities (Holt 1990) the 'pioneer' species already favoured by several other change factors.

\subsection{MODELLING CURRENT AND CHANGING DISTRIBUTIONS}

\subsubsection{Broad model types}

Changes in distribution through time can be simulated using pattern analysis, process models or empirical observations of functionally or taxonomically similar species. The last option may be useful in evaluating model outcomes. There are a range of options between a static pattern analysis model and a dynamic process model of individual outcomes, with increasing requirements for data and processing time per spatial unit as the model grows more complex.

Analytical models (section 4.3.6) give single-state outcomes, whilst process models simulate an ongoing response whose results are dependent on the rate as well as the extent of change. Process models can include more sophisticated environmental events, and variation within a population of organisms. They include mechanistic (functional) and demographic models (section 4.3.7). In general, analytical models require fewer parameters and have more transparent outcomes, while process models require detailed empirical information (derived from observations or manipulative field experiments on the systems of interest) and have complex outputs. In both types, extrapolation beyond the calibrating dataset is subject to uncertainty: the response of complex systems is inherently difficult to predict (Passioura 1996, Lawton 1999, Gaston \& Blackburn 1999).

Ecological processes can be modelled deterministically or stochastically. In a deterministic model, repetition with the same parameters always leads to the same result. Stochastic models assign probabilities to each event and randomise the results accordingly. The random component has two sources (Kendall 1998): environmental stochasticity represents unpredictable variation that affects all individuals in a population similarly, and demographic stochasticity represents unparameterised variation within the responding population (e.g. in nutritional status or genotype). Stochastic models are repeated many times to produce central and extreme results for each scenario, with a measure of variance. A deterministic model can be likened to a repeatable experiment, while a set of stochastic runs can be treated more like a population of results, where the proportion of outcomes gives an estimated probability. 
Sensitivity testing can provide an estimate of error in either case. Both model types can give rise to apparently chaotic processes - deterministic chaos follows mathematical rules, but is difficult to distinguish from a stochastic outcome (Lüttge 1997, p. 59).

\subsubsection{Model selection criteria}

The following criteria are desirable in a model to map present and projected tropical biodiversity:

- A basis in established ecological theory;

- Production of objective, repeatable results from a standardised method;

- Functionality with presence-only, as opposed to presence-absence (P/A), data;

- Tolerance of sampling bias (i.e. a non-random distribution of points);

- Speed and ease of interpretation to enable replication with multiple species and scenarios;

- Ability to project the rate as well as the outcome of species response to change.

\subsubsection{General form of plant-climate relationships}

There are two paradigms relating species to climate or other environmental variables. The first states that plant response, whether presence, abundance or growth, is determined by the most limiting factor (MLF). This multiple-constraints approach specifies relationships with individual variables (Booth 1998, Hackett \& Vanclay 1998).

The second states that interacting factors can produce a unique response, with one variable mitigating or exacerbating the effect of another (Ter Braak \& Prentice 1988, Carpenter et al. 1993). Measured variables may be correlated so that it is difficult to distinguish which is the controlling variable, or may act independently. When responses to individual variables are dependent upon the value of other variables, the variables are said to be complementary in explanatory power.

A symmetric unimodal curve is frequently applied whether variables are treated individually or together, though the 'curve' may be splined in practice (Carpenter et al. 1993, Hackett \& Vanclay 1998). Many pattern analyses use a Gaussian (normal) response curve, based on the logarithm of species abundance (Ter Braak \& Prentice 1988). Fig. 4:2 illustrates unimodal limitations on reproduction, growth and survival for a single continuous variable. The peak of the curve indicates the species' optimum. Environments that are optimal for reproduction are also likely to be optimal for growth (e.g. Nicotra 1999). 
If the variable falls below the survival threshold, increased mortality occurs. An isolated population can only persist over more than one lifespan in the uppermost part of the curve, where reproduction is possible. It may survive for a shorter time in lower parts of the curve. This contributes to a lag in the distributional response to climate change.

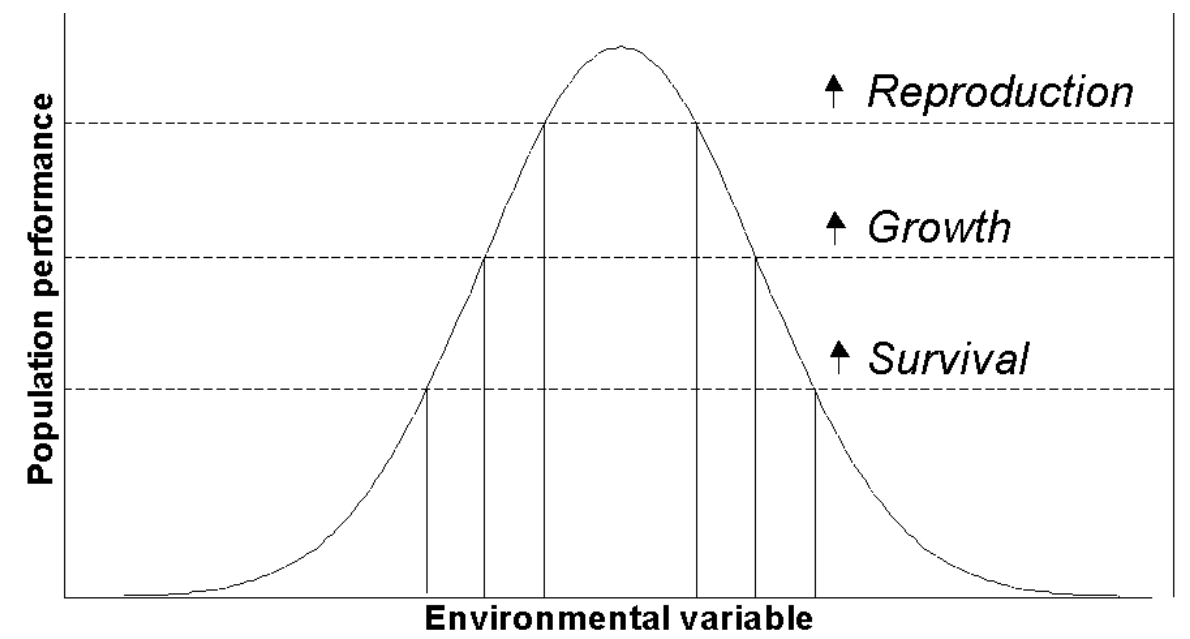

Fig. 4:2: Generalised unimodal response to a single variable

Plant responses to climate can be strongly skewed (Austin \& Smith 1989), requiring a cubic (or higher factor) rather than a linear or quadratic function, but the unimodal curve is accepted as a likely default response (Ter Braak \& Prentice 1988). When a monotonic curve is identified, it is generally inferred to be part of a broader, unseen unimodal curve.

In an ideal model, the distributional response to the environment would be fitted to the set of interacting factors, for example, by using general linear modelling. This requires a minimum of presence/absence (P/A) data. One approach to estimating curve shape from presence-only data is to use a histogram of occurrence frequency (ter Braak \& Looman 1987).

\subsubsection{Scale}

The study of ecological patterns and processes over large areas, especially the division of energy and space between species, has been named macroecology (Brown \& Maurer 1989, Brown 1995). It is assumed that the properties of populations and species can be studied separately from individual responses, just as the study of an individual organism's behaviour does not require a cell-by-cell analysis. Unnecessary complexity increases model time, which reduces applicability to multiple species. The introduction of additional parameters also increases the scope for error when the species' ecology is not well known. There is a trade-off between error resulting from simplification of reality in the model structure, and error in the autecological assumptions underlying the structure (Passioura 1996). 
Extending this concept, a coarse-scale model need not depend upon theories of local biodiversity maintenance (section 2.2.1). The different hypotheses attempt to explain the same local pattern, producing the same higher-level outcome. For example, most modelling approaches assume that a given species assemblage is primarily determined by environmental factors, rather than chance colonisation from a pool of species of equal competitive ability (the 'drift' hypothesis). At a macroscale, the determinist model in effect describes the drift hypothesis' species pool rather than the local assemblages that derive from it - so it is compatible with either theory.

This view is supported by drift hypothesis proponents, whose theory applies within relatively homogenous habitats (Condit et al. 1996c):

"The communities are not subject to pure drift at large scales... we speculate that the Panama Canal area behaves much like [a single large community] at drift with only moderate habitat heterogeneity, whereas across all of Panama, sharp habitat boundaries affect many species' ranges".

The level at which processes are simulated thus depends upon the spatial scale of the study (May 1993, Brown 1995, Lawton 1999). Stochastic individual-level models are appropriate for small-area processes, such as gap dynamics in a stand of trees (section 4.3.7.3.1). If large-area modelling is intended, the vital rates can be applied deterministically at the population level. The probability that an event occurs to an individual is interpreted as a proportion of the population affected, giving an equivalent to the mean result of a large set of stochastic models, and reducing model complexity.

There remains a question over the extent to which some distributions can be predicted using determining variables at low resolution (Stoms 1992). Where a species' environmental niche is very patchily distributed in space (such as river margins), the aggregated variables at a coarser scale may not discriminate between cells that do and do not contain this microhabitat. The challenge is to determine how much information is lost as the level of spatial aggregation for each variable increases (ideally, maximum and minimum recorded values across the cell would be obtained). The potential error arising from the possibility that the species is more reliant on or tolerant to the extreme rather than mean values from its recorded cells would then be estimable. However, this exercise is more data-intensive than the current project can support. 


\subsubsection{Vegetation modelling}

This section considers the impacts of climate change on vegetation distributions as simulated in biome (biosphere) models, both 'offline' and coupled with GCMs. Biome models are generally based on the climate tolerances of a set of component PFTs such as grasses, shrubs and trees. Biotic inputs to GCMs are often simulated in this way (section 3.5.1.3).

The simplest biome models use pre-defined vegetation types able to undergo instantaneous change (Prentice et al. 1992), or have pre-defined transition times (Belotelov et al. 1996). Other models have fixed vegetation types, with climate- or $\mathrm{CO}_{2}$-responsive physiological characteristics such as LAI or stomatal conductance (Sellers et al. 1996a). Dynamic vegetation models use a set of PFTs to build up vegetation types according to the prevailing climate, and may include physiological components such as carbon sequestration, stomatal conductance and optimum temperature for photosynthesis (Cramer et al. 2001). Some recent vegetation models have been coupled with GCMs (Betts et al. 1997, Cox et al. 2000), with feedbacks to the LSP (land surface parameterisation).

Table 4:1 is intended to supply a comprehensive survey of global vegetation models run using HADCM2, with a selection of models forced by other GCMs for comparison. Most modern models demonstrate a reduction in forested area or NPP in the Amazon region. The combination of the type of response to $\mathrm{CO}_{2}$ and the degree of drying over Amazonia produce a wide variation in the spatial extent of change. Other variation is derived from differences in model complexity and in process formulations, which are not easy to untangle (Cramer et al. 2001).

The older biome models tend to have more optimistic outcomes, but this is not universal. For example, the Mapped Atmosphere Plant-Soil System (MAPSS) biogeographic model calculates a supportable LAI for each cell from variables related to water balance and transpiration, on a $0.5^{\circ} \times 0.5^{\circ}$ grid (Neilson 1993). Under five doubled $\mathrm{CO}_{2}$ scenarios, there was a mean $10 \%$ decrease in tropical forest LAI, due to drought stress. Decreases in area were also simulated, with a mean of $3,200,000 \mathrm{~km}^{2}$ changing from tropical forest to savanna. A variant included increases in water use efficiency with $\mathrm{CO}_{2}$ by reducing the stomatal conductance in the model by $35 \%$ (Neilson \& Marks 1994). Tree LAI loss in tropical and temperate areas lessened, but the movement of forest towards the poles persisted.

The changes seen in the set of vegetation models run under HADCM2 are of particular interest. SDGVM alone (Betts et al. 1997) underwent much smaller change than the DGVM 'ensemble mean' (Cramer et al. 2001). In the SDGVM vegetation model, coupled to HADCM2 under doubled $\mathrm{CO}_{2}$, LAI change was negligible for tropical forests, but transpiration was reduced (Betts et al. 1997). This equilibrium vegetation model simulated $\mathrm{CO}_{2}$ fertilisation and water-use efficiency 
effects. A lag would be expected in LAI change, but not in these physiological adaptations. For the Amazon region, this model showed small anomalies in temperature compared to a version that considered physiological changes only, indicating that changing LAI played a role. Both incurred temperature rises within $1{ }^{\circ} \mathrm{C}$ of the $4-6^{\circ} \mathrm{C}$ increase in the non-vegetation GCM.

Table 4:1: Summary of biome models' response to GCM forcing

\begin{tabular}{|c|c|c|c|c|}
\hline Model & GCM \& scenario & Vegetation & Amazon change & Reference \\
\hline $\begin{array}{l}\text { TRIFFID. } \\
\text { Synchronous- } \\
\text { ly coupled to } \\
\text { the GCM }\end{array}$ & $\begin{array}{l}\text { HADCM3 IS92a } \\
\text { with HADCM2 } \\
\text { ocean grid \& flux } \\
\text { adjustments, single } \\
\text { run }\end{array}$ & $\begin{array}{l}\text { Dynamic, } \\
\text { physiological } \\
\mathrm{CO}_{2} \text { response }\end{array}$ & $\begin{array}{l}\text { Loss of forest (broad- } \\
\text { leaved tree PFT) over } \\
\text { most of Amazonia, } \\
\text { leaving Andean and } \\
\text { northwest forests }\end{array}$ & $\begin{array}{l}\text { Cox et al. } \\
2000, \\
\text { Huntingford } \\
\text { \& Cox 2000, } \\
\text { Collins } \\
\text { 2001a, 2001b }\end{array}$ \\
\hline \multirow[t]{2}{*}{$\begin{array}{l}\text { Dominant } \\
\text { response of } 6 \\
\text { models: } \\
\text { HYBRID, } \\
\text { IBIS, LP J, } \\
\text { SDGVM, } \\
\text { TRIFFID, } \\
\text { VECODE }\end{array}$} & \multirow[t]{2}{*}{$\begin{array}{l}\text { HADCM2 IS92a } \\
\text { with sulphur } \\
\text { aerosol forcing, one } \\
\text { run. }\end{array}$} & $\begin{array}{l}\text { 'T': dynamic, } \\
\text { no } \mathrm{CO}_{2} \\
\text { response }\end{array}$ & $\begin{array}{l}\text { Eastern Amazonia } \\
\text { switches from } \\
\text { evergreen to mixed } \\
\text { forest, savanna and } \\
\text { grassland; intrusion of } \\
\text { patches of savanna } \\
\text { into western Amazonia }\end{array}$ & \multirow[t]{2}{*}{$\begin{array}{l}\text { Cramer et al. } \\
2001\end{array}$} \\
\hline & & $\begin{array}{l}\text { 'CT': Dynamic, } \\
\text { physiological } \\
\mathrm{CO}_{2} \text { response }\end{array}$ & $\begin{array}{l}\text { Intrusion of savanna } \\
\text { into north eastern } \\
\text { Amazonia }\end{array}$ & \\
\hline \multirow[t]{2}{*}{ HYBRID } & $\begin{array}{l}\text { HADCM2 IS92a, } \\
\text { excluding sulphate } \\
\text { aerosols, four runs }\end{array}$ & $\begin{array}{l}\text { Dynamic, } \\
\text { physiological } \\
\mathrm{CO}_{2} \text { response }\end{array}$ & $\begin{array}{l}\text { Incursions of savanna, } \\
\text { grassland or desert into } \\
\text { northeastern } \\
\text { Amazonia. }\end{array}$ & \multirow[t]{2}{*}{$\begin{array}{l}\text { White et al. } \\
1999\end{array}$} \\
\hline & $\begin{array}{l}\text { HADCM3 IS92a, } \\
\text { excluding sulphate } \\
\text { aerosols, one run }\end{array}$ & $\begin{array}{l}\text { Dynamic, } \\
\text { physiological } \\
\mathrm{CO}_{2} \text { response }\end{array}$ & $\begin{array}{l}\text { Loss of forest over } \\
\text { most of Amazonia, } \\
\text { resulting in desert }\end{array}$ & \\
\hline $\begin{array}{l}\text { SDGVM. } \\
\text { Asynchronous } \\
\text { ly coupled to } \\
\text { GCM }\end{array}$ & $\begin{array}{l}\text { HADCM2 IS92a, } \\
\text { excluding sulphate } \\
\text { aerosols, fixed } \\
\text { OGCM, single run }\end{array}$ & $\begin{array}{l}\text { Equilibrium, } \\
\text { instantaneous, } \\
\text { physiological } \\
\mathrm{CO}_{2} \text { response. }\end{array}$ & $\begin{array}{l}\text { Little change to } \\
\text { Amazonian forest LAI, } \\
\text { reduced evaporation } \\
\text { from reduced canopy } \\
\text { conductance leads to } \\
\text { increased warming }\end{array}$ & $\begin{array}{l}\text { Betts et al. } \\
1997\end{array}$ \\
\hline SiB2 & $\begin{array}{l}\text { CSU-GCM, } 30 \text { year } \\
\text { simulation at } \\
\text { doubled } \mathrm{CO}_{2}\end{array}$ & $\begin{array}{l}\text { Fixed, } \\
\text { physiological } \\
\mathrm{CO}_{2} \text { response }\end{array}$ & \multirow[t]{2}{*}{$\begin{array}{l}\text { Reduced evaporation } \\
\text { from reduced canopy } \\
\text { conductance leads to } \\
\text { increased warming } \\
\text { over Amazonia }\end{array}$} & $\begin{array}{l}\text { NASA EOS } \\
\text { IDS BAI } \\
\text { 1995, Sellers } \\
\text { et al. } \\
\text { 1996a, d }\end{array}$ \\
\hline $\begin{array}{l}\text { MOSES . } \\
\text { (HADCM3's } \\
\text { original LSP) }\end{array}$ & $\begin{array}{l}\text { HADCM3 AGCM } \\
\text { forced by HADCM2 } \\
\text { OGCM IS92a } \\
\text { anomalies, one run }\end{array}$ & $\begin{array}{l}\text { Fixed, } \\
\text { physiological } \\
\mathrm{CO}_{2} \text { response }\end{array}$ & & $\begin{array}{l}\text { Cox et al. } \\
1997\end{array}$ \\
\hline $\begin{array}{l}\text { 'Life-form } \\
\text { model' }\end{array}$ & $\begin{array}{l}\mathrm{CO}_{2} \text { from } 350 \text { to } \\
560 \text { ppm, uniform } \\
+2^{\circ} \mathrm{C} \text { temperature } \\
\text { and }+10 \% \\
\text { precipitation }\end{array}$ & $\begin{array}{l}\text { Equilibrium, } \\
\text { instantaneous, } \\
\text { based on LAI } \\
\text { and NPP }\end{array}$ & $\begin{array}{l}\text { Extension of Amazon } \\
\text { forest to south }\end{array}$ & $\begin{array}{l}\text { Woodward } \\
\text { \& Lee } 1995\end{array}$ \\
\hline
\end{tabular}




\begin{tabular}{|l|l|l|l|l|}
\hline MAP S & $\begin{array}{l}\text { Five doubled } \mathrm{CO}_{2} \\
\text { scenarios }\end{array}$ & $\begin{array}{l}\text { Equilibrium, } \\
\text { instantaneous, } \\
\text { based on LAI }\end{array}$ & $\begin{array}{l}\text { Opposite results in } \\
\text { forest area change } \\
\text { from simulations using } \\
\text { different GCM } \\
\text { outputs. }\end{array}$ & Neilson 1993 \\
\hline BATS & $\begin{array}{l}\text { NCAR CCM1-Oz } \\
\text { GCM }\end{array}$ & $\begin{array}{l}\text { Equilibrium, } \\
\text { instantaneous, } \\
\text { no CO } \\
\text { response }\end{array}$ & $\begin{array}{l}\text { Little change to } \\
\text { Amazonian forests }\end{array}$ & $\begin{array}{l}\text { Henderson- } \\
\text { Sellers 1993, } \\
\text { Henderson- } \\
\text { Sellers \& } \\
\text { McGuffie } \\
1995\end{array}$ \\
\hline BIOME & $\begin{array}{l}\text { NASA-GISS GCM, } \\
\text { doubled } \mathrm{CO}_{2}\end{array}$ & $\begin{array}{l}\text { Equilibrium, } \\
\text { instantaneous }\end{array}$ & $\begin{array}{l}\text { Small increase in the } \\
\text { area of tropical forest }\end{array}$ & $\begin{array}{l}\text { Leemans } \\
1992\end{array}$ \\
\hline
\end{tabular}

The 'T' scenario of Cramer et al. (2001), simulating the impacts of climate change without the physiological impacts of $\mathrm{CO}_{2}$ shows a widespread incursion of savanna and $\mathrm{C}^{4}$ grassland into eastern Amazonia. The vegetation in the 'CT' scenario, which includes both reduced stomatal opening and an increase in the optimum temperature for photosynthesis with increased $\mathrm{CO}_{2}$, displays a greater adaptation to climate change. Although the reduced rates of canopy conductance will increase temperature, this does not in itself limit forest survival.

However, when TRIFFID was coupled with HADCM3, the simulated drying over much of Amazonia, resulted in a loss of broad-leaved trees in Amazonia over a large region, leaving Andean forests and those to the northwest (Cox et al. 2000, Collins 2001b). Loss of Amazonian forest starts early in the simulation, responding to an increased frequency of El Niño mean conditions, and accelerating until there is complete climate-driven deforestation over most of the region.

The differences between HADCM2 and HADCM3 response are driven by differences in the Pacific SSTs (Collins 2000b). These in turn are driven by differences in the parameterisations of cloud formation and atmospheric boundary layer processes, which unexpectedly produced much larger differences in patterns of cloud, precipitation and SST (Williams et al. 2001). The extent of the drying in the Amazon at doubled $\mathrm{CO}_{2}$ is therefore subject to much uncertainty.

\subsubsection{Biome models and species modelling}

Vegetation types represent combinations of species or PFTs. These could be used as proxy variables in estimating species' potential distributions. In a biodiverse region such as Amazonia, the broad types used in biome models do not serve as good predictors of individual species. Moreover, the biome simulations result from the same set of input variables used in plant distribution modelling. Given that the same variables are available for vegetation and specieslevel modelling, it is more appropriate to concentrate on the direct impacts of individual continuous variables on species than to use vegetation type as a proxy variable. 
Biome models are not directly useful for species response evaluation, except in the case of providing a dispersal matrix. Vegetation type affects seedling germination through shelter conditions and shading, and dispersal through disperser preference for particular matrices. Predicted forest cover following deforestation trends would provide a more realistic map than the current simulations of natural vegetation. The difficulty in spatially simulating deforestation limits the use of this approach.

In the current context, biome models are most interesting because it is possible to compare their outputs with those of the species model. If there is a disparity between the species projected distribution and its usual vegetation type, it would be sensible to investigate why this occurs.

\subsubsection{Analytical distribution models}

The need for accurate information on species' distributions, together with the variety of approaches to data collection, has led to the development of many analytical interpolation techniques. Very few formal interpolations of South American plant distributions exist.

It is simpler to estimate a species' potential distribution (spatial expression of the fundamental niche) than its realised distribution (spatial expression of the realised niche). The realised distribution is the set of locations in which the species occurs, and the potential distribution is this set together with all additional suitable sites. A species can be excluded from parts of the potential distribution by biotic interactions preventing colonisation, by physical barriers preventing dispersal, or by stochastic events. Direct exploitation by humans and anthropogenic alterations to vegetation and landscape features also limit distributions.

These exclusions, and demographic limits on the species rate of colonisation, exert a historical influence on current range. The extent to which the distributions are still responding to, historical factors (in particular past climates) is uncertain. Several hypotheses concerning the historical maintenance of Amazonian biodiversity are discussed in section 2.2.1. Most distribution studies focus on current environmental factors, as modern data are much easier to obtain and verify than palaeoecological data.

Aside from informal interpolations based on expert knowledge (section 4.3.6.1), species distributions are interpolated using pattern analysis techniques, based primarily on the species' locational records. Correlations or relationships with environmental variable maps may be derived and used in interpolation, or the analysis may be purely spatial. The purest form of pattern analysis is based on recorded distribution alone (sections 4.3.6.3.1, 4.3.6.3.2). This is 
attractive where environmental maps at the resolution of the species data are unavailable or costly. The limitations outside a homogeneous environment are obvious.

Surface pattern analyses that include environmental information (direct gradient analyses, Ter Braak \& Prentice 1988) are more common. These assume that environmental variables can explain or predict species' distributions, and that these distributions are at equilibrium. Species are usually treated as individual units, responding to the environment independent of their communities. Human impacts may be included via a land-use map, or wilderness mapping (Lesslie et al. 1988) can provide an inverse map of human activity.

Distribution models may be formulated according to hypotheses of plant response, or based directly upon the pattern of the observed data. The advantages of using correlations with observed environments as a surrogate for detailed knowledge of species requirements include the relative ease of obtaining environmental data and the ability to model little-known species. The disadvantage is that other factors contributing to the effectiveness of the surrogacy may change over large spatial areas (Bugmann 1996).

The models produced are generally deterministic with no random elements: repeated runs will result in identical outcomes. Most map the multidimensional environmental space occupied by the species and then superimpose the results onto physical space. Some models use constraints to create a simple linear environmental envelope - presence is assumed within the recorded extremes of each variable. Others calculate a probability of presence over a curve, sometimes within a constrained envelope.

The major assumptions common to these methods are that:

- The main determinants of the species' distribution are captured in the variables mapped.

- The extremes of the species' range are included in the survey record set. In particular, methods based on presence data rather than abundance data may not otherwise distinguish species preference from habitat availability or recording bias.

- Each presence in the record set represents a viable reproductive population.

- There are no physical, competitive or physiological barriers to the species' occupation of its potential range, and it does not depend upon the presence or absence of other species.

- The species is incapable of adaptation to environmental conditions outside its current range.

- The distributional response to environmental change is immediate.

The following review considers the potential application of various interpolation techniques to Amazonian plant species. Environmental variables of potential use in interpolation and response modelling are considered in section 4.2. 


\subsubsection{Expert knowledge}

Field botanists and taxonomists produce atlases and monographs for particular taxa, which give species' distributions, descriptions of their identifying traits and often of the environments in which they were recorded (e.g. Henderson et al. 1995, NYBG 1997). These describe rather than explaining the distribution. If an estimated range beyond known observations is included, it is usually based on expert knowledge of species' requirements and of field conditions together with edaphic, climate or vegetation maps. The estimated distributions often show the outermost limits of occurrence (the gross range) rather than a grid-based area of occupancy (van Jaarsveld et al. 1998).

This method has the potential to be fairly accurate, inexpensive and swift. However, different experts' judgements may not concur. It would be preferable to use objective, transparent, repeatable interpolation methods.

\subsubsection{Selection of variables for pattern analysis}

One option is to incorporate many environmental variables, in order to include as many potential influences on distribution as possible. However, there are advantages in undertaking a more careful selection of variables.

- Compliance with 'Occam's razor': the principle of parsimony states that no more causes should be assumed than are necessary to account for the facts.

- Better predictive power: in models employing classical statistics, the number of degrees of freedom increases with the number of variables, and the power of the model decreases.

- Avoidance of conflating the effects of separate variables: some factors will be spatially correlated with one another. A better model will be obtained if an attempt is made to select those with known effects on species survival.

- Selection of variables that affect distribution directly (resource gradients), rather than surrogates such as latitude or altitude, is important in change studies (Austin 1999).

- Data from a more comprehensive source, from an appropriate time period, or at a higher resolution can be chosen preferentially.

- Avoidance of accidental redundancy: some factors are derived from others (e.g. soil characteristics from mapped soil types, Bouwman et al. 1993, Batjes 1997; PET from albedo and climate variables, Ahn \& Tateishi 1994a).

- Time benefits: As the number of inputs increases, so does the processing time.

Some methods, such as forward stepwise regression, deselect variables that have little effect on the explanatory power of the model, which means that different variable sets may be used for 
different species. With other methods, such as 'Enter' regression, the entire set of input variables will be used for every species (Norušis 1990). Here it is particularly important to select variables with general application, expressing the basic requirements of energy, mineral nutrients and water (section 4.2.1.3).

\subsubsection{Pattern analysis without independent variables}

\subsection{Geometric approaches}

A species range is the area inside an observed or calculated spatial boundary. Geometric approaches to range simulation include the use of a minimum circle encompassing all the points, a polygon based on the mean distance from points to their geometric centre ('polygonal tessellation'), or a area around each point based on the mean propinquity (mean or standard deviation of distances between nearest presence points) (Rapoport 1982). It is assumed that the entire internal area is suitable. Disjunct or doughnut shaped distributions can be accommodated by assuming that all land units separating two records of an arbitrary maximum distance apart also contain the species (Rohwer 1993).

These geometric approaches are unsuitable for use in a heterogeneous environment, as adjacent cells within a spatial range boundary may not all contain suitable combinations of environmental variables. They are highly unsuitable for modelling distributional response to climate change, as climate variables are not accounted for.

\subsection{Bayesian image analysis}

A variable is 'autocorrelated' when its value at a point in space can be predicted from the known values at other locations (Legendre \& Fortin 1989). Positive spatial autocorrelation in a raster (grid) map is therefore the propensity for a cell value to be similar to those of surrounding cells. This approach attempts to account for the effects of positive spatial autocorrelation by adapting a method from satellite image classification to simulate a distribution from a set of sampled cells in a grid (Högmander. \& Møller 1995).

Image analysis methods ('maximum marginal posterior estimation' and the 'iterated conditional procedures algorithm') are used to 'classify' the maps, treating them as incomplete binary pixel images. It is assumed that the study area environment is homogenous or has no strong effect on species' distribution. The method scores research effort of the recorders to account for bias from non-random survey location, incorporating this into the conditional probability used in the iterative Bayesian image classification. The results are akin to those of smoothing algorithms that generalise data to remove sharp boundaries (e.g. the FILTER module of the IDRISI GIS, 
Eastman 1997, the 'smooth scores' option of WORLDMAP, Williams 1998b), although the process is more complex. Again, this technique cannot be recommended for use outside homogenous areas and therefore is inappropriate for use over large regions.

\subsection{Kriging}

Another method used to look at autocorrelation in spatial processes is kriging, which is a stochastic interpolation technique designed for use with continuous point data. It uses a local estimator, taking into account only points in the 'observation cone' of the point to be estimated (Legendre \& Fortin 1989). Kriging is based upon a generalised least-squares algorithm and if the data are stationary (either their mean and variance are the same over different parts of the study area or the increments between all pairs of points at a given distance apart have a mean 0 and a finite, constant variance) uses variograms (the mean squared differences between values at different points) as weighting functions. Otherwise, 'generalised intrinsic random functions of order k' are used in weighting. The weighting is split between neighbouring points to ensure that the result does not depend upon the local density of points.

Kriging is often used in the creation of digital elevation maps (DEMs), and has several forms, including co-kriging, which incorporates data from a co-variant. The method has been used in mapping distributions of abundant species (e.g. Maynou et al. 1996, Liebhold et al. 1991, Simard et al. 1992), but has been avoided in this project because like Bayesian image analysis (section 4.3.6.3.2), it ignores available environmental variables. An advantage is that there is no assumption of independent samples, so that autocorrelation in datasets is not a barrier. This type of technique would be more useful if the species data were of a higher resolution than the environmental data - spatially based analyses could be performed what would be effectively areas of homogenous environment. As most records in the Neotropics dataset are at the same $1^{\circ}$ scale as the coarsest environmental maps in use, this criterion is not true for the Neotropics.

\subsubsection{Environmental envelopes}

\subsection{Rectilinear environmental envelopes}

Isotherms or isopleths mapping a threshold of a single environmental variable can be used to bound a species range (e.g. Jeffree \& Jeffree 1996). An extension of this approach uses the rectangular bounds of a multidimensional environmental envelope. This is very close to the original quantitative 'niche' concept of

“a supervolume of n-dimensional resources in which a species lives” (Hutchinson 1957). 
BIOCLIM is a well-known program that uses climate variables to define and map potential species ranges in this way (Busby 1991). The search for similar climate locations is described as 'homoclime analysis' (Nix 1986). Variants have been developed for forestry purposes in other locations including South America (Booth \& Jones 1998).

Each dimension of the rectilinear envelope is defined by the two extremes recorded for species presence for that variable (e.g. the lowest and highest maximum temperature of the warmest month, or precipitation of the wettest quarter). If every variable for a spatial point falls between the maximum and minimum-recorded values for the species, that point is accepted as a presence point. Each variable is treated equally. Levels of increasing likelihood of species presence are allocated based upon decreasing percentile limits for the number of records found within that concentric part of the bounding rectangle. A typical BIOCLIM output offers a core (0-100) and marginal (5-95 percentile limits) bioclimate, which is then mapped.

The BIOCLIM literature includes a study of eight Australian temperate rain forest species, which unusually compares related physiological and environmental parameters (Hill et al. 1988). The temperature dependence of photosynthesis and maximum rate of photosynthesis of seedlings grown at a range of temperatures was measured, and the climate envelopes of the species estimated from herbarium collections using BIOCLIM. All but one of the northern species had a substantially higher optimum temperature for photosynthesis than the species restricted to the cooler southern forests. This corresponds to the climate profiles and simulated distributions of these species in relation to temperature. This demonstrates that simple parameters can accurately reflect plant physiological requirements, and that mechanisms linking species' distribution with climate tolerances can be identified.

The approach is not affected by sampling bias as long as samples include the extremes of the climate range of the species. However, each climate axis is treated independently, which can lead to poor results (Nix 1986, Booth 1990). The model assumes that if a species can cope with each climate extreme one at a time, it can cope with all the extremes together - interactions between variables are not modelled. Sites that are very dissimilar to the training sites will thus be included and the potential range will be over-estimated. In addition, the use of percentile ranges to define a 'core bioclimate' means that some actual record sites are excluded from this set (Walker \& Cocks 1991, Carpenter et al. 1993). The later CSIRO programs HABITAT and DOMAIN seek to reduce these errors (sections 4.3.6.4.1.2 and 4.3.6.12). 


\subsection{Minimum convex polytopes}

A minimum convex polygon (MCP) links the spatial outliers of a set of observations, with all internal angles less than $180^{\circ}$. A minimum convex polytope is a combination of the MCPs for each variable projected into multi-dimensional space. This polytope is the basis of the HABITAT algorithm (Walker \& Cocks 1991), which then segregates the envelope into likelihood zones. As the envelope around the record-sites is tight, the method is liable to errors of omission, but it avoids the large errors of commission seen in rectilinear envelopes.

The envelope rapidly becomes increasingly restricted with the addition of new variables, so that only sites identical to one of the training sites are included in the final output (Carpenter et al. 1993). This is solved in HABITAT by the selection of climate parameters using Classification and Regression Trees (CART, a rule-based algorithm, Breiman et al. 1984). Following the similarity calculation, the CART decision rules are used to zone the envelope into sub-volumes of differing confidence in the probability of species occurrence. As CART uses regression, this invokes the assumptions of regression analysis described below (section 4.3.6.7). The method was initially tested using a $1^{\circ}$ latitude-longitude grid to estimate red kangaroo distributions. The sub-envelope categories are mapped, with species presence being assigned to those where the proportion of record to no-record sites $>0.5$. This implies that the allocation of species presence to a given site is dependent upon the number of other no-record sites offered to the procedure there is a map-size bias.

The requirement for randomly sampled data means that the CART-based processes are in any case not useful for neotropical datasets. Without the use of CART, the tendency to over-fit the dataset and the inability to identify unsuitable areas within the polytope remain as issues.

\subsubsection{Predictive habitat mapping}

This method assumes that a species is present in every occurrence of its preferred habitat (Stoms 1992). A refinement is to calculate the minimum area required for a reproductive unit, and estimate the number of units supportable by a habitat patch (White et al. 1997), which offers a measure of population viability. Where species' requirements are well known and there are no other limiting factors, this will provide a potential range map. The method is not applicable to an Amazonia-wide study, because microhabitat preferences are ill known for many species, and only maps of broad habitat types exist. In addition, to estimate potential distributions under a changing climate, it would be necessary to simulate changing habitat types, which presents as much of a challenge as simulating changing species' distributions. 


\subsubsection{Bayesian inductive spatial modelling}

The use of Bayesian statistical inference in species mapping and climate change impacts formally takes account of information not included in the environment variables (Aspinall 1992, Aspinall \& Matthews 1994). The method takes combines prior and conditional probabilities to form a predicted probability of occurrence for each cell. The prior probabilities of presence may be a mixture of observed and guessed locations. These are modified by the evidence for correlation with environmental variables (conditional probabilities). These conditional probabilities can be derived by dividing the record dataset into a presence/absence (P/A) or presence-random $(\mathrm{P} / \mathrm{R})$ sample, and calculating the frequency of association for each attribute (class) of each environmental variable (predictor dataset) with each record class (P/A or P/R). It thus operates the same manner whether the species' locational data includes (P/A) or presenceonly sites. It would presumably be valid to use probability values generated by other methods.

The significance of each high-scoring attribute is tested using chi-squared tests, and less significant attributes are excluded. The remaining conditional probabilities are combined with the prior probabilities using Bayes theorem, seen in Eqn. 3:

$$
p_{p}=\frac{\left(p_{p p} \cdot p_{c p}\right)}{\left(p_{p p} \cdot p_{c p}\right)+\left(p_{p a} \cdot p_{c a}\right)}
$$

Here $p_{\mathrm{p}}=$ projected probability for the cell, $p_{\mathrm{pp}}=$ prior probability of presence, $p_{\mathrm{cp}}=$ conditional probability of presence, $p_{\mathrm{pa}}=$ prior probability of absence, $p_{\mathrm{ca}}=$ conditional probability of absence.

Bayesian inductive spatial analysis has two main disadvantages in the context of the present study. It simplifies continuous environmental data, using class intervals. Secondly, values outside the original range of data are not catered for in the probability analysis, as no overall relationship is derived between environmental values and the presence probability.

The advantages are that the model does not make assumptions about distribution functions or sample bias because it is a probability technique, it has no requirement for absence data and it includes a mechanism for error measurement. The use of prior probabilities could provide a way of distinguishing presence, absence and unknown squares (by assigning $p_{p p}=1,0$ and 0.5 ), or of masking out squares thought to be beyond the range.

These advantages make the model a possible contender for use in present-day neotropical distribution estimation. However, the failure to relate species presence to the continuous environmental data means that this is a poor method for simulating distributional change: if a climate variable falls outside the recorded range, the conditional probability will be 0 . 


\subsubsection{GLMs: Generalised linear (regression) models}

Generalised linear models (GLMs) are frequently used to explain well-known species' distributions or growth patterns, and to extrapolate response in an unsurveyed area or under a changed climate. A GLM is a response function using one or more environmental variables. The function includes a systematic part, describing the expected response, and an error part, describing the deviation of the observed response from the expected response.

A favoured method of building a parsimonious GLM model is forward stepwise regression (Seber 1977). A set of potential explanatory variables is repeatedly searched for variables to include in the model. First, the best explanatory variable is selected, then the second best, and so on, but there is a backwards elimination of unnecessary variables at each step. Ordinal variables can be translated to Boolean (0/1) dummy variables (as Hill 1991, did for soil type).

Each model makes assumptions about the shape of the species-environment response curve that may not hold for the whole range. Discrepancies may be obvious: for example, the optimum may be inappropriately estimated as outside the measured range of environments (Ter Braak \& Prentice 1988). Regression diagnostics can be used to assess the appropriateness of the curve applied (Nicholls 1989). The residuals are examined, and the influence of individual observations on the model fit and parameter estimation are tested using jack-knifing. An alternative approach is to employ generalised additive models (section 4.3.6.9), which generate closely fitted curves through localised smoothing.

\subsection{Logistic models}

For presence/absence (P/A) data, logistic ('logit') GLMs are used. The response curve gives a probability of presence between 0 and 1, rather than an estimate of abundance. The logistic function is s-shaped when the linear predictor is a first-order polynomial, bell-shaped for second-order polynomials, and more complex for higher orders. The error distribution is the Binomial distribution with total 1 (Ter Braak \& Looman 1987).

Buckland \& Elston (1993) review these models, and suggest a method for modelling distributional change based on datasets from different periods and resolutions. A GLM with binomial errors and a logistic link function - a standard logistic model - is constructed for red deer distributions, and successfully tested using 120 bootstrap replications. In a link function, the transformation does not alter the error structure of the response variable (Crawley 1993). 
Generalised linear modelling tests the contribution of the different variables and creates a minimum adequate model that best explains the data. However, GLMs require a set of randomly sampled or comprehensive presence-absence data with few missing values, conditions that are often not met by tropical datasets. The assumption that all no-record sites represent absence records will lead to an underestimation of the species range if data are limited. With a large number of missing values, the technique may even fail to simulate known presences.

\subsection{Autocorrelation in GLMs}

In spatial data, there will tend to be positive autocorrelation of both environmental and species variables between adjacent patches. This violates the assumption made by many interpolation procedures that the variables are independent. Where there is a spatial gradient, one would also expect to see negative autocorrelation over long distances (Legendre \& Fortin 1989).

Spatial autocorrelation coefficients such as 'Moran's I' are often used to test for the presence of autocorrelation in quantitative variables. Coefficients for ordinal variables also exist (Cliff \& Ord 1981). They can be applied over an entire map or for distance classes to seek patterns at different scales (Legendre \& Fortin 1989). These statistics do not otherwise offer information about local patterns, and the outcome depends upon the map boundaries, whose placement is not usually relevant to the study topic.

Another method of checking for autocorrelation effects uses Mantel tests (Legendre \& Fortin 1989). Two matrices, A \& B, contain the difference in values between two variables of interest for each pair of sample points. A third matrix, C, holds the geographic distances. A partial Mantel statistic is calculated between A \& B, controlling for C. This test can be carried out through multiple permutations, or using Mantel's approximation to the normal distribution. This demonstrates whether correlations between variables result from coincident spatial autocorrelation. This technique is only viable for quantitative distributional data.

The spatial influence of autocorrelation on a species presence distribution can better be assessed by representing it directly as a variable: for example, the proportion of neighbouring cells containing a presence (Smith 1994). One logistic regression model used a set of such variables, each signifying presence in a single order of neighbour cells (Smith 1994). An alternative variable for each order, the proportion of available cells containing presences, was tested with the intention of avoiding distortions at coastal and other boundary cells. This was less successful as an explanatory variable, so the original 'presence' variable was retained.

A later study, apparently unaware of this work, used a very similar technique. An iterative method, the 'Gibbs sampler', was used to estimate species presence at unsampled sites 
(Augustin et al. 1996). The method was tested using a $20 \%$ sample from the red deer dataset mentioned above (Buckland \& Elston 1993), so that neighbour values for most squares were initially missing. This 'autologistic' model added the 'autocov' variable (Eqn. 4), which measures autocorrelation over distance, to the original GLM. Cells at the map boundaries with fewer neighbours are not given any special treatment. The number of neighbour orders is chosen according to the additional improvement in the fit of the model to the dataset per unit of computing time.

$$
\operatorname{autocov}_{i}=\frac{\sum_{j-1}^{k} w_{i j} y_{j}}{\sum_{j-1}^{k_{i}} w_{i j}}
$$

where autocov ${ }_{i}$ is the 'autocovariate' for a set of $k_{\mathrm{i}}$ neighbours to square $i, y$ is the species presence or absence (0/1) and $w$ the weight given to square $j$ - the Euclidean distance between squares i and $j$ (Eqn. 5, Sokal 1961):

$$
w_{i j}=\sqrt{\sum_{a=1}^{p}\left(a_{i}-a_{j}\right)^{2}}
$$

where $a=$ each variable over which distance is measured (in this case latitude and longitude) and $p=$ number of $a$.

Where, as for the $20 \%$ deer distribution subsample, the data are incomplete, neighbour cell values will be unknown for many grid cells. Here, the Gibbs sampler can be used as follows:

1) A logistic model ignoring autocorrelation effects is used to produce initial presence or absence values for the cells.

2) $\operatorname{autocov}_{i}$ for each cell is calculated, and the autologistic model formulated including autocov $_{i}$ as one of the variables.

3) The P/A map is updated according to the autologistic model.

4) The Gibbs sampler is applied:

a) The autocovariates are recalculated using the presence/absence values from step 3 , and the autologistic model is refitted using these new values for autocov ${ }_{i}$.

b) A random unsurveyed cell is selected. Starting at this point, autocov ${ }_{i}$ is calculated for each unsurveyed cell in turn according to the presence/absence values of its neighbour cells. Presence/absence, $y$, for the cell is re-determined using the new $\operatorname{autocov}_{i}$ and the autologistic model from (a), and then the next cell's autocov ${ }_{i}$ is recalculated using the modified value for the previous cell(s).

c) Steps (a) to (b) are repeated until a stopping rule (i.e. convergence) is met, or for a predetermined number of times (e.g. 20). 
A similar iterative method for unstudied zones that omits step (b) was tested by Smith (1994) who described it as derived from image analysis techniques (Besag 1989).

The random element in step (b) means that the process must be repeated many times to verify consistency in the resulting map. Augustin et al. found that in step (4b) it was possible to substitute the estimated probability of occupation from the autologistic model for the recalculated binary P/A data $\left(y_{\mathrm{j}}\right)$ in Eqn. 4 . This improved the speed of convergence.

The Gibbs sampler creates GLMs that perform well with patchy distribution data, but the recordset is still expected to include validated absence cells. A complete spatial dataset was required to produce the initial deer model without the autocovariate. This approach is expected to work less well with presence-only data. This assumption is evaluated in section 7.2.4.

\subsubsection{Notional relationships}

Various authors create response surfaces using notional rather than derived relationships (e.g. Shao \& Halpin, 1995, Rutherford et al. 1996). This requires much less data, but a high level of confidence in the form of the relationships used.

In one approach, unimodal response surfaces are approximated using weighted averaging to determine the optimum (ter Braak \& Prentice 1988). Where there is no information about the shape of the curve, unimodality is a useful assumption (section 4.3.2). The optimum value for any variable is specified as the mean value for presence sites. Tests show that this method simulates a Gaussian curve optimum when a species is rare or has a small ecological amplitude, or the distribution of the environmental variables is even over the species range (Ter Braak and Looman 1987).

The approach has been modified for moisture-limited environments by taking the mean of the minimum and maximum thermal values in the recorded range, but the maximum moisture value, as optimal (Shao \& Halpin, 1995, Rutherford et al. 1996). It is assumed that moisture and temperature conditions are equally weighted and that the response function is symmetric. There was a generally high level of success in simulating known distributions according to the Kappa statistic, but these assumptions on optima are not applicable to all plant species (Austin \& Smith 1989).

\subsection{Rate limiting factors}

In a unimodal response model, suitability is greatest at the peak of the curve. Weighted averaging methods estimate this using the mean value of those sites in which presence is 
recorded (Ter Braak \& Prentice 1988). It may be assumed that where several environmental variables influence plant response, the rate-limiting factor determines the dependent variable. For example, scaled growth rates based on forestry data were assigned to different ranges of soil and climate variables (Booth 1998). The factor for which growth was smallest was identified at each site of interest in each month, and that growth level recorded. The mean growth rate over all months and variables was mapped. This type of application requires the response of the dependent variable to each independent variable to be well known, but is flexible in terms of the relationships employed.

Similarly, Hackett and Vanclay (1998) modelled height growth with an index based on overall suitability, which offered correlations with actual growth as high as $80 \%$. Their index used 'splined' optimal parameters to estimate growth under different conditions. It is a qualitative, ranked 0-9 index (death-optimal growth), with a value being attached to different levels of each limiting factor.

\subsubsection{GAMs: Generalised additive models}

GLM's restriction to parametric curves can make it difficult to apply to distribution data. Generalised additive models (GAM) "have the data suggest the shape of the curve" (Yee \& Mitchell 1991, Huntley et al. 1995, Wiser et al. 1998). The technique uses a scatterplot smoothing function such as the LOWESS smoother (Cleveland 1979). The regression surface is expressed as a sum of the functions of each explanatory variable. Non-parametric bimodal and asymmetric curves, as well as parametric curves, can arise. The freely available program GAIM (Almudevar \& Tibshirani 1991) performs this type of analysis.

No statistical model is assumed, which suits species' distribution data with no prior knowledge of relationships with the environmental variables. Randomly sampled P/A data are expected, and spatial autocorrelation in the environmental variables is not expected. As for GLMs, this technique requires a comprehensive record of species' distribution.

\subsubsection{Ordinations}

An ordination is a multivariate technique used in ecology to arrange sites along axes according to species composition, or species along axes according to 'latent' environmental variables. This is indirect gradient analysis, because the axes are derived from total community data without reference to environmental variables (Ter Braak \& Prentice 1988). Actual variables can be compared with the axes visually or via correlation coefficients. 
Principal component analysis (PCA) reduces a set of variables to two or more principal axes of variation (principal components, PCs) and provides information on the contribution of each variable to the axes. It assumes an underlying linear relationship between variables, which must first be standardised to equalise their variance (Prince 1986). The physical arrangement of the co-ordinates relative to one another gives an approximation of the Euclidean distance between them in environmental space (Eqn. 5, p.101). It is not assumed that data are normally distributed, but the technique works best with continuous data, and performs badly where a large number of zeroes exist. This is sometimes worked around by substituting very small positive numbers for the zeroes.

Correspondence analyses and detrended correspondence analyses (DCAs) are PCA variants that can be used to ordinate multiple species or environmental variables altogether, identifying communities and their environments. They assume an underlying unimodal curve, and use weighted averaging (Ter Braak \& Prentice 1988). DCAs are better suited to point data, and cope better with matrices containing a large number of absences, than PCAs. DCAs re-scale the first axis, aiming to equalise the within-site variance at all points, and detrend the scores such that at any point on the first axis, the mean of the site scores on additional axes approximates to zero. Both methods are most often used to analyse ecological variation in complete datasets, to evaluate or create composite independent variables for use in a GLM (e.g. Jones et al. 1997), rather than to interpolate distributions from limited data.

An ordination creates a set of composite variables that emphasise the variation within the sample, and reduce the amount of duplicated information. The underlying concept is like similarity analysis (section 4.3.6.12), which produces a distance value for each pair of points. DCAs and PCAs also place the points into an n-dimensional environment space, with distances between points based on the contribution of the different variables. The cluster analysis groups them according to their similarity. If PCs were calculated for no-record cells, these could be categorised using a standard similarity metric.

\subsubsection{Constrained ordination: canonical correspondence analysis}

In a constrained ordination, multiple environmental and species variables can be brought together in a single step, identifying those variables that fit the community best. Canonical correspondence analysis (CCA, CANOCO) defines composite environmental variables that explain multiple species abundance. It is a constrained ordination developed by Ter Braak (1986), in which the axes are weighted sums of the environmental variables. The resulting ordination explains environmental variation and species patterns. It can be used to transform the variables to improve a model's fit to the species' distribution. The method is distinct from canonical correlation analysis, a type of constrained ordination using a linear response model. 
Canonical correlations between two sets of multivariate data are analogous to correlation coefficients in regression modelling, and canonical variates are linear combinations of the original measurements which are not correlated with each other (Prince 1986).

The success of the technique in explaining distribution was evaluated using a one-in-nine subset of the $10 \mathrm{~km}$ square dataset for British birds (Hill 1991). A comparative analysis was carried out using DCA, "to assess the effectiveness of CCA in predicting the major axis of biogeographical variation." The British Isles were superimposed onto the axes produced by the CCA to illustrate the ecological transition zones expressed, and individual species presence distributions were estimated using logistic regression on the four major axes. These were of variable quality.

A note of caution comes from Diniz-Filho \& Bini (1994), working with 'Africanised' honeybees (Apis mellifera scutellata L.) in Brazil. They investigated the relationship between naturalised bee populations' morphology and climate and obtained a good canonical correlation between the two sets of variables. It was hypothesised that the morphological differences were adaptive. When the team performed a 'canonical trend surface analysis' (CTS) to exclude the effects of spatial variation, and recalculated the original CCA, they found that there was no effect $^{5}$. The bees had been correlated with geographical rather than climate space. This analysis provides a reminder that environmental correlations with distribution do not automatically imply causation. A common spatial structure may derive from a third unmeasured variable.

\subsubsection{Similarity methods}

Similarity techniques were primarily developed with the aim of modelling distributions from limited data. They use overall similarity between record sites and no-record sites to evaluate the likelihood of species presence.

Similarity measures are frequently used in ecology to compare species associations, using binary or quantitative measures of species presence (Krebs 1989). Their use in comparing the overall environmental similarity of sites to known species records was pioneered by Australian forestry researchers (Booth et al. 1987). The Gower point-to-point similarity metric (Gower

${ }^{5}$ In the CTS, two CCAs evaluated the relationship between morphometric and climatic data with latitude and longitude. A further CCA was carried out using the residuals of each, representing the space-free variation in the data. This method is only applicable to datasets containing simple spatial structures such as linear gradients, when it neatly disaggregates spatial and environmental variation. The extraction of residuals to isolate the effects of spatial autocorrelation in environmental datasets has also been used in regression-based trend surface analysis (Legendre \& Fortin 1989). 
1971) was used to quantify the similarity of all grid cells on an Australian map to any given site. The contribution from each environmental variable is treated equally by using range standardisation before the mean distance is taken (Eqn. 6).

\subsection{The Gower metric}

Where $R_{\mathrm{ij}}$ is the similarity and $d$ the distance between two points $i$ and $j, a$ is an environmental variable and $p$ is the number of variables, the Gower metric is:

$$
R_{i j}=1-\left(d_{i j}=\sqrt{\frac{1}{p} \sum_{a}^{p}=1\left(\frac{\left|a_{i}-a_{j}\right|}{\operatorname{range} a}\right)}\right)
$$

$R_{\mathrm{ij}}$ has values between 0 and 1 for points inside range $a$. $R_{\mathrm{ij}}$ is calculated for each combination of record $(i)$ and no-record $(j)$ points, and the highest value, $S_{\mathrm{A}}$, for each no-record point identified. The threshold $S_{\mathrm{A}}$ for species presence is selected subjectively or by using known absence records to determine the value that minimises the classification error.

This similarity measure is used in the DOMAIN model (Carpenter et al. 1993). DOMAIN is described as a successor to HABITAT (section 4.3.6.4.1.2), but uses distance measures rather than polytopes. DOMAIN includes all environments with a set degree of similarity to those at record sites, so may tend towards errors of commission (all record sites are regarded as optimal for the species).

The original method uses the maximum similarity, $S_{\mathrm{A}}$, between the candidate point and the record sites. This is a form of single linkage clustering, where similarity between a sample and an existing cluster is equal to the similarity between that sample and the nearest member of the cluster (Krebs 1989). An alternative measure in DOMAIN for Windows (CIFOR 1996) is the mean similarity to a user-selected number of the closest points. This is closer to UPGMA (unweighted pair-group method using arithmetic averages, Krebs 1989), which uses the mean of the similarities between the sample and all cluster members. The option decreases the estimated likelihood of presence, and reduces the effect of outliers. The option is recommended for large datasets that are prone to locational error.

This method is a strong candidate for use with irregularly sampled Amazonian species data, offering a suitability measure based on a unimodal response around each known site. It would be less suitable for more comprehensive locational datasets, because any absence data are not used. If a good set of absence data were available, it would be possible to carry out an inverse suitability' model, and compare this with the presence dataset to identify areas of overlap. 


\subsection{Other similarity and distance measures}

One question is whether the similarity metric used is the best alternative. An unbiased measure should be independent of the number of samples (i.e. the size of the map used) and the number of categories (the amount of variation in a continuous variable). It should increase smoothly from a fixed minimum to a fixed maximum value (Wolda 1981, Krebs 1989). The Gower metric meets both these requirements for values inside range $a$, unless range $a=0$, which produces a divisibility problem. This component of the distance metric should be 0 if the value is identical to $a$, and approaching infinity if the value is outside $a$. Many users replace all $0 \mathrm{~s}$ with small positive values (as for PCA). A similar solution can be taken for zero-value ranges in all affected metrics.

The measure should be range-standardised, to ensure that variables with a large range do not have an exaggerated influence. The Gower metric is based upon the Manhattan distance metric, which considers the modulus of the distances without range standardisation (Eqn. 7). The Manhattan metric has no fixed range of outcomes.

$$
d_{i j}=\sum\left|a_{i}-a_{j}\right|
$$

The Bray-Curtis measure standardises the Manhattan metric to give a range from 0 to 1 (Eqn. 8). Bray-Curtis outcomes are dominated by the higher values in each variable (Krebs 1989). It has the same divisibility problem as the Gower metric, but because values for all variables are summed in the denominator, the 0 occurs less frequently.

$$
d_{i j}=\frac{\sum_{a=1}^{p}\left|a_{i}-a_{j}\right|}{\sum_{a=1}^{p}\left(a_{i}+a_{j}\right)}
$$

The Canberra metric (Eqn. 9), like the Gower metric, standardises the distance over the variables rather than the values for each variable. It is not biased by abundance like the BrayCurtis metric, but again has problems when $a=0$. If $a=0$ for both sites, that pair of values cause a division by 0 . If $a=0$ for one site, the index is at its maximum value for that variable.

$$
d_{i j}=\frac{1}{p}\left[\sum_{a=1}^{p}\left(\frac{\left|a_{i}-a_{j}\right|}{\left(a_{i}+a_{j}\right)}\right)\right]
$$


Similarity is calculated for the Bray-Curtis and Canberra metrics as for the Gower metric (Eqn. 6). Both metrics perform best with large sample sizes (number of variables, $p$ ).

Correlation coefficients can be used to measure similarity, but this is not recommended where > $50 \%$ of samples have a zero value (Krebs 1989). This may occur with environmental data: for example, for AET of $0 \mathrm{~mm} / \mathrm{month}$. Correlation coefficients are also inappropriate for use with small samples (values of the environment variables).

Another measure is the Euclidean or interstand distance formula (Eqn. 5, p.101), which is often used in ordination (Goldsmith et al. 1986). It can have values from 0 to infinity, so fails the requirement for a fixed range. Several other indices are often used in determining similarity between species communities (Morista's, Horn's and the Renkonen index), dealing with relative abundance of different species rather than continuous environmental variables.

The Gower and Canberra distance metrics seems most useful for record-site/no-record-site comparisons, as long as environmental variables with zero range in the record-site dataset are treated appropriately.

\subsection{Variation within the niche}

Patches near the edge of a species range frequently support fewer individuals (Whittaker 1967), so that habitat suitability is negatively correlated with distance to the range centre. The use of the range centre as the core niche is problematic for species with dispersed or isopleth ('doughnut') ranges, or simply inhabiting heterogeneous environments, and less useful under a situation of environmental change. Can the core and margins of the environmental niche be identified using a similarity metric?

Where the underlying species dataset is without spatial bias, the core and periphery of the niche could be calculated from the utilisation of different habitats compared to their availability (note that this measure is affected by the map extent). Suitability could then be determined by distance from the core rather than from the record-set. However, if spatial bias is present, then the habitat from the core survey region rather than the core niche appears most frequently. The assumption that all presence sites are equally suitable decreases this source of bias. In addition, patches adjacent to highly suitable 'core' patches may host larger populations than equivalent patches far from the core (Brown 1995), because dispersal into these patches maintains the population. A density count will indicate that these 'sinks' are more suitable than they actually are. In a similarity model based on presence records, suitability is best determined by ecological distance from known record sites, rather than applying this type of weighting. 


\subsubsection{Analytical models of change response}

Individual species' responses are extremely variable, and climate changes are expected to generate new communities (Prentice et al. 1991). The species level of analysis provides a good indication of overall diversity impacts. Change response estimates for single species are usually derived from equilibrium models of current distribution by modifying the values of the independent variables according to GCM results (e.g. Jeffree \& Jeffree 1996, Bartlein et al. 1997, Kienast et al. 1998).

This approach is subject to several criticisms:

- When change in the variables governing the system is unusually rapid, the rate of change is important in estimating response (Woodward \& Beerling 1997, Collingham et al. 1996). The ability of populations to match their migration rate to that of the spatial niche, and the possibility that remnant populations of longer-lived plants may persist for some time in areas that are no longer suitable for reproduction, are not considered. These models only map change in potential distribution or probability of occurrence.

- The extrapolation of an analytical model based on an empirical relationship to environmental values beyond the calibrating dataset should be regarded with caution (Passioura 1996).

- The baseline 'equilibrium' is relative, as all ecosystems are dynamic in composition (Stott 1998), and some display an ongoing response to recent climate changes (Sparks \& Carey 1995). If the methods are considered as modelling a probability of occurrence, whose relationship with environment is constant in time, the assumption that species are currently at equilibrium with their environment can be circumvented (Ostendorf et al. 2001). However, when species' records have been collected over long periods (as for monograph data) it is difficult to avoid making an equilibrium assumption.

- The scale issue (section 4.3.4) may be aggravated by other factors. The individualistic responses of localised microclimates and topographies to generalised warming patterns are not usually considered - changes in climate are applied uniformly over large areas (Halpin 1997). Downscaling techniques may reduce this problem (section 3.6).

- Interspecific interactions are not modelled, but can be critical in deciding success under changing conditions (section 4.2.5).

- Direct response to changing $\mathrm{CO}_{2}$ is difficult to incorporate and rarely considered.

Nonetheless, an analytical model of change response should provide a map of potential distribution that is consistent with the assumptions used to map present-day range. It is therefore 
a good basis for a more complex model of realised distribution, which might incorporate lags in demographic response and limits to dispersal.

\subsubsection{Mechanistic response models}

Mechanistic models address functional issues directly, rather than formulating correlative relationships with environmental variables. Individual physiological and developmental processes are modelled to identify those affected by climate change. Species-level processes are related to demography via individual survival and reproductive success. Can these concepts be adapted for use with tropical forest species or PFTs?

\subsubsection{Productivity and leaf area index (LAI)}

Competition between individual plants can be viewed as a contest to accumulate biomass and horizontal cover. Reproductive fitness is directly related to productivity, which can be viewed as the accumulation of energy with which to generate offspring (Brown 1995). Growth rates of comparable species indicate which is likely to dominate in a particular situation, if other biotic requirements are met.

Interspecies variation in growth rate at a given locale results from differences in optima or niche breadth, and differences in growth strategy. When plants are propagated independently, growth optima will reveal the favoured potential niche. Comparison with other species' growth rates may indicate competitive exclusion. Selection pressure may separate species optima following the 'niche-diversification' hypothesis (section 2.2.2.2.2), but competition occurs in highresource habitats.

Following Eqn. 1, productivity can be described as (Woodward 1987):

$$
w=E \int_{0}^{t} I_{o}\left(1-e^{-k L}\right) d t
$$

where $w=$ accumulated dry mass, $E=$ conversion efficiency (typically $2.5 \%$ ), $I_{0}=$ incident radiation, $k=$ extinction coefficient for solar radiation, $L=$ LAI of plant. Exploitation of water and light are interdependent, with LAI depending on moisture uptake and biomass accumulation (section 4.2.1.2).

Potential LAI is calculable for climate zones, which allows the estimation of climax vegetation types (section 4.3.5), rather than distinguishing between species or PFT responses. This calculates optimal growth for plants able to use all of the resources available. AET is an alternative measure of potential primary production (Stephenson 1998). 
Growth for an individual species classically follows a unimodal curve rather than increasing continuously with increased resources. An adjusted LAI can be calculated for agricultural or forested land devoted to monoculture. Yield models have been constructed for these singlespecies zones, with species responses to particular climate variations parameterised through manipulative experiments. For example, EPIC calculates the maximum daily increase in biomass based on existing LAI, incident photosynthetically active radiation and modelled ET (Brown \& Rosenberg 1999). Processes of photosynthesis, respiration, translocation (movement of water through the plant) and transpiration are modelled for a typical individual in each location. The impacts of change in $\mathrm{CO}_{2}$, temperature, precipitation, humidity, nitrogen content and radiation can be estimated, as each directly affects at least one process.

The adverse consequences of excess moisture, relevant for forest margin plants colonising core areas of moist forest, or terra firme plants colonising flooded habitats, are not covered by these concepts but could be considered separately. However, species-specific predictors of growth rates under different moisture regimes are required, so these methods are not directly useful for the current analysis.

Another approach might be to use potential LAI as a bioclimate variable, directly relating productivity to species success.

\subsubsection{Forest growth models}

Growth may be modelled at the vegetation level, as above, for PFTs or species. When detailed historical data exist for the dependent and independent variable, temporal rather than spatial relationships can be explored. Dendrochronological data provide a record of growth through time, which may be correlated with local climate station data to examine the effects of different climate regimes on the same forest. This type of data can help to assess sensitivity to climate and climate variability.

\subsection{Classical growth models}

Growth-yield models used in forestry research consider the stand or individual tree, and are based on regressions of environmental and tree size data from repeated surveys (Liu \& Ashton 1995). Areas up to thousands of hectares are simulated, based on data from many plots $<1$ ha. Growth is the only vital rate simulated, as mortality and reproduction are managed in plantations. Where individual size is modelled, the measures are timber volume rather than mass-based, i.e. dbh or height. 
Site indexes constructed from environmental variables are expressed as the expected height of dominant trees at a set age. A regression function of site index, species basal area and density is used to determine diameter growth rate (Liu \& Ashton 1995). Additional competition measures may be included to estimate growth of individual trees. Alternative models have used multiple linear functions of environmental variables to express growth. When site index can not be calculated, previous growth rate has been used as a surrogate. A representative sample of growth functions from individual-based models can be found in Liu \& Ashton (1995).

The pattern analysis concept of suitability and the growth model concepts of primary productivity and site index are similar. Site index is another way of formulating suitability, with a specialist application. This is unsurprising, as growth potential is a major factor in determining species' distribution.

\subsubsection{Demographic models}

Demographic models examine lifecycle processes in discrete populations, simulating the 'vital rates' of survival, growth and reproduction. This section assesses their use in a changing environment, applicability to tropical plants and data requirements.

\subsection{Structured population models}

Structured population modellers are usually interested in the form and regulation of population dynamics, including the conditions under which populations expand or decline. These considerations are useful for biological resource management, providing estimates of maximum sustainable yield at different life stages. Traits linked to age or size are allocated to individuals in a single-species, single-site population. Vital rates and lags in change response can then be modelled in detail. Size-based traits are more applicable to plant demography than age-based traits, because vital rates are generally dependent on size, whilst size with age varies between individuals.

The three main types of demographic model are based on matrix algebra, delay differential equations (DDE) and partial differential equations (PDE) (the descriptions here are drawn from Caswell, 1989 and Tuljapurker \& Caswell, 1997). Each type may include stochastic processes. Detailed life-history data may be used in parameterisation (e.g. Olmsted \& Alvarez-Buylla 1995, Bernal 1998). Interactions between populations are not usually considered.

The types are distinguished by the transfer equations used to alter the size or age of individuals. Matrix and DDE models divide the population and time into discrete stages, whilst PDE models describe a continuous distribution of age or size through time. Although there is a size 
continuum between juvenile and mature plants, botanical surveys often employ height or dbh classes, making PDE models more difficult to parameterise from field data.

These approaches do not evaluate the relationship between plants and their environment, but they can be adapted to examine the demographic response to environmental change, and thus to simulate lags in distributional response.

\subsection{Stage-classified models}

In stage classified models, individuals survive, reproduce and are recruited between stages according to probabilities assigned to their stage. Individuals do not possess characteristics independent of the stage. The population $n$ at time $t$ is characterised as a vector composed of $k$ stages, $n_{1} \ldots n_{\mathrm{k}}$ :

$$
n(t)=\left[n_{1}(t), \ldots, n_{\mathrm{k}}(t)\right]
$$

To give a population estimate for time $t+1$, the vector is acted upon by a single projection matrix (matrix models, Eqn. 12), or by a differential equation for each $n_{\mathrm{i}}$ (DDE models, Eqn. 14 for $k=2)$.

$$
n(t+1)=\mathbf{A} n(t)
$$

In the matrix model, Eqn. 12, $\mathbf{A}$ represents a square matrix in which each row and column matches a vector row. The matrix maps the contribution of each stage to each other stage, which may be linear or non-linear through time (e.g. density dependent A). Matrix element $a_{\mathrm{ij}}$ specifies the contribution of column life-stage $j$ to the change in row stage $i$ over a single time unit. The $i j$ th entry of $\mathbf{A}$ represents the number of individuals of stage $i$ at $t+1$ per individual of the contributing stage $j$ at $t$.

For example, a fertility coefficient represents the number of seedlings produced per plant in $j$. The coefficients express survival (rather than mortality) and reproduction. Individuals not assigned to the same or a higher stage are discarded.

Matrix analysis techniques allow equilibrium (asymptotic) outcomes of a linear set of elements to be examined. When $\mathbf{A}$ is multiplied by a vector $n$, and the result is a vector whose elements have all been multiplied by the same constant $(\lambda)$ with respect to the original $n, n$ is an eigenvector for $\mathbf{A}$ (Eqn. 13). $\lambda$ is known as the eigenvalue, the latent root or characteristic value. $\lambda$ indicates whether a transition matrix predicts a stable $(\lambda=1)$, expanding $(\lambda>1)$ or declining $(\lambda<1)$ population. 
When $n$ is the eigenvector:

$$
n(t+1)=\mathbf{A} n(t)=\lambda n
$$

In the two-stage DDE model, Eqn. 14, the number of individuals in $n_{\mathrm{i}}$ depends on recruitment rates ( $m_{2}$, because stage 2 is the reproductive stage), mortality rates $(\mu)$ and the time required for maturation from stage 1 to stage $2\left(T_{1}\right)$. Surviving organisms from $n_{1}$ are recruited to $n_{2}$ when time of length $T_{1}$ has passed. For both stages, 'population change = recruitment - mortality'. The same type of model can be developed for multiple stages.
a) $\frac{d n_{1}}{d t}=m_{2} n_{2}(t)-\mu_{1} n_{1}(t)$
b) $\frac{d n_{2}}{d t}=m_{2} n_{2}\left(t-T_{1}\right) e^{-\mu_{1} T_{1}}-\mu_{2} n_{2}(t)$

Stage-classified models were designed for use with static or consistently changing vital rates. For change response simulation, the vital rates should be altered by the external conditions. The matrix formulation is easy to manipulate in this way, as each element is individually accessible, and may be made dependent on density or site-index. Additional alterations in population structure (such as migrating propagules) may be applied in each temporal iteration. The matrix approach is transparent, but may be computationally intensive.

A recruitment lag may be useful in change modelling. If an area becomes more suitable for the species, the expected increase in population would be rapid in a standard matrix model. In reality, recruitment is dependent upon growth rates. A matrix-based model that incorporated $T$ would be more useful under change conditions. In a more complex model, the direction and magnitude of change could determine the size of $T$.

In a DDE model, the recruitment lag means that if the parameters are made to vary through time in response to climate change, earlier values of $n$ for each stage must be retained until $T$ has passed. Complexity increases with increasing incorporation of ongoing environmental change. The comparative efficiency of this approach is reduced, and opacity increases.

Both types of model assume a good knowledge of the species' transfer probabilities and reproductive rates at each stage. If available data were used to approximate these for PFTs, lags in response could be estimated for less well-known species. 


\subsection{Continuous models}

A PDE model represents the population as a function of continuous age or size, $a$ at time $t, n(a$, $t$ ), rather than as a vector. The effects of mortality, $\mu(a)$ and reproduction, $m(a)$ are represented in Eqn. 15 (a) and (b):

$$
\begin{aligned}
& \text { a) } \frac{\partial n(a, t)}{\partial t}+\frac{\partial n(a, t)}{\partial a}=-\mu(a) n(a, t) \\
& \text { b) } n(0, t)=\int_{0}^{\infty} m(a) n(a, t) d a
\end{aligned}
$$

The parameters in PDE models can be made density dependent, and there is potential for inclusion of detailed vital rate information. They are difficult to parameterise from size-based field data. However, there is a smaller body of theory surrounding this set of models, and they are 'analytically difficult' (Tuljapurker \& Caswell 1997).

\subsection{Stand simulation models}

Forest succession and stand simulation models work at the level of the individual tree in a multi-species system. The characteristics of each individual are tracked and replacement probabilities determined, to simulate community structure through time. These simulations are most often employed in temperate and boreal systems where species richness is low and vital rates well-parameterised (Shugart \& Urban 1989), but a few tropical studies exist (e.g. Bossel \& Krieger 1994, Kammesheidt 1998).

The forest is portrayed as a mosaic of patches, each dominated by single trees. Patches may interact through shading and seed dispersal. The forest boundary may be surrounded by a variable matrix contributing seeds (Liu \& Ashton 1995, 1998), the edges of the plot may wrap or deflect seeds (Liu \& Ashton 1999), or the forest may be modelled as a fragment outside which seeds are lost.

Tree size and growth may be modelled, but over a much smaller area than in growth-yield models ( $<1$ ha, Liu \& Ashton 1995). The death of an adult tree creates a gap, stimulating the growth of seedlings, which compete to fill the gap. 'Physiology-based' gap models model photosynthesis and respiration on an individual level (Friend et al. 1993). Detailed simulation of each species may be used to calculate NPP on a daily basis (e.g. \% live sapwood, canopy light extinction coefficient, leaf dark respiration at $25^{\circ} \mathrm{C}$ ). 
Forest composition in a changing environment has also been simulated - for example the effects of climate change on boreal forests (FORSKA2, Prentice et al. 1991) and alpine forests (FORCLIM, Fischlin \& Gyalistras 1997). Replacement probabilities and growth were directly related to climate, with temperature being a strong controlling variable. In both cases, species assemblages without current analogues were simulated.

Stand simulation models can help to elucidate how different types of species succeed in competition with one another, and the possible impacts of change on species dominance. However, it is only feasible to employ them over a small area, so these models are not suitable for the present study.

\subsection{Metapopulation models}

Stand simulation models consider a single spatial unit or 'patch'. Metapopulation models examine interactions between patches, often using hypothetical species. Population demography, immigration and emigration are modelled for each patch. Source and sink populations with local extinction and recolonisation may arise. Competing species may be simulated together.

The metapopulation environment is usually modelled as an annually variable matrix that is otherwise homogeneous, but a few models consider ongoing environmental change (Bowers \& Harris 1994). Cost-distance between patches and dispersal mechanisms may be included, or the probability of movement between patches may be equal.

Population viability analysis (PVA) applies metapopulation theory to the assessment of species vulnerability. Extinction probabilities and minimum viable populations (MVPs) for small populations are calculated, and management interventions suggested. A standard MVP measure is the population size with a $99 \%$ probability of persistence over 1000 years (Shaffer 1981). The critical patch size is the smallest area required for population persistence (Latore et al. 1998). Accurate modelling requires a good knowledge of the species' initial distribution, population ecology, reproductive strategy and environmental niche. The modelling process and the plausibility of the results can indicate gaps in knowledge of autecology or theory.

Several generic metapopulation models are available. VORTEX, RAMAS and ALEX are reviewed by Lindenmayer et al. (1995). Each models an age-structured population with a maximum carrying capacity per patch, dispersal between patches and catastrophic events. All annual events are stepwise and stochastic, with classical probability distributions (normal, binomial or Poisson) being sampled for different types of event. As small samples show a high variance 
around mean values in a probability distribution, stochastic variation can be as important as the mean values in determining population survival (Lacy 1993).

\subsection{Life history}

Age structure may be modelled using a transition matrix for each population or individual organisms may be modelled. An individual focus limits the model to small populations, but enables simple (one-locus) genetic profiles to be allocated to each individual, simulating genetic drift and inbreeding ${ }^{6}$. Different mortality rates may be applied to different life stages in either case, and may be sampled from a probability distribution or applied as an absolute rate. Sometimes these rates are separately set for individual populations to simulate habitat variability. Reproductive probabilities vary between populations, but not between mature individuals in any one population. Individuals are reproductively active until death. A maximum lifespan is generally specified.

Density-dependent modifiers may be applied to reproductive and death rates. In RAMAS, the possibilities are exponential growth up to the carrying capacity (which may be infinite); 'scramble; or 'contest'. Scramble and contest functions add a maximum population growth rate. Allee effects, where reproductive rate is lower for small population sizes, are also sometimes simulated.

Migration between patches is modelled - in the case of plants, for propagules only. Emigration probabilities may depend upon the density of individuals in the original patch, and in ALEX result in death if the dispersing organism does not encounter a suitable habitat. In RAMAS, it is possible to specify directional dispersal probabilities to simulate one-way boundaries such as slopes or stream currents.

\subsection{Environmental variation}

Most PVA models are not combined with environmental suitability models. It is assumed that environments are more-or-less suitable, and that the population is restricted to an established set of patches. Optimal and marginal suitability are not distinguished.

Interannual environmental variation may be modelled by randomised fluctuations in life-history parameters, patch carrying capacity and catastrophe probability. Different classes of catastrophes with different levels of severity may be simulated. Each extreme event affects the

\footnotetext{
${ }^{6}$ More sophisticated "genetic algorithms", modelling processes of selection and mutation, have been independently developed in evolutionary biology (Mitchell 1996).
} 
reproductive success and mortality rates in the patches where it occurs. In ALEX, catastrophes also have an impact for several subsequent years. Some simulations include density dependent 'catastrophes' to model disease.

\subsection{Cellular automata}

Cellular automata are grid-based models in which a set of rules determines interactions within and between cells. The system is parallel because each cell's values are updated simultaneously in the same iteration of the model; homogeneous because the same rules are used throughout the grid; and local because cell values are influenced only by neighbour cells (Rucker \& Walker 1997). A cell can be in one of a finite number of states. Simple rules can lead to the emergence of complex patterns. Strictly, only the state of the cell and its neighbours should affect its state in the following iteration, but in ecological models, external variables may be allowed an influence (e.g. disturbance, Ellison \& Bedford 1995). This approach to spatial population modelling is generally used at a coarser scale than a metapopulation model, with some exceptions (e.g. stand simulation, Solé \& Manrubia 1995).

Where the metapopulation model concentrates on detailed population dynamics, the cellular automaton focuses on the effects of spatial and temporal variation. Some models seek to identify generalised migration rates for a species, using a fixed initial distribution front over a variety of landscapes (Dyer 1995). Others look at the effect of environmental change on current distributions (Carey 1996, Collingham et al. 1996), or the rate of invasion of a non-native species.

For example, a South African cellular automaton was based on a 1-minute latitude-longitude climate database and the ACKDAT plant abundance database of 3,500 sites (Rutherford et al. 1996). The model simulated climate change impacts on a few opportunist species, whose realised niches were thought likely to correspond with their full potential range, and whose short generation time would permit a faster distributional change response. Unimodal response surfaces for each species were approximated from the three minimum and maximum extremes for each climate parameter. Separate surfaces were formulated for the overall range and the 'core' regions where population density was highest.

Dispersal and longevity indices were estimated, and a landscape permeability coefficient based on productivity devised to simulate barriers to cell colonisation. The dispersal rate estimates seem subjective ("dispersal rate indices are largely based on the morphology of the diaspore and a factor of about 30 was used for conversion to linear kilometre distance over 50 years"). Plant migration is modelled at a constant rate of climate change for landscapes of different 
permeability. The model suggests that some of these fast-moving opportunists will experience a decrease in areal extent under $\mathrm{a}+3^{\circ} \mathrm{C}$ scenario.

This type of study highlights the importance in threat evaluation of species' dispersal needs and abilities under changed conditions. The potential for migration between present realised and new potential niche should be evaluated.

\subsection{Conclusions: Modelling Distribution AND CHANGE}

This review has covered the likely impacts of climate change on species and habitats, and methods of modelling distribution and distributional change. The importance of considering lag in change response and of using ecophysiologically meaningful forcing variables has been noted.

The literature search has identified no models of potential climate change impacts upon South American species' distributions, although there are speculative papers focusing at the forest level (Fearnside 1995c, Laurance 1998). Useful components of a change response model can be drawn from different fields. Biome, community and species-level studies from other regions have been reviewed here. Each approach investigates a different facet of response to climate.

The gap between analytical simulations and more detailed process models that consider the rate of change could be bridged using a pattern analysis model that iterates through time. Changes in patch suitability would be derived from a baseline correlative analysis, and known dispersal rates used to estimate the probability that a species was able to migrate to the new parts of its range during each period.

To better understand the rate of change, a focus on demography is also required. The impact of climate change on different simulated functional or demographic processes may have opposing effects on species' survival. In this case, process simulation gives a more sophisticated overview of change than pattern analysis. However, ill-founded estimation of life history or growth parameters diminishes model accuracy whilst retaining the illusion of a precise simulation, and the potential for error increases with the number of processes. The limitations regarding interspecific interactions, scale and $\mathrm{CO}_{2}$ effects still apply. An additional challenge is that change conditions may bring about unexpected alterations in the processes simulated.

I conclude that for change response simulation, correlative models based on emergent patterns from numerous individuals are to be preferred over process models based on imprecise parameterisations, but that well-founded process models are the ideal. Population-level 
simulation should be sufficient when working over a large area. When data are limited, a demographic model working alongside a correlative model can define the difference between new and old ranges, and generate an estimate of the potential lag in species' response. This approach is taken here.

Models of potential distributions often cover much greater areas than species' realised distributions. It is likely that this is frequently due to dispersal barriers that have prevented species from colonising suitable areas that are not contiguous with existing populations (Peterson \& Vieglais 2001). A model of species current and changing distributions should take these barriers into account.

\subsubsection{Functional classifications}

If species can be grouped by traits indicating a likely common response, common parameters can be developed for response models for use with less-known species (section 4.1.2). Where possible, the traits should be directly related to the parameters.

Any functional classification evaluating change response must consider reproductive rates, dispersal mechanisms and pre-adaptations to the expected stresses. For example, deciduous plants with rapid reproductive cycles are well adapted for seasonal rainfall, whilst evergreen plants are more successful where moisture is continuously available.

This project develops a PFT set using traits chosen to reflect the whole range of response. PFT parameters are substituted for species parameters where necessary.

\subsubsection{Simulating distributions}

The selection criteria set out earlier for a useful simulation model are objectivity, a sound theoretical basis, compatibility with neotropical data, tolerance of sampling bias, speed and parsimony (section 4.3.2). Expert judgement is simply not objective and repeatable. Pattern analysis techniques are based on known correlations with environmental or spatial variables rather than estimated physiological parameters and are thus well-suited for current distribution modelling. The present study uses a suite of climate variables to generate bioclimate variables.

Of the many analytical models available, most have constraints on the type of data required. The spatial bias in and presence-only nature of monograph data prevent the use of modelling techniques with classical statistical requirements. While random or systematic data sampling remains the ideal, some techniques are more tolerant of bias. Four methods fit these restrictions, 
and still take advantage of environmental datasets in estimating species distributions: geometric approaches, Bayesian inductive spatial modelling, similarity models and environmental envelopes.

Table 4:2: Interpolation models' data requirements

\begin{tabular}{|l|l|l|l|l|}
\hline Model & $\begin{array}{l}\text { Presence } \\
\text { data }\end{array}$ & $\begin{array}{l}\text { Absence data/ } \\
\text { Training area }\end{array}$ & $\begin{array}{l}\text { Strict random } \\
\text { sampling }\end{array}$ & $\begin{array}{l}\text { Environmental } \\
\text { variables }\end{array}$ \\
\hline Geometric approaches & Yes & No & No & No \\
\hline Bayesian image analysis & Yes & No & No & No \\
\hline Kriging & Yes & No & No & No \\
\hline Environmental envelopes & Yes & No & No & Yes \\
\hline Predictive habitat mapping & Yes & Yes & No & Yes \\
\hline $\begin{array}{l}\text { Bayesian inductive } \\
\text { modelling }\end{array}$ & Yes & No & No & Yes \\
\hline $\begin{array}{l}\text { GLM; GAM, autologistic } \\
\text { regression }\end{array}$ & Yes & Yes & Yes & Yes \\
\hline $\begin{array}{l}\text { Ordinations: PCA, CCA, } \\
\text { DCA }\end{array}$ & Yes & Yes & No & Yes \\
\hline Similarity methods & Yes & No & No & Yes \\
\hline
\end{tabular}

Geometric approaches are rejected because they do not consider the impact of environment on species, which makes them particularly unsuitable for modelling the impact of change on potential distributions. Bayesian inductive modelling is rejected because of its use of prior probability maps, and its inability to consider variables outside the current range. Were an expert assessment of probable distribution available for each species layer, Bayesian methods would be more applicable, but in the circumstances the prior probabilities could only be based upon known species points. This would be of little value as the points are used in a later step, so the information would simply be duplicated.

Environmental envelopes and similarity methods remain. As discussed earlier, polygonal envelopes are susceptible to errors of omission where the dataset has a large number of norecord sites relative to record sites, and rectilinear envelopes are vulnerable to large errors of commission.

Similarity methods accommodate multiple optima by simulating a unimodal distribution around each presence point's set of values. The Gower metric offers a useful basis for estimation of species' distribution under different scenarios from low-frequency present-day binary data. However, any translation of a continuous suitability gradient into P/A values allows the potential for errors of commission. 


\subsubsection{Simulating change response}

Species' responses to climate change could include an expansion or shift of potential range, or extinction for species with narrow climate preferences and limited dispersal. Pattern analysis is sufficient to estimate present-day distributions or future potential distributions. To simulated future realised distributions we require an evaluation of the response to the degree and rate of change as it affects population survival through its effect on the balance of mortality and recruitment. Ideally, a transient model of climate change (section 3.5.1.1.1) should be matched with such a transient response model.

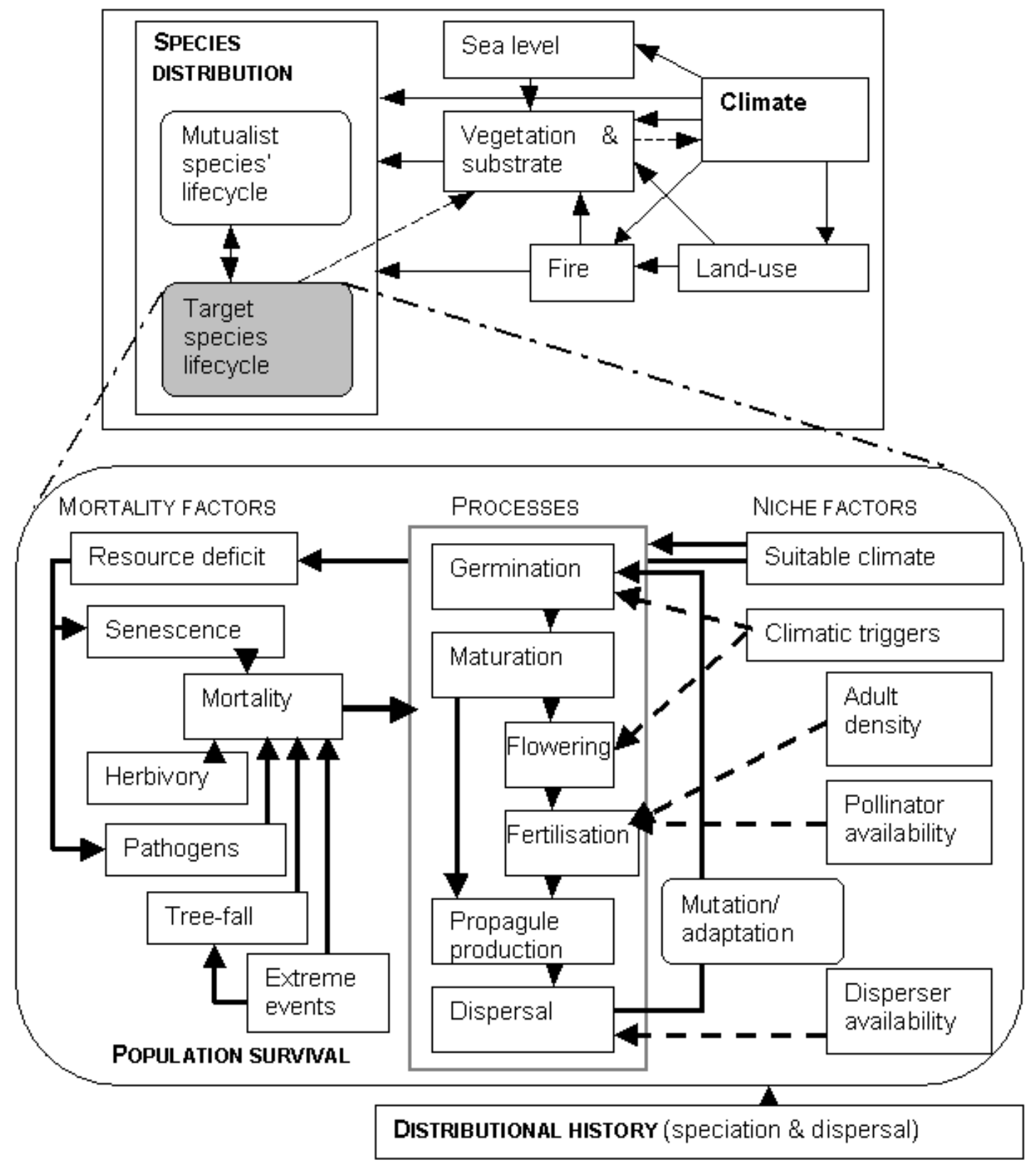

Fig. 4:3: Regional and local processes affecting species' distribution

This thesis considers only the direct impacts of climate on individual species' distribution and demography. Each box in the upper section of Fig. 4:3 represents a regional submodel that would be required for a full simulation of distributional change. The lower section shows local 
factors affecting the vital rates of survival, growth and reproduction in each individual population.

Local demographic processes and interactions between populations of a single species would normally be simulated using a metapopulation model. The impact of regional processes on a species would normally be simulated by an analytical distribution model. This thesis combines the two to produce a novel spatial demographic model, in which each occupied map cell holds its own independently simulated, size structured population. Thus, lags in population response to changes in local environments can be simulated to give an indication of species survival over a large region. 


\section{Materials and methods}

\subsection{METHODOLOGY: CONCEPTS}

The impact of environmental change on plant species richness can be described as the sum of the unique responses of individual species. This section outlines a methodology for assessing their distributional response to climate change. It employs a spatial demographic model to simulate the potential distribution, realised distribution, and change response of individual species. This should produce repeatable, transparent results for any tropical plant species.

\subsubsection{A spatial model of species distribution}

To model the impact of climate change on the biodiversity of an area we begin with a conceptual area surrounded by natural barriers, within which all environmental factors are homogeneous. Its botanical biodiversity is represented by a large number of species, all of which are in equilibrium with their environment in terms of their density and demography. The potential distribution of each species, which is defined by the environmental conditions in which the species can survive and reproduce, then corresponds to the area enclosed by the natural barriers (Fig. 5:1a).

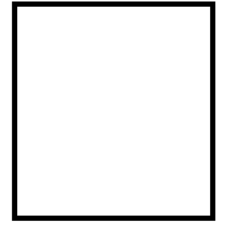

(a)

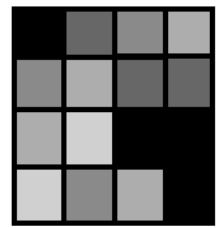

(b)

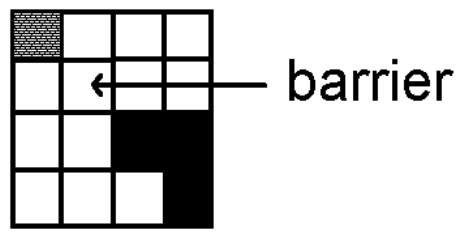

(c)

Fig. 5:1: (a) Homogenous region; (b) Variation in suitability within region; (c) Patches of potential distribution divided by unsuitable zone

As this model is too restrictive to describe reality, the concept of variation in environmental suitability is introduced. The region is divided into patches of equal area, and the probability of a species' occurrence in a patch is correlated with its suitability for that species (Fig. 5:1b).

Some environments are so dissimilar to the species' optimum that the probability of population survival is negligible. The species is only able to populate patches that fulfil its habitat requirements. The potential distribution is then redefined as the full set of patches in which the species can survive and reproduce. The potential distribution can vary between species, and for certain species may be identical to the study area. 
The realised distribution of each species is limited by the presence of dispersal barriers that separate occupied and vacant suitable patches (Fig. 5:1c). Barriers are defined as unsuitable patches separating two suitable areas, which are too wide to be crossed by a single dispersal event, and have been unsuitable for colonisation throughout the time that the species has occupied one of the suitable patches. The probability that the species will cross such a barrier is very low.

It follows that each species' realised distribution depends on:

1) its evolutionary history, which determines the environmental niche occupied and the potential to adapt to new environments

2) biogeographical factors, which describe the species' spatial origin and thus its ability to colonise further suitable habitats

3) environmental factors including dispersal barriers, e.g. annual mean and extreme values for moisture and energy availability, seasonality and soil nutrients

The distribution is determined by local processes of population survival and reproductive success, strongly influenced by regional-scale processes of population interactions and climate. The environmental influence on distribution is exerted via controls on the vital rates (of germination, survival, growth and reproduction) that govern population persistence. These can be separated into relative constraints on distribution and vital rates, and absolute constraints that permit or prevent particular processes (for example, germination). The relative constraints on the potential distribution can be expressed as a suitability index based on climate and other environmental factors. The level of one climate variable may only be important in the context of the value of another - the index indicates the combination of climate conditions that suit the species. Absolute constraints are based on the presence of any species-specific phenological triggers or other absolute limits on distribution.

A positive correlation would be expected between population density and environmental suitability. Population density therefore varies over the realised distribution, and typically declines towards the edge of the spatial range (Brown et al. 1995), as expected if the environmental variables are on a continuous gradient. In this revised model, therefore, not only are the spatial distributions of each species different from one another, but their population sizes differ between species and locations.

Environmental change alters the potential and thus, in time, the realised distributions of each species. Lags in each population's demographic response to change, resulting from individual lifespans and maturation time, slow this distributional response. 
To better understand the demographic response, each species can be represented as a metapopulation composed of a finite set of size-structured populations. A population is defined here as the set of individuals present within a given spatial patch, rather than as an interbreeding unit. Each population is divided into discrete size classes (stages), each with distinct properties including vital rates. For the sake of simplicity, it is assumed that each species behaves independently of all others. Although there are important mutual relationships between species in tropical forests, this seems an acceptable approximation for an initial model.

\subsubsection{Modelling demography at equilibrium}

\subsubsection{The balance between survival, growth and reproduction}

Present-day populations are initiated in all suitable patches connected by others to a species' record-site. This simulates dispersal from the known record points, in a stable environment, until equilibrium. Stable populations are assumed at the end of this process, although this is a simplification of reality.

Population size depends on the balance between survival, growth and reproduction. It is additionally limited by the available land area within the patch ${ }^{7}$, and the observed maximum density $(K)$ per stage $(c), K_{\text {cmax }}$. We know that some species occur sparsely, and others in great numbers. There are various hypothesised mechanisms behind these disparities in number (section 2.2.2). We also know that changes in interspecific competition or herbivory following climate change can affect the relative abundance of different species in unexpected ways (section 4.2.5). The conservative assumption is taken that $K_{\mathrm{cmax}}$ will remain unchanged.

Small, marginal populations are likely to be more susceptible to demographic change than larger ones. They are usually the first to be affected by changing climate, whether it moves the patch towards or away from the core niche.

In a population that is in equilibrium with its environment under present conditions there must, by definition, be a balance between the vital processes of germination, survival, growth between stages, and reproduction (propagule production and dispersal). Every year, survival, growth and reproduction occur in sequence and simultaneously in each stage and patch. All processes are considered in isolation from other patches. For stage $c$ at the end of year $y_{1}$, the number of

\footnotetext{
${ }^{7}$ Patches need not be of equal size - for example, where an implementation of this methodology includes a categorical restriction covering part of a map cell, such as land use or soil class, or when a cell is coastal, patch size can vary.
} 
individuals is determined by the vital rates operating on the population remaining at the end of $y_{0}$ :

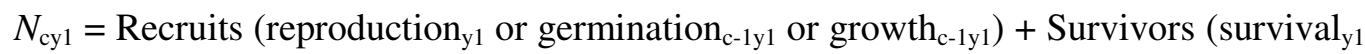

$$
\begin{aligned}
& \left.* N_{\text {cy } 0}\right)- \text { Transition }\left(\text { growth }_{\text {cy } 1} * \text { survival }_{\mathrm{y} 1} * N_{\text {cy } 0}\right)
\end{aligned}
$$

This can be translated into a population matrix equation encompassing all stages from seed to final stage. The vector $\mathrm{n}(t+1)=\mathbf{A n}(t)$ gives the number of individuals that would be encountered in each stage for the interval $t$, with no temporal lag between stages. A changes in response to environmental factors and population density. Each matrix coefficient is altered individually through time, to simulate vital rate response to equilibrium and changing climates.

Eqn. 17 describes a single population with $k$ stages, $c$. Transitions between stages are upwards only ${ }^{8}$, one stage per time interval.

$$
\left(\begin{array}{c}
n_{1} \\
n_{2} \\
n_{3} \\
\ldots \\
\ldots \\
n_{\mathrm{k}-2} \\
n_{\mathrm{k}-1} \\
n_{\mathrm{k}}
\end{array}\right)(t+1)=\left(\begin{array}{cccccccc}
P_{1} & F_{2} & F_{3} & \ldots & \ldots & F_{\mathrm{k}-2} & F_{\mathrm{k}-1} & F_{\mathrm{k}} \\
G_{1} & P_{2} & 0 & \ldots & \ldots & 0 & 0 & 0 \\
0 & G_{2} & P_{3} & \ldots & \ldots & 0 & 0 & 0 \\
\ldots & \ldots & \ldots & \ldots & \ldots & \ldots & \ldots & \ldots \\
\ldots & \ldots & \ldots & \ldots & \ldots & \ldots & \ldots & \ldots \\
0 & 0 & 0 & \ldots & \ldots & P_{\mathrm{k}-2} & 0 & 0 \\
0 & 0 & 0 & \ldots & \ldots & G_{\mathrm{k}-2} & P_{\mathrm{k}-1} & 0 \\
0 & 0 & 0 & \ldots & \ldots & 0 & G_{\mathrm{k}-1} & P_{\mathrm{k}}
\end{array}\right)\left(\begin{array}{c}
n_{1} \\
n_{2} \\
\ldots \\
n_{\mathrm{k}-2} \\
n_{\mathrm{k}-1} \\
n_{\mathrm{k}}
\end{array}\right)(t)
$$

The matrix elements are $F_{\mathrm{c}}=$ propagule production (number of seeds per plant), $G_{\mathrm{c}}=$ recruitment to succeeding stage (proportion of plants undergoing transition) and $P_{\mathrm{c}}=$ persistence (proportion of plants undergoing survival without transition) within stage. The coefficients' values vary through time and between patches. $P_{\mathrm{c}}$ has a maximum level of 1 background mortality, $M_{\mathrm{c}}$. The vital rates of growth and survival are needed to specify $G_{\mathrm{c}}$ and $P_{\mathrm{c}}$, and seed production levels to specify $F_{\mathrm{c}}$.

\footnotetext{
${ }^{8}$ In reality, individuals may occasionally decrease in size, for example when a stem is broken but not killed. This complication is disregarded.
} 


\subsubsection{Variation between patches}

The coefficients of $\mathbf{A}$ for a patch $p$ depend upon the prevailing climate $\left(C_{\mathrm{p}}\right)$ and population density $\left(K_{\mathrm{cp}}\right)$. These factors together with the species' life history characteristics determine the availability of energy, water and minerals required for growth and reproduction ${ }^{9} C_{\mathrm{p}}$ influences vital rates directly, and determines the overall patch 'suitability' $\left(S_{\mathrm{p}}\right)$ for the species, which acts as a relative constraint on distribution. The species can populate patches where $S_{\mathrm{p}}$ is greater than

the minimum level, $S_{\min } . K_{\mathrm{cp}}$ acts as an absolute constraint on recruitment when $K_{\mathrm{cp}}=K_{\mathrm{cmax}}$, and is the only biotic factor external to the individual that is considered.

$C_{\mathrm{p}}$ is simulated using three bioclimate variables: the annual sum of daily $A E T$, annual sum of daily $M D$, and maximum annual $S M A$; plus lowest mean monthly temperature $\left(T_{\min }\right) . A E T$ expresses accessible energy and water, $M D$ expresses drought stress, indicating a lack of moisture to meet evapotranspirative demands (Stephenson 1998), and SMA represents the maximum duration of the stress (section 4.2.1.3). $T_{\min }$ represents resistance to cold temperatures, important for survival and germination (section 4.2.1.1). These are variables relevant to distributions on a geographic scale, rather than microhabitat or substrate variation at a finer scale.

\subsubsection{Survival}

Causes of mortality $(M)$ include senescence, herbivory, disease, and competitive exclusion, as well as the additional impacts of climate stress (Fig. 4:3, p. 122). Additionally, a high proportion of tree and shrub mortality results from localised disturbance events (Bernal 1998, Nicotra 1998). Although survival rate does respond to resource availability, this is expected to be the vital rate least affected by suboptimal conditions, as plants actively reduce rates of growth and reproduction to avoid death.

$M$ varies with species and size. Various $M$ functions allocating mortality between stages exist in the gap model literature (Liu \& Ashton 1995). Concave and sigmoid survivorship curves have been observed in Amazonian tree species (Korning \& Balsev 1994). Smaller plants have a higher mortality rate because they are less able to compete for resources, because of their greater shading, smaller photosynthetic area, shallower root system and less developed defences against herbivory. They can also be subject to biotic mortality causes, which vary across a patch with population density (section 2.2.3.6). This model assumes an even distribution of

\footnotetext{
${ }^{9}$ Mineral availability is not explicitly considered because of the heterogeneous nature of soil type within a patch at this scale. However, soil type does contribute to the estimation of the bioclimate variables through soil water holding capacity.
} 
individuals through the patch, and ignores this spatial variation in $M$. Mature plants can have a higher mortality rate because of increased risks of tree-fall, and also senescence.

\subsubsection{Germination}

$\mathrm{G}_{\mathrm{cs}}$, the germination rate, follows its climate response surface unless recorded germination triggers such as seasonal drought or moisture are absent. Seed survival is treated as for other classes.

\subsubsection{Growth}

Successful growth and reproduction both require biomass accumulation, which depends upon resource levels. Reduced growth is accompanied by reduced initiation of reproductive organs, directly lowering reproductive fitness. Rates of reproduction and growth tend to be correlated in individual plants (e.g. Siparuna grandiflora, Nicotra 1999), unless phenological constraints prevent reproduction. However, there is no generally applicable theory relating the partitioning of biomass between growth and reproduction to resource availability.

$G_{\mathrm{c}}$, representing recruitment to $c$, depends upon $K_{\mathrm{cmax}}$ and $K_{\mathrm{cp}}$ of $c$, and upon the size of individuals in cohorts of the donating stage (c - 1). Survivors from a cohort move to the subsequent stage if:

1. The cohort has gained sufficient biomass: i.e. years * (growth rate/difference between size classes $) \geq 1$

2. The recipient size stage is below maximum density, $K_{\text {cmax }}$.

\subsubsection{Reproduction}

The matrix coefficient $F_{\mathrm{cp}}$ represents the mean number of seeds produced per pollinated individual. For a patch to be suitable for reproduction, any climate triggers (e.g. for flowering, pollen tube fertilisation or mutualist activity) must occur that year. The level of reproductive success for a cohort is then dependent upon the climate variables as specified in the $F_{\mathrm{c}}$ response surface, and on the probability of pollination.

Pollen availability depends on plant mating strategies and sex ratios. For most dioecious species, sex ratios are equal (Bernal 1998). For a non-selfing female flower, pollen availability is estimated from the pollination distance (dependent on mechanism) and the density of mature individuals with male flowers (Eqn. 18). 
$\mathrm{P}($ fert $)=1-\left(1-\left(\left(\pi *((\text { pdist }) / 1000)^{2} / \text { cell area }\left(\mathrm{km}^{2}\right)\right)^{(\text {Lmature } \mathrm{c}(\mathrm{ncp} * \mathrm{P}(\text { reprodv }) \mathrm{c})) / \mathrm{P}\left({ }^{\circ}\right)+1}\right)\right.$

where $\mathrm{P}(\mathrm{fert})=$ probability of fertilisation, $n=$ number of individuals, pdist $=$ pollination distance (metres), $c=$ stage, $p=$ patch, $\mathrm{P}(+)=$ probability that potential mate has only female flowers. This is the probability at least one individual with male flowers falls within a circle of radius pdist around the female flower, were the male individuals randomly distributed within the grid cell. Inter-patch pollination, variation in pollen production, and special circumstances for females within pdist of the grid cell edge are disregarded.

\subsubsection{Modelling perturbed populations}

The perturbation model is a natural extension of the equilibrium model. The equilibrium distribution is perturbed by a gradual change and an increase in interannual variation in the bioclimate variables.

\subsubsection{Climate change}

Expected climate changes in the Neotropics involve increases in the duration of the dry season, in temperature and in frequency of droughts (Mata \& Campos 2001). Climate models suggest that precipitation will increase in some areas and decrease in others. AET, $M D$ and $T_{\min }$ would be affected by these changes. SMA would be affected by changing $M D$ and by increased daily variation in PET and AET.

The local substrate, which is assumed to be fixed, influences the impact of change on $M D$ through soil water holding capacity. Decreased precipitation will most affect populations occupying soils that are the most freely draining. Changes in soil nutrient levels could influence $S_{\mathrm{p}}$, but as there is no consensus on the likely direction of change, this factor is disregarded.

The models also indicate that climate variability over diurnal, monthly, annual and interannual periods will increase, with an associated greater frequency of climate extremes. This change will be reflected in the bioclimate variables controlling potential distribution and vital rates. Climate change may involve increasing frequencies of extreme events, especially drought and fire, in addition to the ongoing shift in annual values. Ongoing change in annual values will produce chronic drought beyond some species' tolerances. Otherwise, these extremes are disregarded, rendering the species model more optimistic than the projected reality. 


\subsubsection{Impacts on population processes}

Climate change alters the vital rates in affected patches according to species' climate tolerances, changing population size, structure and eventually distribution. Patches may leave or enter the habitable niche, creating new areas of potential distribution or changing population status from viable populations to non-viable populations (relicts).

A non-viable population on an extinction trajectory may survive for a long time in this model. Lags in increase will initially be less marked than lags in decrease, as a population at equilibrium should have a supply of fully-grown individuals awaiting recruitment to each successive stage.

Different physiological or physical traits may be employed by different species following the same broad ecological strategy. Recently diverged species are more likely to share strategies, and thus have closer responses than distantly related species.

\subsection{Survival responses}

A species' life history strategy affects the rate at which environmental change affects a plant population. For example, annual plants mature and disperse rapidly, but can be lost from a patch during a single year of hostile conditions unless there is a bank of dormant seeds. Both the maturation time and reproductive lifespan are longer for trees, meaning that there is a greater inertia in tree density in either change direction.

Strategies for survival and reproduction are most relevant in the context of climate response modelling. The survival strategies may permit a wider tolerance to change, whilst the reproductive strategies may offer a shorter generation time or greater dispersal distance. Each external stress may be met with a passive strategy (survival via dormancy), an active strategy (survival via active mechanisms) or no resistance strategy. Passive strategies often involve propagule survival, whilst active strategies involve survival of the mature plant.

\subsection{Reproductive responses}

Strategies for reproduction differ in terms of requirements, output and dispersal capability. For example, a species may employ wide dispersal of a large number of seeds, or selective placement of and greater investment in a few seeds. If a species' potential distribution is shifting, the former strategy is more likely to allow seeds to spread to new patches. 
Populations at low density may also experience limited opportunities for sexual reproduction. The ability to self-fertilise is therefore useful in maintaining decreasing populations, or initiating new ones. Dispersal between patches is beyond the scope of this model.

\subsection{Changes in potential distribution}

The potential distribution changes in response to movement of the patch on its climate surface. Thus, existing populations may now occur in newly suitable sites. Changes in realised distribution are limited by lags in population loss. Established plants may tolerate sub-optimal conditions, but the equilibrium assumption states that populations cannot persist indefinitely where $S_{\mathrm{p}}<S_{\min }$.

Increases in $T_{\min }$ alone would expand the potential distribution of equatorial species with metabolic or reproductive low-temperature limits towards the poles and areas of higher altitude. Mesophytic species may still be limited by $M D$.

\subsection{Changing absolute constraints}

Where patches do not fulfil an absolute constraint, the constrained process is prevented altogether by reducing the matrix coefficient to zero.

Germination constraints evolved to reduce the likelihood of seed death under the conditions experienced by that species: for example, seasonal forest plants frequently germinate at the onset of the wet season. The absence of such triggers prevents colonisation of an otherwisesuitable patch. In addition, to conform to the equilibrium assumptions of eventual extinction under unsuitable conditions, germination is only permitted where $S_{\mathrm{p}}>S_{\min }$.

Some species have phenological constraints on flowering and seed setting. These triggers restrict the reproductive period to a season offering sufficient resources. When the constraints are absent, reproduction is prevented $(F=0)$.

When population density becomes so low that pollination is prevented, sexual reproduction ceases (Eqn. 18). Species able to employ clonal (vegetative) reproduction continue to do so. The arbitrary assumption is taken that a single juvenile is produced by each mature individual, creating a new seedling cohort at replacement levels. Vital rates are applied to this cohort as normal. When the cohort has either attained the sapling stage or succumbed to mortality, another cohort is created in the same manner. 


\subsubsection{Hypotheses}

This methodology simulates the impact of possible future climate change on Amazonian plant species. The Methods section (5.2) details the selection of a set of species to represent biodiversity, using plant taxonomy and functional traits, and a model constructed to simulate change in Amazonia. Hypotheses concerning absolute and comparative impacts on different species and locations can be investigated from the outcomes of the model.

These hypotheses have been formulated to fulfil the thesis aims, in particular to provide a preliminary risk assessment for the individual species and functional groups, and identify areas likely to undergo change in species composition or retain their original species complements. Maps of viable, non-viable and potential distributions are created to help assess levels of species threat, and to identify important areas for monitoring and conservation.

In testing the hypotheses below, relative population density change is used to represent change response. A measure of change that is independent of the original population size is important, because this varies both between species and sites. To obtain a range of responses for each species, a standard (SIS) and reduced impact simulation (RIS) of climate change was applied. In each case, an extra 100 year equilibration period under the 2095 climate was modelled, to identify long-term impacts of the changed climate. The hypotheses are tested separately for each scenario at 2095 and 2195.

\subsubsection{Spatial impacts on individual species}

H1)Individual species' realised distributions will alter under the climate change scenarios. As dispersal is not modelled, this implies that population extinctions will occur.

$\mathrm{H} 2)$ Present day realised and final potential distributions do not overlap one another for some species, so that these will eventually be lost from their entire current range. This hypothesis identifies species under threat of extinction from the Amazon region in the long-term.

\subsubsection{Spatial impacts on the set of species}

H3)There is a significant difference in species composition for many cells between 1990 and 2095 or 2195, and this difference varies consistently over the Amazon region. This is expected because a varying intensity of climate change will be applied to cells with varying initial climates hosting species with different environmental niches. When applied on a cellby-cell basis, this hypothesis identifies cells most and least likely to change.

$\mathrm{H} 4)$ The location of the set of 'best' cells, representing the core of each species' realised Amazonian distribution, will change between 1990 and 2095. The 'best' cells are defined as the set of single cells in which each species is modelled as being most successful. This 
hypothesis is based on the concepts that (a) areas that are presently on the spatial margin of the range are not likely to be optimal under present conditions and (b) as the range shifts with climate change, the 'best' cell may shift towards the current spatial margin. This tests the general prediction that climatic change will cause shifts in species ranges towards the poles and areas of higher altitude (Peters and Darling 1985, Halpin 1997, Still et al. 1999).

\subsubsection{Variation between species}

H5)The functional traits used in species selection are correlated with change response. For example, species with a smaller pollination distance will undergo a greater decline than species with a longer distance (through reduced reproduction in low-density populations). Investigating this hypothesis will help to identify any functional groups most in danger from climate change.

H6)There is a significant difference in change response for species belonging to different families. This may follow from shared climate tolerances, vital rates or distributions.

$\mathrm{H} 7)$ Species' present range size is correlated with change response. That is, population declines are more likely in species with narrow ranges than broad ranges. This is expected because spatial autocorrelation in environmental variables and the smaller number of sites occupied mean that these species are more likely to have a narrow climate niche. The small spatial range also means that the range of new climates encountered will be smaller than for a widespread species. Unless this happens to be similar to that occurring under a new climate, these species are more likely to be in danger of extinction across their entire range.

H8)The variation in each bioclimate variable in occupied cells is correlated with change response. For example, narrow mesophytes with a low variation in tolerated $M D$ will undergo a greater decline than species with a high variation in tolerated $M D$. 


\subsection{METHODS: APPLICATION TO AMAZONIA}

\subsubsection{Scope}

This study is set in Amazonia, a large lowland area within the humid tropics with a relatively limited range of temperature, mean annual rainfall and seasonality (New et al. 1999). The large size and continental position of the study area help to reduce the influence of edge effects and island biogeography on its species' distributions. It contains a large number of species in comparison to temperate regions. Some are more-or-less ubiquitous; others are tightly clustered with limited distributions; others have disjunct distributions comprising multiple clusters. A large spatial range does not necessarily imply a large number of individuals, as population density may be low.

This study considers the impact of climate change on species' populations, over a $1^{\circ}$ latitudelongitude scale, for the period 1990 - 2095 and for a hypothetical post change equilibrium period of 100 years.

\subsubsection{Summary}

The modelling framework adopted here is an adaptation of the methodology to accommodate this project's resource constraints. The process is as follows:

1) Reference and changing bioclimate variables are modelled on a $1^{\circ}$ latitude-longitude grid, as an offline model dependent on GCM outputs (section 5.2.3).

2) A set of species is selected to represent Amazonian angiosperm biodiversity (sections 5.2.55.2.7).

3) For each species:

a) The current distribution of each species is estimated (section 5.2.8.1).

b) A size-structured population is initiated in each $1^{\circ}$ cell in the distribution, and the processes of germination, survival, growth, recruitment and reproduction are simulated for that population for 100 years under a stable baseline climate. This provides the 1990 population set (sections 5.2.8.2 to 5.2.8.8).

c) These populations are subjected to annual climate change from 1991 to 2095 (section 5.2.8.9).

d) The populations are allowed to equilibrate under 2095 conditions for a further 100 years (section 5.2.8.10).

e) Steps 2 and 3 are repeated for a reduced-impact change scenario, 'RIS' (section 5.2.9).

The distribution model (3a) constrained each species to its known environmental niche. It was assumed that each species was adapted to a set of optimal conditions, around which there is a 
band of tolerance within which vital rates are high enough to maintain a viable population. These optimal conditions are distinct from the theoretical optima for mechanisms such as photosynthesis or nutrient transport, because each species has invested in adaptations allowing it to exploit a particular environment.

Current distributions were modelled using a suitability index $\left(S_{\mathrm{p}}\right)$ based on bioclimate variables (AET, MD and SMA). The distributions were additionally limited by estimated absolute tolerances to extreme values and by dispersal barriers. Species were classified into plant functional types (PFTs), which share traits expected to influence their response to climate change. A species' response to change derives from the set of traits possessed, various speciesand family-specific life history parameters, and its initial distribution.

A size-structured population was simulated (3b) for each occupied grid cell, to enable modelling of lags in the response to climate change. Population processes were dependent upon PFT- and life-stage specific parameters. The species' own parameters were used where known. Maximum population density in each cell was simulated using the most limiting factor theory with a Gaussian distribution (ter Braak \& Prentice 1988, Hackett \& Vanclay 1998).

Each population's change response (3c) depends upon the impact on the 'vital rates' of survivorship, growth and reproduction. The best way to model this response would be to simulate the relationship between each vital rate and combination of climate variables, under changing or stable conditions. If there are no reliable data on which to base these relationships, a minimal response model may be formulated by limiting recruitment according to estimated equilibrium population density for conditions in each model year. This approach was taken here.

Population processes were simulated from 1991 to 2095, with changes in the bioclimate variables being applied annually according to anomalies from a GCM run. The level of the most limiting factor (MLF) determined abundance under equilibrium conditions, with a time lag mediated by the interaction between mortality and recruitment rates. A further 100-year run was then undertaken (3d) to assess surviving populations' long-term viability under 2095 conditions.

The range of potential outcomes for each modelled species and PFT was evaluated. The most vulnerable taxonomic groups, PFTs and geographical regions were identified as targets for monitoring and conservation action. Areas estimated to be important for persistence of the most threatened species and PFTs were identified. Those with little distributional data were highlighted for future survey work. 


\subsubsection{Assumptions}

For simplicity's sake, it is assumed that:

1) Each species behaves independently of all others and can be represented as a set of populations, each occupying a single spatial patch. This implies that the species' mutualists share habitat requirements and dispersal abilities, and that its success depends upon resource availability rather than species composition. Although an ideal framework would consider interspecific interactions, the relationships between tropical species are largely undocumented (section 2.2.3.6), and the chosen scale is inappropriate (co-occurring species may occupy very different niches at a finer scale).

2) Species' current distributions are determined by their distributional history and the spatial boundaries of their environmental niche (section 2.2.2.2.2). Thus, the potential distribution includes all patches with environmental conditions in which the species can survive and reproduce, but the dispersal history since speciation may prevent some patches from being occupied today.

3) Species are currently in equilibrium with their environment: all suitable patches within a zone surrounded by dispersal barriers are occupied if the species has been able to colonise the zone in the past. The relatively stable climate and geology supports the contention that present populations are viable, rather than being relicts from a previous climate (sections 2.1.2, 4.3). This may be a poor assumption for some rare populations, which may be in decline (section 2.2.4.2).

4) All populations of a species have the same environmental tolerances and optima - ecotypes resulting from genetic heterogeneity are ignored.

5) Population processes can be simulated at a $1^{\circ}$ spatial scale without obscuring any large-area impacts of finer-scale ecological patterns and events (section 4.3.2).

6) Species occupying a similar environmental and functional niche will have a similar response to change. Closely related species will have a more similar response than distantly related species (section 4.1.2).

7) The maximum density of an area is directly related to the species' probability of occurrence.

8) Interaction between populations of a species may be disregarded at this scale.

9) Individuals are distributed evenly within the patch. This assumption is made to allow calculation of pollination distances.

10) Species have recovered from or been unaffected by human influences - the distribution models do not consider habitat alterations.

11) Evolutionary change operates too slowly to alter species' traits over the time-scale and under the rate of environmental change considered (section 4.2.7).

12) The projected changes from downscaled GCMs provide a reasonable simulation of possible sub-regional climates under doubled $\mathrm{CO}_{2}$ conditions (section 3.7). Sea level rise will not reduce the available land area. 
13) There are no net effects on species from elevated levels of atmospheric chemicals, including $\mathrm{CO}_{2}$, or reduced stratospheric $\mathrm{O}_{3}$ (this assumption is taken because potential responses of individual species are poorly understood, section 4.2.4).

14) It is assumed that the direct effects of temperature on photosynthetic rate are minimal (Scholes \& van Breemen 1997), so that temperature only affects growth through its contribution to the variables used to simulate the model climate.

\subsubsection{Climatology}

\subsubsection{GCM and scenario selection}

It was decided to use the IS92a 'business as usual' scenario, the standard scenario for low levels of international action on climate change. This provided a 'worst case' GCM output for the standard impact scenario (SIS), which was then modified to produce a reduced impact scenario (RIS, section 5.2.9). The 'GSa' variant was chosen to include the effects of sulphate forcing, which mitigates GHG impacts (Mitchell et al. 1995).

The HADCM2 GCM was chosen to provide a model of climate change because it:

1) has a comparatively good representation of current global and Amazon climate

2) falls within the standard model range of global climate sensitivity (Kacholia \& Reck 1997)

3) has precipitation and temperature responses in the Amazon region are well within the range seen in other GCMs (Hulme et al. 1999)

4) has a high spatial resolution in comparison to other GCMs (Hulme \& Viner 1995).

The latest Hadley Centre CGCM, HADCM3, shares these properties but has much more extreme results over the Amazon (section 3.7.3.2). HADCM2 is more compatible with the species model used here, because it does not include a physiological response to change. Under HADCM3, species with a constant relationship with their environment would be modelled as having to endure increased temperatures (and therefore moisture deficit) due to decreased AET, but would not benefit from the increased water use efficiency seen in the GCM vegetation.

The level of uncertainty associated with the variation between ensemble members in regional projections from HADCM2 simulations is low, less than the variation between different scenarios for the same GCM, or different GCMs for the same scenario (Giorgi \& Francisco 2000). Thus, one model run does not have extreme results compared to the others. Outputs from a single run, HADCM2GSa1, rather than an ensemble mean were used because mean outputs can lack internal consistency - spatial patterns of change are obscured. 


\subsubsection{Selection of variables}

The climate variables used to model distributional response to climate were selected to represent the factors that limit species distributions. Bioclimate variables, often based on actual and potential evapotranspiration (AET and PET), combine the effects of temperature and precipitation. This incorporates some interaction between the recorded variables to express the 'effective environment' (Mackey 1996) experienced by plants. PET represents energy input, or the atmospheric demand for water over a saturated surface. AET represents the water returned to the atmosphere, constrained by moisture availability and maximum rates of ET. The difference between PET and AET represents the degree of water stress, or moisture deficit (MD). The combination of AET (measuring biologically available energy and water) and MD (measuring drought stress) is well supported for use in vegetation and species modelling (Shao \& Halpin 1995, Prentice et al. 1992, Stephenson 1998). In the Amazon region, a third strong gradient known to affect vegetation types is seasonality of precipitation. This is measured here as seasonal moisture availability (SMA), the maximum count of consecutive days under which AET $<$ PET.

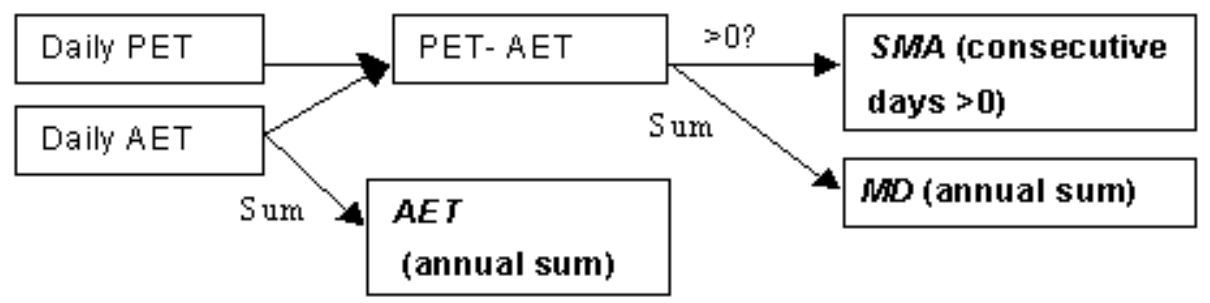

Fig. 5:2: Relationship between variables used to derive cell suitability

These variables are employed here as annual measures. SMA is annual by definition, and AET and $M D$ are annual sums. Each is based on daily AET and PET (Fig. 5:2), which should estimate ET more accurately than using monthly variables. Few GCMs calculate PET, but AET is a common output (Table 3:5). Here, AET and PET were calculated on the same principles. Downscaling GCM AET would have risked inconsistencies such as AET > PET.

The derivation of daily AET and PET is a multistep process. Rainfall is distributed sequentially between different stores and lost through transpiration, canopy evaporation and soil drainage. A large number of input variables and parameters are required (Appendix $1 \& 2$ ). Those derived from global digital maps were aggregated to a $1^{\circ}$ scale where necessary.

\subsubsection{GCM climate data}

Decadal mean values for monthly climate change variables were sourced from run HADCM2GSa1 of the HADCM2 model, following the IS92a scenario of 1\% of 1990 'equivalent $\mathrm{CO}_{2}{ }^{\prime}$ increase per year from 1990-2100 plus negative sulphate forcing (see Table 3:1, p. 38). 
Outputs of precipitation, $10 \mathrm{~m}$ wind speed (NS and EW components), $1.5 \mathrm{~m}$ temperature (mean $\&$ range), net radiation (shortwave \& longwave), $1.5 \mathrm{~m}$ relative humidity, AET (soil \& canopy) and surface pressure for the Amazon region were supplied by the Hadley Centre. $65 \times 2.5^{\circ}$ latitude $\mathrm{x} 3.75^{\circ}$ longitude cells, with a longitudinal grid spacing of $417 \mathrm{~km}$ at the equator, cover the 'inclusive-Amazon' (section 1.4.2). To evaluate spatial variation in climate change, the Amazon region was divided into three longitudinal bands, each three GCM cells wide. Decadal mean annual temperature and precipitation were plotted from the 1960s to 2090s (Fig. 5:3). The western section of the Amazon was overlapped by 20 GCM cells, the eastern section by 19 cells and the central section by 32 cells.

(a)
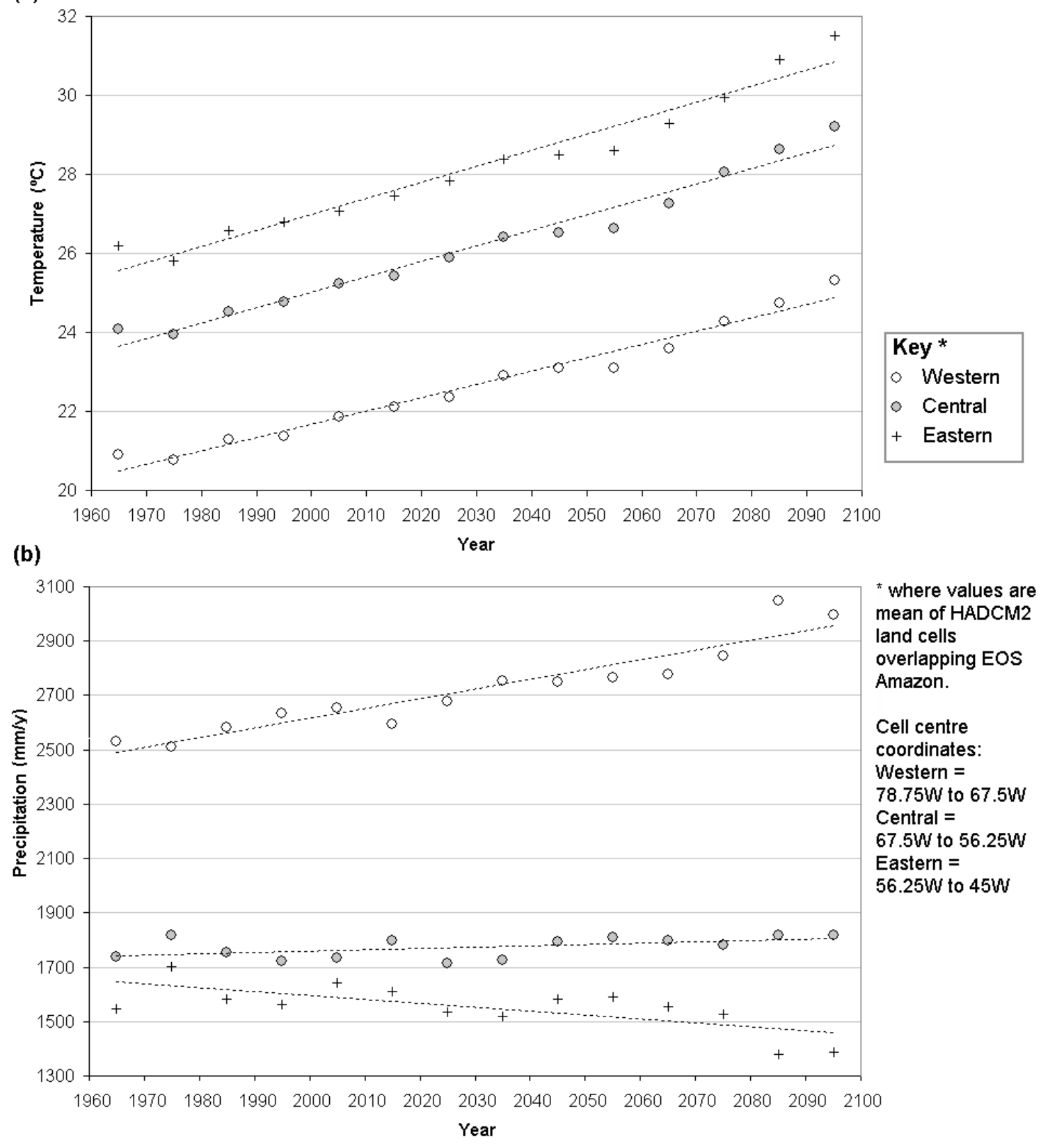

Fig. 5:3: GCM trajectories in (a) temperature and (b) precipitation for Amazon region from HADCM2GSa1, showing mean values for three groups of cells segregated by longitude 
The existing longitudinal gradients in temperature persist with climate change, with all three regions experiencing a mean increase (Fig. 5:3a). The longitudinal gradient in precipitation becomes stronger with change, with mean precipitation increasing dramatically in the west, remaining relatively constant in the centre, and decreasing in the east (Fig. 5:3b). When these data are downscaled, one would expect an increasing longitudinal gradient in moisture deficit to be seen, following the greater eastern increase in temperature and decrease in precipitation.

\subsubsection{Present climate data}

Several datasets for temperature, precipitation, albedo and radiation are available at a global scale (Legates \& Wilmott 1990a, b, Leemans \& Cramer 1991, New et al. 1999). The 1961-1990 Hadley Centre $0.5^{\circ}$ climate datasets (New et al. 1999) were selected because of the following advantages:

1) Availability of mean maximum and minimum monthly temperatures (not available in Leemans \& Cramer (1991) or Legates \& Wilmott (1990 a, b), and required for calculation of radiation variables)

2) Availability of temperature, precipitation, cloud and vapour pressure variables, leaving only the land-surface parameters to be sourced from alternate datasets. This reduces the variability in the criteria and methods used in selecting and interpolating the climate data.

3) The 1961-1990 period is the standard WMO normal period for climate model comparisons (Hulme et al. 1999)

4) Acceptance by the global climate modelling community: this is the set of observed variables distributed by the IPCC website, and the 1901-1961 annual version is used to validate many GCM experiments (Viner, pers. comm.).

A potential disadvantage is the warming trend, starting in the 1960s, observed in some Amazonian data (Victoria et al. 1998). The reference dataset is intended to represent an equilibrium climate for the species. If they are beginning to respond to change, or if the climate is different from that at the time they colonised a site, there will be a small initial anomaly if the climate is already warming. An alternative would be to initiate the model earlier, using only climate and distribution records dating from before the start of change. However, the improvement through the latter half of the $20^{\text {th }}$ century in species distribution and climate records negates this option.

The land-surface parameters required for calculation of PET and AET are sourced from the same vegetation and soils data (Wilson \& Hendersen-Sellers, 1985) and in the same manner (Buckley \& Warrilow 1988) as that used in the Hadley Centre GCMs, except for potential interception of precipitation (section 5.2.4.5.1). 


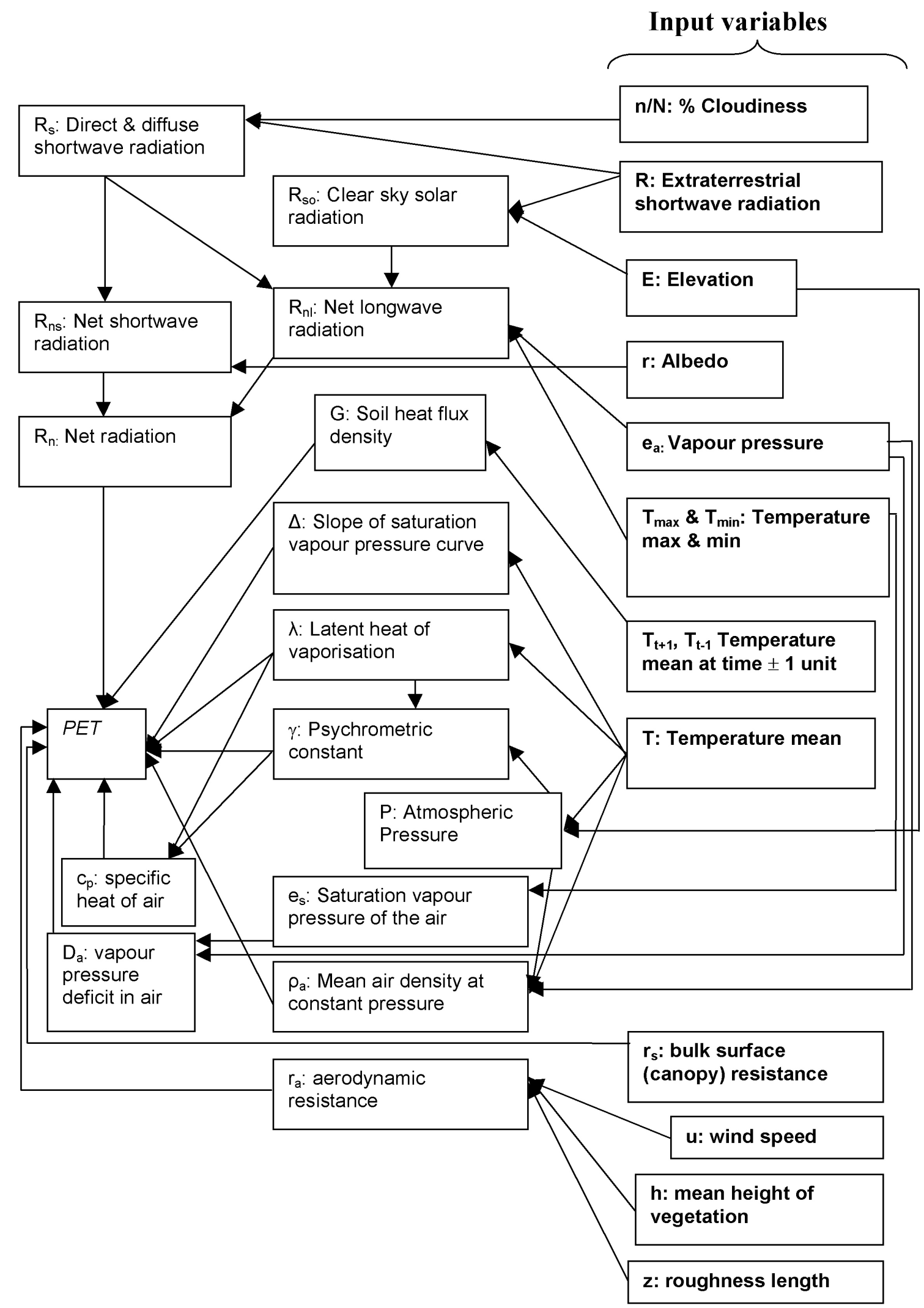

Fig. 5:4: Data flow for daily Penman-Monteith PET 


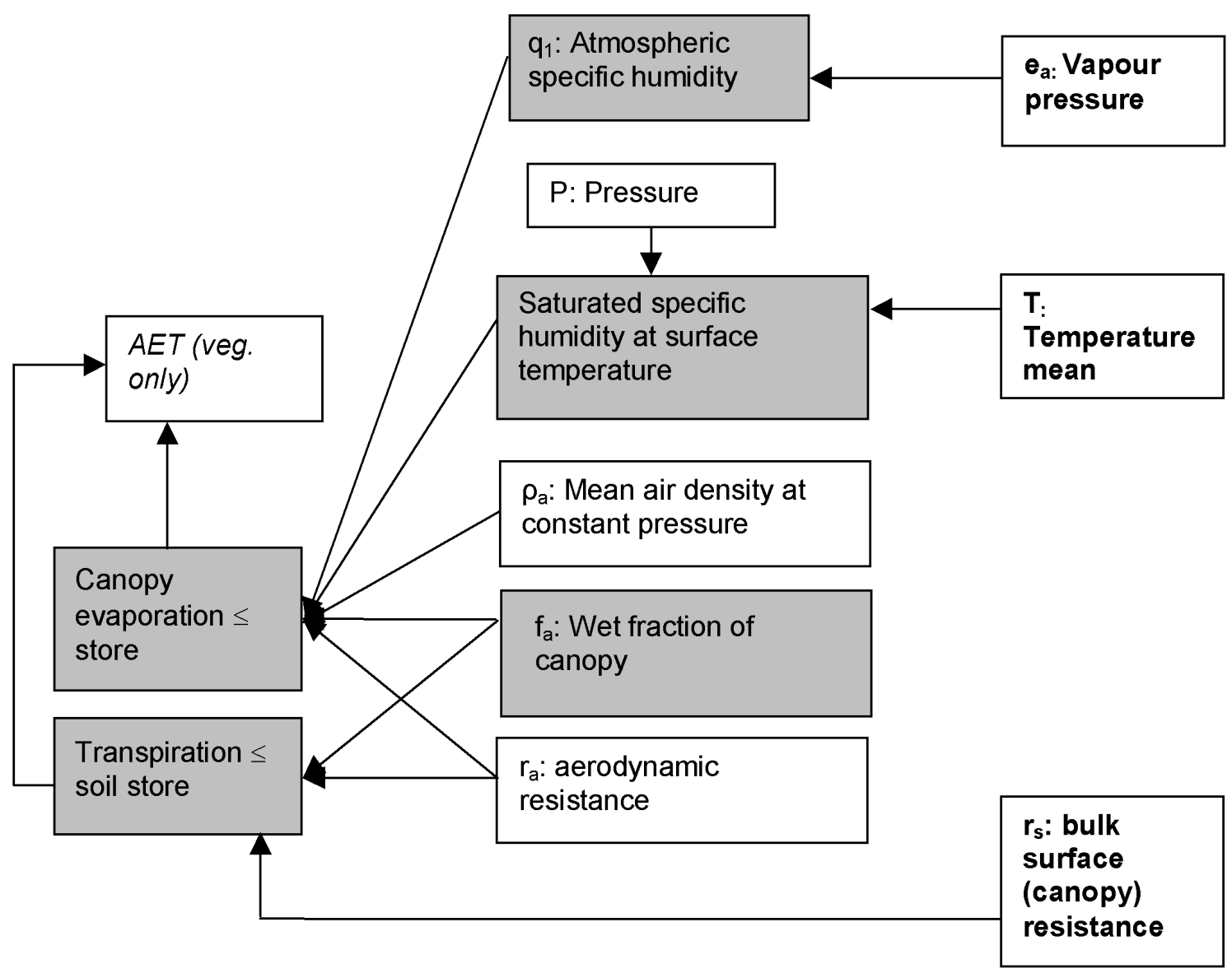

Fig. 5:5: Data flow for daily AET

\subsubsection{Model of present climate}

PET, AET, canopy and soil moisture stores are calculated on a daily basis. Annual AET, MD and SMA are calculated from daily AET and PET.

Of the time-variant inputs required, only daily radiation variables are calculated from first principles. Daily values for precipitation, temperature, vapour pressure, cloudiness and wind speed are estimated from the monthly input variables via linear interpolation. Where an interpolated value is volumetric (e.g. precipitation), interpolation of daily values $(d)$ between the midpoints of months will not conserve the observed monthly volume. Multiple simultaneous equations based on the values of the first and last days of the months have proved insoluble. Therefore Eqn. 19 is used: where $D=$ output daily value, $m=$ sum of interpolated daily values, and $M=$ observed monthly sum:

$$
D=d M / m
$$

For each day:

1) Daily PET is calculated from meteorological variables and surface parameters based on vegetation type (Eqn. 20, Monteith 1964); 


$$
\mathrm{PET}=\left(\frac{\Delta(\mathrm{Rn}-\mathrm{G})+\mathrm{K} \text { time } \rho a \mathrm{c} p \frac{\left(\mathrm{e}_{s}-\mathrm{e} a\right)}{\mathrm{r} a}}{\Delta+\gamma\left(1+\frac{\mathrm{r} s}{\mathrm{r} a}\right)}\right) / \lambda
$$

2) Precipitation $(P)$ is estimated from monthly precipitation (Eqn. 19);

3) Precipitation is supplied to the canopy moisture store $\left(\mathrm{C}_{S}\right)$; the remainder supplied to the soil moisture store $\left(\mathrm{S}_{\mathrm{S}}\right)$; the remainder is lost through surface runoff. Daily store size $=$ store moisture holding capacity + maximum ET capacity from that store;

4) Daily AET is calculated as canopy evaporation ( $E_{c}$, Eqn. 21) plus transpiration ( $E_{v}$, Eqn. 22); as limited by $\mathrm{C}_{\mathrm{S}}$ and $\mathrm{S}_{\mathrm{S}}$.

$$
\begin{aligned}
& \text { Canopy evap. }=\frac{\rho_{\mathrm{a}}}{\mathrm{r}_{\mathrm{a}}}\left(\mathrm{q}_{\mathrm{sat}} \mathrm{T}-\mathrm{q}_{1}\right) \text { where } \leq C_{S}(\text { Cox et al. 1999) } \\
& \text { Transpiration }=\frac{\rho_{\mathrm{a}}}{\mathrm{r}_{\mathrm{a}}+\mathrm{r}_{\mathrm{s}}}\left(\mathrm{q}_{\mathrm{sat}} \mathrm{T}-\mathrm{q}_{1}\right) \text { where } \leq S_{S}(\text { Cox et al. 1999) }
\end{aligned}
$$

where

$\alpha=$ constant value 1.26

$\mathrm{R}_{\mathrm{n}}=$ net radiation, $\mathrm{MJ} / \mathrm{m}^{2} \mathrm{~d}$

$\mathrm{G}=$ soil heat flux density, $\mathrm{MJ} / \mathrm{m}^{2} \mathrm{~d}$

$\Delta=$ slope of saturation vapour pressure curve, $\mathrm{kPa} /{ }^{\circ} \mathrm{C}$

$\lambda=$ latent heat of vaporisation $(\mathrm{MJ} / \mathrm{kg})$

$\gamma=$ psychometric constant $\mathrm{kPa} /{ }^{\circ} \mathrm{C}$

$\mathrm{K}_{\mathrm{time}}=$ units conversion, equal to $86,400 \mathrm{~s} / \mathrm{d}$ for $\mathrm{ET}$ in $\mathrm{mm} / \mathrm{d}$

$\rho_{\mathrm{a}}=$ mean air density at constant pressure, $\mathrm{kg} / \mathrm{m}^{3}$

$\mathrm{e}_{s}=$ saturation vapour pressure of the air, $\mathrm{kPa}$

$\mathrm{e}_{a}=$ actual vapour pressure of the air, $\mathrm{kPa}$

$\mathrm{c}_{p}=$ specific heat of the air, $1.013 \times 10^{-3} \mathrm{MJ} / \mathrm{kg}^{\circ} \mathrm{C}$ for moist air

$\mathrm{r}_{s}=$ bulk surface (canopy) resistance, $\mathrm{s} / \mathrm{m}$

$\mathrm{r}_{a}=$ aerodynamic resistance, $\mathrm{s} / \mathrm{m}$

$\mathrm{q}_{\mathrm{sat}} \mathrm{T}=$ saturated specific humidity at surface temperature $\mathrm{T}$

$\mathrm{q}_{1}=$ atmospheric humidity

The data flow for this process is shown in Fig. 5:4 and Fig. 5:5. Boxes with bold text represent input variables. The white boxes in Fig. 5:5 are derived as in Fig. 5:4. The variables and parameters are sourced as shown in Table A1:1 and A2:1. These also indicate which inputs or procedures are known to be identical to those used in HADCM2. 


\subsection{Derivation of land-surface parameters}

The source vegetation dataset has 53 global vegetation types (Wilson \& Henderson-Sellers 1985, 'WHS'). Buckley \& Warrilow (1988) assigned these to combinations of 24 sub-types, following WHS protocols. Each sub-type has its own values for root depth, albedo, surface resistance, roughness and canopy moisture storage capacity. This system has been used in all subsequent Hadley Centre GCM runs.

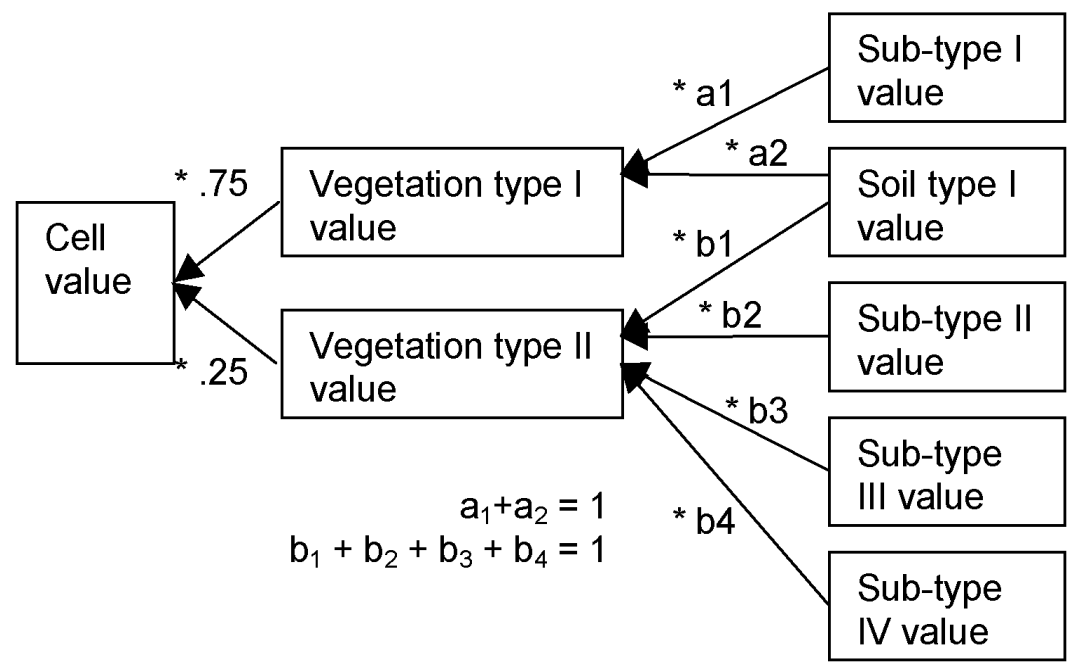

Fig. 5:6: Example of derivation of land-surface parameters

The subjectively assigned primary and secondary land-surface types offered in the WHS dataset were combined to give a single value for each degree cell, being arbritarily set to $75 \%$ of primary $+25 \%$ of secondary type (Fig. 5:6). To improve compatibility with HADCM2, this classification is employed here to designate surface parameters. These do not vary seasonally, but deciduous and evergreen forest types are distinguished.

In HADCM2, a saturated soil moisture concentration value is derived from the WHS soil texture dataset, and root depth is based on vegetation type. Together these give the soil field capacity, which can be used for a soil moisture bucket model. Many WHS cells for the inclusive-Amazon lack soil texture information; these cells were assigned 'medium' values following Warrilow \& Buckley (1988). The soil model occupies four layers in the HADCM2 land surface scheme, but for simplicity, only a single layer is simulated in the downscaled model.

Interception of precipitation was calculated using estimated percentage cover, rather than via an iterative calculation of canopy moisture storage as in HADCM2. LAI for each land cover class was calculated from the LAI of each of the 24 sub-types in the WHS dataset. No equivalent classification of percentage cover was readily available. Instead, a linear interpolation for cover 
between LAI for $0 \%$ cover ( 0 , barren land or open water) and $97 \%$ cover (8.55, equatorial rain forest) was carried out (Fig. 5:7).

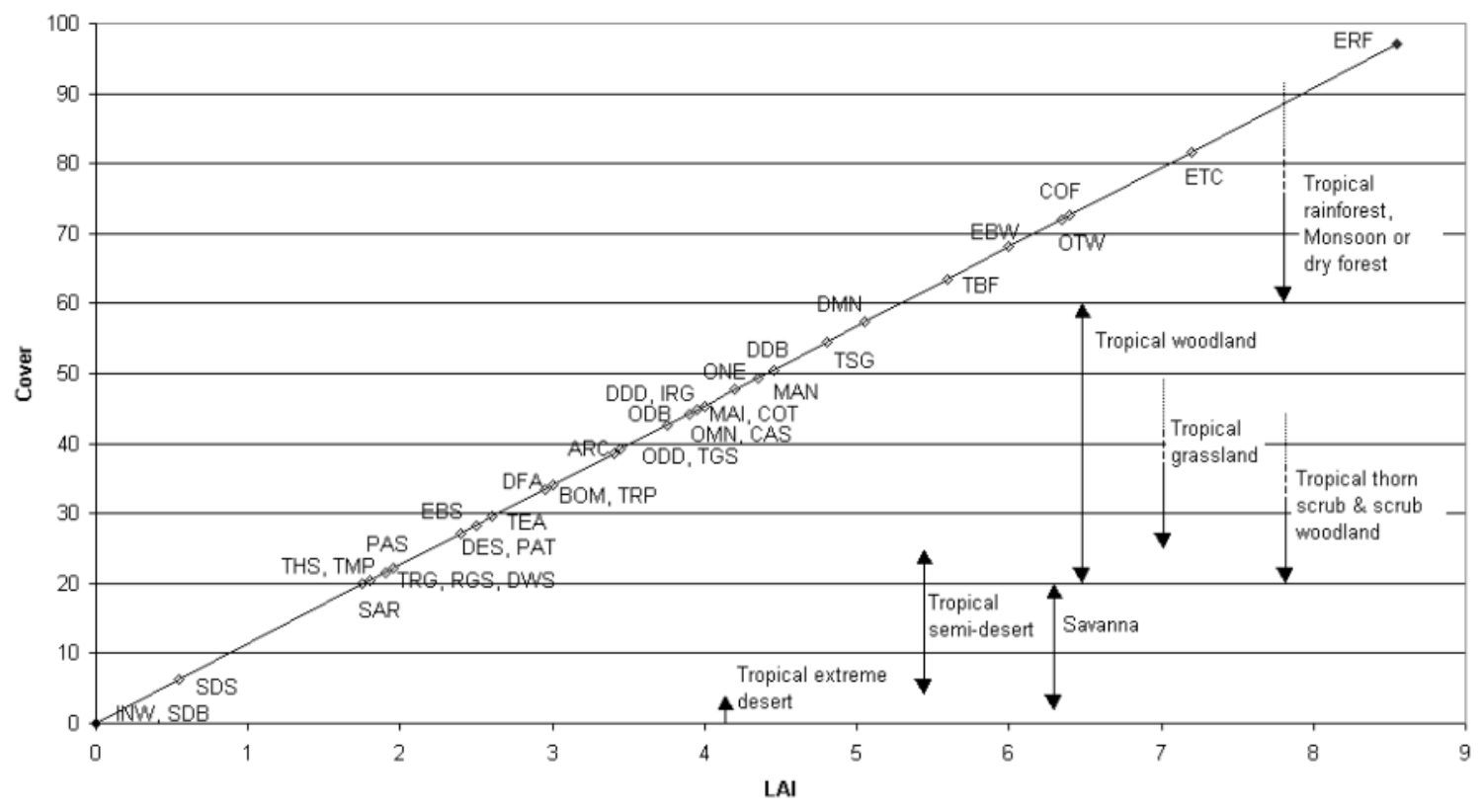

Fig. 5:7: Percentage cover for vegetation classes (coded as in Wilson \& Henderson-Sellers 1985) estimated from LAI, with QEN cover type

Although canopy structure is not considered in this interpolation, the values are largely consistent with the range of cover estimates (shown as arrows) for the vegetation classes developed by a Quaternary Environment Network workshop (Adams \& Faure 1997). An openended arrow represents an open-ended QEN range. Of the 38 classes, the only anomalies are for WHS 'TGS: Tropical Grassland and Shrub' ("39.14\%") and 'OTW: Open Tropical Woodland' ("72.04\%") which have cover values above the QEN ranges for 'Savanna' and 'Tropical Woodland' respectively. The interpolation is therefore deemed acceptable in the absence of better data.

\subsubsection{Simulated change}

As there is no climatic feedback from the species response in these simulations, the climate change simulation was run only once, to record changes in the annual values of $M D, A E T$ and $S M A$. Climate change was only simulated for the inclusive-Amazon region, to comply with the thesis mandate of investigating results of change on Amazonian rather than neotropical biodiversity. This change simulation is known as the DOWNS climate, because it used downscaled GCM variables.

Each decadal variable required for ET modelling was downscaled from the GCM output grid (Fig. 5:8) using an area-weighted projection of anomalies (section 3.6.1). The radiation, wind speed, water- and pressure-based variables must remain positive, and have a ratio scale. Under a 
climate change scenario, a percentage anomaly was added (relative to the original value of that variable at that site). Temperature in ${ }^{\circ} \mathrm{C}$ can be positive or negative, and has an interval scale. Hence, absolute anomaly values were added to temperature. For variables other than temperature, the magnitude of the applied change was therefore dependent on the reference bioclimate value.

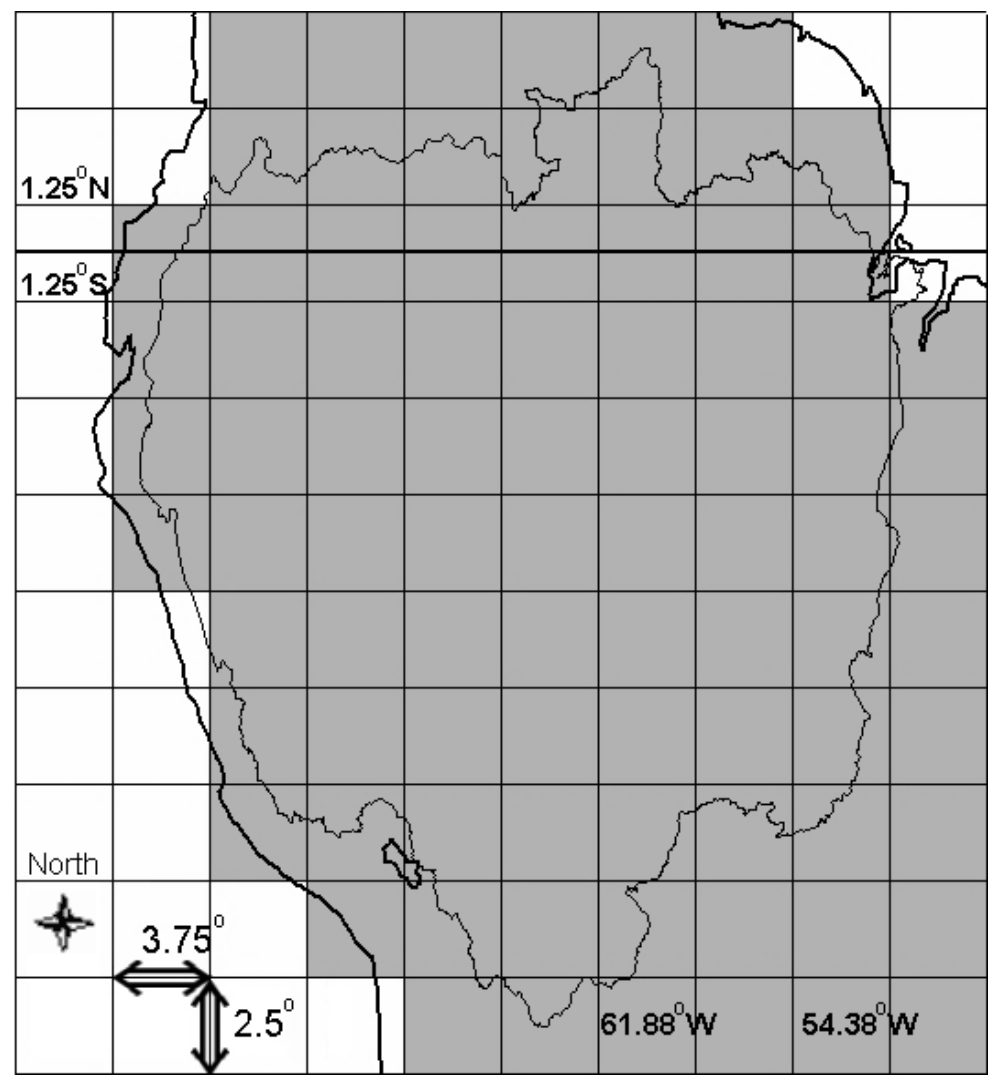

Fig. 5:8: HADCM land grid for Amazon region, with EOS-Amazon boundary

The present-day simulated values for precipitation and temperature are related to elevation through the New et al. (1999) climate data interpolation. Under the reference climate, cells of medium elevation can receive heightened levels of precipitation due to orographic rain, e.g. on the edge of the Andes, and the cells with the greatest elevation receive the least precipitation and lowest temperatures. As the precipitation anomaly was downscaled relative to the reference climate value, a smaller absolute change in precipitation was simulated in the most elevated cells. Any effect of elevation on the simulated temperature change originated in the GCM.

As an example, in the NE Amazonian cell with central co-ordinates $59.5^{\circ} \mathrm{W}, 2.5^{\circ} \mathrm{S}$, local precipitation and temperature both exceeded HADCM2 values throughout the model period. As the GCM mean decadal climate for the cell fluctuates, a different anomaly was applied in each year. The same percentage change had a greater effect on the local precipitation than it did on the smaller HADCM2 value (Fig. 5:9), whilst the temperature anomaly was applied directly (Fig. 5:10). 


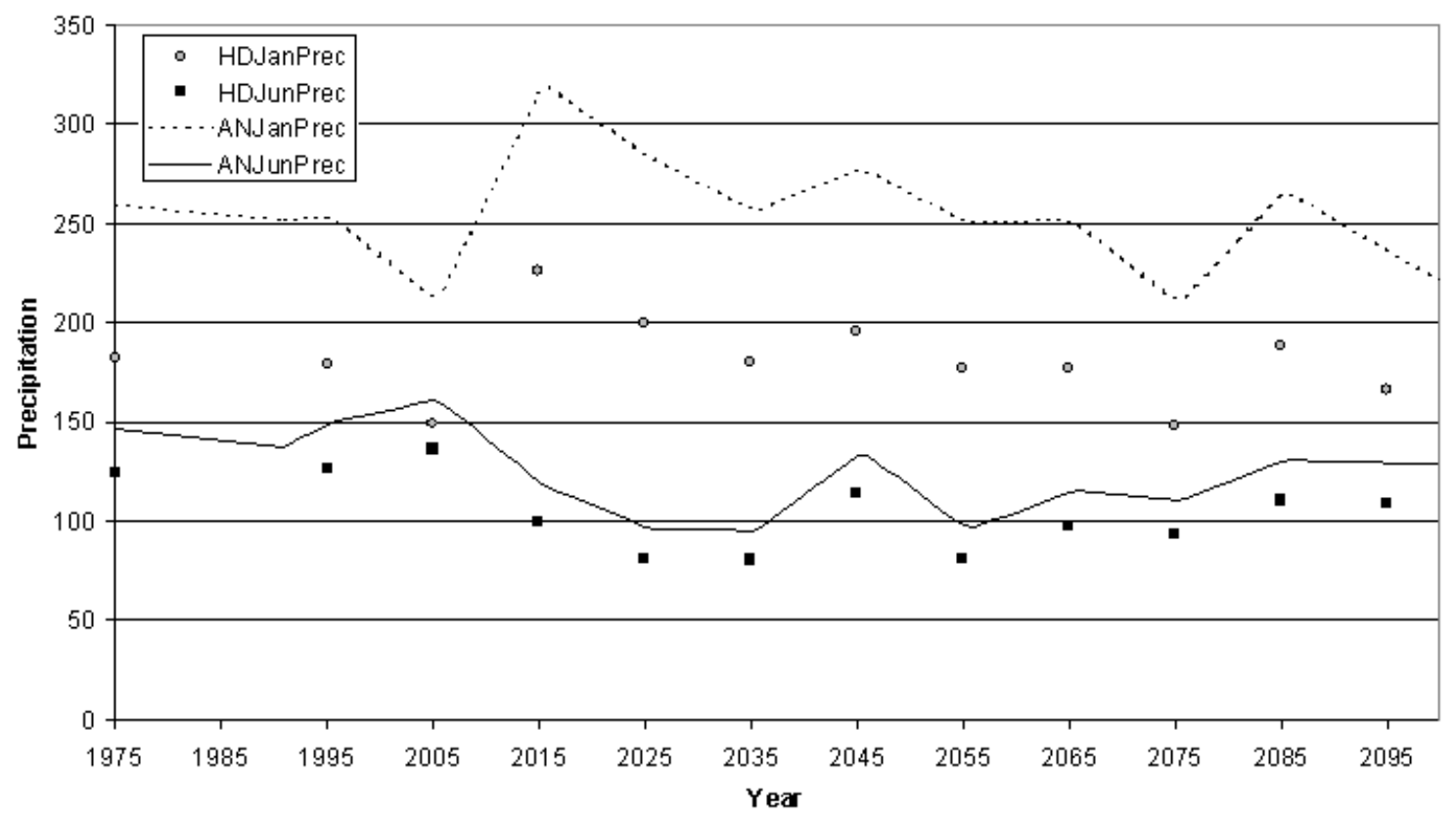

Fig. 5:9: Decadal HADCM2 (HD), and annual downscaled (AN), January \& June precipitation for cell SW002060

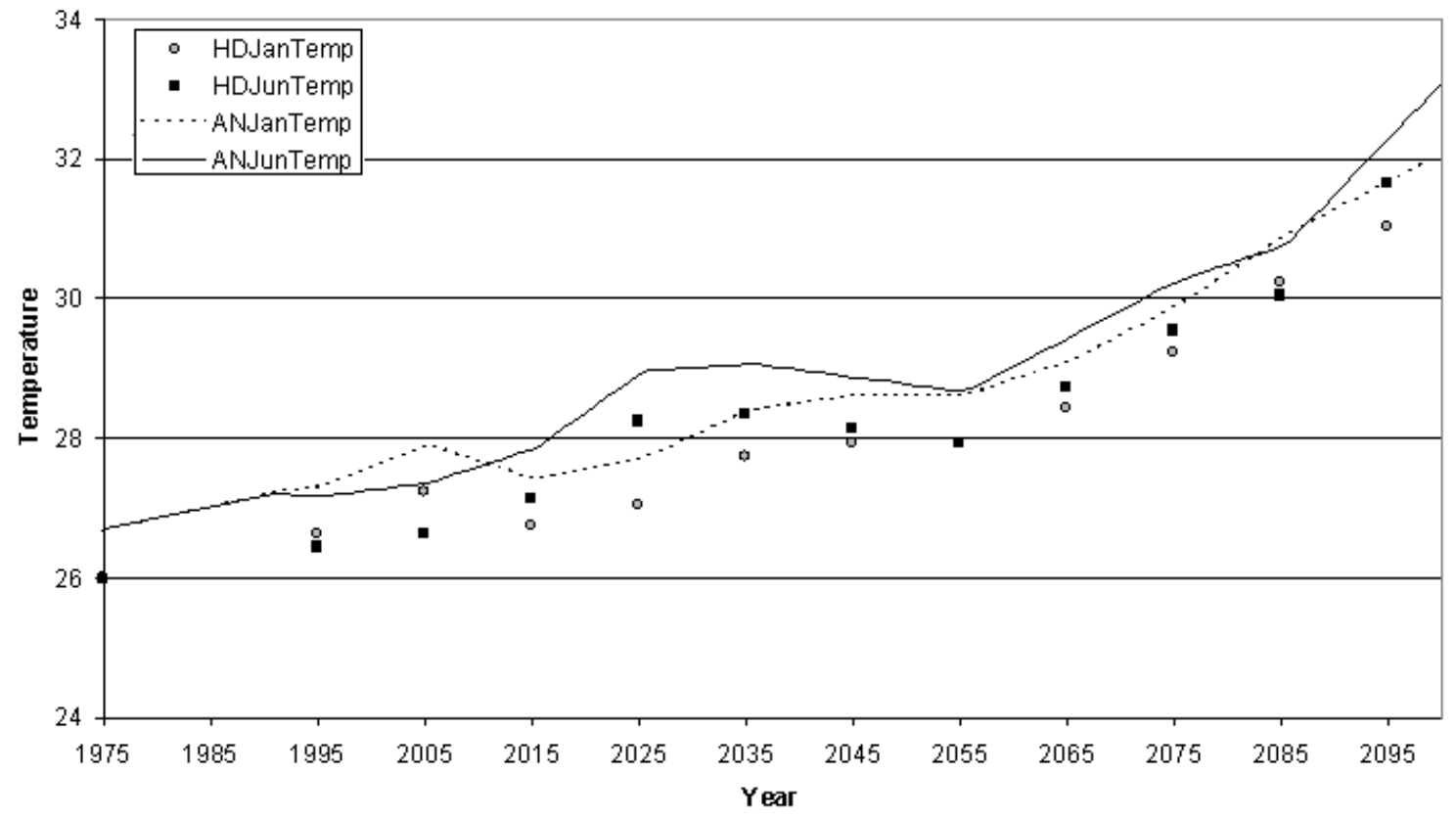

Fig. 5:10: Decadal HADCM2 (HD), and annual downscaled (AN), January \& June temperature for cell SW002060

Other parameters and variables remained constant. Principally, there was no modelled change in land surface parameters as might be expected from a change in vegetation distribution. This is consistent with the GCM simulation. It implies that any change in vegetation type will incur a lag as long as the model period, which would be unrealistic where there is extreme climate change. It could be justified up to a certain point with the concept that lag in mortality and migration of vegetation would delay large-area loss or spread of forests. 
The downscaled anomalies include long and shortwave radiation, simplifying the calculation of net radiation in the change scenario. Saturation vapour pressure $\left(\mathrm{e}_{s}\right)$ was calculated from the relative humidity (RH) anomaly as shown in Table A2:1. The HADCM2 transient run data were obtained as decadal mean monthly values from 1860 to 2100 . Annual change was simulated for each monthly variable through linear interpolation between decades. Anomalies were then calculated versus the control mean data for that scenario for the 1961-1990 mean (the period matching the baseline data, New et al. 1999).

\subsubsection{Neotropical and Amazonian reference bioclimates}

The species model and climate change simulation were carried out for the inclusive-Amazon only, whilst locational records for the entire extended Neotropics were required to define mean, extreme and suitable environments for each species. The bioclimate model was run until equilibrium for the extended Neotropics, using global environment data (especially New et al. 1999). This is the 'PRES' version of the reference climate.

The simulation of species' present populations was carried out using the mean bioclimate calculated using downscaled 1960s to 1980s GCM data, run annually from an initiation with PRES values. The intention was to avoid anomalies with the 1990-2090s bioclimate other than those expected from the GCM. This is the 'DOWNS' version of the reference climate.

The mixed-reference bioclimate for each species was sourced from DOWNS for inclusiveAmazon cells, and from PRES for extra-Amazonian cells.

\subsubsection{Family selection}

The family selection is designed to represent functional and phylogenetic diversity, and ensure that groups endemic to the region are included. Phylogenetic diversity and endemicity are represented through the selection of endemic families from a maximised coverage of the APG classification (1998). Functional diversity is represented through the selection of (i) speciose families with a diversity of life forms, and (ii) species belonging to different PFTs within these families. An ideal selection of families is made according to these guidelines, to show the optimum selection strategy results given perfect knowledge of species distributions, and then a final selection is made to best match this ideal to data availability.

The APG (1998) classification of angiosperms is useful for current purposes because unlike traditional higher taxon systems, the clades are based on genetics rather than morphology. A 
'clade' is a monophyletic group of taxa sharing a closer common ancestry with one another than with members of any other clade; these clades form the branches of a 'cladogram' (Fig. 5:11). Families in the APG classification are grouped into monophyletic orders, which are grouped into 'informal' monophyletic clades according to the estimated time of divergence of different groups. Thus, selecting across all clades ensures a wide representation of genetic diversity.

\subsubsection{Geographic filters}

Only families that occur within Amazonian lowland forest are of interest. Occurrence in the Amazon region, and endemicity within the Neotropics, was ascertained from distributional descriptions and maps (Groombridge 1992, Maas et al. 1998, Heywood 1993). In addition, distributional descriptions in Maas et al. (1998) were used to exclude families whose range is mainly in montane or arid regions (e.g. "Grossulariaceae: Throughout the Neotropics; montane"). These sources were also used to exclude wholly aquatic families.

228 APG families are known to occur in the Amazon region. 203 are known not to occur in the region, including those comprising the orders Acorales (Acoraceae) and Garryales (Garryaceae). For 23 families, there is evidence of occurrence in the Neotropics, but I do not know whether Amazonian species exist. I lack positive or negative evidence of neotropical occurrence for 12 families.

66 families (including all from the Ceratophyllales, Santalales, Saxifragales, Geraniales, Cornales, Alismatales, Apiales, Aquifoliales and Dipsacales orders) were excluded because they occurred mainly in montane or arid regions. This information was not readily available for 13 families, which were also excluded. A further 17 Amazonian families were rejected because all genera are aquatic.

132 families remained, of which 17 are endemic to the Neotropics (Groombridge 1992, Heywood 1993, Watson \& Dallwitz 1992, APG 1998). These families and the selection criteria (see below) are shown in Table A3:1.

\subsubsection{Selection by non-spatial characteristics}

To represent genetic diversity, an ideal selection from the remaining families would include families from each informal group (Fig. 5:12, eurosids I and II, euasterids I and II, commelinoids) and each order outside these groups. Various families were not assigned to an order, being placed as Dicots or Eurosids, or labelled 'Uncertain'. These unplaced families are conservatively treated as having equal taxonomic distinctiveness to any other clade on that level, and included in the ideal selection. 
The families were classified according to presence or absence of trees, shrubs, climbers (vine, liana) \& herbaceous plants among their neotropical species (Watson \& Dallwitz 1992, Maas et al. 1998). Longevity and generation time, which affect the speed of population response to climate change, correspond closely to life form.

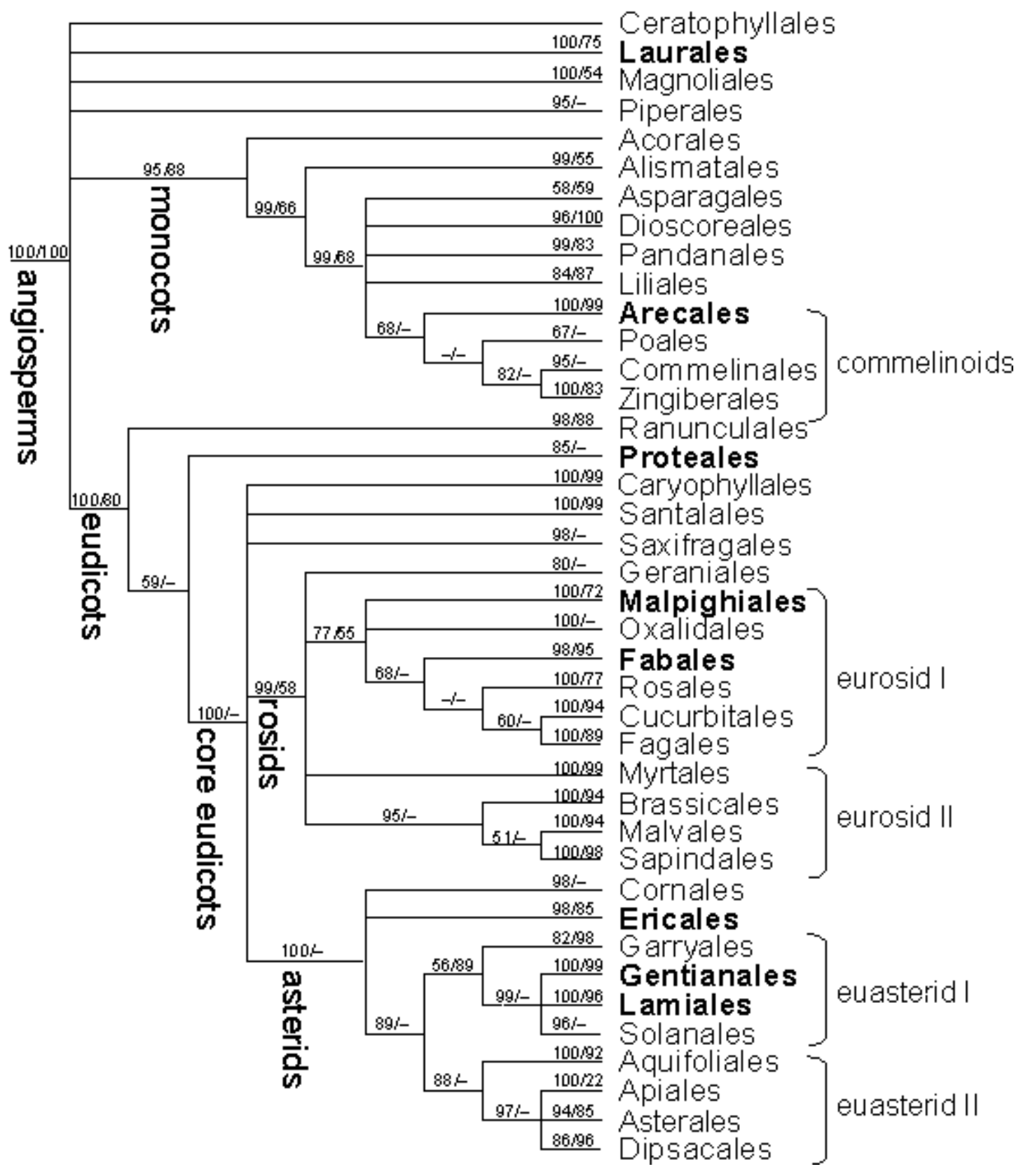

Fig. 5:11: Cladogram (APG 1998). Bold indicates inclusion in the final selection

For each group, those families that are endemic to the Neotropics and those with the most genera are preferentially selected. Endemic groups are of interest because proportionately more global genetic diversity would be lost with their extinction than for a cosmopolitan group. Groups with a large number of genera are of interest because (i) they represent a high proportion of the Amazon basin flora, (ii) they are more likely (all else being equal) to include species with sufficient distributional data for analysis, and (iii) they would hold a greater proportion of 
functional diversity were it randomly allocated between genera. An estimated genus count for each family (Maas et al. 1998) was modified according to taxonomic reassignments (Watson \& Dallwitz 1992).

Fig. 5:12 summarises the selection protocol. The groups from the cladogram were used to determine taxonomic distinctiveness (applied conservatively to include all unplaced orders and families). Within each clade ' $x$ ', families were selected on the bases of neotropical endemicity and of having the maximum number of genera within the group. Additional families are then added to include any unrepresented life forms for that group. Where the families are equally weighted on all counts, a random choice would be made, but this situation did not occur.

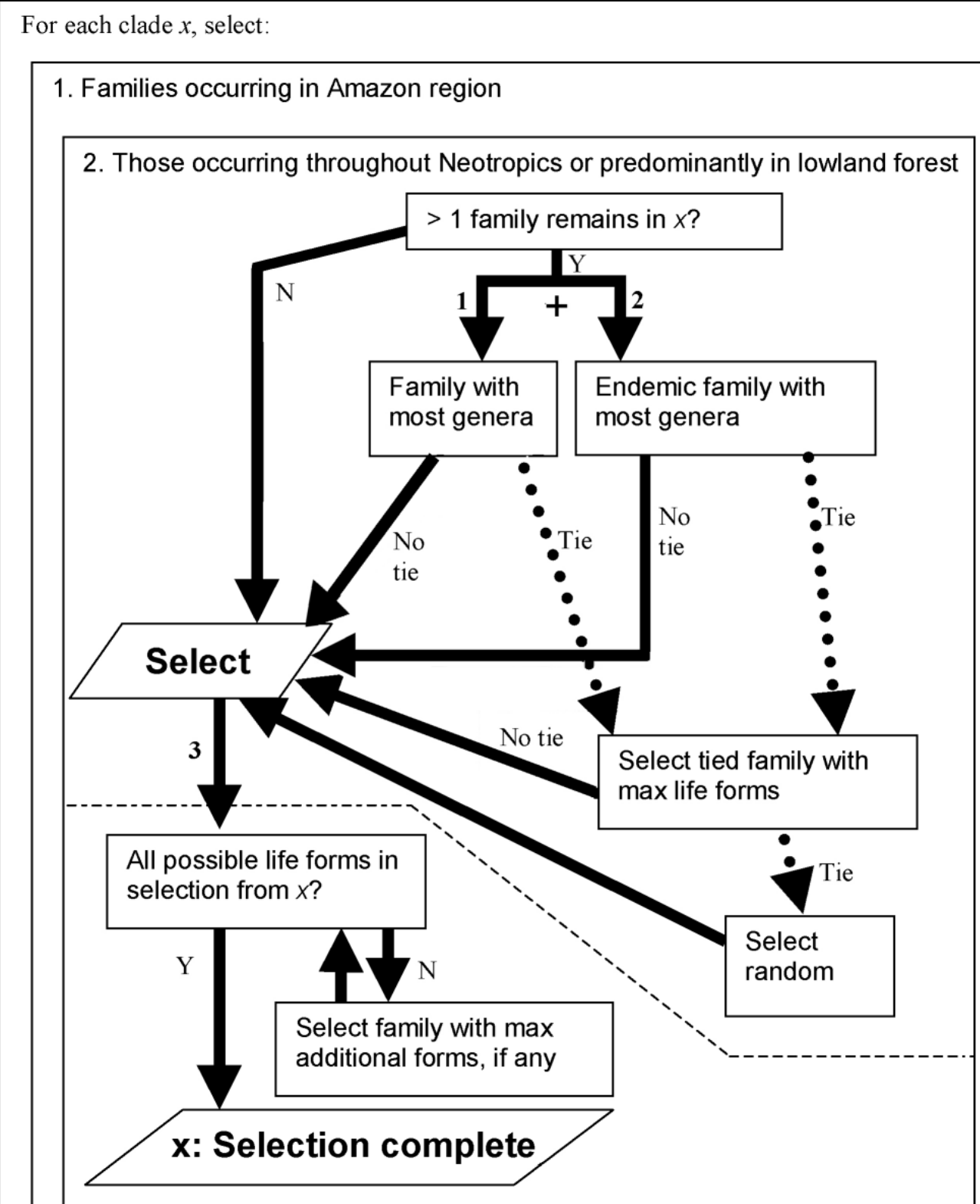

Fig. 5:12: Family selection protocol 
The Schedule of Flora Neotropica Monographs (OFN 1999) was used to identify which families are actively researched. A final selection was then undertaken, following the same criteria but using only these families. Where monograph-quality data were not in fact available, the selection protocol was repeated for that clade.

\subsubsection{Selection outcomes}

An example of the protocol for the Commelinoids follows:

1) Endemicity within group:

a) Thurniaceae, Cannaceae, Heliconiaceae are neotropical endemics

b) Taxonomic diversity between endemics: all are monogeneric

c) Functional diversity: all contain only herbs

d) Data availability: select Heliconiaceae, the only 'OFN active' endemic.

2) Taxonomic diversity within group

a) Most genera: select Poaceae (207 genera, herbs).

3) Additional life forms: select Arecaceae (=Palmae), (trees \& shrubs)

All three ideal families were 'actively researched', but the only comprehensive source of data available was for the Arecaceae (Henderson 1995). Zingiberaceae (Maas 1972) was chosen as a substitute for Heliconiaceae, but its distributional data were insufficient according to the species selection criteria (section 5.2.7.3).

Eight neotropical endemics (Heliconiaceae, Clusiaceae, Tovariaceae, Peridiscaceae, Lissocarpaceae, Marcgraviaceae, Cyclanthaceae and Krameriaceae) were included in the ideal selection. Of these, OFN (1999) states that Marcgraviaceae and Heliconiaceae are currently under revision, and Krameriaceae was monographed in 1989. These three families and Quiinaceae (currently worked on) are the endemics included in the final selection. However, sufficient data for these groups were not available. Hence, Caryocaraceae and Chrysobalanaceae are substituted for Quiinaceae to represent the Malpighiales. Caryocaraceae is the only endemic family in the final selection. Chrysobalanaceae is selected to represent shrubs.

Table 5:1 shows the final selection. The number of species for which monograph-quality data was acquired and which occur within the 'enclosed-Amazon' (section 1.4.2) is shown, as is the number of unique species-cell combinations for these over the Neotropics. Where only a few genera have been databased, these are listed. These values are repeated for the 'pool' species, which meet the record-cell frequency and density criteria. No species from Dioscoreaceae, Myrtaceae (substituted for Melastomataceae), Triuridaceae or Zingiberaceae met these criteria. 
Table 5:1: Family selection, summary with species and records available, and data sources ${ }^{10}$

\begin{tabular}{|c|c|c|c|c|c|c|c|c|c|c|c|}
\hline \multirow{2}{*}{$\begin{array}{l}\text { Ideal or } \\
\text { replacement? }\end{array}$} & \multirow[t]{2}{*}{ Family } & \multirow[t]{2}{*}{ Species databased } & \multirow{2}{*}{$\begin{array}{l}\text { Record } \\
\text {-cells total }\end{array}$} & \multirow[t]{2}{*}{ |'Pool' species } & \multirow{2}{*}{\begin{tabular}{|l|} 
Record \\
-cells total
\end{tabular}} & \multicolumn{6}{|c|}{ Spatial data source } \\
\hline & & & & & & Mono & MPEG & EMBR & INPA & W3T & Kew \\
\hline \multirow[t]{10}{*}{ Ideal } & Arecaceae & 118 & 2922 & 37 & 1674 & $\sqrt{ }$ & Some & Some & Some & & \\
\hline & Balanophoraceae & 8 & 248 & 2 & 202 & $\checkmark$ & & $\checkmark$ & Some & & \\
\hline & Bignoniaceae & 206 & 4218 & 34 & 1824 & $\checkmark$ & $\checkmark$ & $\checkmark$ & Some & Some & \\
\hline & Dioscoreaceae & 4 & 61 & 0 & - & Some & Some & & Some & $\checkmark$ & Some \\
\hline & Fabaceae & 135 (Inga) & 3487 & 21 & 1330 & $\checkmark$ & $\checkmark$ & Some & Some & Some & \\
\hline & Lauraceae & $\begin{array}{c}82 \text { (Endlicheria, Nectandra } \\
\text { Aniba \& Aiouea) }\end{array}$ & 1920 & 17 & 825 & $\checkmark$ & $\checkmark$ & $\checkmark$ & $\checkmark$ & Some & \\
\hline & Proteaceae & 18 & 463 & 3 & 313 & $\checkmark$ & Some & Some & Some & Some & \\
\hline & Rubiaceae & $\begin{array}{c}\text { (Gleasonia, Henriquezia, } \\
\text { Platycarpum) }\end{array}$ & 58 & 5 & 44 & $\checkmark$ & & $\checkmark$ & Some & & \\
\hline & Sapotaceae & 16 & 733 & 6 & 477 & Some & Some & & Some & $\checkmark$ & \\
\hline & Triuridaceae & 7 & 99 & 0 & - & $\checkmark$ & & & & & \\
\hline \multirow[t]{4}{*}{ Replacement } & Caryocaraceae & 17 & 488 & 8 & 351 & $\sqrt{ }$ & & $\sqrt{ }$ & Some & Some & \\
\hline & Chrysobalanaceae & 248 & 6204 & 60 & 3688 & $\checkmark$ & Some & $\checkmark$ & Some & Some & \\
\hline & Myrtaceae & 4 (Campomanesia) & 72 & 0 & - & $\checkmark$ & & & & & \\
\hline & Zingiberaceae & 1 & 34 & 0 & - & Some & Some & & & & \\
\hline
\end{tabular}

10 "Kew" = RBG Kew herbarium, London, "INPA" = INPA herbarium, Manaus, Brazil; "MPEG" = Museu Paraense de Emilio Goeldi herbarium, Belém, Brazil; "EMBR" = EMBRAPA herbarium, Belém, Brazil; "W3T" = records from the Missouri herbarium W3TROPICOS system, USA [online] http://mobot.mobot.org/W3T/Search/vast.html. Last accessed 9-10-01 
The families from the ideal selection not included in the final selection are Annonaceae, Asteraceae, Dilleniaceae, Heliconiaceae, Krameriaceae, Marcgraviaceae, Melastomataceae, Orchidaceae, Poaceae, Rafflesiaceae, Vitaceae, Clusiaceae, Cyclanthaceae, Gunneraceae, Lepidobotryaceae, Lissocarpaceae, Menispermaceae, Mitrastemonaceae, Nyctaginaceae, Piperaceae, Polygonaceae, Primulaceae, Staphyleaceae, Taccaceae, and Tovariaceae.

\subsubsection{PFT definition}

PFTs based on traits that are expected to influence change response can be used to select for (Faith \& Walker 1996) and parameterise a diversity of plant strategies. PFT traits either provide direct inputs to the model for all species (e.g. number of life stages), or offer 'surrogate traits' indicating mean values for poorly documented species (e.g. growth rate between stages).

The traits considered here are intended to identify different response factors (section 4.4.1) reproductive rates, dispersal mechanisms and pre-adaptations to the expected stresses. All are categorical variables: life-form, height class, deciduousness, pollination and dispersal modes. Form and height class are associated with generation time and surface area, which affects drought resistance. The reproductive characteristics determine the speed of recovery in fluctuating environments and the impact of population density on reproductive potential. Deciduousness is an indicator of drought tolerance.

These traits were defined before examining the data available for Amazonian species, but the PFTs were not.

\subsubsection{Identifying functional traits related to vital rates}

In the Amazon region, poorly documented species vastly outnumber well-documented species. If there is a relationship between vital rates (survival, growth, reproduction) and functional traits, standard PFT rates should form a reasonable approximation for less-known species.

Some growth and survival relationships were assessed using a tree and liana diameter dataset from permanent sample plots (PSPs, subset of data in Phillips et al. 1998). Mortality was calculated for species with $>5$ specimens at a 'site'. Records from PSPs in the same $1^{\circ}$ cell were grouped to increase the number of species with $>5$ specimens, to define sites in accordance with the project scale. Even so, most species were excluded by this criterion.

Annual mortality was calculated as the proportion of trees known to exist that year that were first recorded dead that year. The site mean for a species was estimated as the mean of these PSP annual mortalities, including zero value years between records. As the PSPs were not 
surveyed annually, it was assumed that the year of death was that in which the tree was first recorded as dead, and that trees not present during the original census were recruited in the year of first recording. If the levels of recruitment and death are similar, the errors from these assumptions should balance one another. This method risks underestimating mortality by omitting individuals that are recruited and die between census intervals (Sheil \& May 1996).

Mean growth rates were calculated for each species at each site. Mean dbh increments do not offer a standard for comparing between individuals of different initial sizes. Relative increase in biomass (relative growth rate, RGR) is often used for this purpose. Here, relative diameter growth rate (RDGR) was calculated by substituting dbh for biomass (Eqn. 23 derived from RGR, South 1995). Individuals that had apparently decreased in diameter were included with the aim of balancing measurement error.

$$
\text { Mean RDGR }=\frac{\ln d 2-\ln d 1}{t 2-t 1}
$$

Three sets of tests of the relationship between the traits and growth rate or survival were carried out, comparing all species, and comparing palms and dicots separately. The null hypothesis that there is no difference between growth and mortality rates belonging to groups of individuals having different traits was tested for each functional trait variable. Each individual in each 'trait group' was treated as an independent data point. Form, deciduousness and family were treated as nominal variables, and height class, dispersal and pollination mode as ordinal variables. Pollen or seed were assumed to be dispersed farthest by flying vertebrates, then insects, then wind, then arboreal and ground mammals. Species dispersed by unknown vertebrates or water were ignored in dispersal-mode tests.

\subsubsection{Outcomes}

3828 of a sample of 16138 species-PSP records represent 191 of the databased species. 73 species were represented by $>5$ specimens. The plots were all located in degree cells that are simulated as aseasonal (Table 5:2).

Table 5:2: Climate for cells holding PSPs

\begin{tabular}{|l|l|l|l|l|l|l|l|l|}
\hline $\begin{array}{l}\text { Site } \\
\text { ID }\end{array}$ & $\begin{array}{l}\text { Central } \\
\text { latitude }\end{array}$ & $\begin{array}{l}\text { Central } \\
\text { longitude }\end{array}$ & $\begin{array}{l}\text { No of } \\
\text { plots }\end{array}$ & $\begin{array}{l}\text { Minimum mean } \\
\text { monthly } \\
\text { temperature, }{ }^{\circ} \mathbf{C}\end{array}$ & $\begin{array}{l}\text { Annual } \\
\text { AET, } \\
\text { mm }\end{array}$ & $\begin{array}{l}\text { Annual MD } \\
\text { (PET - } \\
\text { AET), mm }\end{array}$ & $\begin{array}{l}\text { SMA } \\
\text { (days) }\end{array}$ & $\begin{array}{l}\text { Records } \\
\text { selected }\end{array}$ \\
\hline 1834 & -3.5 & -72.5 & 2 & 25.5 & 1359.2 & 0 & 0 & 420 \\
\hline 1835 & -3.5 & -73.5 & 4 & 25.475 & 1486.9 & 0 & 0 & 638 \\
\hline 2154 & -11.5 & -71.5 & 5 & 22.725 & 1729.0 & 0 & 0 & 1093 \\
\hline 2193 & -12.5 & -69.5 & 11 & 22.775 & 1771.1 & 0 & 0 & 2089 \\
\hline
\end{tabular}




\subsection{Growth}

Family was strongly related to growth rate when all families were considered together, and also when Arecaceae was treated separately (Table 5:3).

Studies of Costa Rican trees have related diameter growth rate to mature height class, with canopy trees growing more rapidly (Lieberman \& Lieberman 1987, Finegan et al. 1999). However, for this dataset height class was significant for palms only, reflecting their limited dbh increase with increasing height. Dispersal mode was also significant for palms; it may distinguish between pioneer and mature forest species. Deciduousness was significant for dicots (all palms were evergreen), suggesting that growth rates are higher for evergreens.

Record site was significant in determining growth rates only when palms and dicots were treated together. This can be explained by site differences in the proportion of palms in the databased species present (from 0.38 to 0.72 ), so can be disregarded.

Table 5:3: Relationship between mean species growth rates for each site and traits

\begin{tabular}{|l|l|l|l|l|l|l|l|}
\hline \multicolumn{4}{|c|}{} & \multicolumn{3}{l|}{ All } & \multicolumn{2}{l|}{ Palms } & \multicolumn{2}{l|}{ Dicots } \\
\hline Trait & Test & Sig. & Cases & Sig. & Cases & Sig. & Cases \\
\hline Family & Kruskal-Wallis & $\mathbf{. 0 0 0}$ & 291 & - & - & $\mathbf{. 0 0 0}$ & 263 \\
\hline Form (tree, liana) & Mann-Whitney U & .122 & 238 & - & - & .069 & 213 \\
\hline Height & $\begin{array}{l}\text { Jonckheere- } \\
\text { Terpstra 2-tailed }\end{array}$ & .196 & 206 & $\mathbf{. 0 2 6}$ & 26 & .848 & 180 \\
\hline Dispersal mode & $\begin{array}{l}\text { Jonckheere- } \\
\text { Terpstra 2-tailed }\end{array}$ & $\mathbf{. 0 3 9}$ & 94 & $\mathbf{. 0 2 8}$ & 13 & .110 & 81 \\
\hline Deciduousness & Kruskal-Wallis & $\mathbf{. 0 3 7}$ & 143 & - & 28 & $\mathbf{. 0 0 2}$ & 115 \\
\hline Pollination mode & $\begin{array}{l}\text { Jonckheere- } \\
\text { Terpstra 2-tailed }\end{array}$ & .913 & 234 & - & & .110 & 81 \\
\hline Record site & Kruskal-Wallis & $\mathbf{. 0 1 9}$ & 291 & .219 & 28 & .053 & 263 \\
\hline
\end{tabular}

Table 5:4: Relationships between mean species mortality rate for each site and traits

\begin{tabular}{|l|l|l|l|l|l|l|l|}
\hline \multicolumn{4}{|c|}{ All } & \multicolumn{3}{l|}{ Palms } & \multicolumn{2}{l|}{ Dicots } \\
\hline Trait & Test & Sig. & Cases & Sig. & Cases & Sig. & Cases \\
\hline Family & Kruskal-Wallis & $\mathbf{0 . 0 4 5}$ & 79 & - & - & $\mathbf{0 . 0 3 0}$ & 55 \\
\hline $\begin{array}{l}\text { Form (tree, } \\
\text { liana) }\end{array}$ & Mann-Whitney U & 0.709 & 71 & - & - & 0.870 & 49 \\
\hline Height & $\begin{array}{l}\text { Jonckheere- } \\
\text { Terpstra 2-tailed }\end{array}$ & 0.615 & 66 & 0.077 & 24 & 0.571 & 42 \\
\hline $\begin{array}{l}\text { Dispersal } \\
\text { mode }\end{array}$ & $\begin{array}{l}\text { Jonckheere- } \\
\text { Terpstra 2-tailed }\end{array}$ & 0.778 & 28 & 0.829 & 12 & 0.920 & 16 \\
\hline $\begin{array}{l}\text { Deciduous- } \\
\text { ness }\end{array}$ & Kruskal-Wallis & 0.272 & 51 & - & - & 0.348 & 27 \\
\hline $\begin{array}{l}\text { Pollination } \\
\text { mode }\end{array}$ & $\begin{array}{l}\text { Jonckheere- } \\
\text { Terpstra 2-tailed }\end{array}$ & 0.339 & 63 & - & - & 0.254 & 49 \\
\hline Record site & Kruskal-Wallis & $\mathbf{0 . 0 0 0}$ & 79 & 0.018 & 24 & $\mathbf{0 . 0 0 0}$ & 55 \\
\hline
\end{tabular}




\subsection{Mortality}

Mortality was estimated for 52 species from nine databased families were represented by at least five specimens in one or more sites ( 79 species-site combinations). The mean mortality rate was 0.014, within the range of rates cited for tropical forests (Korning \& Balsev 1994, Lugo \& Scatena 1996, Sheil \& May 1996). There was a highly significant relationship between mortality rates and family or site, regardless of whether palms are treated separately.

The variation in mortality between sites suggests that the local environment has a stronger effect on mortality than it does on growth (Fig. 5:13). The PSPs sampled may have been in different stages of recovery from, or undergone different levels of, disturbance, as well as occupying different climates from the mean cell.

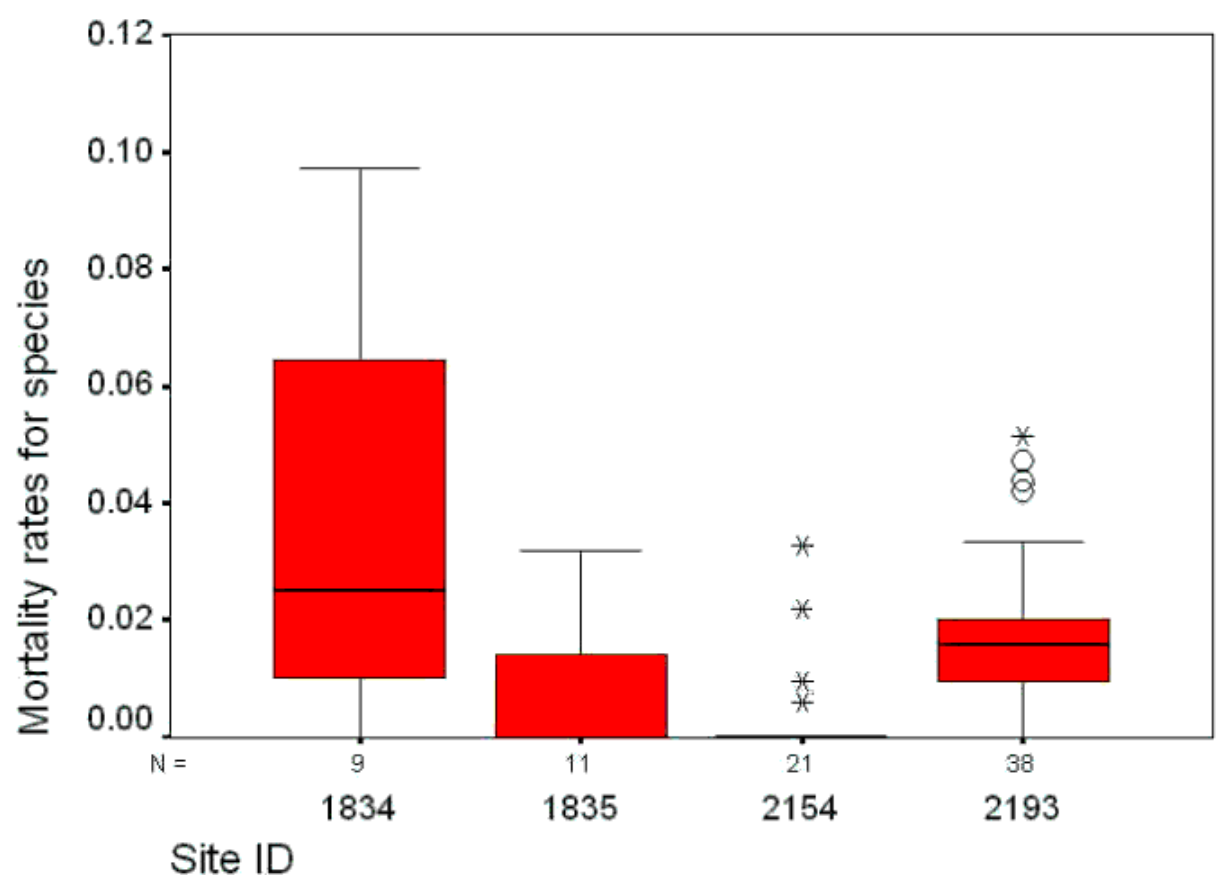

Fig. 5:13: Range of mortality rates in PSPs sampled ${ }^{11}$

\subsection{Density}

The relationship of density with family and deciduousness can be explained by the difference between palms and dicots (Table 5:5). Density can otherwise be only related to record site.

\footnotetext{
${ }^{11}$ The box represents the first to third quartiles of the distribution. Asterisks represent extreme values (further than 3 interquartile ranges from the box edge) and circles outliers (1.5 to 3 interquartile ranges from the box edge). The number of species sampled per site is shown on the y axis as $n$.
} 
Table 5:5: Relationships between species' density per hectare for each site and traits

\begin{tabular}{|l|l|l|l|l|l|l|l|}
\hline \multicolumn{4}{|c|}{ All } & \multicolumn{3}{l|}{ Palms } & \multicolumn{2}{l|}{ Dicots } \\
\hline Trait & Test & Sig. & Cases & Sig. & Cases & Sig. & Cases \\
\hline Family & Kruskal-Wallis & $\mathbf{0 . 0 0 0}$ & 291 & - & - & 0.460 & 263 \\
\hline $\begin{array}{l}\text { Form (tree, } \\
\text { liana) }\end{array}$ & Mann-Whitney U & 0.540 & 238 & - & - & 0.808 & 213 \\
\hline Height & $\begin{array}{l}\text { Jonckheere- } \\
\text { Terpstra 2-tailed }\end{array}$ & 0.090 & 206 & 0.401 & 26 & 0.90 & 180 \\
\hline $\begin{array}{l}\text { Dispersal } \\
\text { mode }\end{array}$ & $\begin{array}{l}\text { Jonckheere- } \\
\text { Terpstra 2-tailed }\end{array}$ & 0.288 & 94 & 0.612 & 13 & 0.937 & 220 \\
\hline $\begin{array}{l}\text { Deciduous- } \\
\text { ness }\end{array}$ & Kruskal-Wallis & $\mathbf{0 . 0 3 4}$ & 143 & - & - & 0.587 & 115 \\
\hline $\begin{array}{l}\text { Pollination } \\
\text { mode }\end{array}$ & $\begin{array}{l}\text { Jonckheere- } \\
\text { Terpstra 2-tailed }\end{array}$ & 0.804 & 234 & - & - & 0.943 & 81 \\
\hline Record site & Kruskal-Wallis & $\mathbf{0 . 0 0 0}$ & 291 & 0.643 & 28 & $\mathbf{0 . 0 0 0}$ & 263 \\
\hline
\end{tabular}

\subsubsection{Summary of traits required for PFTs}

The PFT classification is intended to maximise functional diversity as it relates to modelled change response. Thus, traits that are used directly in the model, and surrogate traits were both included in the classification (Table 5:6). Although consistent patterns of association between traits are seen in documented floras (Grime et al. 1988, Díaz \& Cabido 1997), each combination of selected traits in the dataset was defined as an individual PFT, to maximise inclusivity. If all potential combinations of response traits are viewed as a multi-dimensional matrix, many spaces are likely to be unoccupied (Raup 1966). The occupied places are PFTs that are successful under equilibrium conditions.

Table 5:6: Traits for PFT construction

\begin{tabular}{|l|l|}
\hline Trait & Categories \\
\hline Life form & Annual herb, perennial herb, bush, tree, epiphyte, climber (liana/vine) \\
\hline $\begin{array}{l}\text { Height class on } \\
\text { maturity }\end{array}$ & $\begin{array}{l}\text { Herb }(<1 \mathrm{~m}), \text { bush }(1-4 \mathrm{~m}), \text { understorey }(4-10 \mathrm{~m}), \text { midstorey }(10-20 \mathrm{~m}), \\
\text { subcanopy }(20-25 \mathrm{~m}), \text { canopy }(25 \mathrm{~m}+)\end{array}$ \\
\hline Dispersal mode & $\begin{array}{l}\text { Wind, vertebrate (flying), vertebrate (arboreal), vertebrate (swimming) or } \\
\text { water, vertebrate (ground), invertebrate, unassisted (explosive dehiscence, } \\
\text { gravity, subterranean) }\end{array}$ \\
\hline Pollination mode & Selfing, wind, vertebrate (flying), vertebrate (arboreal), invertebrate \\
\hline Deciduousness & Deciduous, semideciduous, deciduous under drought stress, evergreen \\
\hline
\end{tabular}

\subsubsection{Species selection}

The species selection is a stratified sample, intending to maximise representation of families, functional traits and seasonality tolerance. Species are chosen randomly within each group as a last selection step, but the choice may be so narrow that this is not necessary. 


\subsubsection{Seasonality}

Species from aseasonal and seasonal environments will undergo different pressures following increases in $M D$ or $S M A$. Species adapted to aseasonal moist environments were expected to experience more difficulty than other species. Although deciduousness is an adaptation to seasonal environments, the data show that deciduous and evergreen PFTs occur throughout the range of seasonality. So, rather than selecting a physical trait, species-range mean SMA was used to ensure inclusion of different seasonality response. The species with the maximum, minimum and median mean SMA were selected for each family.

\subsubsection{PFTs}

Unique PFTs are sought within each selected family. Representatives of each PFT found within each family and not already included in the seasonality selection were chosen. Most effort was put into allocating traits to species having sufficient locational data. Where species-level information was not available, genus or family-level information was sought. A category in each trait was identified for each species in the final selection.

The relative density of each PFT in the Amazon region cannot be assessed with this dataset, so there was no attempt at proportional representation of functional diversity.

\subsubsection{Locational data availability}

The choice was restricted to species with monograph-quality locational data, which limits the number available for modelling. The production of a monograph involves identifying a range of herbarium specimens over the region, thus capturing the species' known range, and also establishes an accepted taxonomy to reduce the incidence of misidentifications. Only species with records falling into the enclosed-Amazon (section 1.4.2) are considered. If the inclusiveAmazon were considered instead, there is a chance that these species' distributions would fall entirely outside the Amazon basin.

A small number of points may indicate limited distributional data. Spatial rarity may also indicate that the species is already undergoing a decline or expansion in range, unrelated to changing climate. Very restricted or under-recorded species (recorded in $\leq 5 \times 1^{\circ}$ cells) were excluded from the present analysis. Species whose record-cell density below a minimum value, 0.35, within the recorded range were also excluded, unless their record cell count exceeded an arbritarily defined upper limit of 50(which happens to be supported by Stockwell \& Peterson (2002)). The areal calculations were performed using decimal degrees. The recorded range was defined as an MCP linking the centre-points of the cells on the edge of the recorded distribution. 
This can produce point-in-polygon densities greater than 1 point per degree cell - for example, if the record-cells formed a rectangle, the corners of the MCP being formed by the cell centres.

The 0.35 limit was selected following a test of the similarity interpolation method with European species (section 7.2.4). The mean point-in-polygon density for five $20 \%$ samples from the Quercus coccifera and Salix atrocinerea monographs in Atlas Florae Europaeae (AFE) was found. These had produced good (Salix) and moderate (Quercus) simulations of the remaining $80 \%$ of the species range. The mean densities were 0.262 for Quercus, and 0.376 for Salix. The polygons were calculated in the same projection system, but at different resolutions, so there is scope for further investigation of an appropriate record density limit.

\subsubsection{Protocol}

The procedure for species selection within each family is thus:

1) Filter species according to locational data availability and extraction threat status

2) Group species into PFTs based on traits in Table 5:6, p. 159

3) Select $\leq 3$ species having maximum, minimum and median range-mean $S M A$. Where there is a tie, select species to increase PFT representation; if all are represented, choose at random from the tied group.

4) Add species to represent unique PFTs not already selected. Where an unselected PFT includes $>1$ species, choose species at random from the PFT.

\subsubsection{Species selection outcomes}

Of 193 'pool' species from selected families with sufficient monographed data, 69 were selected to represent the different PFTs (minimum set) and seasonality preferences (maximum, minimum, median of recordset-mean seasonality). The selected species, their traits, mean record-cell SMA and record frequency are listed in Appendix 4.

Cell SW003073, which includes the Peruvian city of Iquitos, held 102 of the pool species (Fig. 5:14i). Each of the cells occupied by the Brazilian city of Manaus (SW002061-60) held 90 species. In all, 70\% of the pool species fell into at least one of these top three cells. Conversely, there are large areas between some of the major rivers without any records. This demonstrates a strong collection bias towards accessible areas, even in species that meet the density criteria. The same biases can be seen in the selected species set (Fig. 5:14ii). 


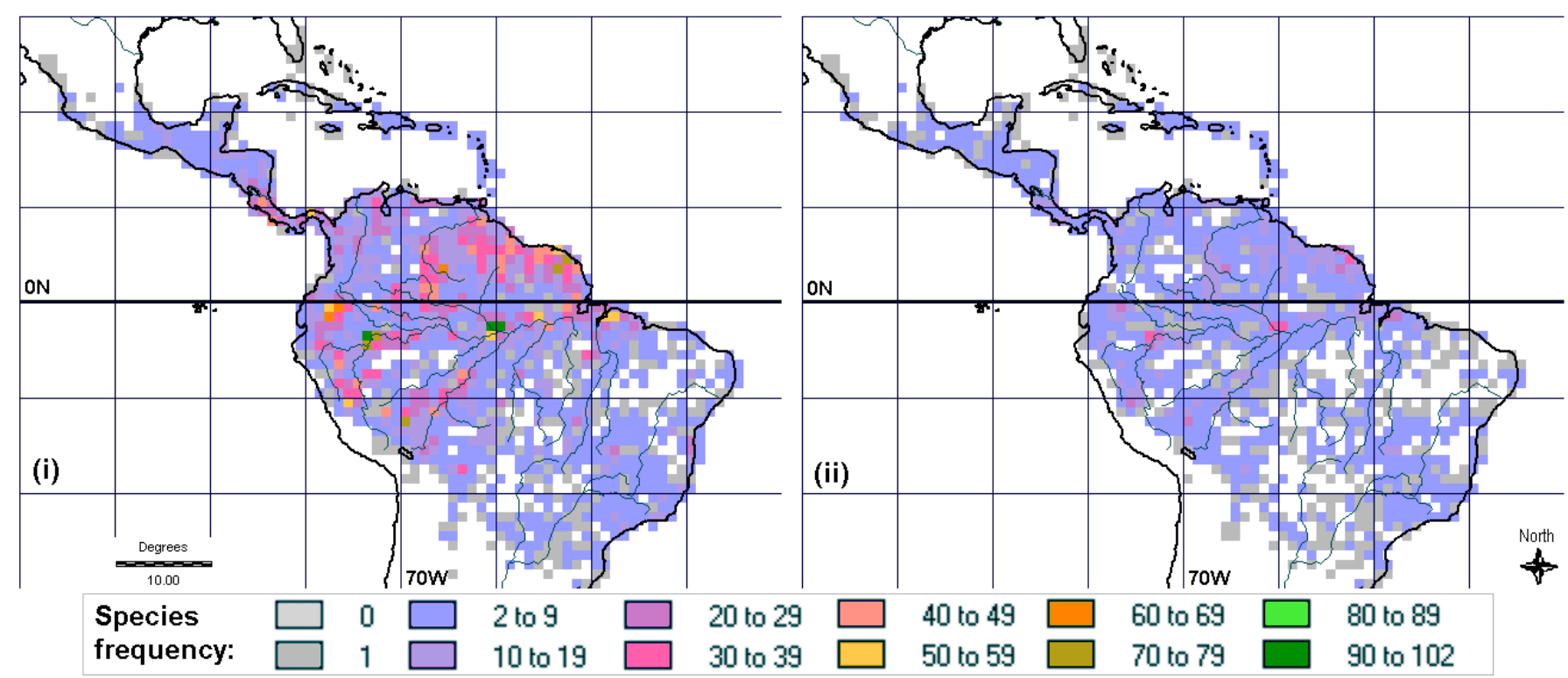

Fig. 5:14: Distribution of records for (i) the 'pool' and (ii) selected species sets

\subsubsection{Model structure}

All populations of selected species in the 560 cells falling within the inclusive-Amazon (section 1.4.2) were modelled. The model simulated a single stage-structured population in each cell. Population processes were run for an equilibrating period, and then subjected to environmental change. The vital rates governed the modelled rate of response to change, with germination and recruitment being limited by environmental conditions. No interactions between populations were included.

\subsubsection{Distribution model}

The bioclimate variables $A E T, M D$ and $S M A$ were used to calculate a suitability index, $S_{\mathrm{p}}$, in each $1^{\circ}$ cell. Presence was simulated where $S_{\mathrm{p}}$ exceeded a minimum boundary, $S_{\min }$. The choice of model for $S_{\mathrm{p}}$ does not affect the population model, whose relationship with climate is determined separately (section 5.2.8.2). It does affect the distributional response to change, because the same boundary is applied to evaluate the suitability of changed environments.

For presence-absence data, $S_{\mathrm{p}}$ is best estimated using a general linear model of the distribution based on the bioclimate variables. In this case, $S_{\min }$ would equal 0.5 (section 4.3.6.7.1). As this is not feasible with presence-only data, an alternative approach was taken. First, the maximum and minimum recorded values of the bioclimate variables for each species, together with minimum temperature ( $T_{\min }$, from lowest mean monthly temperature) were used as absolute constraints on distribution. These 'rectilinear envelopes' limit the simulated range to the extremes known to be tolerated by each species. 
The tolerated values were sourced from a mixed-reference climate (section 5.2.4.7). To allow representation of the variation in climate over recent history, it was decided to use mean values from three decades where possible. This extends the simulated climate tolerance of the species concerned in a realistic manner. Where a record-cell fell within the inclusive Amazon, the mean bioclimate values from DOWNS for the each of the decades 1960s - 1980s were added to the set of tolerated climates. Where the cell fell outside this region, multiple decadal simulations were not available, so the single PRES value was added three times. This prevents the extraAmazonian cells from exerting an artificially reduced influence on the climate means.

Second, a Gower similarity metric was used to calculate $S_{\mathrm{p}}$ inside the absolute constraints (Eqn. 6, p. 106). It simulates a unimodal distribution around each record point in environmental space, which together may produce an overall uni-, bi- or multimodal relationship between the record and no-record cells. This excludes parts of the environmental envelope which might be physiologically optimal for the species, but from which it is competitively excluded. As the impact of climate change on relative competitiveness is highly unpredictable, the conservative assumption is made that present-day competitive exclusion will persist under conditions of change, even if the composition of the excluding community changes, rather than assuming that today's poor competitors will somehow become preferentially advantaged.

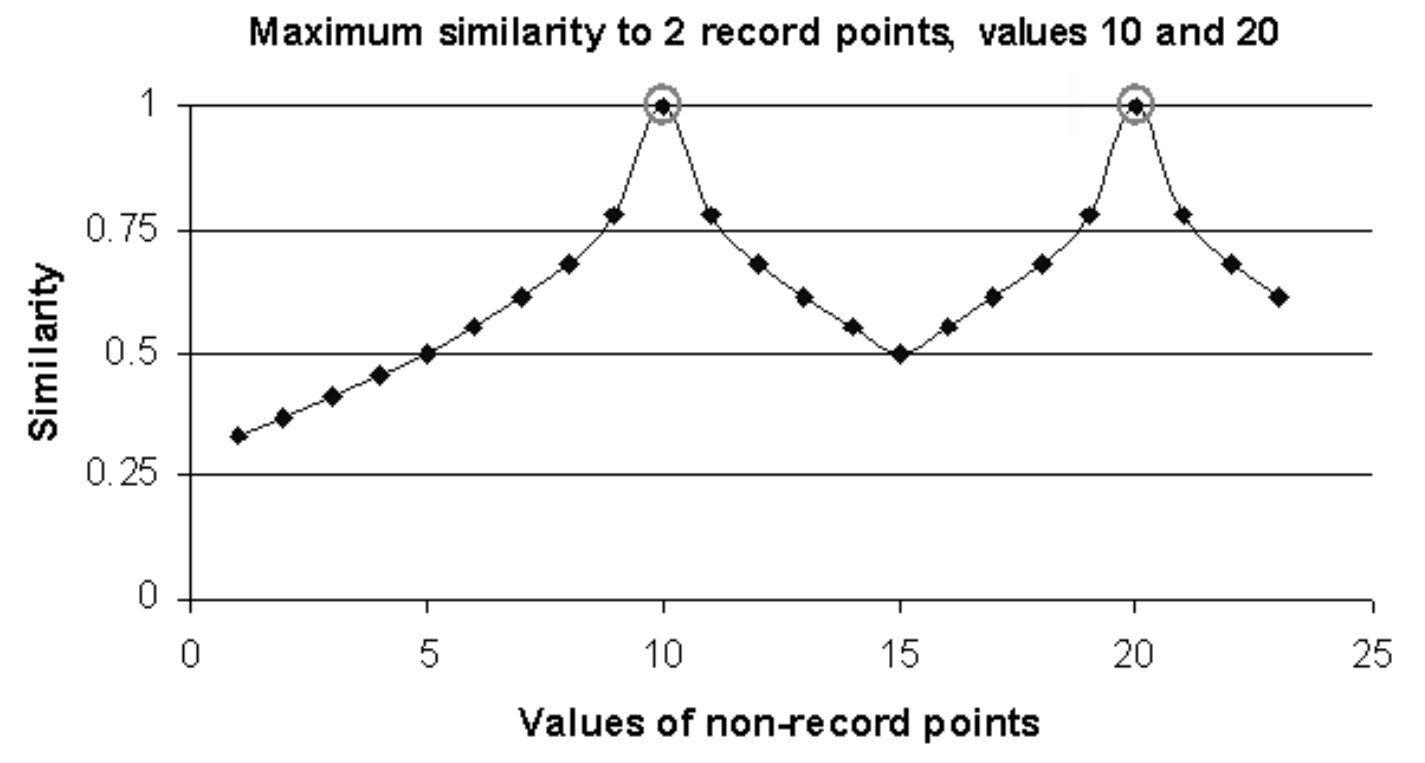

Fig. 5:15: Example of application of Gower similarity metric to a single variable

Fig. 5:15 demonstrates the use of the Gower metric for a single hypothetical variable (x-axis). The graph shows the maximum similarity to each of two record-points (values 10 and 20) of one variable. The metric is applied to all three bioclimate variables together in practice (Eqn. 6). The Gower metric fails if any range $a$ is zero, because division by zero is not possible. Zerovalue ranges are therefore adjusted to $10^{-6}$. 
The spatial scale of the environmental data $\left(1^{\circ}\right)$ relative to the species distribution data (often from 1 ha sites), means that the grid cell value may differ greatly from the record-site conditions. The species' environmental tolerance may therefore be overestimated. Environmental outliers were excluded from the species' recordset to reduce this problem. Outliers for each environmental variable were excluded using the following rule: if a cell value is further than three interquartile ranges from the first or third quartile of the recorded values for that variable, it is excluded from the list of record-cells used to calculate suitability.

As $S_{\mathrm{p}}$ does not equate to a statistical probability, a $90 \%$ or $95 \%$ value for $S_{\min }$ is not necessarily appropriate. The question of selecting an appropriate $S_{\min }$ is resolved using jackknifing. In this process, the experiment is re-run for as many times as there are samples (record-sites), missing one sample out in each run (Manly 1992, Crowley 1992). The minimum positive $S_{\mathrm{p}}$ from the jackknifed distribution was used to quantify $S_{\min }$ for each species. For records falling within the Amazon basin, each climate from the three modelled decades was tested and the minimum positive $S_{\mathrm{p}}$ was taken. Record-sites that fell outside the ranges of extremes of the environmental variables seen in the remaining set were allocated an $S_{\mathrm{p}}$ of 0 , to apply the rectilinear envelope as in the full model. Previously identified outliers were excluded from the set of record-sites during jackknifing.

$S_{\mathrm{p}}$ and the rectilinear envelope were used to construct a potential distribution. The Gower metric is an exact interpolation technique (section 4.3.1), and does not discriminate between marginal and optimal record sites, which makes it more prone to errors of commission than omission. Distributions are also limited by historical biogeography (the species may not have fully recovered from a previous climate change, or may still be expanding its range), biological competition (the species' niche may be filled by a functionally similar species), and dispersal barriers.

With these factors in mind, additional restrictions were placed on the present distribution (Box 5:1). First, the maximum spatial nearest neighbour distance within the recordset was calculated (between cell centres, so that the minimum is $1^{\circ}$ ). This distance, or $5^{\circ}$, whichever is the smaller, was used to buffer the potential distribution, limiting the estimated distribution to within a set distance of the recorded range. This buffering acknowledges that macroclimate is not the only factor to constrain species' distributions, without specifying which of the alternatives listed above is responsible for each species. The $5^{\circ}$ limit was placed to avoid large buffers resulting from spatial outliers, which would overestimate the range relative to more clustered species. The addition or removal of one record may decide the fate of many surrounding patches. 
Second, a spatial overlay between the estimated distribution and the record-cells was performed. Presence was only modelled where there was evidence that the patch is accessible. It is assumed that any contiguous block of suitable cells that includes a species record is fully occupied, but that other suitable blocks are not. Diagonal linkages are included. This simulates dispersal from the known record points, in a stable environment, until equilibrium. Features that diminish but do not prevent dispersal, such as rivers or locally steep altitudinal gradients, are not considered at the $1^{\circ}$ scale.

\section{Box 5:1: Example of distribution simulation}

Couepia dolichopoda (Chrysobalanaceae) has 11 records, restricted to the northwest of the inclusive-Amazon. No record-cell is a climate outlier. $S_{\min }$ on jackknifing was 0.740 , which included 128 cells within the extended Neotropics, found between $8^{\circ} \mathrm{N}$ and $7^{\circ} \mathrm{S}$. This is the potential distribution for the species (Fig. 5:16). The part that fell within the extended Amazon formed a single spatial group, which included all the record-cells. There was thus no need to exclude any Amazonian cell clusters.

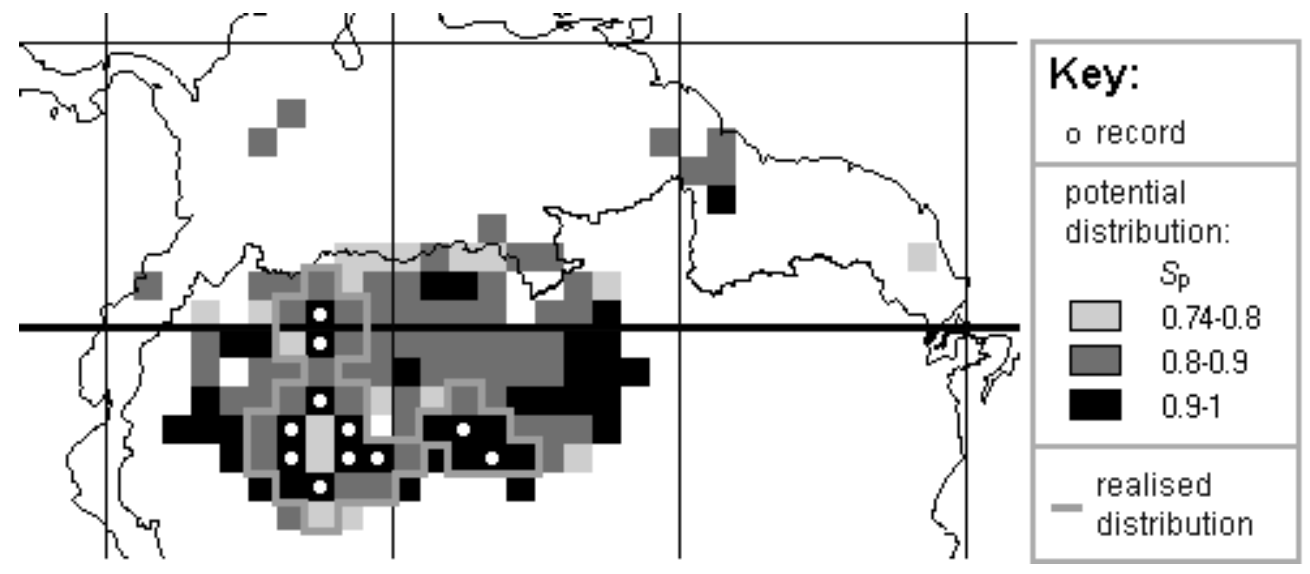

Fig. 5:16: Potential and realised distribution for Couepia dolichopoda, SIS

The maximum nearest neighbour distance was $1.41^{\circ}$. A 1 -cell buffer was applied to the recordcells. Where this spatial envelope corresponded with an area of potential distribution, this was simulated as the realised distribution of the species: 33 cells.

\subsubsection{Population structure and initialisation}

To understand the rate of change as well as the direction, demography must be considered. Population simulation provides a more detailed overview of change than pattern analysis. The detailed data required for an accurate simulation even of present-day populations are not available for Amazonian species, and will not be in the near future. The following approach uses available data to give a 'best guess' at a demographic response to change. 
All life forms were modelled using $k$ size classes (stages, ' $c$ '), where $k$ denotes the maximum size for that species. Different vital rates were allocated to the stages, which were classified by maximum height. A seed (propagule) class and up to six plant stages were included. Reproductive maturity was assigned to at least the maximum stage, with seed output per individual varying by stage. Each stage was populated by a number of cohorts, whose key attributes were germination year, population count and proportion growth achieved towards next stage $(g)$. Other attributes, including vital rates, were shared between all cohorts in a stage. Cohorts moved between stages when $g \geq 1$, and there was space in the subsequent stage. Each stage had a density limit, $K_{\text {cpmax }}$, which depended on the environment for that population.

A single transfer matrix was used to describe the stage-specific rates of survival, death, reproduction and growth for all populations of a species. These rates were based on recorded data (section 5.2.8.12).

$K_{\text {cpmax }}$ (individuals/ha) for each stage above seed and the land area of the grid cell (ha) were multiplied to determine the size of the initial population, $n_{c}$ for each stage $c$. In coastal regions, or if sub-cellular factors were used to determine land availability were used, area would be a more important factor than in this implementation. In any model operation concerning the population size, the number of individuals rather than their density was used, and the outcome rounded down to involve only whole individuals. For example, if 8.9 individuals from a stage were calculated as surviving a model year, this was rounded down to 8 . This rounding speeds the rate of decline of very small populations. In an ideal model, vital rate processes would be implemented as probabilities rather than fractional multipliers, and the entire model would be repeated many times to obtain a frequency distribution of outcomes. As it is, an increased rate of decline in small populations has some basis in ecology (section 2.2.4.2.2).

Initially, $n_{\mathrm{c}}$ for each stage $c$ above seed was divided equally into cohorts of different ages, whose number was equal to the number of years $\left(=1 / G_{c}\right.$, section 5.2.8.7) required to reach the next stage. Any remainder was arbitrarily allocated to the youngest cohort. This type of reallocation was applied in the model whenever the number of individuals affected by a stagelevel procedure did not divide evenly between cohorts. The proportion of growth to the next stage undergone by a cohort was initiated according to its age. The final stage was modelled by a single cohort, as the size or age of these individuals did not affect the model. Maximum lifespan for some species can exceed 1000 years (Condit et al 1995, Martínez-Ramos \& Alvarez-Buylla 1998), which is greater than the timespan simulated here.

Seed cohorts were generated for as many years as the seed viability time. It was assumed that reproductive stages had reproduced at standard rates (section 5.2.8.8) in each year. The number 
of seeds that would have germinated at standard rates in each year following their initialisation was removed from each cohort (section 5.2.8.5).

\subsubsection{Maximum density}

The maximum density for the patch $\left(K_{\text {cpmax }}\right)$ is set by climate conditions. The constraint to $K_{\text {cpmax }}$ via recruitment limitation acts as a surrogate for the level of adversity presented by the climate. Thus, the density relationship reflects the physiological optima for that species, but the spatial distribution is constrained by similarity of environments to the record points.

An exact relationship between population density and environmental variables cannot be inferred from published work for most tropical species. Instead, a 'most limiting factor' (MLF) approach based on notional relationships (Shao \& Halpin 1995, Rutherford et al. 1996, Hackett \& Vanclay 1998) was employed to model $K_{\text {cpmax }}$. An assumption is made about the form of the relationship, and available information is used to formulate a curve.

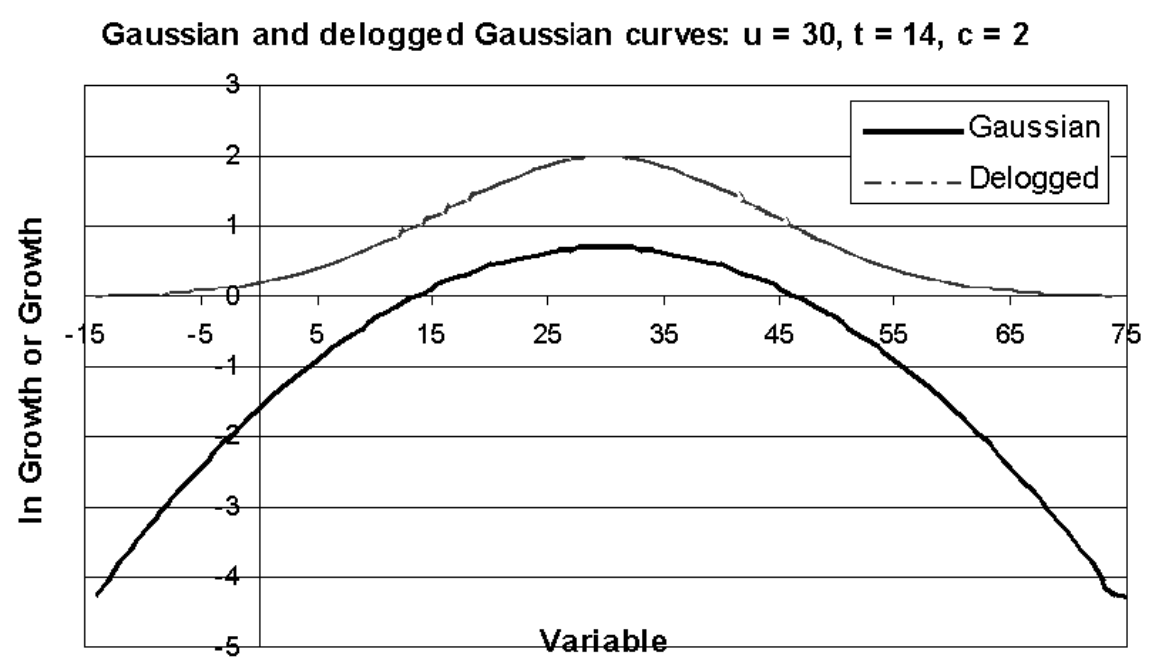

Fig. 5:17: Example of Gaussian curve and underlying relationship

A Gaussian response to each of the three bioclimate variables was assumed for $K_{\mathrm{cp}}$, with the MLF acting in each case. The approach has historically been used to estimate abundance (Ter Braak \& Prentice 1988). From the Gauss curve:

$$
\log K_{\mathrm{cpmax}}=b_{0}+b_{1} x+b_{2} x^{2}
$$

we obtain an ecological interpretation (ter Braak \& Prentice 1988):

$$
\log K_{\mathrm{cpmax}}=a-0.5(x-u)^{2} / t^{2}
$$

where $b_{0}, b_{1}, b_{2}$ are constants, $K_{\text {cpmax }}=$ maximum density for patch $\mathrm{p}, u=$ optimum of variable $x$, $t=$ tolerance of $x, \mathrm{a}=\log \left(K_{\mathrm{cmax}}\right)$, for stage $\mathrm{c}$. 
An example of the procedure that could be used to estimate $t$ with climate-linked density data is given in section 5.2.8.12.3.1. As density data were not available for a sufficient range of environments, $u$ and $t$ were estimated based upon the known environments occupied by each species. A conservative approach to estimating the stage maximum density under optimal conditions, $K_{\text {cmax }}$, was taken here, limiting it to observed levels. Mean $K_{\text {cmax }}$ for the height class was used (Table 5:7) where a density measure was not available for a species.

The response surface optimum $(u)$ and tolerance $(t)$ were approximated using the mean value and standard deviation of the chosen environmental variable for presence sites (ter Braak \& Prentice 1988). Weighting could be applied if quantitative abundance data were available for the entire range, but this was never the case. Weighted averaging approximates the optimum of a Gaussian curve when a species is rare or has a small ecological amplitude, or the distribution of the environmental variables is even over the species range (ter Braak and Looman 1987).

Several disadvantages apply to this technique. First, the assumption that the mean is the optimum can lead to error: if the availability of marginal environments is higher than the availability of optimal environments, this averaging approach will misassign the optimal value for each curve. In addition, a species' likelihood of occurrence often does not translate directly into abundance (van Jaarsveld et al. 1998), and so any method based on presence data will often mis-estimate population size. Second, the greater the number of limiting factors selected, the smaller the abundance at any given site, all else being equal. Finally, the approach doesn't account for interactions between climate variables, which could be especially important under changing conditions.

Any reproductively viable population will attain $K_{\mathrm{cpmax}}$ under equilibrium conditions, unless the vital rates have been specified (section 5.2.8.11) so that mortality exceeds recruitment. The impact of a varying environment on vital rates is disregarded. This approach is therefore conservative with regard to individuals' change tolerance.

\subsubsection{Annual sequence of events}

The model was first run for 100 years under a stable baseline climate. This initial run was performed to allow the intra-stage distribution of cohorts to equilibrate. The DOWNS climate for 1990 was selected as the baseline, to ensure that observed changes in the perturbation model were not a result of a shift from PRES to DOWNS.

In each year, survival, germination, growth, recruitment, and reproduction were modelled in that order for each cohort, and were governed by stage-specific rates, which were not varied between patches. The stages and years were cycled through from eldest to youngest, so that, for example, 
all seedlings must survive for at least one year before being available for recruitment to the $1-4 \mathrm{~m}$ stage.

Where conditions were so restrictive that no individuals were present in the mature stage at the end of the initial equilibrium period, populations were dropped. This situation arose when $K_{\text {cpmax }}$ $=0$ or was so small that pollination limitation prevented reproduction (obligate cross-pollinating species only).

\subsubsection{Germination}

Each seed had an equal probability of germination, P(germ). No limitation was applied to seedling numbers. During the equilibration period, seedling numbers increased or decreased from the initiated level until annual mortality was equal to recruitment.

If $S_{\mathrm{p}}>S_{\min }$, germination was prevented. The implication is that the seed or seedling stage is most intolerant of conditions outside the recorded environment. The intention is to apply the same rules to the projected potential distribution as to the present-day one: a species cannot colonise cells outside its suitable niche.

\subsubsection{Survival}

In each model year, stage-specific survival rates were applied to each cohort prior to recruitment to the stage. Hence, if sufficient recruits were continuously available, $K_{\mathrm{cp}}$ would be maintained

at $K_{\text {cpmax }}$. Individual lifespan was determined by the successive survival probabilities, and the length of time spent, in each stage.

Recorded minimum temperatures for survival were obtained for several selected species (CRFG 1995, Brickell 1998), but the species did not experience temperatures this low under the modelled climates.

\subsubsection{Growth and recruitment}

Growth was modelled at a stage-specific rate $G_{\mathrm{c}}$ (Table 5:7). The proportion of growth to the next stage, $g$, is recorded for each cohort, and reset to 0 upon recruitment to the next stage.

Recruitment was allowed when a cohort's growth was complete and the recipient stage was below maximum density $\left(K_{\text {cpmax }}\right)$. That is, only gaps following the death of conspecifics are modelled as available for recruitment. Recruitment was calculated from the number in the cohort and the deficit in the following stage $\left(N_{\mathrm{c}+1}-n_{\mathrm{c}+1}\right)_{\mathrm{p}}$. If the deficit was not equal to the 
number available, the smaller number was taken. If fewer individuals were recruited than available, the cohort was split between stages. Individuals were recruited from more than one available cohort if the first contained insufficient to supply the deficit.

Immature individuals of pioneer species can only persist in the smaller size classes for a limited period. In some species, individuals that are not recruited within the first year have high $M_{\mathrm{c}}$ in subsequent years (Brokaw 1987), whilst others can persist up to seven years in the understorey. The requirement for early recruitment seems linked to fast growth rates and high light demand, suggesting that shading causes mortality. A simple method of modelling density-dependent mortality to better simulate a shade-intolerant species' growth mode would be to remove individuals for which $g \geq 1$ following recruitment.

\subsubsection{Reproduction}

Reproduction occurred on an annual or interannual basis, according to species. It was calculated last, so that individuals that were recruited to a reproductive stage in that year participated. This would lead to an overestimate where reproduction is limited to the early part of the year. If some current populations are in fact sinks with $\lambda<1$ (section 4.3.7.3.1), then the reproductive niche is a subset of the observed suitable niche. These populations would be lost during equilibration.

Seed production was calculated as:

$$
n(\text { seed cohort })=\Sigma_{\text {mature c }}\left(K_{\mathrm{cp}} * \operatorname{seed}_{c} * \mathrm{P}(\text { reprodv })_{\mathrm{c}} * \mathrm{P}(+) * \mathrm{P}(\text { fert })\right)
$$

where $\operatorname{seed}_{\mathrm{c}}=$ number of propagules per individual, $\mathrm{P}($ reprodv $)=$ the proportion of reproductive plants in the stage, $\mathrm{P}(+)=$ the proportion of plants with female flowers, $\mathrm{P}(\mathrm{fert})=$ the proportion of plants being fertilised.

$\mathrm{P}({ })$ and $\mathrm{P}($ fert $)$ were modelled as equal across all reproductive stages. In selfing or vegetatively reproducing species, $\mathrm{P}($ fert $)=1$. Three species were modelled as selfing and none as vegetatively reproducing. A more detailed model might limit seed production for selfing plants according to recorded levels of seed inviability.

For non-selfing species, the probability that a female flower was within the pollen radius of a male was based on the density of mature individuals with male flowers. Hence:

$$
\begin{aligned}
& \mathrm{P}(\text { fert })=1-\left(1-\left(\left(\pi *((\text { dist }(\mathrm{m})) / 1000)^{2} / \text { cell area }\left(\mathrm{km}^{2}\right)\right)^{(\text {Lmature c }(\mathrm{Kcp} * \mathrm{P}(\text { reprodv }) \mathrm{c})) / \mathrm{P}(P)+1}\right)\right. \\
& \text { where } \text { pdist }=\text { pollination distance. }
\end{aligned}
$$


Individuals were assumed to be distributed randomly within the cell. This is less likely at a low spatial resolution, as internal environmental gradients will increase. It is less realistic for standforming species with clustered local distribution. The alternatives are (I) to simulate annually pollination in a stochastic manner, which incurs an unlikely assumption of mobile individuals, or (II) to track the position of each individual, which is beyond the scope of this project.

Variation in male flower count was disregarded, as was inter-population pollination. $\mathrm{P}$ (fert) would be 0 if a required pollinator or phenological trigger was known to be absent, but these factors were not modelled.

If interactions between patches were to be modelled, adjacent populations would contribute to one another and to newly viable locations via seed dispersal. The fraction of seeds dispersed from a patch would depend upon population density and dispersal distance. However, these processes are not simulated here.

\subsubsection{Perturbation}

Following 100 years of equilibration, the model was perturbed with an annual increment of climate change in the governing variables, from 1991 to 2095. In an ideal model, stochastic variation would be applied to the annual climate variables simulated by a GCM before downscaling to the scale of interest. Multiple repeats of each species model would be run under different randomised climate scenarios to give a range of response.

In this implementation, change was taken from daily calculations of $A E T, P E T$ and $M D$, without a stochastic element, as described in section 5.2.4.6. Species were modelled in the same way under equilibrium and perturbation conditions. Annual change may alter the position of each location on each Gaussian curve, and consequently alter the potential population density of each stage, $K_{\text {cpmax }}$. The curves were assumed to describe a constant relationship with the environmental variables, so were unchanged.

Species were modelled as affected by climate change in two ways. First, $K_{\text {cpmax }}$ in any year was made dependent upon the level of the MLF for that cell. Recruitment was only permitted until that population density was achieved. Second, germination was made dependent upon the suitability index, $S_{\mathrm{p}}$. If the similarity was lower than the cut-off calculated for that species, or an environmental value fell outside the rectilinear envelope, germination was not allowed in that year.

Population loss can therefore occur in two ways: 
- $K_{\mathrm{cp}}$ for reproductive stages (c) may be so small that obligate cross-pollination is prevented, or may be 0 .

- Germination does not occur outside the potential range, but existing individuals may persist for some time.

Lags in response to climate change arose due to model structure. An increase in the limiting factor increases $K_{\text {cpmax }}$, but recruitment to c will not happen until individuals in c-1 have grown to the maximum size for that stage. A decrease in the limiting factor decreases $K_{\text {cpmax }}$, but this lower potential density will not be fulfilled until mortality has removed sufficient individuals from the stage. The changing balance between mortality and recruitment defines the success of a population within any one patch over time. A faster rate of detrimental change would result in faster population decline, and less potential for migration towards newly suitable areas.

Rates of growth, mortality and reproduction were not altered, which lengthens the lag between the species current and long-term potential distribution compared to the alternative of modifying these vital rates according to the environment. This is contradicted by observed mortality responses (e.g. Hubbell \& Foster 1990), but was thought better than applying climate-related changes to vital rates without a basis in empirical data. Survival rates are known to be responsive to environmental change as well as environmental variation. Established plants display acclimatisation to their original environment and find it more difficult to adapt to negative change. This provides scope for future development of the model. Some records of mortality in response to natural change exist (see Lugo \& Scatena 1996 for a review). The BCI plots have yielded information about mortality in response to a gradual change and a drought event (Condit et al 1995, 1996a, 1996b). These data were insufficient to formulate a set of relationships with the bioclimate variables.

\subsubsection{Run under 2095 conditions}

Following the perturbation period, the model run was continued for a further 100 years under a stable 2095 climate. The aim was to investigate species survival under a changed climate in the long-term. Were the GCM continued for such a period, it's simulated climate would be considerably more variable, but would be likely to have a similar decadal mean to the 2095 climate (section 5.2.9).

\subsubsection{Parameters}

The following is a list of all parameters used in species modelling. In estimating these parameters, information was sought from tropical forests throughout the extended Neotropics. 


\section{Species level}

- Life form (tree or liana \& no of stages; bush; herb)

- Stage of reproductive maturity

- Reproductive mode (self-compatibility)

- Pollination distance, pdist

- Reproductive frequency (years)

- Sex ratio (mature plants bearing/not bearing female flowers), $\mathrm{P}($ female)

- Seed viability (years)

- Proportion germinating under field conditions

- Locational data

- Any recorded absolute constraints on vital rates

\section{Stage level}

For these parameters, the field location was recorded, in the hope of accumulating sufficient information to formulate relationships with the environmental variables.

- $\quad$ Maximum recorded density, $K$

- Growth-years required to reach next stage, $G_{\mathrm{c}}\left(Y_{\mathrm{c}} \rightarrow Y_{\mathrm{c}}+1\right)$ (often as dbh growth and maximum dbh for stages)

- Annual survivorship, $1-M_{\mathrm{c}}$

- Proportion of plants reproducing in a given year (rarely recorded), $\mathrm{P}(\text { reprodv })_{\mathrm{c}}$

- Mean seed count per individual (rarely recorded), seed $_{c}$

Trait parameterisation is hierarchical throughout: values from a higher level are substituted if traits from the appropriate level are not available. The ideal is a species-specific parameter derived from autecological study. For example, in the case of pollination by vertebrates, maximum pollination distance may have been recorded for the plant or vertebrate species. It is not unusual to lack this type of basic ecological data for Amazonian species (Pitman et al. 2001). Where autecological data are unavailable, the PFT-level parameter is favoured next. If the trait itself is unknown, the trait most common to the genus or family is allocated.

The derivation of surrogate traits and default values used to supply missing parameters are specified in Table 5:7 and Table 5:8. The PSP analysis identified family, deciduousness, dispersal rate and height class as surrogate traits for growth, family for mortality, and cotyledon class for density. Appendix 5 shows the values and sources of vital rates allocated to PFTs for the different families. 
Table 5:7: Derivation of model parameters from surrogate traits

\begin{tabular}{|l|l|}
\hline Parameter & Derivation \\
\hline$G_{\mathrm{c}}\left(Y_{\mathrm{c}} \rightarrow Y_{\mathrm{c}}+1\right)$ & $\begin{array}{l}\text { Growth/stage: mean for family and deciduousness (dicots) or height } \\
\text { class/dispersal rate combination (palms) }\end{array}$ \\
\hline$M_{\mathrm{c}}$ & Mortality/stage: mean for family \\
\hline$K_{\mathrm{cmax}}$ & Maximum density/stage/unit area: mean for all dicot and all palm species \\
\hline No of stages & Maximum height \\
\hline pdist & Pollination mode (including selfing) \\
\hline
\end{tabular}

Table 5:8: Model parameters with default values in the absence of species data

\begin{tabular}{|c|c|}
\hline Parameter & Derivation \\
\hline $\mathrm{P}($ female $)$ & Tertiary sex ratio (dioecious plants only): 1 unless otherwise recorded \\
\hline $\begin{array}{l}\text { Reproductive } \\
\text { frequency }\end{array}$ & Annual, multiannual, biennial: annual unless otherwise recorded \\
\hline $\mathrm{P}(\text { reprodv })_{\mathrm{c}}$ & $\begin{array}{l}\text { Maximum proportion of plants producing fertile flowers in a reproductive } \\
\text { interval: } 1 \text { unless otherwise recorded }\end{array}$ \\
\hline $\mathrm{L}_{\text {seed }}$ & Mean seed viability (years): 1 unless otherwise recorded \\
\hline seed $_{c}$ & $\begin{array}{l}\text { Propagule count/individual/stage: estimates were available for only six } \\
\text { databased species }{ }^{12} \text {. A regression with height class was carried out to generate } \\
\text { a standard seed count for an individual in any stage }\left(\operatorname{seed}_{c}=791.4 \text { height). }\right.\end{array}$ \\
\hline $\mathrm{P}($ germ $)$ & $\begin{array}{l}\text { Germination rate: estimates were available for only nine databased species. } \\
\text { The mean rate was applied to all other species (based on BCI data from } \\
\text { Garwood et al. 1983) }\end{array}$ \\
\hline
\end{tabular}

\subsubsection{Ecological data}

\subsection{Species ranges}

Most of the species locations come from taxonomic monographs, where the sampling methods are largely opportunistic and there is no distinction between absence records and missing values. The classical statistical assumptions of random spatial sampling and lack of spatial bias are not met by these data. This is typical of species atlases and distribution maps.

Many of the supplementary locations are derived from single-site lists, which may add further to the inherent spatial bias. Monographs have a high taxonomic reliability, and site lists a high spatial reliability. When collecting site data, sites that are environmentally distinct from those in the recorded set are valuable in defining species tolerances, and those that are spatially distant are valuable in defining realised range.

\footnotetext{
${ }^{12}$ Total numbers of seeds produced by trees and bushes are rarely recorded. The number of seeds per fruit, fruits per flower head and flowers per individual are sometimes recorded. Seed counts per stage can be estimated from these values.
} 


\subsection{Diameter-height classes for growth estimates}

The model requires an estimate of the time taken for a plant to grow from one height class to the next. PSP growth data are typically recorded using dbh or basal area, without height records, because this is easier to measure from the ground. There is a standard formula linking dbh and height for a wooden cylinder that is just thick enough to avoid bending under its own weight (Table 5:9, McMahon 1973). In practice, each species has its own dbh-height relationship, related to form and crown size (King 1996), which gives diameters greater than this. As most species appear at low frequencies and as height is rarely recorded with dbh, it is difficult to identify the form of each relationship.

The maximum dbh and height at maturity from herbarium records or monographs were recorded for 250 databased neotropical species. Arecaceae (palms) have limited secondary stem thickening, so after the first growth their diameter would be expected to increase less with height than that of dicotyledonous species (dicots). When grouping by height class, there is a significant difference between palm and dicot maximum dbhs (two-tailed $\mathrm{p}<0.05$ in all cases, Mann-Whitney U-test) for all but the smallest height class (1-4 m), for which there were insufficient data. Kruskal-Wallis tests comparing the dicotyledonous families show no significant difference between them.

The median values from each height class, except $25 \mathrm{~m}+$, are used to specify minimum diameter limits for the subsequent class (Table 5:9). Growth rates at different sizes are later used to estimate the time required to move between height classes. Increased height in canopy trees may increase exposure to light more than increased diameter, making height class a better indicator of growth rate.

Table 5:9: Diameter values for height classes

\begin{tabular}{|c|c|c|c|c|c|}
\hline Height class & $\begin{array}{l}\text { McMahon } \\
\text { diameter at } 0.1 * \text { height }(\mathbf{m})= \\
0.0011 * \text { height }^{1.5}(\mathrm{~m})\end{array}$ & $\begin{array}{l}\text { Arecaceae } \\
\text { median of } \\
\text { max dbh }\end{array}$ & Count & \begin{tabular}{|l|} 
Dicot \\
median of \\
max dbh
\end{tabular} & Count \\
\hline $1-4 \mathrm{~m}$ & $0.88 \mathrm{~cm} @ 0.4 \mathrm{~m}$ & $3 \mathrm{~cm}$ & 10 & $8 \mathrm{~cm}$ & 1 \\
\hline $4-10 \mathrm{~m}$ & $3.48 \mathrm{~cm} @ 1 \mathrm{~m}$ & $7 \mathrm{~cm}$ & 17 & $25 \mathrm{~cm}$ & 6 \\
\hline $10-20 \mathrm{~m}$ & $9.84 \mathrm{~cm} @ 2 \mathrm{~m}$ & $18.5 \mathrm{~cm}$ & 16 & $30 \mathrm{~cm}$ & 64 \\
\hline $20-25 \mathrm{~m}$ & $13.75 \mathrm{~cm} @ 2.5 \mathrm{~m}$ & $23 \mathrm{~cm}$ & 5 & $40 \mathrm{~cm}$ & 25 \\
\hline $25 \mathrm{~m}+$ & $22.78 \mathrm{~cm} @ 3.5 \mathrm{~m}$ for a $35 \mathrm{~m}$ tree & $30 \mathrm{~cm}$ & 5 & $60 \mathrm{~cm}$ & 101 \\
\hline
\end{tabular}

There is some field evidence that height class is a reasonable classifier with which to allocate rates of reproductive success: for example, in Couratari multiflora, flowering levels correlate more with height than with dbh (41 individuals, Lepsch-Cunha \& Mori 1999). 


\subsection{Species functional data sources}

Where species vital rates were available, these were used in the model. Although many traits can vary over a species' range, variations in survival and growth rates are most often recorded. Survivorship and growth under optimum conditions were estimated as the greatest site-mean values. If no mortality was recorded for the species, the PFT mean was substituted.

\subsection{Estimating vital rate relationships with climate}

In an ideal model, vital rate relationships with climate would be specified independently through GLM with the set of environmental variables. This requires vital rate data from a set of plots randomly sampled throughout the species' occupied climate space. As the impact of climate change varies with size (Condit et al. 1995), an empirical formulation of the effect of climate on each stage is preferable. The initial assumption is that the rates would form a unimodal relationship with the climate variables. Computation of the mean and confidence intervals of vital rate data from increasing sample sizes from existing records would be used to ensure a sufficient sampling size with a set degree of confidence for each species combination (Krebs 1989). $F_{\mathrm{c}}$, the mean number of seeds produced, will also be affected by population density for obligate cross-pollinators, so this data should be gathered only from individuals known to have been pollinated. Obtaining all this data for species with a characteristic low density is a challenging task, so approximations need to be made.

It is assumed that species' vital rate responses to the environment can be approximated using a Gaussian curve. Where vital rate information is available over a wide range of environments, the curve would be parameterised using a Gaussian GLM with a log link function and Poisson error distribution (ter Braak \& Prentice 1988). Otherwise, the optimum $(u, \exp a)$ and amplitude $(t)$ of the curve must be sourced from available data.

Hirtella triandra (Sw.) is given here as an example of a well-known species. It is recorded in 214 degree cells, and was selected to represent the 'evergreen tree of $25 \mathrm{~m}+$ whose distributors are known to include arboreal and terrestrial mammals' PFT for the Chrysobalanaceae. It is assumed to be insect pollinated as this is typical for the genus. Records indicate that the species can attain reproductive maturity in the 1-4 m height class. Each fruit contains a single seed, but it is not known how many fruits are produced at different life stages.

Survivorship data were held for cells SW012069 (based on 11 Peruvian individuals: source as Phillips et al. 1998) and NW009079 (based on three surveys on BCI, one using 519 individuals: Welden et al. 1991, Milton et al. 1994, Condit et al. 1995). Growth and density data were held for NW010084 (based on 31 individuals at La Tirimbina Farm, Costa Rica: Finegan et al. 
1999). $100 \%$ of tested seeds germinated in NW009079 (Welden et al. 91). Only the NW009079 mortality data were stage-classified.

To estimate the tolerance of a rate-limiting environment variable for a species' vital rate, the minimum data required are a maximum rate estimate for that species and a rate measurement at a site where that variable is the MLF. The maximum rate is approximated here by its highest recorded value. When the 'best' rate is the only recorded value for a cell, that cell can't be used to calculate tolerance. This emphasises the flaw in the assumption that the highest recorded rate is the actual optimum.

Table 5:10: Maximum recorded vital rates for Hirtella triandra

\begin{tabular}{|c|c|c|c|c|c|}
\hline & \multirow{2}{*}{$\begin{array}{l}\text { Trees / ha, } \\
>10 \mathrm{~cm} \text { dbh }\end{array}$} & \multicolumn{4}{|c|}{ Growth, cm/y, Survival probability / year } \\
\hline & & $>10 \mathrm{~cm} \mathrm{dbh}$ & $1-9.9 \mathrm{~cm} \mathrm{dbh}$ & All $>10 \mathrm{~cm} \mathrm{dbh}$ & All >19 cm dbh \\
\hline Value & 11 & 0.5 & 0.991 & 0.995 & 0.989 \\
\hline Source & Finegan et al. & 1999 & $\begin{array}{l}\text { Condit et al. } \\
1995\end{array}$ & $\begin{array}{l}\text { data as Phillips } \\
\text { et al. } 1998\end{array}$ & Milton et al. 1994 \\
\hline
\end{tabular}

Following Table 5:9 the measurements from Table 5:10 can be roughly assigned to $>4 \mathrm{~m}$ height (based on $8 \mathrm{~cm}$ minimum) if $>10 \mathrm{~cm}$ dbh and $>10 \mathrm{~m}$ height (based on $25 \mathrm{~cm}$ minimum) if $>19$ cm dbh.

To find the MLF, a measure of tolerance needs to be assumed so that the site's location on a Gauss curve can be found. The standard deviation of the occupied environment was used to identify the MLF, which was SMA for SW010084, and AET for SW012069 and NW008080. It was therefore not possible to estimate tolerance to $M D$ for $H$. triandra using this method.

Rearranging Eqn. 25:

$$
t=\sqrt{ }\left(\left(-0.5(x-u)^{2} /(\log y-a)\right)\right.
$$

There was only one source of growth data, but it had values from three plots. The weighted mean rates for the cell $\left(0.36 \mathrm{cmy}^{-1}\right)$ and "optimum" $\left(0.5 \mathrm{cmy}^{-1}\right)$ gave an SMA tolerance of 104.3 days for dbh growth of trees $>10 \mathrm{~cm}$ dbh. There was insufficient information to find stageclassified tolerances for all environmental variables.

There is a reasonable basis for assuming that mortality is constant in trees past the sapling stage, and the similarity of the data support this. The Milton et al. (1994) value of 0.989 can be used to calculate $S M A$ survival tolerance for BCI trees $>10 \mathrm{~m}$ height, assuming that the optimum rate is 0.995 ! However, AET, MD and SMA were not calculated for NW009079 as it is absent from the soil maps (Buckley \& Warrilow 1988). Values from the adjacent Panama cell NW008080 are taken for purposes of this example analysis. This calculation gives an AET tolerance of 1051 
$\mathrm{mmy}^{-1}$ for survival. (Such missing-value cells are not used in the model). For saplings, using BCI data, the mean survival probability is 0.988 , and the optimum is 0.991.This yields an AET tolerance of $2361 \mathrm{mmy}^{-1}$ for survival.

The large values result from the small variation in mortality rates relative to the variation in AET (mean for $H$. triandra $=1409 \mathrm{mmy}^{-1}$, mean for NW008080 $=1276 \mathrm{mmy}^{-1}$ ). H. triandra is widespread tree, so would be expected to have a high tolerance to environmental variation.

Only a few variable/species combinations could be assessed even with this limited level of data. It was therefore decided to use standardised vital rates for species and PFTs, omitting rather than fabricating a response to climate.

\subsection{PFT data sources}

Site-specific life history data was located for only 18 of the selected species, and these data are very scanty (Appendix 4). These species are all widespread, and so assumptions based on them may be inappropriate for those with a narrow range. Forest mensuration data are more often available for mortality and growth of all trees rather than for individual species. Appendix 5 documents the vital rate data used for the PFTs. The locations of sites from which the life history data are sourced are a very limited set.

The ideal approach is to estimate PFT parameters from the mean of those of their species. All databased Amazonian species for which data were held for that parameter were used in the PFT estimates. Species means were used rather than abundance-based means, to prevent the most common species from dominating the results. Growth and mortality rates were calculated using combinations of trait parameters and family (Table 5:7). Data on seed loss from predation and disease were included in seedling mortality estimates. Seeds lost from the parent were excluded from the seed count estimate. Other missing traits or values were estimated as the modal value for the life form for that family.

Where a combination cannot be devised from the available data, summary data for all dicot or all palm species was used instead (Appendix 5). The two groups differ due to greater species richness of dicots and mean density of palm species, as well as in growth mode. 


\subsubsection{Technical description of model}

\subsubsection{Model development}

The model was coded in Microsoft Visual Basic v6.0. Plant and climate data were accessed from and stored in a Microsoft Access 97 database, using a DataEnvironment object. In addition, various spatial functions were accessed through the freely available Applications Programming Interface for IDRISI for Windows v2 (Clark Labs, 1999). The model was run for each species separately.

\subsubsection{Distribution model}

The distribution model compares climate and species distribution, and stores the results. The PRES reference climate (AET, SMA, MD and $T_{\min }$ ) for the $17551^{\circ}$ cells is loaded into an array, arrCells. An array of species' locational records and their mixed reference bioclimate (section 5.2.4.7) is also loaded (arrRecs). Each record-cell is represented in the arrRecs three times, accommodating the three decadal DOWNS climates for extended-Amazon records and duplicating the PRES climate for extra-Amazon records. This latter step was taken to simplify the treatment of both record-cell types. Cells with climate values further than three interquartile ranges from the first or third quartile of any bioclimate variable (AET, SMA and MD) in arrRecs are dropped from arrRecs (Fig. 5:18a).

The array is queried to run the jack-knifing routine, fnJack, to determine a minimum suitability $(S)$ level, $S_{\min }$. fnJack calls the fnSuit function once for each record-cell climate, and stores the lowest positive $S_{\mathrm{p}}$ value for that cell (p) in a temporary array. In fnSuit, arrRecs is crossreferenced with a candidate cell from arrCells (or from arrRecs itself if called by fnJack). If the candidate cell falls outside the record-set's rectilinear envelope for any bioclimate variable, $S_{\mathrm{p}}$ is set to 0 . Otherwise, the candidate cell's Gower similarity to each record-cell climate (excluding any alternative climates for the candidate cell) is calculated (Eqn. 6, p.106). The function returns the maximum $S_{\mathrm{p}}$ value for the candidate cell.

In a small set of record-cells, each jack-knifed cell may fall outside a rectilinear envelope, giving rise to $S_{\mathrm{p}}=0$ and the conclusion that a simulated population must reside in a cell with identical climate values identical to one of the record-cells. To avoid this unlikely outcome, $S_{\min }$ is arbritarily set at 0.99 in this case. Note that cells are not excluded from the accepted recordset because they fall outside the rectilinear envelope in jack-knifing. 


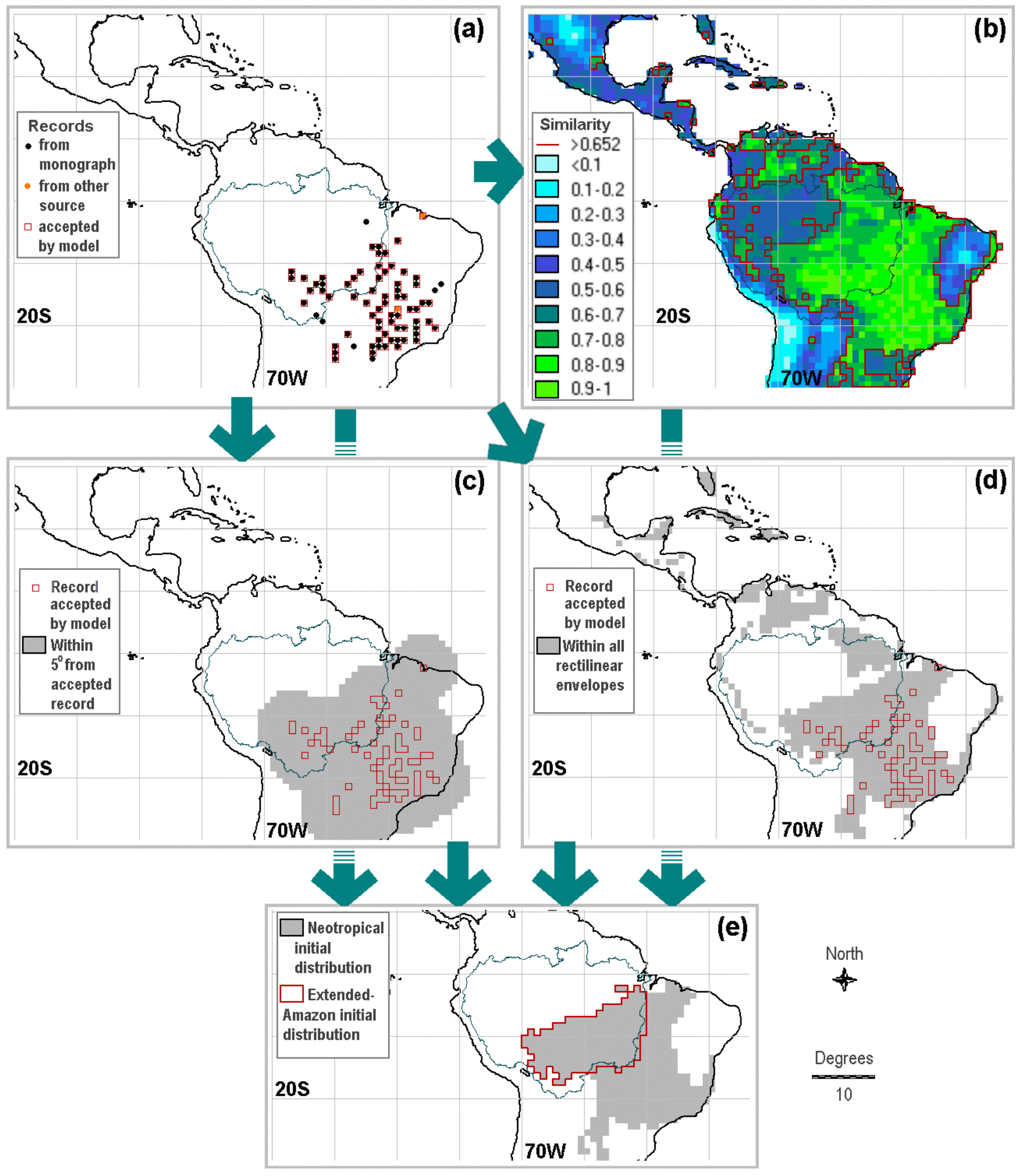

Fig. 5:18: Steps to simulating realised distribution, for Caryocar brasiliensis under SIS:

(a) recorded and accepted records; (b) maximum similarity to accepted records under mixed-reference bioclimate; (c) $5^{\circ}$ spatial buffer from accepted records; (d) areas inside all rectilinear envelopes; (e) Simulated initial distribution for extended Neotropics and extended Amazon. Records: Prance \& Plana (1997), INPA (2000b) \& EMBRAPA (2000b) 


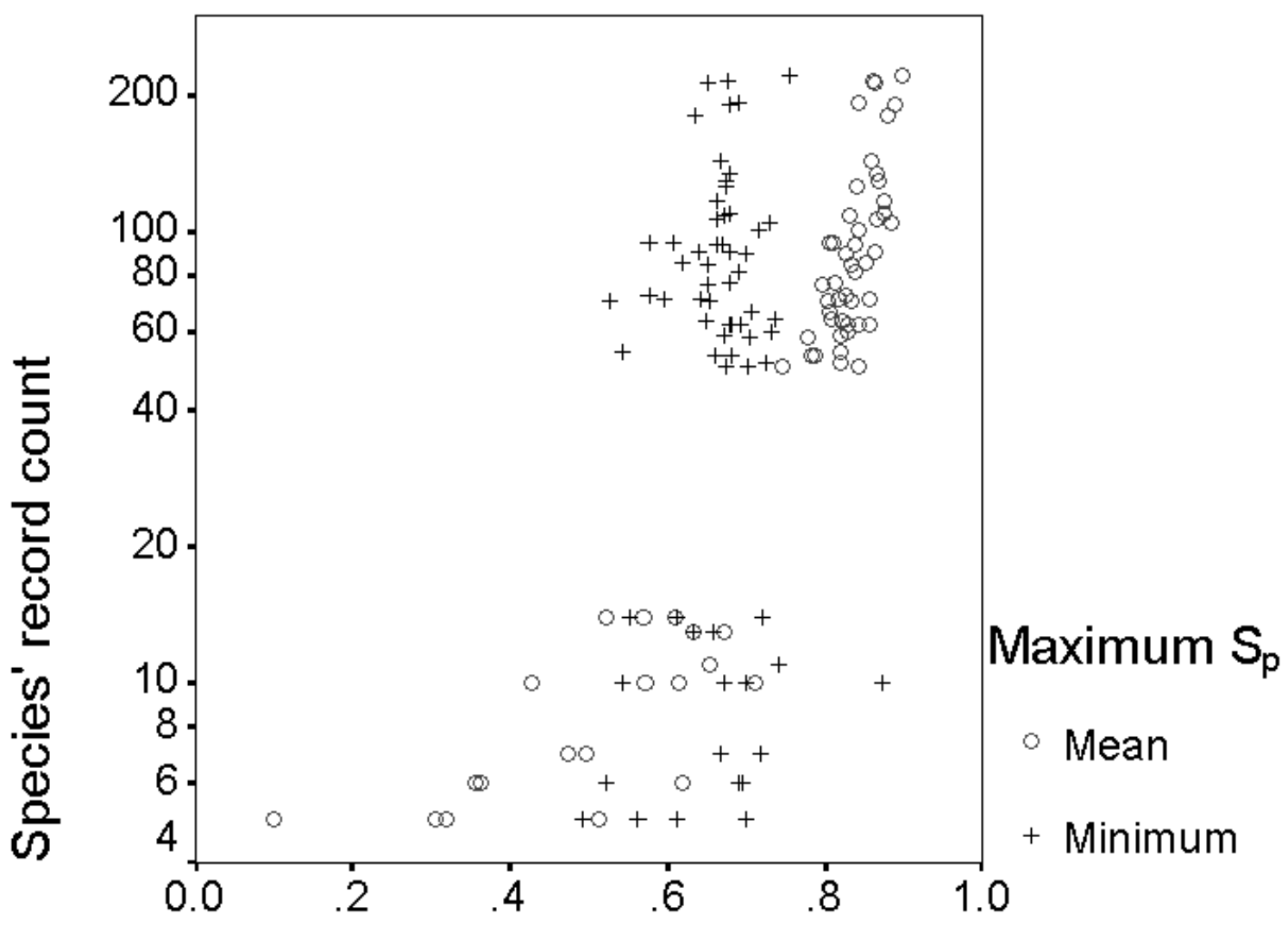

\section{Jack-knifed similarity}

Fig. 5:19: Mean and minimum of the maximum similarity values for each jack-knifed record, plotted against record count, for all 69 species

This jack-knifing method demonstrates (a) that there is great variation in the appropriate $S_{\text {min }}$ value for species of different record counts; (b) that record count has a distribution similar to that of species-mean jack-knifed $S_{\mathrm{p}}$ (Fig. 5:19, Wilcoxon signed ranks test, 1-tailed $\mathrm{p}<0.001$ ), as there is a greater likelihood that any given excluded record will have a similar member in the set when there are more records; but (c) that there is no significant relationship between $S_{\min }$ and record count.

Following the exclusion of outliers and determination of $S_{\min }$, the distribution model itself is run. $S_{\mathrm{p}}$ values are calculated as above, and stored in arrCells alongside the environment variables. Cells where $S_{\mathrm{p}}>S_{\min }$ represent the potential distribution at this point (Fig. 5:18b).

The maximum nearest neighbour distance within the recordset is found via Pythagoras' theorem. This value, or $5^{\circ}$, whichever is the smaller, is set as the buffer distance. A Boolean map of cells within this distance of the accepted records is created using IDRISI modules DISTANCE and RECLASS (Fig. 5:18c). The $S_{\mathrm{p}}$ values and cell IDs are output to a plain text file. ASSIGN and 
GROUP are called to produce a similarity map (Fig. 5:18b), and identify contiguous groups of cells in which $S_{\mathrm{p}}>S_{\min }$ and a record location exists. 'Doughnuts' produced by GROUP are removed in an OVERLAY operation. OVERLAY is again used to produce a final simulated distribution map including only cells that satisfy the similarity, contiguity and proximity criteria (Fig. 5:18e). Cell IDs are identified using EXTRACT. This set of cells represents the realised distribution for the species under present conditions.

The level and identity of the most limiting factor (MLF) for each of these cells is identified, based on the mean and standard deviation of each bioclimate variable in arrRecs, following the method in section 5.2.8.3. Populations are initialised in these cells as described below.

\subsubsection{Population structure and initialisation}

The life history parameters (section 5.2.8.11) allocated to this species are read from the database. The number of life stages, maximum density and typical vital rates of growth, survival and reproduction are treated as standard across the species. There is scope for testing the sensitivity of the model to individual parameters by altering them at this stage.

An object-oriented modelling strategy was adopted to allow a flexible number of cohorts in each cell, with individual properties assigned to each. An object is a combination of code and data that is treated as a unit. Different types of objects are defined by the class that they belong to. Each class determines the methods, events and properties that belong to its objects. Objects from a collection class have the ability to contain other objects of a specified type.

All simulated populations for the species are created in arrPop, an array of objects of userdefined collection type $c$ Patch. These objects have the properties intS4, which stores the cell ID for that population, and boolD, which records whether the patch has lost its population. Each cPatch can hold multiple objects of user-defined collection type $c$ Stage, which in turn can hold multiple user-defined Cohort objects. For each stage, $c$, in each patch, $p$, the initial density of individuals is set as $K_{c p m a x}=K_{c p} *$ MLF level.

Each population is initialised as a cPatch holding a number of stages (cStages) equal to the number of stage classes for that species. Each cStage is initialised with a number of Cohorts equal to the number of growth years $\left(\mathrm{Y}_{\mathrm{c}}=1 / G_{\mathrm{c}}\right)$ required for a newly recruited cohort to reach the next stage (section 5.2.8.2). $\mathrm{Y}_{\mathrm{c}}$ is limited to provide a maximum estimated stage lifespan of the number of years taken for the starting population to be reduced to $1 \%$ of its initial level (Eqn. 29). The final stage is not divided into cohorts, as there is no need to track growth or age.

$$
\text { Cap on } Y_{c}=\log (0.01) / \log \left(M_{c}\right)
$$


A Cohort object has the properties Key, holding the germination year, $d b l G r$, the proportion of growth achieved towards the next stage, and $d b l C t$, the number of individuals in the cohort. For example, for the youngest Cohort in stage 2, Key $=-Y_{1} . d b l C t$ is initialised as $K_{\text {cpmax }} / \mathrm{n}($ Cohort $)$, with any remainder allocated to the youngest Cohort. $d b l G r$ is initialised as $\frac{\left(K e y-\sum_{1}^{c-1} Y\right)}{Y_{c}}$.

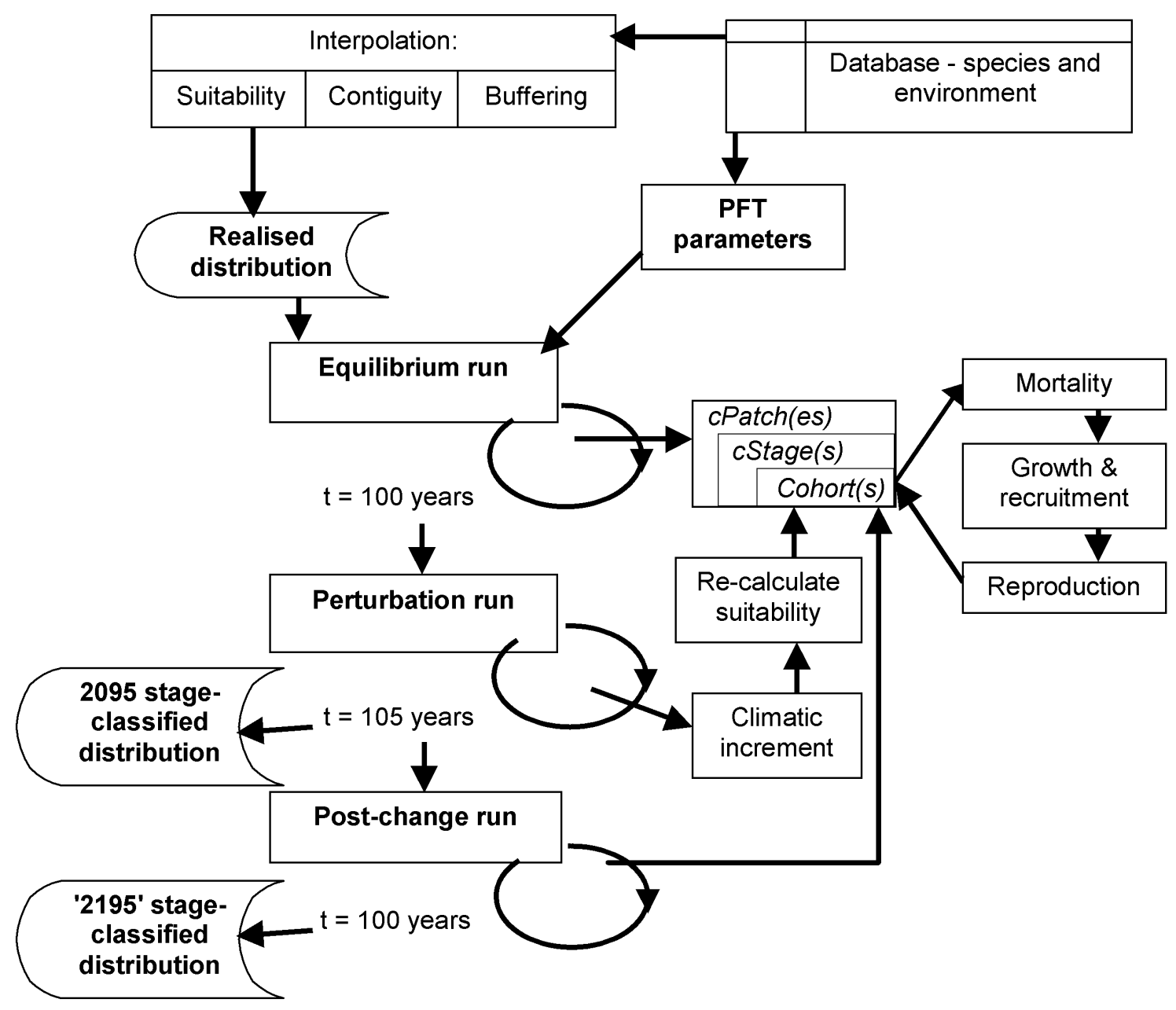

Fig. 5:20: Model processes through simulated time

\subsubsection{Annual sequence of events}

To simulate equilibrium conditions, fnTransfer is called once for each of 100 modelled years (Fig. 5:20). It first checks whether each population has been lost $($ cPatch.boolD $=$ False $)$. Emptied $c$ Patches are ignored; for the remainder, the vital rates are applied as follows:

To simulate survival from the previous model year, the function applies a stage-specific fixed mortality rate to each Cohort. Any emptied Cohorts are dropped from the model. If there is a 
difference between the number of survivors found by applying the rate to the cStage and the sum of survivors obtained through application to Cohorts, the difference is (arbritarily) added to the youngest Cohort. This procedure was followed whenever a stage-specific vital rate was applied to individual Cohorts.

Seed Cohorts older than the seed viability parameter assigned to that species are dropped from the model.

Recruitment is simulated by cycling through the cStages between seed and final stage, largest first. Recruitment occurs where Cohort.dblGr $\geq 1$ and $K_{\mathrm{c}+1 \mathrm{p}}<\mathrm{K}_{\mathrm{c}+1 \mathrm{pmax}}$ (section 5.2.8.7). A new Cohort is created in the following stage with $d b l G r=0$, unless this is the final stage, where growth is not tracked, and recruited individuals are added to the single original Cohort. Depending upon mortality, Cohorts may be completely recruited in this way and dropped from the donor $c$ Stage, or some individuals may remain. Recruits are arbritarily sought first from the youngest Cohort where $d b l G r=0$, until $K_{\mathrm{c}+1 \mathrm{p}}=\mathrm{K}_{\mathrm{c}+1 \mathrm{pmax}}$.

A single new seed Cohort is created by cycling through the $c$ Stages defined as reproductive for this species and summing the propagules produced. If the species is an interannual reproducer, reproduction occurs when (model year)/(reproduction frequency) $=0$. The number of males in all stages and number of females in the stage under consideration are calculated using this species' fixed sex ratio. The probability of fertilisation for any given female is found as in Eqn. 18, p.130. This proportion of plants with female flowers from the cStage is then allowed to produce a fixed number of propagules specified for that stage and species.

Germination is allowed only in years when $S_{\mathrm{p}} \geq S_{\min }$. A fixed species-specific germination rate was applied to all seed Cohorts, creating a single new seedling Cohort. No limit to the number of seedlings produced was applied except for herbaceous species. For these, recruitment was limited to constrain the density of mature individuals as for later stages in other species.

\subsubsection{Perturbation}

At the end of the equilibrium iteration, a perturbation iteration is initiated. This process continues for 105 model years, from 1990 to 2095. This only differs in that the bioclimate variables in arrCells are updated with stored values for each successive year of the change model. Each $S_{\mathrm{p}}$ and MLF is then recalculated via fnSuit.

$K_{\text {cpmax }}$ for that year is reset according to the level of the MLF. The new value may prevent or increase recruitment. If $S_{\mathrm{p}}<S_{\min }$, germination is prohibited in that year. 


\subsubsection{Run under 2095 conditions}

The final iteration is identical to the equilibrium run, with arrCells maintained at its 2095 state.

\subsubsection{Outputs}

The model stores information about the run specifications $\left(S_{\min }\right.$, extreme values and accepted records) and each model year from initiation to 2195 (number of individuals per $c$ Stage for each extant $c$ Patch, with $S_{\mathrm{p}}$ and level and identity of MLF) in a results database. This can be queried to produce maps and statistical outputs.

\subsubsection{Modelling scenarios}

A range of species response can be identified by comparing outcomes for different species under different change scenarios. Ideally, a full range of climate forcing scenarios using models from different centres would be included (Hulme et al. 1999), which would have entailed running both climate and species models multiple times. In practice, two scenarios were used for each of the 69 selected species: a 'standard-impact' climate scenario (SIS) and a 'reducedimpact' scenario (RIS). SIS is an approximately doubled $\mathrm{CO}_{2}$ scenario, and RIS represents a change of exactly half that magnitude.

SIS uses the bioclimate variable outcomes forced by the HADCM2GSa1 model as described in section 5.2.4. For RIS, the effects of climate change for each variable in each cell have been halved. Scaling by a single coefficient (a global mean climate sensitivity value) is also undertaken by climate modellers investigating ranges of change without resorting to further GCM runs (Betts, 14 November 2001), so this approach is compatible with common practice.

Different record sites for a single species may be dropped as outliers under the slightly different climates of the two scenarios. This would be expected to cause some differences between species simulations under SIS and RIS.

A quarter of the increase in $\mathrm{CO}_{2}$ concentrations is still present in the atmosphere several centuries after emissions occur. When concentrations have stabilised global mean temperature rise is expected at a level of a few tenths of a ${ }^{\circ} \mathrm{C}$ per century, rather than several ${ }^{\circ} \mathrm{C}$ (Houghton $e t$ al. 2001). For these reasons, it is assumed to be reasonably valid to hold global climate at a doubled $\mathrm{CO}_{2}$ level for a further century in the equilibrium run following SIS and RIS. The lack of variation is not intended to be climatically accurate - rather, it is a simple way of examining plant's ongoing response to a certain level of mean change. A similar approach was followed in Cramer et al. 2000. 


\subsubsection{Methods for hypothesis testing}

The hypotheses under investigation are listed in section 5.1.4. The present section details the measures of climate impact on species, and the types of test that are appropriate for use with different combinations of data, to avoid repetitions of this information in the Results chapter.

\subsubsection{Measures of impact}

Model outcomes for individual species will be examined using several measures of the impact of change, for use with the numbered hypotheses listed:

1) The proportion of populations lost during the course of the model (H1).

2) The presence of overlapping cells between present and future potential distributions (H2).

3) The proportion of populations that are viable $\left(S_{\mathrm{p}}>S_{\min }\right)$ under 2095 conditions in the longterm, versus the number of non-breeding populations $(\mathrm{H} 1)(\mathrm{H} 2)$.

4) Spatial change in potential $\left(S_{\mathrm{p}}>S_{\min }\right)$ and absolute (buffered record-cell-contiguous $\left.S_{\mathrm{p}}>S_{\min }\right)$ distributions between 1990 and 2095 (H1) (H2).

5) The minimum distance separating 1990 and 2095 potential distribution ( 0 if overlapping, or not applicable if $S_{\mathrm{p} 2095}>S_{\min }$ throughout the inclusive-Amazon) (H2).

6) The population density as a proportion of each species' potential maximum, $K_{\mathrm{kp}} / K_{\mathrm{kmax}}$ for $k$ = last stage, at 1990, 2095 or 2195 (H3).

7) The location of the 'best' cell for the species under 2095 conditions. Species populations were categorised as 'viable', 'potentially migrating' or 'relict', where 'potentially migrating' populations were relicts with an adjacent suitable cell under 2095 conditions. If new potential distribution was simulated in an area separate from the original distribution, it was assumed that the species could not migrate to this area within the 200-year model period.

Where a species had viable populations, the one with the maximum density at 2195 was chosen as 'best'. Otherwise, if it had potentially migrating populations, the pair of relict and receptive adjacent cells with the maximum density in the relict was selected, following the logic that a high density cell was more likely to be able contribute propagules to the new cell. If neither viable nor potentially migrating populations existed, the relict with the highest density was chosen as best. This was assumed to be the population with the best chance of survival until more favourable conditions prevailed. Where there was a tie for density, the tied cell with the highest $S_{\mathrm{p}}$ was chosen - having environmental conditions closest to the recorded distribution $(\mathrm{H} 4)$.

8) Mean population change as a proportion of 1990 population size $\Delta K_{\mathrm{kp}}(1990 \rightarrow 2095 / 2195)$, e.g. $\left(K_{\mathrm{kp}}(2095)-K_{\mathrm{kp}}(1990)\right) / K_{\mathrm{kp}}(1990)$ for $c=$ last stage (H5) (H6) (H7) (H8). 


\subsubsection{Parametric and non-parametric tests employed}

Parametric tests require normality in the datasets tested (Table 5:11). Where the null hypothesis is that the observed distribution function cannot be distinguished from normal, a two-tailed Kolmogorov-Smirnov test can be used to assess this. The t-test ANOVA also assumes that each category comes from an underlying symmetric distribution with homogeneity of groups' variance (tested using Levene statistics). Non-parametric alternatives with exact or Monte Carlo significance were substituted if these assumptions were not met. These tests do not make assumptions about the population distribution.

An exact test takes the groups being compared and repeatedly shuffles the values to calculate the test statistic for every possible combination of the observed numbers. The test statistic for the dataset, $a$, is also calculated as usual. The exact p-value is the frequency at which $a$ occurs within the collection of statistics, rather than the standard p-value for that test statistic distribution. That is, if it occurs less than $5 \%$ of the time, the null hypothesis is rejected at the $95 \%$ level.

Due to computational limitations, there is a 30-case suggested maximum for exact tests (SPSS 1998). A Monte Carlo estimate of the exact significance level is based on a limited number of resamples from a reference set of tables with the same dimensions, row and column margins as the observed table (SPSS 1998). The Monte Carlo option with resampling 10,000 times was used to substitute for exact testing in these circumstances.

Table 5:11: Statistical tests (Siegel \& Castellan 1998, SPSS 1998)

\begin{tabular}{|c|c|c|c|}
\hline Hypothesis type & Data description & $\begin{array}{l}\text { Parametric } \\
\text { alternative }\end{array}$ & $\begin{array}{l}\text { Non-parametric } \\
\text { alternative }\end{array}$ \\
\hline Data normal? & $\begin{array}{l}\text { Single continuous } \\
\text { variable }\end{array}$ & - & $\begin{array}{l}\text { 2-tailed Kolmogorov- } \\
\text { Smirnov test }\end{array}$ \\
\hline Data of equal variance? & $\begin{array}{l}\text { Continuous variable with } \\
\text { categories to compare }\end{array}$ & - & Levene statistics \\
\hline $\begin{array}{l}\text { Difference between two } \\
\text { unrelated groups }\end{array}$ & Two continuous variables & Unpaired t-test & Mann-Whitney U-test \\
\hline $\begin{array}{l}\text { Difference between two } \\
\text { related groups }\end{array}$ & $\begin{array}{l}\text { Two continuous variables } \\
\text { for same cases }\end{array}$ & Paired t-test & $\begin{array}{l}\text { Wilcoxon paired } \\
\text { signed-ranks test }\end{array}$ \\
\hline \multirow{2}{*}{$\begin{array}{l}\text { Difference between } \\
\text { three or more unrelated } \\
\text { categories = } \\
\text { relationship between } \\
\text { continuous dependent } \\
\text { variable and one or } \\
\text { more categorical } \\
\text { independent variables }\end{array}$} & $\begin{array}{l}\text { Continuous variable with } \\
\text { nominal categories }\end{array}$ & $\begin{array}{l}\text { t-test ANOVA } \\
\text { (one-way) }\end{array}$ & $\begin{array}{l}\text { Kruskal-Wallis H-test } \\
\text { ANOVA }\end{array}$ \\
\hline & $\begin{array}{l}\text { Continuous variable with } \\
\text { ordered categories }\end{array}$ & $\begin{array}{l}\text { t-test ANOVA } \\
\text { (one-way) }\end{array}$ & $\begin{array}{l}\text { Jonckheere-Terpstra } \\
\text { ANOVA }\end{array}$ \\
\hline $\begin{array}{l}\text { Linear correlation } \\
\text { between variables? }\end{array}$ & Two continuous variables & $\begin{array}{l}\text { Pearson's } \\
\text { correlation } \\
\text { coefficient }\end{array}$ & $\begin{array}{l}\text { Spearman's rho } \\
\text { coefficient }(+1 \text { to }-1)\end{array}$ \\
\hline
\end{tabular}




\section{Results}

This chapter first examines the bioclimate model outcomes for present and changing climates, and then the results of the species response model forced by these. Species' outcomes are considered separately and shared patterns of change are identified.

Relationships are then sought between species' fate and their family, functional traits, current occupied environment and distribution. Finally, aggregate spatial patterns for the entire set of species are identified, highlighting areas undergoing most compositional change, with the secondary aim of identifying potential refugia for affected species.

\subsection{Climate Simulation}

The reference (baseline) climate was created to simulate species climate preferences, tolerances and present distributions, and as a basis for downscaling of the climate anomalies that allow simulation of change impacts. The climate variables are of interest in their own right, as a similar range of bioclimate variable maps responding to climate change forcing is not available in the public domain. In particular, the seasonality of moisture availability maps (Fig. 6:4, Fig. 6:8) differ from any publicly available variable. An exploration of the climate results indicates which variables vary most under the present and future climate, and which areas and variables undergo the most change.

To obtain a reference climate for the 1755 extended Neotropics cells, the 'PRES' bioclimate model was based on the 1961-91 climate of New et al. (1999). A second 'DOWNS' bioclimate model for the 560 cells enclosed or intersected by the EOS-Amazon boundary (the inclusiveAmazon', section 1.4.2) was based on annual interpolations of the downscaled HADCM2 decadal means from 1960s to 2090s.

\subsubsection{Reference climate}

This section examines the spatial and frequency distributions of the PRES bioclimate variables under current conditions. These variables were used to supply the climate values encountered by the study species in locations outside the Amazon region, so are vital in determining the species' overall climate range. The spatial distributions of the bioclimate variables (Fig. 6:2 to Fig. 6:5) can also be compared with the frequency of recorded and modelled species locations (Fig. 5:14, Fig. 6:11) to identify whether any climates are under-represented in the species set. White cells represent land units for which values were not calculated. 
The neotropical frequency distribution of annual evapotranspiration (AET) at a $1^{\circ}$ scale is $\mathrm{J}$ shaped and unimodal, peaking about $200 \mathrm{mmy}^{-1}$ above the mean (Fig. 6:1a). High AET indicates a hot, moist environment. AET over the inclusive-Amazon ranges from 206 to $1919 \mathrm{mmy}^{-1}$, and is generally higher than in the surrounding area, with particularly high values in the west in the orographic convergence region adjacent to the Andes (Fig. 6:2). However, the three highest values are extra-Amazonian, on the western coast of Colombia. The lowest value in the enclosed-Amazon is $495 \mathrm{mmy}^{-1}$, with the lowest inclusive-Amazon values being sited on the EOS boundary.
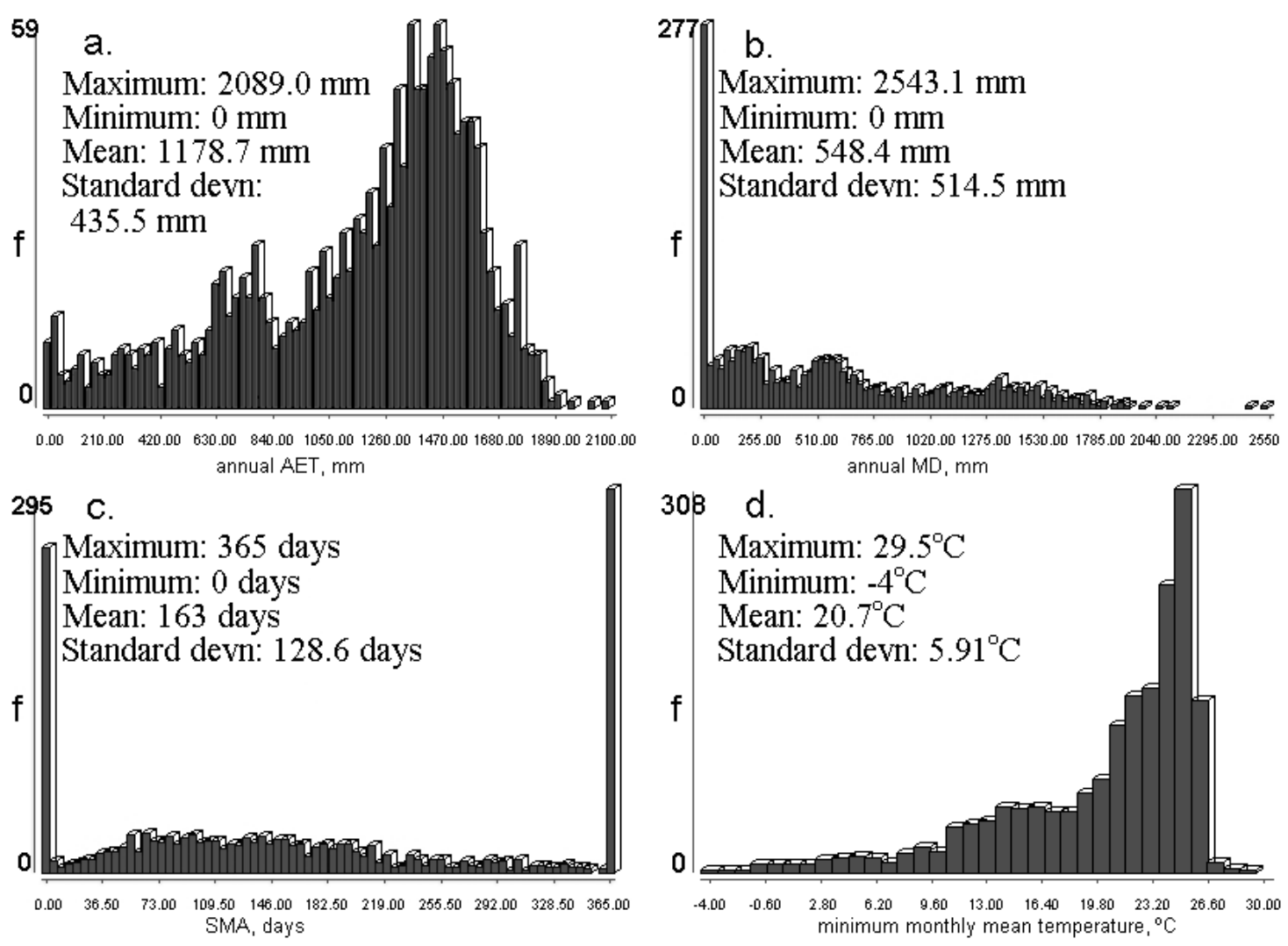

Fig. 6:1: Histograms of values over the extended Neotropics for (a) AET (annual sum of daily evapotranspiration), (b) MD (annual sum of daily PET - AET), (c) SMA (maximum consecutive days where AET $<$ PET), and (d) $T_{\min }$ (lowest mean monthly temperature)

The annual sum of daily moisture deficit $(M D)$ ranges from 0 to $1919 \mathrm{mmy}^{-1}$ over the inclusiveAmazon. The highest neotropical value $\left(2543 \mathrm{mmy}^{-1}\right)$ is found on the coast of Ecuador, in the westernmost cell of South America to be simulated, and the top five $M D$ values are found in surrounding cells. No monographed species were recorded in the top two cells. 


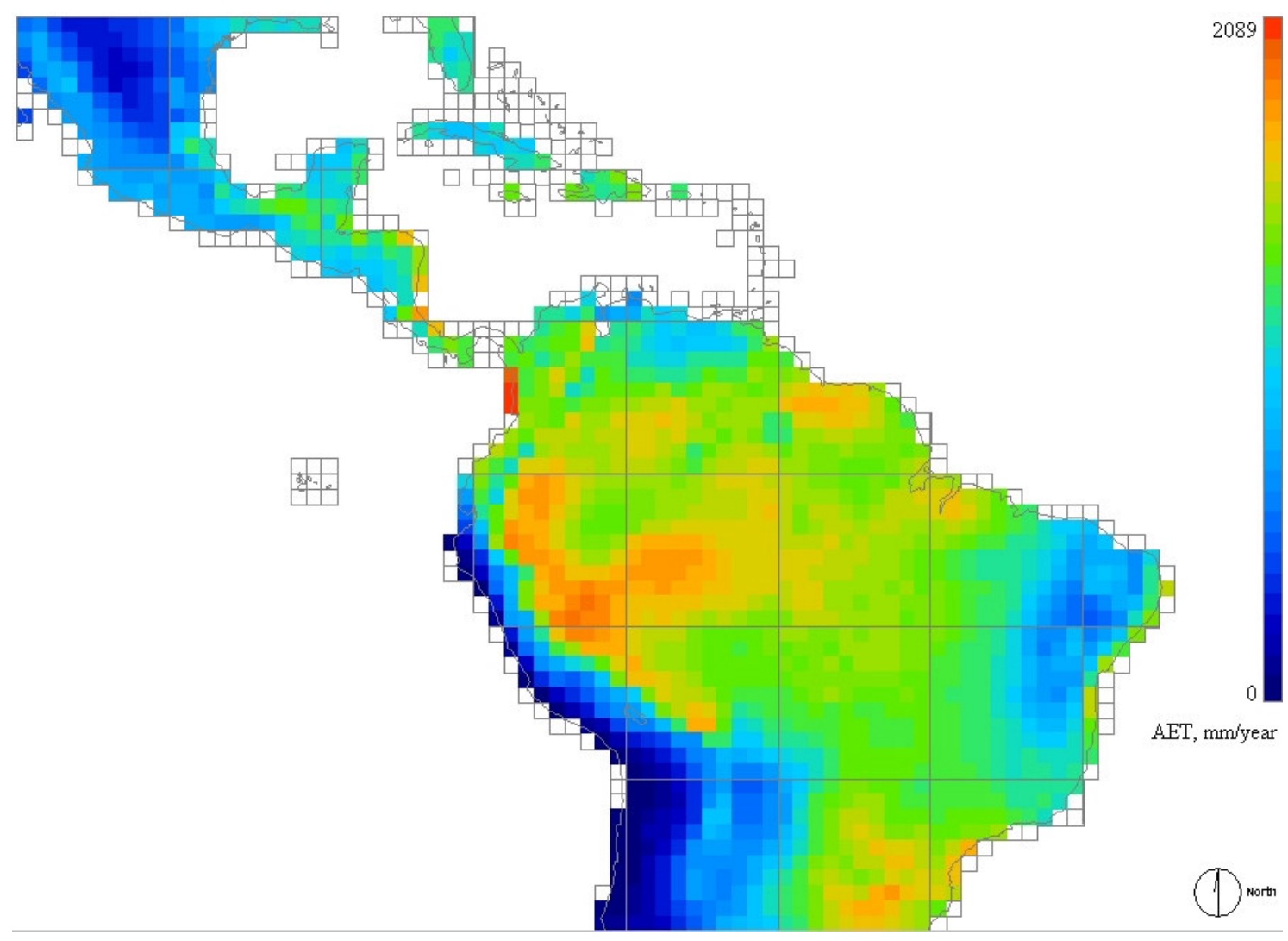

Fig. 6:2: $A E T$, extended Neotropics (linear colour scale)

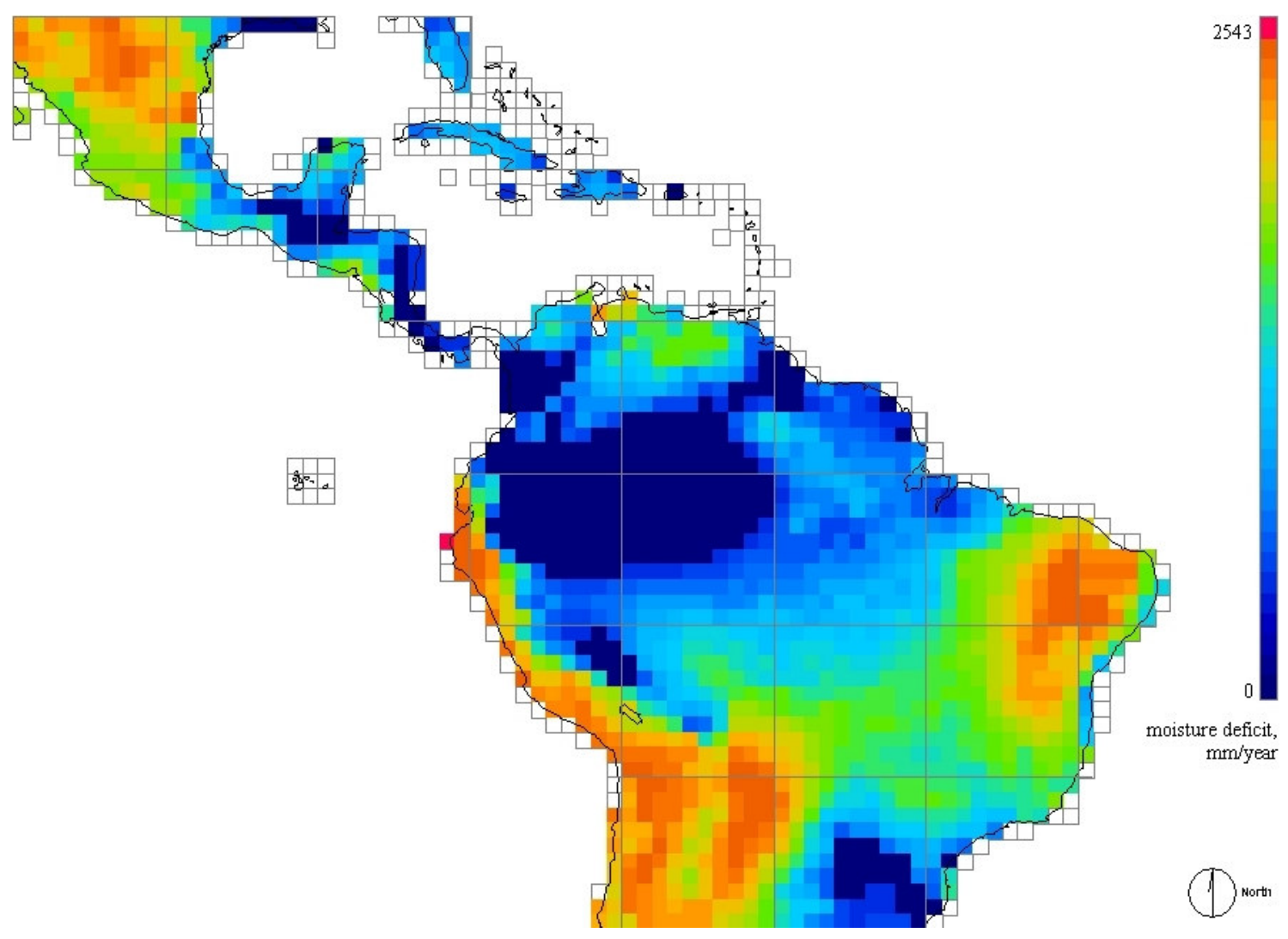

Fig. 6:3: $M D$, extended Neotropics (linear colour scale) 


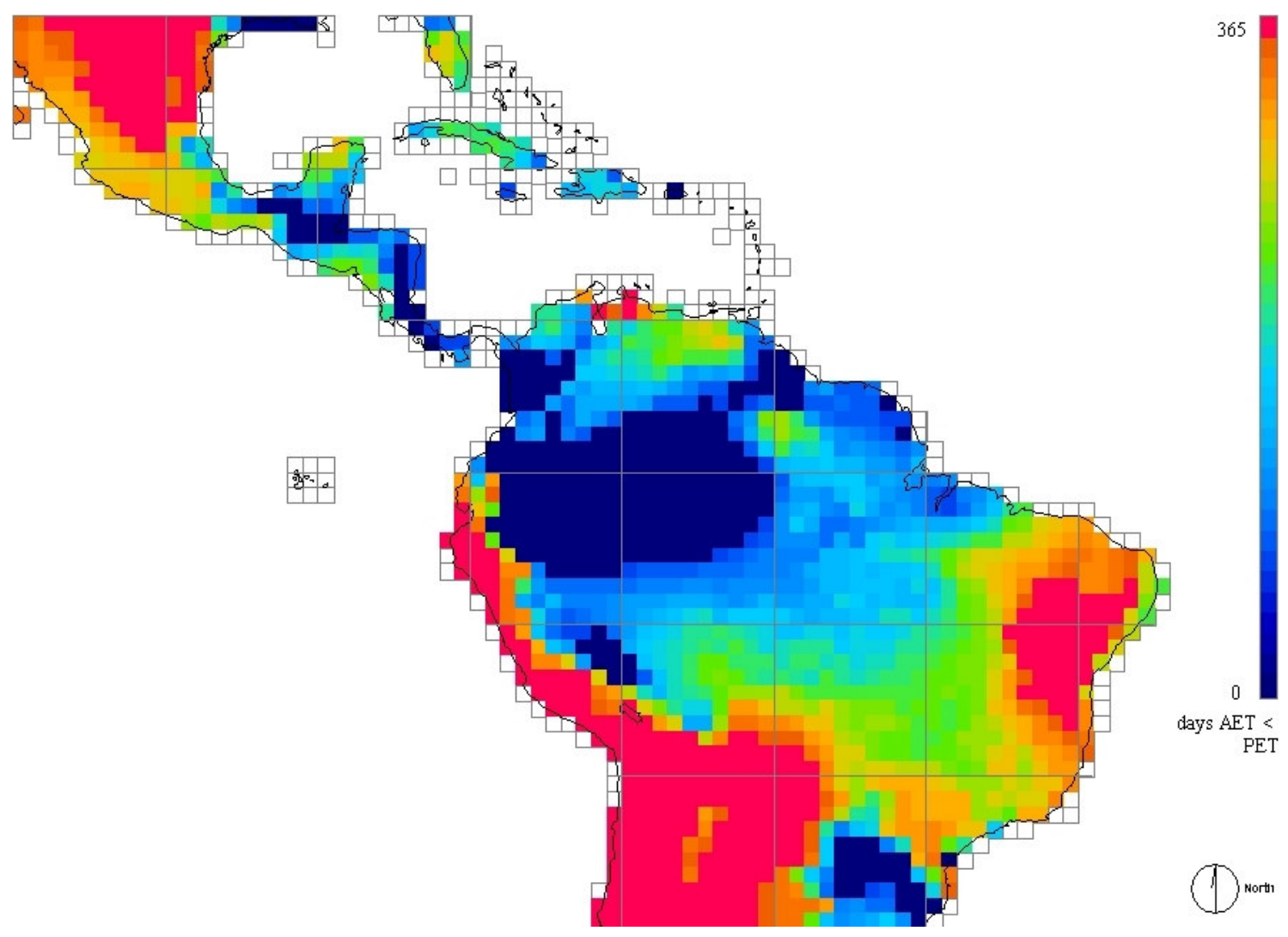

Fig. 6:4: SMA, extended Neotropics (linear colour scale)

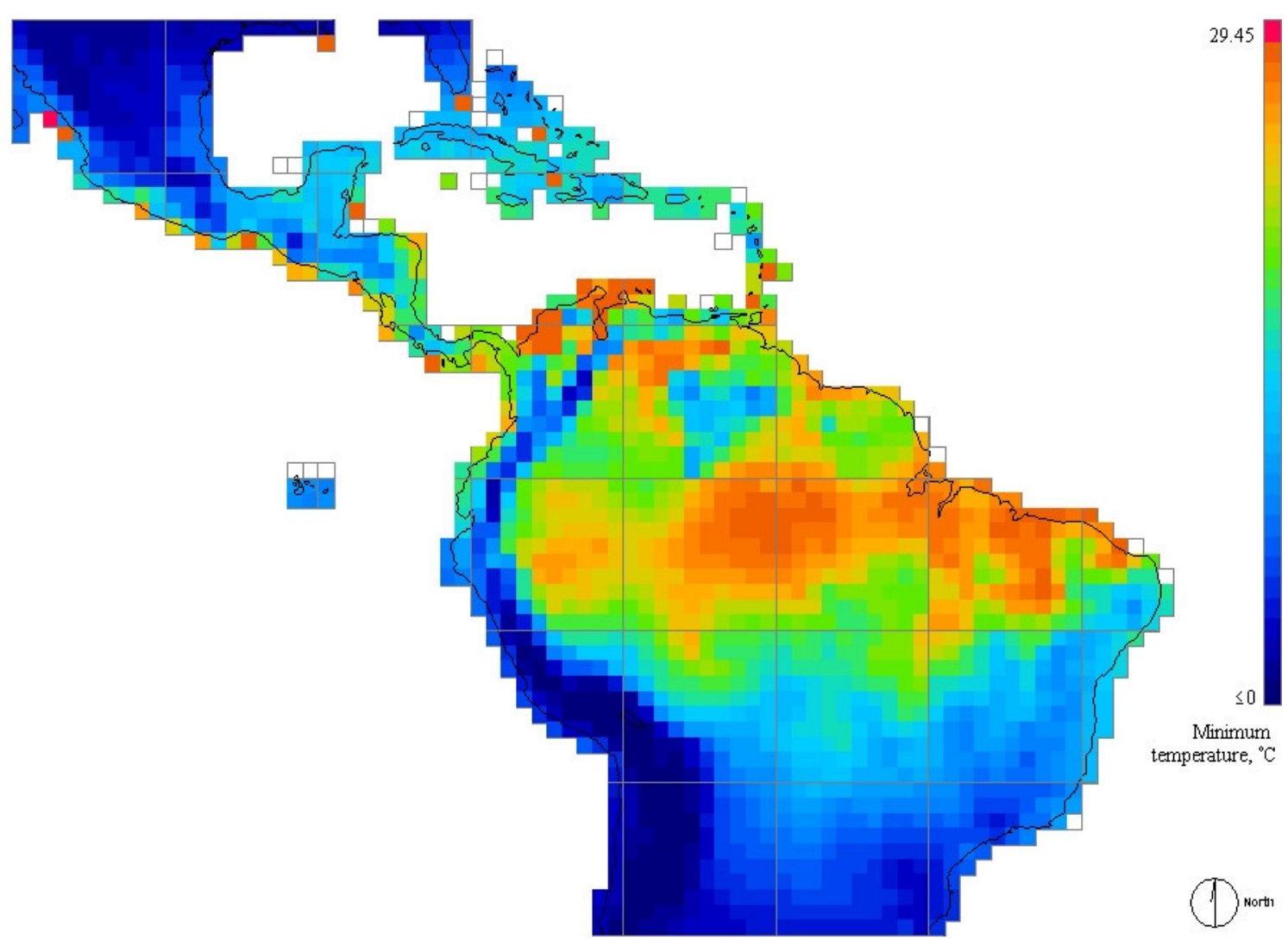

Fig. 6:5: $T_{\text {min }}$, extended Neotropics (linear colour scale) 
The highest value in the enclosed-Amazon is $1397 \mathrm{mmy}^{-1}$. However, over most of the inclusiveAmazon, $M D$ ranges only from 0 to $300 \mathrm{mmy}^{-1}$. Thus, species that are confined to the Amazon basin are likely to have smaller $M D$ tolerances than species with a wider neotropical distribution. Evaporative demand is met by AET in 251 neotropical cells $(M D=0 \mathrm{~mm})$. Much of western and north-central Amazonia has low or 0 MD (Fig. 6:3).

Where the seasonality of moisture availability (SMA) value is 0 days, daily AET always matches PET, hence there is an aseasonal moisture surplus. 365-day cells have a year-round deficit, hence have an aseasonal moisture deficit. A seasonality measure based on rainfall alone would define a dry-aseasonal cell as seasonal where there was annual cyclicity in rainfall intensity. SMA ranges from 0 to 365 days over the enclosed-Amazon (Fig. 6:4). Most aseasonal Amazonian cells are zero-deficit, and occur in the northwest of the basin. Cells with a constant deficit occur only at the western and southern edges of the EOS basin. Aseasonal and almost aseasonal environments dominate the seasonal moisture availability (SMA) distribution for the extended Neotropics, with the high cell frequency at both extremes (Fig. 6:1c).

The spatial pattern of reference $S M A$ and $M D$ are similar, as both measure moisture deficit. SMA measures the temporal clustering of the deficit, whilst $M D$ measures the annual summed deficit. Whilst it is not inevitable that the two are so strongly correlated, zero values of both must coincide and extremely high values are likely to coincide.

The lowest mean monthly temperature $\left(T_{\min }\right)$ map is distinctively different from the ET-based variables, as it is not affected by moisture availability and only represents part of the annual temperature range (Fig. 6:5). The $T_{\min }$ spatial coverage is also more complete than that of the other variables, which are limited by missing coastal values in the soil datasets.

The $T_{\min }$ distribution for the extended Neotropics is J-shaped, with a high mode (Fig. 6:1d). The longitudinal gradient that is seen in the other maps is not visible, rather the clearest gradient is from the warm equatorial centre of the continent to the cooler poleward parts and areas of high altitude. A few cells in the Argentinean Andes do experience sub-zero ${ }^{\circ} \mathrm{C}$ temperatures, but no monographed species were recorded here. $T_{\min }$ ranges from $1.38^{\circ} \mathrm{C}$ to $26.68^{\circ} \mathrm{C}$ over the inclusive-Amazon, with the lowest values on the western (Andes) edge. The lowest value in the enclosed-Amazon is $6.78^{\circ} \mathrm{C}$.

The model assumes that Amazonian species cannot colonise locations where $T_{\min }$ is lower than the minimum record-cell value (for $M D, A E T$ and $S M A$, both upper and lower boundaries are applied). This is implemented as a restriction on initial population and on germination. $T_{\min }$ is not otherwise used as a limiting factor. 


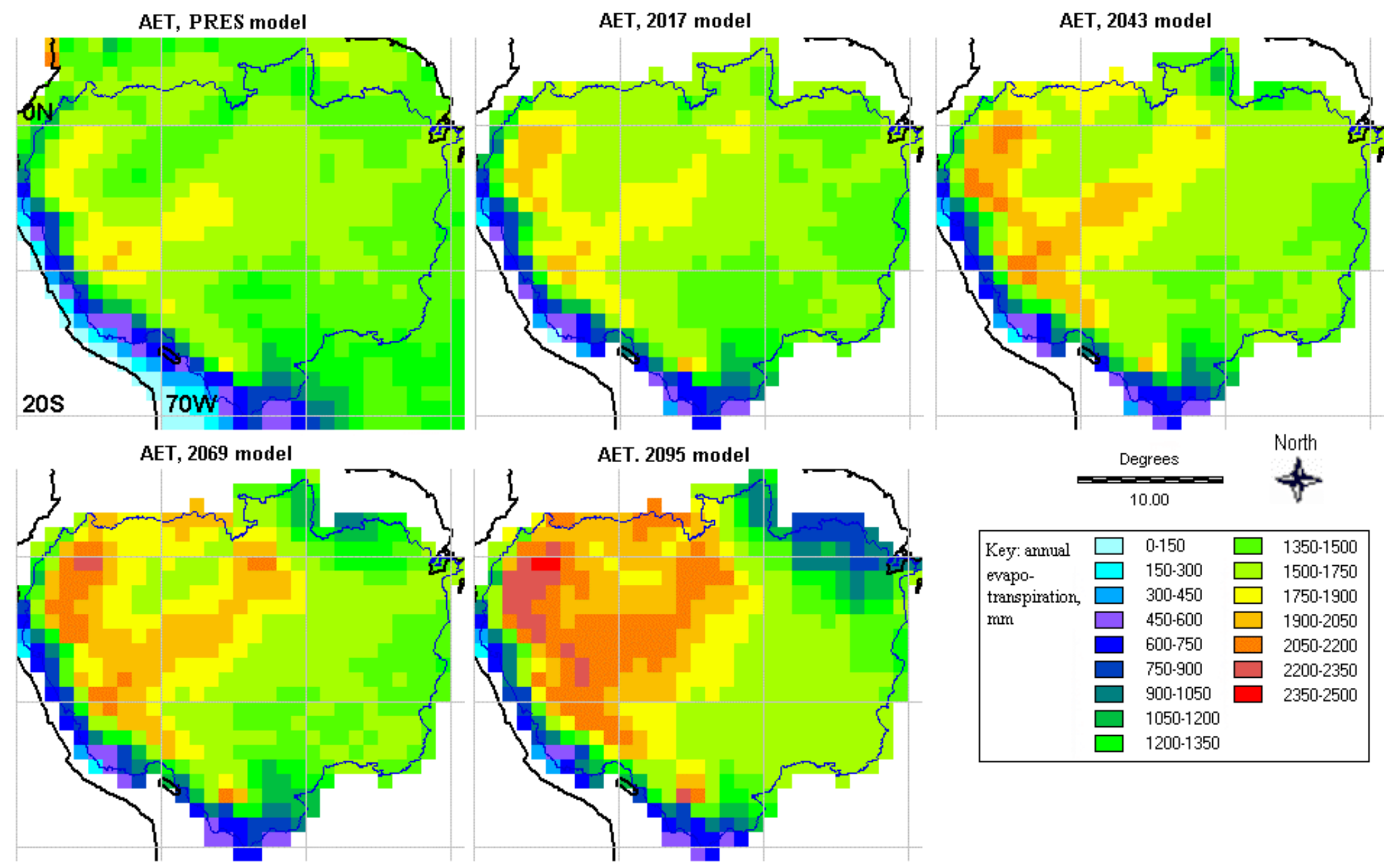

Fig. 6:6: $A E T$, PRES and changing values under SIS 


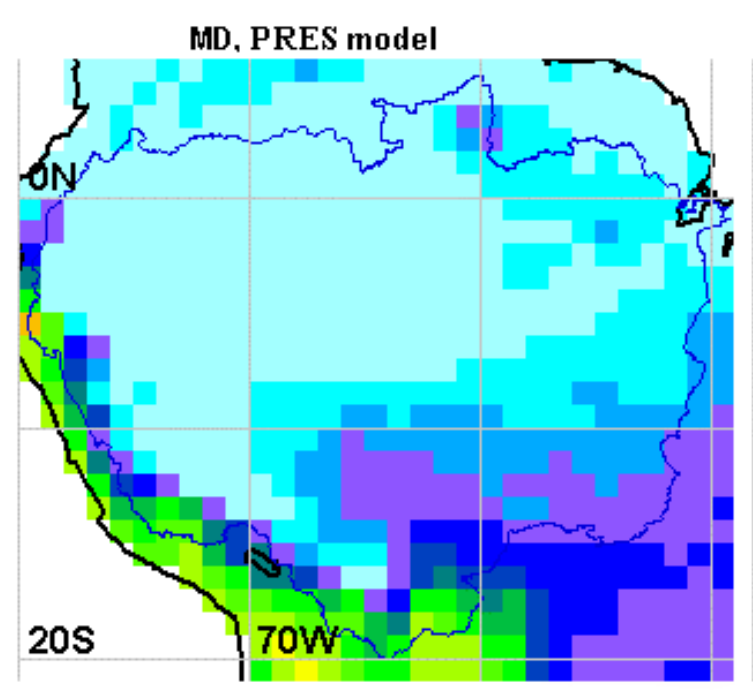

MD, 2069 model

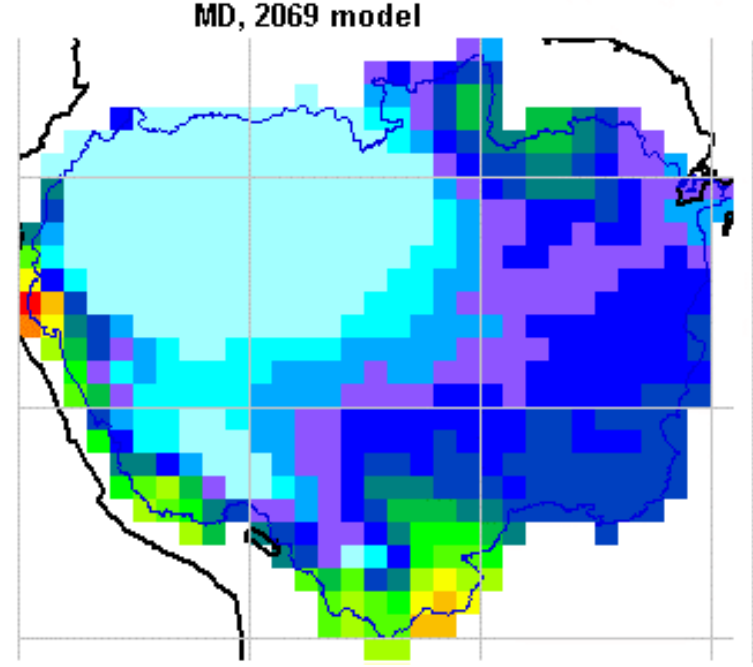

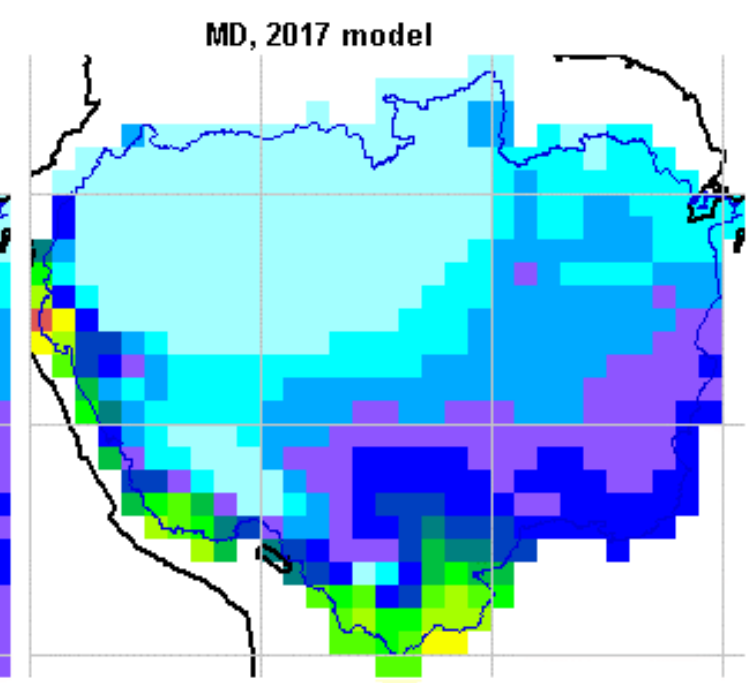

MD, 2095 model

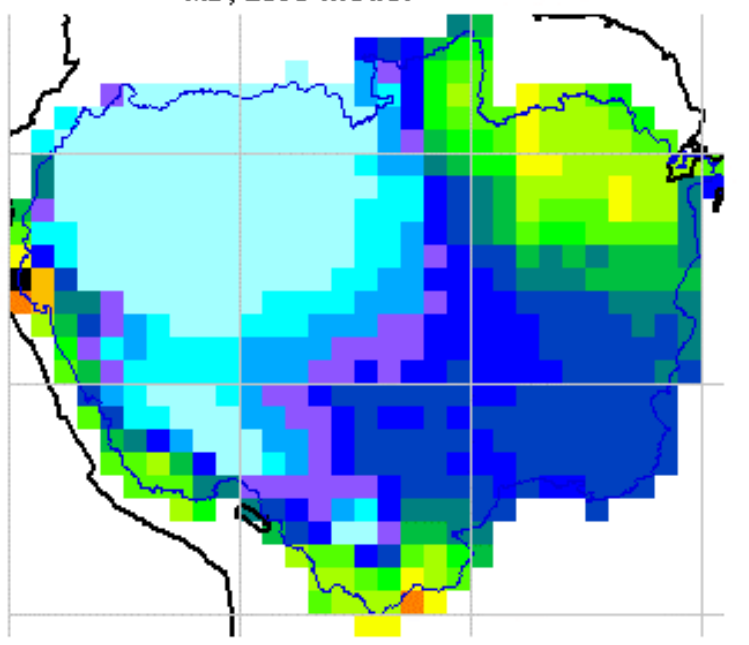

MD, 2043 model
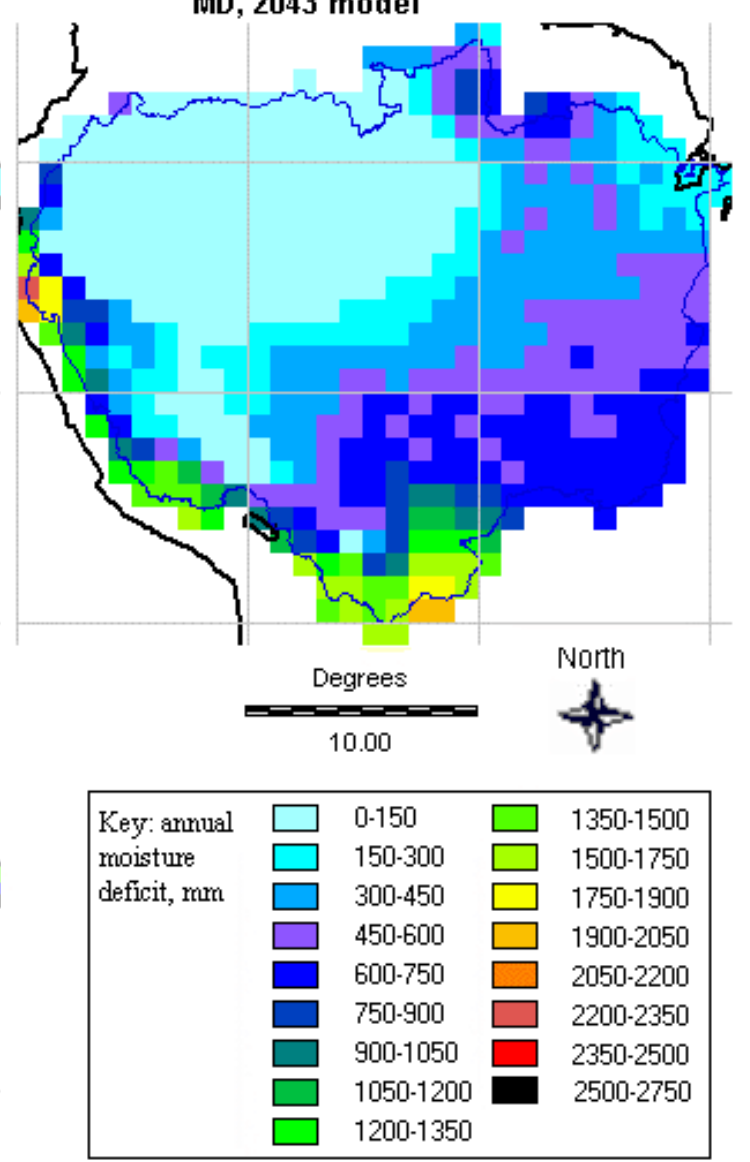

Fig. 6:7: $M D$, PRES and changing values under SIS 

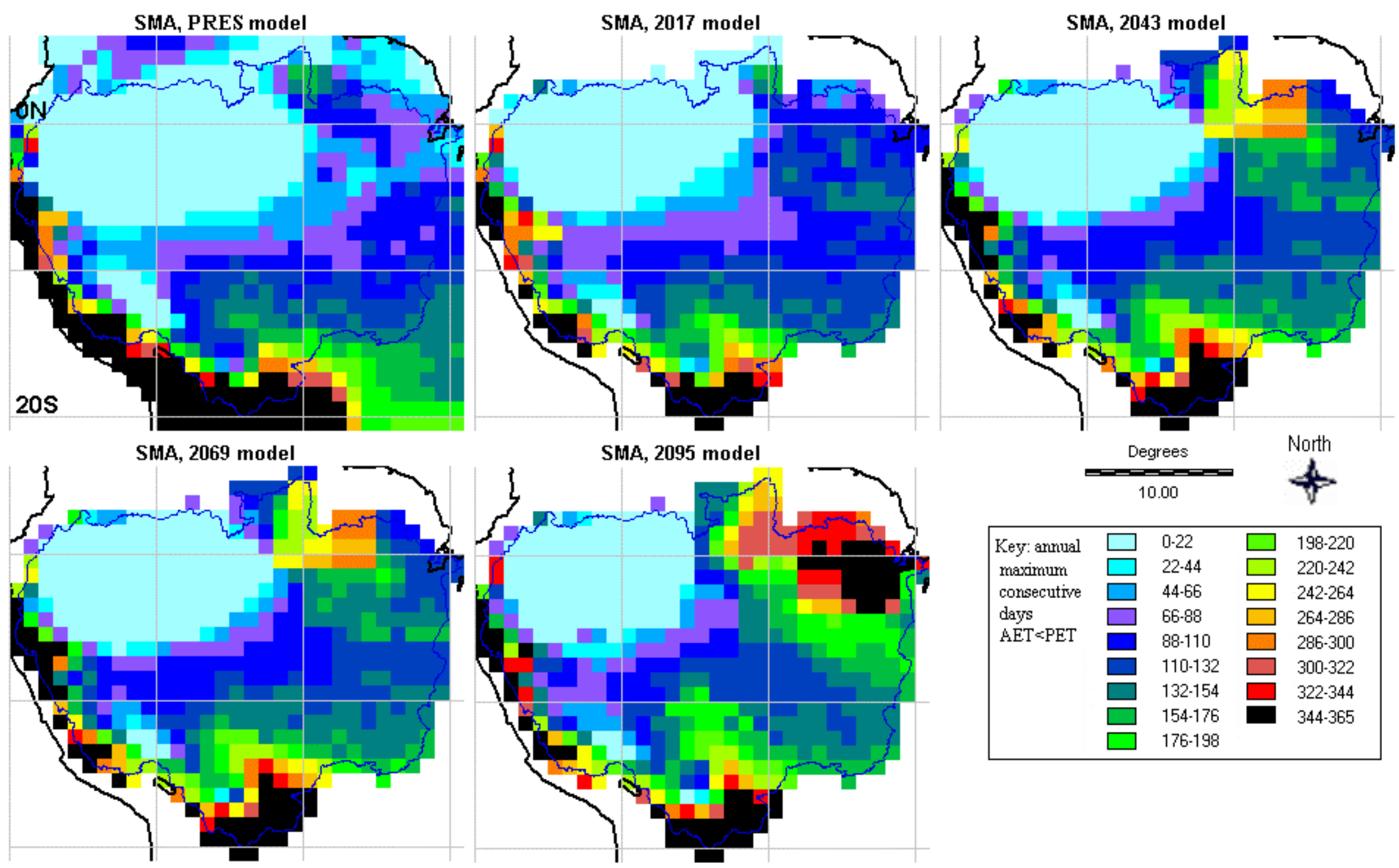

Fig. 6:8: SMA, PRES and changing values under SIS 

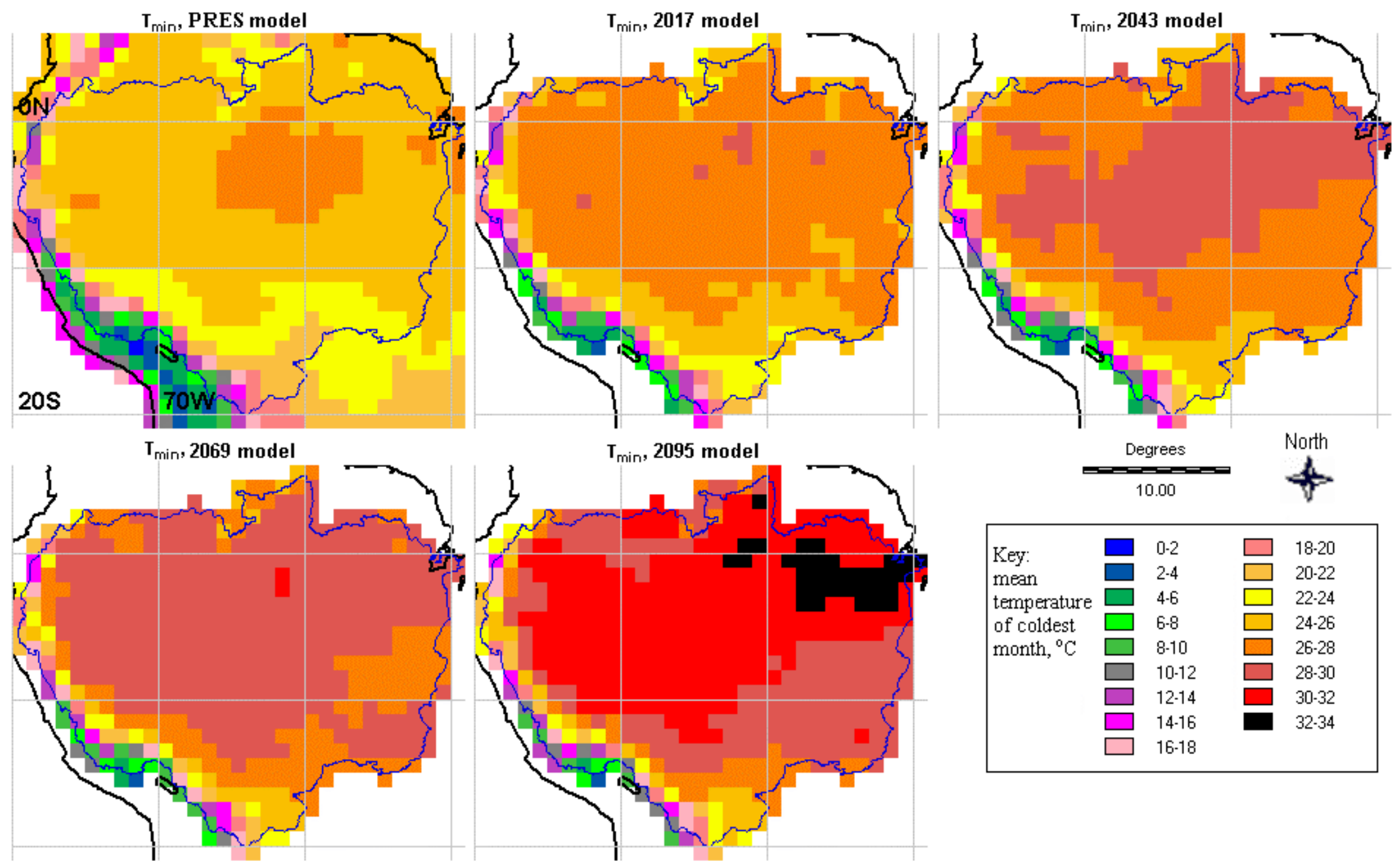

\begin{tabular}{|lllll|}
\hline Key: & $\square$ & $0-2$ & $\square$ & $18-20$ \\
mean & $\square$ & $2-4$ & $\square$ & $20-22$ \\
temperature & $\square$ & $4 \cdot 6$ & $\square$ & $22-24$ \\
of coldest & $\square$ & $6-8$ & $\square$ & $24-26$ \\
month, ${ }^{\circ} \mathrm{C}$ & $\square$ & $8-10$ & $\square$ & $26-28$ \\
& $\square$ & $10-12$ & $\square$ & $28-30$ \\
& $\square$ & $12 \cdot 14$ & $\square$ & $30-32$ \\
& $\square$ & $14-16$ & $\square$ & $32-34$ \\
& $\square$ & $16-18$ & & \\
\hline
\end{tabular}

Fig. 6:9: $T_{\min }$, PRES and changing values under SIS 


\subsubsection{Changing climate}

\subsubsection{Temporal change over the Amazon basin}

The values and spatial patterns of $M D, A E T$ and $S M A$ over the inclusive-Amazon undergo substantial change when the ET model is forced by GCM anomalies (section 5.2.4.6). AET increases in the west, and to a lesser extent in the southeast, and decreases in northeast Amazonia (Fig. 6:6).

Increases in $M D$ can coincide with increases in $A E T$ where a rise in PET coincides with, but exceeds, a rise in precipitation. The highest initial $M D$ is also in the eastern part of the inclusiveAmazon (Fig. 6:7). $M D$ increase is disproportionately high in the northeast. There is an incursion of areas of high $M D$ into central Amazonia, whilst the northwest retains a relatively low deficit until the end of the period. This area, where precipitation keeps step with rising temperature, might be expected to be a refuge for moist forest species.

From the start of the period SMA is higher to the east, with the northeast acquiring an aseasonal moisture-deficient climate at the end of the change period (Fig. 6:8). SMA increases throughout most of Amazonia, indicating increasing moisture stress even on some aseasonal moist forests. $S M A$ is consistently low in the aseasonal northwest, but there is a reduction in the size of the zero-deficit area, from 148 to 99 cells (26\% to $18 \%$ of the inclusive-Amazon).

Increase in $T_{\min }$ is more homogenous, but greatest in northeast Amazonia (Fig. 6:9).

\subsubsection{Variables most affected by climate change}

If some bioclimate variables are more affected by climate change than others, it would be expected that these would play a greater role in limiting or aiding species performance. The 1990 and 2095 values of the Amazonian variables were compared to assess impacts under SIS (Fig. 6:10), looking at their initial variation, relative mean change, and variation in change.

The coefficient of variation $(\mathrm{CV},=$ standard deviation/mean) allows comparison of variables whose units differ. Relatively high initial $\mathrm{CV}$ is found for $A E T$ and $T_{\min }$. This suggests that widespread species should be more likely to be resistant to variation in AET and $T_{\min }$ than in $S M A$ and $M D$, as long as the climate remains within the existing extremes. Between 1990 and 2095 , the CV increases for all variables except AET, indicating a greater spread of climate values across the region. 
The mean relative change for $A E T$ is low, but the coefficient of variation in change is comparable to that of $M D$ and $S M A$ (Fig. 6:10). This value was calculated to assess the variation in the amount of change undergone.

Despite the mean relative change for $M D$ being greatest, no cells exceeded the mixed-reference bioclimate maximum $M D$ or $S M A^{13}$. The reference climate maximum for $A E T$ was exceeded in 83 cells (RIS: 0 cells). This violates the assumption that had been made that no cells would exceed the current range of environments experienced by the whole range of Neotropical species. Where this does occur, no species can remain viable under the present model. This issue is explored further in section 7.2.5.

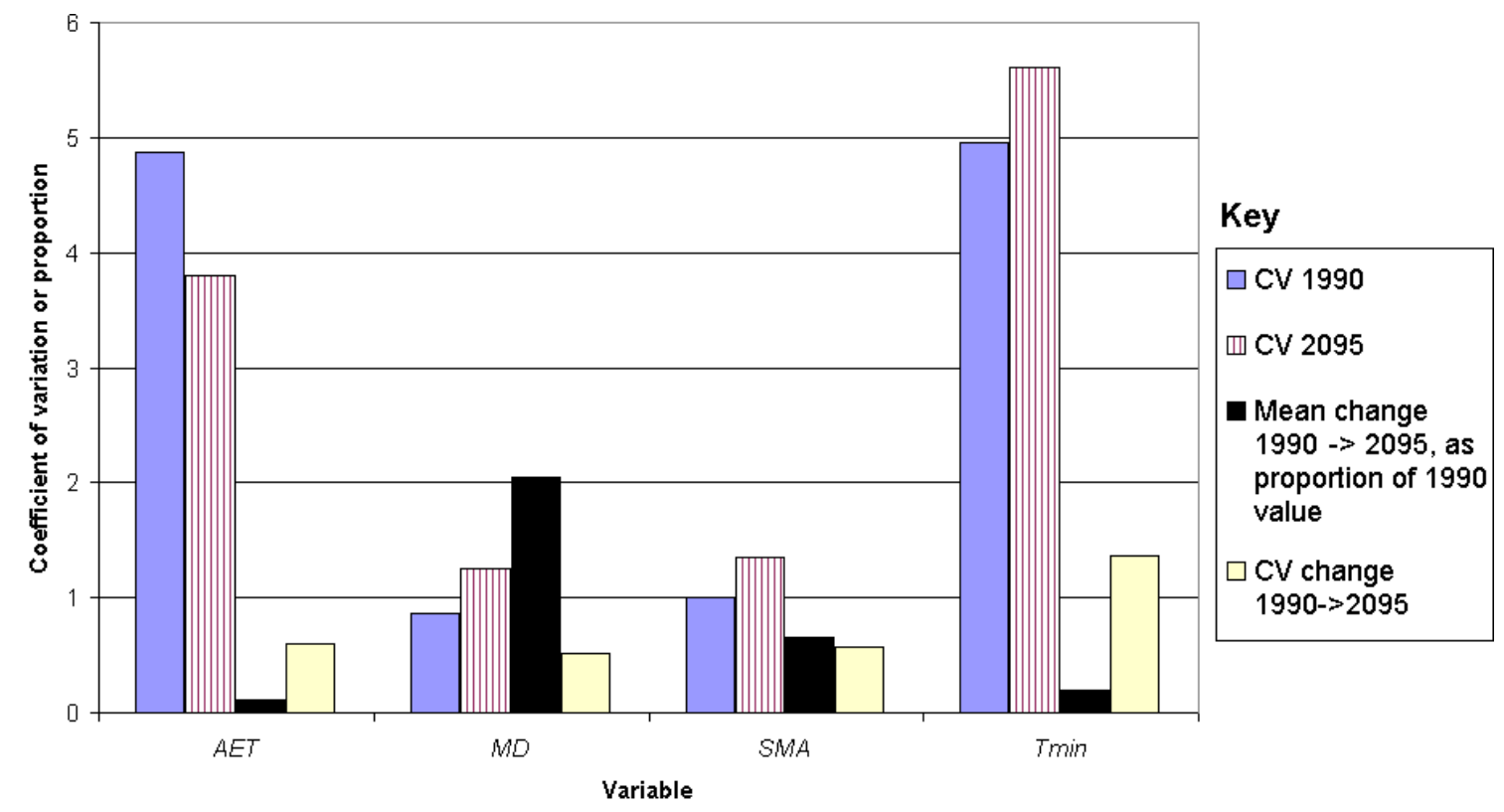

Fig. 6:10: CVs and mean relative change in DOWNS bioclimate variables, SIS

No cells had values that were less than the mixed-reference bioclimate's minimum value for any variable. 284 cells exceeded the maximum $T_{\min }$ value, but as only the minimum value is used in the species model, this did not affect species outcomes. High temperature levels are represented via increased $A E T$ and/or $M D$.

\footnotetext{
${ }^{13}$ When calculating relative (percentage) change, 59 cells with value 0 at 1990 that increased by an infinite percentage were excluded from the analysis for $M D$ and $S M A$.
} 


\subsection{EXPLORING SPECIES OUTCOMES}

The 69 species were simulated under the standard impact scenario (SIS) and reduced impact scenario (RIS). Aspects of the outcome that can be considered for a given cell-based population are whether it retains viability and whether and how much its density changes (including extinctions). The impact of these changes on the metapopulation can be judged by the given population's initial density - loss of a core population would be a more catastrophic outcome for the species' conservation status. Spatial outcomes can be visualised in terms of changes in the species' realised distribution (local extinctions), viable distribution (reproductive populations) and potential distribution (unoccupied suitable cells).

\subsubsection{Modelled present distributions}

The present-day spatial distribution of the modelled species frequency (Fig. 6:11) indicates that species from the western edge and southern tip of the Amazon are underrepresented in the selection. A few cells on the southwestern EOS-Amazon border hold no simulated populations. These cells were not particularly representative of Amazon climates, having AET values lower than any seen in the enclosed-Amazon, but not the lowest value from the inclusive-Amazon.

(i)

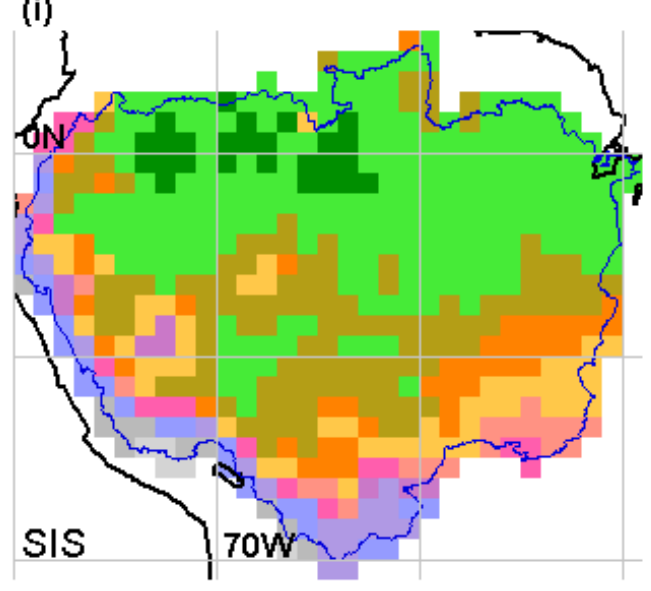

(ii)

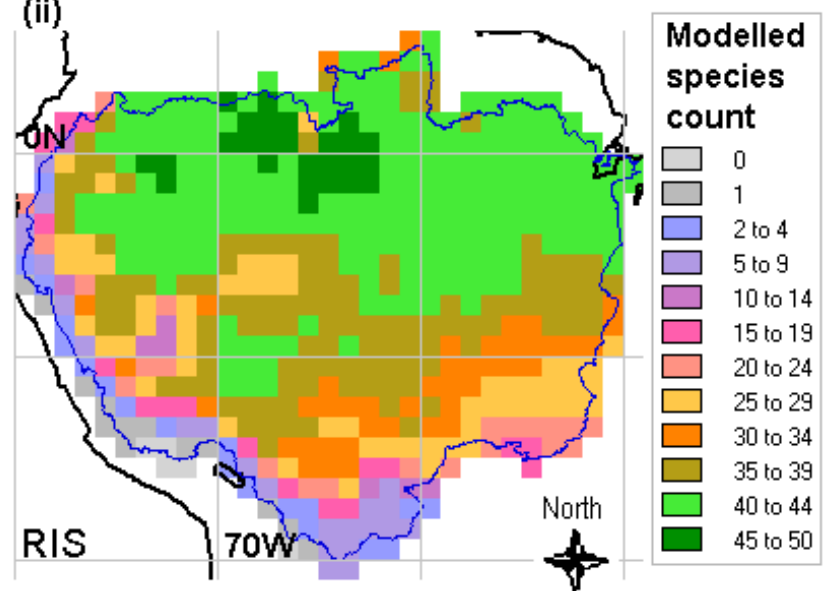

Fig. 6:11: Species frequency (realised distributions) at 1990 under (i) SIS and (ii) RIS

The potential distribution frequently occupied many more cells than the realised distribution (Fig. 6:14 - Fig. 6:25). This reflects the relative homogeneity in climate of the Amazon region, and the assumption that factors such as the species' history have also played a role in limiting colonisation of all suitable sites (section 5.2.8.1). 


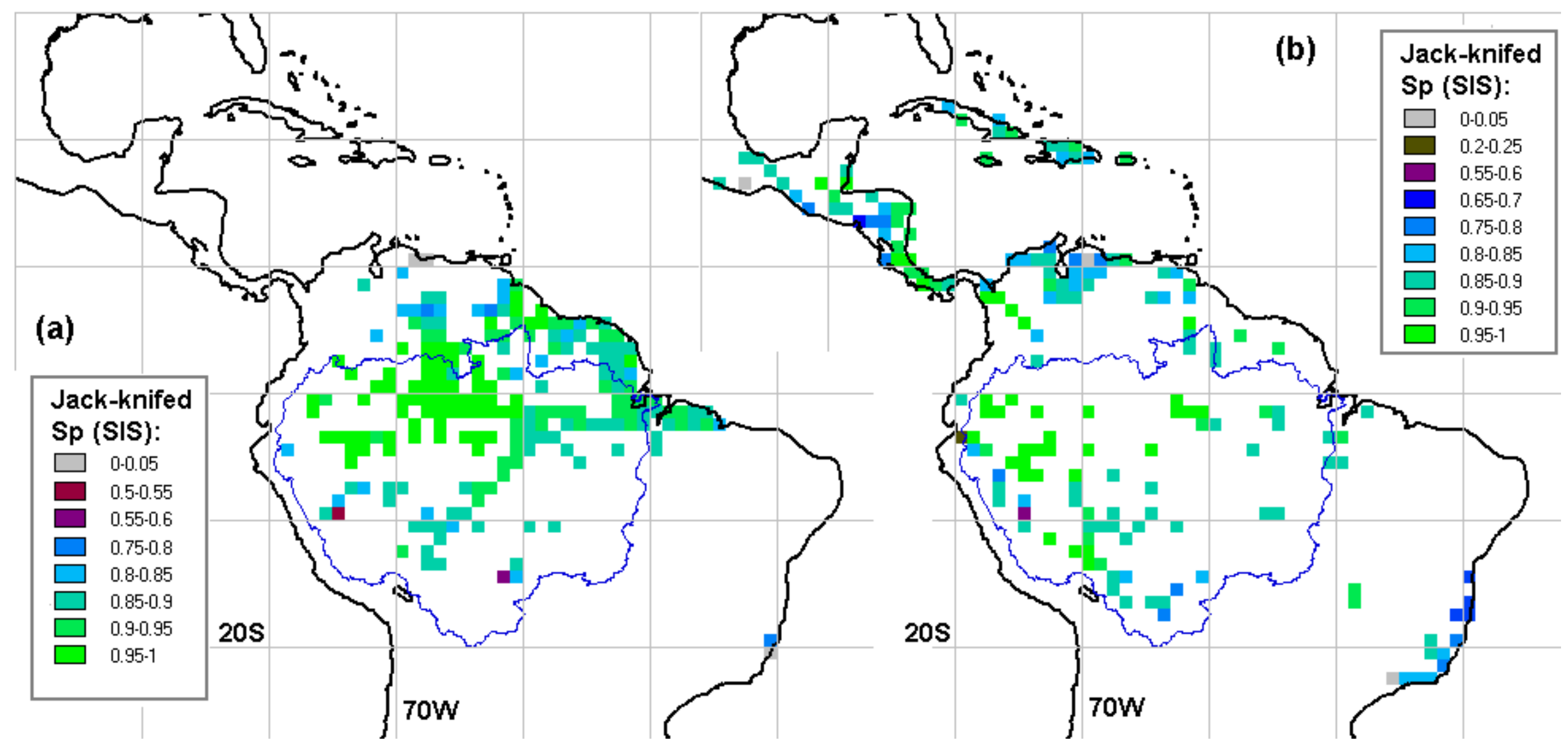

Fig. 6:12: Jack-knifed similarity under SIS for the two species with most record-cells, (a) Licania heteromorpha (220 cells); (b) Hirtella triandra (214 cells) 


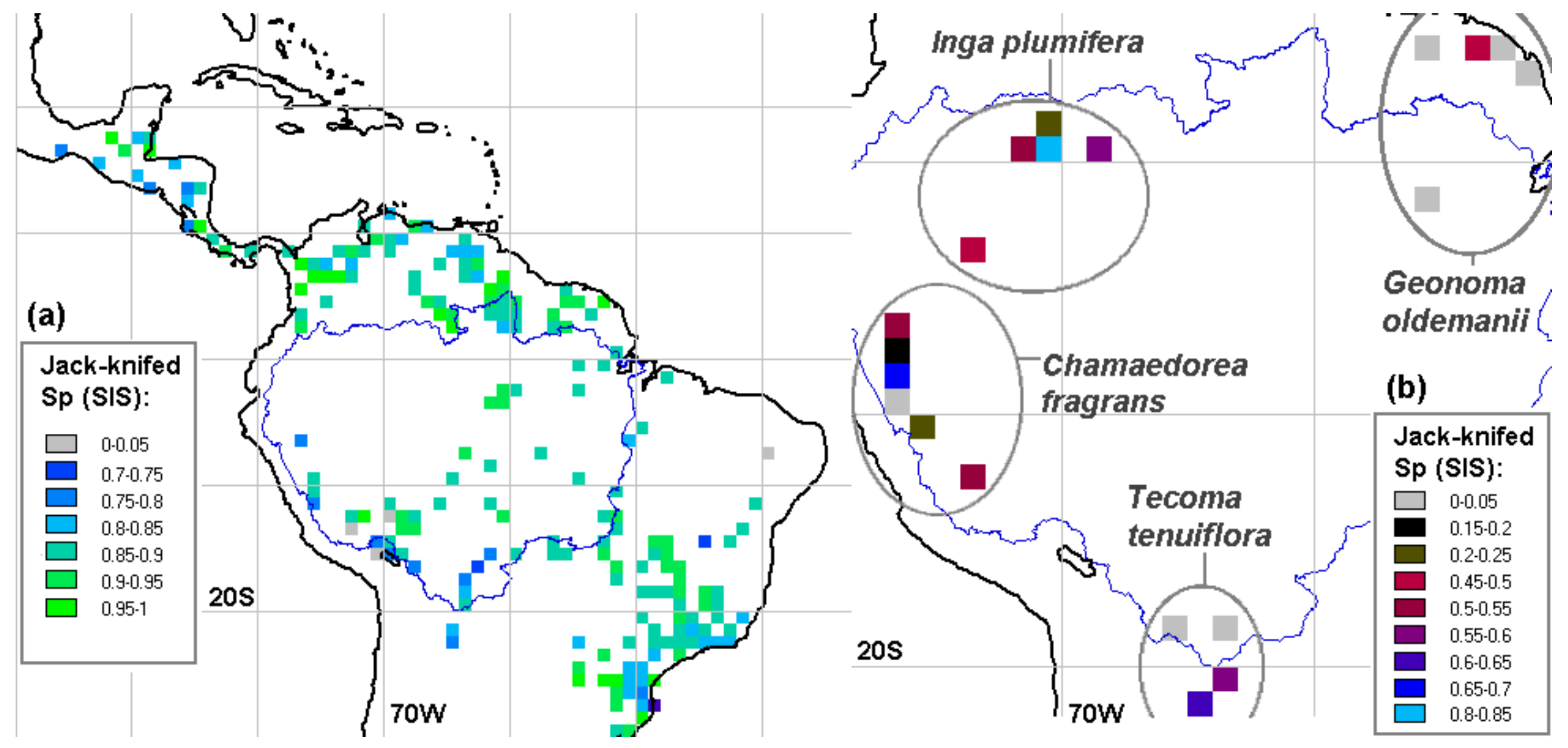

Fig. 6:13: Jack-knifed similarity under SIS for (a) Roupala montana (213 cells); (b) a selection of species with a small number of record-cells: Inga plumifera, Chamaedorea fragrans, Tecoma tenuiflora and Geonoma oldemanii 


\subsubsection{Success in simulating present distributions}

An evaluation of the accuracy of the similarity model using a presence-absence dataset is discussed in section 7.2.4. Where only presence data is available, success in simulating known distributions can be mapped using outcomes from the jack-knifing method used to determine $S_{\min }$ (Fig. 6:12 - Fig. 6:13). $S_{\mathrm{p}}$ is generally higher for more common species, and for the less common species, a high proportion of record cells may fall outside the extreme climate values for the remainder of the recordset, thus obtaining an $S_{\mathrm{p}}$ of 0 (e.g. Geonoma oldemanii, Fig. 6:13). The rectilinear envelope is therefore likely to exclude some cells from the potential distribution that are actually suitable for the species.

\subsubsection{Distributional changes}

The change outcomes were illustrated for individual species, grouped by family, in a set of coded maps (Fig. 6:14 - Fig. 6:25). These identify each cell that falls within the potential distribution at 1990 or 2095 for both SIS and RIS, and whether it was occupied in 1990. For occupied cells, the relative population density at $1990\left(K_{\mathrm{kp}} / K_{\mathrm{kmax}}\right)$, the direction and magnitude of density change by $2095\left(\Delta K_{\mathrm{kp}}(1990 \rightarrow 2095)\right)$, and whether or not the population remained viable were mapped. Not all types of change are encountered in every family.

Absolute loss of populations was relatively infrequent because of the lag in the model, so the hypothesis that individual species' realised distributions would change (H1) is invalidated for the majority of species. However, no species remained viable in all its current locations under either change scenario (Table 6:1). For $43 \%$ of species under SIS and $20 \%$ of species under RIS, all modelled populations became non-viable (non-reproductive) or were extirpated by 2095 . For these species, hypothesis $(\mathrm{H} 2)$, that " present day realised and final potential distributions will not overlap one another, so that the species will eventually be lost from its entire current range", is confirmed. Species-mean percentage viability loss was more severe under SIS than RIS ( $p<$ 0.0001, Wilcoxon signed ranks test).

Differences between the SIS and RIS DOWNS climates caused the simulated distributions to differ for some species. 18,599 populations were modelled under SIS and 18,645 under RIS. Some populations were lost during equilibration because the level of the most limiting factor was too low for population survival, although the environment was sufficiently similar to those in the recordset to be included. Three populations were further discarded from SIS and four from RIS, where no individuals were present in the mature stage at the end of the initial equilibrium period. All these discarded populations were Inga nobilis or Cydista aequinoctialis. One population of I. nobilis was lost following change under RIS, but not SIS. 
Table 6:1: Species outcomes at 2095, SIS and RIS

\begin{tabular}{|c|c|c|c|c|c|c|c|c|c|c|c|c|}
\hline \multirow[t]{2}{*}{ Species id } & \multirow[t]{2}{*}{ Family } & \multirow[t]{2}{*}{ Form } & \multirow{2}{*}{$\begin{array}{l}\text { Height } \\
\text { class }\end{array}$} & \multirow[t]{2}{*}{ Species } & \multicolumn{4}{|l|}{ SIS } & \multicolumn{4}{|l|}{ RIS } \\
\hline & & & & & $\begin{array}{l}\text { Modelled } \\
\text { patches }\end{array}$ & $\begin{array}{l}\% \\
\text { viable }\end{array}$ & $\begin{array}{l}\text { \% non- } \\
\text { viable }\end{array}$ & $\%$ lost & $\begin{array}{l}\text { Modelled } \\
\text { patches }\end{array}$ & $\begin{array}{l}\% \\
\text { viable }\end{array}$ & $\begin{array}{l}\text { \% non- } \\
\text { viable }\end{array}$ & $\%$ lost \\
\hline Amphpani & Bignoniaceae & Liana & $10-20 \mathrm{~m}$ & Amphilophium paniculatum & 529 & 47.8 & 52.17 & 0.0 & 524 & 79.8 & 20.2 & 0.0 \\
\hline Anibpanu & Lauraceae & Tree & $10-20 \mathrm{~m}$ & Aniba panurensis & 432 & 1.9 & 97.92 & 0.2 & 413 & 55.9 & 43.8 & 0.2 \\
\hline Anthobov & Caryocaraceae & Tree & $20-25 \mathrm{~m}$ & Anthodiscus obovatus & 36 & 0.0 & 100.00 & 0.0 & 39 & 0.0 & 100.0 & 0.0 \\
\hline Arracora & Bignoniaceae & Liana & $25+m$ & Arrabidaea corallina & 494 & 25.3 & 74.70 & 0.0 & 489 & 78.7 & 21.3 & 0.0 \\
\hline Astrgyna & Arecaceae & Tree & 4-10 m & Astrocaryum gynacanthum & 330 & 0.0 & 100.00 & 0.0 & 326 & 39.3 & 60.7 & 0.0 \\
\hline Astrmuru & Arecaceae & Tree & $10-20 \mathrm{~m}$ & Astrocaryum murumuru & 399 & 6.3 & 93.73 & 0.0 & 400 & 59.0 & 41.0 & 0.0 \\
\hline Astrscio & Arecaceae & Bush & $1-4 \mathrm{~m}$ & Astrocaryum sciophilum & 21 & 0.0 & 100.00 & 0.0 & 21 & 0.0 & 100.0 & 0.0 \\
\hline Bactaca1 & Arecaceae & Herb layer & $0-1 \mathrm{~m}$ & Bactris acanthocarpa & 450 & 0.0 & 100.00 & 0.0 & 448 & 36.6 & 63.4 & 0.0 \\
\hline Calllati & Bignoniaceae & Liana & $20-25 \mathrm{~m}$ & Callichlamys latifolia & 440 & 41.4 & 58.64 & 0.0 & 440 & 92.5 & 7.5 & 0.0 \\
\hline Carybras & Caryocaraceae & Tree & $4-10 \mathrm{~m}$ & Caryocar brasiliense & 176 & 1.1 & 98.86 & 0.0 & 176 & 76.1 & 23.9 & 0.0 \\
\hline Caryglab & Caryocaraceae & Tree & $25+m$ & Caryocar glabrum & 411 & 0.0 & 98.30 & 1.7 & 403 & 31.3 & 66.3 & 2.5 \\
\hline Carymicr & Caryocaraceae & Tree & $20-25 \mathrm{~m}$ & Caryocar microcarpum & 398 & 0.0 & 100.00 & 0.0 & 394 & 45.2 & 54.8 & 0.0 \\
\hline Chamfrag & Arecaceae & Bush & $1-4 \mathrm{~m}$ & Chamaedorea fragrans & 9 & 33.3 & 66.67 & 0.0 & 9 & 33.3 & 66.7 & 0.0 \\
\hline Chryicac & Chrysobalanaceae & Bush & $1-4 \mathrm{~m}$ & Chrysobalanus icaco & 159 & 28.9 & 71.07 & 0.0 & 158 & 88.6 & 11.4 & 0.0 \\
\hline Clytbina & Bignoniaceae & Liana & $20-25 \mathrm{~m}$ & Clytostoma binatum & 486 & 57.0 & 43.00 & 0.0 & 484 & 98.1 & 1.9 & 0.0 \\
\hline Couedoli & Chrysobalanaceae & Tree & $25+m$ & Couepia dolichopoda & 33 & 0.0 & 100.00 & 0.0 & 32 & 40.6 & 59.4 & 0.0 \\
\hline Couegui2 & Chrysobalanaceae & Tree & $25+m$ & Couepia guianensis & 407 & 0.0 & 100.00 & 0.0 & 394 & 20.8 & 79.2 & 0.0 \\
\hline Couepar2 & Chrysobalanaceae & Tree & $10-20 \mathrm{~m}$ & Couepia paraensis & 393 & 0.0 & 100.00 & 0.0 & 379 & 69.1 & 30.9 & 0.0 \\
\hline Cydiaequ & Bignoniaceae & Liana & 4-10 m & Cydista aequinoctialis & 494 & 45.3 & 52.23 & 2.4 & 491 & 90.0 & 7.5 & 2.5 \\
\hline Geonmacr & Arecaceae & Bush & $1-4 \mathrm{~m}$ & Geonoma macrostachys & 372 & 5.4 & 94.62 & 0.0 & 375 & 37.3 & 62.7 & 0.0 \\
\hline Geonolde & Arecaceae & Bush & $1-4 \mathrm{~m}$ & Geonoma oldemanii & 77 & 0.0 & 100.00 & 0.0 & 65 & 0.0 & 100.0 & 0.0 \\
\hline Gleaduid & Rubiaceae & Tree & 4-10 m & Gleasonia duidana & 14 & 0.0 & 100.00 & 0.0 & 19 & 0.0 & 100.0 & 0.0 \\
\hline Gleauaup & Rubiaceae & Tree & 4-10 m & Gleasonia uaupensis & 1 & 0.0 & 100.00 & 0.0 & 17 & 0.0 & 100.0 & 0.0 \\
\hline Helocaye & Balanophoraceae & Herb layer & $0-1 \mathrm{~m}$ & Helosis cayennensis & 433 & 4.9 & 95.15 & 0.0 & 435 & 49.9 & 50.1 & 0.0 \\
\hline Henrniti & Rubiaceae & Tree & $10-20 \mathrm{~m}$ & Henriquezia nitida & 2 & 0.0 & 100.00 & 0.0 & 9 & 0.0 & 100.0 & 0.0 \\
\hline Henrvert & Rubiaceae & Tree & $25+m$ & Henriquezia verticillata & 28 & 0.0 & 100.00 & 0.0 & 18 & 5.6 & 94.4 & 0.0 \\
\hline Hirtcili & Chrysobalanaceae & Tree & 4-10 m & Hirtella ciliata & 161 & 18.6 & 81.37 & 0.0 & 162 & 93.2 & 6.8 & 0.0 \\
\hline Hirtglan & Chrysobalanaceae & Tree & $20-25 \mathrm{~m}$ & Hirtella glandulosa & 420 & 34.1 & 65.95 & 0.0 & 410 & 77.3 & 22.7 & 0.0 \\
\hline
\end{tabular}




\begin{tabular}{|c|c|c|c|c|c|c|c|c|c|c|c|c|}
\hline Hirtgrac & Chrysobalanaceae & Tree & $10-20 \mathrm{~m}$ & Hirtella gracilipes & 136 & 19.1 & 80.88 & 0.0 & 136 & 75.7 & 24.3 & 0.0 \\
\hline Hirtmuti & Chrysobalanaceae & Tree & $25+m$ & Hirtella mutisii & 1 & 0.0 & 100.00 & 0.0 & 2 & 0.0 & 100.0 & 0.0 \\
\hline Hirtpani & Chrysobalanaceae & Tree & $4-10 \mathrm{~m}$ & Hirtella paniculata & 193 & 0.0 & 100.00 & 0.0 & 201 & 30.4 & 69.7 & 0.0 \\
\hline Hirttris & Chrysobalanaceae & Tree & $25+m$ & Hirtella triandra & 449 & 7.6 & 92.43 & 0.0 & 452 & 80.1 & 19.9 & 0.0 \\
\hline Ingaalba & Fabaceae & Tree & $25+m$ & Inga alba & 434 & 0.5 & 99.54 & 0.0 & 419 & 42.5 & 57.5 & 0.0 \\
\hline Ingaaria & Fabaceae & Tree & $20-25 \mathrm{~m}$ & Inga cayennensis & 423 & 1.9 & 98.11 & 0.0 & 415 & 41.5 & 58.6 & 0.0 \\
\hline Ingacard & Fabaceae & Tree & $20-25 \mathrm{~m}$ & Inga cardozana & 13 & 0.0 & 100.00 & 0.0 & 12 & 25.0 & 75.0 & 0.0 \\
\hline Ingaingo & Fabaceae & Tree & $25+m$ & Inga ingoides & 474 & 6.5 & 93.46 & 0.0 & 467 & 72.4 & 27.6 & 0.0 \\
\hline Ingalaur & Fabaceae & Tree & $20-25 \mathrm{~m}$ & Inga laurina & 451 & 45.9 & 54.10 & 0.0 & 450 & 79.1 & 20.9 & 0.0 \\
\hline Ingamarg & Fabaceae & Tree & $20-25 \mathrm{~m}$ & Inga marginata & 493 & 10.3 & 89.45 & 0.2 & 493 & 75.6 & 24.3 & 0.4 \\
\hline Ingamath & Fabaceae & Tree & $10-20 \mathrm{~m}$ & Inga nobilis & 504 & 28.8 & 71.23 & 0.0 & 508 & 95.9 & 3.9 & 0.2 \\
\hline Ingaplum & Fabaceae & Tree & $4-10 \mathrm{~m}$ & Inga plumifera & 41 & 0.0 & 100.00 & 0.0 & 14 & 0.0 & 100.0 & 0.0 \\
\hline Iriadelt & Arecaceae & Tree & $25+m$ & Iriartea deltoidea & 378 & 24.1 & 75.93 & 0.0 & 378 & 84.4 & 15.6 & 0.0 \\
\hline Jacamacr & Bignoniaceae & Tree & $10-20 \mathrm{~m}$ & Jacaranda macrocarpa & 1 & 0.0 & 100.00 & 0.0 & 8 & 0.0 & 100.0 & 0.0 \\
\hline Langhypo & Balanophoraceae & Herb layer & $0-1 \mathrm{~m}$ & Langsdorffia hypogaea & 414 & 23.4 & 76.57 & 0.0 & 412 & 79.9 & 20.2 & 0.0 \\
\hline Leopmajo & Arecaceae & Tree & $4-10 \mathrm{~m}$ & Leopoldinia major & 34 & 0.0 & 100.00 & 0.0 & 31 & 0.0 & 100.0 & 0.0 \\
\hline Leoppias & Arecaceae & Tree & $4-10 \mathrm{~m}$ & Leopoldinia piassaba & 16 & 0.0 & 100.00 & 0.0 & 25 & 0.0 & 100.0 & 0.0 \\
\hline Licaarbo & Chrysobalanaceae & Tree & $25+m$ & Licania arborea & 106 & 4.7 & 95.28 & 0.0 & 103 & 24.3 & 75.7 & 0.0 \\
\hline Licahete & Chrysobalanaceae & Tree & $20-25 \mathrm{~m}$ & Licania heteromorpha & 429 & 1.2 & 98.83 & 0.0 & 455 & 30.8 & 69.2 & 0.0 \\
\hline Licahumi & Chrysobalanaceae & Bush & $1-4 \mathrm{~m}$ & Licania humilis & 66 & 0.0 & 100.00 & 0.0 & 67 & 35.8 & 64.2 & 0.0 \\
\hline Manibide & Sapotaceae & Tree & $25+m$ & Manilkara bidentata & 393 & 14.8 & 85.24 & 0.0 & 381 & 36.2 & 63.8 & 0.0 \\
\hline Martobov & Bignoniaceae & Liana & $1-4 \mathrm{~m}$ & Martinella obovata & 446 & 5.8 & 93.95 & 0.2 & 439 & 48.5 & 51.5 & 0.0 \\
\hline Nectamaz & Lauraceae & Tree & $20-25 \mathrm{~m}$ & Nectandra amazonum & 264 & 0.0 & 100.00 & 0.0 & 258 & 11.2 & 88.8 & 0.0 \\
\hline Nectegen & Lauraceae & Tree & $10-20 \mathrm{~m}$ & Nectandra egensis & 58 & 0.0 & 98.28 & 1.7 & 51 & 0.0 & 100.0 & 0.0 \\
\hline Necthihu & Lauraceae & Tree & $25+m$ & Nectandra hihua & 491 & 14.2 & 85.74 & 0.0 & 489 & 76.5 & 23.5 & 0.0 \\
\hline Nectrefl & Lauraceae & Tree & $25+m$ & Nectandra reflexa & 15 & 13.3 & 86.67 & 0.0 & 14 & 28.6 & 71.4 & 0.0 \\
\hline Nectturb & Lauraceae & Tree & $20-25 \mathrm{~m}$ & Nectandra turbacensis & 287 & 7.3 & 92.68 & 0.0 & 282 & 60.3 & 39.7 & 0.0 \\
\hline Oenobata & Arecaceae & Tree & $10-20 \mathrm{~m}$ & Oenocarpus bataua & 439 & 23.0 & 76.99 & 0.0 & 439 & 84.5 & 15.5 & 0.0 \\
\hline Panometc & Proteaceae & Tree & $10-20 \mathrm{~m}$ & Panopsis metcalfii & 3 & 0.0 & 100.00 & 0.0 & 3 & 0.0 & 100.0 & 0.0 \\
\hline Panorube & Proteaceae & Tree & $25+m$ & Panopsis rubescens & 415 & 0.0 & 100.00 & 0.0 & 416 & 49.3 & 50.7 & 0.0 \\
\hline Paricamp & Chrysobalanaceae & Tree & $10-20 \mathrm{~m}$ & Parinari campestris & 258 & 0.0 & 100.00 & 0.0 & 259 & 23.9 & 76.1 & 0.0 \\
\hline Platschu & Rubiaceae & Tree & $4-10 \mathrm{~m}$ & Platycarpum schultesii & 35 & 0.0 & 100.00 & 0.0 & 31 & 0.0 & 100.0 & 0.0 \\
\hline Pouteleg & Sapotaceae & Tree & $10-20 \mathrm{~m}$ & Pouteria elegans & 293 & 0.0 & 100.00 & 0.0 & 283 & 27.2 & 72.8 & 0.0 \\
\hline
\end{tabular}




\begin{tabular}{|c|c|c|c|c|c|c|c|c|c|c|c|c|}
\hline Poutguia & Sapotaceae & Tree & $25+m$ & Pouteria guianensis & 359 & 16.7 & 83.29 & 0.0 & 357 & 38.7 & 61.3 & 0.0 \\
\hline Poutreti & Sapotaceae & Tree & $25+m$ & Pouteria reticulata & 390 & 2.1 & 97.95 & 0.0 & 390 & 39.7 & 60.3 & 0.0 \\
\hline Roupmont & Proteaceae & Tree & $20-25 \mathrm{~m}$ & Roupala montana & 468 & 44.2 & 55.77 & 0.0 & 462 & 78.8 & 21.2 & 0.0 \\
\hline Sarcbras & Sapotaceae & Tree & $20-25 \mathrm{~m}$ & Sarcaulus brasiliensis & 355 & 1.1 & 98.87 & 0.0 & 359 & 28.4 & 71.6 & 0.0 \\
\hline Socrexor & Arecaceae & Tree & $20-25 \mathrm{~m}$ & Socratea exorrhiza & 476 & 17.4 & 82.56 & 0.0 & 479 & 54.7 & 45.3 & 0.0 \\
\hline Tabenodo & Bignoniaceae & Tree & $4-10 \mathrm{~m}$ & Tabebuia nodosa & 7 & 28.6 & 71.43 & 0.0 & 9 & 44.4 & 55.6 & 0.0 \\
\hline Tabeserr & Bignoniaceae & Tree & $25+m$ & Tabebuia serratifolia & 480 & 42.1 & 57.92 & 0.0 & 480 & 83.1 & 16.9 & 0.0 \\
\hline Tecotenu & Bignoniaceae & Bush & $1-4 \mathrm{~m}$ & Tecoma tenuiflora & 6 & 0.0 & 100.00 & 0.0 & 6 & 33.3 & 66.7 & 0.0 \\
\hline
\end{tabular}



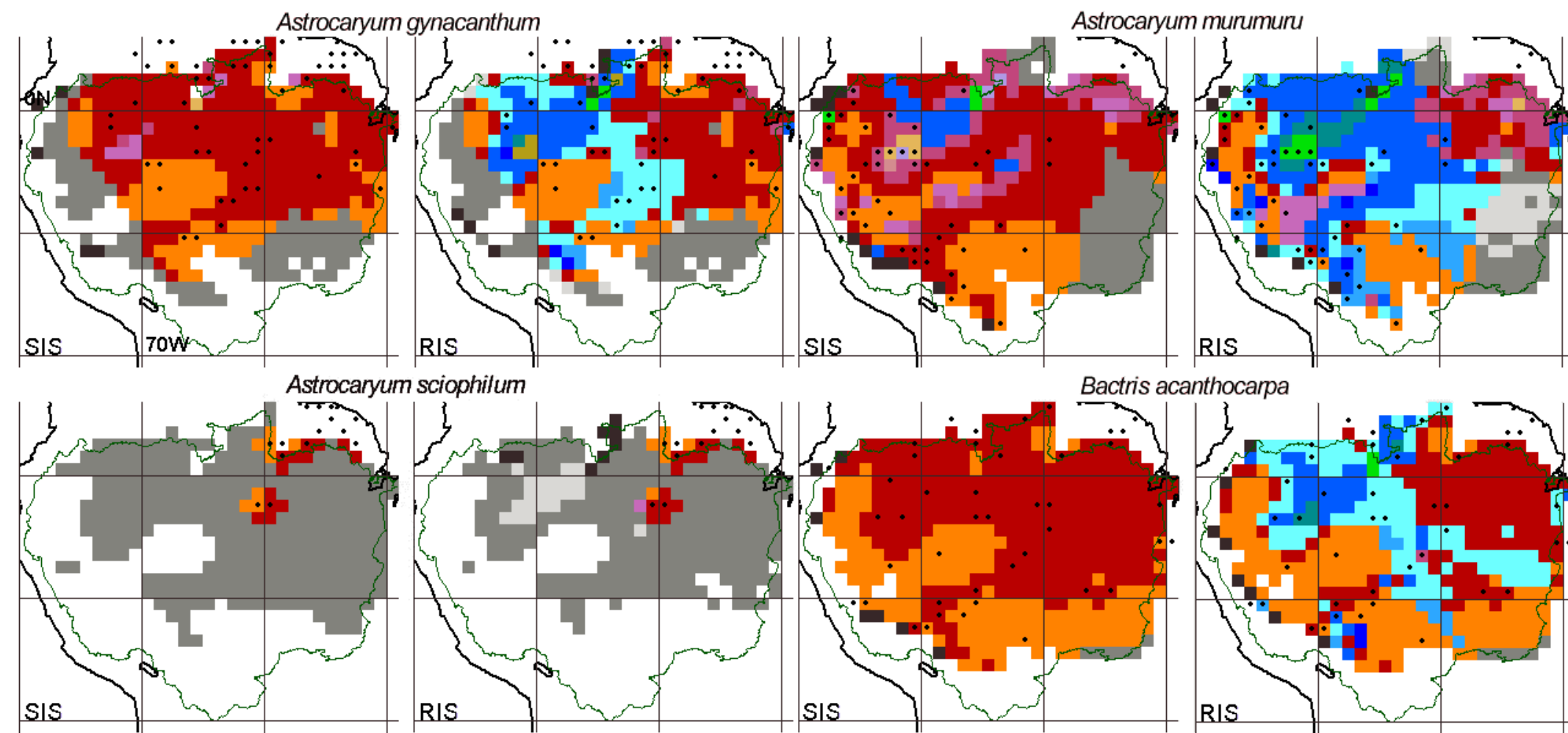

ey to model outcomes where

$a=K_{k p} 1990 / K_{k} \max$ $b=\Delta K_{k p}(1990->2095)$

- Record cell

$\square$ Absent

Viable populations

$\square \quad a>0.5, b<=-0.5$

$a<=0.5, b<=-0.5$

- $a>0.5, b<=0$

a $a<=0.5, b<=0$

$\square \quad a<=0.5, b<=0.5$

$\square \quad a>0.5, b<=0.5$

$\square \quad a<=0.5, b>0.5$

\section{Relicts}

- $a>0.5, b<=-0.5$

$\square \quad a<=0.5, b<=-0.5$

口 $a>0.5, b<=0$

$\square \quad a<=0.5, b<=0$

$\square \quad \mathrm{a}<=0.5, \mathrm{~b}<=0.5$

$\square \quad a>0.5, b<=0.5$
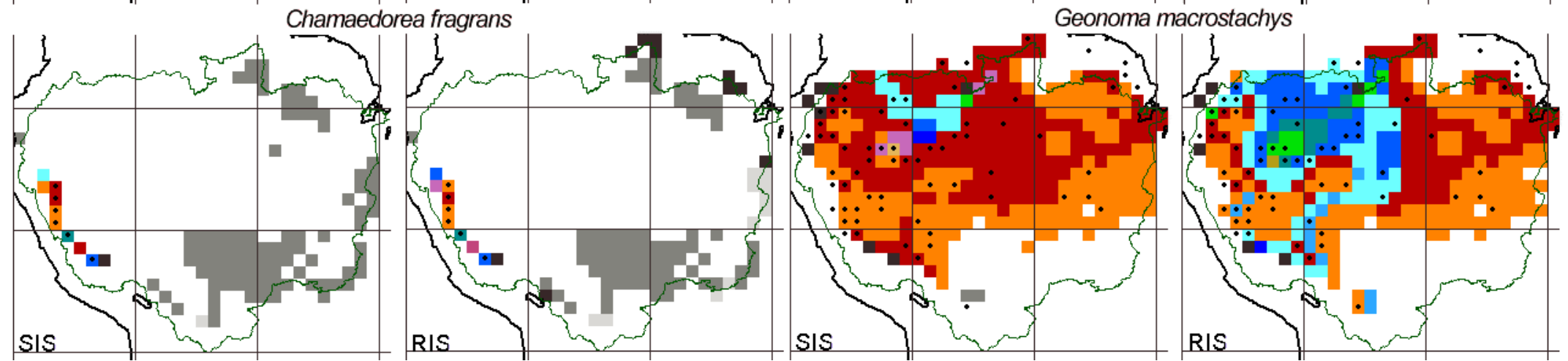

$a<=0.5, b>0.5$

Unoccupied potential distribution

$\square \quad 1990$ \& 2095

- 2095

口 1990

Fig. 6:14: Coded SIS \& RIS outcomes for 6 Arecaceae species 


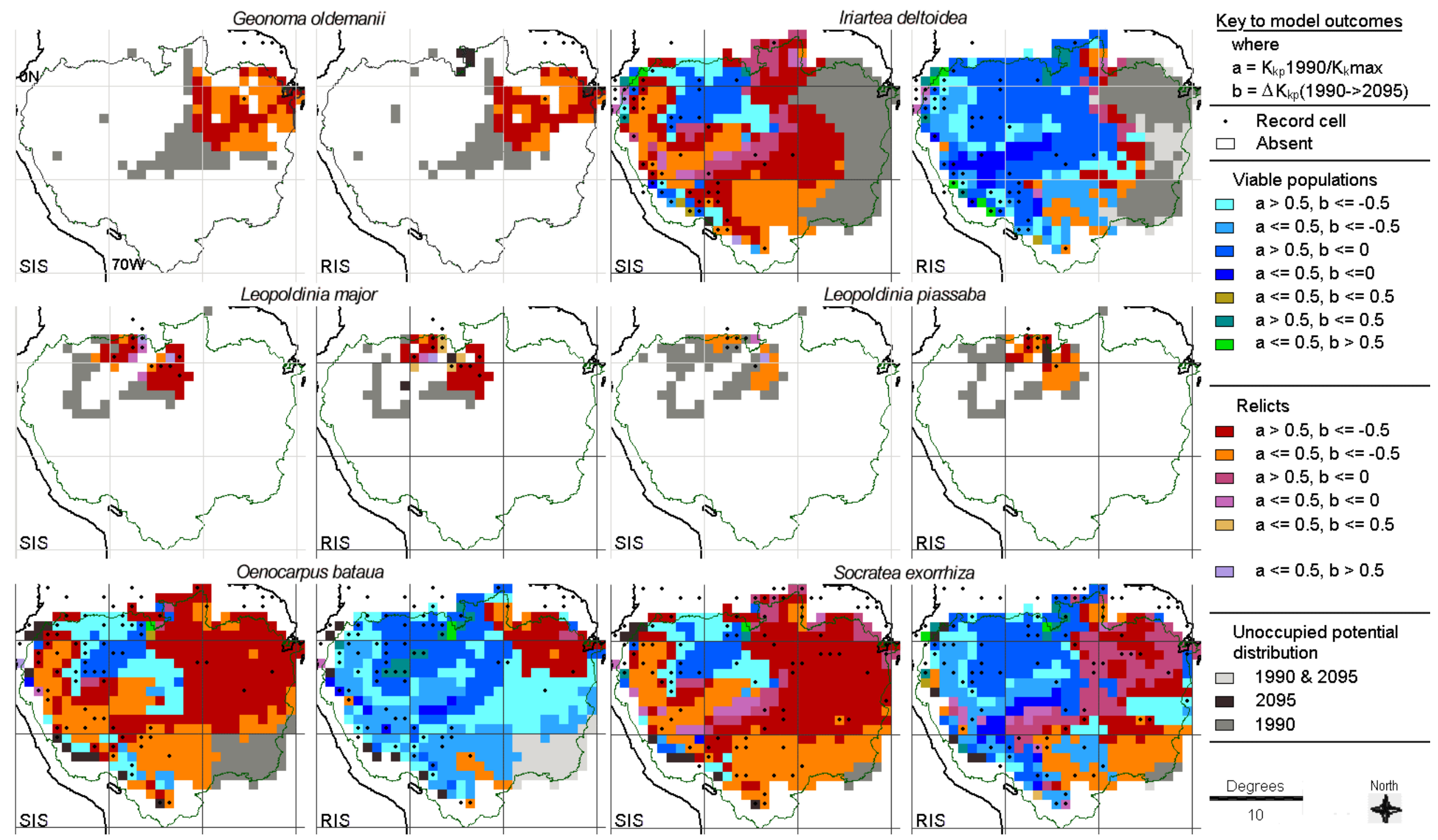

Fig. 6:15: Coded SIS \& RIS outcomes for a further 6 Arecaceae species 

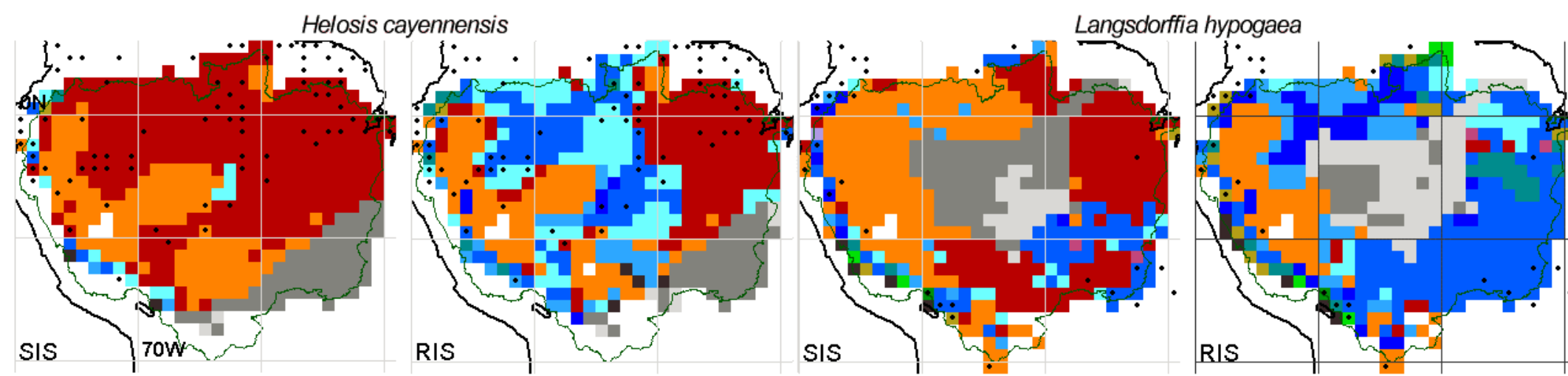

Key to model outcomes where

$a=K_{k p} 1990 / K_{k} \max$

$\mathrm{b}=\Delta \mathrm{K}_{\mathrm{kp}}(1990->2095)$

- Record cell

$\square$ Absent

Viable populations

$\square \quad a>0.5, b<=-0.5$

$a<=0.5, b<=-0.5$

$\square \quad a>0.5, b<=0$
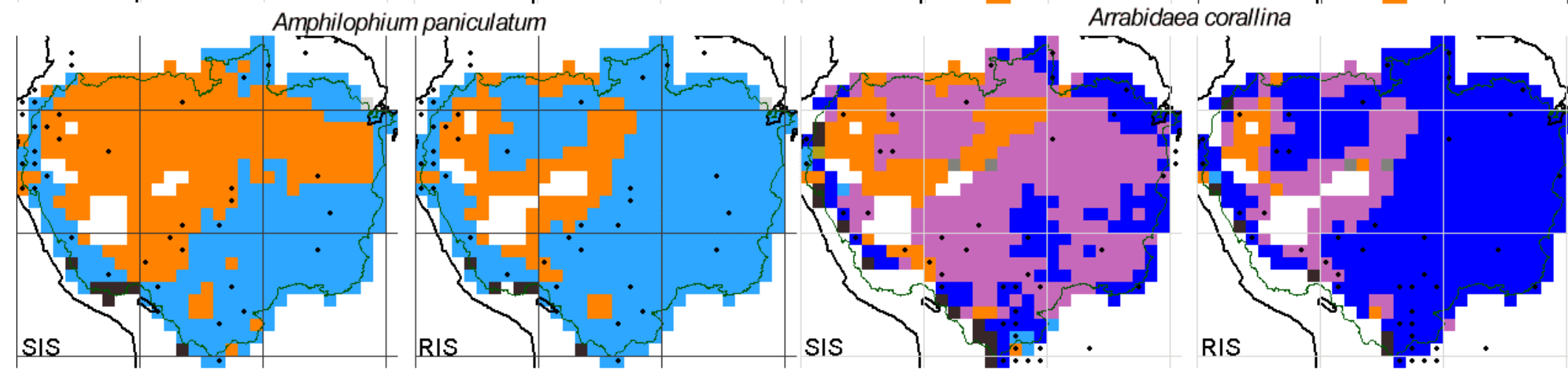

$\square \quad \mathrm{a}<=0.5, \mathrm{~b}<=0.5$

- $a>0.5, b<=0.5$

$\square \quad a<=0.5, b>0.5$

- $a>0.5, b>0.5$
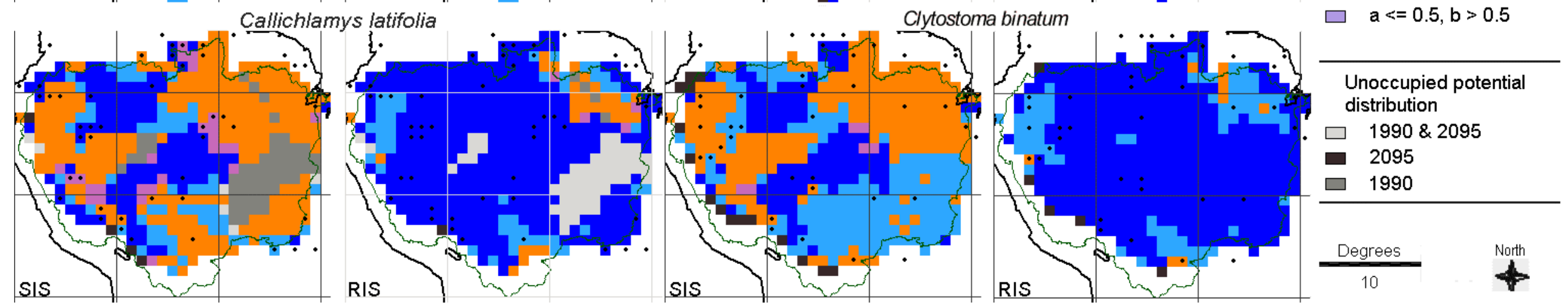

Fig. 6:16: Coded SIS \& RIS outcomes for 2 Balanophoraceae (top row) and 4 Bignoniaceae (centre \& bottom rows) species 

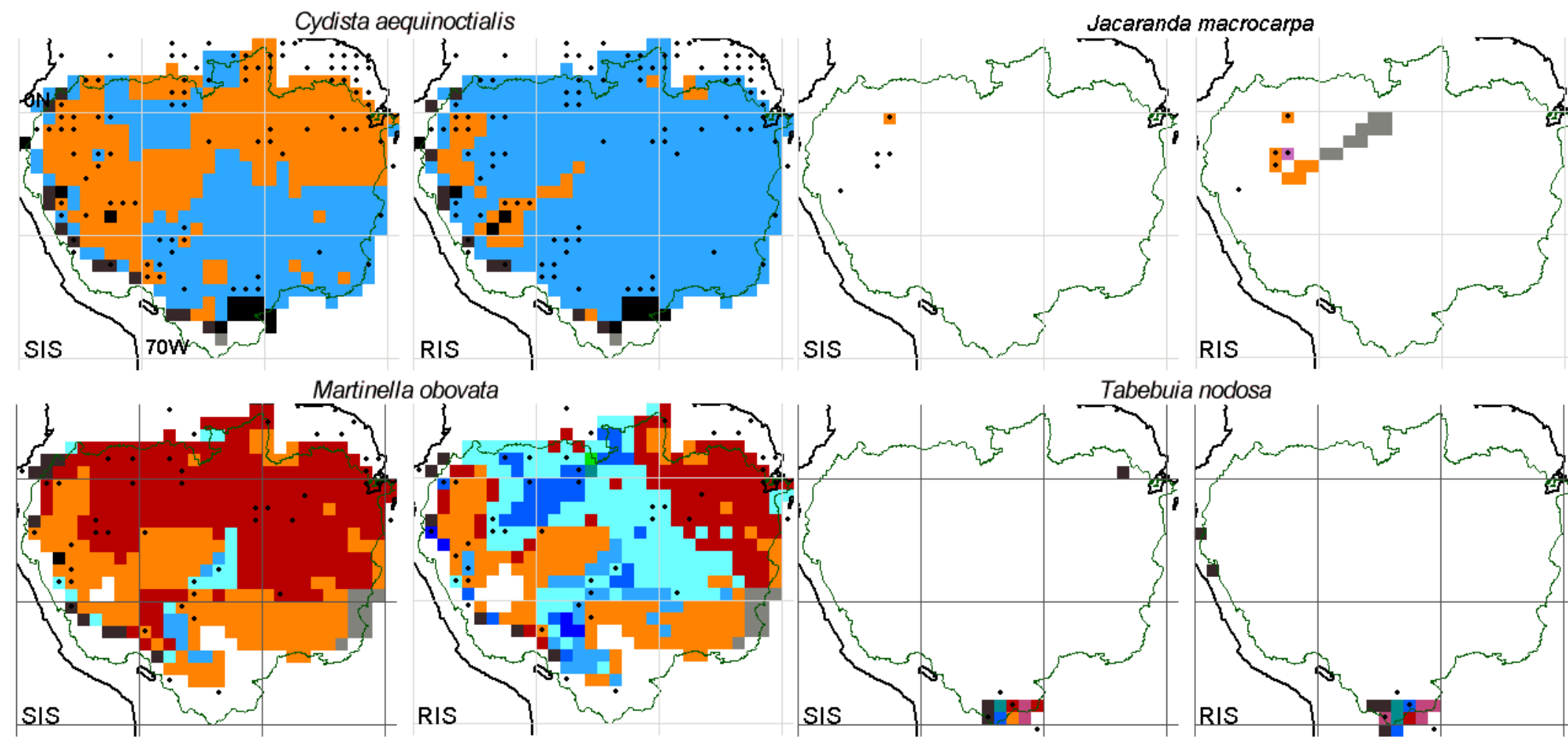

Tabebuia nodosa
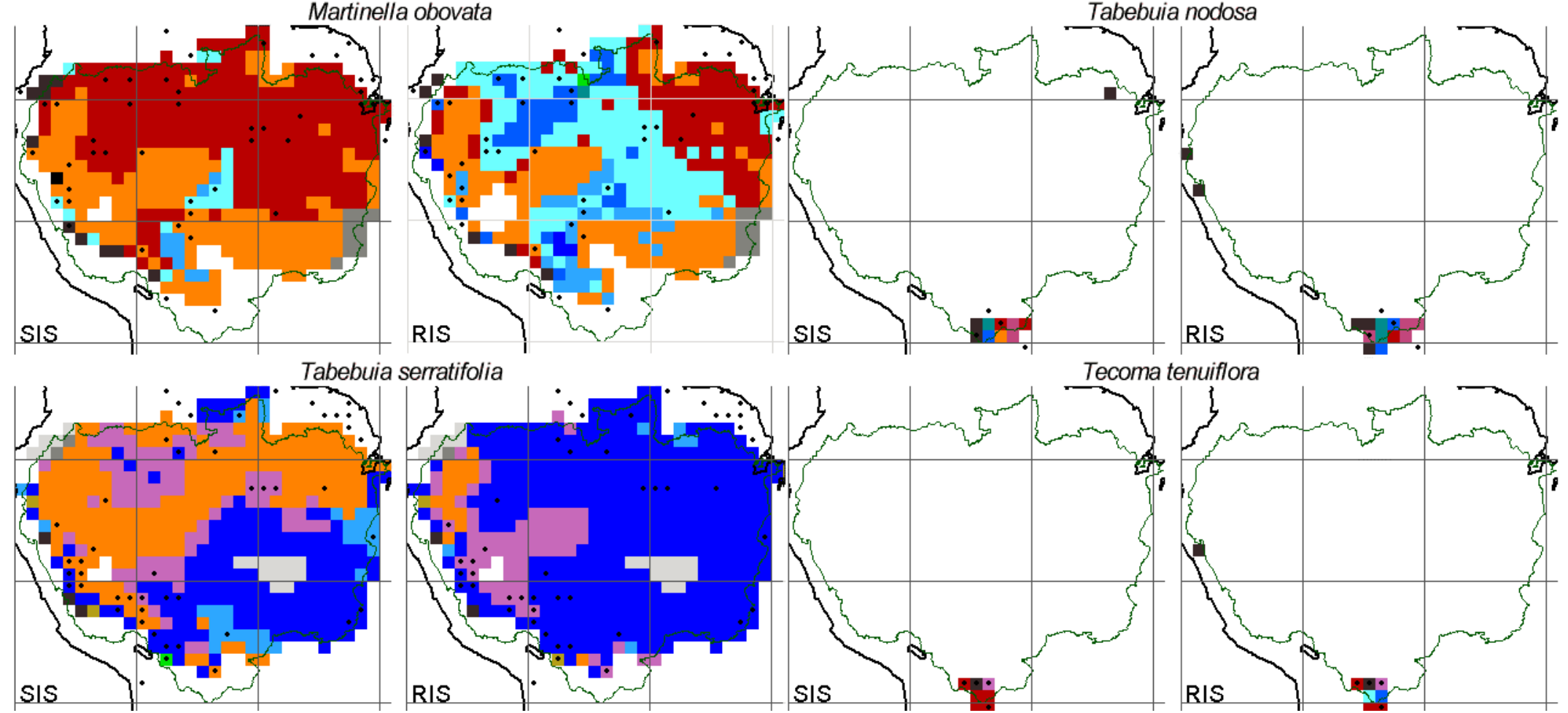

Key to model outcomes where

$a=K_{k p} 1990 / K_{k} \max$ $b=\Delta K_{k p}(1990->2095)$

- Record cell

$\square$ Absent

Viable populations

$\square \quad a>0.5, b<=-0.5$

$\square \quad a<=0.5, b<=-0.5$

- $a>0.5, b<=0$

a $a<=0.5, b<=0$

$\square \quad \mathrm{a}<=0.5, \mathrm{~b}<=0.5$

a $a>0.5, b<=0.5$

$\square \quad a<=0.5, b>0.5$

Relicts

- $a>0.5, b<=-0.5$

$\square \quad a<=0.5, b<=-0.5$

$a>0.5, b<=0$

$\square \quad a<=0.5, b<=0$

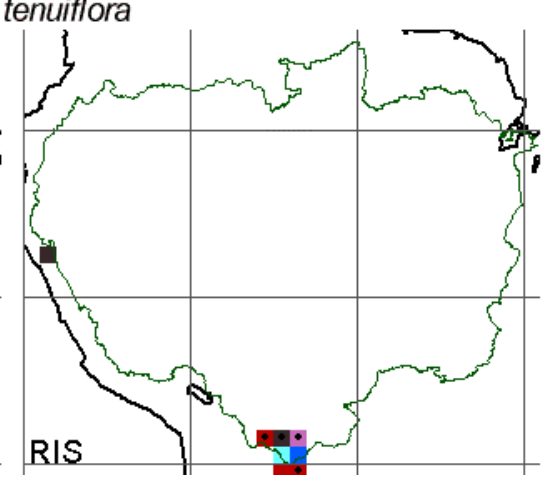

Unoccupied potential distribution

$\square \quad 1990$ \& 2095

- 2095

$\square \quad 1990$

- Lost by 2095

Degrees

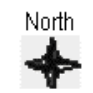

Fig. 6:17: Coded SIS \& RIS outcomes for 6 Bignoniaceae species 


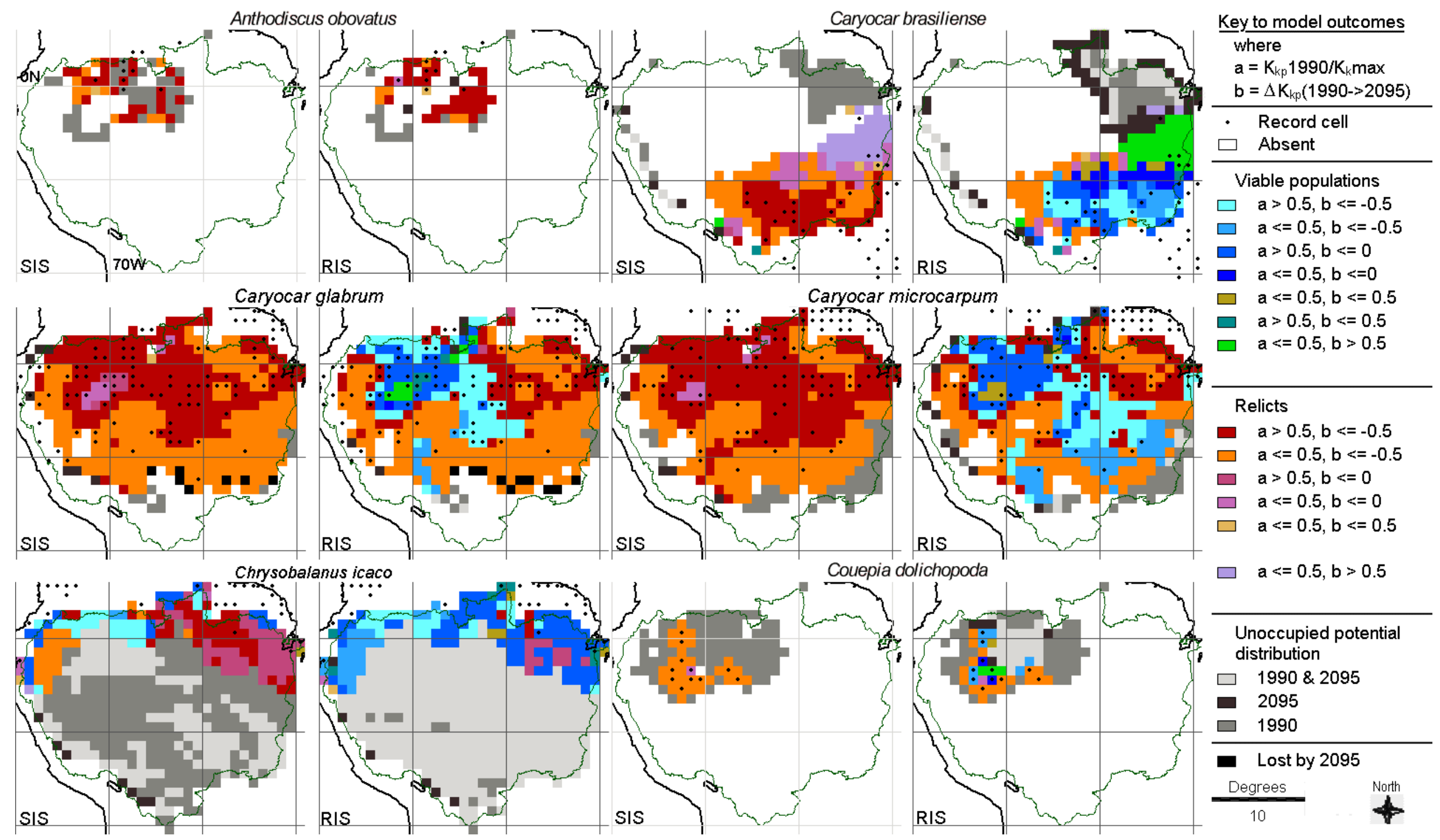

Fig. 6:18: Coded SIS \& RIS outcomes for 4 Caryocaraceae (top \& centre rows) and 2 Chrysobalanaceae (bottom row) species 

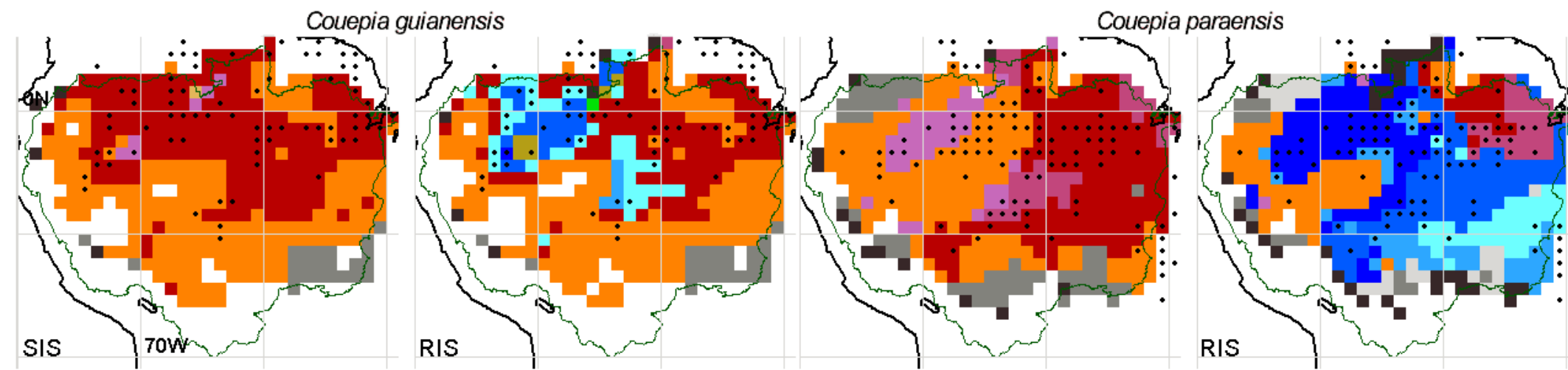

Key to model outcomes
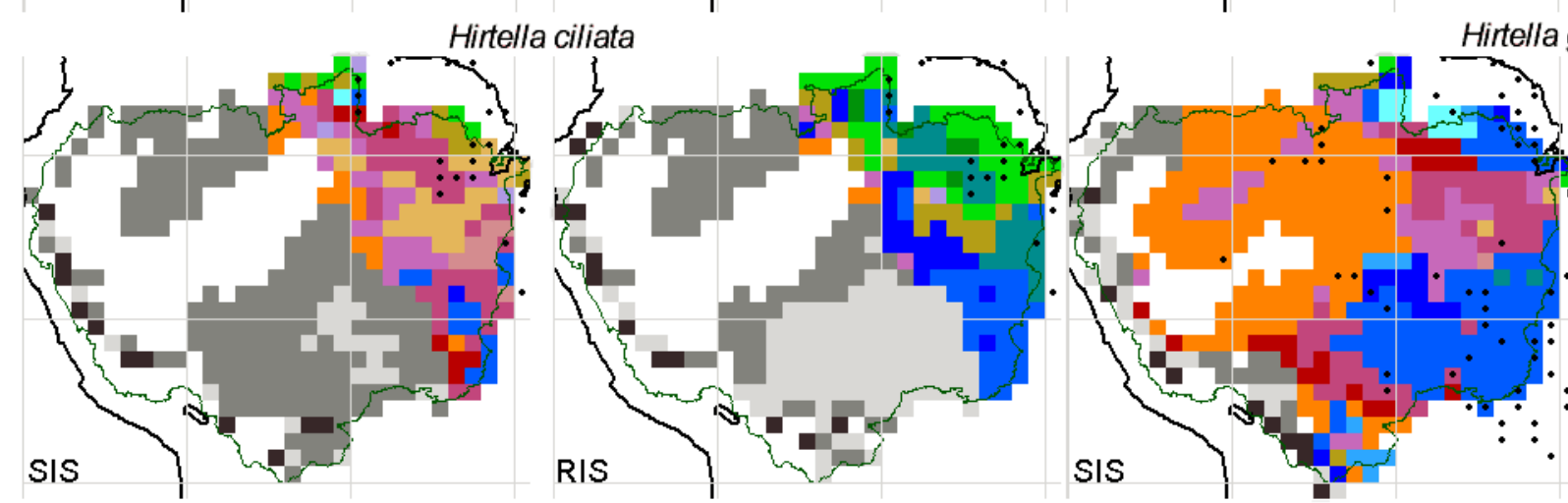

glandulosa $\quad \because \quad \square \quad \begin{aligned} & a<0.5, b<=0.5 \\ & \square\end{aligned}$ where

$\mathrm{a}=\mathrm{K}_{\mathrm{kp}} 1990 / \mathrm{K}_{\mathrm{k}} \max$

$\mathrm{b}=\Delta \mathrm{K}_{\mathrm{kp}}(1990->2095)$

- Record cell

$\square$ Absent

Viable populations

$\square \quad a>0.5, b<=-0.5$

$\square \quad a<=0.5, b<=-0.5$

$\square \quad a>0.5, b<=0$

$\mathrm{a}<=0.5, \mathrm{~b}<=0$

landulosa $\quad \because \quad \square \quad \begin{aligned} & a<0.5, b<=0.5 \\ & a>0.5, b<=0.5\end{aligned}$

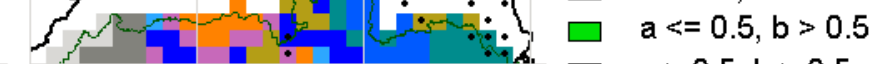

, $a>0.5, b>0.5$

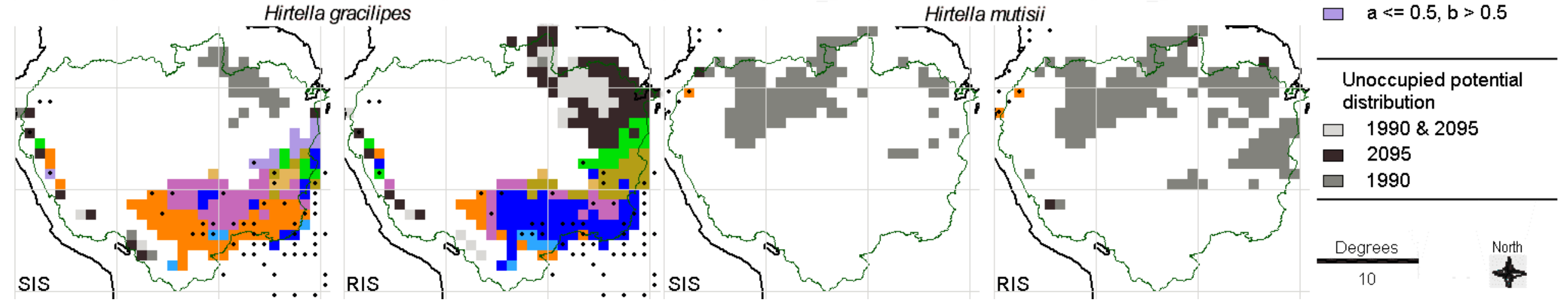

Fig. 6:19: Coded SIS \& RIS outcomes for 6 Chrysobalanaceae species 

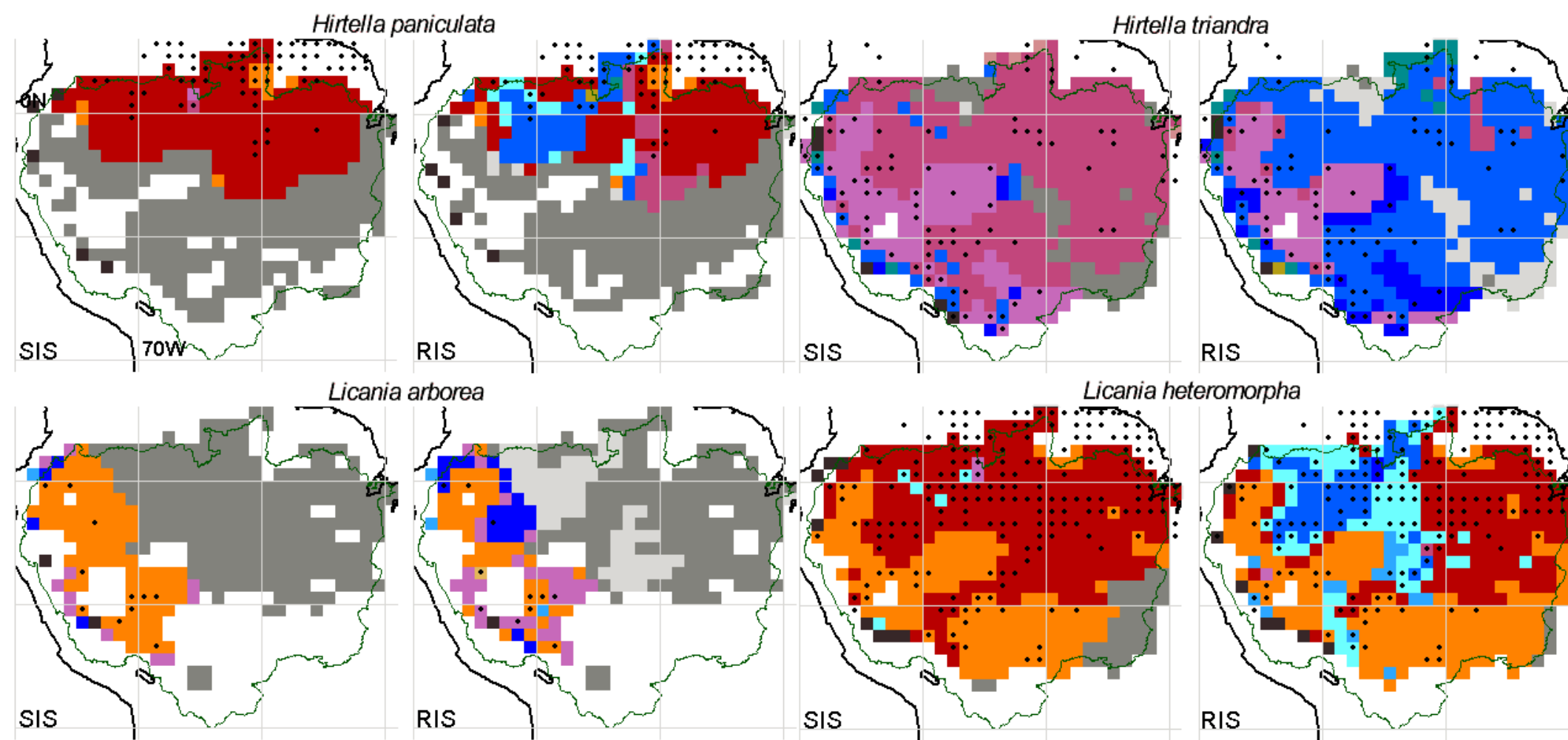

eromorpha
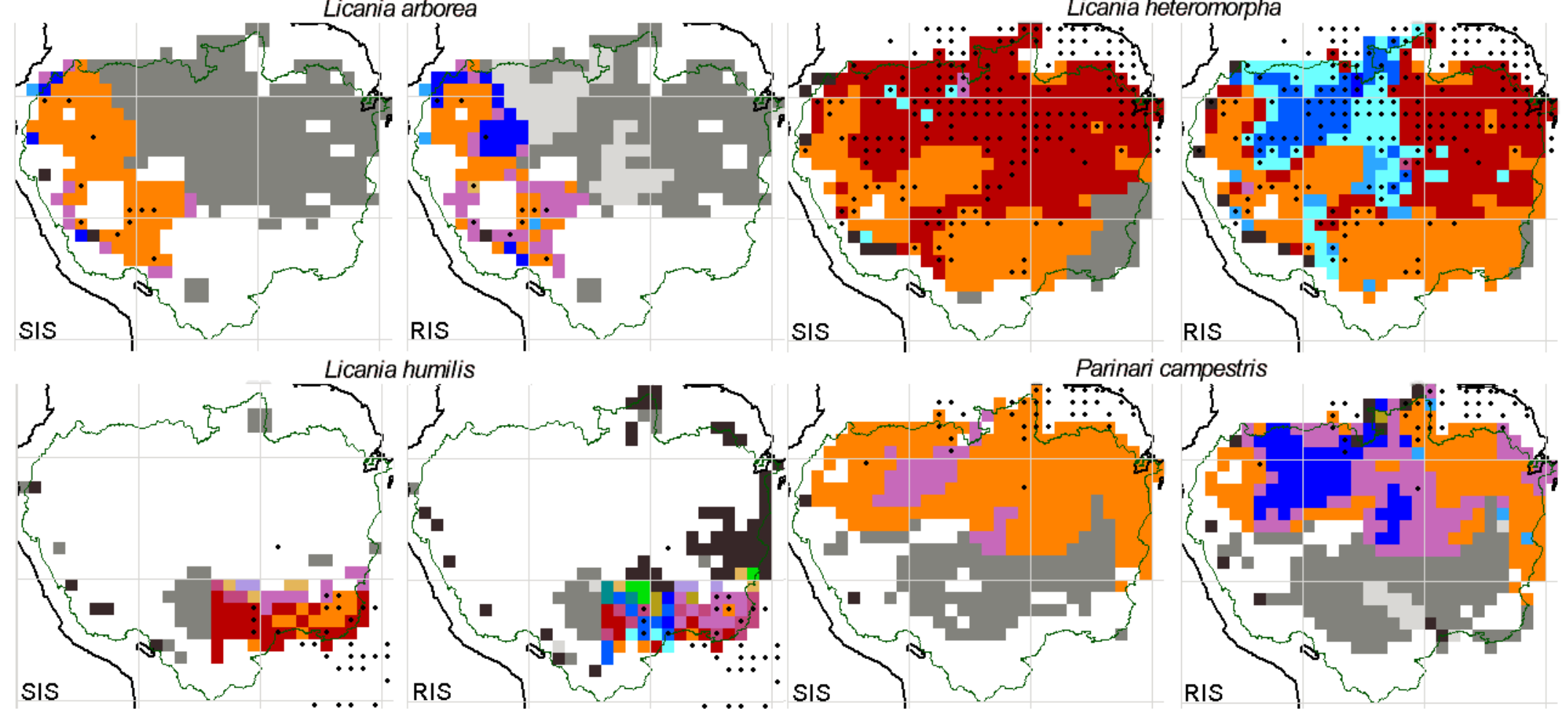

Key to model outcomes where

$a=K_{k p} 1990 / K_{k} \max$ $b=\Delta K_{k p}(1990->2095)$

- Record cell

\section{$\square$ Absent}

Viable populations

$\square \quad a>0.5, b<=-0.5$

$\square \quad \mathrm{a}<=0.5, \mathrm{~b}<=-0.5$

$\square \quad a>0.5, b<=0$

a $a<=0.5, b<=0$

$\square \quad a<=0.5, b<=0.5$

$\square \quad a>0.5, b<=0.5$

$\square \quad a<=0.5, b>0.5$

\section{Relicts}

- $a>0.5, b<=-0.5$

$\square \quad a<=0.5, b<=-0.5$

$a>0.5, b<=0$

$\square \quad a<=0.5, b<=0$

$\square \quad \mathrm{a}<=0.5, \mathrm{~b}<=0.5$

$\square \quad a>0.5, b<=0.5$

$\square \quad a<=0.5, b>0.5$

\section{Unoccupied potential} distribution

$\square \quad 1990 \& 2095$

- 2095

$\square \quad 1990$

Fig. 6:20: Coded SIS \& RIS outcomes for a further 6 Chrysobalanaceae species 

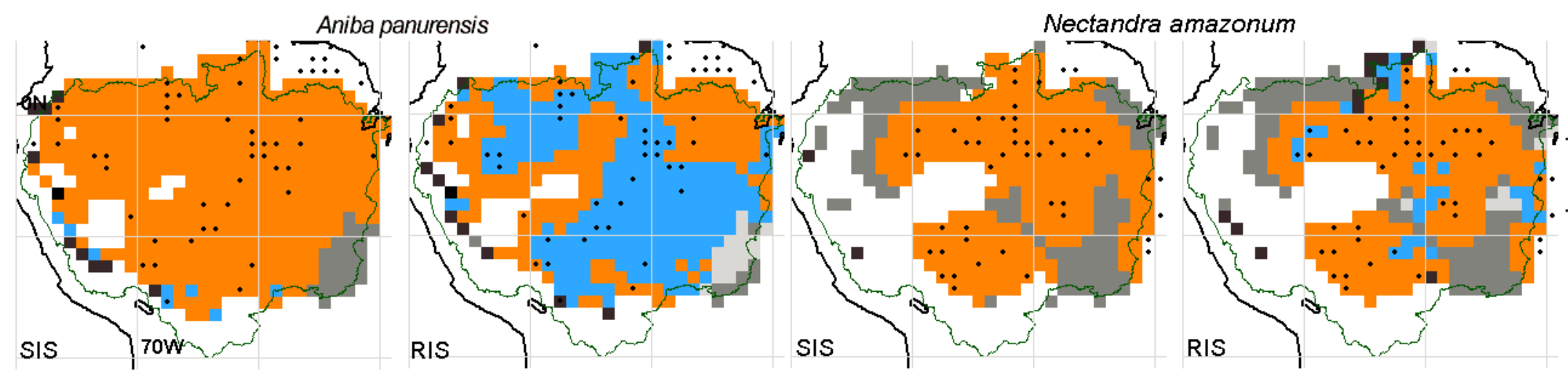

Key to model outcomes where

$a=K_{k p} 1990 / K_{k} \max$ $b=\Delta K_{k p}(1990->2095)$

Record cell

$\square$ Absent

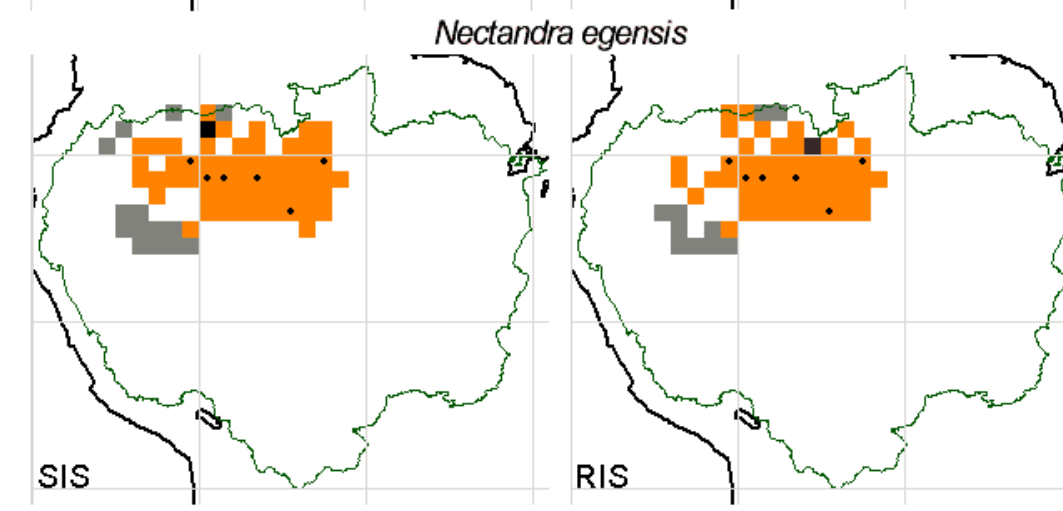

Nectandra hihua

Viable populations

$\square \quad a<=0.5, b<=-0.5$
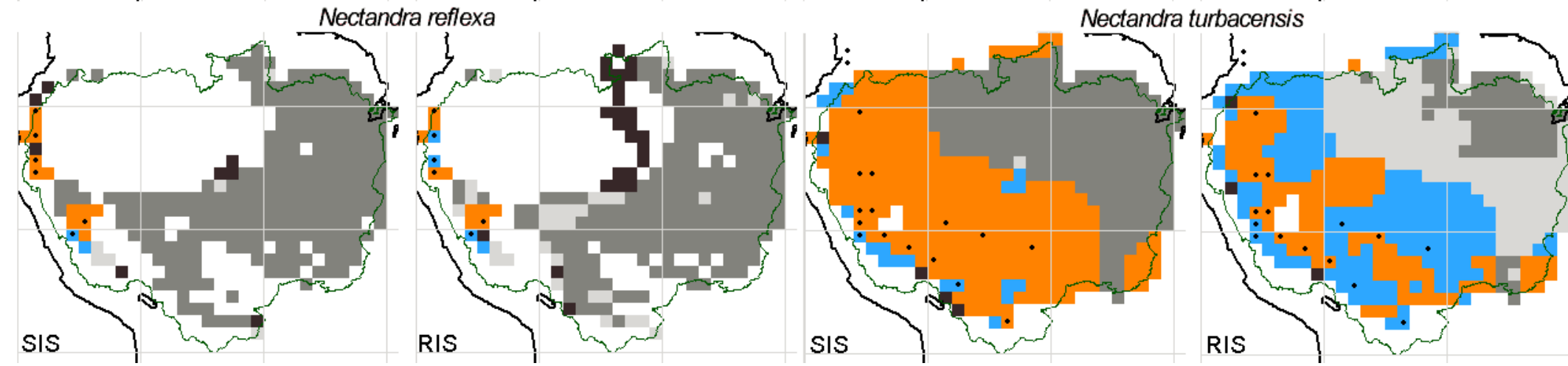

Unoccupied potential distribution

$\square \quad 1990 \& 2095$

- 2095

$\square \quad 1990$

- Lost by 2095

Degrees

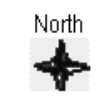

Fig. 6:21: Coded SIS \& RIS outcomes for 6 Lauraceae species 


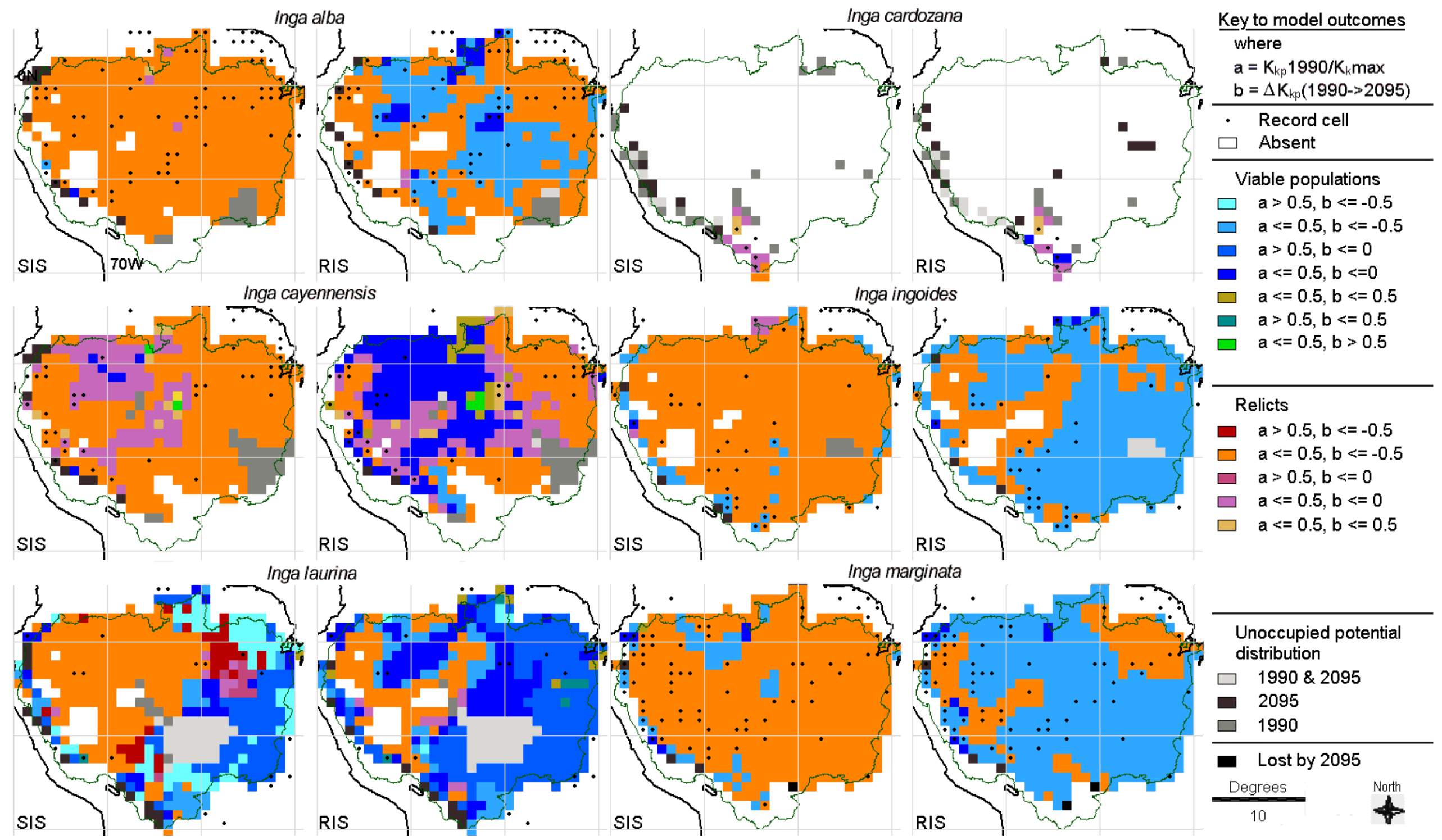

Fig. 6:22: Coded SIS \& RIS outcomes for 6 Fabaceae species 


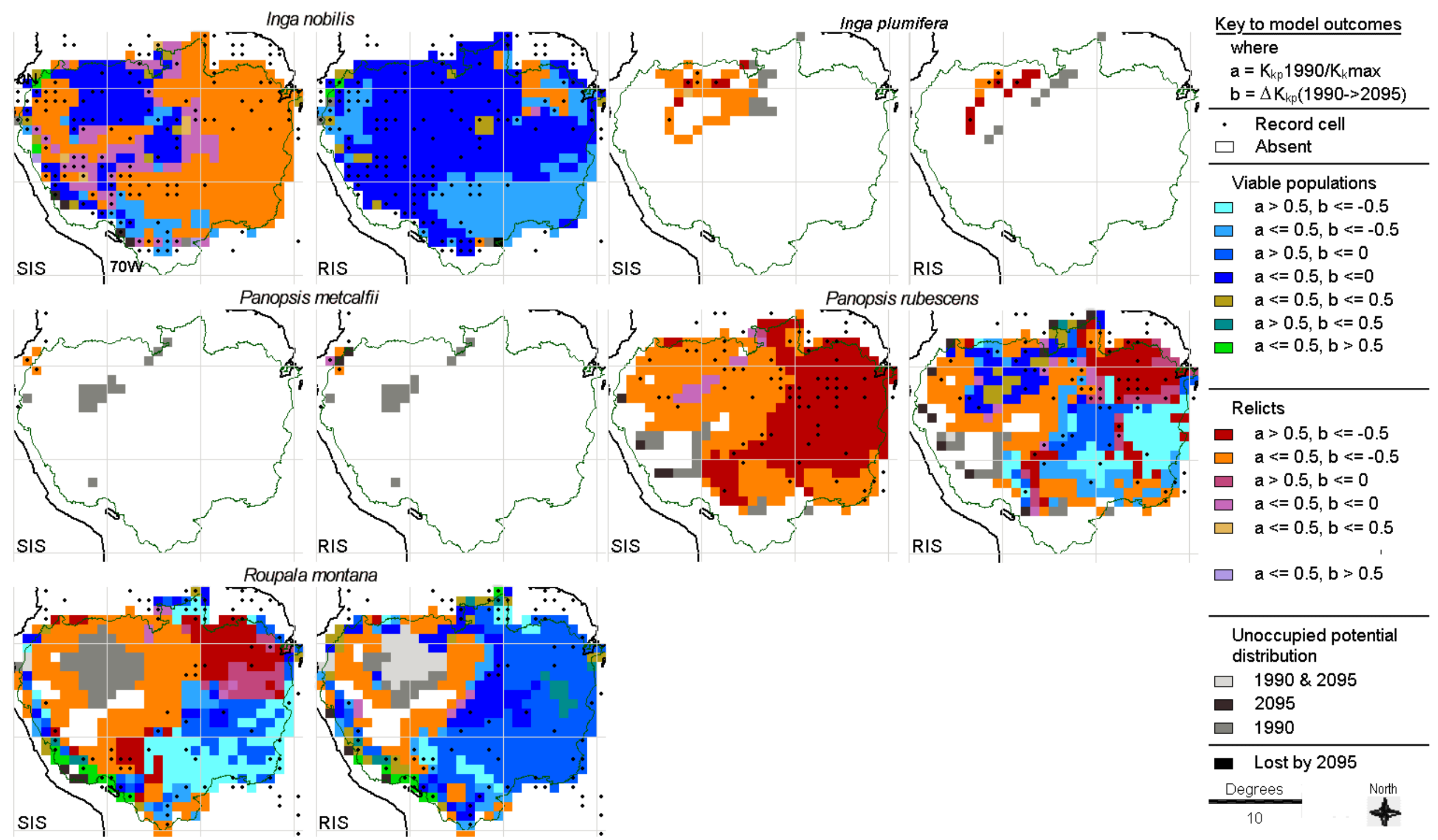

Fig. 6:23: Coded SIS \& RIS outcomes for 2 Fabaceae (top row) and 3 Proteaceae (centre \& bottom rows) species 


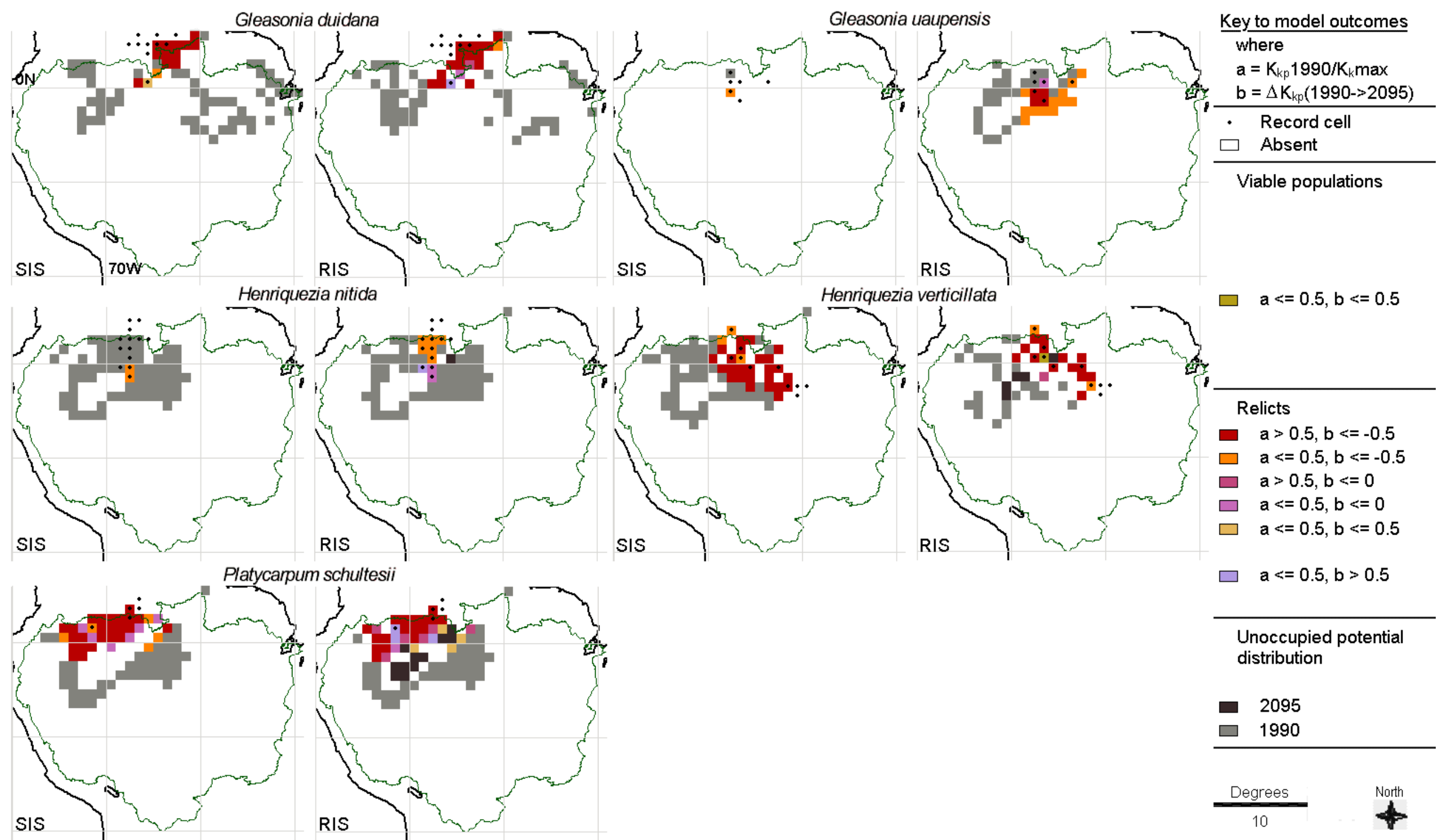

Fig. 6:24: Coded SIS \& RIS outcomes for 5 Rubiaceae species 

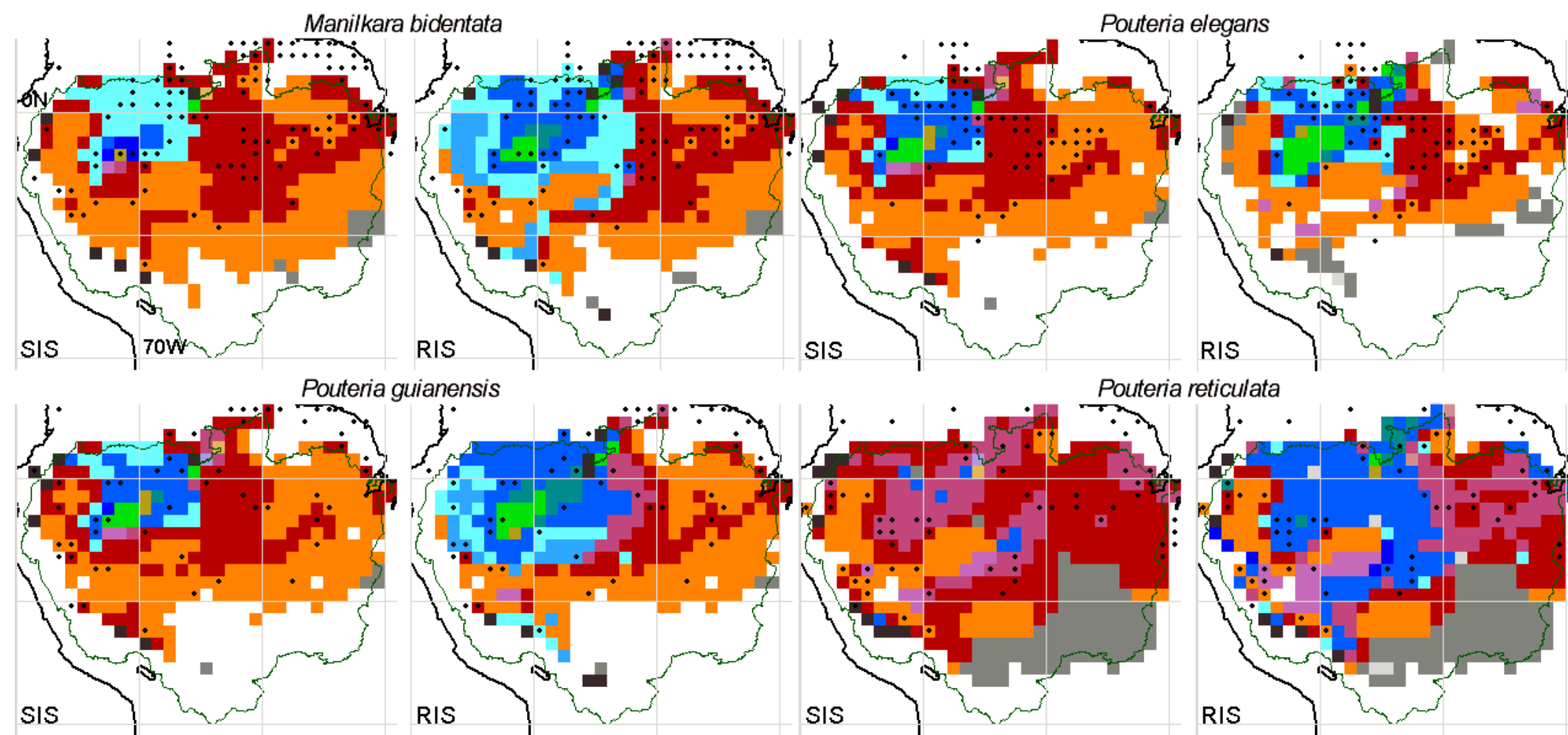

reticulata
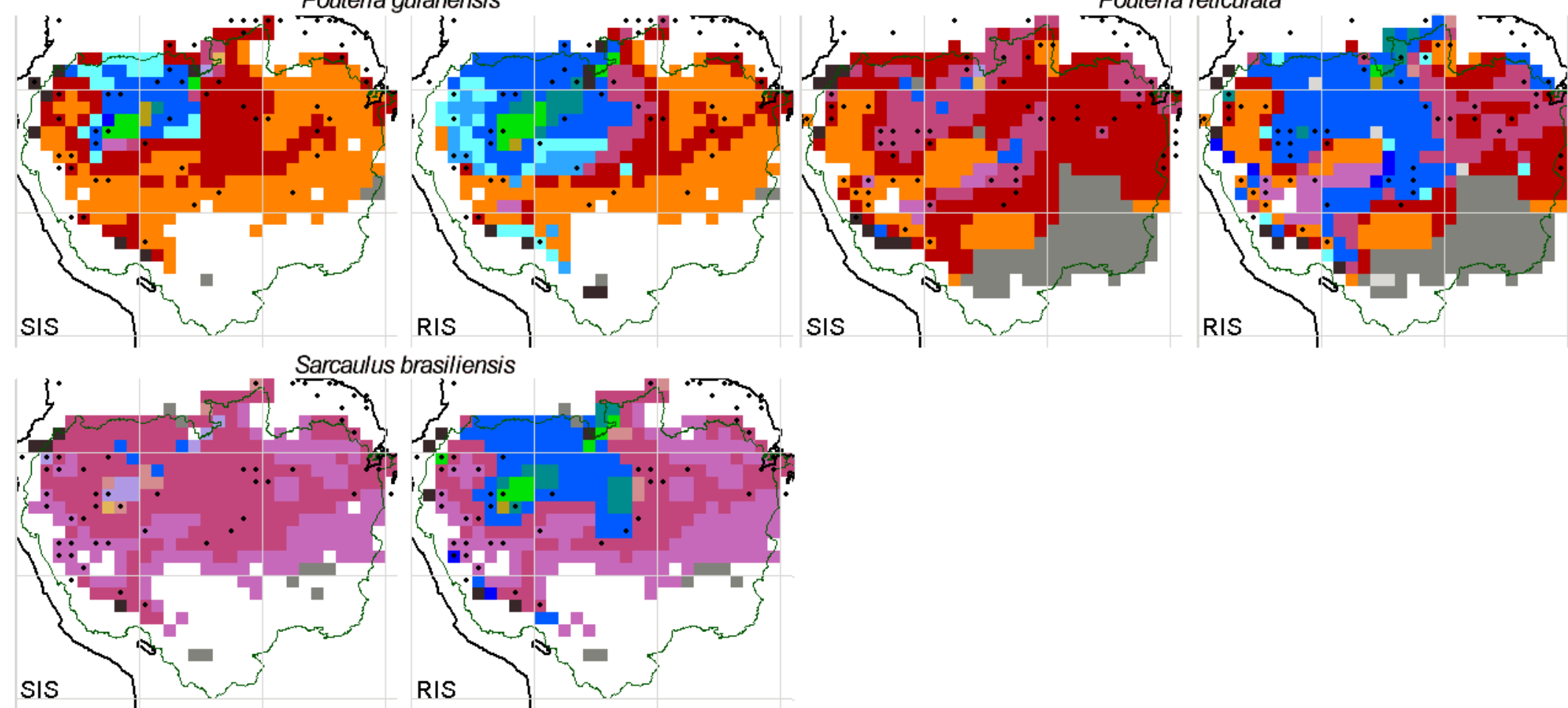

Key to model outcomes where

$a=K_{k p} 1990 / K_{k} \max$

$b=\Delta K_{k p}(1990->2095)$

- Record cell

$\square$ Absent

Viable populations

$\square \quad a>0.5, b<=-0.5$

$a<=0.5, b<=-0.5$

$\square \quad a>0.5, b<=0$

- $\mathrm{a}<=0.5, \mathrm{~b}<=0$

$\square \quad a<=0.5, b<=0.5$

$\square \quad a>0.5, b<=0.5$

$\square \quad a<=0.5, b>0.5$

\section{Relicts}

$a>0.5, b<=-0.5$

$\square \quad a<=0.5, b<=-0.5$

$\square \quad a>0.5, b<=0$

$\square \quad \mathrm{a}<=0.5, \mathrm{~b}<=0$

$\square \quad a<=0.5, b<=0.5$

$\square \quad a>0.5, b<=0.5$

$\square \quad a<=0.5, b>0.5$

Unoccupied potential
distribution
$\begin{aligned} & 1990 \& 2095 \\ & 2095 \\ & 1990\end{aligned}$

- 2095

Fig. 6:25: Coded SIS \& RIS outcomes for 5 Sapotaceae species 


\subsubsection{Shared spatial patterns}

The maps of climate change alone indicate that species in some areas may be at more risk than others. The spatial demographic model offers more information on potential biodiversity change than can be obtained from this broad conclusion, or from projections of changing vegetation types. Adjacent cells sharing a single climate change anomaly may have different initial species compositions, each species having different climate tolerances - not all sympatric species react the same way to a given change. Alternatively, two cells may share the same species composition, but have sufficiently different original climates that the same degree of change removes only one cell from a species' environmental niche. Where different patterns of species response can be identified, areas and species under threat may also be identifiable.

The environmental preferences of each species were estimated from its present Neotropical distribution; hence, its present distribution was key in determining the changes in potential and realised distribution seen in the model. Where new potential distribution was adjacent to existing distribution, there is an increased possibility that the species could migrate to occupy this area, as there are no obvious climate barriers.

Most species' potential ranges $\left(S_{\mathrm{p}}>S_{\min }\right)$ shifted between 1990 and 2095 (Fig. 6:14 - Fig. 6:25). Various types of distribution and shared responses were observed, but shared, frequently encountered patterns and those most interesting in terms of change response are as follows:

\section{4) Restricted to western Amazonia}

The contemporary Amazonian distributions of Hirtella mutisii (Fig. 6:19), Chamaedorea fragrans (Fig. 6:14) and Nectandra reflexa (Fig. 6:21) were restricted to the western edge of the basin. Only H. mutisii had extra-Amazonian records in 1990. Each species had an extensive area of disjunct potential distribution, which the model simulated as unoccupied, but which was largely lost as potential distribution by 2095. All three species retained at least one viable population under the SIS projection, and gained new areas of potential distribution in the far west, although for $H$. mutisii, the only new cell was not adjacent to any existing populations.

Licania arborea (Fig. 6:20) originally had a patchy distribution in the west, and a large potential distribution over much of the rest of the north of Amazonia. The reference climate here was similar to that in the species' record-cells, but the realised distribution was restricted by the species' buffer distance. Few patches remained viable under the SIS change projection; a few more did so under the RIS projection. 
5) Distributed largely in the west

Iriartea deltoidea (Fig. 6:15) occupies such a wide range of environments that its 1990 potential distribution covered all but the southern and western edge of the Amazon. The realised distribution was restricted by buffering to the west and central regions. Under SIS at 2095, this species retained viable populations only in the northwest and southwestern edge. Under the RIS projection, it remained viable over most of its range, with relicts appearing to the east.

The 1990 distribution of Nectandra turbacensis (Fig. 6:21) was also limited by buffering rather than climate. This species inhabited west and south-central Amazonia, with disjunct areas of distribution on the northern edge and southeast corner. These zones are contiguous with some of the species' many extra-Amazonian record-cells. Under the SIS projection, the viable distribution was reduced to a block of cells in central Amazonia, and others along the western edge, where some new potential distribution also arose. Under the RIS projection, $60 \%$ of the range remained viable, with relict cells experiencing increased $A E T$ in the west or increased $M D$ in the south.

6) Restricted to northwest to central region

Thirteen of the 69 selected species were restricted to the most northern part of Amazonia under the reference climate, between approximately $60^{\circ} \mathrm{W}$ and $80^{\circ} \mathrm{W}$. All the Rubiaceae species in the selection inhabit this region (Henriquezia nitida, H. verticillata, Platycarpum schultesii, Gleasonia duidana and G. uaupensis, Fig. 6:24). The two north-central palm species, Leopoldinia major and L. piassaba, had very similar distributions to one another and under SIS and RIS at 1990, and similar responses in 2095 (Fig. 6:15).

The Jacaranda macrocarpa (Fig. 6:17) simulation for SIS was poor, as four of its five recordcells were simulated as outside the 1990 distribution. The most limiting factor in these cells was too stringent to allow population survival at equilibrium. This can occur when the 1960s to 1980s mean DOWNS value for an occupied cell differs substantially from the species mean for a variable, and the range is small. The same did not happen under RIS, but the species still became non-viable throughout its simulated distribution by 2095 .

The remaining north-west to north-central species are Anthodiscus obovatus (Fig. 6:18) and Nectandra egensis (Fig. 6:21), Inga plumifera (Fig. 6:23), and Couepia dolichopoda (Fig. 6:18). Although Panopsis metcalfii (Fig. 6:23) has been included in this set, it has a different distribution pattern to the others, its intrusion into the Amazon basin being the southern part of a coastal range that stretches up to Colombia. C. dolichopoda had a reduced potential distribution under RIS at 2095, but maintained a core of viable cells. The other species did not retain viable 
cells under the SIS or RIS projection. Some gained a small area of adjacent potential distribution under RIS.

Under the reference climate, the north-central area had midrange AET, low $M D$, and low SMA. AET was somewhat higher in the northwest. By 2095, the increase in each of these variables under SIS had caused the climate to leave the tolerated range of species originally restricted to this area. This is a typical projected result of perturbation to narrow-range species.

7) Widespread except for southern tip and/or western edge of Amazonia

About a third of the species were virtually ubiquitous under the reference climate, being restricted only from occupying the cooler southern and/or western edges of the region. These moist forest species all gained new potential distributions on the western edge of their ranges, but lost viability over much of their existing range under the SIS projection. Astrocaryum gynacanthum, Bactris acanthocarpa (both Fig. 6:14), Caryocar glabrum, C. microcarpum (both Fig. 6:18), Couepia guianensis, C. paraensis (both Fig. 6:19) and Panopsis rubescens (Fig. 6:23) all lost viability from their entire realised distributions at 2095.

Many of the other species in this group retained viable cells at the west of their distributions at 2095 (Astrocaryum murumuru (Fig. 6:14), Oenocarpus bataua, Socratea exorrhiza (both Fig. 6:15), Callichlamys latifolia (Fig. 6:16), Hirtella triandra, Licania heteromorpha (both Fig. 6:20), Inga alba, I. cayennensis, I. marginata (all Fig. 6:22) and I. nobilis (Fig. 6:23)). Some species (e.g. C. latifolia) retained a high proportion of viable populations, whilst others (e.g. L. heteromorpha) retained very few. All fared much better under RIS.

To different extents, Clytostoma binatum (Fig. 6:16), Cydista aequinoctialis and Tabebuia serratifolia (both Fig. 6:17) retained a proportion of their viable range in the northwest and a larger portion in the southeast under the SIS projection. The remaining species (Martinella obovata (Fig. 6:17), Aniba panurensis, Nectandra hihua (both Fig. 6:21), and Inga ingoides (Fig. 6:22)) had very variable outcomes.

8) Restricted to south-central region

An opposing pattern of initial distribution was seen in Tecoma tenuiflora, Tabebuia nodosa (both Fig. 6:17) and Inga cardozana (Fig. 6:22), all of which were confined to the southern tip of Amazonia. Only T. nodosa retained any viable cells under SIS at 2095. All had limited new areas of potential distribution at 2095. I. cardozana is found in cloud forests (Pennington 1997b), which was reflected in its potential distribution along the Andes at both points in time. 


\section{9) Restricted to northeast region}

Geonoma oldemanii was simulated as having a surprisingly broad initial distribution, in the northeast (Fig. 6:14). It has only one record in the inclusive-Amazon, but a large buffer distance. Astrocaryum sciophilum had a very broad potential distribution at 1990, but its realised distribution was more limited. Neither species retained viable populations under the SIS or RIS change projections, but both gained new areas of potential distribution in the northcentral regions under RIS.

\section{0) Restricted to southeast region}

Caryocar brasiliense (Fig. 6:18) and Licania humilis (Fig. 6:20) both did reasonably well under the RIS change projection, persisting in large parts of the present range and gaining new potential distribution to the north. C. brasiliense displayed increases in population density over much of its range. However, under the SIS projection, both species lost viability in all cells.

Hirtella gracilipes shared the same type of initial distribution (Fig. 6:19), but also had a disjunct set of populations at the western edge of Amazonia. The greater range of climates occupied enabled the species to increase in density over parts of the southeast distribution under the SIS projection, although many populations still became non-viable. In reality, the two disjunct populations may have undergone different adaptations, and so not share climate tolerances, so there is additional uncertainty in this type of simulation.

\subsubsection{Summary}

We frequently see that narrow-range species are most likely to experience range contraction under an IS92a scenario. The more revealing aspect is the lack of a consistent pattern in species that are widespread over Amazonia. This variation for species with a similar Amazonian range reinforces the importance of obtaining the full environmental range of the study species.

Amazon-endemic species lose viability over significantly more populations than those with cells outside the inclusive-Amazon in RIS but not in SIS ( $p=0.053$ SIS, 0.000, RIS, Mann-Whitney U-test). SIS seems to have such a strong impact that differences between groups of species are more difficult to identify.

Under SIS, many species develop areas of new potential distribution along the western edge of their current simulated range. In general, this follows the longitudinal gradient in moisture deficit and seasonality, which shifts westwards between 1990 and 2095. The consistent loss of viability in the forked western region of increased AET is discussed further in section 7.2.5. 


\subsubsection{Change in population density}

The rate of density change in response to perturbation depends on the climate tolerance and on the demographic parameters of the species concerned.

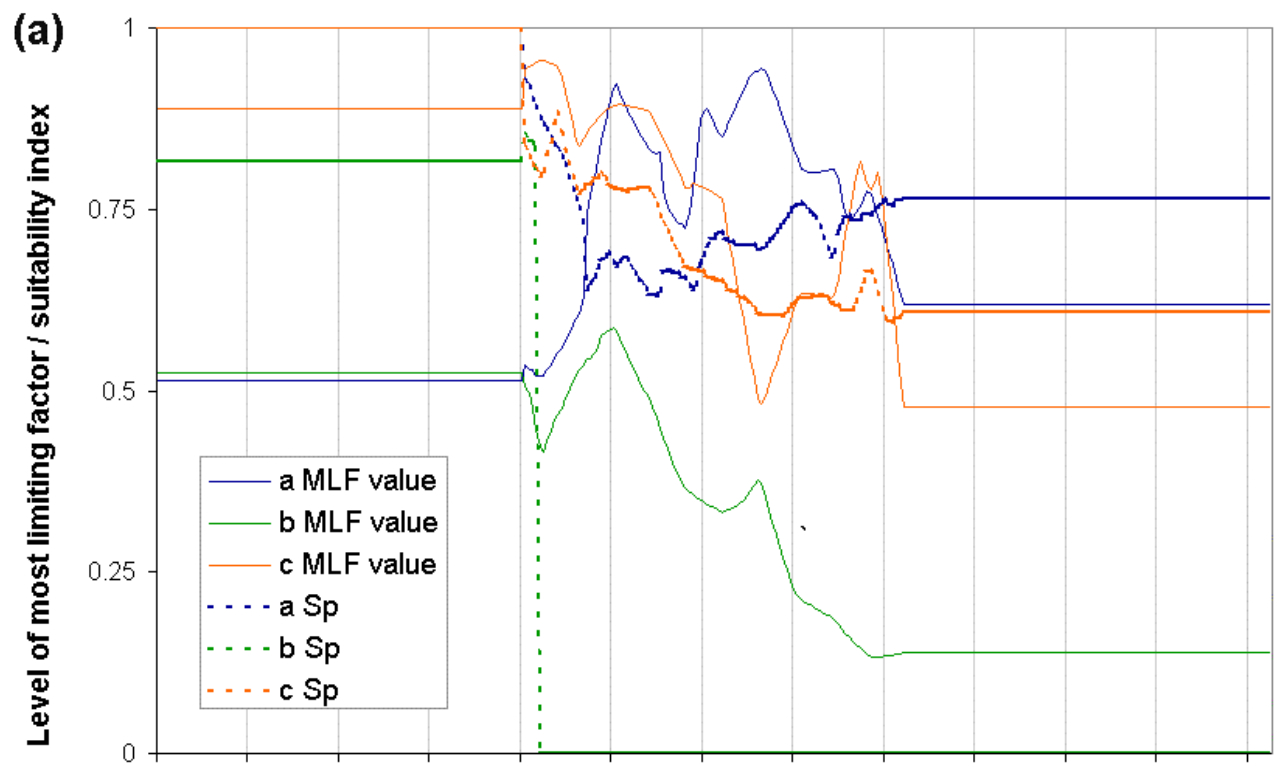

(b)

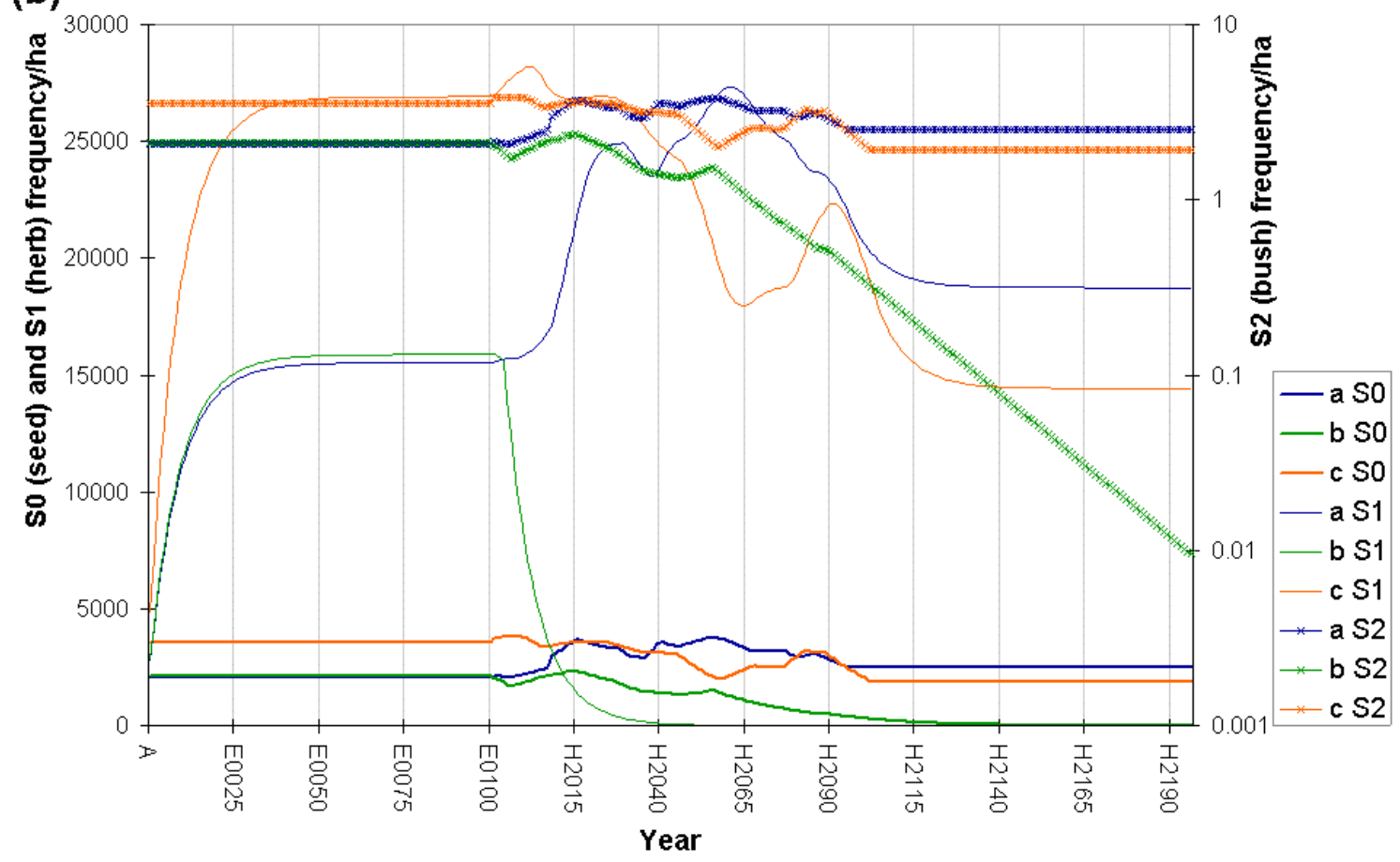

Fig. 6:26: (a) Limiting factor level \& suitability index; (b) Frequency in stage classes (S0 = seed, S1 = seedling, S2 = bush) ('c') both for Chamaedorea fragrans in cells SW010075 ('a'), SW011074 ('b') \& SW012073 ('c')

As an example, the most limiting factor and suitability index for three simulated populations of the small palm Chamaedorea fragrans (Fig. 6:26a) are charted alongside the counts in all stages (Fig. 6:26b) throughout the model period. These group of cells gives a sample of different patterns (see map in Fig. 6:14 for whole range), and in each case shows the lag in population 
response to environmental change. Cell 'a' remains viable, with a decreasing $S_{\mathrm{p}}$ but an increase in the level of the MLF and thus the population. Cell 'b' started with a very similar population count to cell 'a', but loses viability rapidly as one variable moves outside the species' tolerated envelope and undergoes a lagged decline in population. Cell 'c' retains viability, but has a decrease in population as the level of the MLF decreases.

To compare population success for all species at different time intervals, the fraction of the maximum final stage density attained $\left(K_{\mathrm{kp}} / K_{\mathrm{kmax}}\right)$ was calculated (SIS, Fig. 6:27 - Fig. 6:29). This indicator is undistorted by the relative densities of different species. The boxplots represent all cells falling between the first and third quartile. The key to Species ID is in Table 6:1.

All species experienced a mean negative impact in terms of mean density under SIS, though the severity varies widely. Under SIS, 13 species had lost one or more cells from their population by 2195 (Fig. 6:30), with a total of 411 cells lost (RIS: 13 species, 247 cells). A further three species had lost all final stage individuals in at least one cell, but retained immature individuals due to variation in maximum density, growth and mortality rates between the stages.

Although no species became extinct over more than $\sim 1 / 3$ of its estimated range, not all were viable. Eighteen species (RIS: 14 species) retained no cells in which germination was simulated, which places them on an extinction trajectory (Fig. 6:31). Others were reduced to very low population density, which would render them vulnerable to extinction through stochastic external events and genetic drift (section 2.2.4.2.2).

The highest proportion of cells was lost by the herbaceous palm Bactris acanthocarpa, which lost $36 \%$ of the simulated 450 -cell metapopulation by 2195 . Under RIS, the understorey liana Cydista aequinoctialis lost the greatest proportion, $12 \%$ of an original 491-cell population. Under the model, smaller species decline most rapidly under adverse conditions, because once germination ceases, there are no or few population stages to supply a reservoir for adult recruitment, and because annual mortality is set an order of magnitude higher for herbs than for canopy trees(Appendix 5).

There was a more substantial decline in the number of populations for which $S_{\mathrm{p}} \geq S_{\min }$, permitting germination (Fig. 6:31). Only these populations are viable in the long-term under 2095 conditions. However, the lag in population response as portrayed in this model means that most species retained most populations until 2195 under both scenarios (Fig. 6:30). 


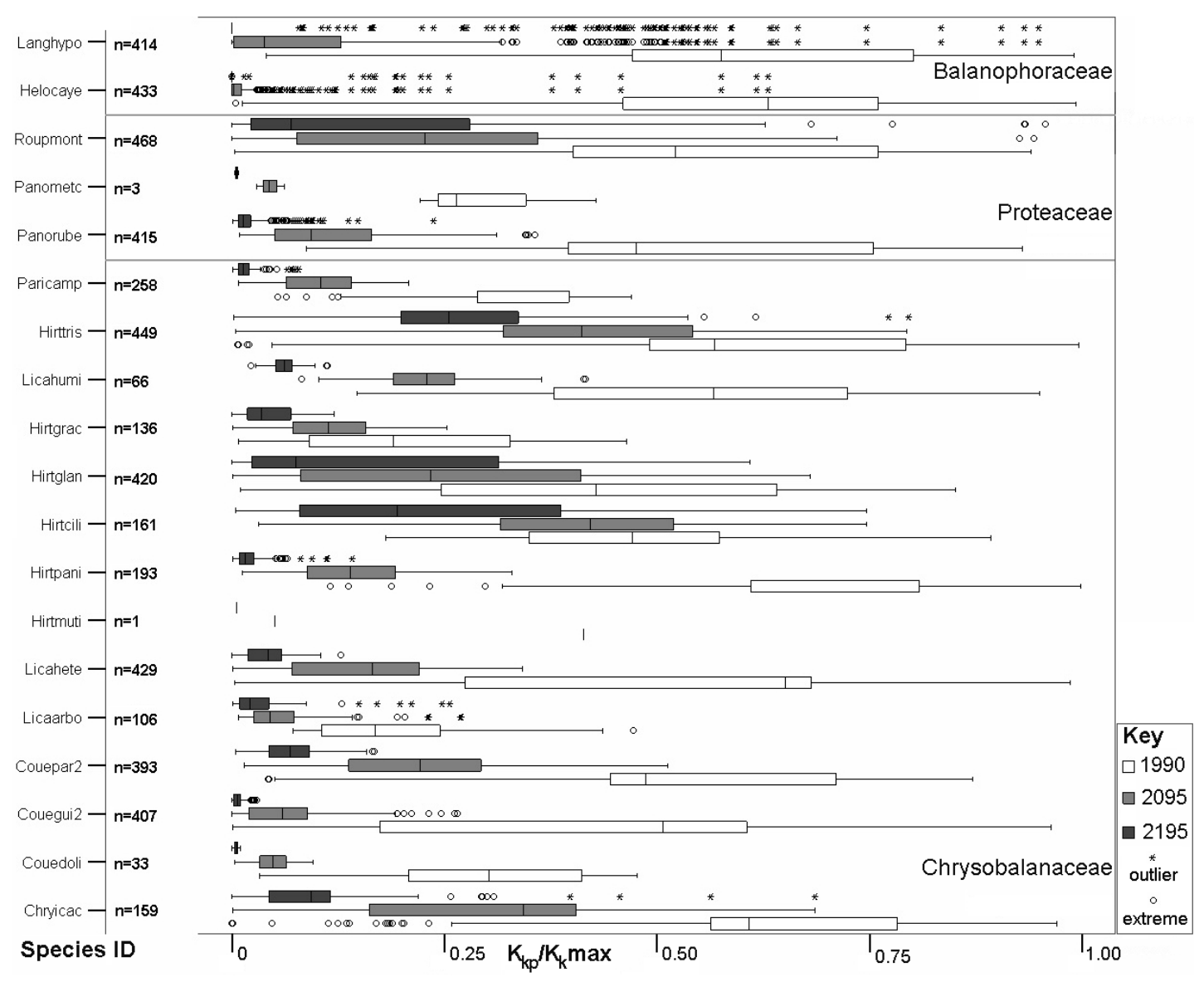

Fig. 6:27: $K_{\mathrm{kp}} / K_{\mathrm{kmax}}$, SIS- Balanophoraceae, Proteaceae \& Chrysobalanaceae

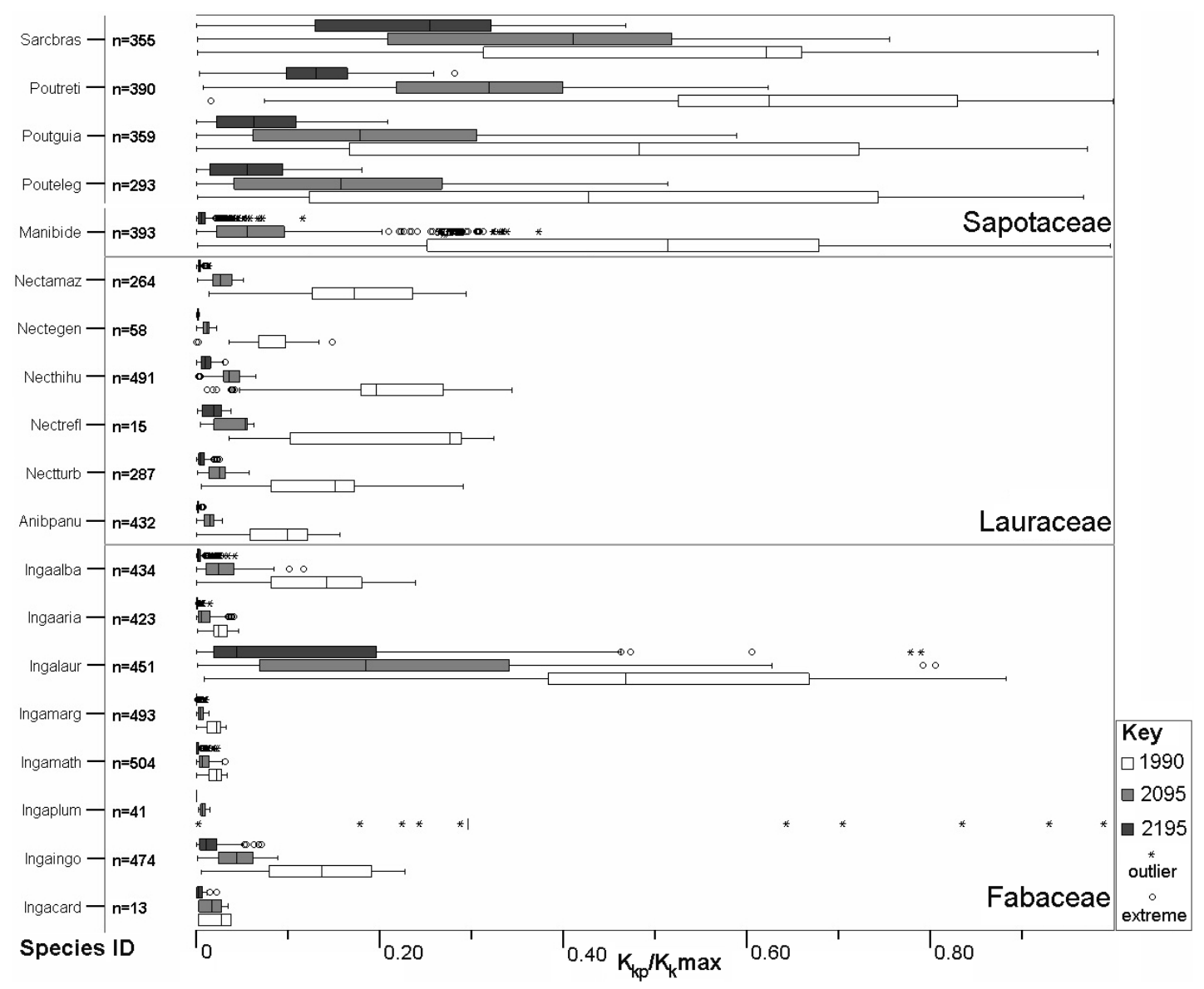

Fig. 6:28: $K_{\mathrm{kp}} / K_{\mathrm{kmax}}$, SIS - Sapotaceae, Lauraceae \& Fabaceae 


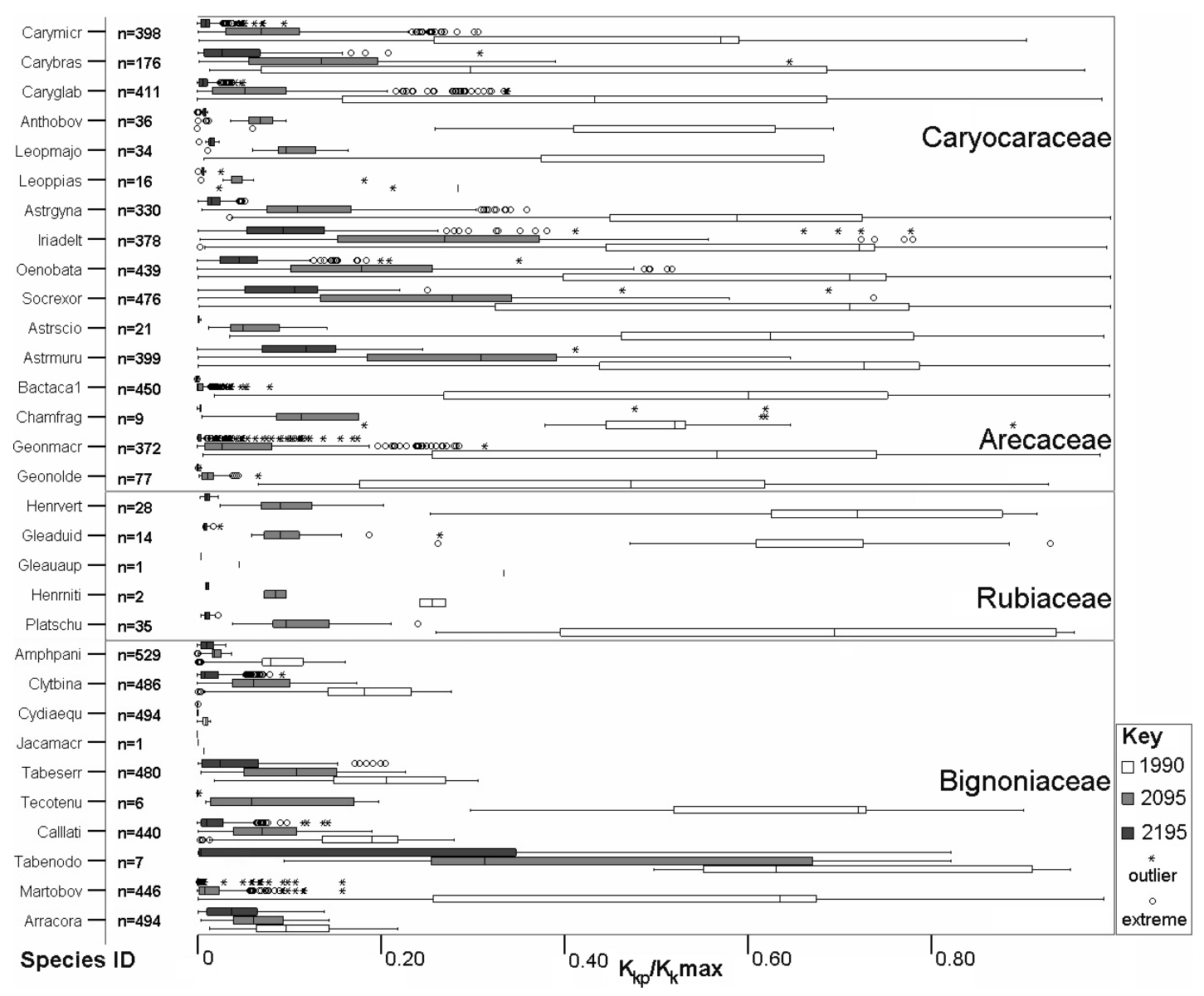

Fig. 6:29: $K_{\mathrm{kp}} / K_{\mathrm{kmax}}, \mathrm{SIS}$ - Caryocaraceae, Arecaceae, Rubiaceae \& Bignoniaceae

The mean relative change in population size, $\Delta K_{\mathrm{kp}}(1990 \rightarrow$ target year), was calculated to allow comparison between species with greatly differing $K_{\text {kmax }}$ (maximum last-stage density), and between species populations with differing initial $K_{\mathrm{kp}}$ (last-stage density in patch) (Fig. 6:32).

There is a 'left wall' to potential outcomes (a population cannot decrease by more than $100 \%$, but it can increase by more than $100 \%$ ). Therefore, if some populations increase it is possible for $\Delta K_{\mathrm{kp}}(1990 \rightarrow$ target year) to be positive when the modal population trajectory is negative. This pattern is shown for Caryocar brasiliensis under SIS, although most of the cells that had experienced increases were not reproductively viable under 2095 conditions (Fig. 6:18). Conversely, for Hirtella triandra under RIS, it was a minority of well-populated cells whose increase was sufficient to produce a positive mean (Fig. 6:20). All other species had a mean negative response by both measures. 


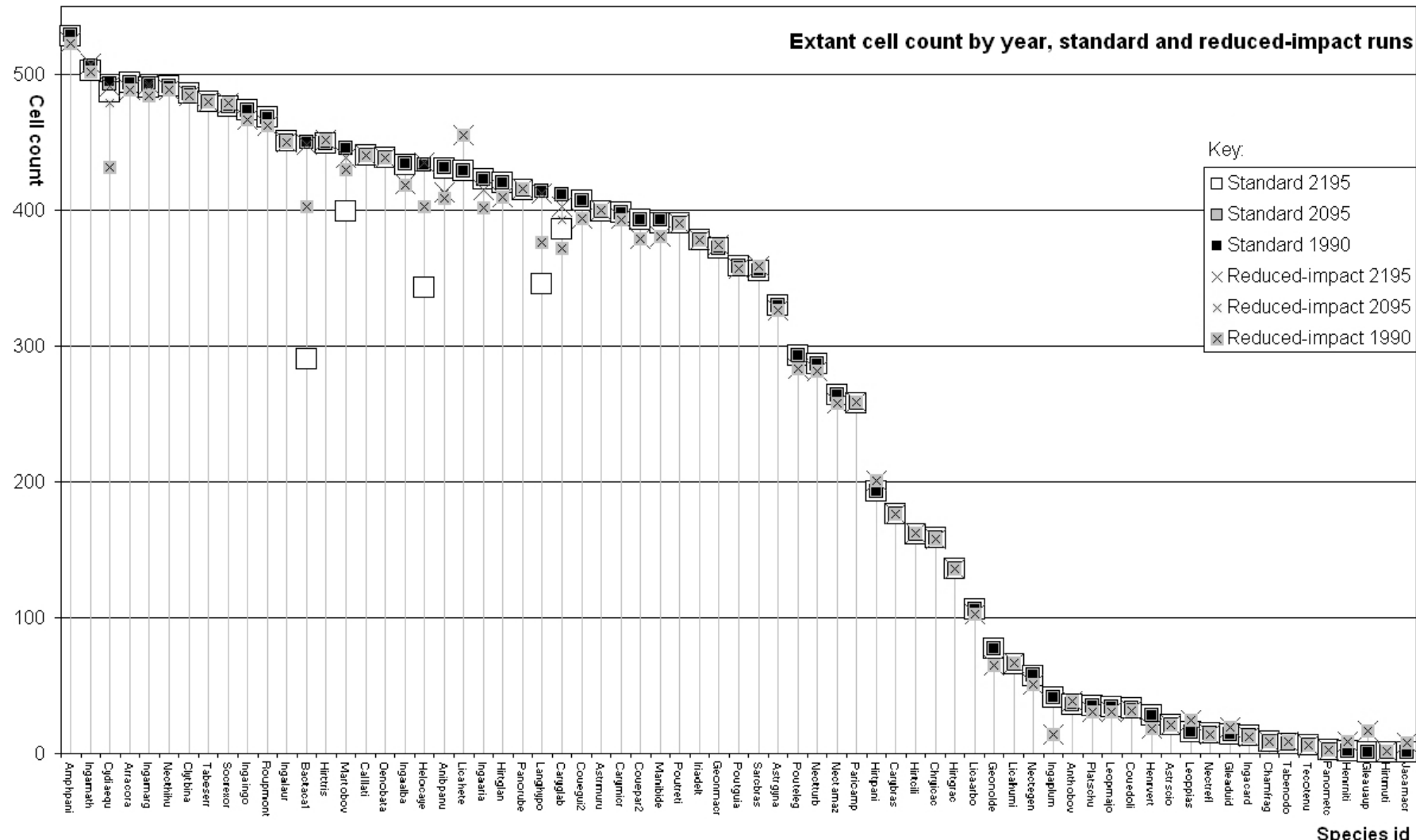

Fig. 6:30: Count of cells with surviving populations at three points in time

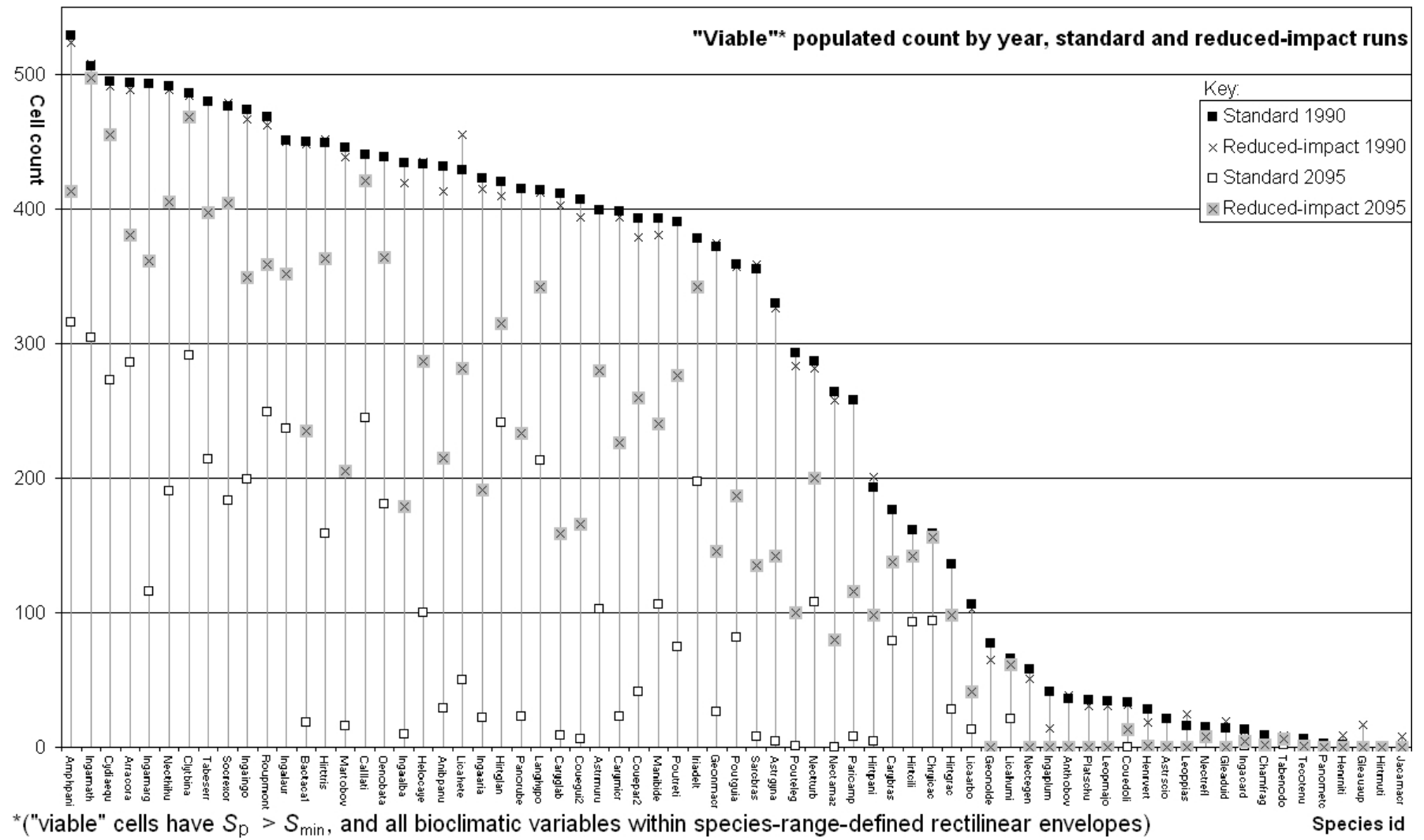

Fig. 6:31: Count of cells with $S_{\mathrm{p}}>S_{\min }$ at 2 points in time 


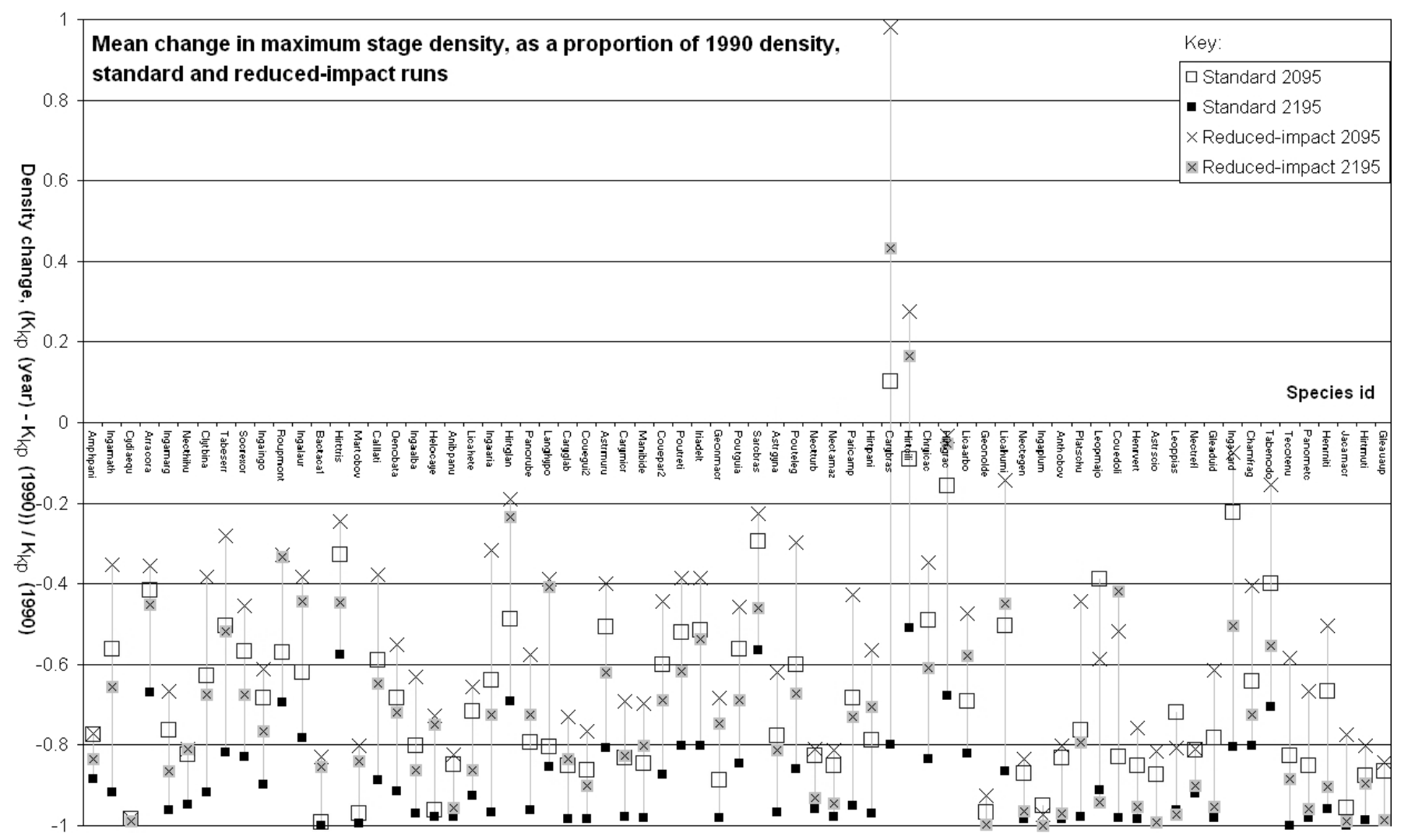

Fig. 6:32: Species-mean change in density relative to 1990 levels

\subsection{EFFECT OF FAMILY AND FUNCTIONAL TRAITS ON SPECIES' OUTCOMES}

Plant functional traits were expected to affect the magnitude of the change response (hypothesis (H5)), because they affect the model demography. Family was expected to affect species response (hypothesis (H6)) because different growth rates were specified for different families, but also because selected species distributions are often more similar within than between families (e.g. Fig. 6:24, Fig. 6:25). In addition to the functional traits used in selection, the effect of self-compatibility was considered, because this allows populations to continue reproducing however low their density. However, as only three selected species were known to be selfcompatible, this is a limited test.

Species were classified by the categorical variables (Appendix 4) regardless of whether these had played a role in model parameterisation. Specifically, dispersal mode had been used in determining mean growth rates for palm PFTs, and deciduousness for angiosperm PFTs. The tests are intended to discover what the model design simulates for species possessing these traits, rather than the direct effects of the traits on the model. Indirect effects could emerge where species' own parameters were used rather than PFT means, or through a relationship between traits and climate space occupied. 
Change in final stage density $\left(\Delta K_{\mathrm{kp}}\right)$ was used to measure species' success when testing these hypotheses. Change in density indicates the cumulative impact on the population by that model year, without consideration of population viability (breeding status) at that time. Thus, it incorporates the effect of lag in response to change, the accumulated effect of any variation in change direction over the model period, and indicates a relict's status were Amazon climate patterns to return to present-day conditions. The rate of decline in $K_{\mathrm{kp}}$ should be related to species longevity, and thus in practice to the survival rates assigned to each height class.

For each species, the mean relative density change in each population's mature stage, $k$, was calculated from 1990 to $2095\left(\Delta K_{\mathrm{kp}}(1990 \rightarrow 2095)\right)$, and 1990 to $2195\left(\Delta K_{\mathrm{kp}}(1990 \rightarrow 2195)\right)$. Density change was calculated relative to the 1990 value as in section 6.2.4. Using speciesmean percentages treats the results for species equally, regardless of population count.

Table 6:2: One-way ANOVA results - traits versus 2095 and 2195 mean outcomes

\begin{tabular}{|l|l|l|l|l|l|l|}
\hline \multirow{2}{*}{ Trait } & \multicolumn{2}{l|}{ SIS } & \multicolumn{2}{l|}{ RIS } & \multirow{2}{*}{ Categories } & \multirow{2}{*}{ Cases } \\
\cline { 2 - 5 } & \multicolumn{2}{|l|}{ Monte Carlo p-value } & \multicolumn{2}{l|}{ Monte Carlo p-value } & & \\
\cline { 2 - 5 } & $\mathbf{2 0 9 5}$ & $\mathbf{2 1 9 5}$ & $\mathbf{2 0 9 5}$ & $\mathbf{2 1 9 5}$ & & \\
\hline Family & 0.260 & 0.443 & $\mathbf{0 . 0 1 6}$ & $\mathbf{0 . 0 1 3}$ & 10 & 69 \\
\hline Form & 0.196 & 0.512 & 0.815 & 0.892 & 4 & 69 \\
\hline Height class & $(1$-tailed) & $(1$-tailed) & $(1$-tailed) & $(1$-tailed) & 6 & 69 \\
& 0.07 & $\mathbf{0 . 0 3 6}$ & 0.254 & 0.078 & & \\
\hline Dispersal mode & 0.724 & 0.675 & 0.873 & 0.370 & 4 & 51 \\
\hline Pollination mode & 0.569 & 0.107 & 0.774 & 0.925 & 3 & 69 \\
\hline Deciduousness & 0.520 & 0.249 & 0.410 & 0.166 & 3 & 36 \\
\hline Selfing & $(1$-tailed) & $(1$-tailed) & $(1$-tailed) & $(1$-tailed $)$ & 2 & 69 \\
& 0.140 & 0.138 & 0.052 & $\mathbf{0 . 0 2 2}$ & & \\
\hline
\end{tabular}

Under SIS, the only categorical variable to significantly affect the mean density outcomes was height class (Fig. 6:33), which is a strong determinant of generation time, and thus lag in population change. Smaller stature species were more rapidly affected by negative change. Under RIS, family and selfing were more important - affecting generation time, through familyparameterised growth and death rates, and the survival of small populations, through ability to reproduce at low density, respectively. It was concluded that these traits were operating at different levels of impact; survival-related traits being more important under severe change and reproductive traits under lesser change. However, these conclusions carry a high level of uncertainty, because the vital rates that the traits specify are not themselves modelled as responding to climate change.

To further examine the results at family level, the proportion of populations becoming 'relicts' can be compared (Fig. 6:34). All families had high percentages of non-viable populations under SIS, with the smallest mean over all species being $70 \%$ (Bignoniaceae), and the greatest being Rubiaceae and Caryocaraceae (all populations of all and virtually all species respectively). The difference between families was significant under SIS, and just failed to be so for RIS ( $p=$ 
0.047 SIS, $\mathrm{p}=0.051$, RIS, Kruskal-Wallis 1-way ANOVA). The within-family distribution of viability was much more variable under RIS (Table 6:1).

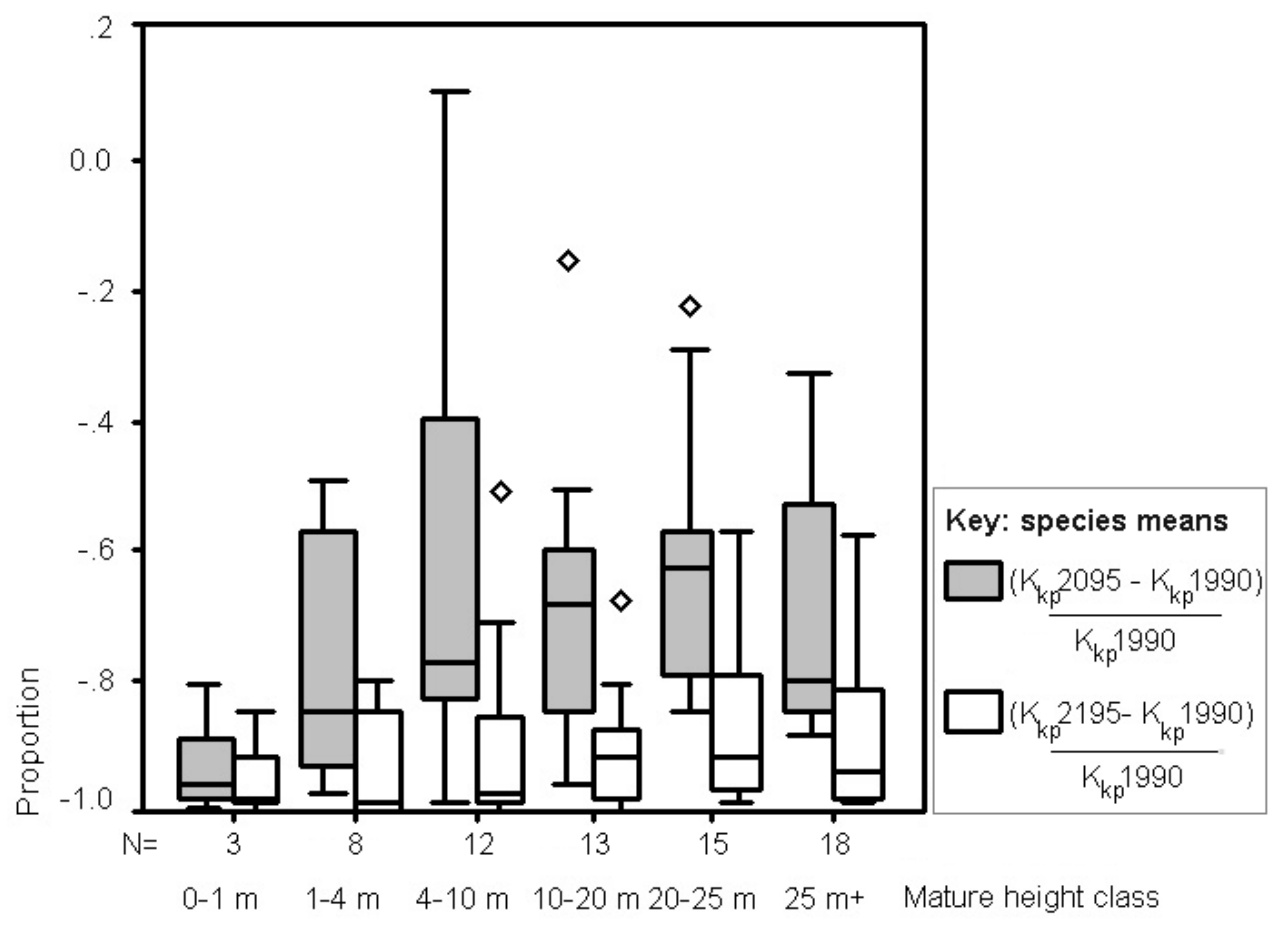

Fig. 6:33: Species-mean $\Delta K_{\mathrm{kp}}(1990 \rightarrow 2095$ or 2195$)$, by height class, SIS

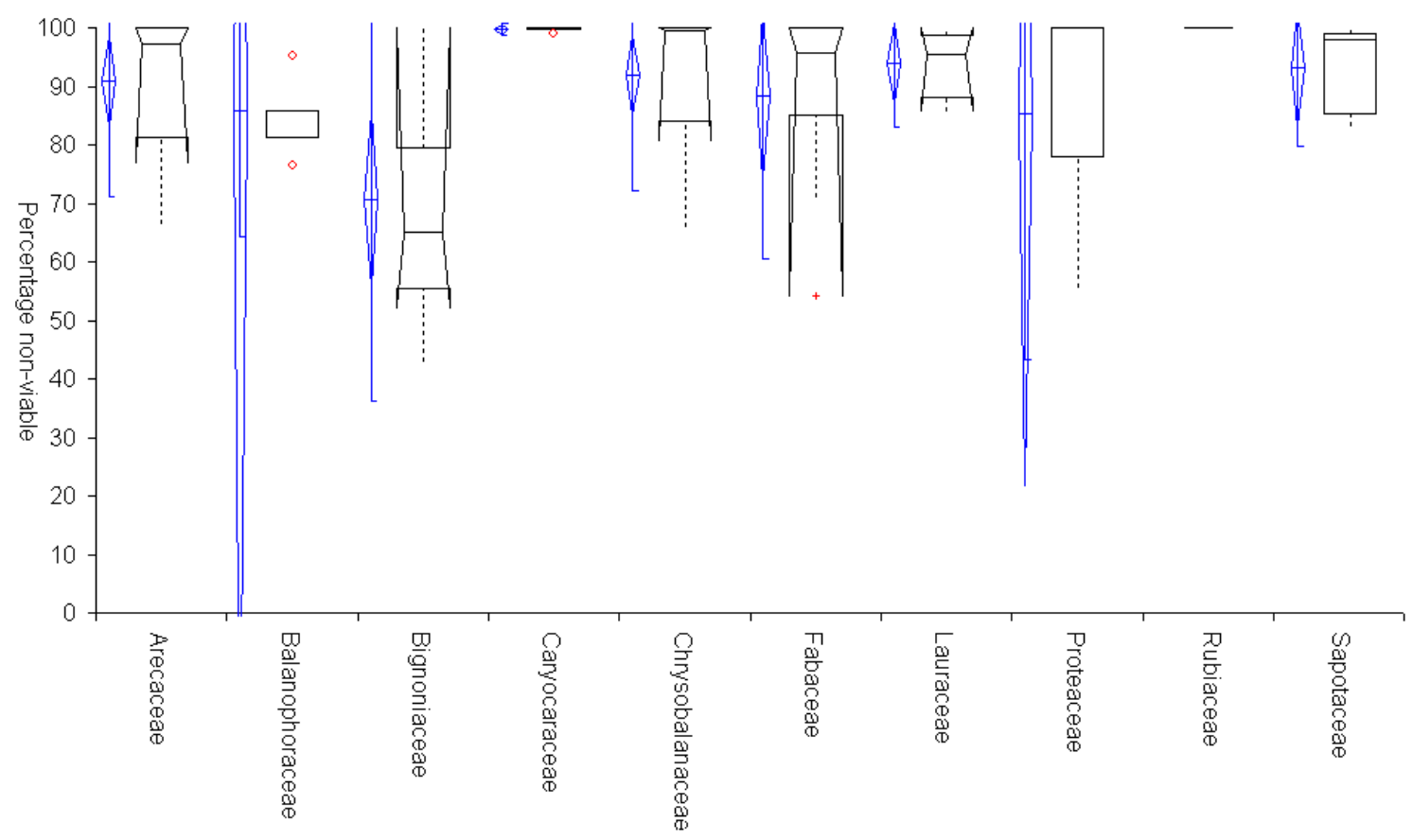

Fig. 6:34: Distribution of species-mean population non-viability by family, SIS, 2095; mean with confidence limits (left series) and median with boxplots (right series) 


\subsection{EFFECT OF DISTRIBUTIONS ON SPECIES' OUTCOMES}

Hypothesis (H7) suggests that plants with a wider distribution would have a greater tolerance to environmental variation and thus a greater mean population resistance to change (Janzen 1967). They should also be more likely to encounter a range of projected alterations to climate. The species outcome maps strongly suggest that spatial distribution is the most decisive factor in this change model (section 6.2). As distribution is partially a surrogate for environmental range, it was also expected that species with a greater variation in each bioclimate variable (expressed as coefficients of variation) would be more resistant to change (hypothesis (H8)).

Correlations between populated cell count or recorded environmental variables and density change $\left(\Delta K_{\mathrm{kp}}\right)$ were sought. Record-cells with extreme values of environmental variables were excluded as in the species model.

\subsubsection{Record-cell count}

There was no significant correlation between species' 1990 population counts and mean $\Delta K_{\mathrm{kp}}$ by 2095 under either scenario (Spearman's $\rho=0.124$ SIS, 0.116 RIS; $p=0.309$ SIS, 0.342 RIS). There was a small positive correlation by 2195 (Spearman's $\rho=0.254$ SIS, 0.303 RIS, $p=$ 0.035 SIS, 0.011 RIS). By 2195, species with larger occupied ranges experienced a less negative mean relative density change than species with smaller ranges. In other words, there was a lag in expressing this difference, providing only limited support for hypothesis (H7).

There is a slight trend towards higher mean simulated population density for range-restricted species under reference conditions. Mean $K_{\mathrm{kp}} 1990 / K_{\mathrm{kmax}}$ is negatively correlated with 1990 population size in both runs, but the correlation is only significant for RIS (Spearman's $\rho=$ -0.189 SIS, -0.267 RIS; $p=0.121$ SIS, 0.026 RIS). This indicates that the simulations tended to include less marginal habitat for these species. It was thought possible that this contributed to the limited relationship between range size and density change. However, there is no relationship between initial population size and the extent of the decline in either run (no significant correlation between mean $K_{\mathrm{kp}} 1990 / K_{\mathrm{kmax}}$ and $\left.\Delta K_{\mathrm{kp}}(1990 \rightarrow 2195)\right)$.

There is a stronger negative correlation between the percentage of populations to become nonviable at 2095 and 1990 population count (Spearman's $\rho=-0.59$ SIS, -0.73 RIS; $p=0.000$ ). That is, narrow-range species lost viability over a greater proportion of their range (Fig. 6:35). Under SIS, species from throughout the cell count range began to lose viability in all cells, reducing the strength of the correlation. 


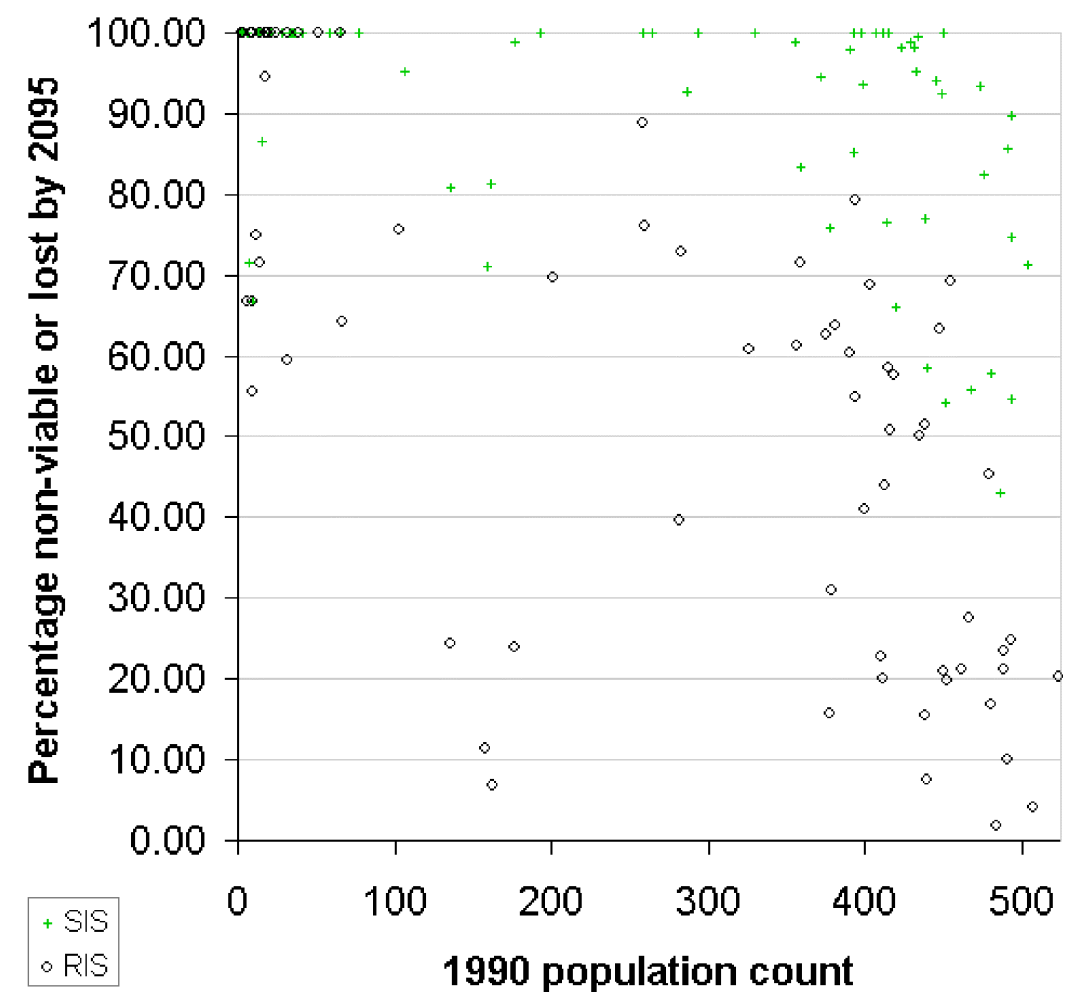

Fig. 6:35: Percentage of non-viable populations (2095) by population count (1990), per species. SIS \& RIS

\subsubsection{Environment}

Recorded climate had a strong effect on the density outcomes (Table 6:3). Minimum AET was negatively correlated with $\Delta K_{\mathrm{kp}}$ in all cases - that is, more drought-adapted species, which had a greater tolerance of low $A E T$ or high $M D$, performed better. It is interesting that there is no significant correlation with maximum AET for SIS, or the coefficients of variation for $M D$ and SMA for either scenario. Although AET did increase (Fig. 6:6), generalist species with a wide range of tolerance were more successful than those specialising in high evaporation environments. It seems that the latter set of species did less well because they were located in cells where $2095 A E T$ exceeds all values from the neotropical reference climate.

Conversely, tolerance to a range of $M D$ or $S M A$ was not important for species-mean outcomes, presumably because the strong trend to increasing values mainly favoured adaptation to longer periods of moisture deficit. Species tolerant to higher $S M A$ and $M D$ were more successful; and there was a smaller tendency for species with a higher minimum SMA to do better. Again, the more successful species tended to be those that specialise in moisture-stressed environments. 
Table 6:3: Spearman's correlation coefficients ( $\rho$ ) between environment variables and species-mean relative change in final-stage density $\left(\Delta K_{\mathrm{kp}}\right)$

\begin{tabular}{|c|c|c|c|c|}
\hline & $\begin{array}{l}\text { SIS: mean } \\
\Delta K_{\mathrm{kp}}(1990 \rightarrow \\
2095)\end{array}$ & $\begin{array}{l}\text { SIS: mean } \\
\Delta K_{\mathrm{kp}}(1990 \rightarrow \\
2195)\end{array}$ & $\begin{array}{l}\text { RIS: mean } \\
\Delta K_{\mathrm{kp}}(1990 \rightarrow \\
2095)\end{array}$ & $\begin{array}{l}\text { RIS: mean } \\
\Delta K_{\mathrm{kp}}(1990 \rightarrow \\
2195) \\
\end{array}$ \\
\hline AET maximum & $\begin{array}{l}\rho=0.110 \\
p=0.367\end{array}$ & $\begin{array}{l}\rho=0.199 \\
p=0.102\end{array}$ & $\begin{array}{l}\rho=0.098 \\
p=0.423\end{array}$ & $\begin{array}{l}\rho=0.260 \\
p=0.031\end{array}$ \\
\hline$A E T$ minimum & $\begin{array}{l}\rho=-0.399 \\
p=0.001\end{array}$ & $\begin{array}{l}\rho=-\mathbf{0 . 5 5 8} \\
p=0.000\end{array}$ & $\begin{array}{l}\rho=-0.443 \\
p=0.000\end{array}$ & $\begin{array}{l}\rho=-0.580 \\
p=0.000\end{array}$ \\
\hline $\begin{array}{l}A E T \text { coefficient } \\
\text { of variation }\end{array}$ & $\begin{array}{l}\rho=0.401 \\
p=0.001\end{array}$ & $\begin{array}{l}\rho=0.592 \\
p=0.000\end{array}$ & $\begin{array}{l}\rho=0.405 \\
p=0.001\end{array}$ & $\begin{array}{l}\rho=0.569 \\
p=0.000\end{array}$ \\
\hline$M D$ maximum & $\begin{array}{l}\rho=0.489 \\
p=0.000\end{array}$ & $\begin{array}{l}\rho=0.632 \\
p=0.000\end{array}$ & $\begin{array}{l}\rho=0.495 \\
p=0.000\end{array}$ & $\begin{array}{l}\rho=0.618 \\
p=0.000\end{array}$ \\
\hline$M D$ minimum & $\begin{array}{l}\rho=0.176 \\
p=0.149\end{array}$ & $\begin{array}{l}\rho=0.093 \\
p=0.447\end{array}$ & $\begin{array}{l}\rho=0.228 \\
p=0.059\end{array}$ & $\begin{array}{l}\rho=0.118 \\
p=0.335\end{array}$ \\
\hline $\begin{array}{l}\text { MD coefficient } \\
\text { of variation }\end{array}$ & $\begin{array}{l}\rho=-0.230 \\
p=0.058\end{array}$ & $\begin{array}{l}\rho=-0.235 \\
p=0.052\end{array}$ & $\begin{array}{l}\rho=-0.168 \\
p=0.168\end{array}$ & $\begin{array}{l}\rho=-0.148 \\
p=0.226\end{array}$ \\
\hline$S M A$ maximum & $\begin{array}{l}\rho=0.404 \\
p=0.001\end{array}$ & $\begin{array}{l}\rho=0.548 \\
p=0.000\end{array}$ & $\begin{array}{l}\rho=0.389 \\
p=0.001\end{array}$ & $\begin{array}{l}\rho=0.519 \\
p=0.000\end{array}$ \\
\hline$S M A$ minimum & $\begin{array}{l}\rho=0.302 \\
p=0.012\end{array}$ & $\begin{array}{l}\rho=0.241 \\
p=0.046\end{array}$ & $\begin{array}{l}\rho=0.327 \\
p=0.006\end{array}$ & $\begin{array}{l}\rho=0.240 \\
p=0.047\end{array}$ \\
\hline $\begin{array}{l}S M A \text { coefficient } \\
\text { of variation }\end{array}$ & $\begin{array}{l}\rho=-0.120 \\
p=0.326\end{array}$ & $\begin{array}{l}\rho=-0.071 \\
p=0.559\end{array}$ & $\begin{array}{l}\rho=-0.028 \\
p=0.819\end{array}$ & $\begin{array}{l}\rho=0.017 \\
p=0.893\end{array}$ \\
\hline
\end{tabular}

To sum up, species adapted to high evapotranspiration environments did worse under the model, whilst the more drought-adapted species were less deleteriously affected by change. All significant correlations were stronger by 2195 , when the lag in population response to change had reduced, except for the correlation of $\Delta K_{\mathrm{kp}}$ with minimum tolerated $S M A$, which reduced in both size and significance level.

\subsection{SPATIAL PATTERNS OF CHANGE}

This section seeks to identify regions of better or worse population survival over the set of species, both after the change period and in the longer-term. Tests for significant change in population density for the set of species were performed, and then changes in the mean density and viability of populations were mapped. As study species' density varies over the region, the measure used was relative density change.

\subsubsection{Spatial changes in population density}

To test the cell-by-cell null hypothesis that species composition does not change in response to climate (H3), the success of each modelled population at model years 1990, 2095 and 2195 was estimated as $K_{\mathrm{kp}} / K_{\mathrm{kmax}}$ (Fig. 6:27 - Fig. 6:29). This variable indicates which species are present at a point in time, rather than which are reproductively viable. 

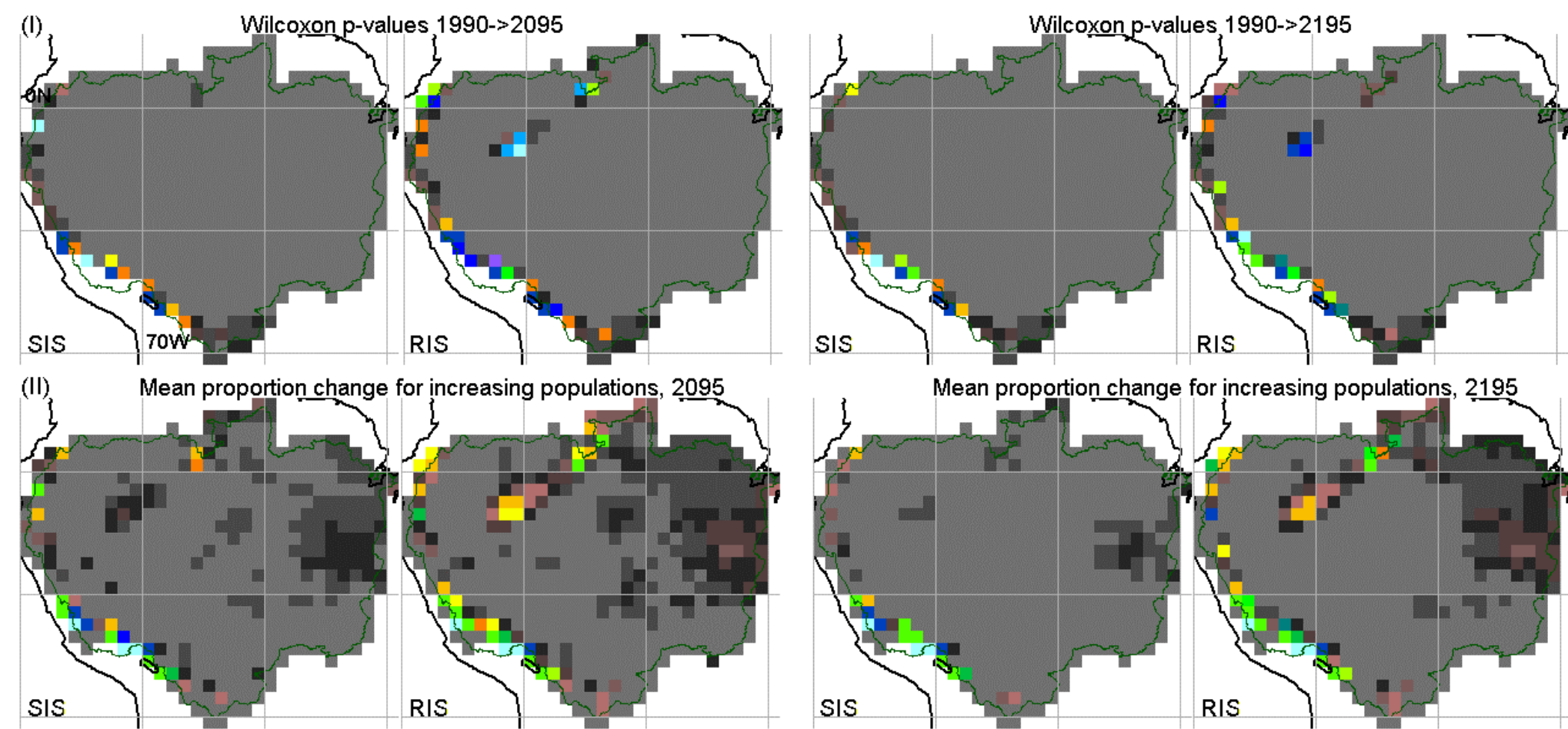

Key:

$\square 0.9-1$

$\square \quad 0.85-0.9$

$\square 0.8-0.85$

$\square \quad 0.75-0.8$

口 $0.7-0.75$

$\square \quad 0.65-0.7$

$\square \quad 0.6-0.65$

$\square \quad 0.55-0.6$

$\square \quad 0.5-0.55$

$\square \quad 0.45-0.5$

$\square \quad 0.4-0.45$

$\square \quad 0.35-0.4$

$\square \quad 0.3-0.35$

$\square \quad 0.25-0.3$

$\square 0.2-0.25$

$\square 0.15-0.2$

$\square 0.1-0.15$

- $0.05-0.1$

口 $0.01-0.05$

$\square \quad 0-0.01$
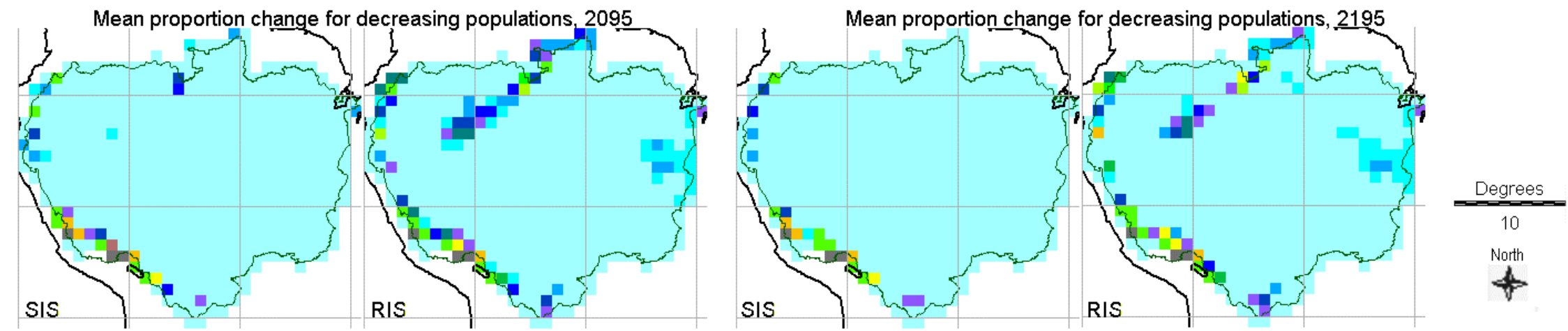

Fig. 6:36: (I) Wilcoxon tests comparing $K_{\mathrm{kp}} / K_{\mathrm{kmax}}$ for 1990 versus 2095 and 2195, (II) mean change by 2095 and 2195 for increasing and decreasing populations. SIS \& RIS 
Under both scenarios, this 'community change' was highly significant ( $\mathrm{p} \leq 0.01$ Wilcoxon paired signed-ranks test) in almost every cell, for both 2095 and 2195 (Fig. 6:36 I). That is, there was a significant change in composition as defined by population density of the set of modelled species present. Under SIS, the few cells that did not experience significant change were restricted to the western edge and southern tip of the Amazon basin. There was a small number of study species in these areas in 1990, with many widespread species modelled as absent, and so the species set was probably less representative of the community there. However, these areas also experienced relatively low AET and high SMA under the reference climate. Under RIS, small northwest and north-central areas also escaped significant change.

As these tests did not discriminate regional differences within most of Amazonia, the mean trajectories of the increasing and decreasing populations were calculated separately for each cell. Over most of the map, the mean proportional change in decreasing populations was large $(>0.9)$, while the change in increasing populations was small $(<0.05)$. On the western boundary, increasing populations increased more and decreasing populations decreased less, on average (Fig. 6:36 II). A corridor of the same trends was visible from the north-central to northwestern Amazonia under RIS. This region was intermediate between the zones of very high AET and very high SMA under the 2095 climate.

\subsubsection{Spatial changes in population viability}

The long-term regional impacts can be seen more clearly by mapping the proportion of viable populations (Fig. 6:37a). The same general pattern appears under SIS and RIS, though the frequency of cells with low species viability is much higher under SIS.

The areas undergoing most negative change are the northeast corner and much of the west. The forked band in the west for which no viable species are simulated under SIS corresponds to the band of highest $A E T$ at 2095 (Fig. 6:6), and the northeastern patch corresponds to a region of much increased $M D$ and $S M A$. This suggests that these variables have acted to limit species viability in these zones.

The remainder of the northwest experiences less severe impact under SIS, as does much of the periphery. Under RIS, the band in the north-central region, where all populations remained viable, matches the band with higher relative increase for those populations that were doing better under change. This area has a relatively high modelled species frequency. The few populations modelled along the southwestern edge remained viable under both scenarios. The bioclimate variables remained relatively stable in this region, with some decrease in SMA. 
a. Proportion of species that retain viable populations at $\mathbf{2 0 9 5}$

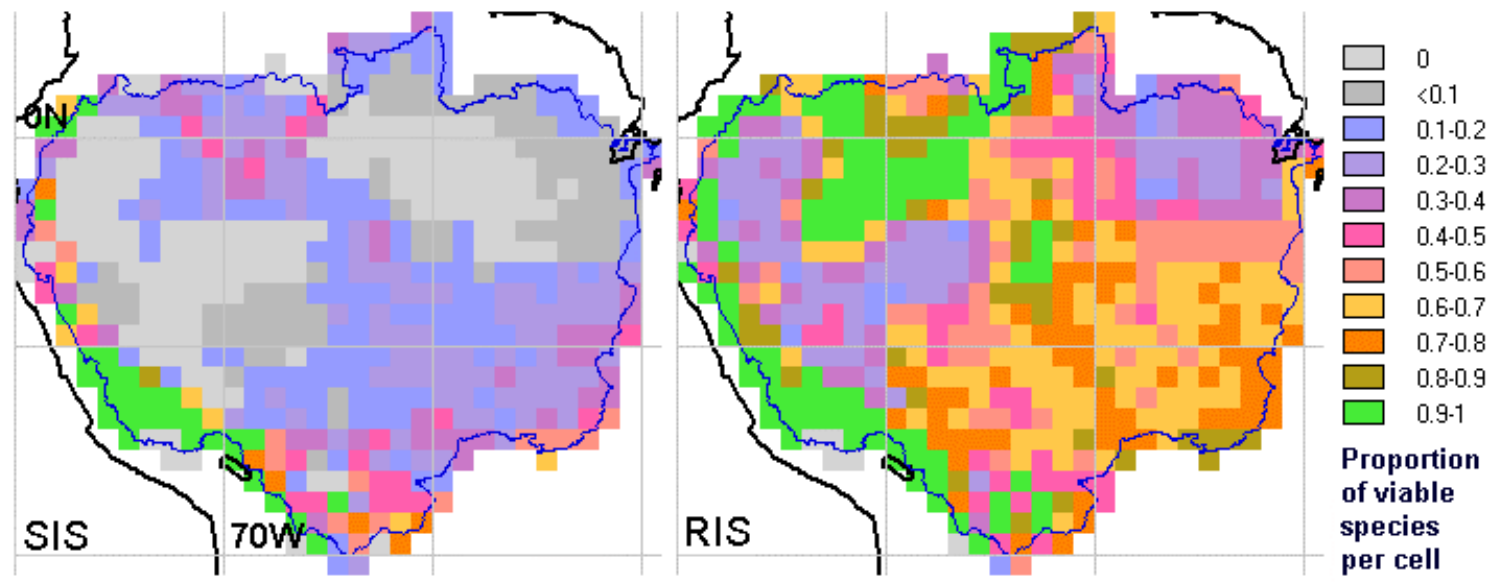

b. Single 'best' cell for each of 69 species under present conditions

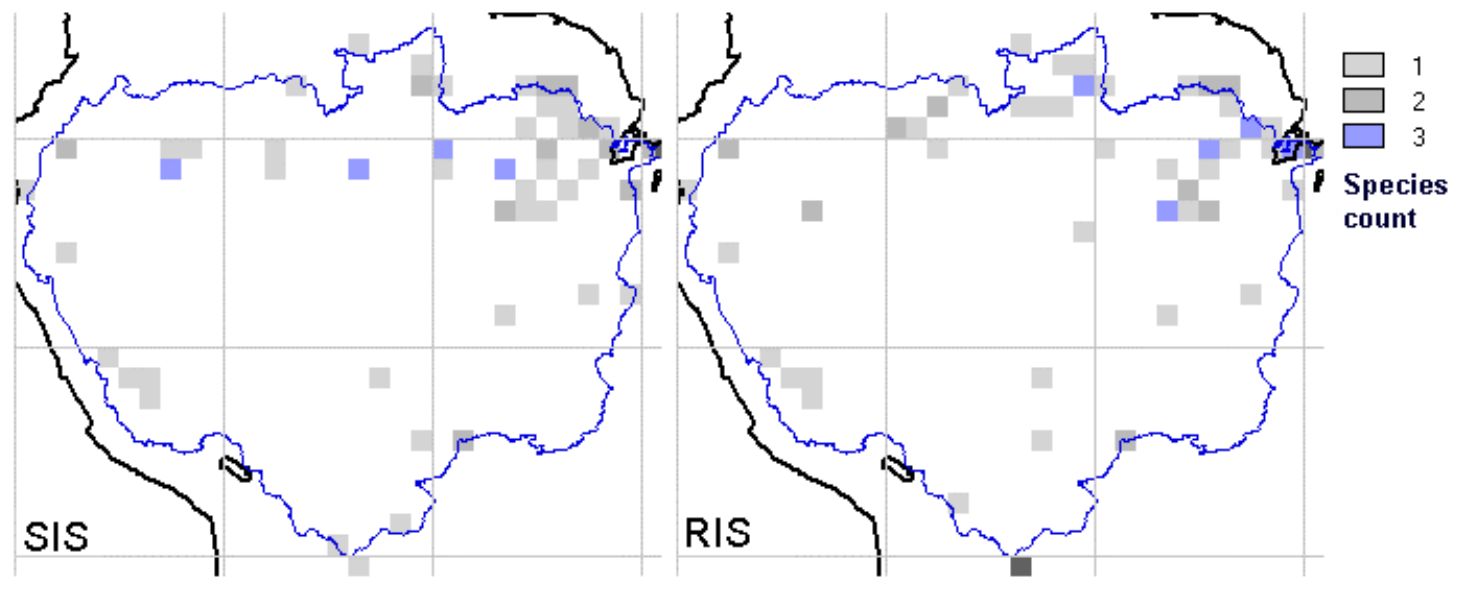

c. 'Best' cells for each of 69 species under '2195' conditions

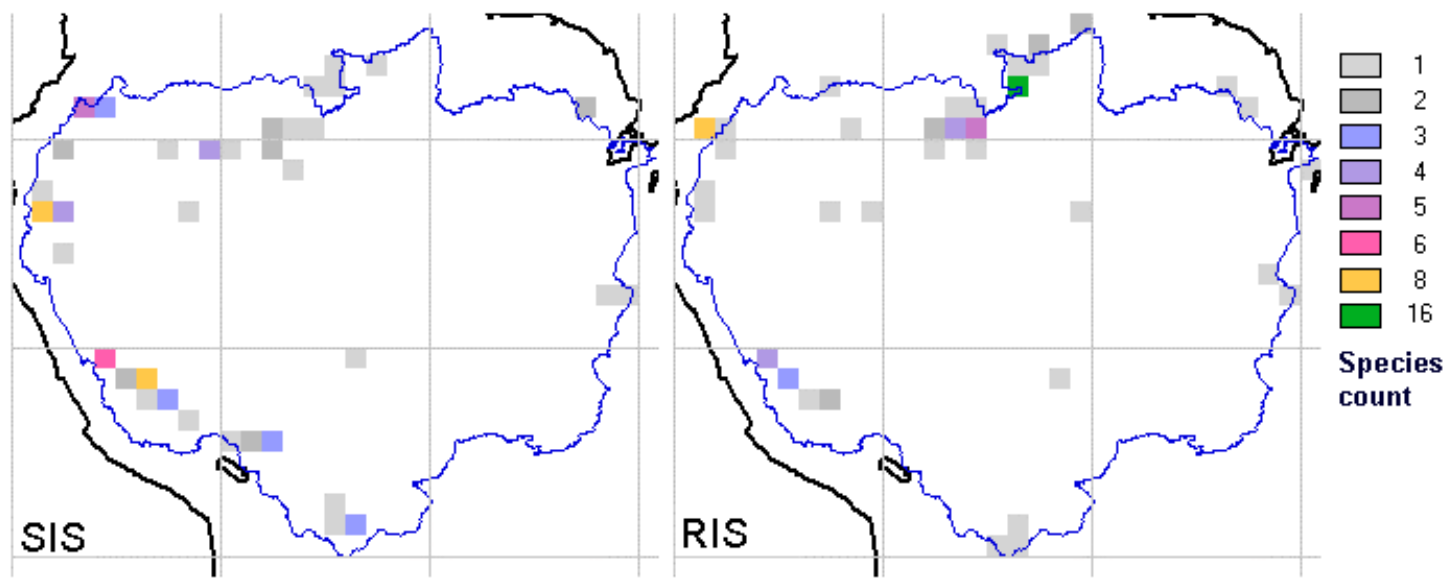

Fig. 6:37: (a) Proportion of viable populations at 2095, and 'best' cells for (b) 1990 climate and (c) 2195. SIS \& RIS

The distribution of the 'best' cells within each species range at 1990 and 2095 is of interest, because this cell would in theory be that best suited for the species' conservation. The location of the 'best' cell (defined as that holding the largest population) from each species' 1990 distribution is similar for SIS and RIS, with some disparities resulting from the different 
downscaled climates (Fig. 6:37b). Despite one species selection criterion being for a range of occupied SMA, many of these 'best' cells are located in the seasonal northeast.

Hypothesis (H4) is that the distribution of these 'best' cells for the set of species will change - it is concerned with the distribution of the cells required for the best representation of all study species. To identify the best' areas under changed conditions, population viability must be taken into account as well as density. Following the procedure in section 5.2.11.1, the best attainable positions for these species were mapped (Fig. 6:37c), rather than the locations of their climate optima over the whole region. There is a westward shift in the position of best cells under both SIS and RIS such that the cluster of cells in the northeast is replaced by a cluster of cells along the southwestern edge of the inclusive-Amazon.

Under RIS, there is a striking cluster of species preferring the north-central region, with 16 species favouring one cell ("Serra dos Surucucus", Roraima, NW002063). All but one of the 16 are viable in this cell. Eleven have mean occupied $M D$ and $S M A$ values (i.e. modelled optima) lower than the cell's values of 58 days and $134 \mathrm{mmy}^{-1}$. No species have mean AET lower than this cell's RIS 2095 value of $1621 \mathrm{mmy}^{-1}$. AET is the most limiting factor (MLF) for 12, MD for one and SMA for three species. Of the nine neighbouring cells, seven have higher AET, all have higher $S M A$, and only one has a lower $M D$. The model thus simulates the cell as a local refuge for more aseasonal species. Under SIS, the cell has a more seasonal climate (SMA = 116 days, $A E T=1888.5 \mathrm{mmy}^{-1}$ ) so does not fulfil the same function.

For the cell which is modelled as best for 16 species under RIS, the mean elevation is several hundred metres higher than all but one of its neighbours $(868 \mathrm{~m}$, data derived from Row \& Hastings 1989). It is on the border of an elevated region (the Guiana Highlands) with higher precipitation and lower temperature than the closest Amazonian cells. The same type of pattern is seen in the southwest.

If a cell adjacent to the realised range was classified as potential distribution in both 1990 and 2095, having been excluded from the realised distribution through buffering, there is a chance that the this exclusion is erroneous. The assumption that such a species had no viable populations at 2095 would be incorrect. However, this pattern only occurred for species which retained viable cells in other parts of the range (e.g. Roupala montana under RIS, Fig. 6:23), so did not affect any species' overall outcome.

60 species have record-cells outside the inclusive-Amazon. For some, it is likely that extraAmazonian areas within their realised distribution would be considered the 'best' extant areas 
were the entire Neotropics modelled at 2095. The concentration of 'best' areas on the northcentral border of the inclusive-Amazon suggests this.

Where a new potential distribution cell was diagonally adjacent to the species set, it was not considered for migration. If these cells were included, Astrocaryum gynacanthum and Inga cardozana would have benefited under SIS, whilst Anthodiscus obovatus and Astrocaryum sciophilum would have benefited under RIS. The A. sciophilum cell was classified as potential distribution both in 1990 and 2095 - this is the only instance in which such a possible error of omission affects a species final status. 


\section{Discussion}

\subsection{MODEL OUTCOMES}

When investigating biodiversity change, species modelling should provide a valuable alternative perspective to modelling climate alone or vegetation response to climate, because the species' unique patterns of distribution are likely to be distinct from those of a vegetation type. Vegetation modelling can indicate the likely structural diversity seen in a cell after equilibration. It cannot reveal detailed change in species composition, which depends on the range of tolerances of the original species set and the ability of new species to disperse to that cell. Models of species' demography and dispersal are also required to elucidate the potential rate of change in vegetation type, as there will be a lag between the establishment of a new equilibrium climate and a new equilibrium vegetation. New transitional and 'climax' vegetation types may appear, different from those already seen.

Ideally, thousands of species would be modelled. As this is not possible, the sample of 69 species was chosen to represent functional and taxonomic biodiversity. Both had an affect on the modelled demography, and family was also linked to the species' spatial distributions and therefore their tolerated climates. The model was used to examine the hypotheses on species' response stated in the methodology, in the hope of making some generalisations about the determinants of species' vulnerability to change. This section summarises these results.

\subsubsection{Spatial impacts}

Hypothesis (H1), that "individual species' distribution patterns will alter under the climate change scenarios" can be examined at the level of species, scenario and time period. The demographic lag included in the model means that relatively few populations were lost, although under the projected change many ceased to be viable. In the worst-case scenario, the standard impact scenario (SIS) at 2195, some populations were lost in almost a quarter of species, but no species lost viability over more than $1 / 3$ of its range.

Potential distribution changed much more visibly than realised distribution by 2095 or 2195 . Hypothesis (H2), that "present day realised and final potential distributions will not overlap one another, so that the species will eventually be lost from its entire current range", is supported for $43 \%$ of species under SIS, and $20 \%$ of species under the reduced impact scenario (RIS). These species (Table 6:1) are in danger of eventual extinction from the Amazon. 
Areas simulated as unoccupied potential distribution under current conditions offer targets for search for species of interest, for example those most endangered by change.

Often, new potential distribution appears at the western border of a species' range, following the westward shift in both annual and seasonal moisture availability. Many of the species that lost all viable populations still had the opportunity to migrate to adjacent new or persisting areas of potential distribution.

\subsubsection{Spatial impacts on the set of species}

Hypothesis (H3) was that "there is a significant difference in species composition for many cells between 1990 and 2095 or 2195, and this difference varies consistently over the Amazon region". There was indeed a significant difference in composition in nearly all cells in SIS and RIS (when measured as the relative density of the set of species modelled). However, the only spatial pattern was that some cells on the western border and north-central areas of Amazonia did not experience significant change.

When the distribution of the most viable cells was examined, the varying pattern of threat to species was clearer. The western and north-central areas retained viability for most species under RIS, and a relatively high proportion of species under SIS. The north-central area hosted many more species, and represents an area of aseasonal moist forest, which although it underwent an increase in annual actual evapotranspiration (AET), retained a climate within the present Amazonian range. The western and northernmost areas represent zones of higher altitude, which either underwent little change or moved towards climates currently typical of downslope areas.

Under present conditions, many of the 'best' cells were found in the northeast region, though there was also a distribution of cells throughout Amazonia. This means that many of the widespread species modelled have a mean climate (including extra-Amazonian distributions) that is more seasonal. Hypothesis (H4) is that "the set of best cells, together representing the areas that would be required to conserve the most successful populations of each species, will change under climate change conditions". It is important to identify the location of the best cells, because there is such uncertainty about the speed and severity of the demographic response to change - as this model maintains a fixed mortality rate, it is rather optimistic about population survival.

Under SIS and RIS there is a westward movement of best cells (where environment least limits density), and a marked clustering in the north-central and western edges of Amazonia. This 
location of the best occupied or accessible sites in areas of higher altitude suggests that under doubled $\mathrm{CO}_{2}$ conditions, montane forests would be invaded by lowland species. This is as expected, and consistent with the results from reduced- $\mathrm{CO}_{2}$ Pleistocene conditions, when palynological data suggests that cooler temperatures allowed lowland forests to be invaded by montane species (Colinvaux 1998).

\subsubsection{Correlates of variation between species}

In ideal circumstances, it would be possible to relate vital rates of survival, growth and reproduction to climate. As this could not be achieved for the study species, the full power of the demographic model was not realised. Species density would be expected to rise or fall more rapidly in a such a scenario, as the rates of growth and survival would be related to climate directly, rather than through a limit on recruitment as here. The ability to model the relationship between mortality and climate for individual species is particularly desirable in a rapidly changing environment.

It was expected that at least some of the functional traits used in species selection would be correlated with change response (hypothesis (H5)). Population density change was associated with height class and self-fertilisation ability, and also with family, environmental and spatial distributions. This follows from the model parameterisation, which assigned different vital rates and population densities to different families, limiting the relative response of their component species, and from the environmental niches of the species concerned, which were also related to family. It is difficult to separate out the combined impacts of this mixture of categorical and continuous variables on the species' performance under change. A set of tests and correlations was carried out to examine individual relationships.

Under SIS, height class alone was important, affecting the lag in population change through generation time. Height class was well distributed through the families. Under RIS, selfing was important, acting through the survival of small populations at low density. The difference seems to derive from the lesser magnitude of change under RIS, which allowed populations the opportunity to reproduce for longer and had a smaller impact on $K_{\text {cpmax }}$, so that the reproductive traits had a greater impact. It was tentatively concluded that smaller stature was the only trait correlated with a more immediate threat from climate change, whilst species' ability to reproduce at low density may be more important where change is less severe.

Hypothesis (H6), that there is a significant difference in change response for species belonging to different families, was supported. Interestingly, for SIS there was a significant difference in the proportion of cells remaining viable, and for RIS, there was a significant difference in the 
percentage density change. Here, it is suggested that family affected generation time in increasing or decreasing populations through its influence on the vital rates. RIS displayed much greater interspecific variation in viability, which was not so obviously related to family. For SIS, the spatial clustering of species from individual families seems to have played a strong role in their shared fortunes. The viability result was not always a good representation of the family per se - for example, all the Rubiaceae species experienced $100 \%$ loss in viability, but those monographed happened to be narrow-range species from the same general location. Caryocaraceae also experienced a uniformly high loss of viability under SIS, despite having much more variation in distribution. It is therefore suggested that these families are more at risk because of their limited spatial distribution, and hence environmental tolerances, rather than because of variation in their vital rates.

If this is so, it was expected that initial range size might be correlated with change response (hypothesis (H7)). Initial range size was better correlated with viability than with density change. It was thought that the strength of the relationship with density change might have been reduced by the greater mean initial relative density of the narrow-range species. As no relationship was found between initial density and density change, this suggestion was rejected. Narrow range species were more likely to lose viability over the whole of their distributions, but the mean relative reduction in density could not be related to range size.

The final aspect of change to be examined had the most direct impact on species in the model: there were good correlations between density change and recorded bioclimate (hypothesis (H8)). Although AET increased in the model, species with a broad AET tolerance did better than those specialising in high AET conditions. Under SIS, some of the species most under threat are narrow-range species restricted to the northwest, where $A E T$ increases beyond the level so far experienced. Species whose current distribution also includes areas of higher AET may outcompete these species (section 7.2.6). However, as the outcome of competitive interactions under increasing resource availability is notoriously difficult to predict (section 4.2.5), I do not place a high level of confidence in this result.

Various correlations showed that species adapted to low AET, higher annual moisture deficit $(M D)$ and seasonal moisture availability (SMA) were performing best in terms of relative density change, and that this trend increased with time spent under the doubled $\mathrm{CO}_{2}$ conditions.

Overall, the severity of the impacts of climate change on modelled populations at this low latitude is higher than might have been intuitively expected, given that change is smaller in the equatorial region is smaller than in temperate zones. There is a strong impact on potential distributions and population viability, even when the impacts are halved under RIS. The impact 
of increased $M D$ in northeastern Amazonia is particularly notable for both scenarios, and does not hold the same degree of uncertainty as the impact of increased AET in northwestern Amazonia.

\subsection{METhod VALidation}

Choices have been made throughout the methods, especially when choosing feasible methods to approximate to the methodological ideal. This section mainly uses the study data and outcomes to consider the impact of some of these choices in an Amazonian context.

\subsubsection{Bioclimate variable selection}

\subsubsection{Relationships between bioclimate variables}

The bioclimate variables were selected from first principles to represent different aspects of current and changing moisture availability, seasonality and temperature (section 5.2.4.2). It was expected that relationships between the variables might change with increasing temperature and humidity. Decadal means for each cell for the last reference climate decade (1980s) and last complete change decade (2080s) are compared here to evaluate the extent to which variables explain one another - are any superfluous for Amazonian modelling?

Table 7:1: Spearman's correlation coefficients ( $\rho$ ) for $A E T, S M A, M D$ and $T_{\min }, 1980 \mathrm{~s}$ and 2080s cell means (two-tailed, $p<0.01$ in each case). SIS

\begin{tabular}{|l|l|l|l|l|}
\hline $\begin{array}{l}1980 \mathrm{~s} \\
/ 2080 \mathrm{~s}\end{array}$ & $\boldsymbol{M D}$ & $\boldsymbol{A E T}$ & $\boldsymbol{S M A}$ & $\boldsymbol{T}_{\min }$ \\
\hline $\boldsymbol{M D}$ & - & -0.708 & 0.976 & -0.539 \\
\hline $\boldsymbol{A E T}$ & -0.904 & - & -0.687 & 0.611 \\
\hline $\boldsymbol{S M A}$ & 0.955 & -0.878 & - & -0.504 \\
\hline $\boldsymbol{T}_{\min }$ & -0.132 & 0.320 & -0.170 & - \\
\hline
\end{tabular}

Under both the 1980s and 2080s climates, there are strong positive correlations between $M D$ and SMA, and strong negative correlations between AET and MD or SMA (Table 7:1). There is an absolute increase in correlation between $A E T$ and both $M D$ and SMA over the century. A visual analysis suggests that $A E T$ and $M D$ have a more consistent pattern between the decades, being closely correlated at high $A E T$ levels, where $A E T$ is most likely to approach $P E T$, and more divergent at low levels (Fig. 7:1a). All three ET-related variables have a fairly strong correlation with $T_{\min }$ in the 1980s, and a weak correlation by the 2080s (e.g. Fig. 7:1b). Despite the high $\rho$ value for $S M A$ versus $M D$, there is an increase in the spread of $S M A$ values for midrange $M D$ by the 2080s (Fig. 7:1c). 
(a) AET and MD: simulated means for Amazon cells

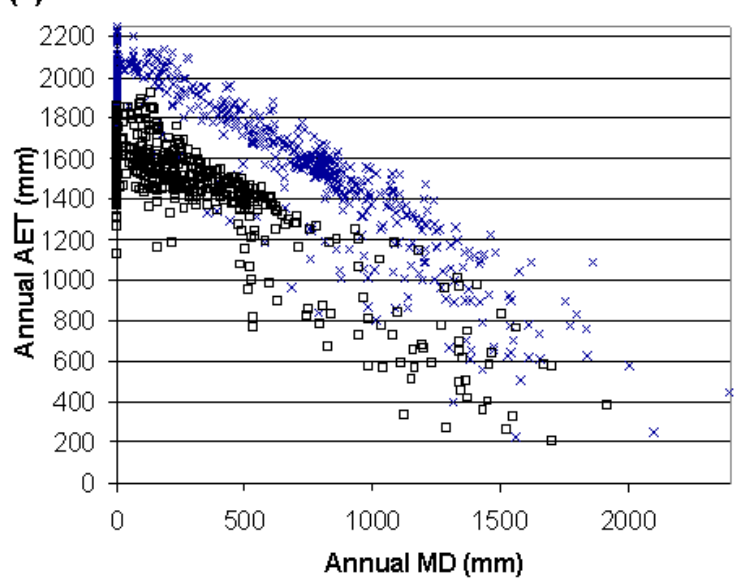

(c) MD and SMA: simulated means for Amazon cells

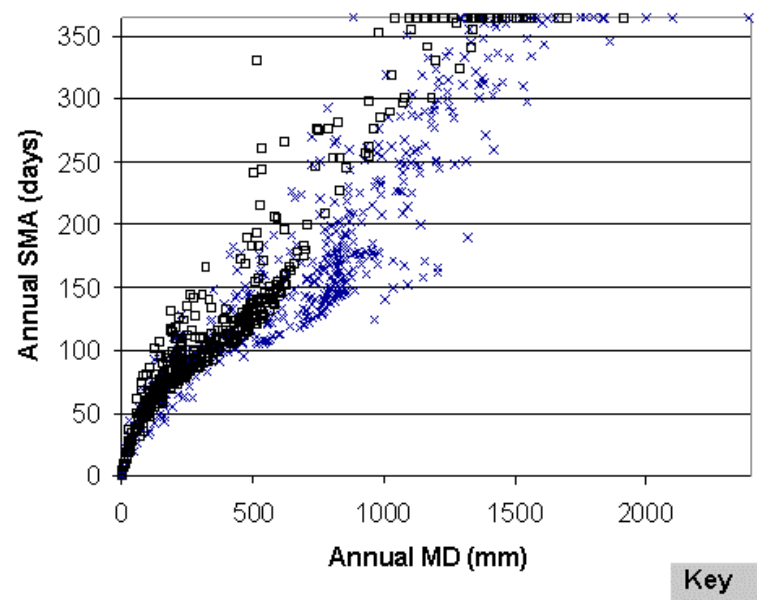

(b) MD and Tmin: simulated means for Amazon cells

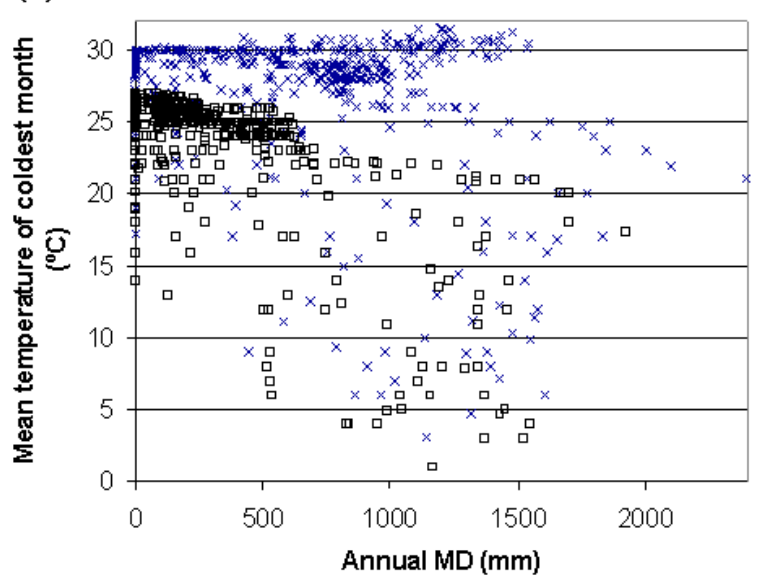

(d) GCMv Amazon model annual AET: means

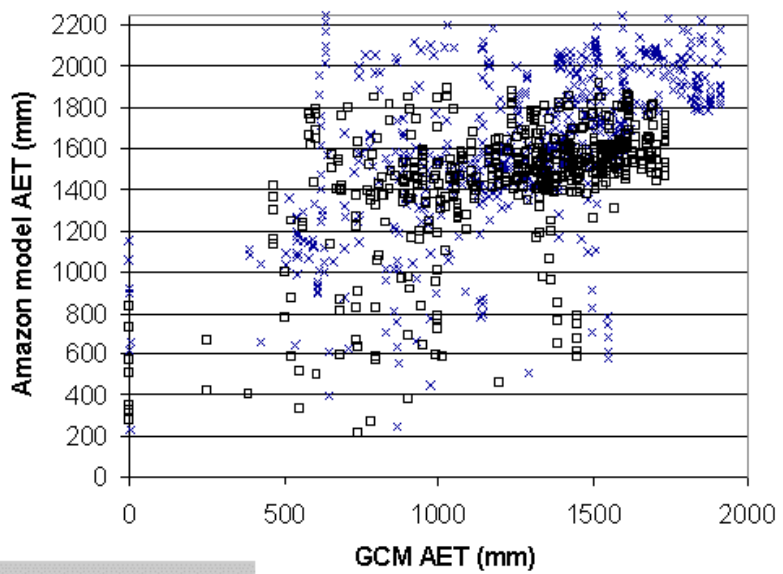

Fig. 7:1: DOWNS $M D$ versus (a) $A E T$, (b) $T_{\min }$ and (c) $S M A$ for 1980 s and 2080s decadal means, and (d) HADCM2 versus DOWNS AET. SIS

Before discarding one of the bioclimate variables, it would be useful to verify that small populations were not influenced by instances of that variable that stray from the main trend of the relationship with the other variables. Combinations of the variables far from this set are possible (high $M D$, low $S M A$, for example), and the methodology is intended to cater for all types of climate change. If a variable was omitted to reduce duplication in the model, SMA would be the most sensible option as it is a bounded variable, which limits its ability to discriminate the effects of change at high levels.

\subsubsection{Species frequency and bioclimate variables}

For the selected species set, record frequency is positively correlated with low moisture deficit, aseasonal, high-AET and high minimum temperature under the mixed-reference bioclimate (Fig. 7:2). Although there is only a medium correlation between reference $A E T$ and $T_{\min }$, there is a strong similarity in their species frequency curves. 
(a) Species records by mixed-reference MD

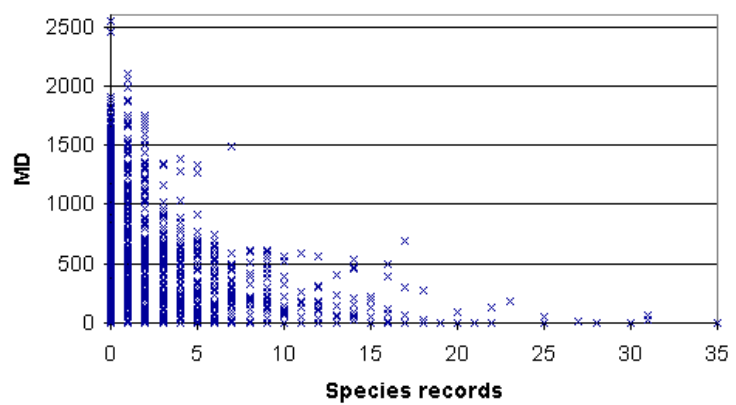

(c) Species records by mixed-reference AET

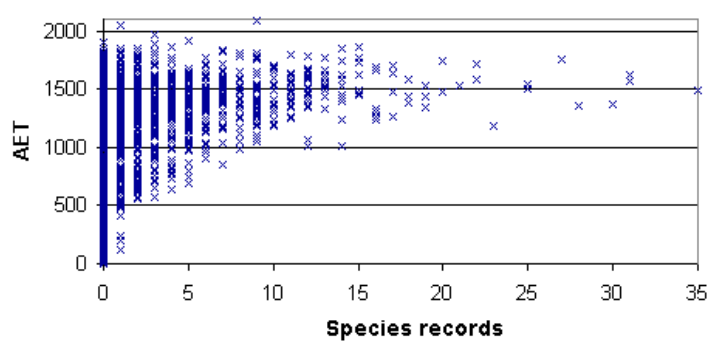

(b) Species records by mixed-reference SMA

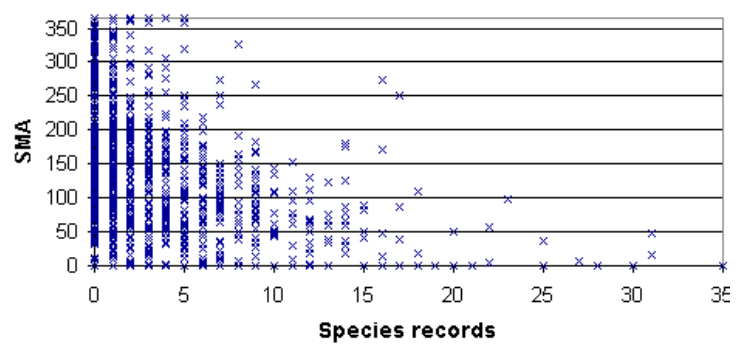

(d) Species records by mixed-reference $T_{\min }$

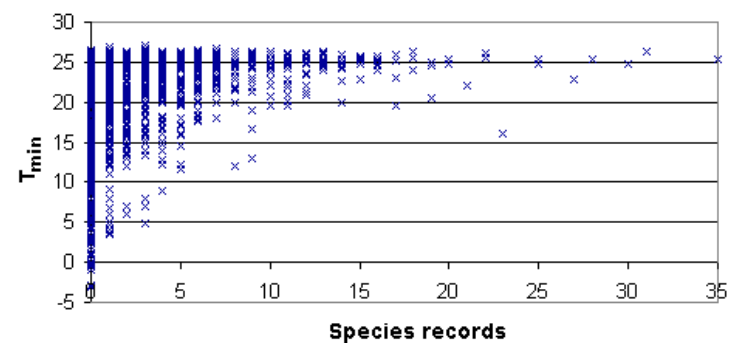

Fig. 7:2: Observed record count versus mixed-reference (a) AET, (b) SMA, (c) MD, (d)

$T_{\text {min. }}$ Extended Neotropics, SIS

\subsubsection{Bioclimate variable calculation}

Daily potential and actual ET calculations provide the basis for all three moisture-related bioclimatic variables. To test the success of the evapotranspiration (ET) model, output values should be compared with field measurements. Limited data are available. One study has estimated AET as the difference between rainfall and stream discharge over 1981-1983 at the Ducke reserve, $03^{\circ} 08^{\prime} \mathrm{S}, 60^{\circ} 02^{\prime} \mathrm{W}$ (Leopoldo et al. 1995).

Table 7:2: Evapotranspiration and precipitation estimates for cell SW003060

\begin{tabular}{|l|l|l|l|l|l|}
\hline Model & $\begin{array}{l}\text { AET mean } \\
(\mathbf{m m})\end{array}$ & $\begin{array}{l}\text { AET st. dev. } \\
(\mathbf{m m})\end{array}$ & $\begin{array}{l}\text { Precipitation } \\
\text { mean }(\mathbf{m m})\end{array}$ & $\begin{array}{l}\text { Precipitation } \\
\text { st. dev. }(\mathbf{m m})\end{array}$ & $\begin{array}{l}\text { Sample } \\
\text { interval }\end{array}$ \\
\hline $\begin{array}{l}\text { Leopoldo } \text { et } \\
\text { al. 1995 }\end{array}$ & 1493.1 & 38.02 & 2209 & 226.5 & years \\
\hline $\begin{array}{l}\text { DOWNS SIS, } \\
\text { mean 1960s }- \\
\text { 1980s values }\end{array}$ & 1626.2 & 41.21 & 2038.7 & 108.8 & decades \\
\hline PRES & 1653.2 & - & 2234.6 & - & - \\
\hline
\end{tabular}

When these values are compared with the reference DOWNS and PRES AET and precipitation estimates for this cell (Table 7:2), estimated AET is about $8-11 \%$ higher than the measured value. The standard deviation of $A E T$ is very similar, despite the difference in estimated precipitation. An exact concurrence between measured and modelled ET for any single site is not expected, because there is only a loose correspondence between the two measurements in both space and time. The measurement site is not likely to equal the cell mean, and the Ducke values are based on only three years of the 1961-1990 reference climate period. General 
conclusions cannot be drawn from one example, but it is reassuring to see that the model is performing well in estimating evaporative demand at this site (c.f. section 3.7.2.3.1).

Comparisons with general circulation model (GCM) AET outputs provide a way to assess the regional model's compatibility with the GCM (Fig. 7:1d). The pattern at 1980 and 2080 is similar, though the DOWNS AET consistently exceeds the range of $A E T$ from the GCM, and AET increases more in DOWNS than in HADCM2. Likely causes of this difference include:

- The coarser scale of the GCM predisposes it to a smaller variation in AET.

- The GCM cells cover a greater area than the inclusive-Amazon, which is generally surrounded by lower AET (Fig. 6:2).

- The AET calculation algorithms in the GCM and DOWNS are not absolutely identical.

- The GCM cells are much larger than the DOWNS cells. Spatial scale changes frequently lead to different model outcomes (e.g. Stoms 1992).

- The GCM timestep is much smaller, and the GCM operates in a stochastic rather than smoothed deterministic manner.

If there is a reasonable concordance with field values, differences between modelled AET and GCM AET are less important, but it is reassuring that the trend and scale of AET change are similar in the two models.

\subsubsection{Mixed-reference bioclimate}

If there is a consistent difference between inclusive-Amazon DOWNS (1960s to 1980s mean) and PRES, the mixed-reference bioclimate (section 5.2.4.7), then this will cause species with records from Amazonia to be simulated as having different tolerances to those occupying similar climates outside Amazonia.

To assess the impact, PRES and mean 1960s - 1980s DOWNS AET, SMA, MD and $T_{\min }$ were compared for Amazonian cells. In each case, the PRES values tended to be greater than the DOWNS values than vice versa, and this difference was significant $(\mathrm{p}=0.000$, Wilcoxon test (Monte Carlo option)). However, the mean paired differences (MD, $0.18 \mathrm{~mm}, A E T, 2.86 \mathrm{~mm}$, $S M A, 0.7$ days, $T_{\min }, 0.24^{\circ} \mathrm{C}$ ) were less than $1 \%$ of the mean values of either variable set. Species with records outside Amazonia would therefore be allocated slightly more resilience to positive change in the variables. Given the magnitude of the overall change, this effect should be relatively unimportant. 


\subsubsection{Species selection}

\subsubsection{Representativeness of sample}

The range of cell counts has been unintentionally narrowed by the selection. For every family, the databased mean for all monographed species was less than the mean count of the selected species. This is unsurprising, because of the lower density limit and the exemption of species with greater than 50 record-cells from the density criterion. Narrow-range species will also have been underrepresented in the species selection because it is more likely that widespread species' traits will be well known, which increases their likelihood of selection.

\subsubsection{Bias in data distribution}

For the selected set of species, the majority of cells in the extended Neotropics hold few records per cell - but the majority of records occur in these cells (Fig. 7:3). The high frequency cells do have high $A E T$, low $M D$, low $S M A$ and high $T_{\min }$ (Fig. 7:2), suggesting that the record frequency reflects Amazonian species' preferences as well as selection bias. If aseasonal forests were overrepresented, this would distort model outcomes for the set of species, but there is a reasonable spread of records over the east and west Amazon (Fig. 5:14).

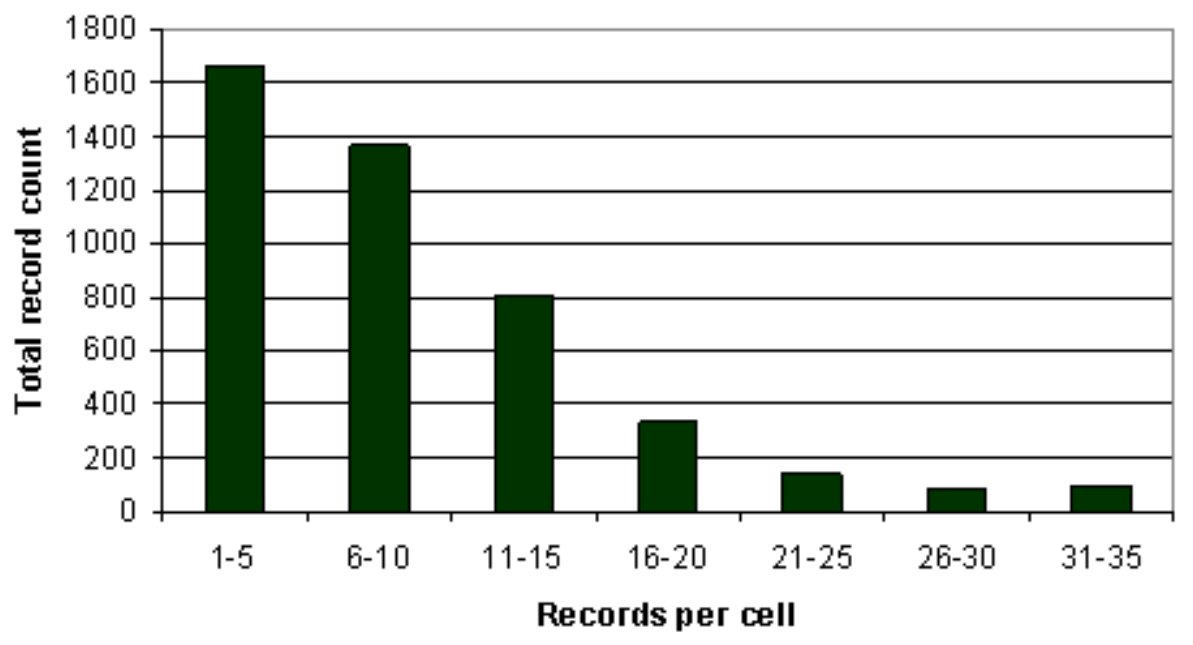

Fig. 7:3: Total number of records found in cells with few or many records

\subsubsection{Potential range estimation}

The accuracy of the similarity model (SM, section 4.3.6.12) in small-scale interpolation of limited botanical datasets was compared with a logistic and an autologistic model (LM \& AM, section 4.3.6.7). To compare modelled outputs with observed data, it was necessary to obtain a near-complete botanical dataset (presence-absence rather than presence-only). Temperate data were used, as these are much more comprehensive than tropical data. The trials measured 
success at predicting presences and absences using a $20 \%$ sample from a well-known distribution (the same percentage used by Augustin et al. 1996). This is intended to be a rigorous test at a recording density lower than or similar to that found in tropical plant datasets. They were devised and conducted in collaboration with Miguel Araújo of the Natural History Museum (NHM), London, who carried out the logistic model runs (Miles \& Araújo, in prep.).

Selected species from a 50-km grid tree database from the Atlas Florae Europaeae (Jalas et al. 1972-1996), provided by the Biogeography and Conservation Laboratory of the Natural History Museum in London, were used. These have well-documented distributions with a variety of spatial patterns (Table 7:3).

Table 7:3: European tree species used in the tests

\begin{tabular}{|l|l|l|}
\hline Species & Record count & Distribution \\
\hline Betula pubescens & $2684(20 \%=537)$ & Widespread north of Mediterranean \\
\hline Fagus sylvatica & $1123(20 \%=225)$ & $\begin{array}{l}\text { Widespread in central and southern } \\
\text { Europe }\end{array}$ \\
\hline Quercus coccifera & $311(20 \%=62)$ & Mediterranean \\
\hline Salix atrocinerea & $508(20 \%=102)$ & North and west Europe \\
\hline
\end{tabular}

Seven environmental variables were used: annual precipitation (Legates \& Wilmott 1990a), January \& July mean temperatures (Legates \& Wilmott 1990b), January \& July mean PET (Ahn \& Tateishi 1994), altitude above sea level (Row \& Hastings 1989), and soil water holding capacity (Bouwman et al. 1993). Altitude was included because the climate source maps were not adjusted for elevation. As climate change is not an issue in this analysis, the relationship between altitude and climate variables is constant. Soil water holding capacity gives a continuous measure of soil type that is directly related to plant function. Note that these tests were carried out before the selection of variables for the thesis method itself. No variables are excluded in the regression models in order to provide a fair comparison with the similarity model, which uses all variables available.

The environmental variables use a latitude-longitude grid, whilst the AFE grid is an unusual adaptation of the Military Grid Reference System UTM grid, which is difficult to convert to a standard raster format for GIS map analysis. Instead, environmental values were extracted using the latitude-longitude co-ordinates of the centre point of each AFE cell. Cells holding missing values were excluded from the analysis.

One hundred $20 \%$ random samples without replacement were taken from each dataset and supplied to each model to assess their success at recreating the original distribution maps. This process is a form of bootstrapping (Krebs 1989, Fielding \& Bell 1996), usually used to assess the effectiveness of a single model at explaining a well-known distribution, but here evaluating the usefulness of the modelling technique itself. A probability value of 0.5 or greater was used 
to denote predicted presence for LM and AM, and a similarity value of 0.95 or greater for SM. For consistency in the tests of the models, the threshold value was not altered through bootstrapping as it was in the Methods (section 5.2.8.1).

With high record densities it was expected that AM would provide the closest fit to the data, and SM is most likely to present errors of commissions. With low densities, it was expected that SM would perform better, as a logistic model does not cope well with a large number of 0 s.

It was assumed that:

1. The AFE dataset is an accurate representation of species presence and absence (so that presences modelled outside the known distribution would be false predictions);

2. The environmental data are of sufficient accuracy, relevancy and resolution to estimate species' distributions;

3. The performance of the models will be similar in boreal-Mediterranean and neotropical environments.

Autocovariates for AM were created using an adapted version of WORLDMAP provided by Paul Williams of NHM (Williams 1998b). The formula is based on presences in first and second order neighbour cells, weighting first order neighbours twice as heavily:

$$
\operatorname{autocov}_{i}=\frac{2 * 9 * p_{i(1 \mathrm{st})}}{c_{i(1 \mathrm{st})}}+\frac{16 * p_{i(2 \mathrm{nd})}}{c_{i(2 \mathrm{nd})}}
$$

where $i=$ cell of interest, 1 st $=$ rectangular first order neighbours, 2 nd $=$ second order neighbours, $p=$ record count and $c=$ cell count.

The 300 maps for each species were assessed according to the proportion of errors of omission and commission as compared to the original distributions (Fig. 7:4).

At the 20\% level, SM consistently outperforms LM and AM in the proportion of presences correctly predicted. LM and AM barely managed to locate any presences. When provided with a complete $(100 \%)$ distribution, AM was most accurate in reproducing it in three out of four cases. B. pubescens, a species distributed very widely throughout Europe, caused problems for SM at the $20 \%$ level, with a very high mean proportion of false presences simulated $(0.589$ for B. pubescens versus $0.144,0.026$ and 0.012 for the other species). This indicates that the 0.95 threshold of $S_{\min }$ was too high in this case.

AM, while very successful at explaining well-known distributions, copes poorly with a dataset that has many more absences than presences in locations that are broadly similar to the presence 
points. As AM performed so poorly at the $20 \%$ level, it was judged that the small improvement seen by Augustin et al. (1996) using the iterative routine based on the Gibbs sampler (section 4.3.6.7.2) would not be sufficient to justify the additional effort involved in its use.

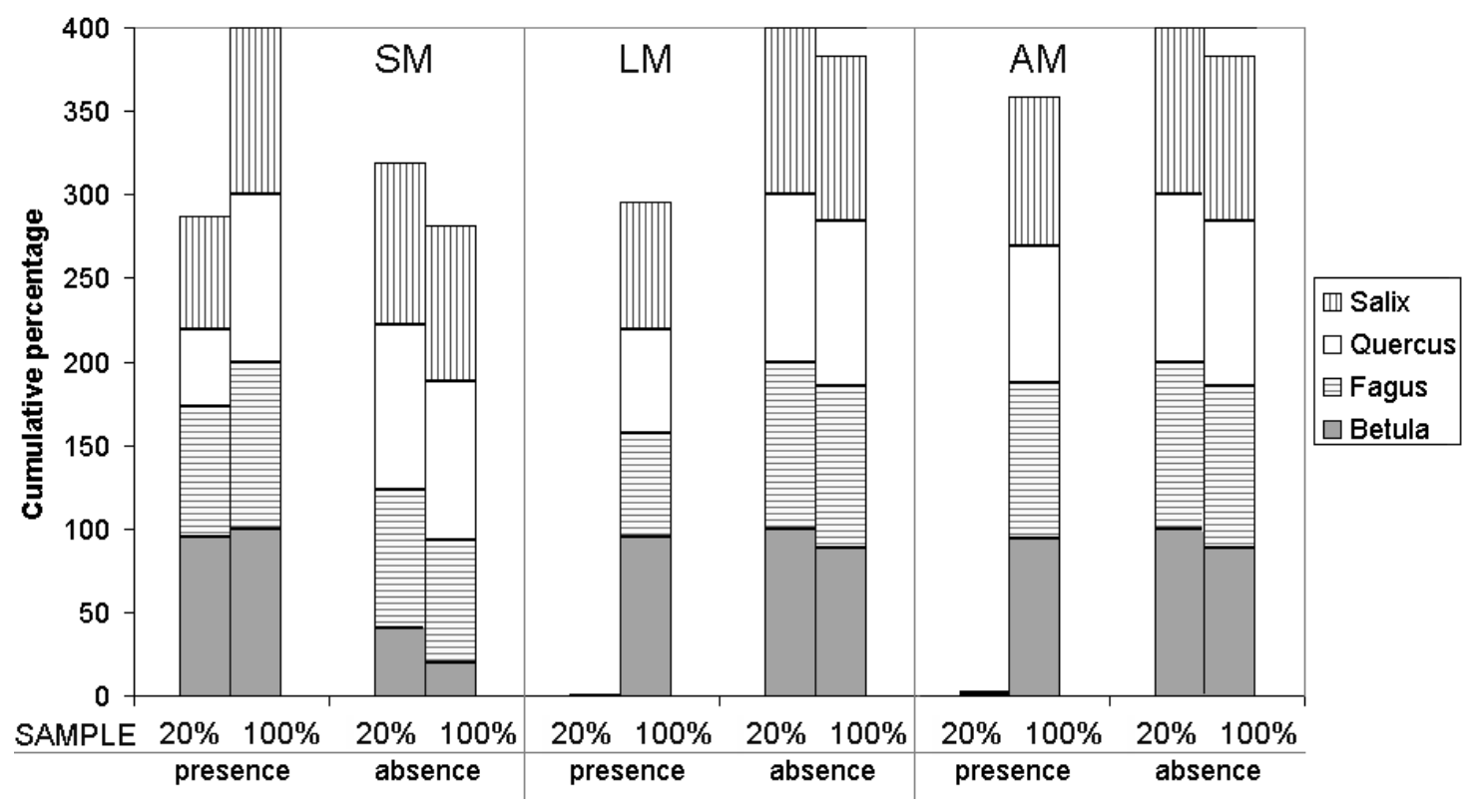

Fig. 7:4: Success of simulation of actual presences and absences by SM, LM and AM, based on bootstrapping of $20 \%$ samples, for four European tree species

With a $20 \%$ random sample of the species distribution, SM was by far the most successful technique. The tendency to overestimate species presence seen for B. pubescens shows that the technique can be overoptimistic in allocating potential distributions. In the context of the current project, SM therefore provides a conservative model of the impacts of climate change on biodiversity, because estimated range under the new conditions will err towards errors of commission. This serves to emphasise the severity of the change modelled here - even with an optimistic interpolation, most species suffered large reductions in potential distribution.

\subsubsection{Impact of variables on species}

The model makes two major assumptions for any given species, one on 'suitability' of environment, denoting areas that the species can tolerate and is not competitively excluded from, and the other on 'optimality', denoting the areas in which the species does best when it appears there. For example, a set of species might all take one part of climate space as their ideal, but those specialised to occupy it would be expected to be better competitors there than those with a broader niche (section 2.2.2.2). Neither assumption considers the possibility that species' true optima may fall outside the observed range. As there has been no experimental assessment of the study species' climate tolerances, the observed range is the only basis upon which these values can be estimated. 
This section investigates the degree of influence of the different variables on population viability (suitability) and density (optimality).

\subsubsection{Population viability}

Population viability was allocated according to the similarity calculation $\left(S_{\mathrm{p}}\right)$ and the species recorded maxima and minima for each variable (rectilinear envelope). For $S_{\mathrm{p}}$ it is the combination of values of the variables that is important, whilst in the rectilinear envelope variables act independently. Absolute exclusions under SIS at 2095 account for $81 \%$ of 15694 non-viable or lost populations (RIS: $78 \%$ of 7478); the others are caused by low $S_{\mathrm{p}}$ or loss of reproductive capacity through small density. I therefore concentrate on absolute exclusions when investigating how much viability loss was caused by the different variables.
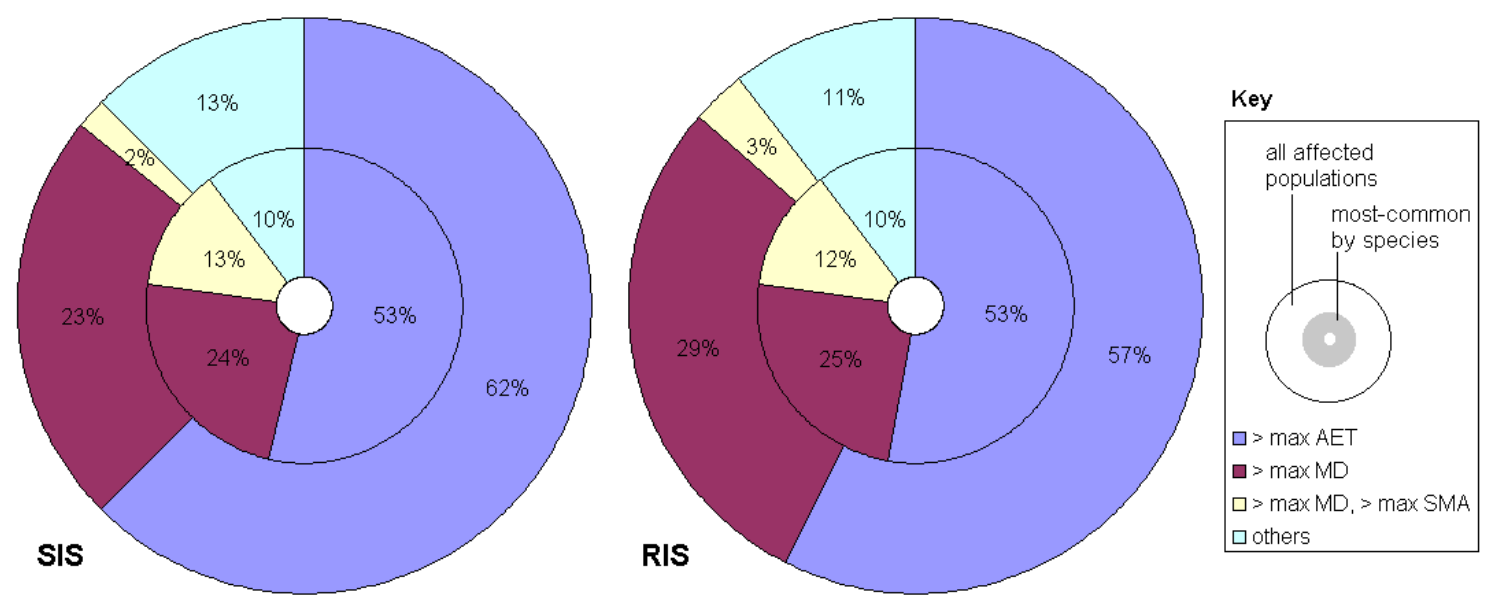

Fig. 7:5: Causes of absolute exclusion by rectilinear envelope, all non-viable populations and most-common cause per species, 2095 SIS \& RIS

Excessive $A E T$ alone was the most common cause of populations losing viability by leaving the rectilinear bioclimate envelope (Fig. 7:5). This seems counterintuitive - the model is suggesting that warmer, wetter climates than now experienced are unsuitable for tropical forest species.

The limitation arises from the assumption that species cannot tolerate climates outside the set of climates currently experienced (where 'current' is represented by the maxima and minima from 3 downscaled decadal means), or that they are excluded from these climates by competition. The model therefore predicts that no Amazonian species can tolerate values of bioclimate variable that are greater than those currently existing in the Neotropics, and this does occur for many cells for $A E T$ (section 6.1.2.2). An additional scenario has been created to explore the impact of relaxing this conservative assumption for maximum AET tolerated (section 7.2.6). 


\subsubsection{Population density}

The most limiting factor (MLF) controlled the maximum equilibrium density in viable cells. In relicts, population size was also limited by the absence of germination. The identity of the MLF was recorded for each species, cell and model year. Change in the most common MLF across the set of viable populations indicates that a different variable had more impact on density under reference and doubled $\mathrm{CO}_{2}$ conditions.
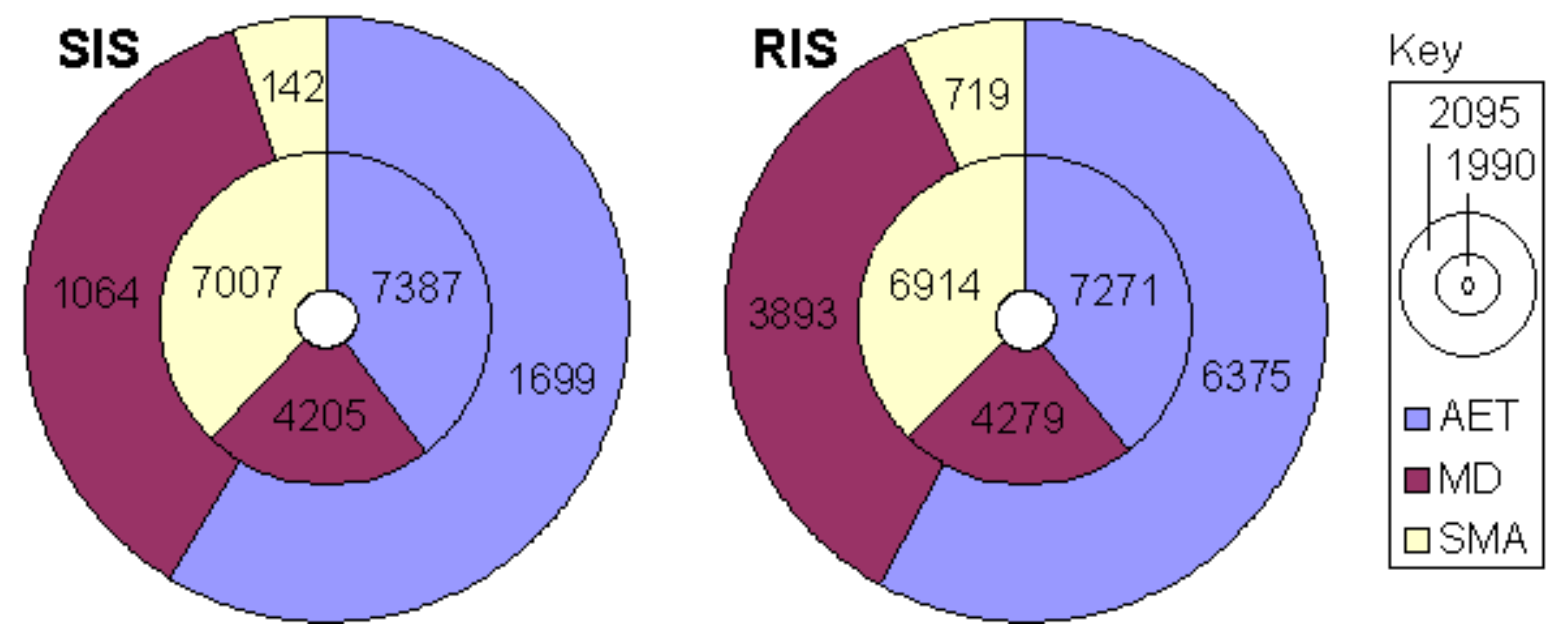

Fig. 7:6: Distribution of MLF across viable populations at 1990 and 2095, SIS \& RIS

(population frequency)

The initial and 2095 frequency distributions of MLF follow the same pattern for SIS and RIS (Fig. 7:6). The influence of AET on population density increases, and that of SMA decreases. The change in AET affects site optimality more frequently than the change in seasonality or moisture deficit. In some regions $A E T$ is more likely to be limiting because it is lower than the species optimum (Fig. 6:6); these MLF diagrams include both causes of limitation.
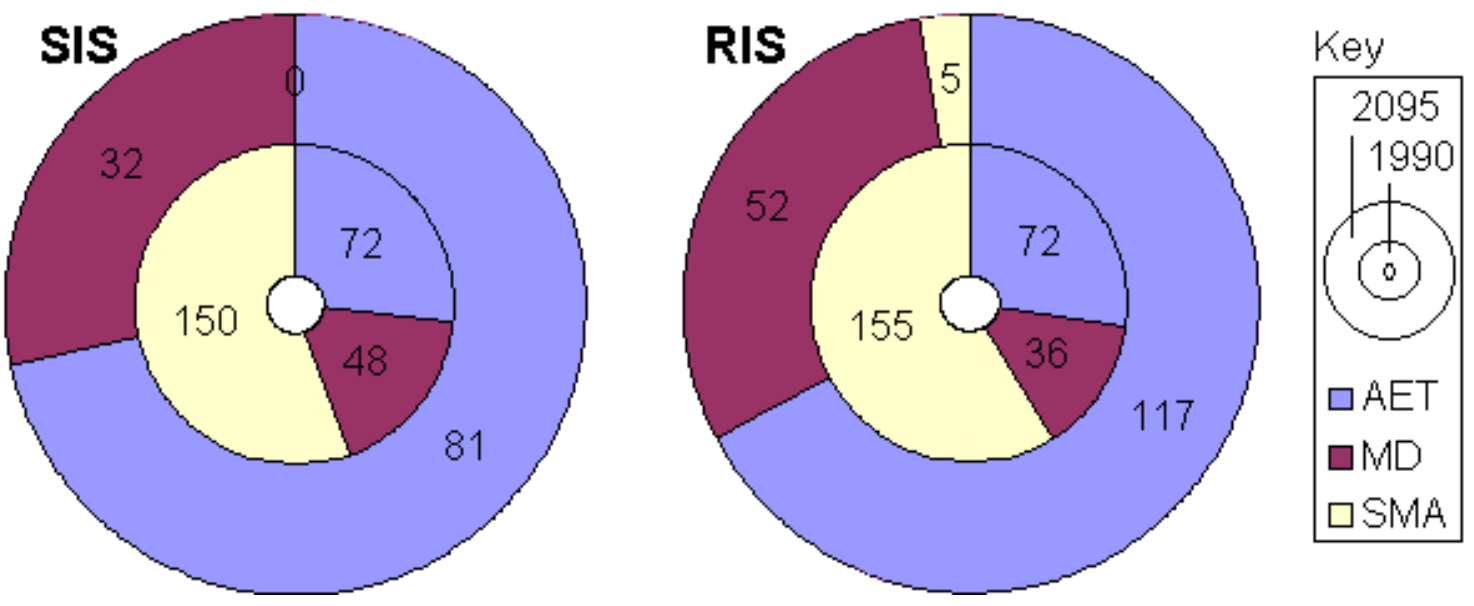

Fig. 7:7: Most common MLF per species for viable populations at 1990 and 2095, SIS \& RIS (species frequency)

When considering species' most common MLF for viable populations, the shift to AET is more pronounced (Fig. 7:7). Under the reference climate, an absolute majority of species were most 
commonly SMA-limited, but in 2095, AET was the dominant MLF. The nature of the variables has an effect - SMA is a bounded variable, whilst $A E T$ and $M D$ are not, and unlike $A E T$, the full range of $S M A$ and $M D$ were already experienced at some location in the reference climate. Thus, all else being equal, it is more likely that new values of $A E T$ should be further from the species' mean than for the other variables.

\subsubsection{Relaxed AET scenario (AIS)}

Species from different parts of Amazonia would be expected to tolerate and/or be competitively excluded from different $A E T$ ranges. However, it is likely that for moist forest species, the only maximum AET limitation would be indirect, through ecological processes such as competitive exclusion. Hence, if $A E T$ exceeds the range experienced by all Neotropical species, the outcome is uncertain. The best-guess scenario is that those species most competitive under present high $A E T$ would also be most successful under heightened AET (c-species, sensu Grime 1977).

When AET exceeded the reference climate's Neotropical range in SIS, the stringent assumption that species cannot survive long-term in environments outside the range of their current exposure was applied by preventing germination. (RIS does not produce climate values exceeding the current Neotropical range, and therefore some species were able to tolerate all simulated AET values). An alternative impact scenario ("AIS") has been devised to consider the outcomes if this assumption were relaxed for AET for some c-species. There is no need to do likewise for $M D$, because high $M D$ limits species physiologically as well as through competition. In any case, $M D$ values remain within the reference climate range.

The species occupying the highest $A E T$ sites under the reference climate are the best candidates for survival and/or expansion under the new high-AET climate, as their actual optimal AET may be higher than the reference climate mean. These sites were arbritarily defined as those occupying the top $10 \%$ of the mixed-reference bioclimate $A E T$ range $\left(>1880 \mathrm{mmy}^{-1}\right)$ for the Neotropics. All 15 selected species with records in these six sites were designated 'c-species' (Table 7:4). The c-species were all widespread, with a high record-count, and had retained some viable cells under SIS at 2095. Several are known to appear in ecologically diverse habitats (Cydista aequinoctialis, Steyermark et al. 1997; Inga cayennensis, Pennington 1997b, Nectandra hihua, Rohwer 1993, Pouteria guianensis, Pennington 1990). This indicates that they are universal competitors rather than specialised high-AET competitors.

The reference climate's high-AET cells formed two clusters of three, one located within the enclosed-Amazon around SW008073 in Colombia, and one set outside the inclusive-Amazon 
around NW005077 in the Brazilian state of Acre. The Colombian cluster cells exceed reference climate AET under SIS; the extra-Amazonian cells were not modelled under SIS.

The distribution of viable and non-viable populations under this relaxed AIS can be examined by allowing these species to ignore maximum $A E T$ limitation wherever $A E T$ exceeds the maximum value from the reference climate, without regard to $S_{\mathrm{p}}$ at those locations. The other assumptions of the model are retained. If maximum AET limitation is ignored, other excluding factors sometimes exist (included in 'others' in Fig. 7:5).

Table 7:4: 'c-species' ( $A E T$ assumption relaxed in AIS)

\begin{tabular}{|l|l|l|l|}
\hline Family & Species & Species ID & Records \\
\hline \multirow{4}{*}{ Arecaceae } & Astrocaryum murumuru & Astrmuru & 50 \\
\cline { 2 - 4 } & Geonoma macrostachys & Geonmacr & 71 \\
\cline { 2 - 4 } & Iriartea deltoidea & Iriadelt & 62 \\
\cline { 2 - 4 } & Oenocarpus bataua & Oenobata & 90 \\
\cline { 2 - 4 } & Socratea exorrhiza & Socrexor & 109 \\
\hline \multirow{3}{*}{ Bignoniaceae } & Callichlamys latifolia & Calllati & 84 \\
\cline { 2 - 4 } & Clytostoma binatum & Clytbina & 72 \\
\cline { 2 - 4 } & Cydista aequinoctialis & Cydiaequ & 181 \\
\hline Fabaceae & Chrysobalanus icaco & Chryicac & 193 \\
\hline Lauraceae & Inga cayennensis & Ingaaria & 53 \\
\cline { 2 - 4 } & Inga marginata & Ingamarg & 93 \\
\cline { 2 - 4 } & Inga nobilis & Ingamath & 191 \\
\hline Sapotaceae & Manilkara bidentata & Necthihu & 126 \\
\cline { 2 - 4 } & Pouteria guianensis & Panibide & 104 \\
\hline
\end{tabular}

The initial distribution of species under SIS was retained for AIS, as the assumptions governing current competitive exclusion are unchanged. Only the new set of conditions is conjectured to offer new opportunities to the 'c-species'. In these high-AET cells (Fig. 7:8a), the SIS 2095 results were reclassified for these 15 species (Fig. 7:8b). Where another bioclimate variable caused an absolute exclusion, the SIS result was retained. Otherwise, if there was no existing population, the area was classified as within the potential distribution, and if a population existed, it was classified as viable. Few populations of c-species were limited by extreme values of other variables. SIS results were retained for areas outside the high-AET zone. In this way, AIS results were approximated without re-running the model.

To see the combined outcome for the $15 \mathrm{c}$-species and the remaining species under AIS, the overall proportion of viable populations was mapped (Fig. 7:8c). Northeast Amazonia is still severely impacted by the increase in $M D$ and SMA. The uncertain outcome in the western high$A E T$ areas is now represented as a decrease in the proportion of viable populations, which is largely less severe than that of the surrounding forest. The better prognosis for species survival 
on higher ground to the west of Amazonia is still clear. The proportion of species remaining viable in the southern part of the region is now matched by a number of cells in the northwest.

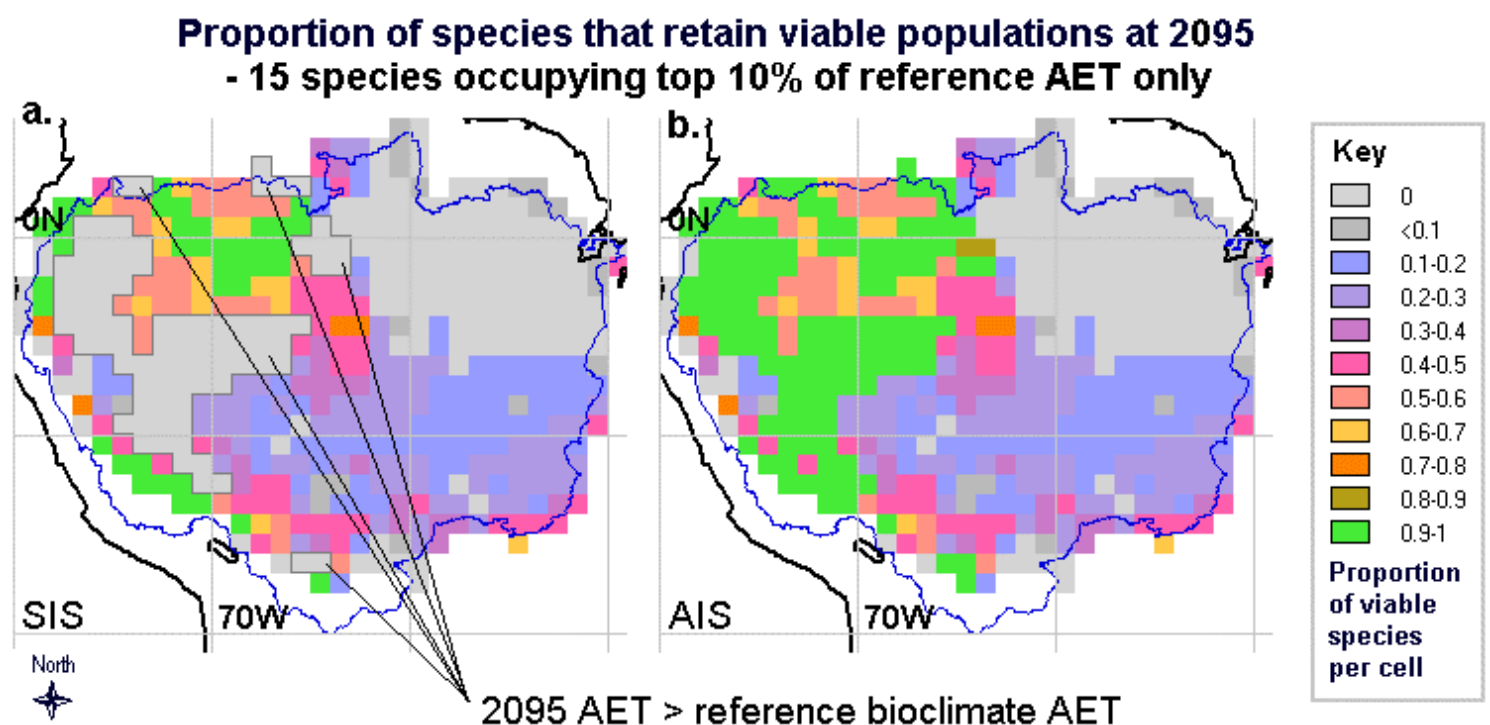

\section{c. Proportion of species that retain viable populations at $2095 \cdot 69$ species under AIS}

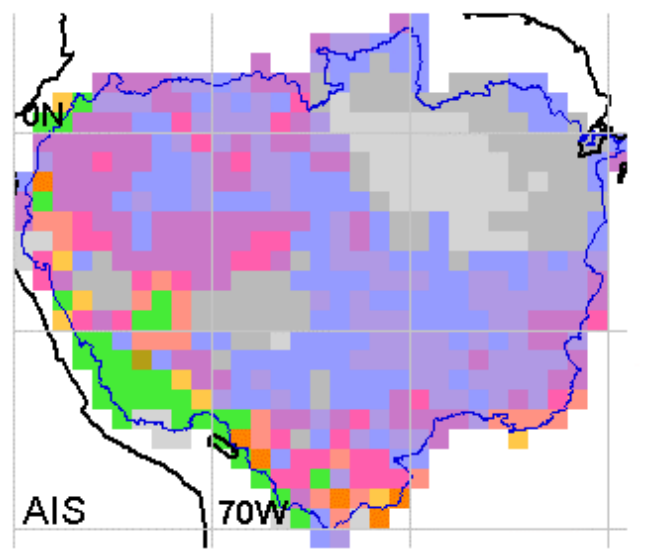

\section{d. 'Best' cells for 69 species under AIS at 2095}

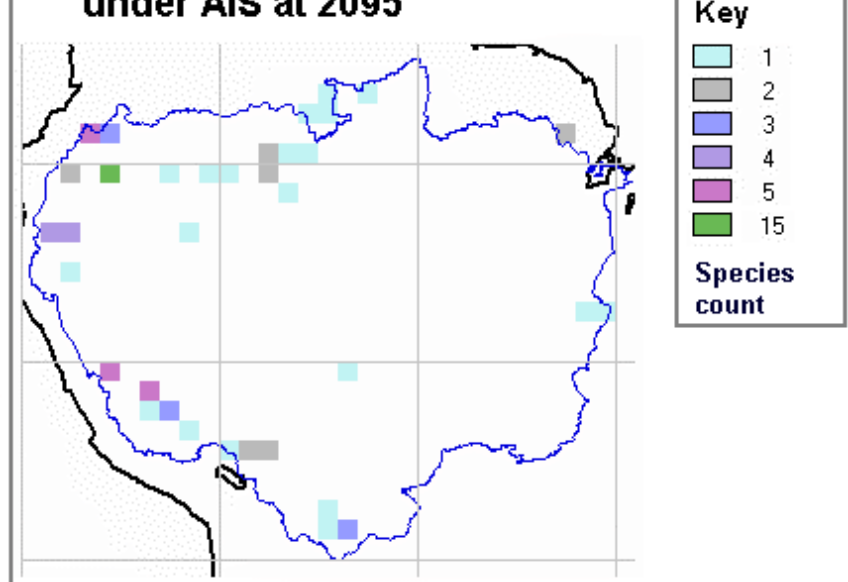

Fig. 7:8: (a) Outcomes for the 15 'c-species', SIS \& AIS; (b) Outcomes for all species, formed by replacing SIS with AIS outcomes set for those in (a); (c) 'Best cells under AIS.

The set of 'best' cells was also recalculated (Fig. 7:8d). The c-species are so widespread that all 15 occurred in many of the high AET cells. As they were assumed to be most successful under high-AET conditions, cell SW000075, which had the highest AET at 2095, and also held populations of all c-species, was allocated as their best cell. This northwestern cell is the location of Yasuní Parque Nacional, in Ecuador, and has a much lower mean altitude $(167 \mathrm{~m}$ above sea level) than the 'best' cells for most of the other species. 


\subsection{IMPLICATIONS OF STUDY}

It is clear that the most information about Amazonian species' likely response to climate change can be gained from their environmental tolerances. These determine species' long-term response to change. Changes in population viability are subject to less uncertainty than the models of equilibrium demography or population response to change. It is much easier to obtain a reasonable approximation of a species' environmental niche than of its vital rates' interaction with climate. Changes in distributions, however, are expected to be subject to potentially long delays before non-viable populations are finally extinguished, and the demographic aspect to this model provides a basis for estimating the length of the lag. Both components of climate response are affected by a lack of knowledge about changing competitive interactions.

The clustering seen in the spatial distributions and response of many species, and the correlations of change response with the climate variables, show that environmental tolerances also manifest as the key to species success in the model.

\subsubsection{Most vulnerable species}

Vulnerable groups can thus be defined by their spatial distributions and their likely demography. By 2095, $29 \%$ of species under SIS and 13\% of species under RIS had no viable distribution, and no cells adjacent to potential distribution (Table 7:5). These 'victim' species have a smaller modelled range size than the rest of the set $(\mathrm{p}=0.000$, Mann-Whitney U-test, SIS). All but three have fewer than 15 record cells - for example, all the restricted-range Rubiaceae species in the north-central area. Seven had no records outside the Amazon region (Table 7:5), so would become extinct if their Amazon populations were lost.

Table 7:5: Most threatened species: SIS \& AIS (not bold); SIS, AIS \& RIS (bold); endemic

to Amazonia (underlined)

\begin{tabular}{|l|l|l|l|}
\hline Family & Species & Family & Species \\
\hline Arecaceae & Astrocaryum gynacanthum & Fabaceae & Inga cardozana \\
\hline Arecaceae & Astrocaryum sciophilum & Fabaceae & $\underline{\text { Inga } \text { plumifera }}$ \\
\hline Arecaceae & Geonoma oldemanii & Lauraceae & Nectandra amazonum \\
\hline Arecaceae & Leopoldinia major & Lauraceae & Nectandra egensis \\
\hline Arecaceae & Leopoldinia piassaba & Proteaceae & Panopsis metcalfii \\
\hline Bignoniaceae & Jacaranda macrocarpa & Rubiaceae & Gleasonia duidana \\
\hline Caryocaraceae & Anthodiscus obovatus & Rubiaceae & Gleasonia uapensis \\
\hline Chrysobalanaceae & Couepia dolichopoda & Rubiaceae & Henriquezia nitida \\
\hline Chrysobalanaceae & Hirtella mutisii & Rubiaceae & Henriquezia verticillata \\
\hline Chrysobalanaceae & Licania humilis & Rubiaceae & Platycarpum schultesii \\
\hline
\end{tabular}

The areas in which a high proportion of species present are projected to be vulnerable over the entire Amazon region were mapped (Fig. 7:9a). The northwest to north-central region is highlighted as an area with a high proportion of 'victims'. The southern tip of the Amazon 
region also has a high proportion, but this is a less reliable result because of the small number of species initially modelled in this zone (Fig. 6:11).

(a)

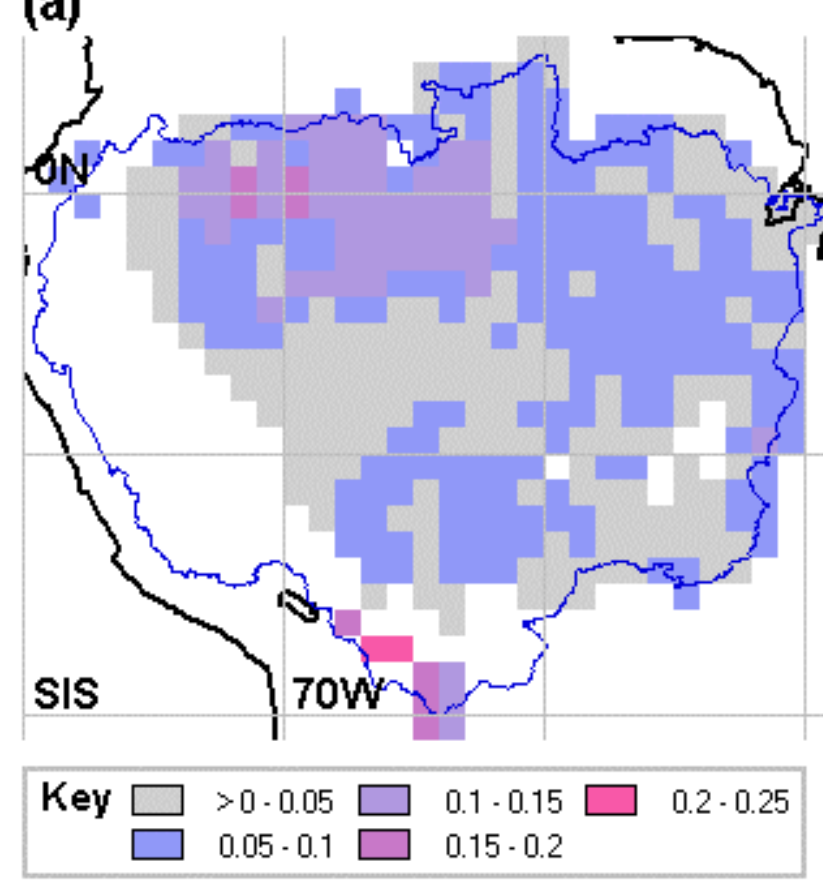

(b)

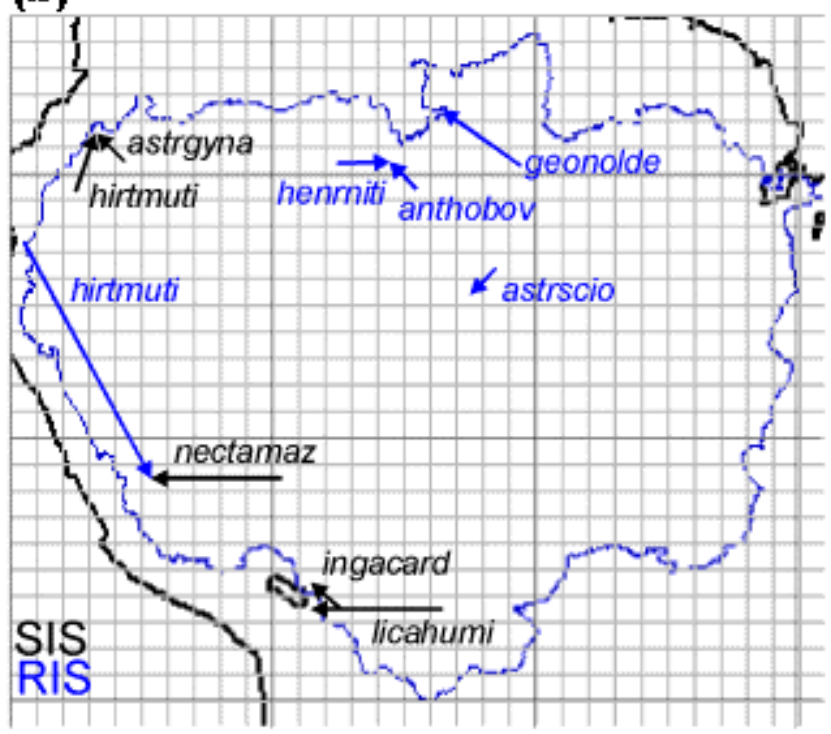

Key

RIS shortest path

Fig. 7:9: (a) Proportion of modelled species to fall into 'victim' category under SIS (and AIS); (b) minimum distance between existing and new potential distribution for 'victims'

Some 'victim' species did have areas of potential distribution at 2095 that was not adjacent to existing populations, or only adjacent on a diagonal. This situation occurred five times under both SIS and RIS. The shortest spatial trajectory between the 1990 populations and 2095 relicts was mapped (Fig. 7:9b). Ideally, the least cost pathway in terms of environmental suitability would also have been calculated, during several model-years in case the intervening patches might have been more suitable at some point. As the crow flies, most species would be required to migrate between two cells that are both close to the edge of the Amazon basin. Astrocaryum sciophilum is the most extreme exception, moving towards the centre of the region under RIS. By definition, each migration would have to traverse cells modelled as not suitable for the species, so would have to occur via long distance dispersal rather than spread. For some species, the dispersal events required to facilitate this would have to cover hundreds of kilometres, which is very great in comparison with the $300 \mathrm{my}^{-1}$ historical migration rate for European tree species (Huntley \& Birks 1983).

The minimum cell criterion for species selection excluded the most narrow-range species from the model, to avoid conflation between narrow range and locational data scarcity. These very narrow-range species are even more likely to be threatened by change, because they are climate specialists or simply because the probability that part of their distribution occupies an area of 
less substantial change is reduced. This exclusion will therefore affect simulation of the true distribution of concentrations of threatened species.

\subsubsection{Correlates of vulnerability}

To create a predictive profile for the most vulnerable species, demography and environmental niche should be considered. Spatial range size offers a good surrogate for environmental niche species with smaller population counts were more at risk of viability loss. The rate of density change is also important, but has been modelled with a greater degree of uncertainty. Climate will not remain stable under 2095 conditions, and may return towards 1990 conditions with time - so non-viable populations that persist to 2095 have a chance of survival.

The demographic model suggests that self-incompatible species of smaller mature size class will be most vulnerable to rapid density change under declining conditions. Short-lived species would also be expected to be at more risk under increasing climate variability, as a run of missed reproductive years can have a greater demographic impact. Hence, on a geographic scale, narrow-range species are of concern, and on a local scale, species with higher background mortality rates and density-dependent reproduction are most vulnerable under negative change.

The correlation of impact level with taxonomy seems to follow more from the connection between taxonomy and niche breadth than from the different vital rates assigned to families. The worst affected family, the Rubiaceae, happened to be represented by narrow-range species. The only Neotropical endemic family included, Caryocaraceae, was badly affected in terms of viability loss under SIS; most species were classified as 'potentially migrating'. Its response was much more variable under RIS, with many populations increasing in density.

AIS proposes that the most robust species for much of western Amazonia under change conditions are those that occur in the most productive sites at present, and so are likely to be the most competitive. These are also some of the most widespread species modelled. The northwestern region where the c-species are so successful even under SIS (Fig. 7:8a) largely overlaps an area where there is a high concentration of 'victim' species (Fig. 7:9a), suggesting the possibility that these are in danger of being displaced.

For northeastern Amazonia, no species were modelled as viable under SIS or AIS change conditions. In southeastern Amazonia, up to about a third of species remained viable. These again tended to be widespread species (e.g. Roupala montana, Fig. 6:23) rather than those presently confined to the region. 


\subsubsection{Areal targets for conservation and monitoring}

Traditionally, species range centres have been identified as important areas for conservation, as these are likely to hold the highest density and least variable populations (Brown et al. 1995). However, this does not consider the effects of climate change, or of human impacts which usually drive species to their range peripheries (Channell \& Lomolino 2000). The 'best' cells under doubled $\mathrm{CO}_{2}$ conditions were frequently located on the edge of the species' Amazonian range. As the typical pattern of anthropogenic range contraction leads to core-extinctions first (Channell \& Lomolino 2000), a strategy of prioritising reserves on the edge of the species' current range would be a 'no regrets' strategy (sensu McCarthy et al. 2001).

The 'best' cells are the areas of least uncertainty for those species' survival. As they have been produced using limited, interpolated data, these areas should be considered as indicating regions to represent rather than exact geographical locations for a reserve network. One approach would be to define the selected cells as core areas around which buffer zones would also be desirable (Araújo \& Williams 2000).

These were often areas of higher altitude, in the north or west of the region, as climate change impacts shift the isotherms upslope (temperature typically decreases by $2^{\circ} \mathrm{C}$ for every $300 \mathrm{~m}$ in altitude). Neotropical montane forests currently host their own high concentrations of endemics - the 'tropical Andes' region has been ranked as the top global hotspot for endemic plant and vertebrate species (Churchill et al. 1995, Myers et al. 2000). The Guiana Shield forest is also rich in endemic species and relatively undisturbed (Myers et al. 2000). At present, agricultural practices are removing Andean high-montane forests (Hartshorn 1992). If lowland species migrate upwards, montane forest species will be caught between the two pressures. Thus, providing reserves that span an altitudinal gradient is particularly important to allow both lowland and montane species the best opportunity to adapt to climate change. A broad buffer zone above the forested area should be provided to allow montane forest expansion.

The high species turnover rate with altitude also argues that 'corridor' reserves spanning a range of elevations provide a good protection for existing distribution of biodiversity. The likely existence of vertebrate species that rely on habitats at different altitudes to supply different resources further supports this argument (Diamond 1986). If montane forest areas were also chosen to protect lowland species in the event of climate change, this would again be a no regrets strategy.

Not all species distributions are currently adjacent to any area that would be suitable for them under changed conditions. Twenty species under SIS and AIS, and nine species under RIS were left in this situation (Table 7:5). In addition, species that are currently confined to the eastern 
Amazon would not benefit from the proposed montane reserves - the best cells for four species are located on the eastern boundary of Amazonia (Fig. 7:8d).

The AIS scenario suggests that many c-species of aseasonal moist forest would find climate change refugia in the zone of projected increased AET in the west. It is concluded that these regions and the regions of increased $M D$ in the northeast are key targets for monitoring the incidence and impact of climate change. They are modelled as undergoing the most extreme increases in $A E T$ and $M D$ respectively. Competitive displacements by c-species would be expected in the western zone, and decline of forest species in the northeastern zone.

\subsubsection{Comparison with vegetation model outcomes}

Under the IS92a scenario, 1.4 billion hectares of tropical forests are cleared by 2100 (section 3.4). This contributes to the increase in $\mathrm{CO}_{2}$ specified in IS92a, and would impact directly on species' distributions. Several models have explored GCM sensitivity to total deforestation of the Amazon basin (section 3.5.1.3.2), but GCMs have not incorporated land surface change as a part of implementing IS92a. It appears that no vegetation model has explicitly included this deforestation projection when working with GCM outputs based on IS92a - neither have the GCM's land-surface parameterisations, or the present study. When physiological vegetation models are forced with a level of $\mathrm{CO}_{2}$ consistent with the IS92a deforestation scenario, and go on to simulate a climate-induced forest dieback, they then release the $\mathrm{CO}_{2}$ into the atmosphere again! The integration of projected land-use change into the GCM is a vital next step to understanding both regional climate change and threats to species distributions.

In the meantime, it is interesting to compare the spatial outcomes for the 69 species with some vegetation model results. The results from the Hadley Centre models are most relevant, as it is a HADCM2 run that drives the species model.

All other models coupled to HADCM2 showed changes in vegetation type over at least part of Amazonia (Cramer et al. 2001). The climate-only ('T') scenario is most comparable with the present project's simulation, as it does not include the physiological impacts of $\mathrm{CO}_{2}$ increase. In this scenario, eastern Amazonia is invaded by savanna and grassland vegetation. When the physiological impacts of $\mathrm{CO}_{2}$ were introduced in the climate-and- $\mathrm{CO}_{2}$ ('CT') scenario, the change was less severe, but still affected much of the northeast Amazon. Under HADCM3, with its more frequent El Niño-like conditions, most of the forest was lost, even with a physiological $\mathrm{CO}_{2}$ response to mitigate the climate change (White et al. 1999, Cox et al. 2000). 
Taking AIS as the most realistic species-impact scenario, the overall impacts on viability produce a map (Fig. 7:8b) which visually falls between the Cramer et al. (2001) 'C' and 'CT' evergreen forest maps. The species and vegetation models are responding to a similar pattern of change, despite the process simulation being completely different. The models therefore support one another's conclusions about the impact of climate on biodiversity.

If SIS was considered as the most realistic scenario, the division between high- $A E T$ and high$M D$ limitation on the combined species viability map (Fig. 6:37a) is unlike anything the vegetation models have simulated. TRIFFID coupled with HADCM3 projects a greater area of deforestation than this, but it is clear that it originates from increased $M D$ originating in the northeast and spreading westward. RIS has a similar differentiation between the two sides.

The unique aspect to the species-based outcome map is that the different components can be examined individually. Similar maps could be created to investigate the effect of climate change on a subset of species - for example, to find out how the impact of change on a particular region contributes to biodiversity loss over the entire Amazon basin.

\subsubsection{Implications for GCM land surface parameterisations}

Species models of the type pursued here may provide guidelines for the variation in response within the PFTs now employed in climate models. For instance, they can help to answer questions such as "to what extent can a tree community from one area be parameterised as having the tolerances of a broader type such as 'moist forest tree'?" If an area of forest occupied by narrow-range species from the high- $M D$ end of the spectrum becomes suitable only for high$A E T$ species, or vice versa, there may be a deforested period between the loss of the seasonal and colonisation of the aseasonal species. The demographic half of the model can help to assess the likelihood of a lag based on the mortality and growth rates of the relevant species.

The species models also indicate the extent to which forests may decrease in species richness. The great variation between individual species' new distributions raises questions about the resilience of the vegetation matrix that they inhabit. A new, impoverished forest type brings an additional uncertainty to questions of carbon storage by forest. If trees do react to changed conditions with increased mortality, will the uptake of carbon by their successors exceed the loss of carbon from their demise? In the west, the new simulated community of c-species may have higher growth rates than the species they replace, as their competitive ability would be expected to be based on resource acquisition. In the northeast, the decline in all the simulated species indicates a loss of forest with concomitant decrease in carbon storage. 
If forest area does decrease, there is also a potential risk of ecological catastrophe caused by fragmentation. Is a critical minimum area of high-evapotranspiration forest required to preserve the local precipitation cycle? Is there a deforestation threshold beyond which fragments are too vulnerable to fire and drought to survive as moist forest? Both catastrophic outcomes have a basis in ecological theory (Shukla et al. 1990, Laurance \& Williamson 2001).

\subsection{DIRECTIONS FOR FUTURE RESEARCH}

The strength of the response to even the less severe of the scenarios indicates that climate change is an additional pressure that needs to be considered when planning conservation initiatives in the region. To help in this process, it would be useful to continue this line of research, improving the realism of both the scenarios and the model responses. The range of possible advances in this multidisciplinary field is vast. Some interesting and feasible approaches are discussed here.

\subsubsection{Modelled environment}

The climate model that drives the species change could be improved in structure and its underlying assumptions varied to give a greater range of potential futures. The regional bioclimate model used here is a relatively simple deterministic one, based on linear interpolation of decadal monthly GCM means. If a stochastic model were implemented, based on the interannual variability seen on the ground and in the GCM, multiple runs would be required to obtain a mean species response to change. Stochastic daily outcomes of the GCM output variables could be based on a frequency distribution derived from a series of matched monthly means for different years of an equilibrium GCM run (c.f. Cramer et al. 2001).

Such a stochastic climate model should be evaluated in terms of its simulation of the ENSO cycle return frequency, which in the current project is subsumed into the decadal climate means. The variation in the climate data used to configure species tolerance is sourced from the same decadal mean scale. Species tolerance of interannual variation and the return frequency of climate extremes would have to be carefully evaluated in line with the new model.

Perhaps a more pressing need is to carry out Amazonian species response models based on different GCMs' projections; because these produce different local patterns of change even when the regional results are broadly similar. It is important to ensure compatibility between the GCM and species model treatments of evapotranspiration and vegetation response as in the current model. The 'land surface scheme' in the Amazon ET model should be comparable with that of the GCM, and results from GCMs with a physiological vegetative response to change 
should only be used for species models whose climate tolerances are equally $\mathrm{CO}_{2}$-mediated. If the areas expected to undergo most change in species composition are similar, we can place a higher degree of confidence in the current set of results.

As discussed above (section 7.3.5), integration of ongoing land-use change into GCMs would improve both the simulation of regional climate change and threats to species distributions. The current regional bioclimate model is based on ET from natural cover, and the species model assumes that the entire basin is available for species populations. Existing deforestation and agricultural expansion already falsify this assumption. A simple remedy that falls short of further model integration would be to overlay existing and projected (e.g. Laurance et al. 2001) land use change onto the species model outcomes. The percentage of land in cultivation within each cell, estimated from satellite data (e.g. USGS et al. 1998), could be used to reduce the area available to species rather than applying an overall exclusion within affected cells.

A more detailed scenario could include the impacts of fire in areas adjacent to agricultural land. Feedbacks from fuel provision by previous fires cause mortality to be proportional to the fire return time (Kellman \& Meave 1997, Cochrane \& Schulze 1998, Fulé \& Covington 1999). Tolerance to fire varies between species in seasonal areas (Uhl \& Kaufman 1990) and is lower in aseasonal moist forests that do not usually burn. As land is deforested, the proportion of cells exposed to regular fire incursion would increase.

\subsubsection{Model sensitivity}

Further evaluation of many aspects of this type of model is possible. Some of the more fundamental avenues for exploration are:

1) Effect of scale on outcome: if the model run using finer-scale climate and species data, are more areas of potential refuge under change conditions uncovered? As model scale decreases, localised variations in topography and soil type are expected to have an influence on local climates, which may differ substantially from the mean climate of the degree cell.

2) Sensitivity to life history parameters: if one density model parameter is varied at a time, being set to recorded extreme values for the modelled species' PFT, which has the greatest influence on population stability under a stable and changing climate? When a population is decreasing in size, underestimated mortality should have a greater lag effect than poorly specified recruitment rates. As mortality rate effectively determines the rate of decline of a non-reproductive population, and as most populations underwent eventual declines, the model outcomes would be expected to be most sensitive to mortality variation.

3) Sensitivity of species to climate: it would be interesting to find out what is the minimum change in global climate to extinguish a metapopulation of the most and least sensitive 
species. For example, forcings of $10 \%, 25 \%, 150 \%$ of the climate anomalies applied in SIS could be generated (RIS was $50 \%$ of SIS). If the most vulnerable species fail under a climate analogous to that tolerated in the past, the model may have specified too limited a climate tolerance for these species.

4) Sensitivity to deterministic versus stochastic implementation: would the stochastic climate model described above, and/or a stochastic representation of species response (through application of processes on a probabilistic basis for individuals rather to a fraction of a stage class), generate a different mean outcome from that seen with this deterministic model? A stochastic model has the advantage of generating a range of responses and a confidence level for the mean, but a deterministic model is more computationally efficient so can be readily repeated for a greater range of species. If the mean outcomes using the two approaches for a test set of species are similar, this would support the continued use of a deterministic model.

\subsubsection{Response model advances}

\subsubsection{Scope of species and spatial area included}

Species that are projected to be lost from the Amazon basin may have climate change refugia in other parts of their Neotropical range. An extension of the potential distribution model to cover the extended Neotropics at 1990 and 2095 would present a full picture of viability over the species range, without necessitating a full model run for the extra area. The only omission in terms of evaluating realised distribution would be that those populations that were simulated as non-reproductive under 1990 conditions would not be excluded.

Similarly, the repetition of the potential distribution model for a larger species set would allow further assessment of whether the conclusions presented here are representative of the families, and gradients in species' spatial and environmental range under consideration. This extension requires only locational data for the additional species and minimal additional modelling time.

A fuller locational dataset for the existing species would be useful in ground-truthing the distribution model and improving its accuracy, particularly as regards buffering around known record points. Data from the areas falling between the major rivers of the region are particularly sparse (Fig. 5:14), due to access limitations, and would be valuable in this respect. 


\subsubsection{Processes included in the species model}

Potential improvements to the spatial demographic model include improvements in representation of existing processes, and the addition of new processes such as migration. The vital rate simulation could be improved by including density-dependence in growth and mortality, and direct relationships between vital rates and climate. For example, a greater sensitivity to desiccation would be expected in individuals that had reached their mature size, so were less able to adapt by altering the ratio of above and below-ground biomass.

An approach that would help to identify potential rates of species replacement is to treat pioneer and 'old-growth' species separately, by increasing pioneers' relative growth rate and decreasing their shade tolerance as immature individuals through density dependent mortality. These changes would provide a more realistic representation of the processes that lead to density limitation, and remove the need to specify maximum density per cell. However, these advances are dependent on improvements in autecological data for the individual species involved.

Finally, the ability of metapopulations to occupy their new areas of potential distribution could be assessed by simulating dispersal between cells. As the overwhelming majority of dispersal events would occur within rather than between cells, some care should be taken in simulating migration speed.

One option would be to model population advance for individual species at a much finer scale (e.g. Dyer 1995, Malanson \& Cairns 1997), and apply this as a maximum rate of spread from the time that an adjacent cell becomes suitable. Dispersal rates are little known for Amazonian species, so would be estimated from dispersal mode. Rare, very long distance dispersal events can strongly influence migration speed (Neubert \& Caswell 2000), though pollen availability remains a necessity for obligate outcrossing species. In a simple implementation, it would be possible to colonise cells adjacent to a reproductive initial population almost immediately they became suitable, but dispersal from these new cells to their neighbours would be subject to a lag in the migration front. This lag would correspond to the species-specific maturation time multiplied by the number of dispersal events required to cross the breadth of the cell.

\subsection{CONCLUSIONS}

When modelling species outcomes at the scale of the Amazon basin, for the foreseeable future it will be necessary to work with very limited data on distributions and relationships with climate variables. At the least, it will be a long time before more comprehensive and systematically gathered material is available. 
This project has developed a simple model to simulate the impact of global climate change on Amazonian plant species with a minimum of information. The modelling framework is flexible enough to incorporate more detailed demographic information when this is available.

Though the data are sparse and the species response modelling is the first done for this region, the topic is important and the results are striking. Similar spatial patterns occur for scenarios of different strengths, and in vegetation models by other authors. This suggests that even if the details on species rate of response and exact modelled locations are inaccurate, the general conclusions are robust. Thus action is urgent, not just in developing the science but also in ensuring that monitoring for potential climate change impacts is initiated, and that these impacts are taken into account when allocating areal priorities for biodiversity conservation. The monitoring and new survey work needed to complement these practical actions - identifying the response to change and species' actual presence or absence from proposed climate refugia should itself provide reservoirs of data for the science.

The spatial demographic model has been applied to 69 plant species under standard (SIS) and reduced impact (RIS) climate change scenarios based on HADCM2 IS92a outputs. Substantial impacts on species' potential range and population density resulted from both scenarios. Various shared patterns of spatial response in potential, viable and relict distributions were readily identifiable.

Annual actual evapotranspiration (AET) was the only bioclimate variable whose 1990 maximum value over the Neotropics was exceeded under SIS. The original model assumptions stated that specie populations were only viable within the range of each variable encountered under present conditions. An alternative impact scenario (AIS) was devised to allocate a positive population response to novel levels of AET to a set of "c-species", which were those inhabiting the $1^{\circ}$ cells with the highest $A E T$ under 1990 conditions. More weight was given to this scenario than to SIS when considering the spatial distribution of change impacts.

For most species, the hypothesis that the projected climate change would change the realised distributions between 1990 and 2095 could not be validated. However, the potential distribution of all species was affected by the projected change, leaving many populations as non-viable relicts. The hypothesis that all populations of a species would be rendered non-viable following change was confirmed for 30 species under SIS and 14 under RIS. The disparity in outcome between these hypotheses arises from the model's incorporation of a demographic lag in population response to change. 
Species' new potential distributions reflect climate change patterns as might be expected. However, the variation in response to these patterns is marked, and argues against simplistic treatments of plant functional types in vegetation models.

Almost every cell underwent a significant change in composition during the model period, as measured by the relative density of the set of modelled species present. The most extreme changes in bioclimate were projected to occur over much of western Amazonia, which experienced an increase in AET by 2095, and northeastern Amazonia, which experienced a strong increase in annual moisture deficit $(M D)$ and seasonality of moisture availability (SMA). Whilst the western region is likely to undergo compositional change, the outcomes for individual species were uncertain. The northeastern area of Amazonia appears to be the most likely region to undergo severe long-term change in species density and composition, with all modelled populations losing viability here under SIS and AIS, and only a small proportion remaining viable under RIS. The high- $A E T$ western and high- $M D$ northeastern zones are highlighted as targets for those wishing to monitor climate change impacts in Amazonia.

The set of cells modelled to be most favourable for each species under each scenario underwent a shift to the more aseasonal west, and to areas of high altitude, by 2095. Areas of moderate to high altitude on the western and centre-northern boundaries of the Amazon basin emerged as potential refugia for moist forest species affected by change in other parts of their range. These zones are therefore highlighted as important for conservation not only for the wide range of montane habitats and species, but also for many typical lowland species.

The species most at risk from change were defined as those that had no potential distribution at 2095, or were spatially separated from this new potential distribution. Under SIS, these most vulnerable species included seven of the nine Amazon endemics modelled. The hypotheses that vulnerability was correlated with range size and environmental tolerance were confirmed, with species of smaller ranges or poor tolerance of moisture deficit undergoing most change in population density. These species are least likely to benefit from the proposed refugia.

The demographic factors to most influence change response may differ depending upon the severity and rate of change. Height class and the combination of family and selfing were density response indicators under SIS and RIS respectively. Essentially, if the impact of change is to reduce population density, reproductive traits can influence population survival. If reproduction is prevented altogether by a more negative change, mortality rates (related to mature size) are most important. The longer the lag in population response to negative change, the better prospect of recovery if the climate change trajectory reverses, but there is also likely to be a delay between greenhouse gas emissions reduction and climate return to current conditions. 
A major conclusion is that the species most under threat of extinction following climate change are narrow-range Amazonian endemics. Those of small adult stature are probably under a more immediate threat than larger plants. This group of species is highlighted as targets for monitoring potential climate change impacts on population density and distribution. The small size and short generation time of bush and understorey species compared to canopy trees conveniently makes them relatively accessible subjects for demographic study.

This model, and the comparison with various vegetation models for Amazonia under doubled$\mathrm{CO}_{2}$, indicates that species confined to northeast Amazonia are most likely to be threatened, as this area most consistently undergoes a change in vegetation type. The only selected species to be restricted to this part of the Amazon (Astrocaryum sciophilum and Geonoma oldemanii) also had extra-Amazonian locations, which were not modelled, so their overall status is unknown.

The vegetation model comparison offers some support for the assumption that this model gives a representative view of projected change impacts on individual species. However, it would be useful to support the conclusions presented here with a wider range of scenarios, embodying the sub-regional impacts of different GCM simulations. The integration of major new elements such as stochasticity in climate and species response and future land use change, and an improved parameterisation of species' life histories and relationships with climate would also increase the level of confidence in the model results.

Although much further research could be done on this topic, it is thought appropriate to offer conservation management oriented conclusions because the short time scale for climate change demands for urgent action. Furthermore, ongoing land use change may already be threatening target areas. The high frequency of 'best' sites for predominantly lowland species that appear on the Amazon edge of the Andes and Guiana Highlands following climate change, and the possibility that these species may displace the existing biodiverse montane forest flora, suggest that 'climate change reserves' should encompass a wide altitudinal gradient.

This analysis does not imply that reserves outside these areas should be discarded. The high level of uncertainty attached to the climate change projection, the small fraction of Amazonian species that have been considered here, and the long list of ecosystem services provided by tropical forest argue strongly for conserving the maximum possible forest area. Nevertheless, it does suggest that to help protect Amazonian biodiversity from climate change impacts, a high priority should be given to existing and new reserves that span lowland to high montane forests. 


\section{Glossary of abbreviations}

For acronyms used in evapotranspiration calculation, see Table A1:1 and A2:1.

\begin{tabular}{|c|c|}
\hline $2 \mathrm{D}, 3 \mathrm{D}$ & $\begin{array}{l}\text { Two-dimensional, three- } \\
\text { dimensional }\end{array}$ \\
\hline$\alpha$-diversity & $\begin{array}{l}\text { Diversity found within a site or } \\
\text { community }\end{array}$ \\
\hline$\beta$-diversity & Diversity found between sites \\
\hline$\gamma$-diversity & Regional diversity \\
\hline ABRACOS & $\begin{array}{l}\text { Anglo-Brazilian Amazonian } \\
\text { Climate Observation Study }\end{array}$ \\
\hline $\begin{array}{l}\text { AET, } \mathrm{AET}_{\mathrm{a}}, \\
\mathrm{AET}_{\mathrm{r}}\end{array}$ & Actual ET, $\mathrm{ET}_{\mathrm{a}}, \mathrm{ET}_{\mathrm{r}}$ \\
\hline AIS & Alternative impact scenario \\
\hline AFE & Atlas Flora Europaeae \\
\hline AGCM & Atmospheric GCM \\
\hline ARPEGE & $\begin{array}{l}\text { Action de Recherche Petite } \\
\text { Echelle Grande Echelle GCM } \\
\text { (Meteo-France/ECMWF) }\end{array}$ \\
\hline BATS & $\begin{array}{l}\text { Biosphere-Atmosphere } \\
\text { Transfer Scheme }\end{array}$ \\
\hline $\mathrm{BCI}$ & $\begin{array}{l}\text { Barro Colorado Island, } \\
\text { Panama }\end{array}$ \\
\hline BMRC & $\begin{array}{l}\text { Bureau of Meteorology } \\
\text { Research (Australia) }\end{array}$ \\
\hline $\mathrm{BP}$ & (Years) before present \\
\hline $\mathrm{C}^{3}$ & $\mathrm{C}^{3}$ photosynthetic mechanism \\
\hline $\mathrm{C}^{4}$ & $\mathrm{C}^{4}$ photosynthetic mechanism \\
\hline CAM & Crassulacean acid metabolism \\
\hline CART & $\begin{array}{l}\text { Classification and regression } \\
\text { trees }\end{array}$ \\
\hline $\mathrm{CCA}$ & $\begin{array}{l}\text { Canonical correspondence } \\
\text { analysis }\end{array}$ \\
\hline $\mathrm{CCC}$ & Canadian Climate Centre \\
\hline $\mathrm{CCM}$ & Community Climate Model \\
\hline $\mathrm{CFC}$ & Chlorofluorocarbon \\
\hline CGCM & Coupled GCM \\
\hline $\mathrm{CH}_{4}$ & Methane \\
\hline CIC & $\begin{array}{l}\text { Climate Impacts Centre, } \\
\text { Sydney, Australia }\end{array}$ \\
\hline CIFOR & $\begin{array}{l}\text { Center for International } \\
\text { Forestry Research (Indonesia) }\end{array}$ \\
\hline $\mathrm{CO}_{2}$ & Carbon dioxide \\
\hline CRU & Climatic Research Unit (UK) \\
\hline CSIRO & $\begin{array}{l}\text { Commonwealth Scientific and } \\
\text { Industrial Research } \\
\text { Organization (Australia) }\end{array}$ \\
\hline CSR & $\begin{array}{l}\text { Competitive-stress tolerant- } \\
\text { ruderal }\end{array}$ \\
\hline CSU-GCM & $\begin{array}{l}\text { Colorado State University } \\
\text { General Circulation Model }\end{array}$ \\
\hline CTS & $\begin{array}{l}\text { Canonical trend surface } \\
\text { analysis }\end{array}$ \\
\hline
\end{tabular}

\begin{tabular}{|c|c|}
\hline DBH & $\begin{array}{l}\text { Diameter at breast height (1.3 } \\
\mathrm{m})\end{array}$ \\
\hline DCA & $\begin{array}{l}\text { Detrended correspondence } \\
\text { analysis }\end{array}$ \\
\hline DDE & Delay differential equations \\
\hline DEM & Digital elevation map \\
\hline DGVM & $\begin{array}{l}\text { Sheffield University Dynamic } \\
\text { Global Vegetation Model }\end{array}$ \\
\hline DKRZ & $\begin{array}{l}\text { Deutsches } \\
\text { Klimarechenzentrum } \\
\text { (Germany) }\end{array}$ \\
\hline DOWNS & $\begin{array}{l}\text { This project's bioclimate } \\
\text { model based on downscaled } \\
\text { climate values from GCM, for } \\
\text { inclusive-Amazon (see PRES) }\end{array}$ \\
\hline EBM & Energy balance model \\
\hline ECHAM & $\begin{array}{l}\text { European Centre/Hamburg } \\
\text { GCM (ECMWF/MPI) }\end{array}$ \\
\hline ECMWF & $\begin{array}{l}\text { European Centre for Medium- } \\
\text { Range Weather Forecasts } \\
\text { (UK) }\end{array}$ \\
\hline EM & Ectomycorrhizae \\
\hline ENSO & El Niño Southern Oscillation \\
\hline $\mathrm{ET}, \mathrm{ET}_{\mathrm{a}}, \mathrm{ET}_{\mathrm{r}}$ & $\begin{array}{l}\text { Evapotranspiration }(\mathrm{a}=\text { based } \\
\text { on actual vegetation, } \mathrm{r}=\text { based } \\
\text { on reference vegetation })\end{array}$ \\
\hline ETS & Effective temperature sum \\
\hline FACE & Free-air carbon enrichment \\
\hline FAO & $\begin{array}{l}\text { Food and Agriculture } \\
\text { Organisation of the United } \\
\text { Nations }\end{array}$ \\
\hline GAM & Generalised additive model \\
\hline GCM & General Circulation Model \\
\hline GDD & Growing degree days \\
\hline GFDL & $\begin{array}{l}\text { Geophysical Fluid Dynamics } \\
\text { Laboratory (USA) }\end{array}$ \\
\hline GGa & $\begin{array}{l}\text { Greenhouse forcing with } \\
\text { negative forcing from sulphate } \\
\text { aerosols }\end{array}$ \\
\hline GHG & Greenhouse gas \\
\hline GIS & $\begin{array}{l}\text { Geographic Information } \\
\text { System }\end{array}$ \\
\hline GISS & $\begin{array}{l}\text { Goddard Institute for Space } \\
\text { Studies (USA) }\end{array}$ \\
\hline GLM & Generalised linear model \\
\hline GSa & $\begin{array}{l}\text { Greenhouse forcing without } \\
\text { negative forcing from sulphate } \\
\text { aerosols }\end{array}$ \\
\hline $\mathrm{GtC}$ & Giga-tons of carbon \\
\hline
\end{tabular}




\section{Glossary of abbreviations}

For acronyms used in evapotranspiration calculation, see Table A1:1 and A2:1.

\begin{tabular}{|c|c|}
\hline $2 \mathrm{D}, 3 \mathrm{D}$ & $\begin{array}{l}\text { Two-dimensional, three- } \\
\text { dimensional }\end{array}$ \\
\hline$\alpha$-diversity & $\begin{array}{l}\text { Diversity found within a site or } \\
\text { community }\end{array}$ \\
\hline$\beta$-diversity & Diversity found between sites \\
\hline$\gamma$-diversity & Regional diversity \\
\hline ABRACOS & $\begin{array}{l}\text { Anglo-Brazilian Amazonian } \\
\text { Climate Observation Study }\end{array}$ \\
\hline $\begin{array}{l}\text { AET, } \mathrm{AET}_{\mathrm{a}}, \\
\mathrm{AET}_{\mathrm{r}}\end{array}$ & Actual ET, $\mathrm{ET}_{\mathrm{a}}, \mathrm{ET}_{\mathrm{r}}$ \\
\hline AIS & Alternative impact scenario \\
\hline AFE & Atlas Flora Europaeae \\
\hline AGCM & Atmospheric GCM \\
\hline ARPEGE & $\begin{array}{l}\text { Action de Recherche Petite } \\
\text { Echelle Grande Echelle GCM } \\
\text { (Meteo-France/ECMWF) }\end{array}$ \\
\hline BATS & $\begin{array}{l}\text { Biosphere-Atmosphere } \\
\text { Transfer Scheme }\end{array}$ \\
\hline $\mathrm{BCI}$ & $\begin{array}{l}\text { Barro Colorado Island, } \\
\text { Panama }\end{array}$ \\
\hline BMRC & $\begin{array}{l}\text { Bureau of Meteorology } \\
\text { Research (Australia) }\end{array}$ \\
\hline $\mathrm{BP}$ & (Years) before present \\
\hline $\mathrm{C}^{3}$ & $\mathrm{C}^{3}$ photosynthetic mechanism \\
\hline $\mathrm{C}^{4}$ & $\mathrm{C}^{4}$ photosynthetic mechanism \\
\hline CAM & Crassulacean acid metabolism \\
\hline CART & $\begin{array}{l}\text { Classification and regression } \\
\text { trees }\end{array}$ \\
\hline $\mathrm{CCA}$ & $\begin{array}{l}\text { Canonical correspondence } \\
\text { analysis }\end{array}$ \\
\hline $\mathrm{CCC}$ & Canadian Climate Centre \\
\hline $\mathrm{CCM}$ & Community Climate Model \\
\hline $\mathrm{CFC}$ & Chlorofluorocarbon \\
\hline CGCM & Coupled GCM \\
\hline $\mathrm{CH}_{4}$ & Methane \\
\hline CIC & $\begin{array}{l}\text { Climate Impacts Centre, } \\
\text { Sydney, Australia }\end{array}$ \\
\hline CIFOR & $\begin{array}{l}\text { Center for International } \\
\text { Forestry Research (Indonesia) }\end{array}$ \\
\hline $\mathrm{CO}_{2}$ & Carbon dioxide \\
\hline CRU & Climatic Research Unit (UK) \\
\hline CSIRO & $\begin{array}{l}\text { Commonwealth Scientific and } \\
\text { Industrial Research } \\
\text { Organization (Australia) }\end{array}$ \\
\hline CSR & $\begin{array}{l}\text { Competitive-stress tolerant- } \\
\text { ruderal }\end{array}$ \\
\hline CSU-GCM & $\begin{array}{l}\text { Colorado State University } \\
\text { General Circulation Model }\end{array}$ \\
\hline CTS & $\begin{array}{l}\text { Canonical trend surface } \\
\text { analysis }\end{array}$ \\
\hline
\end{tabular}

\begin{tabular}{|c|c|}
\hline DBH & $\begin{array}{l}\text { Diameter at breast height (1.3 } \\
\mathrm{m})\end{array}$ \\
\hline DCA & $\begin{array}{l}\text { Detrended correspondence } \\
\text { analysis }\end{array}$ \\
\hline DDE & Delay differential equations \\
\hline DEM & Digital elevation map \\
\hline DGVM & $\begin{array}{l}\text { Sheffield University Dynamic } \\
\text { Global Vegetation Model }\end{array}$ \\
\hline DKRZ & $\begin{array}{l}\text { Deutsches } \\
\text { Klimarechenzentrum } \\
\text { (Germany) }\end{array}$ \\
\hline DOWNS & $\begin{array}{l}\text { This project's bioclimate } \\
\text { model based on downscaled } \\
\text { climate values from GCM, for } \\
\text { inclusive-Amazon (see PRES) }\end{array}$ \\
\hline EBM & Energy balance model \\
\hline ECHAM & $\begin{array}{l}\text { European Centre/Hamburg } \\
\text { GCM (ECMWF/MPI) }\end{array}$ \\
\hline ECMWF & $\begin{array}{l}\text { European Centre for Medium- } \\
\text { Range Weather Forecasts } \\
\text { (UK) }\end{array}$ \\
\hline EM & Ectomycorrhizae \\
\hline ENSO & El Niño Southern Oscillation \\
\hline $\mathrm{ET}, \mathrm{ET}_{\mathrm{a}}, \mathrm{ET}_{\mathrm{r}}$ & $\begin{array}{l}\text { Evapotranspiration }(\mathrm{a}=\text { based } \\
\text { on actual vegetation, } \mathrm{r}=\text { based } \\
\text { on reference vegetation })\end{array}$ \\
\hline ETS & Effective temperature sum \\
\hline FACE & Free-air carbon enrichment \\
\hline FAO & $\begin{array}{l}\text { Food and Agriculture } \\
\text { Organisation of the United } \\
\text { Nations }\end{array}$ \\
\hline GAM & Generalised additive model \\
\hline GCM & General Circulation Model \\
\hline GDD & Growing degree days \\
\hline GFDL & $\begin{array}{l}\text { Geophysical Fluid Dynamics } \\
\text { Laboratory (USA) }\end{array}$ \\
\hline GGa & $\begin{array}{l}\text { Greenhouse forcing with } \\
\text { negative forcing from sulphate } \\
\text { aerosols }\end{array}$ \\
\hline GHG & Greenhouse gas \\
\hline GIS & $\begin{array}{l}\text { Geographic Information } \\
\text { System }\end{array}$ \\
\hline GISS & $\begin{array}{l}\text { Goddard Institute for Space } \\
\text { Studies (USA) }\end{array}$ \\
\hline GLM & Generalised linear model \\
\hline GSa & $\begin{array}{l}\text { Greenhouse forcing without } \\
\text { negative forcing from sulphate } \\
\text { aerosols }\end{array}$ \\
\hline $\mathrm{GtC}$ & Giga-tons of carbon \\
\hline
\end{tabular}




\begin{tabular}{|c|c|}
\hline $\begin{array}{l}\text { HADCM2, } \\
\text { HADCM3 }\end{array}$ & $\begin{array}{l}\text { Hadley Centre Coupled Model } \\
\text { v. } 2 \text { and v. } 3\end{array}$ \\
\hline $\mathrm{HCFC} / \mathrm{HFC}$ & Hydrogenated halocarbon \\
\hline HCFC-22 & $\begin{array}{l}\text { Chlorodifluoromethane } \\
(\mathrm{CF} 2 \mathrm{HCl})\end{array}$ \\
\hline HFC-134a & $\mathrm{CH} 2 \mathrm{FCF} 3$ \\
\hline IBAMA & $\begin{array}{l}\text { Brazilian Institute for the } \\
\text { Environment \& Renewable } \\
\text { Natural Resources }\end{array}$ \\
\hline IBIS & $\begin{array}{l}\text { Integrated Biosphere } \\
\text { Simulator }\end{array}$ \\
\hline IPCC & $\begin{array}{l}\text { Intergovernmental Panel on } \\
\text { Climate Change }\end{array}$ \\
\hline IS92a-f & $\begin{array}{l}\text { IPCC scenarios for potential } \\
\text { future GHG emissions }\end{array}$ \\
\hline LAI & Leaf area index \\
\hline LGM & Last Glacial Maximum \\
\hline LMD & $\begin{array}{l}\text { Laboratoire du Météorologie } \\
\text { Dynamique (France) }\end{array}$ \\
\hline $\begin{array}{l}\text { LOESS / } \\
\text { LOWESS }\end{array}$ & $\begin{array}{l}\text { Locally weighted linear } \\
\text { regression }\end{array}$ \\
\hline LSG & $\begin{array}{l}\text { Large Scale Geostrophic OCM } \\
\text { (DKRZ) }\end{array}$ \\
\hline LSP & Land-surface parameterisation \\
\hline MA & Moisture availability \\
\hline MAPSS & $\begin{array}{l}\text { Mapped Atmosphere Plant- } \\
\text { Soil System }\end{array}$ \\
\hline $\mathrm{MCP}$ & Minimum convex polygon \\
\hline $\mathrm{MD}$ & Moisture deficit \\
\hline MPI & $\begin{array}{l}\text { Max-Planck Institute for } \\
\text { Meteorology (Germany) }\end{array}$ \\
\hline MVP & Minimum viable population \\
\hline $\mathrm{N}_{2} \mathrm{O}$ & Nitrous oxide \\
\hline NASA & $\begin{array}{l}\text { National Aeronautics \& Space } \\
\text { Administration (USA) }\end{array}$ \\
\hline NCAR CCM & $\begin{array}{l}\text { National Center for } \\
\text { Atmospheric (Research) } \\
\text { Community Climate Model } \\
\text { (USA) }\end{array}$ \\
\hline NDVI & $\begin{array}{l}\text { Normalised difference } \\
\text { vegetation index }\end{array}$ \\
\hline NGO & $\begin{array}{l}\text { Non-governmental } \\
\text { organisation }\end{array}$ \\
\hline NOAA & $\begin{array}{l}\text { National Oceanic and } \\
\text { Atmospheric Administration } \\
\text { (USA) }\end{array}$ \\
\hline $\mathrm{NOx}$ & Nitrogen oxides \\
\hline NMC & $\begin{array}{l}\text { National Meteorological } \\
\text { Center }\end{array}$ \\
\hline NMVOCs & $\begin{array}{l}\text { Non-methane volatile organic } \\
\text { compounds }\end{array}$ \\
\hline NPP & Net primary productivity \\
\hline $\mathrm{O}_{2}$ & Oxygen \\
\hline $\mathrm{O}_{3}$ & Ozone \\
\hline $\mathrm{OCM}$ & Ocean circulation model $(=$ \\
\hline
\end{tabular}

\begin{tabular}{ll}
\hline & OGCM) \\
\hline OECD & $\begin{array}{l}\text { Organisation for Economic } \\
\text { Co-operation and } \\
\text { Development }\end{array}$ \\
\hline OGCM & Ocean GCM \\
\hline OPYC & $\begin{array}{l}\text { Ocean and Isopycnal Co- } \\
\text { ordinates GCM }\end{array}$ \\
\hline P/A & Presence/absence \\
\hline PAR & $\begin{array}{l}\text { Photosynthetically active } \\
\text { radiation }\end{array}$ \\
\hline
\end{tabular}

PBDFF Biological Dynamics of Forest Fragments Project, Brazil

PCA Principal component analysis

\begin{tabular}{ll}
\hline PDE & Partial differential equations
\end{tabular}

PET, PET $_{a}, \quad$ Potential ET, $E_{a}, E T_{r}$

PET

\begin{tabular}{ll}
\hline PFT & Plant functional type \\
\hline P-M & Penman-Monteith \\
\hline ppmv & Parts per million by volume \\
\hline P/R & Presence/random \\
\hline PRES & This project's bioclimate
\end{tabular}

PRES This project's bioclimate model based on present climate values from global datasets, for extended Neotropics (see DOWNS)

\begin{tabular}{ll}
\hline P-T & Priestley-Taylor \\
\hline PVA & Population viability analysis \\
\hline RegCM & Regional climate model \\
\hline RH & Relative humidity \\
\hline RIS & Reduced impact scenario \\
\hline SD & Statistical dynamical (model) \\
\hline SiB, SiB2 & Simple Biosphere model, v2 \\
\hline SIS & Standard impact scenario \\
\hline SO 2 & Sulphur dioxide \\
\hline SMA & $\begin{array}{l}\text { Seasonality of moisture } \\
\text { availability }\end{array}$ \\
\hline SRES & $\begin{array}{l}\text { Special Report on Emissions } \\
\text { Scenarios of the IPCC }\end{array}$ \\
\hline SST & Sea surface temperature \\
\hline TCG & Tropical cyclone generation \\
\hline TWINSPAN & $\begin{array}{l}\text { Two-way indicator species } \\
\text { analysis }\end{array}$ \\
\hline UKMO & UK Meteorological Office \\
\hline UPGMA & $\begin{array}{l}\text { Unweighted pair-group } \\
\text { method using arithmetic } \\
\text { averages }\end{array}$ \\
\hline VAM & $\begin{array}{l}\text { Vesicular arbuscular } \\
\text { mycorrhizae }\end{array}$ \\
\hline WHC & (Soil) water holding capacity \\
\hline WUE & Water use efficiency \\
\hline &
\end{tabular}




\section{Appendix 1 Input variables for ET}

Table A1:1: Input variables for daily P/AET simulation

\begin{tabular}{|c|c|c|c|c|}
\hline Code & Variable & Derivation & Reference & \begin{tabular}{|l|} 
As \\
HADCM2
\end{tabular} \\
\hline & $\begin{array}{l}\text { Vegetation classes } \\
\text { ( } 24 \text { types) }\end{array}$ & (input variable) & $\begin{array}{l}\text { Wilson \& } \\
\text { Hendersen- } \\
\text { Sellers, } 1985\end{array}$ & Yes \\
\hline & $\begin{array}{l}\text { Soil texture class } \\
\text { (3 types) }\end{array}$ & (input variable) & $\begin{array}{l}\text { Wilson \& } \\
\text { Hendersen- } \\
\text { Sellers, 1985; } \\
\text { Buckley \& } \\
\text { Warrilow } \\
1988\end{array}$ & Yes \\
\hline & $\begin{array}{l}\text { Soil colour class } \\
\text { (3 types) }\end{array}$ & (input variable) & $\begin{array}{l}\text { Wilson \& } \\
\text { Hendersen- } \\
\text { Sellers, } 1985, \\
\text { Buckley \& } \\
\text { Warrilow } \\
1988 \\
\end{array}$ & Yes \\
\hline $\mathrm{E}$ & Topology, m & (input variable) & $\begin{array}{l}\text { Row \& } \\
\text { Hastings, } \\
1989\end{array}$ & $?$ \\
\hline $\mathrm{e}_{a}$ & $\begin{array}{l}\text { Monthly mean } \\
\text { actual vapour } \\
\text { pressure of the air, } \\
\mathrm{kPa}\end{array}$ & $\begin{array}{l}\text { (input variable). Annual change is calculated } \\
\text { from GCM anomaly for relative humidity } \\
(\mathrm{RH}): \\
\mathrm{e}_{\mathrm{a}}=\frac{\mathrm{e}_{\mathrm{s}} \mathrm{RH}}{100}\end{array}$ & $\begin{array}{l}\text { New et al., } \\
1999\end{array}$ & No \\
\hline $\mathrm{n} / \mathrm{N}$ & $\begin{array}{l}\text { Relative sunshine } \\
\text { duration }\end{array}$ & $\begin{array}{l}\text { (input variable - monthly mean cloud cover, } \\
\text { fraction). Radiation is updated directly using } \\
\text { GCM anomaly, so that there is no need for } \% \\
\text { cloud. }\end{array}$ & $\begin{array}{l}\text { New et al., } \\
1999\end{array}$ & No \\
\hline $\mathrm{P}$ & $\begin{array}{l}\text { Monthly daily } \\
\text { precipitation, } \\
\mathrm{mmd}^{-1}\end{array}$ & $\begin{array}{l}\text { (input variable). \% GCM anomaly added } \\
\text { annually. }\end{array}$ & $\begin{array}{l}\text { New et al., } \\
1999\end{array}$ & No \\
\hline $\begin{array}{l}\mathrm{T}, \mathrm{T}_{\mathrm{x}} \\
\mathrm{T}_{\mathrm{n}}\end{array}$ & $\begin{array}{l}\text { Monthly daily } \\
\text { temperature mean } \\
(\mathrm{T}), \text { maximum } \\
\left(\mathrm{T}_{\mathrm{x}}\right) \text { and minimum } \\
\left(\mathrm{T}_{\mathrm{n}}\right),{ }^{\circ} \mathrm{C}\end{array}$ & $\begin{array}{l}\text { (input variable). Absolute GCM anomaly } \\
\text { added annually to } \mathrm{T} \text { (at } 1.5 \mathrm{~m}) \text {; monthly range } \\
\text { of } \mathrm{T}\left(\mathrm{T}_{\text {range }}\right) \text { is also available from GCM. } \mathrm{T}_{\mathrm{x}} \\
\text { and } \mathrm{T}_{\mathrm{n}} \text { are required for } \mathrm{e}_{s} \text { calculation. The } \\
\text { Hargreaves equation based on } \mathrm{r}_{s} \text { (Allen } 2000 \text { ), } \\
\text { estimates } \mathrm{T}_{\text {range }} \text { and assumes that it is } \\
\text { distributed equally around the mean The } \\
\text { following formulae are therefore used to } \\
\text { estimate } \mathrm{T}_{\mathrm{x}} \text { and } \mathrm{T}_{\mathrm{n}} \text { : } \\
\mathrm{T}_{\mathrm{x}}=\mathrm{T}+\frac{\mathrm{T}_{\text {range }}}{2} ; \mathrm{T}_{\mathrm{n}}=\mathrm{T}-\frac{\mathrm{T}_{\text {range }}}{2}\end{array}$ & $\begin{array}{l}\text { New et al., } \\
1999 \text {, Allen } \\
2000\end{array}$ & No \\
\hline $\mathrm{v}$ & $\begin{array}{l}\text { Monthly mean } \\
\text { wind speed, } \mathrm{ms}^{-1}\end{array}$ & $\begin{array}{l}\text { (input variable). \% GCM anomaly added } \\
\text { annually. }\end{array}$ & $\begin{array}{l}\text { New et al., } \\
1999\end{array}$ & No \\
\hline
\end{tabular}




\section{Appendix 2 Calculated parameters for ET}

This table demonstrates how the input variables are used to calculate PET and AET.

Notation of the form $\mathrm{z}=[\mathrm{f}(\mathrm{x}) \leq \mathrm{y}]$ indicates that if $\mathrm{f}(\mathrm{x})<\mathrm{y}, \mathrm{z}=\mathrm{f}(\mathrm{x})$, else $\mathrm{z}=\mathrm{y}$.

Table A2:1: Calculated parameters and variables for daily P/AET simulation

\begin{tabular}{|c|c|c|c|c|}
\hline Code & Variable & Derivation & Reference & $\begin{array}{l}\text { As } \\
\text { HAD- } \\
\text { CM2 }\end{array}$ \\
\hline$\partial$ & $\begin{array}{l}\text { Solar declination } \\
\text { (radians) }\end{array}$ & $0.409 \sin \left(\frac{2 \pi \mathrm{dn}}{365}-1.39\right)$ & $\begin{array}{l}\text { Allen } \\
2000\end{array}$ & $?$ \\
\hline$\gamma$ & $\begin{array}{l}\text { Psychometric constant, } \\
\mathrm{kPa}^{\circ} \mathrm{C}^{-1}\end{array}$ & $\frac{0.00163 \mathrm{PR}}{\lambda}$ & $\begin{array}{l}\text { Allen } \\
2000\end{array}$ & $?$ \\
\hline$\Phi$ & Latitude (radians) & - & - & Yes \\
\hline$\Delta$ & \begin{tabular}{|l} 
Slope of saturation \\
vapour pressure curve, \\
$\mathrm{kPa}^{\circ} \mathrm{C}^{-1}$
\end{tabular} & $\frac{2504 * \exp (17.27 \mathrm{~T} / 237.2+\mathrm{T})}{(237.3+\mathrm{T})^{2}}$ & $\begin{array}{l}\text { Allen } \\
2000\end{array}$ & ? \\
\hline$\lambda$ & $\begin{array}{l}\text { Latent heat of } \\
\text { vaporisation, } \mathrm{MJkg}^{-1}\end{array}$ & $2.501-0.002361 \mathrm{~T}$ & $\begin{array}{l}\text { Allen } \\
2000\end{array}$ & $?$ \\
\hline$\rho_{\mathrm{a}}$ & $\begin{array}{l}\text { Mean air density at } \\
\text { constant pressure, } \\
\mathrm{kgm}^{-3}\end{array}$ & $\frac{3.486 \mathrm{PR}}{\left((\mathrm{T}+273.16)\left(1-\frac{0.378 \mathrm{e}_{\mathrm{a}}}{\mathrm{PR}}\right)^{-1}\right)}$ & $\begin{array}{l}\text { Allen } \\
2000\end{array}$ & $?$ \\
\hline$\psi$ & $\begin{array}{l}\text { Soil moisture potential } \\
\text { (suction), mm }\end{array}$ & $\psi_{\mathrm{s}} \mathrm{W}^{-\mathrm{B}}$ & $\begin{array}{l}\text { Sellers } \\
1992 \\
\end{array}$ & $?$ \\
\hline$\psi_{\mathrm{s}}$ & $\begin{array}{l}\text { Saturated soil moisture } \\
\text { potential, mm }\end{array}$ & Assigned according to soil texture class & $\begin{array}{l}\text { Cox et al. } \\
1999\end{array}$ & Yes \\
\hline$\omega s$ & $\begin{array}{l}\text { Sunset hour angle } \\
\text { (radians) }\end{array}$ & $\operatorname{acos}(-\tan (\Phi) \cdot \tan (\partial))$ & $\begin{array}{l}\text { Allen } \\
2000\end{array}$ & $?$ \\
\hline$a, b$ & $\begin{array}{l}\text { Regression constants } \\
\text { in Angstrom formula }\end{array}$ & $a=0.25 ; b=0.5$ & $\begin{array}{l}\text { Allen } \\
2000\end{array}$ & $?$ \\
\hline AET & $\begin{array}{l}\text { Actual } \\
\text { evapotranspiration, } \\
\text { mmd }^{-1}\end{array}$ & $\mathrm{E}_{\mathrm{c}}+\mathrm{E}_{\mathrm{v}}$ & - & No \\
\hline $\mathrm{B}$ & $\begin{array}{l}\text { Clapp-Hornberger } \\
\text { exponent }\end{array}$ & Assigned according to soil texture class. & $\begin{array}{l}\text { Cox et al. } \\
1999 \\
\end{array}$ & Yes \\
\hline $\mathrm{C}_{\mathrm{M}}$ & $\begin{array}{l}\text { Canopy moisture } \\
\text { holding capacity, mm }\end{array}$ & $\begin{array}{l}\text { Assigned according to vegetation class, }(0.75 \\
\text { primary subclass }+0.25 \text { secondary subclass })\end{array}$ & $\begin{array}{l}\text { Cox et al. } \\
1999\end{array}$ & Yes \\
\hline $\mathrm{c}_{p}$ & $\begin{array}{l}\text { Specific heat of the air, } \\
\mathrm{MJkg}^{-1} \mathrm{C}^{-1}\end{array}$ & $0.622 \lambda \gamma / \mathrm{PR}\left(=1.013 \times 10^{-3}\right.$ for moist air $)$ & $\begin{array}{l}\text { Allen } \\
2000\end{array}$ & $?$ \\
\hline $\mathrm{C}_{\mathrm{R}}$ & $\begin{array}{l}\text { Canopy throughput, } \\
\mathrm{mmd}^{-1}\end{array}$ & $\begin{array}{l}0.03 \mathrm{P}+\left[\left(0.97 \mathrm{P}-\mathrm{C}_{\mathrm{M}}+\mathrm{C}_{\mathrm{Sd}-1}-\mathrm{E}_{\mathrm{c}}(\max )\right) \geq 0\right] \\
\text { where } \mathrm{d}-1=\text { previous day }\end{array}$ & $\begin{array}{l}\text { pers. } \\
\text { comm. } \\
\text { Gash } \\
2000^{14} \\
\end{array}$ & $?$ \\
\hline $\mathrm{C}_{\mathrm{S}}$ & $\begin{array}{l}\text { Canopy moisture store, } \\
\mathrm{mm}\end{array}$ & $\begin{array}{l}{\left[\mathrm{C}_{\mathrm{Sd}-1}+\mathrm{P}-\mathrm{C}_{\mathrm{R}}-\mathrm{E}_{\mathrm{c}} \leq \mathrm{C}_{\mathrm{M}}\right] \text {, where d-1 }=} \\
\text { previous day. Initiated at } \mathrm{C}_{\mathrm{M}}\end{array}$ & \begin{tabular}{|l} 
Cox et al. \\
1999
\end{tabular} & $?$ \\
\hline $\mathrm{D}_{\mathrm{a}}$ & $\begin{array}{l}\text { Vapour pressure } \\
\text { deficit }\end{array}$ & $\mathrm{e}_{s}-\mathrm{e}_{a}$ & $\begin{array}{l}\text { Allen } \\
2000 \\
\end{array}$ & $?$ \\
\hline
\end{tabular}

${ }^{14} 0.97$ = estimated central Amazonian rainforest canopy cover, restricting direct throughfall 


\begin{tabular}{|c|c|c|c|c|}
\hline $\mathrm{dn}$ & Day number & $(1-365)$ & $\begin{array}{l}\text { Allen } \\
2000\end{array}$ & Yes \\
\hline $\mathrm{E}_{\mathrm{c}}$ & $\begin{array}{l}\text { Canopy evaporation, } \\
\mathrm{mmd}^{-1}\end{array}$ & $\mathrm{fa}\left(\frac{\rho \mathrm{a}}{\mathrm{r}_{\mathrm{a}}}\right)(\mathrm{qsat}(\mathrm{T})-\mathrm{q} 1) * \mathrm{~K}_{\text {time }}$ & $\begin{array}{l}\text { Cox et al. } \\
1999\end{array}$ & Yes \\
\hline Eo & $\begin{array}{l}\text { Inverse relative earth- } \\
\text { sun distance }\end{array}$ & $1+0.033 \cos (\mathrm{dn} * 2 \pi / 365)$ & $\begin{array}{l}\text { Allen } \\
2000 \\
\end{array}$ & $?$ \\
\hline $\mathrm{e}_{s}$ & \begin{tabular}{|l|} 
Saturation vapour \\
pressure of the air, $\mathrm{kPa}$
\end{tabular} & $\begin{array}{l}0.3054 \exp \left(\frac{17.27 \mathrm{~T}_{\mathrm{x}}}{\mathrm{T}_{\mathrm{x}}+237.3}\right)+0.3054 \exp \left(\frac{17.27 \mathrm{~T}_{\mathrm{n}}}{\mathrm{T}_{\mathrm{n}}+237.3}\right) \\
\text { This finds the mean of } \mathrm{e}_{s} \text { at } \mathrm{T}_{\mathrm{x}} \text { and } \mathrm{T}_{\mathrm{n}} . \\
\text { HADCM2 provides temperature range } \\
\text { anomalies from which these can be estimated } \\
\text { (see } \mathrm{T}, \mathrm{T}_{\mathrm{x}}, \mathrm{T}_{\mathrm{n}} \text { in Table A1:1) }\end{array}$ & $\begin{array}{l}\text { Mark } \\
1999 \\
\text { Allen } \\
2000\end{array}$ & $?$ \\
\hline$\overline{\mathrm{E}_{\mathrm{v}}}$ & $\begin{array}{l}\text { Canopy } \\
\text { evapotranspiration, } \\
\text { mmd }^{-1}\end{array}$ & $(1-f a)\left(\frac{\rho a}{r a+r s}\right)\left(q_{s a t} T-q 1\right) * \mathrm{~K}_{\mathrm{time}}$ & $\begin{array}{l}\text { Cox et al. } \\
1999\end{array}$ & Yes \\
\hline $\mathrm{F}$ & $\begin{array}{l}\text { Runoff of water, } \\
\mathrm{mmd}^{-1}\end{array}$ & $\mathrm{~F}_{\mathrm{r}}+\mathrm{F}_{\mathrm{s}}$ & - & Yes \\
\hline$f_{a}$ & Wet fraction of cell & $\mathrm{C}_{\mathrm{S}} / \mathrm{C}_{\mathrm{M}}$ & $\begin{array}{l}\text { Cox et al. } \\
1999\end{array}$ & Yes \\
\hline$\overline{F_{r}}$ & $\begin{array}{l}\text { Slow runoff via } \\
\text { drainage, } \mathrm{mmd}^{-1}\end{array}$ & $\begin{array}{l}\text { 'Darcy's Law' - vertical transport of water in } \\
\text { soil column. } \\
{\left[-\mathrm{K}\left(\frac{\delta \psi}{\delta \mathrm{z}}+0.01\right) \mathrm{K}_{\text {time }} \leq \mathrm{S}_{\mathrm{S}}\right]} \\
\text { where } \frac{\delta \psi}{\delta \mathrm{z}}=-\psi_{\mathrm{s}} \mathrm{BW}^{-\mathrm{B}-1}\end{array}$ & \begin{tabular}{|l|} 
Sellers \\
1992, \\
Ward \& \\
Robinson \\
2000
\end{tabular} & $?$ \\
\hline $\mathrm{F}_{\mathrm{s}}$ & $\begin{array}{l}\text { Fast runoff via surface, } \\
\mathrm{mmd}^{-1}\end{array}$ & $\begin{array}{l}\mathrm{C}_{\mathrm{R}}-\mathrm{IC} \text { (all water incident on soil that is not } \\
\text { absorbed) }\end{array}$ & & \\
\hline $\mathrm{G}$ & $\begin{array}{l}\text { Soil heat flux density, } \\
\mathrm{MJm}^{-2} \mathrm{~d}^{-1}\end{array}$ & $0.07\left(\mathrm{~T}_{\mathrm{d}+1}-\mathrm{T}_{\mathrm{d}-1}\right)$, where $\mathrm{d}-1=$ previous day & $\begin{array}{l}\text { Allen } \\
2000\end{array}$ & ? \\
\hline $\mathrm{h}$ & Canopy height, $\mathrm{m}$ & $\begin{array}{l}\text { Assigned according to vegetation class, }(0.75 \\
\text { primary subclass }+0.25 \text { secondary subclass })\end{array}$ & $\begin{array}{l}\text { Buckley } \\
\& \\
\text { Warrilow } \\
1988 \\
\end{array}$ & Yes \\
\hline $\mathrm{h}_{\mathrm{d}}$ & \begin{tabular}{|l|} 
Zero plane \\
displacement value, $\mathrm{m}$
\end{tabular} & $2 \mathrm{~h} / 3$ & $\begin{array}{l}\text { Allen } \\
2000\end{array}$ & $?$ \\
\hline $\mathrm{h}_{\mathrm{T}}$ & $\begin{array}{l}\text { Measurement height } \\
\text { for temperature, } \mathrm{m}\end{array}$ & $\mathrm{h}+2($ was 1.5$)$ & $\begin{array}{l}\text { New pers. } \\
\text { comm. } \\
2000\end{array}$ & ? \\
\hline $\mathrm{h}_{\mathrm{W}}$ & $\begin{array}{l}\text { Measurement height } \\
\text { for wind, } m\end{array}$ & $h+10$ & $\begin{array}{l}\text { New pers. } \\
\text { comm. } \\
2000\end{array}$ & $?$ \\
\hline IC & $\begin{array}{l}\text { Infiltration capacity, } \\
\mathrm{mmd}^{-1}\end{array}$ & $\begin{array}{l}{\left[\mathrm{S}_{\mathrm{M}}-\mathrm{S}_{\mathrm{Sd}-1}+\mathrm{PET}+\mathrm{F}_{\mathrm{r}}(\max ) \leq \mathrm{C}_{\mathrm{R}}\right] \text { where } \mathrm{d}-1=} \\
\text { previous day. Soil moisture holding capacity }+ \\
\text { PET + potential drainage - soil moisture store }\end{array}$ & \begin{tabular}{|l|} 
Ward \& \\
Robinson \\
$2000^{15}$ \\
\end{tabular} & $?$ \\
\hline ISc & $\begin{array}{l}\text { Solar constant, } \\
\mathrm{MJm}^{-2} \mathrm{~h}^{-1}\end{array}$ & 4.921 & $\begin{array}{l}\text { Allen } \\
2000\end{array}$ & $?$ \\
\hline $\mathrm{K}$ & $\begin{array}{l}\text { Soil hydraulic } \\
\text { conductivity, } \mathrm{mms}^{-1}\end{array}$ & $\mathrm{~K}_{\mathrm{s}} \mathrm{W}^{2 \mathrm{~B}+3}$ & $\begin{array}{l}\text { Sellers } \\
1992\end{array}$ & $?$ \\
\hline
\end{tabular}

15 "In well-vegetated areas, both temperate and tropical, most soils can absorb the rainfall of all but the most intense storms",p.207 


\begin{tabular}{|c|c|c|c|c|}
\hline $\mathrm{K}_{\mathrm{s}}$ & \begin{tabular}{|l|}
$\begin{array}{l}\text { Saturated soil } \\
\text { hydraulic conductivity, } \\
\mathrm{mms}^{-1}\end{array}$ \\
\end{tabular} & Assigned according to soil texture class & $\begin{array}{l}\text { Cox et al. } \\
1999\end{array}$ & Yes \\
\hline $\mathrm{K}_{\text {time }}$ & Units conversion, $\mathrm{sd}^{-1}$ & 86,400 (for output values in $\mathrm{d}^{-1}$ ) & $\begin{array}{l}\text { Allen } \\
2000 \\
\end{array}$ & Yes \\
\hline$M D$ & $\begin{array}{l}\text { Annual moisture } \\
\text { deficit }\end{array}$ & Annual sum of PET - AET & - & N/A \\
\hline$\overline{\text { PET }}$ & $\begin{array}{l}\text { Penman-Monteith } \\
\text { potential } \\
\text { evapotranspiration, } \\
\mathrm{mmd}^{-1}\end{array}$ & $\frac{\Delta(\mathrm{Rn}-\mathrm{G})+\mathrm{K}_{\text {time }} \rho_{\mathrm{a}} \mathrm{c}_{\mathrm{p}} \frac{\left(\mathrm{D}_{\mathrm{a}}\right)}{\mathrm{r}_{\mathrm{a}}}}{\lambda\left(\Delta+\gamma\left(1+\frac{\mathrm{r}_{\mathrm{s}}}{2}\right)\right)}$ & $\begin{array}{l}\text { Monteith, } \\
1964 \\
\text { Allen, } \\
2000\end{array}$ & N/A \\
\hline$\overline{P R}$ & $\begin{array}{l}\text { Atmospheric pressure, } \\
\mathrm{kPa}\end{array}$ & $101.3\left(\frac{273.16+\mathrm{T}-0.0065 \mathrm{E}}{273.16+\mathrm{T}}\right)^{\frac{9.807}{0.0065 * 287}}$ & $\begin{array}{l}\text { Allen } \\
2000\end{array}$ & $?$ \\
\hline $\mathrm{q}_{1}$ & $\begin{array}{l}\text { Atmospheric specific } \\
\text { humidity, } \mathrm{gkg}^{-1}\end{array}$ & $\begin{array}{l}\frac{621.97 \mathrm{e}_{\mathrm{a}}}{\mathrm{PR}-0.37803 \mathrm{e}} \\
\% \text { GCM anomaly added annually to RH from } \\
\text { which } \mathrm{e}_{a} \text { may be calculated. }\end{array}$ & $\begin{array}{l}\text { Mark } \\
1999\end{array}$ & $?$ \\
\hline $\mathrm{q}_{\mathrm{sat}} \mathrm{T}$ & $\begin{array}{l}\text { Saturated specific } \\
\text { humidity at surface } \\
\text { temperature }(\mathrm{T}), \mathrm{gkg}^{-1}\end{array}$ & $1000\left(\frac{(1.004 * 6.112 * 0.622)}{10 \mathrm{PR}} \exp \left(\frac{17.502 \mathrm{~T}}{240.97+\mathrm{T}}\right)\right)$ & $\begin{array}{l}\text { Buck } \\
1981\end{array}$ & $?$ \\
\hline $\mathrm{R}$ & $\begin{array}{l}\text { Daily extra-terrestrial } \\
\text { solar radiation, } \\
\mathrm{MJm}^{-2} \mathrm{~d}^{-1}\end{array}$ & $\begin{array}{l}\left(\frac{24}{\pi}\right) \mathrm{E}_{\mathrm{o}} * \\
\mathrm{Isc}(\omega \mathrm{s} \cdot \sin (\Phi) \cdot \sin (\partial)+\cos (\Phi) \cdot \cos (\partial) \cdot \sin (\omega \mathrm{s}))\end{array}$ & $\begin{array}{l}\text { Allen } \\
2000\end{array}$ & $?$ \\
\hline $\mathrm{r}_{a}$ & $\begin{array}{l}\text { Aerodynamic } \\
\text { resistance, } \mathrm{sm}^{-1}\end{array}$ & $\begin{array}{l}\ln \left(\frac{\mathrm{h}_{\mathrm{W}}-\mathrm{h}_{\mathrm{d}}}{\mathrm{z}}\right) \ln \left(\frac{\mathrm{h}_{\mathrm{T}}-\mathrm{h}_{\mathrm{d}}}{0.1 \mathrm{z}}\right) \\
\text { - the denominator } 0.1 \mathrm{z} \text { ' approximates the } \\
\text { roughness length for heat and vapour transfer } \\
\text { as } 0.1 * \text { the roughness length for momentum } \\
\text { transfer, } \mathrm{z}\end{array}$ & $\begin{array}{l}\text { Allen } \\
2000\end{array}$ & $?$ \\
\hline RH & Relative humidity, \% & $\begin{array}{l}100 \mathrm{e}_{a} / \mathrm{e}_{s} \\
\% \mathrm{GCM} \text { anomaly added annually }\end{array}$ & $\begin{array}{l}\text { Mark } \\
1999\end{array}$ & Yes \\
\hline $\mathrm{R}_{\mathrm{n}}$ & $\begin{array}{l}\text { Net radiation, } \\
\mathrm{MJm}^{-2} \mathrm{~d}^{-1}\end{array}$ & $\begin{array}{l}\mathrm{R}_{\mathrm{n}}=\mathrm{R}_{\mathrm{ns}}-\mathrm{R}_{\mathrm{nl}} \\
\% \mathrm{GCM} \text { anomaly added annually }\end{array}$ & $\begin{array}{l}\text { Allen } \\
2000\end{array}$ & $?$ \\
\hline $\mathrm{R}_{\mathrm{nl}}$ & $\begin{array}{l}\text { Net (outgoing) } \\
\text { longwave radiation, } \\
\mathrm{MJm}^{-2} \mathrm{~d}^{-1}\end{array}$ & $\begin{array}{l}4903 * 10^{-9}\left(\frac{\left(\mathrm{T}_{\mathrm{n}}+273.16\right)^{4}+\left(\mathrm{T}_{\mathrm{x}}+273.16\right)^{4}}{2}\right. \\
*\left(0.34-0.14 \sqrt{\mathrm{e}_{\mathrm{a}}}\right)\left(1.35 \frac{\mathrm{R}_{\mathrm{s}}}{\mathrm{R}_{\mathrm{so}}}-0.35\right)\end{array}$ & $\begin{array}{l}\text { Allen } \\
2000\end{array}$ & $?$ \\
\hline $\mathrm{R}_{\mathrm{ns}}$ & $\begin{array}{l}\text { Total shortwave solar } \\
\text { radiation, } \mathrm{MJm}^{-2} \mathrm{~d}^{-1}\end{array}$ & $(1-\alpha) r_{s}$ & $\begin{array}{l}\text { Allen } \\
2000\end{array}$ & $?$ \\
\hline rootd & Root-depth, mm & $\begin{array}{l}\text { Assigned according to vegetation class, }(0.75 \\
\text { primary subclass }+0.25 \text { secondary subclass })\end{array}$ & $\begin{array}{l}\text { Buckley } \\
\& \\
\text { Warrilow } \\
1988\end{array}$ & Yes \\
\hline $\mathrm{r}_{s}$ & $\begin{array}{l}\text { Solar radiation, } \\
\mathrm{MJm}^{-2} \mathrm{~d}^{-1}\end{array}$ & Angstrom formula: $\left(a+\frac{b n}{N}\right) R$ & $\begin{array}{l}\text { Allen } \\
2000\end{array}$ & $?$ \\
\hline $\mathrm{r}_{s}$ & $\begin{array}{l}\text { Bulk surface (canopy) } \\
\text { resistance, } \mathrm{sm}^{-1}\end{array}$ & $\begin{array}{l}\text { Assigned according to vegetation class }(0.75 \\
\text { primary subclass }+0.25 \text { secondary subclass })\end{array}$ & $\begin{array}{l}\text { Buckley } \\
\& \\
\text { Warrilow } \\
1988\end{array}$ & Yes \\
\hline
\end{tabular}




\begin{tabular}{|c|c|c|c|c|}
\hline $\mathrm{R}_{\mathrm{so}}$ & $\begin{array}{l}\text { Clear sky solar } \\
\text { radiation, } \mathrm{MJm}^{-2} \mathrm{~d}^{-1}\end{array}$ & $\begin{array}{l}\text { If } \mathrm{E}<300 \mathrm{~m} \text { then } \mathrm{R}_{\mathrm{so}}=0.75 \mathrm{R} \\
\text { else } \mathrm{R}_{\mathrm{so}}=(0.75+0.00002 \mathrm{E}) \mathrm{R}\end{array}$ & $\begin{array}{l}\text { Allen } \\
2000\end{array}$ & $?$ \\
\hline $\mathrm{S}_{\mathrm{M}}$ & $\begin{array}{l}\text { Soil moisture holding } \\
\text { capacity, mm }\end{array}$ & Assigned according to soil texture class & $\begin{array}{l}\text { Cox et al. } \\
1999\end{array}$ & Yes \\
\hline$S M A$ & $\begin{array}{l}\text { Seasonal moisture } \\
\text { availability, } \mathrm{d}\end{array}$ & $\begin{array}{l}\text { Maximum number of consecutive days where } \\
\text { AET < PET }\end{array}$ & - & N/A \\
\hline $\mathrm{S}_{\mathrm{S}}$ & $\begin{array}{l}\text { Soil moisture store, } \\
\mathrm{mm}\end{array}$ & $\begin{array}{l}{\left[\mathrm{S}_{\mathrm{Sd}-1}+\mathrm{IC}-\mathrm{F}_{\mathrm{r}}-\mathrm{E}_{\mathrm{v}} \leq \mathrm{S}_{\mathrm{M}}\right], \text { where } \mathrm{d}-1=} \\
\text { previous day. Initiated for current climate at } \\
\mathrm{S}_{\mathrm{M}}\end{array}$ & - & $?$ \\
\hline $\mathrm{W}$ & Wet fraction of soil & $\mathrm{S}_{\mathrm{S}} / \mathrm{S}_{\mathrm{M}}$ & - & $?$ \\
\hline $\mathrm{z}$ & Roughness length, m & $\begin{array}{l}\text { Assigned according to vegetation class. }(0.75 \\
\text { primary subclass }+0.25 \text { secondary subclass; } \\
\text { averaging 'y' }=1 /(\ln (550 / \mathrm{z}))^{2}\end{array}$ & \begin{tabular}{|l} 
Buckley \\
$\&$ \\
Warrilow \\
$1988 ;$ \\
Warrilow \\
$\&$ \\
Buckley \\
1989 \\
\end{tabular} & Yes \\
\hline$\alpha$ & Albedo & $\begin{array}{l}\text { Assigned according to vegetation (and soil } \\
\text { colour) class, }(0.75 \text { primary subclass }+0.25 \\
\text { secondary subclass) }\end{array}$ & $\begin{array}{l}\text { Buckley } \\
\& \\
\text { Warrilow } \\
1988 \\
\end{array}$ & Yes \\
\hline
\end{tabular}




\section{Appendix 3 Family selection}

Bold rows indicate families in the final selection. Grey rows indicate families in ideal selection.

Table A3:1: Families with APG higher taxonomy, known distribution ('endemic' = neotropical endemic) and selection criteria

\begin{tabular}{|c|c|c|c|c|c|c|c|c|c|}
\hline Group & Order & Family & Distribution & $\begin{array}{l}\text { Neotropical } \\
\text { genera }\end{array}$ & Trees & Shrubs & Herbs & Climbers & $\begin{array}{l}\text { OFN } \\
\text { genera }\end{array}$ \\
\hline Commelinoids & Arecales & Arecaceae & Cosmopolitan & 60 & Yes & Yes & No & No & $>1$ \\
\hline Commelinoids & Commelinales & Haemodoraceae & Pantropical & 4 & No & No & Yes & No & $>1$ \\
\hline Commelinoids & Commelinales & Commelinaceae & Cosmopolitan & 17 & No & No & Yes & No & 0 \\
\hline Commelinoids & Unplaced Families & Bromeliaceae & Pantropical & 50 & Yes & Yes & Yes & No & $>1$ \\
\hline Commelinoids & Unplaced Families & Rapateaceae & Pantropical & 16 & No & No & Yes & No & $>1$ \\
\hline Commelinoids & Poales & Thurniaceae & Endemic & 1 & No & No & Yes & No & 0 \\
\hline Commelinoids & Poales & Xyridaceae & Pantropical & 4 & No & No & Yes & No & $>1$ \\
\hline Commelinoids & Poales & Poaceae & Cosmopolitan & 207 & No & No & Yes & No & $>1$ \\
\hline Commelinoids & Poales & Cyperaceae & Cosmopolitan & 26 & No & No & Yes & No & $>1$ \\
\hline Commelinoids & Poales & Typhaceae & Cosmopolitan & 1 & No & No & Yes & No & 0 \\
\hline Commelinoids & Zingiberales & Cannaceae & Endemic & 1 & No & No & Yes & No & 0 \\
\hline Commelinoids & Zingiberales & Heliconiaceae & Endemic & 1 & No & No & Yes & No & 1 \\
\hline Commelinoids & Zingiberales & Marantaceae & Pantropical & 13 & No & Yes & Yes & No & $>1$ \\
\hline Commelinoids & Zingiberales & Costaceae & Pantropical & 3 & No & No & Yes & No & 0 \\
\hline Commelinoids & Zingiberales & Zingiberaceae & Pantropical & 1 & No & No & Yes & No & 1 \\
\hline Euasterids I & Unplaced Families & Boraginaceae & Cosmopolitan & 25 & Yes & Yes & Yes & Yes & $>1$ \\
\hline Euasterids I & Gentianales & Rubiaceae & Cosmopolitan & 213 & Yes & Yes & Yes & No & $>1$ \\
\hline Euasterids I & Gentianales & Apocynaceae & Cosmopolitan & 66 & Yes & Yes & Yes & Yes & $>1$ \\
\hline Euasterids I & Gentianales & Loganiaceae & Cosmopolitan & 13 & Yes & Yes & No & Yes & 0 \\
\hline Euasterids I & Lamiales & Avicenniaceae & Pantropical & 1 & Yes & Yes & No & No & 0 \\
\hline Euasterids I & Lamiales & Bignoniaceae & Cosmopolitan & 70 & Yes & Yes & Yes & Yes & $>1$ \\
\hline Euasterids I & Lamiales & Gesneriaceae & Cosmopolitan & 40 & No & Yes & Yes & No & $>1$ \\
\hline Euasterids I & Lamiales & Oleaceae & Cosmopolitan & 7 & Yes & Yes & No & No & 0 \\
\hline Euasterids I & Lamiales & Acanthaceae & Cosmopolitan & 61 & Yes & Yes & Yes & Yes & 1 \\
\hline Euasterids I & Lamiales & Verbenaceae & Cosmopolitan & 24 & No & No & Yes & Yes & 1 \\
\hline Euasterids I & Lamiales & Schlegeliaceae & Amazonian \& ? & 2 & No & No & Yes & No & $>1$ \\
\hline Euasterids I & Solanales & Solanaceae & Cosmopolitan & 69 & Yes & Yes & Yes & Yes & $>1$ \\
\hline
\end{tabular}




\begin{tabular}{|c|c|c|c|c|c|c|c|c|c|}
\hline Euasterids I & Solanales & Convolvulaceae & Cosmopolitan & 21 & No & No & Yes & Yes & $>1$ \\
\hline Euasterids Ii & Asterales & Asteraceae & Cosmopolitan & 589 & Yes & Yes & Yes & Yes & $>1$ \\
\hline Euasterids Ii & Unplaced Families & Icacinaceae & Pantropical & 12 & Yes & Yes & No & Yes & $>1$ \\
\hline Eurosids I & Cucurbitales & Anisophylleaceae & Pantropical & 2 & Yes & No & No & No & 0 \\
\hline Eurosids I & Cucurbitales & Begoniaceae & Pantropical & 1 & Yes & No & Yes & Yes & 1 \\
\hline Eurosids I & Cucurbitales & Cucurbitaceae & Cosmopolitan & 55 & No & No & Yes & Yes & 1 \\
\hline Eurosids I & Unplaced Families & Celastraceae & Pantropical & 26 & Yes & Yes & No & Yes & 0 \\
\hline Eurosids I & Fabales & Fabaceae & Cosmopolitan & 205 & Yes & Yes & Yes & Yes & $>1$ \\
\hline Eurosids I & Fabales & Polygalaceae & Cosmopolitan & 9 & Yes & Yes & Yes & Yes & 1 \\
\hline Eurosids I & Fagales & Juglandaceae & Pantropical & 4 & Yes & No & No & No & 0 \\
\hline Eurosids I & Malpighiales & Quiinaceae & Endemic & 4 & Yes & Yes & No & Yes & $>1$ \\
\hline Eurosids I & Malpighiales & Lacistemataceae & Endemic & 2 & No & Yes & No & No & $>1$ \\
\hline Eurosids I & Malpighiales & Caryocaraceae & Endemic & 2 & Yes & No & No & No & $>1$ \\
\hline Eurosids I & Malpighiales & Clusiaceae & Endemic & 24 & Yes & Yes & Yes & Yes & 0 \\
\hline Eurosids I & Malpighiales & Goupiaceae & Endemic & 1 & Yes & Yes & No & No & 0 \\
\hline Eurosids I & Malpighiales & Malpighiaceae & Pantropical & 44 & Yes & Yes & No & Yes & $>1$ \\
\hline Eurosids I & Malpighiales & Chrysobalanaceae & Pantropical & 8 & Yes & Yes & No & No & $>1$ \\
\hline Eurosids I & Malpighiales & Passifloraceae & Pantropical & 4 & Yes & Yes & Yes & Yes & $>1$ \\
\hline Eurosids I & Malpighiales & Dichapetalaceae & Pantropical & 3 & Yes & Yes & No & Yes & $>1$ \\
\hline Eurosids I & Malpighiales & Rhizophoraceae & Pantropical & 3 & Yes & Yes & No & No & $>1$ \\
\hline Eurosids I & Malpighiales & Turneraceae & Pantropical & 3 & No & Yes & Yes & No & $>1$ \\
\hline Eurosids I & Malpighiales & Trigoniaceae & Pantropical & 2 & Yes & Yes & No & Yes & $>1$ \\
\hline Eurosids I & Malpighiales & Humiriaceae & Pantropical & 8 & Yes & Yes & No & No & 0 \\
\hline Eurosids I & Malpighiales & Ochnaceae & Pantropical & 10 & Yes & Yes & Yes & No & 1 \\
\hline Eurosids I & Malpighiales & Erythroxylaceae & Pantropical & 1 & Yes & Yes & No & No & 1 \\
\hline Eurosids I & Malpighiales & Euphorbiaceae & Cosmopolitan & 92 & Yes & Yes & Yes & Yes & $>1$ \\
\hline Eurosids I & Malpighiales & Flacourtiaceae & Cosmopolitan & 29 & Yes & Yes & No & No & $>1$ \\
\hline Eurosids I & Malpighiales & Violaceae & Cosmopolitan & 13 & Yes & Yes & Yes & Yes & $>1$ \\
\hline Eurosids I & Malpighiales & Linaceae & Cosmopolitan & 3 & Yes & Yes & Yes & No & 0 \\
\hline Eurosids I & Malpighiales & Salicaceae & Cosmopolitan & 1 & Yes & No & No & No & 0 \\
\hline Eurosids I & Oxalidales & Connaraceae & Pantropical & 4 & Yes & Yes & No & Yes & $>1$ \\
\hline Eurosids I & Oxalidales & Elaeocarpaceae & Cosmopolitan & 5 & Yes & Yes & No & No & $>1$ \\
\hline Eurosids I & Rosales & Cecropiaceae & Endemic & 3 & Yes & Yes & No & No & 1 \\
\hline Eurosids I & Rosales & Moraceae & Cosmopolitan & 20 & Yes & Yes & Yes & No & $>1$ \\
\hline Eurosids I & Rosales & Ulmaceae & Cosmopolitan & 5 & Yes & Yes & No & No & $>1$ \\
\hline
\end{tabular}




\begin{tabular}{|c|c|c|c|c|c|c|c|c|c|}
\hline Eurosids I & Rosales & Urticaceae & Cosmopolitan & 14 & Yes & Yes & Yes & No & 0 \\
\hline Eurosids I & Rosales & Rhamnaceae & Cosmopolitan & 23 & Yes & Yes & No & Yes & 1 \\
\hline Eurosids II & Brassicales & Tovariaceae & Endemic & 1 & No & Yes & Yes & No & 0 \\
\hline Eurosids II & Malvales & Bixaceae & Pantropical & 1 & Yes & Yes & No & No & 0 \\
\hline Eurosids II & Malvales & Malvaceae & Cosmopolitan & 50 & Yes & Yes & Yes & No & $>1$ \\
\hline Eurosids II & Malvales & Thymelaeaceae & Cosmopolitan & 8 & No & Yes & Yes & Yes & 0 \\
\hline Eurosids II & Myrtales & Melastomataceae & Pantropical & 130 & Yes & Yes & Yes & Yes & $>1$ \\
\hline Eurosids II & Myrtales & Combretaceae & Pantropical & 7 & Yes & Yes & No & Yes & $>1$ \\
\hline Eurosids II & Myrtales & Vochysiaceae & Pantropical & 5 & Yes & Yes & No & No & 0 \\
\hline Eurosids II & Myrtales & Myrtaceae & Cosmopolitan & 26 & Yes & Yes & No & No & $>1$ \\
\hline Eurosids II & Myrtales & Lythraceae & Cosmopolitan & 16 & Yes & Yes & Yes & No & $>1$ \\
\hline Eurosids II & Sapindales & Meliaceae & Pantropical & 8 & Yes & Yes & No & No & $>1$ \\
\hline Eurosids II & Sapindales & Simaroubaceae & Pantropical & 5 & Yes & Yes & No & No & $>1$ \\
\hline Eurosids II & Sapindales & Sapindaceae & Pantropical & 32 & Yes & Yes & Yes & Yes & 0 \\
\hline Eurosids II & Sapindales & Burseraceae & Pantropical & 7 & Yes & Yes & No & No & 1 \\
\hline Eurosids II & Sapindales & Anacardiaceae & Cosmopolitan & 27 & Yes & Yes & No & Yes & $>1$ \\
\hline Eurosids II & Sapindales & Rutaceae & Cosmopolitan & 17 & Yes & Yes & Yes & No & 1 \\
\hline Uncertain & Uncertain Families & Lissocarpaceae & Endemic & 1 & Yes & No & No & No & 0 \\
\hline Uncertain & Uncertain Families & Balanophoraceae & Pantropical & 7 & No & No & $\overline{\text { Yes }}$ & No & $>1$ \\
\hline Uncertain & Uncertain Families & Lepidobotryaceae & Pantropical & 1 & Yes & $\mathrm{No}$ & No & No & 0 \\
\hline Uncertain & Uncertain Families & Mitrastemonaceae & Cosmopolitan & 1 & No & No & Yes & No & 0 \\
\hline Unplaced Asterids & Ericales & Marcgraviaceae & Endemic & 5 & No & Yes & No & Yes & $>1$ \\
\hline Unplaced Asterids & Ericales & Theophrastaceae & Endemic & 5 & Yes & Yes & No & No & 0 \\
\hline Unplaced Asterids & Ericales & Sapotaceae & Pantropical & 12 & Yes & No & No & No & $>1$ \\
\hline Unplaced Asterids & Ericales & Lecythidaceae & Pantropical & 11 & Yes & No & No & No & $>1$ \\
\hline Unplaced Asterids & Ericales & Ebenaceae & Pantropical & 1 & Yes & Yes & No & No & 1 \\
\hline Unplaced Asterids & Ericales & Primulaceae & Cosmopolitan & 5 & No & No & Yes & No & 0 \\
\hline Unplaced Asterids & Ericales & Theaceae & Cosmopolitan & 3 & Yes & Yes & No & No & 0 \\
\hline Unplaced Asterids & Ericales & Ternstroemiaceae & Amazonian and? & 1 & No & No & No & No & 0 \\
\hline Unplaced Core Eudicots & Caryophyllales & Rhabdodendraceae & Endemic & 1 & Yes & Yes & No & No & $\overline{0}$ \\
\hline Unplaced Core Eudicots & Caryophyllales & Aizoaceae & Pantropical & 5 & No & Yes & Yes & No & 0 \\
\hline Unplaced Core Eudicots & Caryophyllales & Basellaceae & Pantropical & 4 & No & No & No & Yes & 0 \\
\hline Unplaced Core Eudicots & Caryophyllales & Opiliaceae & Pantropical & 1 & Yes & Yes & No & Yes & 1 \\
\hline Unplaced Core Eudicots & Caryophyllales & Olacaceae & Cosmopolitan & 14 & Yes & Yes & No & No & $>1$ \\
\hline Unplaced Core Eudicots & Caryophyllales & Nyctaginaceae & Cosmopolitan & 30 & Yes & Yes & Yes & No & 0 \\
\hline
\end{tabular}


Unplaced Core Eudicots Caryophyllales Unplaced Core Eudicots Caryophyllales Unplaced Core Eudicots Caryophyllales Unplaced Core Eudicots Caryophyllales Unplaced Core Eudicots Caryophyllales Unplaced Core Eudicots Caryophyllales Unplaced Core Eudicots Caryophyllales Unplaced Core Eudicots Caryophyllales Unplaced Core Eudicots Ungrouped Families

Unplaced Core Eudicots Ungrouped Families Unplaced Core Eudicots Ungrouped Families Unplaced Dicots Ungrouped Families

Unplaced Dicots

Unplaced Dicots

Unplaced Dicots

Unplaced Dicots

Unplaced Dicots

Unplaced Dicots

Unplaced Dicots

Unplaced Dicots

Unplaced Dicots

Unplaced Eudicots

Unplaced Eudicots

Unplaced Monocots

Unplaced Monocots

Unplaced Monocots

Unplaced Monocots

Unplaced Monocots

Laurales

Laurales

Laurales

Laurales

Magnoliales

Magnoliales Magnoliales

Piperales

Piperales

Proteales

Ranunculales

Asparagales

Asparagales

Dioscoreales

Dioscoreales

Unplaced Monocots $\quad$ Ungrouped Families

Unplaced Monocots Pandanales

Unplaced Rosids

Ungrouped Families

Ungrouped Families

Unplaced Rosids
Phytolaccaceae

Amaranthaceae

Polygonaceae

Santalaceae

Portulacaceae

Molluginaceae

Loranthaceae

Droseraceae

Dilleniaceae

Vitaceae

Gunneraceae

Rafflesiaceae

Hernandiaceae

Monimiaceae

Siparunaceae

Lauraceae

Annonaceae

Myristicaceae Magnoliaceae

Piperaceae

Aristolochiaceae

Proteaceae

Menispermaceae

Orchidaceae

Amaryllidaceae

Dioscoreaceae

Taccaceae

Smilacaceae

Triuridaceae

Cyclanthaceae

Krameriaceae

Staphyleaceae

Cosmopolitan

Cosmopolitan

Cosmopolitan

Cosmopolitan

Cosmopolitan

Cosmopolitan

Cosmopolitan

Cosmopolitan

Pantropical

Cosmopolitan

Cosmopolitan

Pantropical

Pantropical

Pantropical

Pantropical

Cosmopolitan 15

Pantropical

Pantropical

Cosmopolitan

Cosmopolitan

Cosmopolitan

Cosmopolitan

Cosmopolitan

Cosmopolitan

Cosmopolitan

Pantropical

Pantropical

Cosmopolitan

\begin{tabular}{|l|l|l|l|l|l}
15 & Yes & Yes & Yes & No & 0
\end{tabular}

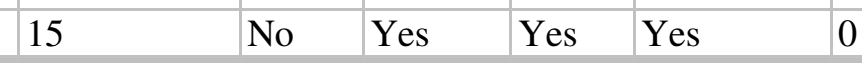

15 Yes Yes Yes Yes 0

$\begin{array}{llllll}10 & \text { Yes } & \text { Yes } & \text { Yes } & \text { No } & 0\end{array}$

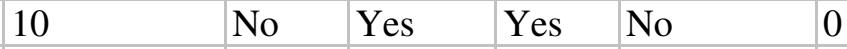

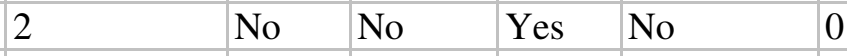

\begin{tabular}{|l|l|l|l|l|l|}
\hline & No & Yes & No & No & 0 \\
\hline 1 & No & No & Yes & No & 1 \\
\hline
\end{tabular}

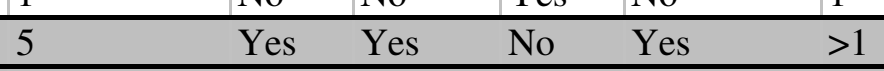

$3 \quad \mathrm{No}$ Yes

$1 \quad$ No $\quad$ No

No Yes

Yes No

\begin{tabular}{lll}
3 & No & No \\
\hline 3 & Yes & No
\end{tabular}

Yes No

No $\quad$ Yes

$3 \quad$ Yes $\quad$ Yes

No

No No

No No

\begin{tabular}{ll} 
Yes & Yes \\
Yes & Yes \\
\hline & Yes
\end{tabular}

No Yes

Yes $>1$

38 Yes Yes No Yes $>1$

\begin{tabular}{l|l|l|l|l|l|}
\hline 5 & Yes & No & No & No & $>1$
\end{tabular}

\begin{tabular}{l|l|l}
\hline 1 & Yes & Yes \\
\hline 6 & Yes
\end{tabular}

6

3 Yes Yes

No No

Yes Yes

Yes

0

$3 \quad$ Yes $\quad$ Yes

Yes Yes

$\begin{array}{lll}5 & \text { Yes } & \text { Yes } \\ 18 & \text { Yes } & \text { Yes }\end{array}$

No $\quad$ No $\quad>1$

No $\quad$ Yes $\quad 0$

Yes No $\quad>1$

\begin{tabular}{|c|c|c|c|}
\hline 300 & No & No & Yes \\
\hline 19 & No & No & Yes \\
\hline
\end{tabular}

\begin{tabular}{lllllll}
19 & No & No & Yes & No & $?$ \\
\hline 2 & No & No & No & Yes & $>1$
\end{tabular}

$\begin{array}{lll}1 & \text { No } & \text { No } \\ 1 & \text { No } & \text { No }\end{array}$

$1 \quad$ No No

Yes $-\mathrm{No}$

Yes No

No Yes

Pantropical 4

Endemic 12

Endemic

$\frac{12}{1}$

$\begin{array}{lll}1 & \text { No } & \text { No } \\ 1 & \text { No } & \text { Yes }\end{array}$

Yes No

Yes Yes No

$\begin{array}{llll}\text { Yes } & \text { Yes } & \text { No } & \text { No }\end{array}$

\begin{tabular}{l} 
No \\
Yes \\
\hline No \\
No \\
No \\
No \\
\hline
\end{tabular}

$\frac{0}{0}$

$\frac{0}{0}$

1
0




\section{Appendix 4 Species selection}

Underlined traits in the table were assigned according to family level data; italicised traits according to genus level data.

Table A4:1: Selected set, with traits, summary of vital rate data available and distribution references

\begin{tabular}{|c|c|c|c|c|c|c|c|c|c|c|c|}
\hline Species & Species id & Form & \begin{tabular}{|l|} 
Height \\
class; \\
Size on \\
maturity \\
where \\
known \\
\end{tabular} & $\begin{array}{l}\text { Pollination } \\
\text { mode }\end{array}$ & Dispersal mode & Selfing & \begin{tabular}{|l|} 
Deciduous- \\
ness
\end{tabular} & $\begin{array}{l}\text { Record } \\
\text { cells }\end{array}$ & \begin{tabular}{|l} 
Mean \\
SMA \\
(PRES), \\
days
\end{tabular} & $\begin{array}{l}\text { Vital } \\
\text { rate } \\
\text { data }\end{array}$ & $\begin{array}{l}\text { Distributional data } \\
\text { sources }^{A}\end{array}$ \\
\hline \multicolumn{12}{|l|}{ Arecaceae } \\
\hline Astrocaryum gynacanthum & Astrgyna & Tree & $4-10 \mathrm{~m}$ & Insect & Bird \& mammal & no & Evergreen & 63 & 47.1 & & $10,27,30,31,34,51$ \\
\hline Astrocaryum murumuru & Astrmuru & Tree & $\begin{array}{l}10-20 \mathrm{~m} \\
4-10 \mathrm{~m}\end{array}$ & Insect & Mammal - ground & $?$ & Evergreen & 50 & 4.4 & $\mathrm{~K}, \mathrm{M}$ & $\begin{array}{l}1,24,30,31,34,51, \\
58\end{array}$ \\
\hline Astrocaryum sciophilum & Astrscio & Bush & $\begin{array}{l}1-4 \mathrm{~m} ; 0-1 \\
\mathrm{~m}\end{array}$ & Insect & Mammal - ground & $?$ & Evergreen & 14 & 53.8 & & $10,30,31,37,51$ \\
\hline Bactris acanthocarpa & Bactacal & $\begin{array}{l}\text { Perennial } \\
\text { herb }\end{array}$ & $\begin{array}{l}0-1 \mathrm{~m} ; 0-1 \\
\mathrm{~m}\end{array}$ & Insect & Bird \& mammal & $?$ & Evergreen & 53 & 59.9 & & $30,31,34,66,67$ \\
\hline Chamaedorea fragrans & Chamfrag & Bush & $1-4 \mathrm{~m}$ & Wind & Bird \& mammal & no & Evergreen & 6 & 174.7 & & 30 \\
\hline Geonoma macrostachys & Geonmacr & Bush & $1-4 \mathrm{~m}$ & Insect & Bird & yes & Evergreen & 71 & 33.2 & & 30 \\
\hline Geonoma oldemanii & Geonolde & Bush & $1-4 \mathrm{~m}$ & $\underline{\text { Insect }}$ & Bird \& mammal & $?$ & Evergreen & 5 & 34.2 & & 30 \\
\hline Iriartea deltoidea & Iriadelt & Tree & $25+\mathrm{m}$ & Insect & Mammal - arboreal & $?$ & Evergreen & 62 & 2.4 & M & $1,24,30,58,74$ \\
\hline Leopoldinia major & Leopmajo & Tree & $4-10 \mathrm{~m}$ & $\underline{\text { Insect }}$ & Water (or fish) & $?$ & Evergreen & 13 & 13.2 & & 30,48 \\
\hline Leopoldinia piassaba & Leoppias & Tree & $4-10 \mathrm{~m}$ & Insect & Mammal - ground & $?$ & Evergreen & 7 & 0.0 & & 30,44 \\
\hline Oenocarpus bataua & Oenobata & Tree & $\begin{array}{l}10-20 \mathrm{~m} \\
4-10 \mathrm{~m}\end{array}$ & Insect & Bird \& mammal & $?$ & Evergreen & 90 & 13.9 & M & $\begin{array}{l}1,9,10,11,24,30 \\
31,51,52,58,64,70\end{array}$ \\
\hline Socratea exorrhiza & Socrexor & Tree & $\begin{array}{l}20-25 \mathrm{~m} \\
10-20 \mathrm{~m}\end{array}$ & Insect & Bird \& mammal & $?$ & Evergreen & 109 & 20.6 & $\mathrm{~K}, \mathrm{M}$ & $\begin{array}{l}1,2,9,10,11,12,13, \\
30,31,34,37,40,42, \\
43,48,51,69,70,72, \\
73,74\end{array}$ \\
\hline
\end{tabular}


Balanophoraceae

\begin{tabular}{|c|c|c|c|c|c|c|c|c|c|c|c|}
\hline Helosis cayennensis & Helocaye & $\begin{array}{l}\text { Perennial } \\
\text { herb }\end{array}$ & $\begin{array}{l}0-1 \mathrm{~m} ; 0-1 \\
\mathrm{~m}\end{array}$ & Insect & Vertebrate & no & Evergreen & 143 & 65.5 & & $\begin{array}{l}10,17,28,34,38,45 \text {, } \\
47,71\end{array}$ \\
\hline Langsdorffia hypogaea & Langhypo & $\begin{array}{l}\text { Perennial } \\
\text { herb }\end{array}$ & $\begin{array}{l}0-1 \mathrm{~m} ; 0-1 \\
\mathrm{~m}\end{array}$ & Insect & Vertebrate & no & Evergreen & 59 & 128.6 & & $17,28,34,71$ \\
\hline \multicolumn{12}{|l|}{ Bignoniaceae } \\
\hline $\begin{array}{l}\text { Amphilophium } \\
\text { paniculatum }\end{array}$ & Amphpani & Liana & $10-20 \mathrm{~m}$ & Insect & Wind & ? & & 108 & 144.2 & Germ & $\begin{array}{l}1,3,13,21,23,33 \\
43,49,53,63,71,74\end{array}$ \\
\hline Arrabidaea corallina & Arracora & Liana & $\begin{array}{l}25+\mathrm{m} ; 1- \\
4 \mathrm{~m}\end{array}$ & Insect & Wind & $?$ & & 101 & 175.8 & & $\begin{array}{l}1,6,13,17,23,32 \\
33,49,71\end{array}$ \\
\hline Callichlamys latifolia & Calllati & Liana & $20-25 \mathrm{~m}$ & Insect & Wind & $?$ & & 84 & 58.0 & Germ & $\begin{array}{l}13,17,21,23,27,31, \\
33,43,74\end{array}$ \\
\hline Clytostoma binatum & Clytbina & Liana & $\begin{array}{l}20-25 \mathrm{~m} \\
10-20 \mathrm{~m}\end{array}$ & Insect & Water (or fish) & ? & Evergreen & 72 & 64.9 & & $\begin{array}{l}13,17,23,32,33,38, \\
39,49,53,74\end{array}$ \\
\hline Cydista aequinoctialis & Cydiaequ & Liana & $4-10 \mathrm{~m}$ & Insect & Wind & ? & & 181 & 68.4 & Germ & $\begin{array}{l}1,10,13,17,21,23, \\
31,32,33,34,43,49, \\
53\end{array}$ \\
\hline Jacaranda macrocarpa & Jacamacr & Tree & $10-20 \mathrm{~m}$ & Insect & Wind & $?$ & & 5 & 8.6 & & 1,23 \\
\hline Martinella obovata & Martobov & Liana & $\begin{array}{l}1-4 \mathrm{~m} ; 1-4 \\
\mathrm{~m}\end{array}$ & Bird & Wind & $?$ & & 70 & 54.2 & & $\begin{array}{l}1,23,17,23,31,33 \\
38,43,49,74\end{array}$ \\
\hline Tabebuia nodosa & Tabenodo & Tree & $4-10 \mathrm{~m}$ & Insect & Wind & no & Deciduous & 14 & 355.1 & & 23 \\
\hline Tabebuia serratifolia & Tabeserr & Tree & $25+m$ & Insect & Wind & $?$ & Deciduous & 71 & 88.9 & & $\begin{array}{l}1,9,10,14,17,23 \\
27,31,34,68\end{array}$ \\
\hline Tecoma tenuiflora & Tecotenu & Bush & $\begin{array}{l}1-4 \mathrm{~m} ; 1-4 \\
\mathrm{~m}\end{array}$ & Bird & Wind & ? & Evergreen & 5 & 345.1 & & 23 \\
\hline \multicolumn{12}{|l|}{ Caryocaraceae } \\
\hline Anthodiscus obovatus & Anthobov & Tree & $\begin{array}{l}20-25 \mathrm{~m} \\
1-4 \mathrm{~m}\end{array}$ & $\underline{B a t}$ & Vertebrate & ? & Evergreen & 10 & 0.0 & & 57 \\
\hline Caryocar brasiliense & Carybras & Tree & $\begin{array}{l}4-10 \mathrm{~m} ; 1- \\
4 \mathrm{~m}\end{array}$ & Bat & Bat & yes & Evergreen & 76 & 177.2 & $\mathrm{~K}$ & $17,20,34,57$ \\
\hline Caryocar glabrum & Caryglab & Tree & $\begin{array}{l}25+\mathrm{m} \\
10-20 \mathrm{~m}\end{array}$ & Bat & Mammal & ? & Evergreen & 106 & 44.7 & & $\begin{array}{l}1,9,10,17,22,26 \\
31,34,36,57,64,70\end{array}$ \\
\hline Caryocar microcarpum & Carymicr & Tree & $20-25 \mathrm{~m}$ & Bat & Mammal & ? & Evergreen & 117 & 47.4 & $\mathrm{~K}$ & $17,18,34,38,57$ \\
\hline \multicolumn{12}{|l|}{ Chrysobalanaceae } \\
\hline Chrysobalanus icaco & Chryicac & Bush & $\begin{array}{l}1-4 \mathrm{~m} ; 1-4 \\
\mathrm{~m}\end{array}$ & $\underline{\text { Insect }}$ & Bird & $?$ & Evergreen & 193 & 91.5 & & $\begin{array}{l}5,17,27,34,48,57 \\
59,65,68\end{array}$ \\
\hline
\end{tabular}




\begin{tabular}{|c|c|c|c|c|c|c|c|c|c|c|c|}
\hline Couepia dolichopoda & Couedoli & Tree & $25+m$ & Insect & Vertebrate & $?$ & & 11 & 0.0 & & $1,57,59$ \\
\hline Couepia guianensis & Couegui2 & Tree & $\begin{array}{l}25+\mathrm{m} ; 4- \\
10 \mathrm{~m}\end{array}$ & Insect & Bird \& mammal & $?$ & & 90 & 45.5 & & $\begin{array}{l}1,9,10,17,24,31 \\
36,48,57\end{array}$ \\
\hline Couepia paraensis & Couepar2 & Tree & $\begin{array}{l}10-20 \mathrm{~m} \\
1-4 \mathrm{~m}\end{array}$ & Bird & Vertebrate & $?$ & & 129 & 64.6 & K & $17,18,34,48,57$ \\
\hline Hirtella ciliata & Hirtcili & Tree & $\begin{array}{l}4-10 \mathrm{~m} ; 1- \\
4 \mathrm{~m}\end{array}$ & Insect & Mammal & $?$ & & 66 & 170.2 & & $17,48,57$ \\
\hline Hirtella glandulosa & Hirtglan & Tree & $\begin{array}{l}20-25 \mathrm{~m} \\
1-4 \mathrm{~m}\end{array}$ & Insect & Bird \& mammal & no & & 134 & 140.4 & & $7,10,17,57$ \\
\hline Hirtella gracilipes & Hirtgrac & Tree & $\begin{array}{l}10-20 \mathrm{~m} \\
1-4 \mathrm{~m}\end{array}$ & Insect & Bird \& mammal & yes & & 94 & 166.6 & & $7,17,57,67$ \\
\hline Hirtella mutisii & Hirtmuti & Tree & $25+m$ & Insect & Mammal & ? & & 14 & 52.6 & & 57 \\
\hline Hirtella paniculata & Hirtpani & Tree & $\begin{array}{l}4-10 \mathrm{~m} ; 1- \\
4 \mathrm{~m}\end{array}$ & Insect & Bird & $?$ & & 89 & 53.6 & & $17,27,34,57$ \\
\hline Hirtella triandra & Hirttris & Tree & $\begin{array}{l}25+\mathrm{m} ; 1- \\
4 \mathrm{~m}\end{array}$ & Insect & Mammal - arboreal & $?$ & Evergreen & 214 & 85.0 & $\begin{array}{l}\mathrm{K}, \\
\mathrm{M}, \\
\text { dbh } \\
\text { grow } \\
\text { th }\end{array}$ & $\begin{array}{l}1,12,13,17,19,21, \\
24,32,40,43,57,69, \\
73\end{array}$ \\
\hline Licania arborea & Licaarbo & Tree & $25+m$ & Insect & Bat & $?$ & Evergreen & 64 & 95.1 & & $8,25,29,32,53,57$ \\
\hline Licania heteromorpha & Licahete & Tree & $20-25 \mathrm{~m}$ & Insect & Mammal & $?$ & Evergreen & 220 & 37.4 & M & $\begin{array}{l}1,9,10,17,24,27 \\
31,34,35,38,57\end{array}$ \\
\hline Licania humilis & Licahumi & Bush & $\begin{array}{l}1-4 \mathrm{~m} ; 1-4 \\
\mathrm{~m}\end{array}$ & Insect & Vertebrate & $?$ & & 58 & 174.5 & & 17,57 \\
\hline Parinari campestris & Paricamp & Tree & $\begin{array}{l}10-20 \mathrm{~m} \\
10-20 \mathrm{~m}\end{array}$ & Insect & Mammal - arboreal & ? & & 50 & 51.2 & & $24,27,36,57$ \\
\hline \multicolumn{12}{|l|}{ Fabaceae } \\
\hline Inga alba & Ingaalba & Tree & $\begin{array}{l}25+\mathrm{m} ; 4- \\
10 \mathrm{~m}\end{array}$ & Insect & Bird \& mammal & $?$ & Evergreen & 85 & 48.2 & & $\begin{array}{l}1,4,9,10,17,19,31, \\
34,49,54,58,61,74\end{array}$ \\
\hline Inga cardozana & Ingacard & Tree & $20-25 \mathrm{~m}$ & Insect & Vertebrate & & Evergreen & 10 & 290.9 & & 55 \\
\hline Inga cayennensis & Ingaaria & Tree & $\begin{array}{l}20-25 \mathrm{~m} \\
1-4 \mathrm{~m}\end{array}$ & Insect & Vertebrate & & Evergreen & 53 & 63.7 & $\begin{array}{l}\text { dbh } \\
\text { grow } \\
\text { th }\end{array}$ & $\begin{array}{l}1,10,17,31,34,49 \\
55,61\end{array}$ \\
\hline Inga ingoides & Ingaingo & Tree & $\begin{array}{l}25+\mathrm{m} ; 4- \\
10 \mathrm{~m}\end{array}$ & Insect & Mammal - arboreal & & Evergreen & 77 & 109.3 & & $17,34,49,55$ \\
\hline Inga laurina & Ingalaur & Tree & $20-25 \mathrm{~m}$ & Insect & Vertebrate & $?$ & Deciduous & 94 & 90.5 & M & $1,12,13,31,40,43$ \\
\hline
\end{tabular}




\begin{tabular}{|c|c|c|c|c|c|c|c|c|c|c|c|}
\hline & & & $1-4 \mathrm{~m}$ & & & & & & & & $45,49,55,69$ \\
\hline Inga marginata & Ingamarg & Tree & $\begin{array}{l}20-25 \mathrm{~m} \\
4-10 \mathrm{~m}\end{array}$ & Insect & Mammal & $?$ & Evergreen & 93 & 72.3 & M, K & $\begin{array}{l}1,6,9,10,12,13,15, \\
17,31,34,40,43,46, \\
49,50,54,56,58,61, \\
69,70,73,74\end{array}$ \\
\hline Inga nobilis & Ingamath & Tree & $\begin{array}{l}10-20 \mathrm{~m} \\
4-10 \mathrm{~m}\end{array}$ & Insect & Mammal - arboreal & ? & Evergreen & 191 & 63.4 & & $\begin{array}{l}1,12,17,34,38,43 \\
45,49,55,73\end{array}$ \\
\hline Inga plumifera & Ingaplum & Tree & $4-10 \mathrm{~m}$ & Insect & Vertebrate & & Evergreen & 5 & 0.0 & & 55 \\
\hline \multicolumn{12}{|l|}{ Lauraceae } \\
\hline Aniba panurensis & Anibpanu & Tree & $\begin{array}{l}10-20 \mathrm{~m} \\
1-4 \mathrm{~m}\end{array}$ & Insect & Bird & no & & 54 & 53.1 & & $1,31,34,41,49,71$ \\
\hline Nectandra amazonum & Nectamaz & Tree & $\begin{array}{l}20-25 \mathrm{~m} \\
4-10 \mathrm{~m}\end{array}$ & Insect & Water (or fish) & $?$ & & 51 & 68.7 & & $17,34,49,62,71$ \\
\hline Nectandra egensis & Nectegen & Tree & $\begin{array}{l}10-20 \mathrm{~m} \\
1-4 \mathrm{~m}\end{array}$ & Insect & Bird & $?$ & & 6 & 0.0 & & 62 \\
\hline Nectandra hihua & Necthihu & Tree & $\begin{array}{l}25+\mathrm{m} ; 1- \\
4 \mathrm{~m}\end{array}$ & Insect & Bird & $?$ & & 126 & 79.2 & & $49,62,71$ \\
\hline Nectandra reflexa & Nectrefl & Tree & $\begin{array}{l}25+m ; \\
10-20 m\end{array}$ & Insect & Bird & $?$ & & 6 & 173.4 & & 62,71 \\
\hline Nectandra turbacensis & Nectturb & Tree & $\begin{array}{l}20-25 \mathrm{~m} \\
1-4 \mathrm{~m}\end{array}$ & Insect & Bird & $?$ & & 70 & 72.4 & & $1,62,71$ \\
\hline \multicolumn{12}{|l|}{ Proteaceae } \\
\hline Panopsis metcalfii & Panometc & Tree & $\begin{array}{l}10-20 \mathrm{~m} \\
4-10 \mathrm{~m}\end{array}$ & Insect & Mammal - ground & $?$ & & 7 & 61.1 & & 57,71 \\
\hline Panopsis rubescens & Panorube & Tree & $\begin{array}{l}25+\mathrm{m} ; 4- \\
10 \mathrm{~m}\end{array}$ & Insect & & $?$ & & 93 & 69.3 & $\mathrm{~K}$ & $16,18,33,34,48,57$ \\
\hline Roupala montana & Roupmont & Tree & $\begin{array}{l}20-25 \mathrm{~m} \\
1-4 \mathrm{~m}\end{array}$ & Insect & Wind & $?$ & & 213 & 121.6 & & $\begin{array}{l}1,9,10,16,33,34, \\
38,45,48,51,57,58\end{array}$ \\
\hline \multicolumn{12}{|l|}{ Rubiaceae } \\
\hline Gleasonia duidana & Gleaduid & Tree & $\begin{array}{l}4-10 \mathrm{~m} ; 4- \\
10 \mathrm{~m}\end{array}$ & Insect & Water (or fish) & & & 10 & 22.7 & & 60,71 \\
\hline Gleasonia uaupensis & Gleauaup & Tree & $\begin{array}{l}4-10 \mathrm{~m} ; 1- \\
4 \mathrm{~m}\end{array}$ & Insect & Wind & & & 6 & 0.0 & & 17,60 \\
\hline Henriquezia nitida & Henrniti & Tree & $\begin{array}{l}10-20 \mathrm{~m} \\
4-10 \mathrm{~m}\end{array}$ & Insect & Water (or fish) & & & 13 & 4.7 & & 17,60 \\
\hline Henriquezia verticillata & Henrvert & Tree & $25+\mathrm{m} ; 4-$ & Insect & Water (or fish) & & & 10 & 7.6 & & $17,31,60$ \\
\hline
\end{tabular}




\begin{tabular}{|c|c|c|c|c|c|c|c|c|c|c|c|}
\hline & & & $10 \mathrm{~m}$ & & & & & & & & \\
\hline Platycarpum schultesii & Platschu & Tree & $4-10 \mathrm{~m}$ & Insect & Wind & & & 5 & 5.0 & & 60 \\
\hline \multicolumn{12}{|l|}{ Sapotaceae } \\
\hline Manilkara bidentata & Manibide & Tree & $\begin{array}{l}25+\mathrm{m} \\
10-20 \mathrm{~m}\end{array}$ & Bat & Bat & $?$ & Deciduous & 104 & 40.9 & $\mathrm{~K}$ & $\begin{array}{l}1,9,10,14,31,34 \\
48,54\end{array}$ \\
\hline Pouteria elegans & Pouteleg & Tree & $\begin{array}{l}10-20 \mathrm{~m} \\
4-10 \mathrm{~m}\end{array}$ & Insect & Mammal & & & 62 & 27.8 & & $9,31,34,48,54$ \\
\hline Pouteria guianensis & Poutguia & Tree & $\begin{array}{l}25+\mathrm{m} ; 4- \\
10 \mathrm{~m}\end{array}$ & Insect & Mammal - arboreal & & $\begin{array}{l}\text { Annual loss } \\
\text { of part: } \\
\text { semidecidu } \\
\text { ous }\end{array}$ & 62 & 36.4 & & $\begin{array}{l}1,9,10,27,31,34 \\
48,54\end{array}$ \\
\hline Pouteria reticulata & Poutreti & Tree & $\begin{array}{l}25+\mathrm{m} ; 4- \\
10 \mathrm{~m}\end{array}$ & Insect & Bird & no & & 81 & 54.3 & $\begin{array}{l}\mathrm{K}, \\
\text { germ } \\
\mathrm{M}\end{array}$ & $\begin{array}{l}1,9,10,12,21,24, \\
31,34,43,45,48,54 \\
67,68,69,73,74\end{array}$ \\
\hline Sarcaulus brasiliensis & Sarcbras & Tree & $\begin{array}{l}20-25 \mathrm{~m} \\
4-10 \mathrm{~m}\end{array}$ & Insect & Bird \& mammal & no & & 60 & 30.9 & M & $1,9,10,31,45,48,54$ \\
\hline
\end{tabular}

A 1 Data as Phillips et al. 1998; 2 Abbott et al. 1997; 3 ABIS 1997; 4 ABRACOS 1999; 5 Aguilar et al. 1992; 6 Aqua-RAP 1997; 7 Arista et al. 1997; 8 Arriaga et al. 1998; 9 BDFFP 2000; 10 Belbenoit et al. 2000; 11 Boom 1986; 12 Condit et al. 1996b; 13 Croat 1978; 14 de Graaf et al. $1999 ; 15$ Delich \& Greer 1996; 16 EMBRAPA. 2000a; 17 EMBRAPA. 2000b; 18 Ferreira \& Prance 1998; 19 Finegan et al. 1999; 20 Garcia Collevatti et al. 1997; 21 Garwood 1983; 22 Gentry 1998a; 23 Gentry 1998b; 24 Gentry 1990; 25 Gillespie 1999; 26 Guehl et al. 1998; 27 Hammond \& Brown 1995; 28 Hansen 1980; 29 Heithaus \& Fleming 1978; 30 Henderson 1995; 31 Hopkins 2000; 32 INBIO 1998-2000; 33 INPA 2000a; 34 INPA 2000b; 35 Johnston 1998; 36 Julien-Laferrière 1999; 37 Kahn 1986; 38 Kelloff \& Funk 1999; 39 Killen et al. 1998; 40 Knight 1975; 41 Kubitzki \& Renner 1982; 42 La Suerte Biological Field Station 1998; 43 Leigh et al. 1996; 44 Lescure et al. 1992; 45 McPherson 1998; 46 Molina Max et al. 1998; 47 Mori \& Boom 1987; 48 MPEG. 2000a; 49 MPEG. 2000b; 50 Nakajima et al. $1996 ; 51$ NYBG 2000; 52 NYBG 2001; 53 OTS 2000; 54 Pennington 1997a; 55 Pennington 1997b; 56 Perez et al. 1996; 57 Prance \& Plana 1997; 58 Rapid Biological Inventory Team 2000; 59 RBG Kew 1998; 60 Rogers 1984; 61 Roggy et al. 1999; 62 Rohwer 1993; 63 Sagástegui \& Dillon. 1991; 64 Saldarriaga et al. 1988; 65 Tellez Valdes \& Cabrera Cano 1987; 66 Thomas et al. 2000a; 67 Thomas et al. 2000b; 68 Thomas et al. 2000c; 69 Thomsen \& Brimer 1997; 70 Vester \& Cleef 1998; 71 W3 TROPICOS 1997-2001; 72 Weissenhofer 2000; 73 Welden et al. 1991; 74 Wilbur et al. 1990. 


\section{Appendix 5 Sources of life history information}

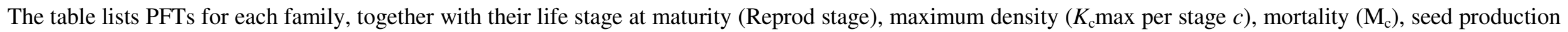

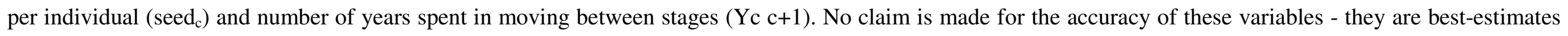

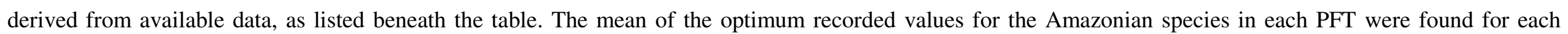

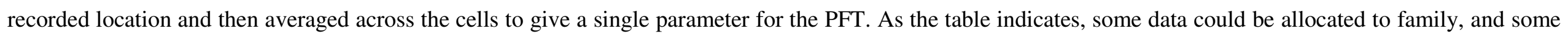
only to dicot/monocot groups.

Table A5:1: Plant functional type parameterisation

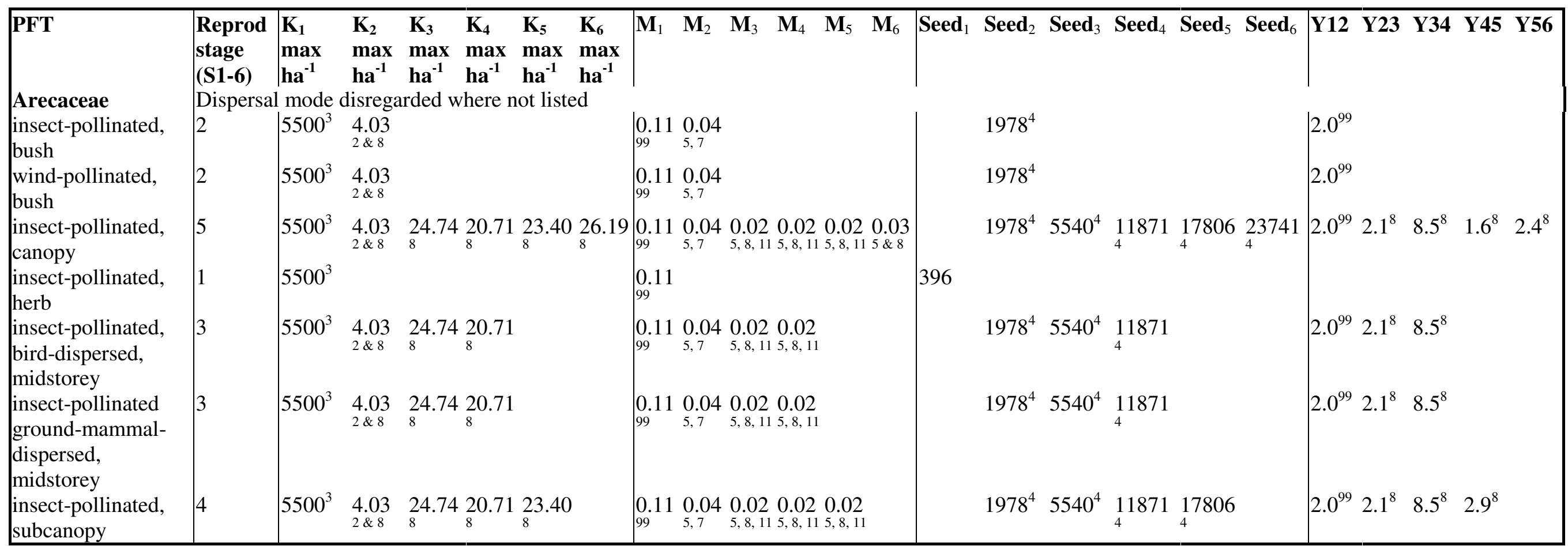




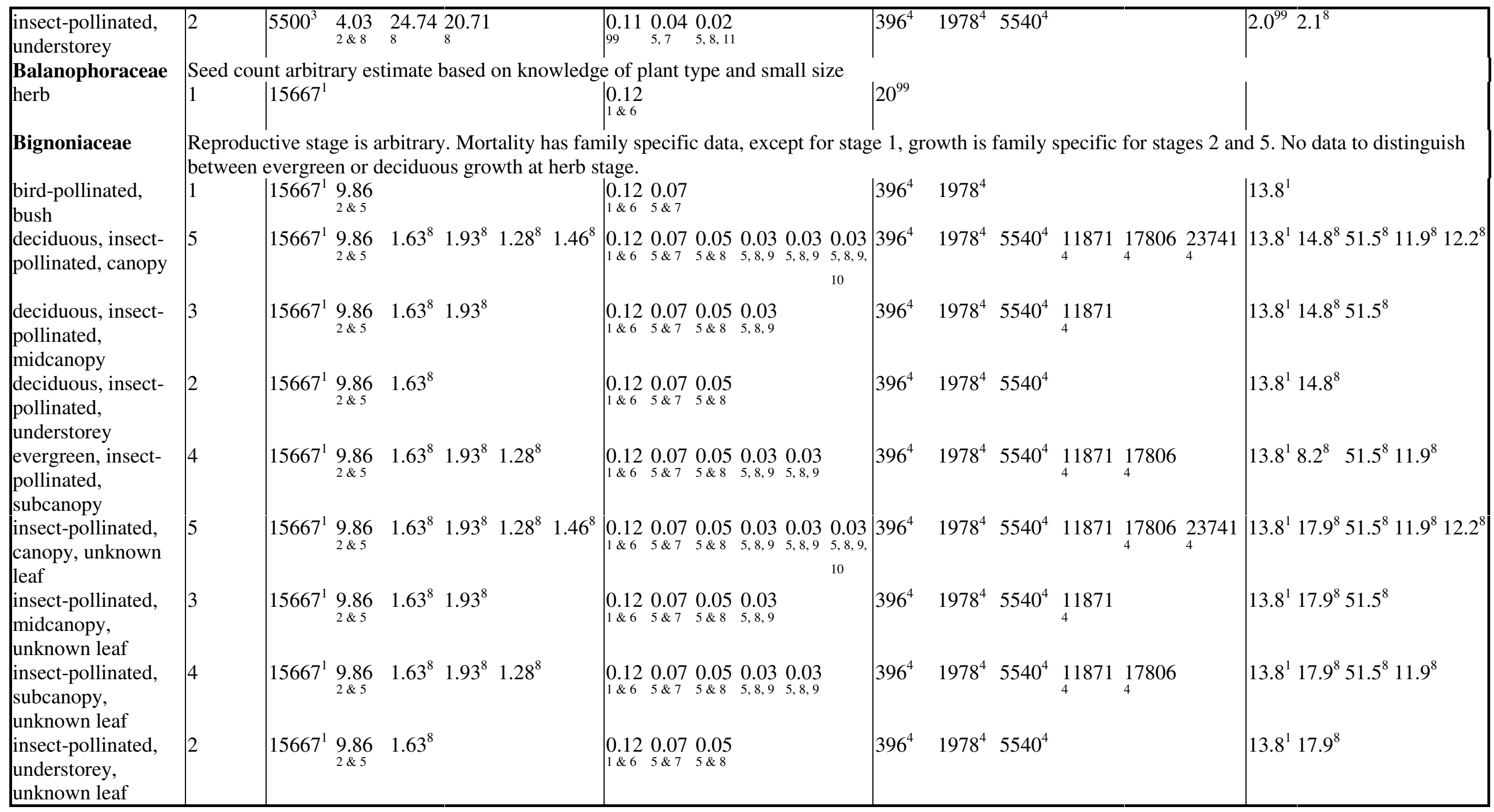




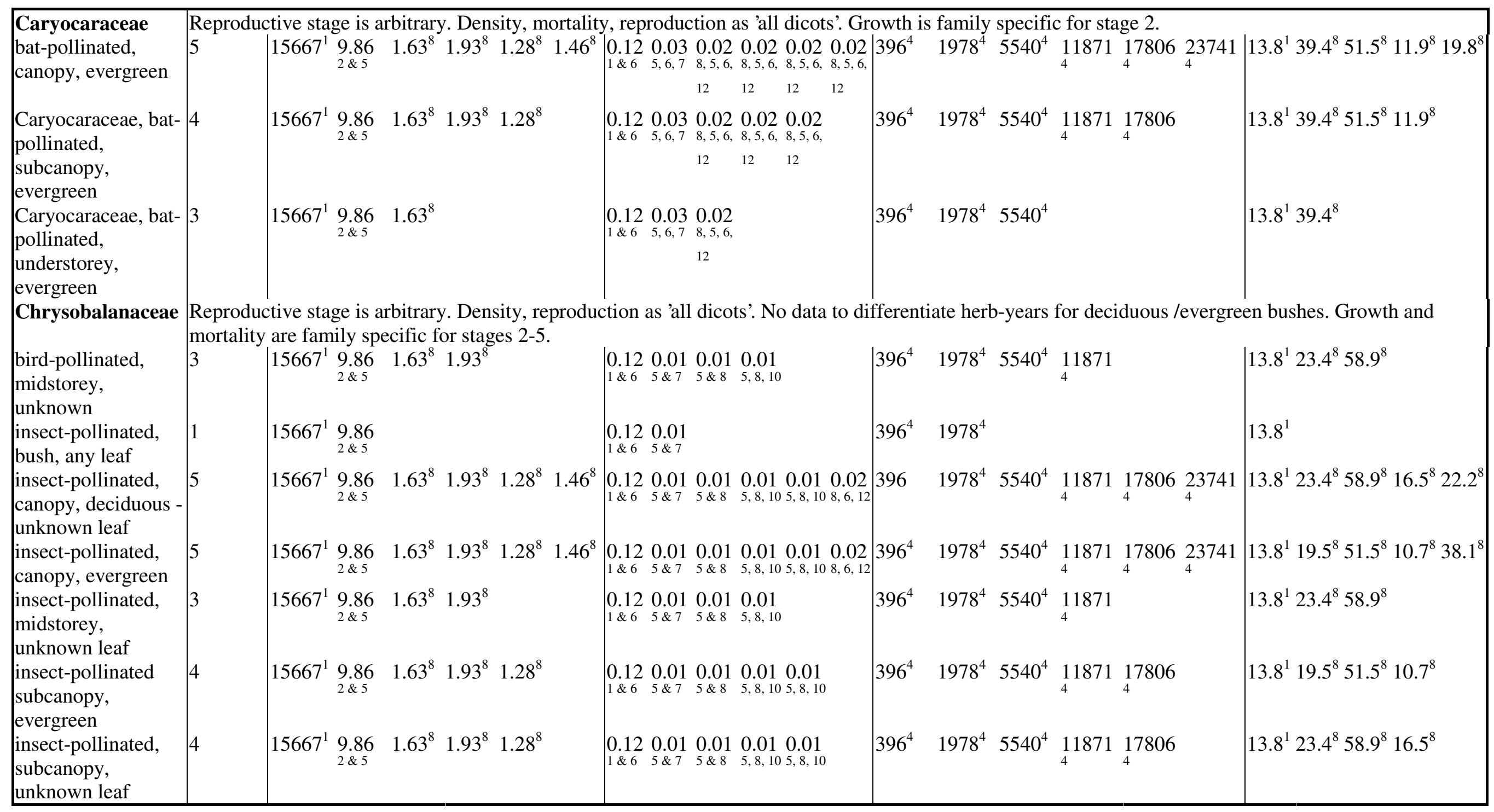




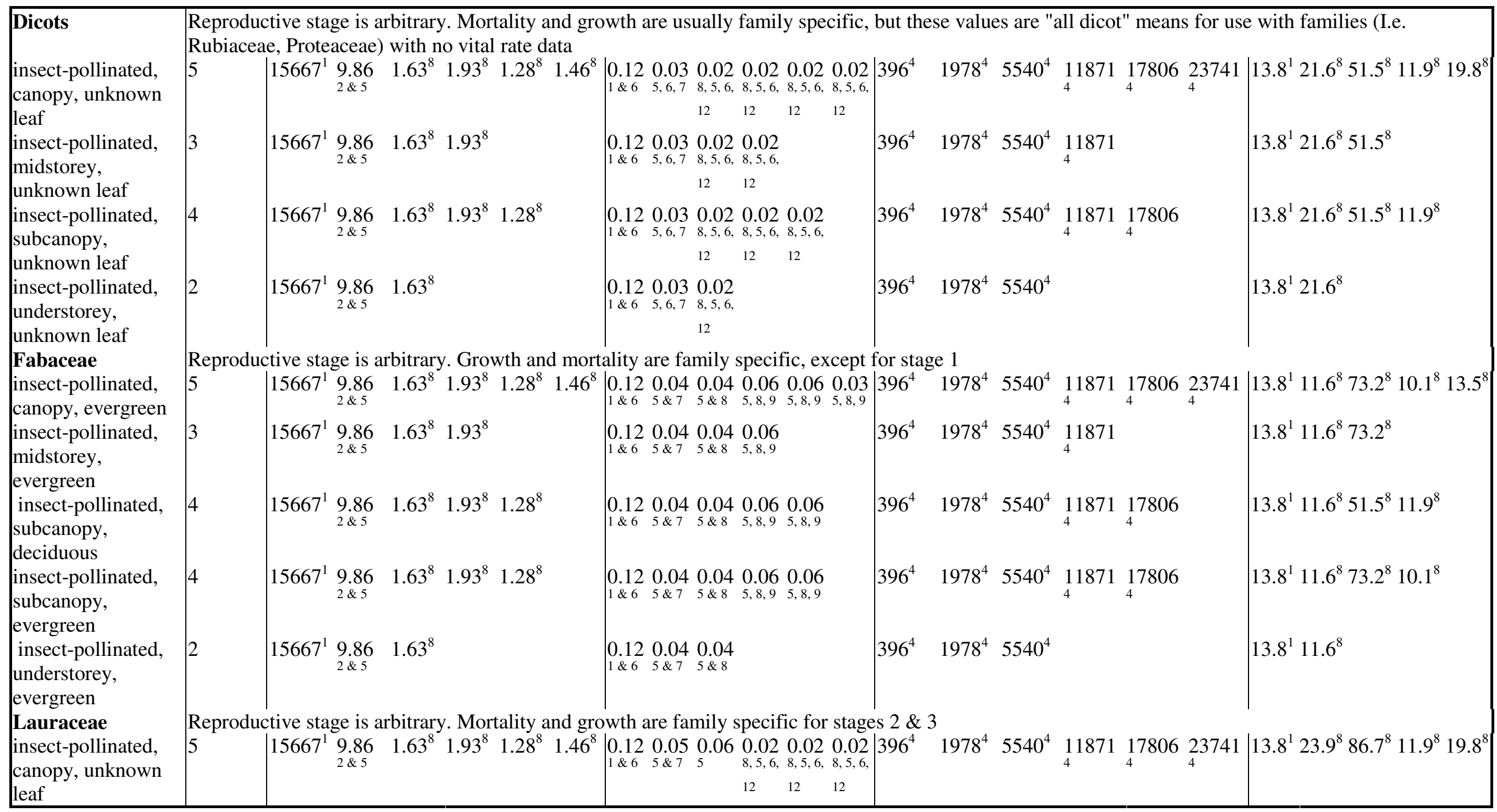




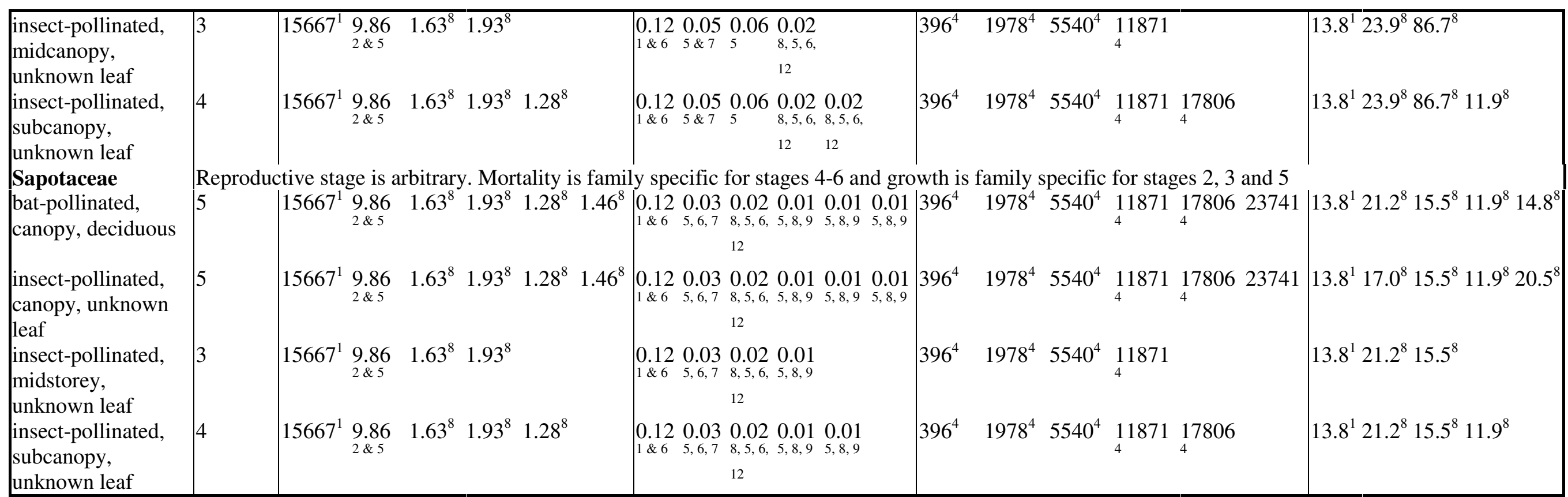

${ }^{1}$ Nascimento \& Proctor 1997; ${ }^{2}$ Condit et al. 1996b; ${ }^{3}$ Cintra 1997a (Astrocaryum murumuru only); ${ }^{4}$ Seed counts derived by linear regression with median height class from several Amazon species with seed counts recorded in Cintra 1997b, de Araújo 1994, Listabarth 1993, Oliveira 1997, Ortiz et al. 1997, Storti 1993; van Roosmalen \& da Cruz Gomes Garcia 2000; ${ }^{5}$ Condit et al. 1995; ${ }^{6}$ Clark \& Clark 1992; ${ }^{7}$ Welden et al. 1991; ${ }^{8}$ Data from sites as: Phillips et al. $1998 ;{ }^{9} \mathrm{de}$ Graaf et al. 1999; ${ }^{10}$ Milton et al. $1994 ;{ }^{11}$ Williamson et al. $2000,{ }^{12}$ Clark \& Clark $1996 ;{ }^{99}$ no data - arbitrary assignment of value 
Table A5:2: Data sources for traits assigned to species

\begin{tabular}{|l|l|}
\hline Subject & References \\
\hline Life form, height class & Erikson \& Bremer 1992, Clark \& Clark 92, Condit et al. 1995, Hammond \& Brown 1995, Gentry 1996, Borchert 1988 \\
\hline $\begin{array}{l}\text { Dispersal mode, seed count } \\
\text { (or other indicators of } \\
\text { reproductive effort) }\end{array}$ & $\begin{array}{l}\text { Erikson \& Bremer 1992, Gorchov } \text { et al. 1993, Guevara \& Laborde 1993, da Cruz Alencar 1994, Hammond \& Brown 1995, Ramirez } \\
\text { Ro5, Killen } \text { et al. 1998, Brewer \& Rejmánek 1999, Gillespie 1999, Küchmeister } \text { et al. 1998, Ruiz \& da Cruz Alencar 1999, van }\end{array}$ \\
\hline Pollination mode, selfing & Erikson \& Bremer 1992, Listabarth 1993, Gentry 1996, Küchmeister et al. 1998, Gillespie 1999 \\
\hline Pollen dispersal distance & Bush \& Rivera 1998; Roubik 2000 \\
\hline Deciduousness & Williams-Linera 1997, Borchert 1988, Huante et al. 1998, Ruiz \& da Cruz Alencar 1999 \\
\hline
\end{tabular}




\section{References}

Abbott, R., Bass, M., Clark, J., KuepPers, L. \& PitM, N. (1997). Bilsa Preliminary Plant

List. [online] Equafor, Leeds, UK. Available from: http://www.bchip.com/equafor/text/ lib03.htm. [accessed 16 January 2002].

ABIS. (1997). Floristic Checklist of Cutervo National Park. [online] Andean Botanical Information System, The Field Museum, Chicago, Illinois, USA. Available from: http://www.sacha.org/envir/bosques/cutervo/cutervo.html [accessed 17 January 2001]

ABRACOS. (1999). Anglo-Brazilian Amazonian Climate Observation Study: Available Data.

[online]. Instituto Nacional de Pesquisas Espaciais, Brazil. Available from: http://yabae.cptec.inpe.br/abracos/index.html. [accessed 17 January 2002].

ADAMS, J.M. \& FAURE, H. (eds) (1997). Review and atlas of palaeovegetation: preliminary land ecosystem maps of the world since the Last Glacial Maximum. [online]. Oak Ridge National Laboratory, TN, USA. Available from: http://www.esd.ornl.gov/projects/qen/ adams1.html. [accessed 15 October 2001].

Aguilar C., María J. \& Aguilar C.M.A. (1992). Árboles de la Biosfera Maya Peten. Guía para las especies del Parque Nacional Tikal. [extract online]. Centro de Estudios Conservacionistas, Universidad de San Carlos de Guatemala, Guatemala. Available from: http://www.nps.gov/centralamerica [accessed 19 April 1999].

AHN, C.-H. \& TATEISHI, R. (1994a). Development of a global 30-minute grid potential evapotranspiration data set. Journal of the Japan Society Photogrammetry and Remote Sensing 33(2): 12-21.

AHN, C.-H. \& TATEISHI, R. (1994b). Development of global land surface evapotranspiration and water balance data sets. Journal of the Japan Society Photogrammetry and Remote Sensing 33(5): 48-61.

ALLEN, R.G. (2000). REF-ET: Reference Evapotranspiration Calculation Software for FAO and ASCE Standardised Equations. Version 2.0 for Windows. Manual. [online]. University of Idaho, USA. Available from: http://www.kimberly.uidaho.edu/ref-et/. [accessed 14 June 2000].

Allen, R.G., Pereira, L.S., RAES, D. \& SMITH, M. (1998). Crop evapotranspiration Guidelines for computing crop water requirements. FAO Irrigation and Drainage Paper $\underline{56}$. [online]. Available from: http://www.fao.org/docrep/x04090E/x04090e00.htm\#Contents. [accessed 26 January 2000].

AlmudeVar, T. \& TibShiRAnI, R.J. (1991) Generalized Additive Interactive Modelling. Software and User's Manual. Tibshirani Enterprises, Inc., Toronto, Canada.

ANALYSE-IT (2001) v. 1.62. Leeds, UK. Available from: http://www.analyse-it.com/ [accessed 15 January 2002]. 
APG (The Angiosperm Phylogeny Group : Bremer, K., Chase, M.W., Stevens, P.F., Anderberg, A.A., Backlund, A., Bremer, B., Briggs, B.G., Endress, P., FAy, M.F., Goldblatt, P., Gustafsson, M.H.G., HoOt, S.B., JudD, W.S., Källersjö, M., KellogG, E.A., Kron, K.A., Les, D.H., Morton, C.M., Nickrent, D.L., Olmstead, R.G., Price, R.A., Quinn, C.J., Rodman, J.E., Rudall, P.J., Savolainen, V., Soltis, D.E., Soltis, P.S., SyTSMA, K.J. \& Thulin, M.) (1998). An ordinal classification for the families of flowering plants. Annals of the Missouri Botanical Garden 85: 531-553.

AQUA-RAP. (1997). Rió Paraguay/Rió Apa Expedition September 2-22, 1997. [online]. Aquatic

Rapid Assessment Program, Conservation International, USA. Available from: http://www.conservation.org/aquarap/Priority/sites/Paraguay/fldobs. [accessed 18 May 1999]

ARAúJO, M.B. \& WiLliams, P.H. (2000). Selecting areas for species persistence using occurrence data. Biological Conservation 96(3): 331-345.

Arista, M., Oliveira, P.E., GibBs, P.E. \& Talavera, S. (1997). Pollination and breeding system of two co-occurring Hirtella species (Chrysobalanaceae) in central Brazil. Botanica Acta 110: 496-502.

Arriaga, L., Aguilar, V., Alcocer, J., Jiménez, R., Muñoz, E. \& Vázquez, E. (coordinators). (1998). Regiones hidrológicas prioritarias. 87. MOTOZINTLA. [online]. Comisión Nacional para el Conocimiento y Uso de la Biodiversidad, Mexico Available from: http://www.conabio.gob.mx/rhp/87.html. [accessed 16 January 2002].

AsPINALL, R. (1992). An inductive modelling procedure based on Bayes' theorem for analysis of pattern in spatial data. International Journal of Geographical Information Systems $\underline{6}(2)$ : 105-121.

ASPINALL, R. \& MATtHeWs, K. (1994). Climate change impact on distribution and abundance

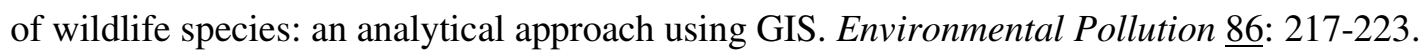

AugsPurger, C.K. (1983). Seed dispersal distance of the tropical tree, Platypodium elegans, and the escape of its seedlings from fungal pathogens. Journal of Ecology 71: 759-771.

AugSPURGER, C.K. (1984). Light requirements of Neotropical tree seedlings: a comparative study of growth and survival. Journal of Ecology 72: 777-795.

Augustin, N.H., Mugglestone, M.A. \& BucKland, S.T. (1996). An autologistic model for the spatial distribution of wildlife. Journal of Applied Ecology 33: 339-347.

Austin, G.E., Thomas, C.J., Houston, D.C. \& Thompson, D.B.A. (1996). Predicting the spatial distribution of buzzard Buteo buteo nesting areas using a Geographical Information System and remote sensing. Journal of Applied Ecology 33(6): 1541-1550.

AUSTIN, M.P. (1980). Searching for a model for vegetation analysis. Vegetatio 43:11-21.

Austin, M.P. (1999). The potential contribution of vegetation ecology to biodiversity research. Ecography 22: 465-484. 
Austin, M.P., Nicholls, A.O. \& MArgules, C.R. (1990). Measurement of the realized qualitative niche: environmental niches of five Eucalyptus species. Ecological Monographs 60(2): 161-177.

Austin, M.P. \& SMITH, T.M. (1989). A new model for the continuum concept. Vegetatio 83 : $35-47$.

BÁRDOSsY, A. (1997). Downscaling from GCMs to local climate through stochastic linkages. Journal of Environmental Management 49(1): 7-17.

BARry, R.G. \& ChORley, R.J. (1998). Atmosphere, Weather and Climate. Seventh Edition. Routledge, London, UK.

Bartlein, P.J., Whitlock, C. \& Shafer, S.L. (1997). Future climate in the Yellowstone National Park region and its potential impact on vegetation. Conservation Biology 11(3): 782792.

BATJES, N.H. (1996). Development of a world data set of soil water retention properties using pedotransfer rules. Geoderma $\underline{71}$ : 31-5.

Batjes, N.H., Fischer, G., Nachtergaele, F.O., Stolbovoy, V.S. \& Van Velthuizen, H.T. (1997). Soil Data Derived from WISE for Use in Global and Regional AEZ Studies. International Institute for Applied Systems Analysis, Laxenburg, Austria.

BAWA, K.S. \& MARKham, A. (1995). Climate change and tropical forests. Trends in Ecology and Evolution 10: 348-349.

BAZZAZ, F.A. (1998). Tropical forests in a future climate: changes in biological diversity and impact on the global climate cycle. Climatic Change 39(2-3):317-336.

BDFFP. (2000). BDFFP tree database. Unpublished data. Biological Dynamics of Forest Fragment Project, INPA, Manaus, Brazil. [accessed March 2000].

Begon, M., HARPer, J.L. \& Townsend, C.R. (1996). Ecology: Individuals, Populations and Communities. Blackwell Science, Oxford, UK.

Belbenoit, P., Poncy, O. \& RierA, B. (2000). List of plant species, Nouragues area. In: Nouragues: Dynamics and Plant-Animal Interactions in a Neotropical Rainforest (eds. F. Bongers, P. Charles-Dominique, P.M. Forget \& M. Thery). [online version]. Kluwer, Dordrecht, Pays Bas. Available from: http://www.cnrs.fr/nouragues/NourPlantes.htm. [accessed 16 January 2002].

Belotelov, N.V., Bogatyrev, B.G., Kirilenko, A.P. \& Venevsky, S.V. (1996). Modelling of time-dependent biome shifts under global climate changes. Ecological Modelling 87: 29-40.

Bengtsson, L., BotZet, M. \& EsCH, M. (1996). Will greenhouse gas induced warming over the next 50 years lead to higher frequency and greater intensity of hurricanes? Tellus 48A: 5773.

Bernal, R. (1998). Demography of the vegetable ivory palm Phytelephas seemannii in Colombia, and the impact of seed harvesting. Journal of Applied Ecology 35(1): 64-74. 
BESAG, J. (1989). Towards Bayesian image analysis. Journal of Applied Statistics 16: 395-407.

BETTS, R. (14 November 2001). GCM scaling. E-mail to L.J. Miles (1.miles@geography.leeds.ac.uk).

BetTS, R.A., Cox, P.M. \& Woodward, F.I. (1997). Contrasting physiological and structural vegetation feedbacks in climate change simulations. Nature 387: 796-799.

BetTs, R.A., Cox, P.M. \& WoOdWARd, F.I. (2000). Simulated responses of potential vegetation to doubled- $\mathrm{CO}_{2}$ climate change and feedbacks on near-surface temperature. Global Ecology and Biogeography 9(2): 171-180.

BLACKBURN, T.M. \& GASTON, K.J. (1996). A sideways look at patterns in species richness, or why there are so few species outside the tropics. Biodiversity Letters $\underline{3}$ : 44-53.

Boom, B. (1986). A forest inventory in Amazonian Bolivia. Biotropica 18(4): 287-294.

BоотH, T.H. (1990). A climatic analysis method for expert systems assisting three species introductions. Agroforestry Systems 10: 33-45.

Bоотн, T.H. (1998). A broadscale land evaluation program to assess the potential for growing particular trees in Africa. Agroforestry Systems 40: 125-138.

BоOTH, T.H. \& JONES, P.G. (1998). Identifying climatically suitable areas for growing particular trees in Latin America. Forest Ecology and Management 108(1-2): 167-173.

Booth, T.H., NiX, H.A., Hutchinson, M.F. \& Busby, J.R. (1987). Grid matching: a new method for homoclime analysis. Agricultural and Forest Meteorology 39: 241-255.

BORCHERT, R. (1998). Responses of tropical trees to rainfall seasonality and its long-term changes. Climatic Change 39: 381-393.

Bossel, H. \& KRIEGER, H. (1994). Simulation of multi-species tropical forest dynamics using a vertically and horizontally structured model. Forest Ecology and Management 69(1-3): 123144.

Boumman, A. F., I. FunG, E. Matthews \& John, J. (1993). Global analysis of the potential for nitrous oxide $\left(\mathrm{N}_{2} \mathrm{O}\right)$ production in natural soils. Global Biogeochemical Cycles $\underline{7}(3)$ : 557597.

BOWERS, M.A. \& HARRIS, L.C. (1994). A large-scale metapopulation model of interspecific competition and environmental change. Ecological Modelling 72: 251-273.

BOX, E.O. (1981). Macroclimate and plant forms: an introduction to predictive modelling in phytogeography. Junk, The Hague, Netherlands.

Breiman, L., Friedman, J.H., Olshen, R.A., \& Stone, C.J. (1984). Classification and Regression Trees. Wadsworth International Group, Belmont, USA.

BREWER, S.W. \& REJMÁNEK, M. (1999). Small rodents as significant dispersers of tree seeds in a Neotropical forest. Journal of Vegetation Science 10: 165-174.

BRICKELl, C. (1998). The Royal Horticultural Society A-Z Encyclopaedia of Garden Plants. Dorling Kindersley, London. UK. 
BROKAW, N.V.L. (1987). Gap-phase regeneration of three pioneer species in a tropical forest. Journal of Ecology 75(1): 9-19.

Brown, J.H. (1995). Macroecology. University of Chicago Press, Chicago, USA: 269 pp.

BROWN, J.H. \& MAURER, B.A. (1989). Macroecology: the division of food and space among species on continents. Science 243: 1145-1150.

Brown, J.H., Mehlman, D.W. \& Stevens, G.C. (1995). Spatial variation in abundance. Ecology 76(7): 2028-2043.

Brown, JR., K.S. \& Prance, G.T. (1987). Soils and vegetation. In Biogeography and quaternary history in tropical America (eds. G.T. Prance \& T.C. Whitmore). Clarendon Press, Oxford, UK: 19-45.

BROwn, R.A. \& RoSENBERG, N.J. (1999). Climate change impacts on the potential productivity of corn and winter wheat in their primary United States growing regions. Climatic Change 41 : 73-107.

Brown, S., Lugo, A.E., Silander, S. \& Liegel, L. (1983). Research history and opportunities in Luquillo Experimental Forest. USDA Forest Service General Technical Report SO-44. Cited in Swaine et al. (1987)

BUCK, A.L. (1981). New equations for computing vapor pressure and enhancement factor. Journal of Applied Meteorology 20(12): 1527-1532.

BuCKLAND, S.T. \& ElSTON, D.A. (1993). Empirical models for the spatial distribution of wildlife. Journal of Applied Ecology 30: 478-495.

BUCKLEY, E. \& WARRILOW, D.A. (1988) Derivation of land surface parameter datasets for use in the Met O 20 GCMs. Met. O 20 Internal Note: $\underline{81}$. United Kingdom Meteorological Office, Bracknell, Berkshire, UK.

BugmanN, H. (1996). Functional types of trees in temperate and boreal forests: Classification and testing. Journal of Vegetation Science 7(3): 359-370.

BUSBY, J.R. (1991). BIOCLIM - a bioclimatic analysis and prediction system. In: Nature conservation: cost effective biological surveys and data analysis (eds. C.R. Margules \& M.P. Austin). CSIRO, Melbourne, Australia 3-6.

BUSH, M.B. (1996). Amazonian conservation in a changing world. Biological Conservation 76 : 219-228.

Bush, M.B. \& RiverA, R. (1998). Pollen dispersal and representation in a neotropical rain forest. Global Ecology and Biogeography Letters $\underline{7}$ (5): 379-392.

BYRNE, M.M. \& LEVEY, D.J. (1993). Removal of seeds from frugivore defecations by ants in a Costa Rican rain forest. Vegetatio 107/108: 363-374.

CAMMELL, M.E. \& KNIGHT, J.D. (1992). Effects of climatic change on the population dynamics of crop pests. Advances in Ecological Research 22: 117-162. Cited in Fearnside 1995c. 
CAMPBELl, D.G. (1993). Scale and patterns of community structure in Amazonian forests. In:

Large-Scale Ecology and Conservation Biology. The 35th Symposium of the British Ecological Society with the Society for Conservation Biology. (eds. P.J. Edwards, R.M. May \& N.R. Webb). University of Southampton, Southampton, UK: 179-198.

CANE, J.H. (2001). Habitat fragmentation and native bees: a premature verdict?. Conservation Ecology 5 (1): U149-U161.

Cane, M.A., Clement, A.C., Kaplan, A., Pozdnyakov, D., Seager, R., Zebiak, S.E. \& Murtugudde, R. (1997). Twentieth-century sea surface temperature trends. Science 275(5302): 957-960.

CAO, G. (1995). The definition of the niche by fuzzy set theory. Ecological Modelling 77: 6571.

Carey, E.V., Brown, S., Gillespie, A.J.R. \& Lugo, A.E. (1994). Tree mortality in mature lowland tropical moist and tropical lower montane moist forests of Venezuela. Biotropica 26: 255-265.

CAREY, P.D. (1996). DISPERSE: A cellular automation for predicting the distribution of species in a changed climate. Global Ecology and Biogeography Letters $\underline{5}$ : 217-226.

CAREY, P.D. \& BROWN, N.J. (1994). The use of GIS to identify sites that will become suitable for a rare orchid, Himantoglossum hircinum L., in a future changed climate. Biodiversity Letters 2: 117-123.

CARlQuist, S. (1988). Comparative Wood Anatomy: Systematic, Ecological, and Evolutionary Aspects of Dicotyledon Wood. Springer-Verlag, Berlin, Germany. Cited in Díaz \& Cabido (1997).

CARPenter, G., Gillison, A.N. \& Winter, J. (1993). DOMAIN: a flexible modelling procedure for mapping potential distributions of plants and animals. Biodiversity and Conservation 2: 667-680.

CARTER, T.R., PARRY, M.L. \& PORTER, J.H. (1991). Climatic change and future agroclimatic potential in Europe. International Journal of Climatology 11: 251-269.

Caswell, H. (1989). Matrix Population Models. Sinauer Associates, Sunderland, Mass., USA.

Снавот, B.F. \& Hicks, D.J. (1982). The ecology of leaf lifespans. Annual Review of Ecology and Systematics 13: 229-259.

Channell, R \& Lomolino, M.V. (2000). Dynamic biogeography and conservation of endangered species. Nature 403: 84-96.

ChaO, A. (1984). Non-parametric estimation of the number of classes in a population. Scandinavian Journal of Statistics 11: 265-270. Cited in Condit et al. (1996b). 
Chen, T.H., Henderson-Sellers, A., Milly, P.C.D., Pitman, A.J., BeljaArs, A.C.M., Abramopolous, A.F., Boone, A., Chang, S, Chen, F., Dai, Y., Desborough, C.E., Dickinson, R.E., DuMENIL, L., EK, M., GARRATt, J.R., GedNEY, N., GuSEV, Y.ML, KiM, J., Koster, R., Kowalczyk, E.A., Laval, K., Lean, J., Lettenmaier, D., Liang, X., Mahfouf, J.-F., Mengelkamp, H.-T., Nasonova, O.N., Noilhan, J., Polcher, J., Robock, A., Rosenzweig, C., SchaAke, J., Schlosser, C.A., Schulz, J.-P., ShaO, Y., Shmakin, A.B., Verseghy, D., Wetzel, P., Wood, E.F., Xue, Y., Yang, Z.-L. \& Zeng, Q. (1997). Cabauw experiment results from the project for intercomparison of land-surface parameterization schemes (PILPS). Journal of Climate 10(6):1194-1215.

Churchill, S.P., BAlslev, H., Forero, E. \& Luteyn, J. (1995). Biodiversity and conservation of Neotropical montane forests. New York Botanical Garden, New York, USA.

CIC (1996). Research projects: Progress in 1995. Climatic model intercomparisons. [online]. Climate Impacts Centre, Sydney, Australia. Available from: http://cic.mq.edu.au/htdocs_96/ GCM_comp.html. [accessed 26 August 1999].

CIFOR (1996). DOMAIN v. 1.3 for Windows. Center for International Forestry Research, Indonesia.

CinTRA, R. (1997a). A test of the Janzen-Connell model with two common tree species in Amazonian forest. Journal of Tropical Ecology 13(5): 641-658.

CinTRA, R. (1997b). Leaf litter effects on seed and seedling predation of the palm Astrocaryum murumuru and the legume tree Dipteryx micrantha in Amazonian forest. Journal of Tropical Ecology 13: 709-725.

Clark, D.A. \& Clark, D.B. (1992). Life history diversity of canopy and emergent trees in a neotropical rain-forest. Ecological Monographs 62(3): 315-344.

Clark, D.A. \& Clark, D.B. (1994). Climate-induced annual variation in canopy tree growth in a Costa Rican tropical rain forest. Journal of Ecology 82(4): 865-872.

ClARK, D.B. \& CLARK, D.A. (1996). Abundance, growth and mortality of very large trees in neotropical lowland rain forest. Forest Ecology and Management 80(1-3): 235-244.

Clark, J.S., LEWIS, M. \& HoRVATH, L. (2001). Invasion by extremes: Population spread with variation in dispersal and reproduction. American Naturalist 157(5): 537-554.

Clark LABS (1999). IDRISI for Windows API. [online]. Clark University, Worcester, MA, USA. Available from: http://www.clarklabs.org/13dnlds/13dnlds.htm [accessed 19 February 2000].

Clement, C.R. (1999). 1492 and the loss of Amazonian crop genetic resources. I. The relation between domestication and human population decline. Economic Botany 53(2): 188-202.

Cleveland, W.S. (1979). Robust locally weighted regression and smoothing scatterplots. Journal of the American Statistical Association 74: 829-836.

CLIFF, A.D. \& ORD, J.K. (1981). Spatial processes: models and applications. Pion Ltd. 
Clinebell, R.R. II, Phillips, O.L., Gentry, A.H., Stark, N. \& Zuuring, H. (1995). Prediction of Neotropical tree and liana species richness from soil and climatic data. Biodiversity and Conservation 4: 56-90.

Cochrane, M.A. \& Schulze, M.D. (1998). Forest fires in the Brazilian Amazon. Conservation Biology 12(5): 948-950.

ColinvauX, P.A. (1998). A new vicariance model for Amazonian endemics. Global Ecology and Biogeography Letters 7(2): 95-96.

Colinvaux, P.A., Bush, M.B., Steinitz-Kannan, M. \& Miller, M.C. (1997). Glacial and postglacial pollen records from the Ecuadorian Andes and Amazon. Quaternary Research 48(1): 69-78.

Colinvaux, P.A., De Oliveira, P.E., Moreno, J.E., Miller, M.C. \& Bush, M.B. (1996). A long pollen record from lowland Amazonia: forest and cooling in glacial times. Science 274: 85-88.

Collingham, Y., Hill, M.O. \& Huntley, B. (1996). The migration of sessile organisms: a simulation model with measurable parameters. Journal of Vegetation Science 7: 831-846.

COLlins, M. (2000a). The El Niño-Southern Oscillation in the second Hadley Centre coupled model and its response to greenhouse warming. Journal of Climate 13(7): 1299-1312.

COLLINS, M. (2000b). Understanding uncertainties in the response of ENSO to greenhouse warming. Geophysical Research Letters 27(21): 3509-3512.

Collins, M. (2001a). Climate change in the tropical Pacific: understanding and quantifying uncertainties. [online]. IGBP newsletter $\underline{47}$. Available from: http://www.met.reading.ac.uk/ $\sim$ mat/igbp/igbp.html [accessed 15 January 2002]

Collins, M. (2001b). Climate change in the tropical Pacific: understanding and resolving uncertainties. [online]. Presented to: Open Science Conference. Challenges of a Changing Earth. 10-13 July 2001. Available from: http://www.sciconf.igbp.kva.se/files/Presentations/ A3.6_Collins.PPT [accessed 15 January 2002].

CONDIT, R. (1996). Defining and mapping vegetation types in mega-diverse tropical forest. Trends in Ecology and Evolution 11(1): 4-5.

CONDIT, R. (1998). Ecological implications of changes in drought patterns: Shifts in forest composition in Panama. Climatic Change 39(2-3): 413-427.

Condit, R., Hubbell, S.P. \& Foster, R.B. (1995). Mortality rates of 205 neotropical tree species and the responses to a severe drought. Ecological Monographs $\underline{65}$ (4): 419-439.

CONDIT, R., HubBEll, S.P. \& Foster, R.B. (1996a). Assessing the response of plant functional types to climatic change in tropical forests. Journal of Vegetation Science 7(3): 405-416.

Condit, R., Hubbell, S.P. \& Foster, R.B. (1996b). Changes in tree species abundance in a neotropical forest - impact of climate change. Journal of Tropical Ecology 12(2): 231-256. 
Condit, R., Hubbell, S.P., Lafrankie, JR., J.V., Sukumar, R., Manokaran, N., Foster, R.B. \& ASHTON, P.S. (1996c). Species-area and species-individual relationships for tropical trees - a comparison of 3 50-ha plots. Journal of Ecology 84: 549-562.

ConNell, J.H. (1978). Diversity of tropical rain forests and coral reefs. Science 199: 13021310 .

CONSERVATION INTERNATIONAL (1991). Workshop 90: Biological Priorities for Conservation in Amazonia (map and explanatory text). C.I., Washington DC, USA. Cited in Kress (1998).

CoOK, R.E. (1985). Growth and development in clonal plant populations. In: Population Biology and Evolution of Clonal Organisms (eds. J.B.E. Jackson, L.W. Buss \& R.E. Cook). Yale University Press, New Haven, USA: 259-296. Cited in Caswell (1989).

CostA, M.H. \& Foley, J.A. (1997). Water balance of the Amazon Basin: Dependence on vegetation cover and canopy conductance. Journal of Geophysical Research - Atmospheres 102(D20): 23973-23989.

Cox, P.M., Betts, R., Bunton, C., Essery, R., Rowntree, P.R. \& SMith, J. (1999). The impact of new land surface physics on the GCM simulation of climate and climate sensitivity. Climate Dynamics 15: 183-203.

Cramer, W., Bondeau, A., Woodward, F.I., Prentice, I.C., Betts, R.A., Brovkin, V., CoX, J., Fisher, V., Foley, J.A., Friend, A.D., KuCharik, C., LOMAS, M.R., RamankutTy, N., Sitch, S., SMith, B., White, A. \& Young-Molling, C. (2001). Global response of terrestrial ecosystem structure and function to $\mathrm{CO}_{2}$ and climate change: results from six dynamic global vegetation models. Global Change Biology 7(4): 357-373.

CRAWLEY, M.J. (1993). GLIM for Ecologists. Blackwell Scientific Publications, Oxford, UK.

CRFG (1995). CRFG Publications 1969-1989. [online]. California Rare Fruit Growers,

California, USA. Available from: http://www.crfg.organism/fg/xref/index.html. Accessed 14 November 2000.

CROAT, T.B. (1978). Flora of Barro Colorado Island. Stanford University Press, Stanford, CA, USA.

Crowley, P.H. (1992). Resampling methods for computation-intensive data analysis in ecology and evolution. Annual Review of Ecology and Systematics 23: 405-447.

CRU (1999). The Hadley Centre's Third Generation Coupled Ocean-Atmosphere GCM: HadCM3. [online]. Climatic Research Unit, Norwich, U.K. Available from: http://www.cru.uea.ac.uk/link. [accessed 26 August 1999].

Cubasch, U., Hasselmann, K., Höck, H., Maier-Reimer, E., Mikolajewicz, U., SANTER, B.D. \& SAUSEN, R. (1992). Time-dependent greenhouse warming computations with a coupled ocean-atmosphere model. Climate Dynamics $\underline{8}$ : 55-69.

Cubasch, U., Waszkewitz, J., Hegerl, G. \& Perlwitz, J. (1995). Regional climate changes as simulated in time-slice experiments. Climatic Change 31: 273-304. 
Culf, A.D., Fisch, G., LeAn, J. \& POlChER, J. (1998). A comparison of Amazonian climate data with general circulation model simulations. Journal of Climate 11(11): 2764-2773.

CURE, J.D. \& ACOCK, B. (1986). Crop responses to carbon dioxide doubling: a literature survey. Agricultural and Forest Meteorology 38: 127-145. Cited in Parry (1990).

DARnell, J., Lodish, H. \& BALtimore, D. (1990). Molecular Cell Biology. Scientific American Books, W.H.Freeman \& Co., New York, USA.

DA CRuZ Alencar, J. (1994). Fenologia de cinco espécies arbóreas tropicais de Sapotaceae correlacionada a variáveis climáticas na Reserva Ducke, Manaus. Acta Amazonica 24: 161182.

DA SiLVA, M.N.F. \& PATTON, J.L. (1998). Molecular phylogeography and the evolution and conservation of Amazonian mammals. Molecular Ecology 7: 475-486.

DAVIS, A.J., Jenkinson, L.S., LAWTON, J.H., SHORROCKS, B. \& WoOD, S. (1998a). Making mistakes when predicting shifts in species range in response to global warming. Nature 391(6669): 783-786.

Davis, A.J., Lawton, J.H., Shorrocks, B. \& Jenkinson, L.S. (1998b). Individualistic species responses invalidate simple physiological models of community dynamics under global environmental change. Journal of Animal Ecology 67: 600-612.

DE ARAÚJO, F.D. (1994). The ecology, ethnobotany and management of Caryocar brasiliense Camb. around Montes Claros, MG, Brazil. Oxford Forestry Institute, Oxford, UK. (DPhil thesis).

DE GRAAF, N.R., POELS, R.L.H. \& VAN ROMPAEY, R.S.A.R. (1999). Effect of silvicultural treatment on growth and mortality of rainforest in Surinam over long periods. Forest Ecology and Management 124: 123-135.

DE Groot, R., Ketner, P. \& OvaA, A.H. (1995). Selection and use of bio-indicators to assess the possible effects of climate change in Europe. Journal of Biogeography 22: 935-943.

DELICH, L.V. \& GREER, P. (1996). Itaipu Dam (OTOMI Case). [online]. Trade Environment Database, The School of International Service, American University, Washington DC, USA. Available from: http://www.american.edu/projects/mandala/TED/ITAIPU.HTM. [accessed 17 January 2002].

DENEVAn, W.M. (1992). The pristine myth: the landscape of the Americas in 1492. Annals of the Association of American Geographers 82(3) 369-385.

DiAmOND, J. (1986). The design of a nature reserve system for Indonesian New Guinea. In: Conservation Biology: the science of scarcity and diversity (ed. M.E. Soule). Sinauer Associates, Sunderland, Mass., USA: 485-503.

DiAmond, J. (1998). Guns, Germs and Steel. A Short History of Everybody for the Last 13,000 years. Vintage Books, New York, USA. 
DíAZ, S. \& CABIDO, M. (1997). Plant functional types and ecosystem function in relation to global change. Journal of Vegetation Science $\underline{8}$ : 463-474.

DíAZ, S., Fraser, L.H., Grime, J.P. \& FALCZUK, V. (1998). The impact of elevated $\mathrm{CO}_{2}$ on plant-herbivore interactions: experimental evidence of moderating effects at the community level. Oecologia 117(1-2): 177-186.

DICK, C.W. (2001). Genetic rescue of remnant tropical trees by an alien pollinator. Proceedings of the Royal Society of London Series B-Biological Sciences 268(1483): 2391-2396.

Dickinson, R.E. Henderson-Sellers, A., Kennedy, P.J. \& Wilson, M.F. (1986). Biosphere-Atmosphere Transfer Scheme (BATS) for the NCAR Community Climate Model. Tech. Note TN275+STR, National Center for Atmospheric Research, Boulder, CO, USA.

DiETRICH, W.E., WindSOR, D.M. \& DUNNE, T. (1982). Geology, climate and hydrology of Barro Colorado Island. In: The Ecology of a Tropical Forest: Seasonal Rhythms and LongTerm Changes (eds. E.G. Leigh, Jr., A.S. Rand \& D.M. Windsor) Smithsonian Institution Press, Washington DC, USA.

DinIZ-FILHO, J.A.F. \& BINI, L.M. (1994). Space-free correlation between morphometric and climatic data: a multivariate analysis of Africanized honey bees (Apis mellifera L.) in Brazil. Global Ecology and Biogeography Letters 4: 195-202.

DYER, J.M. (1995). Assessment of climatic warming using a model of forest species migration. Ecological Modelling 79: 199-219.

EAMUS, D. (1991). The interaction of rising $\mathrm{CO}_{2}$ and temperatures with water use efficiency. Plant Cell and Environment 14: 843-852.

EASTMAN, J.R. (1997). IDRISI for Windows User's Guide Version 2.0. Clark University, Worcester, MA, USA.

ELLISON, A.M. \& BEDFORD, B.L. (1995). Response of a wetland vascular plant community to disturbance: a simulation study. Ecological Applications 5: 109-123.

EMBRAPA. (2000A). Herbarium records. Unpublished. Empresa Brasileira de Pesquisa Agropecuária herbarium, Belem, Brazil [accessed March 2000].

EMBRAPA. (2000B). BRAHMS database. Unpublished. Empresa Brasileira de Pesquisa Agropecuária herbarium, Belém, Brazil. [accessed March 2000].

EOS-AMAZON PROJECT (1998). EOS Amazon Data Information System. University of Washington, Washington DC, USA. Unpublished.

ERIKSON, O. \& BREMER, B. (1992). Pollination systems, dispersal models, life forms and diversification rates in angiosperm families. Evolution 46: 258-266.

Evans, G.C., Bainbridge, R. \& RACKHAM, O. (1974). Light as an Ecological Factor II. Blackwell Scientific Publications, Oxford, UK. 
FAITH, D.P. \& WALKER, P.A. (1996). How do indicator groups provide information about the relative biodiversity of different sets of areas?: on hotspots, complementarity and patternbased approaches. Biodiversity Letters $\underline{3}$ : 18-25.

Farrar, C.D., Sorey, M.L., Evans, W.C., Howle, J.F., Kerr, B.D., Kennedy, B.M., King, C.-Y. \& SOUTHON, J.R. (1995). Forest-killing diffuse $\mathrm{CO}_{2}$ emission at Mammoth Mountain as a sign of magmatic unrest. Nature 376(6542): 675-678.

FEARNSIDE, P.M. (1995a). Global warming response options in the Brazil forest sector comparison of project-level costs and benefits. Biomass and Bioenergy $\underline{8}(5)$ : 309-322.

FEARNSIDE, P.M. (1995b). Hydroelectric dams in the Brazilian Amazon as sources of greenhouse gases. Environmental Conservation 22(1): 7-19.

FEARNSIDE, P.M. (1995c). Potential impacts of climate change on natural forests and forestry in Brazilian Amazonia. Forest Ecology and Management 78: 51-70.

Federer, C.A., VÖrÖSMARTY, C. \& FeKETE, B. (1996). Intercomparison of methods for calculating potential evaporation in regional and global water balance models. Water Resources Research 32 (7): 2315-2321.

FERREIRA, L.V. \& PrANCE, G.T. (1998). Structure and species richness of low diversity floodplain forest on the Rio Tapajós, Eastern Amazonian Brazil. Biodiversity and Conservation 7(5): 585-596.

FIELDING, A.H. \& BELL, J.F. (1997). A review of methods for the assessment of prediction errors in conservation presence/absence models. Environmental Conservation 24(1): 38-49.

FINEGAN, B. (1996). Pattern and process in Neotropical secondary rain forests: the first 100 years of succession. Trends in Ecology and Evolution 11(3): 119-124.

Finegan, B., CAMACHO, M. \& ZAMORA, N. (1999). Diameter increment patterns among 106 tree species in a logged and silviculturally treated Costa Rican rain forest. Forest Ecology and Management 121(3): 159-176.

Fischlin, A. \& GYALISTRAS, D. (1997). Assessing the impacts of climatic change on forests in the Alps. Global Ecology and Biogeography Letters $\underline{6}(1):$ 19-37.

FLENLEY, J.R. (1998). Tropical forests under the climates of the last 30,000 years. Climatic Change 39(2-3): 177-197.

Foley, J.A., Levis, S., Prentice, I.C., Pollard, D., \& Thompson, S.L. (1998). Coupling dynamic models of climate and vegetation. Global Change Biology 4(5): 561-579.

Fragoso, J.M.V. (1997). Tapir-generated seed shadows: scale-dependent patchiness in the Amazon rain forest. Journal of Ecology 85(4): 519-529.

FRIEND, A.D., Shugart, H.H. \& RUNNING, S.W. (1993). A physiology-based gap model of forest dynamics. Ecology 74(3): 792-797.

FulÉ, P.Z. \& Covington, W.W. (1999). Fire regime changes in La Michilía Biosphere Reserve, Durango, Mexico. Conservation Biology 13(3): 640-652. 
FundaÇão InStituto Brasilieiro De GeOgrafia E EstatístiCa (1995). Amazônia Legal. 1:3,000,000 Diretoria de Geociências, Fundação Instituto Brasilieiro de Geografia e Estatística, Ministério do Planejamento e Orçamento, Brazil.

Garcia Collevatti, R. Grattaplagia, D. \& Hay, J.D. (1997). Effects of habitat fragmentation on genetic variability and conservation of the Brazilian "cerrado" tree species, Caryocar brasiliense Camb. (Caryocaraceae): preliminary results. In: Tropical Diversity Origins, Maintenance and Conservation: Symposium of ATB and OTS. [online] Association for Tropical Botany \& Organization for Tropical Studies, San José, Costa Rica. Available from: http://www.ots.duke.edu/en/atb/iatb0013.htm. [accessed 17 May 1999].

GARWOOD, N.C. (1983). Seed germination in a seasonal tropical forest in Panama: a community study. Ecological Monographs 53 (2): 159-181

GASTON, K.J. (1994). Biodiversity measurement. Progress in Physical Geography 18(4): 565574. Cited in Williams et al. (1994).

GASTON, K.J. \& BLACKBURN, T.M. (1999). A critique for macroecology. Oikos 84(3): 353-368.

GASTON, K.J., BLACKBURN, T.M. \& SPICER, J.I. (1998). Rapoport's rule: time for an epitaph? Trends in Ecology and Evolution 13: 70-74.

GATES, W.L. (1992). AMIP: The Atmospheric Model Intercomparison Project. Bulletin of the American Meteorological Society 73: 1962-1970.

GENTRY, A.H. (1982). Neotropical floristic diversity: phytogeographical connections between Central and South America, Pleistocene climatic fluctuations, or an accident of Andean orogeny? Annals of the Missouri Botanical Garden 69: 557-593. Cited in Rose (1996), Gentry (1992).

GENTRY, A.H. (1988). Tree species richness of upper Amazon forests. Proceedings of the National Academy of Science, USA 85: 156-159.

GENTRY, A.H. (1992). Tropical forest biodiversity: distributional patterns and their conservational significance. Oikos 63: 19-28.

GENTRY, A.H. (1996). A field guide to the families and genera of Woody Plants of Northwest South America. Chicago University Press, Chicago, USA.

GENTRY, A.H. (1998a). Alwyn H. Gentry forest transect data. [online]. Missouri Botanical Garden, St. Louis, USA. Available from: http://www.mobot.org/MOBOT/research/ applied_research/gentry.html. [accessed 17 January 2002].

GENTRY, A.H. (1998b). The Alwyn H. Gentry Bignoniaceae Specimen Dataset. [online] Missouri Botanical Garden, St. Louis, USA. http://www.mobot.org/MOBOT/research/gentry/ welcome.html. [accessed 16 January 2002].

Gentry, A.H. \& Terborgh, J.W. (1990). Composition and dynamics of the Cocha Cashu 'mature' floodplain forest. In: Four Neotropical Rainforests (ed. A.H. Gentry). Yale University Press, New Haven, USA: 542-564. 
GENTRY, A.H. (ed). (1990). Four Neotropical Rainforests. Yale University Press, New Haven, USA.

Gignoux, J., Clobert, J. \& Menaut, J.-C. (1997). Alternative fire resistance strategies in savanna trees. Oecologia 110(4): 576-583.

GILLESPIE, T.W. (1999). Life history characteristics and rarity of woody plants in tropical dry forest fragments of Central America. Journal of Tropical Ecology 15: 637-649.

Gillison, A.N. \& CARPENTER, G. (1997). A generic plant functional attribute set and grammar for dynamic vegetation description and analysis. Functional Ecology 11(6): 775-783.

GIORGI, F. \& FRANCISCO, R. (2000). Uncertainties in regional climate prediction: a regional analysis of ensemble simulations with the HADCM2 coupled AOGCM. Climate Dynamics 16 : 169-182.

Goldammer, J.G. \& PRICE, C. (1998). Potential impacts of climate change on fire regimes in the tropics based on MAGICC and a GISS GCM-derived lightning model. Climatic Change 39(2-3): 273-296.

Goldsmith, F.B., Harrison, C.M. \& Morton, A.J. (1986). Description and analysis of vegetation. In Methods in Plant Ecology (2nd edition), (eds. P.D. Moore \& S.B. Chapman). Blackwell Scientific Publications, Oxford, UK: 437-524.

Goodess, C.M. \& PALUTIKOF, J.P. (1992). The development of regional climate scenarios and the ecological impact of greenhouse gas warming. Advances in Ecological Research 22: 3362.

Gorchov, D.L., Cornejo, F., Ascorra, C. \& Jaramillo, M. (1993). The role of seed dispersal in the natural regeneration of rain forest after strip-cutting in the Peruvian Amazon. Vegetatio 107/108: 339-349.

GORDON, H.B. \& O'FARRELL, S.P. (1997). Transient climate change in the CSIRO coupled model with dynamic sea ice. Monthly Weather Review 125: 875-907.

Gordon, C., CoOper, C., Senior, C.A., BAnks, H., Gregory, J.M., Johns, T.C., Mitchell, J.F.B. \& WOOD, R.A. (2000). The simulation of SST, sea ice extents and ocean heat transports in a version of the Hadley Centre coupled model without flux adjustments. Climate Dynamics 16(2-3): 147-168.

Gourlet-Fleury, S., BAR-Hen, A. \& FAUVET, N. (1999). The silvicultural experimental plots of Paracou in French Guiana: example of use and limits of the available data for developing individual growth models. In: Long-Term Observations and Research in Forestry. Proc. IUFRO S4.11 International Symposium, held at CATIE, Costa Rica, Feb. 23-27 1999.

GOWER, J.C. (1971). A general coefficient of similarity and some of its properties. Biometrics 27: 857-871.

GRIME, J.P. (1977). Evidence for the existence of three primary strategies in plants and its relevance to ecological and evolutionary theory. American Naturalist 111: 1169-1194. 
Grime, J.P. \& Callaghan, T.V. (1987). Direct and Indirect Effects of Climate Change on Plant Species, Ecosystems and Processes of Conservation and Amenity Interest. Contract report to the Department of the Environment, Sheffield, UK: 26 pp. Cited in de Groot et al. (1995).

Grime, J.P., Hodgson, J.G. \& HUNT, R. (1988). Comparative Plant Ecology: a Functional Approach to Common British Plants. Chapman \& Hall, London, UK: 742 pp.

GrIMM, A.M., FERRAZ, S.E.T. \& GOMES, J. (1998). Precipitation anomalies in Southern Brazil associated with El Niño and La Niña events. Journal of Climate 11(ii): 2863-2880.

GroOmBrIDGE, B. (1992). Global Biodiversity. Chapman and Hall, London, UK: 542 pp.

Guehl, J.M., Domenach, A.M., Bereau, M., Barigah, T.S., Casabianca, H., Ferhi, A. \& GARBAYE, J. (1998). Functional diversity in an Amazonian rainforest of French Guyana: a dual isotope approach (delta N-15 and delta C-13). Oecologia 116(3): 316-330.

GueVARA, S. \& LABORDE, J. (1993). Monitoring seed dispersal at isolated standing trees in tropical pastures: consequences for local species availability. Vegetatio 107/108: 319-338.

HACKETT, C. \& VANCLAY, J.K. (1998). Mobilizing expert knowledge of tree growth with the PLANTGRO and INFER systems. Ecological Modelling 106(2-3): 233-246.

HAFFER, J. (1987). Quaternary history of tropical America. In Biogeography and quaternary history in tropical America (eds. G.T. Prance \& T.C. Whitmore). Clarendon Press, Oxford, UK: 1-18.

HAFFER, J. (1997). Alternative models of vertebrate speciation in Amazonia: An overview. Biodiversity and Conservation 6 (3): 451-476.

HALPIN, P.N. (1997). Global climate change and natural area protection: management responses and research directions. Ecological Applications 7(3): 828-843.

HAMMOND, D.S. \& BROWN, V.K. (1995). Seed size of woody plants in relation to disturbance, dispersal, soil type in wet Neotropical forests. Ecology 76 (8): 2544-2561.

HANSEN, B. (1980). Balanophoraceae. Flora Neotropica Monograph 23. Organisation for Flora Neotropica/New York Botanical Garden, New York, USA.

HARTSHORN, G.S. (1992). Possible effects of global warming on the biological diversity in tropical forests. In Global Warming and Biodiversity (eds. R.L. Peters \& T.E. Lovejoy). Yale University Press, New Haven, USA: 137-146.

HATTENSCHWILER, S. \& KöRNER, C. (1996). Effects of elevated $\mathrm{CO}_{2}$ and increased nitrogen deposition on photosynthesis and growth of understory plants in spruce model ecosystems. Oecologia 106(2): 172-180.

Heithaus, E.R. \& Fleming, T.H. (1978). Foraging movement of a frugivorous bat, Carollia perspicillata (Phyllostomatidae). Ecological Monographs 48: 127-143.

Henderson, A. (1995). The Palms of the Amazon. WWF/Oxford University Press, Oxford, UK. 
Henderson, A., Galeano, G. \& Bernal, R. (1995). Field Guide to the Palms of the Americas. Harper-Collins, New York, USA.

HENDERSON-SELLERS, A. (1993). Continental vegetation as a dynamic component of a global climate model: a preliminary assessment. Climatic Change 23: 337-377.

HENDERSON-SELlERs, A. \& MCGuFFIE, K. (1995). Global climate models and 'dynamic' vegetation changes. Global Change Biology 1: 63-75.

Hendrey, G.R., Ellsworth, D.S., Lewin, K.F. \& NAGY, J. (1999). A free-air enrichment system for exposing tall forest vegetation to elevated atmospheric $\mathrm{CO}_{2}$. Global Change Biology 5 (3): 293-309.

HEYwOOD, V.H. (1993). Flowering Plants of the World. B.T. Batsford, London, UK: 335 pp.

HILl, M.O., BUNCE R.G.H. \& SHAW M.W. (1975). Indicator species analysis, a divisive polythetic method of classification and its application to a survey of native pinewoods in Scotland. Journal of Ecology 63: 597-613.

HILL, M.O. (1991). Patterns of species distribution in Great Britain elucidated by canonical correspondence analysis. Journal of Biogeography 18: 247-255.

HILL, R.S., READ, J. \& BUSBY, J.R. (1988). The temperature-dependence of photosynthesis of some Australian temperate rainforest trees and its biogeographical significance. Journal of Biogeography 15: 431-449.

Hirst, A.C., Gordon, H.B., AND O'FARrell, S.P. (1996). Global warming in a coupled climate model including oceanic eddy-induced advection. Geophysical Research Letters 23:3361-3364.

HofFMAN, W.A. (1998). Post-burn reproduction of woody plants in a neotropical savanna: the relative importance of sexual and vegetative reproduction. Journal of Applied Ecology 35(3): 422-433.

Hogan, K.P., Smith, A.P. \& Zuska, L.H. (1991). Potential effects of elevated $\mathrm{CO}_{2}$ and changes in temperature on tropical plants. Plant Cell and Environment 14(8): 763-778.

HÖGMANDER, H. \& MølleR, J. (1995) Estimating distribution maps from atlas data using methods of statistical image analysis. Biometrics 51: 393-404.

HOLDRIDGE, L.F. (1947). Determination of world plant formations from simple climatic data. Science 105 (2727): 367.f

HOLDRIDGE, L.R. (1962). The determination of atmospheric water resources. Ecology 43: 1-9. HoLT, R.D. (1990). The microevolutionary consequences of climatic change. Trends in Ecology and Evolution 5(9): 311-315.

HOPKINS, M. (ed.) (2000). Database of specimens identified from the Reserva Ducke. Unpublished. INPA, Manaus, Brazil. [accessed April 2000].

HORN, M.H. (1997). Evidence for dispersal of fig seeds by the fruit-eating characid fish Brycon guatemalensis Regan in a Costa Rican tropical rain forest. Oecologia 109: 259-264. 
Houghton, J.T., Meira Filho, L.G., Callander, B.A., Harris, N., Kattenberg, A. \& MASKELl, K. (1996). Climate change 1995 : The Science of Climate Change. Contribution of Working Group I to the second assessment report of the Intergovernmental Panel on Climate Change. Cambridge University Press, Cambridge, UK.

Houghton, J.T., Ding, Y., Griggs, D.J., Noguer, M., VAn Der Linden, P.J., DAi, X., Maskell, K. \& Johnson, C.A. (eds) (2001). Climate Change 2001: The Scientific Basis. Contribution of Working Group I to the Third Assessment Report of the Intergovernmental Panel on Climate Change. [online] Cambridge University Press, Cambridge, UK. Available from http://www.ipcc.ch/pub/tar/wg1/index.htm. [accessed 10 November 2001].

Howe, H.F. \& SMALlwood, J. (1982). Ecology of seed dispersal. Annual Review of Ecology and Systematics 13: 201-228.

Hoyt, D.V. \& Schatten, K.H. (1997). The Role of the Sun in Climate Change. Oxford University Press, Oxford, UK.

Hsiao, T.C., Acevedo, E., Fereres, E. \& Henderson, D.W. (1976). Water stress, growth and osmotic adjustment. Philosophical Transactions of the Royal Society of London. Series B 273: 479-500. Cited in Wright (1991).

HuAnte, P., Rincon, E. \& Chapin, F.S. III (1998). Foraging for nutrients, responses to changes in light, and competition in tropical deciduous tree seedlings. Oecologia 117(1-2): 209-216.

HuBBELL, S.P. (1997). A unified theory of biogeography and relative species abundance and its application to tropical rain forests and coral reefs. Coral Reefs 16(SS): 9-21.

HubBell, S.P. \& FosTER, R.B. (1986). Commonness and rarity in a Neotropical forest: implications for tropical tree conservation. In: Conservation Biology: the science of scarcity and diversity (ed. M.E. Soule). Sinauer Associates, Sunderland, Mass., USA: 205-231.

HuBbLE, S.P. \& FOSTER, R.B. (1990). Structure, dynamics and equilibrium status of old-growth forest on Barro Colorado Island. In: Four Neotropical Rainforests (ed. A.H. Gentry). Yale University Press, New Haven, USA: 522-541.

Hubbell, S.P., Foster, R.B., O’Brien, S.T., Harms, K.E., Condit, R., WeChSler, B., WRIGHT, S.J. \& LOO DE LAO, S. (1999). Light-gap disturbances, recruitment limitation, and tree diversity in a Neotropical forest. Science 283: 554-557.

Hulme, M., OsBorn, T.J. \& JOHnS, T.C. (1998). Precipitation sensitivity to global warming: comparison of observations with HadCM2 simulations. Geophysical Research Letters 25: 3379-3382. Cited in Cramer et al. (2001).

Hulme, M., Mitchell, J., Ingram, W., Lowe, J., Johns, T., New, M. \& Viner, D. (1999). Climate change scenarios for global impacts studies. Global Environmental Change 9: S3S19. 
Hulme, M. \& Viner, D. (1995). A Climate Change Scenario for Assessing the Impact of Climate Change on Tropical Rain Forests. CRU-WWF(US) Report. CRU, Norwich, UK: 34 pp.

Huntley, B. \& BIRKS, H.J.B. (1983). An Atlas of Past and Present Pollen Maps for Europe: 0 -13000 BP. Cambridge University Press, Cambridge, UK.

Huntley, B., Berry, P.M., Cramer, W. \& McDonald, A.P. (1995). Modelling present and potential future ranges of some European higher plants using climate response surfaces. Journal of Biogeography 22: 967-1001.

HunTingFORD, C. \& COX, P.M. (2000). An analogue model to derive additional climate change scenarios from existing GCM simulations. Climate Dynamics 16: 575-586.

HUTCHINSON, G.E. (1957). Concluding remarks. Cold Spring Harbor Symposia on Quantitative Biology 22: 65-71.

INBIO (1998-2000). Biodiversity Information Management System, Costa Rica. [online]. INBIO, Costa Rica. Accessed from http://www.inbio.ac.cr/bims/. Now available from: http://atta.inbio.ac.cr/ (accessed 16/01/02).

INPA (2000a). Herbarium records. Unpublished. Instituto Nacional de Pesquisas da Amazônia herbarium, Manaus, Brazil. [accessed April 2000].

INPA (2000b). BRAHMS database. Unpublished. Instituto Nacional de Pesquisas da Amazônia herbarium, Manaus, Brazil. [accessed April 2000].

Jalas, J., SuOMinen, J. \& LAmpinen, R. (1972-1996). Atlas Florae Europaeae Vols. 1-11. Societas Biologica Fennica Vanamo, Helsinki, Finland.

JANZEN, D.H. (1967). Why mountain passes are higher in the tropics. American Naturalist 102: 297-306.

JEFFREE, C.E. \& JEFFREE, E.P. (1996). Redistribution of the potential geographical ranges of Mistletoe and Colorado Beetle in Europe in response to the temperature component of climate change. Functional Ecology 10: 562-577.

Johns, T.C., Carnelll R.E., Crossley, J.F., Gregory, J.M., Mitchell, J.F.B., Senior, C.A., TetT, S.F.B. \& Wood, R.A. (1997). The second Hadley Centre coupled oceanatmosphere GCM: Model description, spinup and validation. Climate Dynamics 13: 103-134.

JOHNSTON, M. (1998). Tree population studies in low-diversity forests, Guyana. II. Assessments on the distribution and abundance of non-timber forest products. Biodiversity and Conservation 7: 73-86.

Jones, P.G., Beebe, S.E., Tohme, J. \& Galwey, N.W. (1997). The use of geographical information systems in biodiversity exploration and conservation. Biodiversity and Conservation 6: 947-958.

JULIEN-LAFERRIÈRE, D. (1999). Foraging strategies and food partitioning in the neotropical frugivorous mammals Caluromys philander and Potos flavus. Journal of Zoology 247: 71-80. 
KACHOLIA, K. \& RECK, R.A. (1997). Comparison of global climate change simulations for 2 x $\mathrm{CO}_{2}$-induced warming. An intercomparison of 108 temperature change projections published between 1980 and 1995. Climatic Change 35: 53-69.

KAHN, F. (1986). Life forms of Amazonian palms in relation to forest structure and dynamics. Biotropica 18(3): 214-218.

KAMMESHEIDT, L. (1998). Stand structure and spatial pattern of commercial species in logged and unlogged Venezuelan forest. Forest Ecology and Management 109(1-3).

Kattenberg, A., Giorgi, F., Grassl, H., Meehl, G.A., Mitchell, J.F.B., Stouffer, R.J., TOKioKA, T., WeAVER, A.J. \& Wigley, T.M.L. (1996). Climate models - projections of future climate. In: Climate change 1995: The Science of Climate Change. Contribution of Working Group I to the second assessment report of the Intergovernmental Panel on Climate Change (eds. J.T. Houghton, L.G. Meira Filho, B.A. Callander, N. Harris, A. Kattenberg \& K. Maskell). Cambridge University Press, Cambridge, UK.

KAUFMAN, J.B. (1991). Survival by sprouting following fire in tropical forests of the eastern Amazon. Biotropica 23: 219-224.

Kellman, M. \& Meave, J. (1997). Fire in the tropical gallery forests of Belize. Journal of Biogeography 24: 23-34.

Kellman, M., TACKaberry, R. \& RigG, L. (1998). Structure and function in two tropical gallery forest communities: implications for forest conservation in fragmented systems. Journal of Applied Ecology 35(2): 195-206.

KELlOFF, C.L. \& FUnK, V.A.. (1999). Preliminary checklist of the plants of Kaieteur National Park, Guyana [online]. Centre for the Study of Biological Diversity, University of Guyana, Georgetown, Guyana. Available from: http://mason.gmu.edu/ ckelloff/cover.html [accessed 20 October 1999].

KELLY, P.M. \& WRIGLEY, T.M.L. (1990). The influence of solar forcing trends on global mean temperatures since 1861. Nature 347: 460-462.

KENDALL, B.W. (1998). Estimating the magnitude of environmental stochasticity in survivorship data. Ecological Applications 8(1):184-193.

Kenny, G.J., Warrick, R.A., Mitchell, N.D., Mullan, A.B. \& Salinger, M.J. (1995). CLIMPACTS: an integrated model for assessment of the effects of climate change on the New Zealand environment. Journal of Biogeography 22: 835-895.

KienAst, F., WiLDI, O. \& BRZEZIECKI, B. (1998). Potential impacts of climate change on species richness in mountain forests - an ecological risk assessment. Biological Conservation 83(3): 291-305.

Killen, T.J., Jardim, A., Mamani, F. \& RoJas, N. (1998). Diversity, composition and structure of a tropical semideciduous forest in the Chiquitania region of Santa Cruz, Bolivia. Journal of Tropical Ecology 14: 803-827. 
KIM, C.P. \& ENTEKHABI, D.A. (1997). Examination of two methods for estimating regional evaporation using a coupled mixed layer and land surface model. Water Resources Research 33(9): 2109-2116.

KING, D.A. (1994). Influence of light level on the growth and morphology of saplings in a Panamanian forest. American Journal of Botany 81(8): 948-957.

KING, D.A. (1996). Allometry and life history of tropical trees. Journal of Tropical Ecology 12:25-44.

KitTEL, T.G.F., GiORGI, F. \& MeEhl, G.A. (1998). Intercomparison of regional biases and doubled $\mathrm{CO}_{2}$-sensitivity of coupled atmosphere-ocean general circulation model experiments. Climate Dynamics 14: 1-15.

KNIGHT, D.H. (1975). A phytosociological analysis of species-rich tropical forest on Barro Colorado Island, Panama. Ecological Monographs 45: 259-284.

KORNING, J. \& BALSLEV, H. (1994). Growth rates and mortality patterns of trees in Amazonian tropical rain forest in Ecuador. Journal of Vegetation Science 5: 77-86.

KREBS, C.J. (1989). Ecological Methodology. Harper-Collins, New York, USA.

Kress, W.J., Heyer, W.R., Acevedo, P., Coddington, J., Cole, D., Erwin, T.L., Meggers, B.J., Pogue, M., Thorington, R.W., Vari, R.P., Weitzman, M.J. \& Weitzman, S.H. (1998). Amazonian biodiversity: assessing conservation priorities with taxonomic data. Biodiversity and Conservation 7(12): 1577-1587.

KRICHER, J. (1997). A Neotropical Companion. Princeton University Press, Princeton, USA.

Ǩ̌IVEN, V. (1996). Optimal foraging and predator-prey dynamics. Theoretical Population Biology 49: 265-290.

KUbitzKI, K. \& RenNer, S. (1982). Lauraceae I. (Aniba and Aiouea). Flora Neotropica Monographs 31: Organisation for Flora Neotropica/New York Botanical Garden, New York, USA: $125 \mathrm{pp}$.

KÜChmeister, H., Webber, A.C., Silberbauer-Gottsberger, I. \& Gottsberger, G. (1998). A polinização a sua relação com a termogênese em espécies de Arecaceae e Annonaceae da Amazônia Central. Acta Amazonica 28(3): 217-245

Kunin, W.E. \& GASTON, K.J. (1997). The Biology Of Rarity: Causes and Consequences of Rare-Common Differences. Chapman \& Hall, London, UK.

KURSAR, T.A. (1998). Relating tree physiology to past and future changes in tropical rainforest tree communities. Climatic Change 39:363-379.

LABRAGA, J.C. \& LÓPEZ, M. (1997). A comparison of the climate response to increased carbon dioxide simulated by general circulation models with mixed- layer and dynamic ocean representations in the region of South America. International Journal of Climatology 17(15): $1635-1650$. 
LANG, G.E. \& KNIGHT, D.H. (1983). Tree growth, mortality, recruitment and canopy gap formation in a ten-year period in a tropical moist forest. Ecology 64: 1075-1080.

La Suerte Biological Field Station. (1998). List of Plant Species Identified at La Suerte. [online]. La Suerte Biological Field Station, Costa Rica. http://www.studyabroad.com/lasuerte/plantspec.html. [accessed 17 January 2002].

LAtore, J., Gould, P. \& Mortimer, A.M. (1998). Spatial dynamics and critical patch size of annual plant populations. Journal of Theoretical Biology 190(3): 277-285.

LAURANCE, W.F. (1998). A crisis in the making: responses of Amazonian forests to land use and climate change. Trends in Ecology and Evolution 13(10): 411-415.

Laurance, W.F., Cochrane, M.A., Bergen, S., Fearnside, P.M., Delamônica, P., Barber, C., D'Angelo, S. \& Fernandes, T. (2001). Environment - The future of the Brazilian Amazon. Science 291(5503): 438-439.

LAURANCE, W.F. \& Williamson, G.B. (2001). Positive feedbacks among forest fragmentation, drought, and climate change in the Amazon. Conservation Biology 15(6): 1529-1535.

LAWTON, J.H. (1999). Are there general laws in ecology? Oikos 84(2): 177-192.

Lawton, R.O., Nair, U.S., Pielke SR., R.A. \& Welch, R.M. (2001). Climatic impact of tropical lowland deforestation on nearby montane cloud forests. Science 294(5542): 584-587.

LEEMANS, R. (1992). Biodiversity and global climate change. In Global Biodiversity (ed. B. Groombridge), WCMC, Cambridge, UK.

LEEMANS, R. \& CRAMER, W.P. (1991). The IIASA Database for Mean Monthly Values of Temperature, Precipitation, and Cloudiness on a Global Terrestrial Grid. International Institute for Applied Systems Analysis, Austria.

Legates, D.R. \& WiLmotT, C.J. (1990a). Mean seasonal and spatial variability in gaugecorrected, global precipitation. International Journal of Climatology 10(2): 111-127.

LEGATES, D.R. \& WiLMOTT, C.J. (1990b). Mean seasonal and spatial variability in global surface air temperature. Theoretical and Applied Climatology 41: 11-21.

LEGENDRE, P. \& FoRTIN, M.-J. (1989). Spatial pattern and ecological analysis. Vegetatio $\underline{80}$ : 107-138.

LEGGett, J., PEPPER, W.J. \& SWART, R.J. (1992). Emissions scenarios for the IPCC: an update. In: Climate change 1992. The supplementary report to the IPCC scientific assessment (eds. J.T. Houghton, B.A. Callander \& S.K. Varney). Cambridge University Press, Cambridge, UK: 75-95.

LEIGH, JR., E.G., RAND, A.S. \& WindSOR, D.M. (1996). The Ecology of a Tropical Forest: Seasonal Rhythms and Long-Term Changes. Smithsonian Institution Press, Washington, USA. 
LENIHAN, J.M. \& NEILSON, R.P. (1995). Canadian vegetation sensitivity to projected climatic change at three organizational levels. Climatic Change 30: 27-56.

LeOPOldo, P.R., Franken, W.K. \& Villa Nova, N.A. (1995). Real evaporation and transpiration through a tropical rain forest in central Amazonia as estimated by the water balance method. Forest Ecology and Management 73(1-3): 185-195.

LEPSCH-CUNHA, N. \& MORI, S.A. (1999). Reproductive phenology and mating potential in a low density tree population of Couratari multiflora (Lecythidaceae) in central Amazonia. Journal of Tropical Ecology 15:97-121.

LESCURE, J.P., EMPERAIRE, L. \& FRANCISCON, C.. (1992). Leopoldinia piassaba Wallace (Arecaceae): a few biological and economic data from the Rio Negro region (Brazil). Forest Ecology and Management 55: 83-86.

LeSSLIE, R.B., MACKEY, B.G. \& PREECE K.M. (1988). A computer based method of wilderness evaluation. Environmental Conservation 15(3): 225-232.

LIEBERMAN, D. \& LIEBERMAN, M. (1987). Forest tree growth and dynamics at La Selva, Costa Rica (1969-1982). Journal of Tropical Ecology 3: 347-358.

Lieberman, D., Lieberman, M., Peralta, R. \& Hartshorn, G.S. (1985). Mortality patterns and stand turnover rates in wet tropical forest in Costa Rica. Journal of Ecology 73: 915-924.

Liebhold, A.M., Xu, Z., Hohn, M.E., Elkinton, J.S., Ticehurst, M., Benzon, G.L. \& CAMPBELl, R.W. (1991). Geostatistical analysis of Gypsy Moth (Lepidoptera, Lymantriidae) egg mass populations. Environmental Entomology 20(5): 1407-1417.

Lincoln, R., Boxshall, G. \& Clark, P. (1998). A Dictionary of Ecology, Evolution and Systematics. Cambridge University Press, Cambridge, UK.

Lindenmayer, D.B., Burgman, M.A., AKÇAKaya, H.R., LaCy, R.C. \& Possingham, H.P. (1995). A review of the generic computer-programs ALEX, RAMAS/space and VORTEX for modelling the viability of wildlife metapopulations. Ecological Modelling 82: 161-174.

Listabarth, C. (1993). Pollination in Geonoma macrostachys and 3 congeners, G. acaulis, G. gracilis and G. interrupta. Botanica Acta 106(6): 496-506

LIU, J. \& AsHTON, P.S. (1995). Individual-based simulation models for forest succession and management. Forest Ecology and Management 73(1-3): 157-175.

LIU, J. \& ASHTON, P.S. (1998). FORMOSAIC: an individual-based spatially explicit model for simulating forest dynamics in landscape mosaics. Ecological Modelling 106: 177-200.

LiU, J. \& ASHTON, P.S. (1999). Simulating effects of landscape context and timber harvest on tree species diversity. Ecological Applications 9(1): 186-201.

LOEHLE, C. (1998). Height growth rate tradeoffs determine northern and southern range limits for trees. Journal of Biogeography 25(4): 735-742. 
Lord, J., Egan, J., Clifford, T., JuRAdo, E., Leishman, M., Williams, D. \& Westoby, M. (1997). Larger seeds in tropical floras: consistent patterns independent of growth form and dispersal mode. Journal of Biogeography 24: 205-211.

LugO, A.E. \& SCATENA, F.N. (1996). Background and catastrophic tree mortality in tropical moist, wet and rain forests. Biotropica 28(4a): 585-599.

Lusk, C.H., Contreras, O. \& FigueroA, J. (1997). Growth, biomass allocation and plant nitrogen concentration in Chilean temperate rainforest tree seedlings: effects of nutrient availability. Oecologia 109(1): 49-58.

LÜTTGE, U. (1997). Physiological Ecology of Tropical Plants. Springer-Verlag, Berlin, Germany: $384 \mathrm{pp}$.

MAAS, P.J.M. (1972). Costoideae (Zingiberaceae). Flora Neotropica Monograph $\underline{8}$, Organisation for Flora Neotropica/New York Botanical Garden, New York, USA.

MAAs, P.J.M., WeStRA, L.Y.Th. \& FARJON, A. (1998). Neotropical Plant Families. Koeltz Scientific Books, Koenigstein, Germany.

MACARTHUR, R.H. \& WiLsOn, E.O. (1967). The Theory of Island Biogeography. Princeton University Press, USA.

MacArthur, R.H. (1972). Geographical Ecology: Patterns in the Distribution of Species. Harper \& Row, New York, USA. Cited in Brown (1995) \& Peterson (2001).

MACKEY, B.G. (1996). The role of GIS and environmental modelling in the conservation of biodiversity. In: Third International Conference/Workshop on Integrating GIS and Environmental Modeling. January 21-25, 1996. Santa Fe, New Mexico, USA. CD-ROM. NCGIA -National Center for Geographic Information and Analysis, USA.

MALANSON, G.P. \& CAIRNS, D.M. (1997). Effects of dispersal, population delays, and forest fragmentation on tree migration rates. Plant Ecology 131(1): 67-79.

Manabe, S., Stouffer, R.J., Spelman, M.J. \& Bryan, K. (1991). Transient response of a coupled ocean-atmosphere model to gradual changes of atmospheric $\mathrm{CO}_{2}$. Part I: annualmean response. Journal of Climate 4 : 785-818.

MANLY, B.F.J. (1992). Computer intensive statistics. In: The design and analysis of research studies (ed. B.F.J. Manly). Cambridge University Press, Cambridge, UK: 286-311.

MARK, E. (1999). Weather Programs and Algorithms. Weather Algorithms Index. [online]. Available from: http://snowball.frogspace.net/js/. [accessed 15 August 2000].

Martinelli, L.A., Pessenda, L.C.R., Espinoza, E., Camargo, P.B., Telles, E.C., Cerri, C.C., Victoria, R.L., Aravena, R., Richey, J. \& Trumbore, S. (1996). Carbon-13 variation with depth in soils of Brazil and climate change during the Quaternary. Oecologia 106: 376-381. 
MATA, L.J. \& CAMPos, M. (eds) (2001). Latin America. In: Climate Change 2001: Impacts, Adaptation and Vulnerability. Contribution of Working Group II to the Third Assessment Report of the Intergovernmental Panel on Climate Change (eds. J.J. McCarthy, O.F. Canziani, N.A. Leary, D.J. Dokken \& K.S. White). Cambridge University Press, Cambridge, UK.

MAURER, B.A. (1994). Geographical Population Analysis: Tools for the Analysis of Biodiversity. Blackwell Science, Oxford, UK.

MAY, R.M. (1990). How many species? Philosophical Transactions of the Royal Society of London. Series B Biological Sciences 330: 293-304.

MAY, R.M. (1993). The effects of spatial scale on ecological questions and answers. In: LargeScale Ecology and Conservation Biology. (eds. P.J. Edwards, R.M. May, \& N.R. Webb). Blackwell Science, Oxford, UK: 1-18.

Maynou, F., Conan, G.Y., Cartes, J.E., Company, J.B. \& Sarda, F. (1996). Spatial structure and seasonality of decapod crustacean populations on the North-western Mediterranean slope. Limnology and Oceanography 41 (1): 113-125

MCANeney, K.J. \& ITIER, B. (1996). Operational limits to the Priestley-Taylor formula. Irrigation Science 17(1): 37-43.

McCarthy, J.J., Canziani, O.F., Leary, N.A., DokKen, D.J. \& White, K.S. (eds.) (2001). Climate Change 2001: Impacts, Adaptation and Vulnerability. Contribution of Working Group II to the Third Assessment Report of the Intergovernmental Panel on Climate Change. Cambridge University Press, Cambridge, UK.

McGrath, K., Gachot, R. \& Gallant, N.M. (1953). The Amazon Valley. Unasylva 7. [online edition]. The Food And Agriculture Organization, Rome, Italy. Available from: http://www.fao.org/docrep/x5368e/x5368e00.htm\#Contents. [Accessed 20 January 2002].

McGuffie, K. \& Henderson-Sellers, A. (1996). A Climate Modelling Primer. Second Edition. John Wiley \& Sons, New York, USA.

McGuffie, K., Henderson-Sellers, A., Zhang, H., Durbidge, T.B. \& Pitman, A.J. (1995). Global climate sensitivity to tropical deforestation. Global and Planetary Change 10: 97-128.

MCKenNeY, M.S. \& RoSENBERG, N.J. (1993). Sensitivity of some potential evapotranspiration estimation methods to climate change. Agricultural and Forest Meteorology 64: 81-110.

MCMahon, T.A. (1973). Size and shape in biology. Science 179: 1201-1204. Cited in King (1996).

MCPherson, G.D. (1998). A Checklist of the Fortuna Watershed, Chiriqui, Panama [online]. Missouri Botanical Garden, St. Louis, USA. http://www.mobot.org/MOBOT/Research/ fortuna.shtml. [accessed 16 January 2002]

MeEhL, G.A. \& WAShington, W.M. (1996). El Niño-like climate change in a model with increased atmospheric $\mathrm{CO}_{2}$ concentrations. Nature 382: 56-60. 
MegGers, B.J. (1994). Archaeological evidence for the impact of mega-Niño events on Amazonia during the past two millennia. Climatic Change 28(4): 321-338.

Melillo, J.M, Prentice, I.C., Farquhar, G.D., Schulze, E.-D. \& Sala., O.E. (1996). Terrestrial biotic responses to environmental change and feedbacks to climate. In: Climate change 1995 : The Science of Climate Change. Contribution of Working Group I to the second assessment report of the Intergovernmental Panel on Climate Change (eds. J.T. Houghton, L.G. Meira Filho, B.A. Callander, N. Harris, A. Kattenberg \& K. Maskell). Cambridge University Press, Cambridge, UK: 448-481.

MeltZer, D.J. (1995). Clocking the first Americans. Annual Review of Anthropology 24: 2145.

Milton, K., LACA, E.A. \& Demment, M.W. (1994). Successional patterns of mortality and growth of large trees in a Panamanian lowland forest. Journal of Ecology 82(1): 79-87.

MisHLER, B.D. (1999). Getting rid of species? In: Species: New Interdisciplinary Essays (ed. R.A. Wilson). MIT Press, Cambridge, Massachusetts, USA.

Mitchell, M. (1996). An Introduction to Genetic Algorithms. MIT Press, Cambridge, Massachusetts, USA.

Mitchell, J.F.B., Johns, T.C., GregOry, J.M. \& TeTt, S.F.B. (1995). Climate response to increasing levels of greenhouse gases and sulphate aerosols. Nature 376: 501-504.

MitCheLL, N.D. (1993). Potential greenhouse effects and terrestrial ecosystems: case studies from New Zealand. In: Conservation Biology in Australia and Oceania (eds. C. Moritz \& J. Kikkawa). Surrey Beatty \& Sons, Chipping Norton, New Zealand: 209-217.

Mitchell, N.D. \& Williams, J.E. (1996). The consequences for native biota of anthropogenic-induced climate change. In: Greenhouse. Coping with Climate Change (eds. W.J. Bouma, G.I Pearman \& M.R. Manning). CSIRO Publishing, Melbourne, Australia: 308324.

Molina MaX, J.C., De Oliveira Garrido, M.A., Cabelo Miras, A.L. \& Da Mota Bordin, R. (1998). Série Áreas Naturais: Estação Ecológica dos Caetetus [online]. Instituto Florestal, Sao Paulo, Brazil. Available from: http://www.bdt.org.br/bdt/galia/listagalia?index. [accessed 10 December 1998].

MONTEITH, J.L. (1964). Evaporation and environment. In: The state and movement of water in living organisms. 19th Symp. Soc. Exp. Biol., Academic Press, NY: 205-234.

MOORE, P.D. (1996). Biodiversity and climate change dominate environmental research. Scientist 10(23): 14 .

Morawetz, W., Henzl, M. \& WallnöFER, B. (1992). Tree killing by herbicide producing ants for the establishment of pure Tococa occidentalis populations in the Peruvian Amazon. Biodiversity and Conservation 1: 19-33. 
Mori, S.A. \& Boom, B. (1987). The Forest. Memoirs of the New York Botanical Garden 44: 929.

MPEG. (2000A). Herbarium records. Unpublished. Museu Paraense de Emilío Goeldi herbarium, Belém, Brazil. Accessed March 2000.

MPEG. (2000B). BRAHMS database. Unpublished. Museu Paraense de Emilío Goeldi herbarium, Belém, Brazil. Accessed March 2000.

MuRPhy, J.M. (1995). Transient response of the Hadley Centre coupled ocean-atmosphere model to increasing carbon dioxide. Part I: Control climate and flux adjustment. Journal of Climate 8: 36-56.

MYERS, N. (1980). Conversion of Tropical Moist Forests. National Academy of Science, Washington DC, USA. Cited in Groombridge (1992).

Myers, N., Mittermeier, R.A., Mittermeier, C.G., DA Fonseca, G.A.B. \& Kent, J. (2000). Biodiversity hotspots for conservation priorities. Nature 403(6772): 853-858.

NaKajima, J.N., SoAres Silva, L.H., Medri, M.E., Goldenberg, R., Correa, G.T. (1996). Floristic composition and phytosociological structure in a riparian forest of Tibagi river, Telemaco Borba, Parana, Brazil. [abstract] Arquivos de Biologia e Tecnologia 39(4):933-939.

NASA EOS IDS BAI (1995). Remote Sensing Science: Major Findings of Research to Date. [online]. NASA EOS Interdisciplinary Science Biosphere-Atmosphere Interactions, Berkeley Atmospheric Sciences Center, University of California, Berkeley, USA. Available from: http://www.atmos.berkeley.edu/ids/Report.95/

Results.html. [accessed 12 April 2001].

NAscimento, M.T. \& Proctor, J. (1997). Population dynamics of five tree species in a monodominant Peltogyne forest and two other forest types on Maracá Island, Roraima, Brazil. Forest Ecology and Management 94(1-3): 115-128.

NASON, J.D., ALDRICH, P.R. \& HAMRICK, J.L. (1997). Dispersal and the dynamics of genetic structure in fragmented tropical tree populations. In: Tropical Forest Remnants: Ecology, Management and Conservation of Fragmented Communities (eds. W.F. Laurance, R. Bierregaard \& C. Moritz). University of Chicago Press, Chicago, USA: 304-320.

NEILSON, R.P. (1993). Vegetation redistribution: a possible biosphere source of $\mathrm{CO}_{2}$ during climatic change. Water, Air and Soil Pollution 70: 659-673.

NEILSON, R.P. \& MARKS, D. (1994). A global perspective of regional vegetation and hydrologic sensitivities from climatic change. Journal of Vegetation Science 5 : 715-730.

Nelson, B.W., Ferreira, C.A., Da Silva, M.F. \& KaWasaKi, M.L. (1990). Refugia, endemism centers and collecting density in Brazilian Amazonia. Nature 345: 714-716.

NePstad, D.C., De Carvalho, C. R., Davidson, E.A., JiPP, P.H., LefebVRe, P., Negreiros, G.H., Trumbore, S. \& ViEIRA, S. (1994). The role of deep roots in the hydrological and carbon cycles of Amazonian forests and pastures. Nature 372: 666-669. 
Nepstad, D.C., Verissimo, A., Alencar, A., Nobre, C., Lima, E., Lefebvre, P., Schlesinger, P., Potter, C., Moutinho, P., Mendoza, E., Cochrane, M. \& Brooks, V. (1999). Large-scale impoverishment of Amazonian forests by logging and fire. Nature 398(6727): 505-508.

NeUbert, M.G. \& CASWELl, H. (2000). Demography and dispersal: calculation and sensitivity

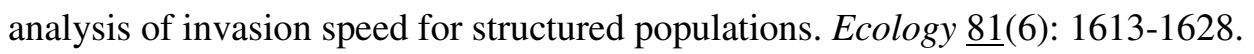

New, M.G., Hulme, M. \& Jones, P.D. (1999). Representing twentieth century space-time climate variability. Part 1: development of a 1961-90 mean monthly terrestrial climatology. Journal of Climate 12(3): 829-856.

NiCHOLLS, A.O. (1989). How to make biological surveys go further with Generalised Linear Models. Biological Conservation 50: 51-75.

NiCOTRA, A.B. (1998). Sex ratio variation and spatial distribution of Siparuna grandiflora, a tropical dioecious shrub. Oecologia 115: 102-113.

NiCOTRA, A.B. (1999). Sexually dimorphic growth in the dioecious tropical shrub, Siparuna grandiflora. Functional Ecology 13(3): 322-331.

NIX, H.A. (1986). A biogeographic analysis of Australian elapid snakes. In: Atlas of Elapid Snakes of Australia (ed. R. Longmore). Australian Flora and Fauna Series 7. Australian Government Publishing Service, Canberra, Australia. Cited in Busby (1991).

NOAA (1997). NCEP. [online]. NOAA-CIRES Climate Diagnostic Center, USA. Available from: http://www.cdc.noaa.gov.

Norby, R.J., EdWARdS, N.T., Riggs, J.S., ABNER, C.H., WUllschleger, S.D. \& GUNDERSON, C.A. (1997). Temperature-controlled open-top chambers for global change research. Global Change Biology 3: 259-267.

NORUŠIS, M.J. (1990). Logistic regression analysis: Procedure LOGISTIC REGRESSION. In: SPSS Advanced Statistics User's Guide (M.J. Norušis). SPSS Inc., Chicago, Illinois, USA: 4469.

NYBG. (2000). Fungal and Plant Diversity of Central French Guiana. [online]. New York Botanical Garden, New York, USA. http://www.nybg.org/bsci/french_guiana/. [accessed 07 March 2001].

NYBG. (2001). Arecaceae specimens in the Flora of Acre database of the New York Botanical Garden. [online]. New York Botanical Garden, New York, USA. Available from: http://www.nybg.org/bsci/acre/arecaceae.html. [accessed 17 January 2002]

O'BRIEN, K.L. (1996). Tropical deforestation and climate change. Progress in Physical Geography 20(3):311-335.

Oberhuber, J.M. (1993). Report No. 7. The OPYC Ocean General Circulation Model. The Description of a Coupled Snow, Sea-Ice, Mixed Layer and Isopycnal Ocean Model. Deutsches Klimarechenzentrum (DKRZ), Hamburg, Germany. 
OFN (ORgANiSATION FOR FlORA NEOTROPICA) (1999). Schedule of Flora Neotropica Monograph (Phanerogamic Plants). [online]. New York Botanical Garden, New York, USA. Available from: http://www.nybg.org/bsci/ofn/. [accessed 10 March 2000].

OLIVEIRA, P.S. (1997). The ecological function of extrafloral nectaries: Herbivore deterrence by visiting ants and reproductive output in Caryocar brasiliense (Caryocaraceae). Functional Ecology 11(3): 323-330.

Olmsted, I. \& Alvarez-BuYlla, E.R. (1995). Sustainable harvesting of tropical trees: demography and matrix models of two palm species in Mexico. Ecological Applications $\underline{5}(2)$ : 484-500.

OLSON, C.E. (1992). World Ecosystems (WE1.4). Digital raster data on a 10-minute Geographic 1080x2160 grid. In: Global Ecosystems Database, Version 1.0: Disc A. National Geophysical Data Center, USA.

Ortiz, E.G., Forsyth, A. \& Rubio, F. (1999). Brazil Nut Home Page. [online] Amazon Conservation Team. Available from: http://www.amazonconservation.organism/index htm. [accessed 21 October 1999].

Ostendorf, B., HilberT, D.W. \& Hopkins, M.S. (2001). The effect of global change on tropical vegetation pattern. Ecological Modelling 145: 211-224.

OTS (2000). Palo Verde. List of Plants. [online]. Organization for Tropical Studies, Costa Rica Available from: http://www.ots.ac.cr/en/paloverde/species/plants.shtml. [accessed 20/12/00].

PARry, M. (1990). Climate Change and World Agriculture. Earthscan Publications, London, UK: 157 pp.

PASSIOURA, J.B. (1996). Simulation models: snake oil, education or engineering? Agronomy Journal 88: 690-694.

Pennington, T.D. (1990). Sapotaceae. Flora Neotropica Monograph 52. Organisation for Flora Neotropica/New York Botanical Garden, New York, USA.

Pennington, T.D. (1997a). Distribution data for selected Sapotaceae and species of the Leguminosae genus Inga. Unpublished. Royal Botanic Gardens, Kew, London, UK

Pennington, T.D. (1997b). The genus Inga. Botany. Royal Botanic Gardens, Kew, London, UK: 844 pp.

Perez, R., Condit, R., Aguilar, S., Hernandez, A. \& Villareal, A. (1996). Inventory of vegetation on Coiba Island, Panama: composition and floristics. Revista de Biologia Tropical $\underline{44}(1): 31-40$.

PETERS, R.L. \& DARLING, J.D. (1985). The greenhouse effect and nature reserves. BioScience 35: 707-717.

Peterson, A.T. \& Vieglais, D.A. (2001). Predicting species invasions using ecological niche modeling: new approaches from bioinformatics attack a pressing problem. BioScience $\underline{51}$ (5): 363-371. 
Petit, J.R., Jouzel, J., RAYnAud, D., BARKov, N.I., BARnOla, J.M., BASIle, I., Bender, M., Chappellaz, J., Davis, M., Delaygue, G., Delmotte, M., Kotlyakov, V.M., Legrand, M., Lipenkov, V.Y., Lorius, C., Pepin, L., Ritz, C., SaltzMan, E. \& Stievenard, M. (1999). Climate and atmospheric history of the past 420,000 years from the Vostok ice core, Antarctica. Nature 399(6375): 429-436.

PHILLIPS, O.L. (1997). The changing ecology of tropical forests. Biodiversity and Conservation $\underline{6}(2): 291-311$.

Phillips, O.L., Malhi, Y., Higuchi, N., Laurance, W.F., Nunez, P.V., Vasquez, R.M., Laurance, S.G., Ferreira, L.V., Stern, M., Brown, S. \& Grace, J. (1998). Changes in the carbon balance of tropical forests: Evidence from long-term plots. Science 282(5388): 439442 .

PigotT, C.D. \& HunTley, B. (1981). Factors controlling the distribution of Tilia cordata at the northern limit of its geographical range. III. Nature and cause of seed sterility. New Phytologist $\underline{87}$ : 817-839.

Pitman, N.C.A., Terborgh, J.W., Silman, M.R., Núñez V., P., Neill, D.A., Cerón, C.E., Palacios, W.A. \& Aulestia, M. (2001). Dominance and distribution of tree species in upper Amazonian terra firme forests. Ecology 82(8): 2101-2117.

Pollard, D. \& SCHUlz, M. (1994). A model for the potential locations of Triassic evaporite basins driven by palaeoclimatic GCM simulations. Global and Planetary Change 9: 233-249.

POORTER, H. (1998). Do slow-growing species and nutrient-stressed plants respond relatively strongly to elevated $\mathrm{CO}_{2}$ ? Global Change Biology 4(6): 693-697.

Pounds, J.A., Fogden, M.P.L. \& CAMPBELL, J.H. (1999). Biological response to climate change on a tropical mountain. Nature 398(6728): 611-615.

PRANCE, G (1987). Biogeography of Neotropical plants. In: Biogeography and quaternary history in tropical America (eds. G.T. Prance \& T.C. Whitmore). Clarendon Press, Oxford, UK: 46-65.

PRANCE, G. \& PlanA, V. (1997). Distribution data for Caryocaraceae, Chrysobalanaceae, Dichapetalaceae, Lecythidaceae \& Proteaceae. Unpublished. Royal Botanic Gardens, Kew, London, UK.

PRIESTlEY, C.B. \& TAYloR, R.J. (1972). On the assessment of surface heat flux and evaporation using large scale parameters. Monthly Weather Review 100: 81-92.

PrentiCE, I.C., SyKeS, M.T. \& CRAMER, W. (1991) The possible dynamic response of northern forests to global warming. Global Ecology and Biogeography Letters 1: 129-135.

Prentice, I.C., Cramer, W., Harrison, S.P., LeEmans, R., Monserud, R.A. \& SolOmOn, A.M. (1992). A global biome model based on plant physiology and dominance, soil properties and climate. Journal of Biogeography 19: 117-134. 
PRESTON, F.W. (1948). The commonness and rarity of species distribution. Ecology 29(3): 254283.

Price, G.D., VAldes, P.J. \& Sellwood, B.W. (1997). Quantitative palaeoclimate GCM validation: Late Jurassic and mid-Cretaceous case studies. Journal of the Geological Society 154: 769-772.

PRIESTLEY, C.H.B. \& TAYLOR, R.J. (1972). On the assessment of surface heat flux and evaporation using large-scale parameters. Monthly Weather Review 100: 81-92.

PRINCE, S.D. (1986). Data analysis. In: Methods in Plant Ecology (2 $2^{\text {nd }}$ edition), (eds. P.D. Moore \& S.B. Chapman.). Blackwell Scientific Publications, Oxford, UK: 345-375.

RAHBEK, C. (1995). The elevational gradient of species richness: a uniform pattern? Ecography 18: 200-205.

RAMíREZ, N. (1995). Producción y costo de frutos y semillas entre modos de polinización en 232 especies de plantas tropicales. Revista de biologia tropical 43 (1-3): 151-159.

RAPID Biological InVEnTORY TEAM. (2000). Rapid Biological Inventories Report 1, March 2000. Appendix 1. Species of vascular plants recorded for the proposed Tahuamanu Ecological Reserve, Pando, Bolivia, from 17 to 24 October, 1999. Field Museum, Chicago, USA. Available from: http://www.fieldmuseum.org/research_collections/ecp/ecp_sites/ rapidinventories/pando/pdfs/pandoapp.PDF. [accessed 13 June 2001]

RAPOPORT, E.H. (1982). Areography, geographical strategies of species. Pergamon Press, Oxford, UK.

RAUnKiaer, C. (1934). The Life Forms of Plants and Statistical Plant Geography. Clarendon Press, Oxford, UK.

RAUP, D.M. (1966). Geometric analysis of shell coiling: general problems. Paleontology $\underline{40}$ : 1178-1190.

RBG KEW. (1998). Herbarium records, Royal Botanic Gardens herbarium, Kew, London, UK. [accessed December 1998].

Rees, P.M., Ziegler, A.M., Gibbs, M.T., KutZBach, J.E., Behling, P.J. \& Rowley, D.B. (2002). Permian phytogeographic patterns and climate data/model comparisons. Journal of Geology 110(1): 1-31.

REICH, P.B. (1995). Phenology of tropical forests: patterns, causes and consequences. Canadian Journal of Botany 73: 164-174.

Richey, J.E., Wilhelm, S.R., McClain, M.E., Victoria, R.L., Melack, J.M. \& ARaúJo LIMA, C. (1997). Organic matter and nutrient dynamics in river corridors of the Amazon basin and their response to anthropogenic change. LBA/AMBIACE White Paper on Rivers and Land Use Change. [online]. Large Scale Biosphere-Atmosphere Experiment in Amazonia, INPE, Brazil. Available from: http://boto.ocean.washington.edu/lba/restricted/whitepaper.txt. Accessed 19 January 2002. 
Rogers, G.K.. (1984). Gleasonia, Henriquezia and Platycarpum (Rubiaceae). Flora Neotropica Monograph 39. Organisation for Flora Neotropica/New York Botanical Garden, New York, USA.

Roggy, J.C., Prevost, M.F., Gourbiere, F., Casabianca, H., Garbaye, J. \& Domenach, A.M.. (1999). Leaf natural N-15 abundance and total N concentration as potential indicators of plant $\mathrm{N}$ nutrition in legumes and pioneer species in a rain forest of French Guiana. Oecologia 120(2): 171-182

ROHWER, J.G. (1993) Lauraceae: Nectandra. Flora Neotropica Monograph 60. Organisation for Flora Neotropica/New York Botanical Garden, New York, USA.

Rose, S. (1996). A Classification System for Mapping Tropical Rain Forest Biodiversity. PhD thesis, School of Geography, University of Leeds, Leeds, UK.

RoubIK, D.W. (2000). Pollination system stability in tropical America. Conservation Biology 14(5): 1235-1236.

Row, L.W. III \& HASTINGS, D. (1989). National Geophysical Data Center TerrainBase Global DTM Version 1.0: Integrated Global Elevation and Bathymetry. National Geophysical Data Center, Boulder, Colorado, USA.

Royer, J.-F., Chauvin, B., Timbal, P., Araspin, P. \& Grimal, D. (1998). A GCM study of the impact of greenhouse gas increase on the frequency of occurrence of tropical cyclones. Climatic Change 38: 307-343.

RUCKER, R. \& WALKer, B. (1997). Cellular Automata Laboratory. [online]. San José State University Department of Mathematics and Computer Science, San José, CA, USA. Available from: http://www.mathcs.sjsu.edu/faculty/rucker/celdoc/cellab.html.

RUIZ, J.E.A. \& DA CRUZ ALENCAR, J. (1999). Interpretação fenológica de cinco espécies de Chrysobalanaceae no Reserva Florestal Adolpho Ducke, Manaus, Amazonas, Brasil. Acta Amazonica 29(2): 223-242

Ruokolainen, K., LinnA, A. \& TuOMisto, H. (1997). Use of Melastomataceae and pteridophytes for revealing phytogeographical patterns in Amazonian rain forests. Journal of Tropical Ecology 13: 243-256.

RUSSO, J.M. \& ZACK, J.W. (1997). Downscaling GCM output with a mesoscale model. Journal of Environmental Management 49(1): 19-29.

Rutherford, M.C., O'Callaghan, M., Hurford, J.L., Powrie, L.W., Schulze, R.E., KunZ, R.P., DAVIS, G.W., HOFFMAN, M.T. \& MACK, F. (1995). Realized niche spaces and functional types - a framework for prediction of compositional change. Journal of Biogeography 22(2-3): 523-531.

Rutherford, M.C., O'Callaghan, M., Powrie, L.W., Hurford, J.L. \& Schulze, R.E. (1996). Predicting survival in new environments through analytical GIS application. Environmental Software 11(1-3):113-121. 
RYLANDS, A.B. (1990). Priority areas for conservation in the Amazon. Trends in Ecology and Evolution 5: 240-241.

SAgÁstegui A. \& Dillon, M.O. (1991). Inventario preliminar de la flora del Bosque Monteseco. Arnaldoa 1(1): 35-52.

SAlAti, E. \& NoBre, C.A. (1991). Possible climatic effects of tropical deforestation. Climatic Change 19: 177-196.

Saldarriaga, J.G., West, D.C., TharP, M.L. \& UhL, C. (1988). Long term chronosequence of forest succession in the upper Rio Negro of Colombia and Venezuela. Journal of Ecology 76: 938-958.

Sato, N., Sellers, P.J., Randall, D.A., Schneider, E.K., Shukla, J., Kinter III, J.L., Hou, Y.-T. \& AlBERTAZZI, E. (1989). Effects of implementing the Simple Biosphere Model in a general circulation model. Journal of the Atmospheric Sciences 46 (18): 2757-2782.

SCHOLES, R.J. \& VAN BREEMEN, N. (1997). The effect of global change on tropical ecosystems. Geoderma 79: 9-24.

SEBER, G.A.F. (1977). Linear Regression Analysis. Wiley, New York, USA.

SELLERS, P.J. (1992). Biophysical models of land surface processes. In: Climate System Modeling (ed. K.E. Trenberth). Cambridge University Press, Cambridge, UK: 451-.

Sellers, P.J., Bounoua, L., Collatz, J., Randall, D.A., Dazlich, D.A., Los, S., Berry, J.A., Fung, I., TuCKer, C.J., Field, C.B. \& JENSEN, J.R. (1996a). Comparison of radiative and physiological effects of doubled atmospheric $\mathrm{CO}_{2}$ on climate. Science 271: 1402-1406.

Sellers, P.J., Los, S.O., Tucker, C.J., Justice, C.O., Dazlich, D.A., Collatz, G.J. \& RANDALL, D.A. (1996b). A revised land surface parameterization (SiB2) for atmospheric GCMs. Part II: the generation of global fields of terrestrial biophysical parameters from satellite data. Journal of Climate 9 (4): 706-737.

Sellers, P.J., Meeson, B.W., Closs, J., Collatz, J., Corprew, F., Dazlich, D., Hall, F.G., Kerr, Y., Koster, R., Los, S., Mitchell, K., McManus, J., MYers, D., Sun, K. -J. $\&$ TRY, P. (1996c). The ISLSCP Initiative I global datasets: surface boundary conditions and atmospheric forcings for land-atmosphere studies. Bulletin of the American Meteorological Society 77(9): 1987-2001.

SEllers, P.J., MinTZ, Y., Sud, Y.C. \& DALCHER, A. (1986). A simple biosphere model for use within general circulation models. Journal of the Atmospheric Sciences 43: 505-531.

Sellers, P.J., Randall, D.A., Collatz, G.J., Berry, J.A., Field, C.B., DaZlich, D.A.., ZhANG, C., Collelo, G.D. \& BounOUA, L. (1996d). A revised land surface parameterization (SiB2) for atmospheric GCMs. Part I: model formulation. Journal of Climate 9(4): 676-705.

SEllers, P.J., ShUtTleworth, W.J., Dorman, J.L. \& RoberTS, J.M. (1989). Calibrating the Simple Biosphere Model for Amazonian tropical forest using field and remote sensing data. Part I: average calibration with field data. Journal of Applied Meteorology 28(8): 727-759. 
SHAFFER, M.L. (1981). Minimum population sizes for species conservation. BioScience $\underline{31}$ : 131-134.

SHAO, G. \& HALPIN, P.N. (1995). Climatic controls of eastern North American coastal tree and shrub distributions. Journal of Biogeography 33: 1083-1089.

SHEIL, D. \& MAY, R.M. (1996). Mortality and recruitment rate evaluations in heterogeneous tropical forests. Journal of Ecology 84(1): 91-100.

Shindell, D.T., Miller, R.L., SchmidT, G.A. \& PANDOlFO, L. (1999). Simulation of recent northern winter climate trends by greenhouse-gas forcing. Nature 399(6735): 452-455.

SHUGART, H.H. \& URBAN, D.L. (1989). Factors affecting the relative abundances of forest trees. In Toward a More Exact Ecology (eds. P.J. Grubb \& J.B. Whittaker). Blackwell Scientific Publications, Oxford, UK: 249-273.

ShuKla, J., Nobre, C.A. \& Sellers, P.J. (1990). Amazon deforestation and climate change. Science 247: 1322-1325.

SHUTTLEWORTH, W.J. (1991). Insight from large scale observational studies of land atmosphere interactions. Surveys in Geophysics 12(1-3): 3-30. Cited in Culf et al. (1998).

SiEgEl, S. \& CASTELlan, N.J. (1988). Nonparametric statistics for the Behavioral Sciences. Second Edition. McGraw-Hill International Editions Statistics Series, Singapore.

Silva, J.N.M., DE CARvalho, J.O.P., LoPes, J. do C.A., DE Almeida, B.F., Costa, D.H.M., DE Oliveira, L.C., VANClay, J.K. \& SkovsgaARD, J.P. (1995). Growth and yield of a tropical rain forest in the Brazilian Amazon 13 years after logging. Forest Ecology and Management 71: 267-274.

Silver, W.L., Brown, S. \& LUGO, A.E. (1996). Effects of changes in biodiversity on ecosystem function in tropical forests. Conservation Biology 10(1): 17-24.

Simard, Y., Legendre, P., Lavoie, G. \& Marcotte, D. (1992). Mapping, estimating biomass, and optimizing sampling programs for spatially autocorrelated data: Case study of the Northern Shrimp (Pandalus borealis). Canadian Journal of Fisheries and Aquatic Sciences 49: 32-45.

SMITH, P.A. (1994). Autocorrelation in logistic regression modelling of species distributions. Global Ecology and Biogeography Letters 4: 47-61.

SOLÉ, R.V. \& MANRUBIA, S.C. (1995). Are rainforests self-organized in a critical state? Journal of Theoretical Biology 173(1): 31-40.

SOKAL, R.R. (1961). Distance as a measure of taxonomic similarity. Systematic Zoology 10: 7079.

SouTH, D.B. (1995). Relative growth rates: a critique. South African Forestry Journal 173: 4348.

SPARKS, T.H. \& CAREY, P.D. (1995). The responses of species to climate over two centuries an analysis of the Marsham phenological record. Journal of Ecology 83(2): 311-329. 
SPICER, R.A. (1993). Paleoecology, past climate systems, and C3/C4 photosynthesis. Chemosphere 27(6): 947-978.

SPSS (1998). SPSS for Windows 9.0.0 (18 Dec 1998) Standard Version Help. SPSS Inc., Chicago, Illinois, USA

StAdDON, P.L. \& FitTER, A.H. (1998). Does elevated atmospheric carbon dioxide affect arbuscular mycorrhizas? Trends in Ecology and Evolution 13(11): 457-461.

STANGE, G. (1997). Effects of changes in atmospheric carbon dioxide on the location of hosts by the moth, Cactoblastis cactorum. Oecologia 110(4): 539-545.

STEIN, A. \& CORSTEN, I.C.A. (1991). Universal kriging and cokriging as a regression procedure. Biometrics 47: 575-587.

STEPHENSON, N.L. (1998). Actual evapotranspiration and deficit: biologically meaningful correlates of vegetation distribution across spatial scales. Journal of Biogeography 25(5): 855870.

Steyermark, J.A., Berry, P.E., Holst, B. K. \& YAtSkievyCh, K. (eds.) (1997). Flora of the Venezuelan Guayana, Volume 3, Araliaceae-Cactaceae [online version]. Missouri Botanical Garden Press, St. Louis, USA. Available from: http://www.mobot.org/MOBOT/research/venguayana/ [accessed 10 January 2002]. 774 pp.

StILl, C.J., Foster, P.N. \& SCHNEIDER, S.H. (1999). Simulating the effects of climate change on tropical montane cloud forests. Nature 398(6728): 608-610.

StOcKWELl, D.R.B. \& PETERSON, A.T. (2002). Effects of sample size on accuracy of species distribution models. Ecological Modelling 148(1): 1-13.

STOMS, D.M. (1992). Effects of habitat map generalisation on biodiversity assessment. Photogrammetric Engineering and Remote Sensing 58(11): 1587-1591.

StORTI, E.F. (1993). Biologia floral de Mauritia flexuosa Lin. Fil., na região de Manaus, AM, Brasil. Acta Amazonica 23(4): 371-381.

STOTT, P. (1998). Biogeography and ecology in crisis: the urgent need for a new metalanguage. Journal of Biogeography 25: 1-2.

Stute, M., Forster, M., Frischkorn, H., Serejo, A., Clark, J.F., Schlosser, P., BroecKer, W.S. \& BonANI, G. (1995). Cooling of tropical Brazil $\left(5^{\circ} \mathrm{C}\right)$ during the last glacial maximum. Science 269(5222): 379-383.

Sud, Y.C., WAlKer, G.K., KiM, J.-H., Liston, G.E., Sellers, P.J. \& LAU, W.K.-M. (1996). Biogeophysical consequences of a tropical deforestation scenario: a GCM simulation study. Journal of Climate 9(12): 3225-3247.

Swaine, M.D., Lieberman, D. \& PUTZ, F.E. (1987). The dynamics of tree populations in tropical forest: a review. Journal of Tropical Ecology $\underline{3}$ : 359-366. 
Sykes, M.T., Prentice, I.C. \& Cramer, W. (1996). A bioclimatic model for the potential distributions of north European tree species under present and future climates. Journal of Biogeography 23: 203-233.

Tellez Valdes, O. \& Cabrera Cano, E.F. (1987). Listados floristicos de Mexico VI. Florula de la Isla de Cozumel, Q. R. [online]. Instituto de Biologia, Universidad Nacional Autonoma De Mexico, Mexico. Available from: http://biblio68.ibiologia.unam.mx/FullText/lfl6.html. [accessed 17 January 2002].

TER BRAAK (1986). Canonical correspondence analysis - a new eigenvector technique for multivariate gradient analysis. Ecology 67(5): 1167-1179.

TER BRAAK, C.J.F. \& LOOMAN, C.W.N. (1987). Regression. In: Data analysis in community and landscape ecology (eds. R.H.G. Jongman, C.J.F. Ter Braak \& O.F.R. van Tongeren): 2977 .

TER BRAaK, C.J.F. \& PrentiCe, I.C. (1988). A theory of gradient analysis. Advances in Ecological Research 18: 271-319.

ter Steege, H., Jansen-Jacobs, M.J. \& Datadin, V.K. (2000). Can botanical collections assist in a National Protected Area Strategy in Guyana? Biodiversity and Conservation 9(2): 215-240.

TETT, S., JOHNS, T.C. \& MitchelL, J.F.B. (1997). Global and regional variability in a coupled AOGCM. Climate Dynamics 13: 303-323. Cited by Cramer et al. ('2001).

THOMAs, W.W. DE CARVAlHo, A.M. \& AMORIM, A.M. (co-ordinators/collaborator). (2000a). Preliminary checklist of the plants of the Mata de Esperanca. New York Botanical Garden, New York, USA. Available from: http://www.nybg.org/bsci/res/bahia/ME-chkl.html. [accessed 16 January 2002].

Thomas, W.W. DE CARVAlho, A.M. \& AMORIM, A.M. (co-ordinators/collaborator). (2000b). Serra Grande Forest Checklist [online]. New York Botanic Gardens, New York, USA. Available from: http://www.nybg.org/bsci/res/bahia/SG-chkl.html. [accessed 16 January 2002].

Thomas, W.W., de Carvalho, A.M., Amorim, A. \& Garrison, J. (2000c). Preliminary checklist of the flora of the Una Biological Reserve [online]. New York Botanic Gardens, New York, USA. Available from: http://www.nybg.org/bsci/res/una.html. [accessed 16 January 2002].

THOMSEN, K. \& BRIMER, K. (1997). Cyanogenic constituents in woody plants in natural lowland rain forest in Costa Rica. Botanical Journal of the Linnean Society 124(3): 273-294.

Thornthwaite, C.W., Mather, J.R. \& CARTer, D.B. (1957). Instructions and tables for computing potential evapotranspiration and the water balance. Publications in Climatology 10 : 183-311. Cited in Stephenson (1998). 
Timmermann, A., Oberhuber, J., Bacher, A., Esch, M., LAtif, M. \& Roeckner, E. (1999). Increased El Niño frequency in a climate model forced by future greenhouse warming. Nature 398(6729): 694-697.

Trenberth, K.E. (1992). Climate System Modeling. Cambridge University Press, Cambridge, UK.

TulJAPURKAR, S. \& CASWELL, H. (1997). Structured-population models in marine, terrestrial, and freshwater systems. Chapman \& Hall, London, UK: 643 pp.

Tuomisto, H. \& Poulsen, A.D. (1996). Influence of edaphic specialization on pteridophyte distribution in neotropical rain forests. Journal of Biogeography 23:283-293.

TuOmisto, H. \& RuOKOLAinen, K. (1997). The role of ecological knowledge in explaining biogeography and biodiversity in Amazonia. Biodiversity and Conservation 6: 347-357.

UHL, C. \& BUSCHBACHER, R. (1985). A disturbing synergism between cattle-ranch burning practices and selective tree harvesting in the eastern Amazon. Biotropica 17(4): 165-168.

UHL, C. \& KAUfMAN, J.B. (1990). Deforestation, fire susceptibility and potential tree responses to fire in the eastern Amazon. Ecology 71(2): 437-449.

USGS, UNL \& JRC (1998). Global Land Cover Characterization. [online]. USGS, USA. Available from: http://edcwww.cr.usgs.gov/landdaac/landdaac.html. [accessed 15 March 1999].

van JaArsveld, A.S., Gaston, K.J., Chown, S.L. \& Freitag, S. (1998). Throwing biodiversity out with the binary data? South African Journal of Science 94(5): 210-214.

VANCLAY, J.K. \& SKOVSGAARD, J.P. (1997). Evaluating forest growth models. Ecological Applications 98(1): 1-12.

VANE-Wright, R.I., HumPhries, C.J. \& Williams, P.H. (1991). What to protect? Systematics and the agony of choice. Biological Conservation 55: 235-254.

Van Roosmalen, M.G.M. \& DA CRuz Gomes Garcia, O.M. (2000). Fruits of the Amazon Forest. Part II: Sapotaceae. Acta Amazonica 30(2): 187-290

Vester, H.F.M. \& ClEeF, A.M. (1998). Tree architecture and secondary tropical rain forest development - A case study in Araracuara, Colombian Amazonia. Flora 193(1): 75-97.

Victoria, R.L., Martinelli, L.A., Moraes, J.M., Ballester, M.V., Krusche, A.V., Pellegrino, G., AlmeidA, R.M.B. \& RiCHEY, J.E. (1998). Surface air temperature variations in the Amazon region and its borders during this century. Journal of Climate 11: 1105-1110.

VINER, D. \& Hulme, M. (1997). The climate impacts LINK project. Climatic Research Unit, Norwich, UK: 24.

W3 TROPICOS. (1997-2001). W3 Tropicos database [online]. Missouri Botanical Garden, St. Louis, USA. Available from: http://mobot.mobot.org/W3T/Search/vast.html. [accessed 16 January 2002].

WALKER, B.H. (1992). Biodiversity and ecological redundancy. Conservation Biology 6: 18-23. 
WALKER, P.A. \& COCKS, J.D. (1991). HABITAT - a procedure for modelling a disjoint environmental envelope for a plant or animal species. Global Ecology and Biogeography Letters 1: 108-118.

WALSH, R.P.D. (1996). Drought frequency changes in Sabah and adjacent parts of northern Borneo since the late nineteenth century and possible implications for tropical rain forest dynamics. Journal of Tropical Ecology 12: 385-407.

WALSH, R.P.D. \& Newbery, D.M. (1999). The ecoclimatology of Danum, Sabah, in the context of the world's rainforest regions, with particular reference to dry periods and their impact. Philosophical Transactions of the Royal Society of London. Series B Biological Sciences 354: 1869-1883.

WALSH, S.J., LIGHTFOOT, D.R. \& BUTLER, D.R. (1987). Recognition and assessment of error in geographic information systems. Photogrammetric Engineering and Remote Sensing 53(10): 1423-1430.

WALTERS, S.M. (1986). The name of the rose: a review of ideas on the European bias in angiosperm classification. New Phytologist 104: 527-546.

WARD, R.C. \& Robinson, M. (2000). Principles of Hydrology. McGraw Hill, London.

WARRILOW, D.A. \& BUCKLEY, E. (1989). The impact of land surface processes on the moisture budget of a climate model. Annales Geophysicae 7(5): 439-450.

Watson, L. \& Dallwitz, M. J. (1992). The Families of Flowering Plants: Descriptions, Illustrations, Identification, and Information Retrieval. Version: 19th August 1999. [online]. Available from: http://biodiversity.uno.edu/delta/angio/index.htm [accessed 1 March 2000].

WCMC (1992). Protected areas database. [online]. WCMC, Cambridge, UK. Available from: http://www.wcmc.org.uk/protected_areas/data/.

WEISSENHOFER, A. (2000). Summary of the thesis "Structure and ecology of a tropical lowland rainforest in the Pacific region of Costa Rica (Esquinas Forest)". Available from: http://www.regenwald.at/RGS/ESSumWeiss.html. [Accessed 29-03-01]. Regenwald der Osterreicher, Austria.

Welden, C.W., Hewett, S.W., Hubbell, S.P. \& Foster, R.B. (1991). Sapling survival, growth, and recruitment: relationship to canopy height in a neotropical forest. Ecology $72(1)$ : $35-50$.

White, A., CANNELl, M.G.R. \& FrIEND, A.D. (1999). Climate change impacts on ecosystems and the terrestrial carbon sink: a new assessment. Global Environmental Change 9: S21-S30.

White, D., Minotti, P.G., Barczak, M.J., Sifneos, J.C., Freemark, K.E., Santelmann, M.V., SteinitZ, C.F., Kiester, A.R. \& PREStOn, E.M. (1997) Assessing risks to biodiversity from future landscape change. Ecological Applications 7(3): 349-360.

WhitMORE, T.C. (1990). An introduction to tropical rain forests. Oxford University Press, Oxford, UK. 
WHITTAKER, R.H. (1965). Dominance and diversity in land plant communities. Science 147: 250-260.

WhITTAKER, R.H. (1967). Gradient analysis of vegetation. Biological Reviews of the Cambridge Philosophical Society 42: 207-269.

WILBUR, R.L. \& collaborators. (1990). Lista Preliminar de las Plantas Vasculares de La Selva. [online]. Organization for Tropical Studies, Costa Rica. http://www.ots.ac.cr/ese/laselva/ ls_plant.htm [accessed 16 January 1999].

WiLBY, R.L. \& Wigley, T.M.L. (1997). Downscaling general circulation model output: a review of methods and limitation. Progress in Physical Geography 21(4): 530-548.

WILKINSON, D.M. (1998). Mycorrhizal fungi and Quaternary plant migrations. Global Ecology and Biogeography Letters: 7(2): 137-140.

WiLLIAMS, D.W. \& LIEBHOLD, A.M. (1995). Herbivorous insects and global change - potential changes in the spatial-distribution of forest defoliator outbreaks. Journal of Biogeography 22(4-5): 665-671.

Williams, L.J.,Shaw, D. \& MENDElsohn, R. (1998). Evaluating GCM output with impact models. Climatic Change 39: 111-133.

Williams, K.D., Senior, C.A. \& Mitchell, J.F.B. (2001). Transient climate change in the Hadley Centre models: The role of physical processes. Journal of Climate 14: 2659-2674. Cited in Collins 2001.

WILLIAMS, P.H. (1998a). Key sites for conservation: area-selection methods for biodiversity. In Conservation in a changing world: integrating processes into priorities for action. Symposia of the Zoological Society of London no. 72 (eds. G.A. Mace, A. Balmsford \& J.R. Ginsberg). Cambridge University Press, Cambridge, UK.

WilliaMS, P.H. (1998b). WORLDMAP version 4.19.10/11. Software and help document. Privately distributed, Natural History Museum, London, UK.

WiLLIAMS, P.H. \& GASTON, K.J. (1994). Measuring more of biodiversity: can higher-taxon richness predict wholesale species richness? Biological Conservation 67: 211-217.

Williams, P.H., GASTON, K.J. \& HUMPHRIES, C.J. (1994). Do conservationists and molecular biologists value differences between organisms in the same way? Biodiversity Letters $2: 67$ 78.

Williams, P.H., Prance, G.T., Humphries, C.J. \& Edwards, K.S. (1996). Promise and problems in applying quantitative complementary areas methods for representing the diversity of some Neotropical plants (families Dichapetalaceae, Lecythidaceae, Caryocaraceae, Chrysobalanaceae and Proteaceae). Biological Journal of the Linnean Society 58: 125-157. 
WiLliams-LinerA, G. (1997). Phenology of deciduous and broadleaved-evergreen tree species in a Mexican tropical lower montane forest. Global Ecology and Biogeography Letters $\underline{6}(2)$ : $115-127$.

Williamson, G.B., Laurance, W.F., Oliveira, A.A., Delamonica, P., Gascon, C., LOVEJOY, T.E. \& POHL, L. (2000). Amazonian tree mortality during the 1997 El Niño drought. Conservation Biology 14(5): 1538-1542.

Wills, C., Condit, R., Hubbell, S.P. \& Foster, R.B. (1998). Strong density- and diversityrelated effects help to maintain tree species diversity in a neotropical forest. Proceedings of the National Academy of Sciences of the United States of America 94(4): 1252-1257.

WILSON, M.F. \& HENDERSON-SELLERS, A. (1985). A global archive of land cover and soils data sets for use in general circulation models. International Journal of Climatology $\underline{5}$ : 119143.

WiSER, S.K., PEET, R.K. \& WHITE, P.S. (1998). Prediction of rare-plant occurrence: A southern Appalachian example. Ecological Applications $\underline{8}(4)$ : 909-920.

WolDA, H. (1981). Similarity indices, sample size and diversity. Oecologia 50: 296-302.

Woodward, F.I. (1987). Climate and Plant Distribution. Cambridge University Press, Cambridge, UK: 174 pp.

WoOdWARD, F.I. \& BeERling, D.J. (1997). The dynamics of vegetation change: health warnings from equilibrium 'dodo' models. Global Ecology \& Biogeography Letters $\underline{6}$ : 413418.

WOODWARD, F.I. \& LEE S.E. (1995). Global scale forest function and distribution. Forestry 68(4): 317-325.

WoRBES, M. (1999). Annual growth rings, rainfall-dependent growth and long-term growth patterns of tropical trees from the Caparo Forest Reserve in Venezuela. Journal of Ecology 87: 391-403.

YeE, T.W. \& MitChELL, N.D. (1991). Generalized additive models in plant ecology. Journal of Vegetation Science 2: 587-602.

Zhao, M., Pitman, A.J. \& Chase, T. (2001). The impact of land cover change on the atmospheric circulation. 17: (5-6): 467-477.

Zimmerman, J.K., Everham, E.M. III, Waide, R.B., Lodge, D.J., TaYlor, C.M. \& BROKAW, N.V.L. (1999). Responses of tree species to hurricane winds in subtropical wet forest in Puerto Rico: Implications for tropical tree life histories. Journal of Ecology 82(4): 911-922. 